\title{
SLUDGE BATCH 3 PHASE 1 VARIABILITY STUDY
}

\author{
D.K. Peeler \\ T.B. Edwards \\ C.C. Herman \\ T.H. Lorier \\ D.R. Best \\ I.A. Reamer \\ R.J. Workman
}

Westinghouse Savannah River Company

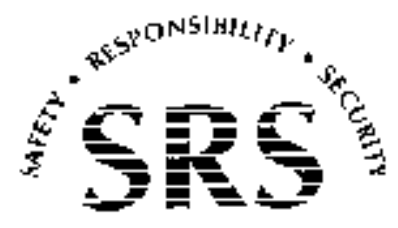


This document was prepared in conjunction with work accomplished under Contract No. DE-AC09-96SR18500 with the U. S. Department of Energy.

\section{DISCLAIMER}

This report was prepared as an account of work sponsored by an agency of the United States Government. Neither the United States Government nor any agency thereof, nor any of their employees, makes any warranty, express or implied, or assumes any legal liability or responsibility for the accuracy, completeness, or usefulness of any information, apparatus, product or process disclosed, or represents that its use would not infringe privately owned rights. Reference herein to any specific commercial product, process or service by trade name, trademark, manufacturer, or otherwise does not necessarily constitute or imply its endorsement, recommendation, or favoring by the United States Government or any agency thereof. The views and opinions of authors expressed herein do not necessarily state or reflect those of the United States Government or any agency thereof.

This report has been reproduced directly from the best available copy.

Available for sale to the public, in paper, from: U.S. Department of Commerce, National Technical Information Service, 5285 Port Royal Road, Springfield, VA 22161, phone: (800) 553-6847, fax: (703) 605-6900

email: orders@ntis.fedworld.gov

online ordering: http://www.ntis.gov/help/index.asp

Available electronically at http://www.osti.gov/bridge

Available for a processing fee to U.S. Department of Energy and its contractors, in paper, from: U.S. Department of Energy, Office of Scientific and Technical Information, P.O. Box 62, Oak Ridge, TN 37831-0062,

phone: (865)576-8401,

fax: (865)576-5728

email: $\underline{\text { reports@ adonis.osti.gov }}$ 


\title{
SLUDGE BATCH 3 (SB3) PHASE 1 VARIABILITY STUDY
}

\author{
D.K. Peeler \\ T.B. Edwards \\ C.C. Herman \\ T.H. Lorier \\ D.R. Best \\ I.A. Reamer \\ R.J. Workman
}

Westinghouse Savannah River Company

Savannah River Technology Center

Aiken, South Carolina 
Immobilization Technology Section

WSRC-TR-2002-00549

Savannah River Technology Center

Rev. 0

Westinghouse Savannah River Company

This page intentionally left blank. 


\section{Executive Summary}

The objective of this task was to assess the applicability of the current Product Composition Control System (PCCS) durability model $\left(\Delta \mathrm{G}_{\mathrm{P}}\right)$ to the anticipated compositional region of interest to Sludge Batch 3 (SB3). The assessment of applicability was accomplished via a compositional variation study in which the durability response of various glasses was experimentally determined and compared to model predictions. Typically, variability studies are compositionally focused on a specific frit coupled with a known sludge composition over a waste loading range of interest. In the present study, various candidate frits were used with different sludge compositional views representing possible washing scenarios. Operating windows were defined for various glass systems using Defense Waste Processing Facility (DWPF) measurement acceptability criteria that formed the basis for the targeted glass compositions.

The experimental results demonstrated applicability (or conservatism) of the $\Delta \mathrm{G}_{\mathrm{P}}$ model for each of the 42 SB3 test matrix glasses when fabricated under oxidizing conditions. The Product Consistency Test (PCT) response for all glasses (both quenched and centerline cansiter cooled) were also acceptable being at least an order of magnitude more durable than the $16.695 \mathrm{~g} / \mathrm{L}$ value for normalized boron release as reported for the Environmental Assessment (EA) glass.

In this phase of the variability study, only a limited assessment of the impact of reduction / oxidation (redox) on the PCT response was experimentally determined. Once again, the limited results indicated that the PCT responses from glasses that bound the acceptable redox range for DWPF were very acceptable with respect to the EA glass. In terms of predictability, the $\Delta \mathrm{G}_{\mathrm{P}}$ model was either predictable or conservative. However, model predictions suggest that the impact of redox on processability (i.e., the predicted $\Delta \mathrm{G}_{\mathrm{P}}$ value in relation to the acceptance criterion value) could have a detrimental effect on the projected operating windows. The projected negative impact would be most severe for glass systems whose upper waste loading is (or is nearly) PCT limited as redox transitions from the oxidized to the reduced state. 
Immobilization Technology Section

WSRC-TR-2002-00549

Savannah River Technology Center

Rev. 0

Westinghouse Savannah River Company

This page intentionally left blank. 


\section{Acronyms}

\begin{tabular}{|c|c|}
\hline AES & atomic emission spectroscopy \\
\hline $\mathrm{Am} / \mathrm{Cm}$ & americium/curium \\
\hline ANOVA & analysis of variance \\
\hline ARM & Approved Reference Material \\
\hline ASTM & American Society for Testing and Materials \\
\hline $\operatorname{ccc}$ & centerline canister cooled \\
\hline$\Delta \mathrm{G}_{\mathrm{p}}$ & $\begin{array}{l}\text { preliminary glass dissolution estimator based on free energy of hydration } \\
\text { (in } \mathrm{kcal} / \mathrm{mol} \text { ) }\end{array}$ \\
\hline DWPF & Defense Waste Processing Facility \\
\hline DWPF PE & Defense Waste Processing Facility Process Engineering \\
\hline EA & Environmental Assessment \\
\hline HLW & high-level waste \\
\hline HLW PE & High Level Waste - Process Engineering \\
\hline $\mathrm{ICP}$ & inductively coupled plasma \\
\hline $\mathrm{LM}$ & lithium-metaborate \\
\hline MAR & Measurement Acceptability Region \\
\hline MRF & dry feed melt rate furnace \\
\hline MST & monosodium titanate \\
\hline $\mathrm{Na}_{2} \mathrm{C}_{2} \mathrm{O}_{4}$ & sodium oxalate \\
\hline NL & normalized leachate \\
\hline PAR & Property Acceptability Region \\
\hline PCCS & Product Composition Control System \\
\hline PCT & Product Consistency Test \\
\hline PF & (Sodium) Peroxide Fusion \\
\hline
\end{tabular}


Immobilization Technology Section

WSRC-TR-2002-00549

Savannah River Technology Center

Rev. 0

Westinghouse Savannah River Company

redox reduction/oxidation

SB sludge batch

SME Slurry Mix Evaporator

SRAT Sludge Receipt and Adjustment Tank

SRS Savannah River Site

SRTC Savannah River Technology Center

SRTC-ML Savannah River Technology Center - Mobile Laboratory

$\mathrm{T}_{\mathrm{L}} \quad$ liquidus temperature

THERMO $^{\mathrm{TM}}$ Thermodynamic Hydration Energy Reaction Model

TTR technical task request

$\mathrm{u}_{\mathrm{STD}} \quad$ uranium standard glass

$\eta_{1150^{\circ} \mathrm{C}} \quad$ melt viscosity at $1150^{\circ} \mathrm{C}$

WCSystem Waste Characterization System

WL waste loading

WQR Waste Qualification Report

XRD X-ray diffraction 


\section{Contents}

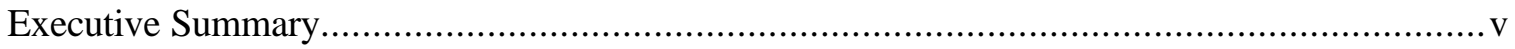

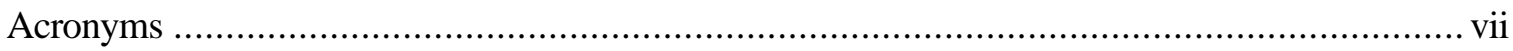

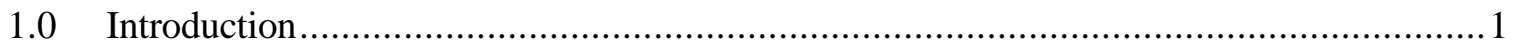

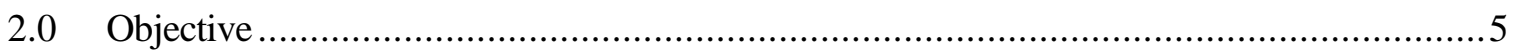

3.0 Compositional Basis for the Glass Selection Process .................................................6

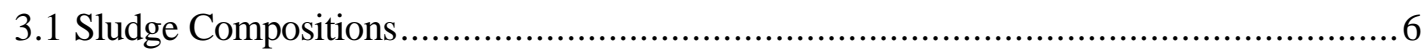

3.1.1 SB3 Compositions Based on Linear Washing Scenarios ..................................6

3.1.2 SB3 Composition as a Function of Decant................................................. 7

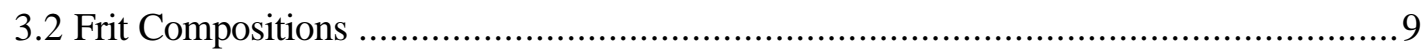

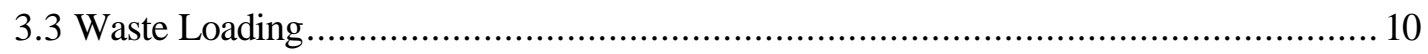

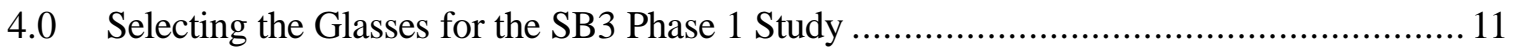

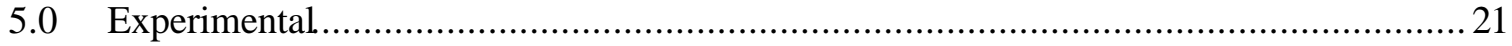

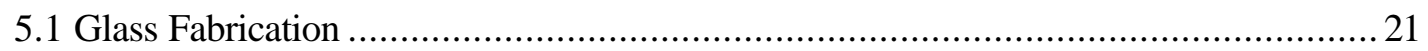

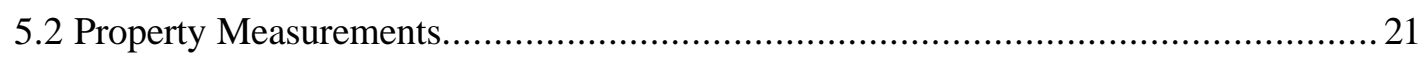

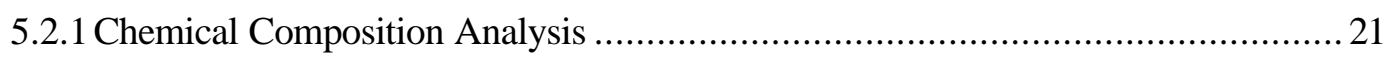

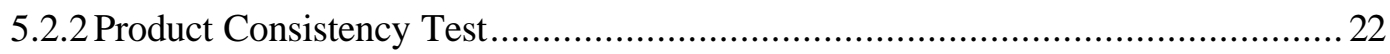

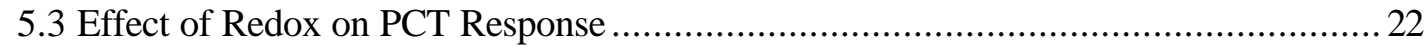

5.3.1 Experimental Assessment of PCT as a Function of Redox ............................. 23

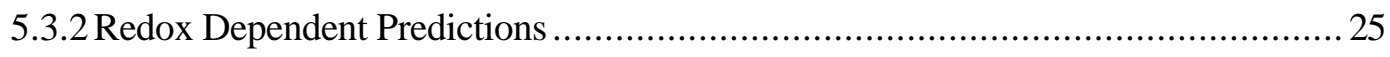

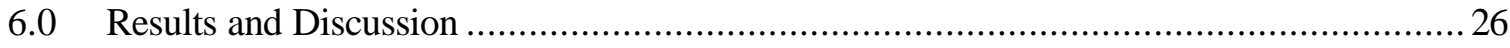

6.1 A Statistical Review of the Chemical Composition Measurements .......................... 26

6.1.1 Measurements in Analytical Sequence ................................................... 26

6.1.2 Batch 1 and Uranium Standard Results....................................................... 27

6.1.3 Composition Measurements by Glass Number............................................. 28

6.1.4 Measured versus Targeted Compositions ...................................................... 28

6.2 A Statistical Review of the PCT Measurements .................................................... 29

6.2.1 PCT Measurements in Analytical Sequence.................................................... 30

6.2.2 Measurements of the Multi-Element Solution Standard................................... 30

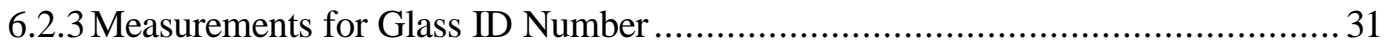

6.2.4Quenched versus Centerline Canister Cooled................................................... 31

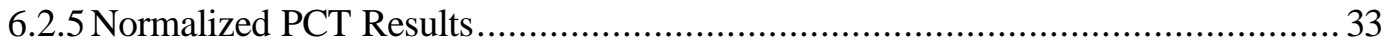

6.3 Applicability of $\Delta \mathrm{G}_{\mathrm{P}}$ to Projected SB3 Compositional Region............................. 33

6.4 "Unacceptable Glasses” Based on Model Predictions .......................................... 43 
Immobilization Technology Section

Westinghouse Savannah River Company

6.4.1 Challenges to the Homogeneity Constraint (SB3-13 and SB3-35) ...................... 43

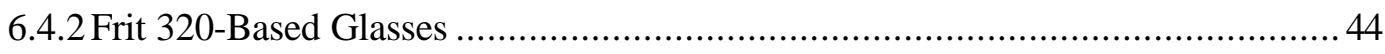

6.5 Experimental Assessment of PCT as a Function of Redox................................... 45

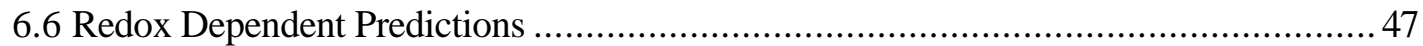

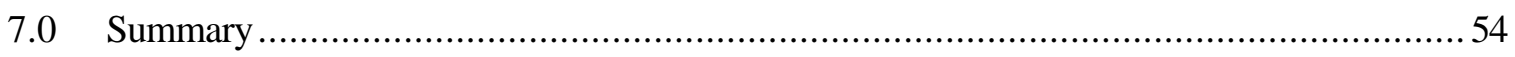

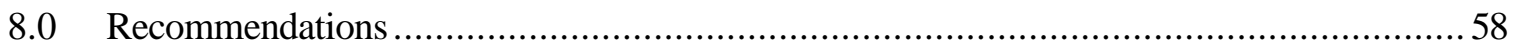

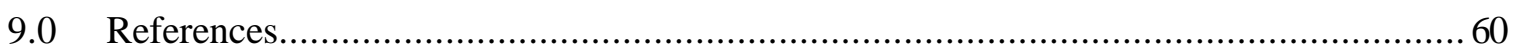

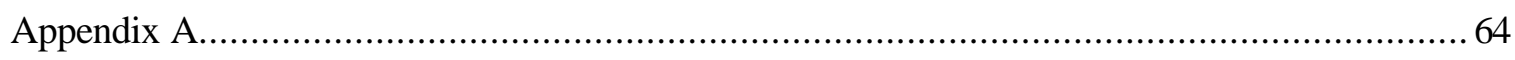

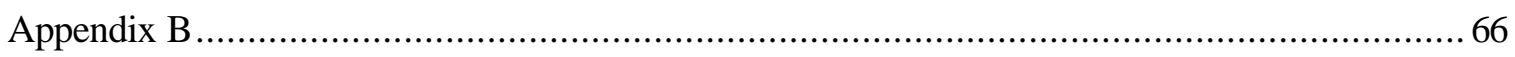

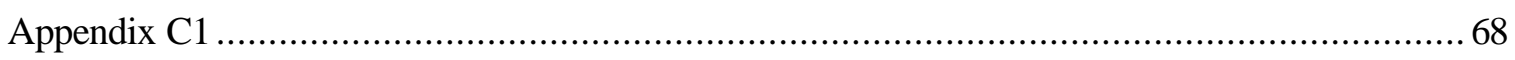

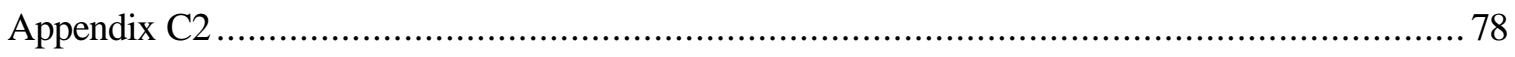

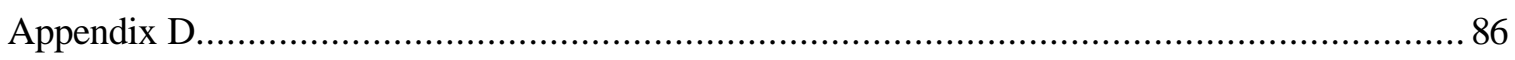

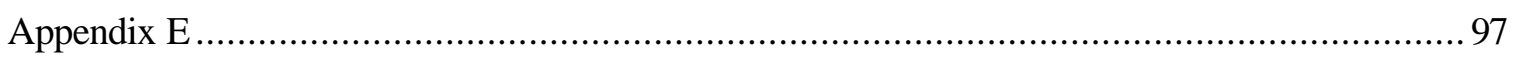

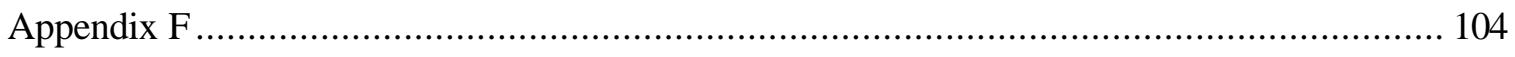

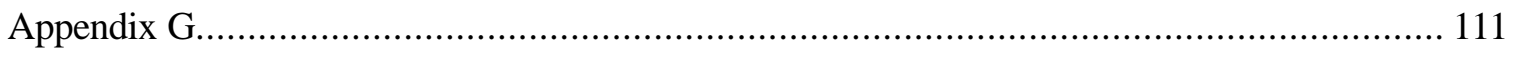

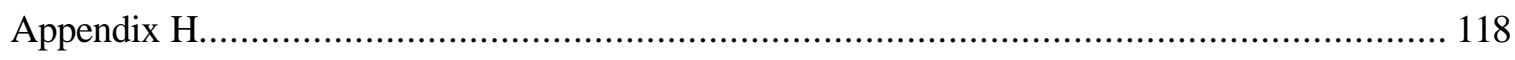

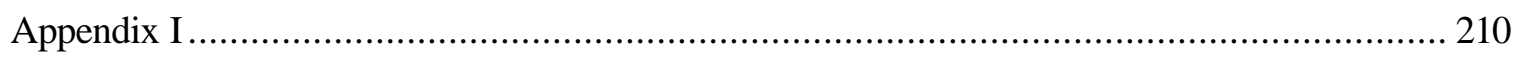

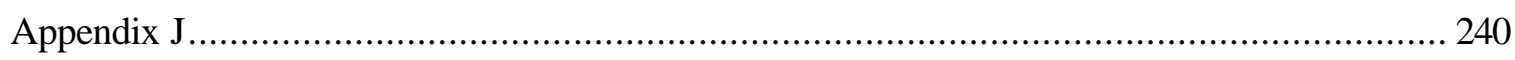


Immobilization Technology Section

WSRC-TR-2002-00549

Savannah River Technology Center

Rev. 0

Westinghouse Savannah River Company

\section{List of Figures}

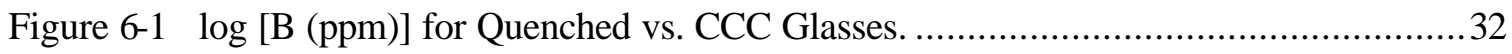

Figure 6-2. $\log \mathrm{NL}[\mathrm{B}]$ Versus $\Delta \mathrm{G}_{\mathrm{P}}$. (both quenched and ccc glasses as well as the three compositional views are shown)

Figure 6-3. Schematic of $\Delta \mathrm{G}_{\mathrm{P}}$ vs. $\log \mathrm{NL}[\mathrm{B}]$ and Potential Prediction Scenarios....................50 
Immobilization Technology Section

WSRC-TR-2002-00549

Savannah River Technology Center

Rev. 0

Westinghouse Savannah River Company

This page intentionally left blank. 


\section{List of Tables}

Table 3-1. Nominal Compositions of SB3 as a Function of Linear Washing Scenario (wt\%, oxide basis).

Table 3-2. Projected SB3 Compositions as a Function of Decant (oxide basis, wt $\%$ )................

Table 3-3. Nominal Compositions of Candidate Frits (in wt\% on an oxide basis). (primary frit

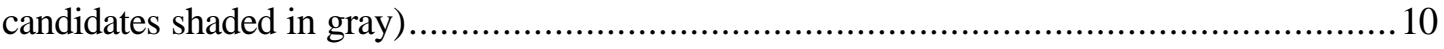

Table 4-1. MAR Assessment for Frit 202 and Decant \#5. (from Appendix C1) ....................... 12

Table 4-2. Summary of Waste Loading Ranges for Select Frit/Sludge Combinations Using the

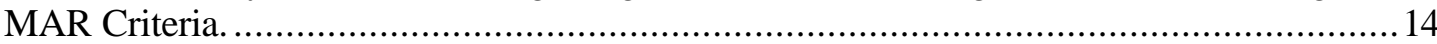

Table 4-3. MAR Assessment for Frit 320 and 75\% Linear Washed Sludge. (from Appendix C2)

Table 4-4. SB3 Phase 1 Glass Identifiers and Compositional Basis.................................... 16

Table 4-5. Target Glass Compositions for the Forty-Two SB3 Phase 1 Variability Study Glasses.

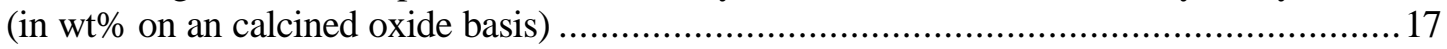

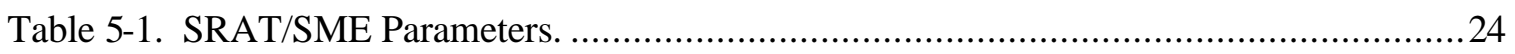

Table 6-1. Results from Samples of the Multi-Element Solution Standard. ............................... 31

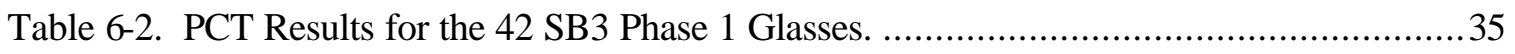

Table 6-3. Summary of PCT Results for SB3-42 ................................................... 44

Table 6-4. Measured Compositions of the Tank 8 Glasses............................................45

Table 6-5. PCTs (in ppm) for Tank 8 Glass Products and Standards..................................46

Table 6-6. Redox and PCT Response of Tank 8 Glass Products....................................... 46

Table 6-7. Summary of Predicted and Measured NL [B] Releases (in g/L) for the SB3 Phase 1 Glasses for Various Redox Values Based Upon Targeted Compositional View..................49

Table 6-8. Predicted $\Delta \mathrm{G}_{\mathrm{P}}$ Values for SB3 Glasses Assuming $\mathrm{Fe}^{2+} / \Sigma \mathrm{Fe}=0.00$ (Fully Oxidized). 52

Table 6-9 Predicted $\Delta \mathrm{G}_{\mathrm{P}}$ Values for SB3 Glasses Assuming $\mathrm{Fe}^{2+} / \Sigma \mathrm{Fe}=0.2$ (assumed targeted value).

Table 6-10. Predicted $\Delta \mathrm{G}_{\mathrm{P}}$ Values for SB3 Glasses Assuming $\mathrm{Fe}^{2+} / \Sigma \mathrm{Fe}=0.33$ (Upper Acceptance Limit). 


\subsection{Introduction}

Approximately $130 \mathrm{M} \mathrm{L}$ of sludge/supernate high-level radioactive waste (HLW) is currently stored in underground carbon steel tanks at the Savannah River Site (SRS) in Aiken, South Carolina. The Defense Waste Processing Facility (DWPF) began immobilizing these wastes in borosilicate glass in 1996. Currently, the radioactive glass is being produced as a "sludge-only" composition by combining washed high-level sludge with glass frit and melting. The molten glass is poured into stainless steel canisters that will eventually be disposed of in a permanent geological repository.

Currently, DWPF is processing Sludge Batch 2 (SB2) and is planning to start processing Sludge Batch 3 (SB3) in the spring of 2004 (WSRC 2001). ${ }^{1}$ A sludge batch is defined as a single tank of sludge slurry or a combination of sludge slurries from different tanks that has been or will be qualified for eventual transfer to DWPF. Sludge Batch 3 will be primarily Tank 7 sludge mixed with the heel of Sludge Batch 1B (SB1B), contributions from Tanks 18 and 19, an H-Canyon slurry containing precipitated Pu with Gd (Jilani 2002), and Americium/Curium (Am/Cm) precipitate from F-Canyon (Patel 2002). The sludge from Tank 7 is expected to contain several components that are considered atypical of DWPF sludge to date including higher levels of noble metals than previously processed sludge batches (Peeler et al. 2002a), sand, coal, sodium oxalate, and zeolite (Jantzen et al. 2002). Based on the process history for Tank 7, it is estimated that significant quantities of sand/coal $(\sim 7723 \mathrm{~kg})$ and sodium oxalate $(\sim 300,000 \mathrm{~kg})$ have been added to this tank (Goslen 1984; Fowler 1980).

The quantities of sand, coal, and sodium oxalate may impact several processing parameters at the DWPF. DWPF Process Engineering (DWPF PE) has issued a Technical Task Request (TTR) requesting the Savannah River Technology Center (SRTC) to address these processing impacts (Rios-Armstrong 2002a). Fellinger (2002) provided a list of the various tasks that are currently being addressed prior to DWPF's acceptance of SB3. Studies have been and are being performed by SRTC to assess the effects of sand, coal, sodium oxalate, the Pu/Gd stream, and the higher levels of noble metals on various SB3 issues [Herman et al. (2002a); Peeler et al. (2002a); Bronikowski et al. (2002); Jantzen (2002); Herman (2002a); Herman (2002b); Lorier et al. 2003); Peeler and Edwards (2002)].

One of the tasks identified by Fellinger (2002) involved an evaluation of potential frits for SB3. Rios-Armstrong (2002b) issued a more specific TTR to address the frit development activity as well as a subsequent variability study.

Peeler and Edwards (2002) outlined an integrated methodology for developing and ultimately selecting a frit for SB3. Several key aspects or criteria that form the basis underlying this methodology include:

- Maximizing the Product Composition Control System (PCCS) projected operational window size over the anticipated SB3 composition region

- Providing a frit that is robust or insensitive to anticipated sludge composition variation

- Improving or maintaining high waste loadings (WLs)

\footnotetext{
1 Although the current HLW System Plan (WSRC 2001) projects the initiation of SB3 processing in the spring of 2004, plans to expedite processing of SB3 are currently being assessed. If proven feasible, processing of SB3 could begin as soon as the spring/summer of 2003 .
} 
- Improving or maintaining high melt rates

- Providing a "frittable" additive or frit composition ${ }^{2}$

The criteria listed above are being used to guide the SB3 frit development task in an effort to establish a baseline flowsheet for SB3 in terms of ease of processing, waste loading, and/or melt rate. $^{3}$ The ability to maximize the size of the DWPF PCCS operational window provides flexibility in targeting waste loadings to meet processing goals. This is strictly an ease-ofprocessing goal targeted to provide as large of a compositional operating window and as much flexibility as possible. Given the five key criteria can be competing, the basis for not only developing but ultimately selecting a frit for SB3 is complex. The selection process should not be made based on a single criterion but on a collection of criteria that provide insight into the ease of processing SB3 while still making an acceptable glass.

Peeler and Edwards (2002) utilized model-based predictions to provide insight into the projected PCCS operational windows as a function of frit and sludge composition over a WL interval of interest. A unique, but technically sound, methodology was developed and implemented for that study to guide frit development activities. The methodology utilized was a sequential, iterative process capable of discerning the effects of frit composition on the projected PCCS operational windows and evaluating its robustness to sludge variation. That is, providing a frit that is robust or insensitive to relatively large variations in sludge composition (yields a relatively large processing window when accounting for composition variation) is a major advantage. A "robust" frit will reduce uncertainties or questions associated with how the frit will respond to SB3 once the qualification sample is obtained and compared to what is being used as the nominal or targeted composition in current testing. A viable frit should not only be able to process the nominal SB3 composition being used but should also be able to process (i.e., be robust to) realistic variations of that composition while still maintaining adequate processing and product characteristics. The degree of tolerance can be measured by the ability to produce acceptable glasses as one transitions from the nominal sludge case to compositions representing larger and larger variation about the nominal. Comparisons among both existing (Frit 202 and Frit 320) and alternative frits (the 400 series) were conducted using objective metrics that were developed to aid in this decision making process.

The model-based assessments indicated that judicious selection of the frit could yield processable and durable products at attractive waste loadings for all washing scenarios. The results provided support for the concept of developing unique frits for specific sludges to optimize PCCS operational windows and waste throughput. The results suggested that an aggressive washing strategy may not be required to assure processability or product quality as long as alternative frits are considered (assuming there are no other glass- or process-related restrictions such as anion solubility, $\mathrm{H}_{2}$ generation, redox control, or rheological control issues). Peeler and Edwards (2002) stress that the assessments were based solely on PCCS model predictions, which did not include assessments of, melt rate or frittability that are part of the integrated testing methodology.

Including assessments of melt rate (Lorier et al. 2003) and frittability in the integrated strategy should lower the risk of introducing a feed into DWPF that although on paper is very attractive (in terms of waste loading) results in a very difficult feed to process (in terms of melt rate). In fact, this strategy should provide the basis for developing a decision matrix in which optimum waste throughput could be targeted.

2 The term of "frittable" refers to the ability to produce a prefabricated frit (glass) from the proposed glass additives.

3 Waste loading (WL) in this report is simply calculated as the HLW oxide fraction of the final glass. 
The last criterion is the desire to have a "frittable" frit (i.e., the targeted frit composition will produce a glass that can be manufactured by a vendor). Use of a prefabricated frit instead of batch chemicals stems primarily from waste acceptance issues. The DWPF process control system imposes several constraints on the composition of the contents of the Slurry Mix Evaporator (SME) to define acceptability. These constraints relate process or product properties to composition via prediction models. A batch is deemed acceptable if its composition measurements lead to acceptable property predictions after accounting for modeling, analytic, and measurement uncertainties. The baseline document guiding the use of these data and models is Revision 4 of "SME Acceptability Determination for DWPF Process Control (U)" by Brown, Postles, and Edwards (2002). Currently, DWPF uses a frit that is ultimately blended with the sludge. Samples of this blend are taken from the SME, the compositions determined, and properties are predicted from the measured composition and are verified to a high confidence level to be within the processing window. Given the feed is acceptable in terms of various property predictions, the feed is transferred to the melter, converted to glass, and poured into canisters. This feed-forward process control strategy has been very effective in terms of assuring processability and product quality. Hence, frittability is an important criterion.

Although not a specific criterion listed above, the focus of this report is to demonstrate the applicability of the current PCCS durability model (referred to as $\Delta \mathrm{G}_{\mathrm{P}}$ - a preliminary glass dissolution estimator based on free energy of hydration (Jantzen et al. 1995)) to the anticipated composition region for SB3. Prior to acceptance and processing of a new sludge batch in DWPF, it must be confirmed that the sludge batch produces an acceptable glass via the PCCS. One of the phases of this waste qualification process is to complete a glass variability study as required by the DWPF Glass Product Control Program (Plodinec et al. 1995). In general, the objective of the variability study is to determine if the durability $-\Delta \mathrm{G}_{\mathrm{P}}$ correlation currently utilized by DWPF applies to the projected compositional region for the next sludge batch - for this study SB3.

Herman, Peeler, and Edwards (2002) indicated that the SB3 variability study might be performed in two phases. The first phase (henceforth referred to as Phase 1 and the subject of this report) will be based on the general assessments performed by Peeler and Edwards (2002). Phase 1 will assess applicability over a bounding or global composition region. The general composition region that will be assessed will be developed based on the latest Tank 7 compositional estimates or projections, candidate frit compositions, and a waste loading interval of interest. Due to the number of uncertainties in the SB3 integrated flowsheet, a more focused study may not be bounding in terms of compositional applicability. Phase 2 (if required) will be a more compositionally focused task. More specifically, as the washing scenario, acid addition and reduction/oxidation (redox) control strategies, and frit selection become more definitive, and the results of the SB3 waste qualification sample analysis are received, Phase 2 will be performed to back fill any data gaps resulting from the Phase 1 analysis or gaps not covered by existing and qualified data. The Phase 2 effort will focus in on a specific frit (or frits), Tank 51 waste qualification results (based on a targeted washing scenario), and a waste loading interval of interest. Uncertainties associated with the sludge composition will be addressed in that study as well. The results of Phase 2 are not covered in this report but will be documented in a subsequent report. ${ }^{4}$

4 The Phase 2 Variability study may not be required. This decision would be based on a review of the Tank 51 waste qualification analysis, the primary frit(s) of interest and the existing composition-property database. More specifically, the compositional space for SB3 would be projected and a review of existing data would be performed (including that from the current SB3 Phase 1 study) to assess the need to fill compositional gaps. One possible outcome of that review is a determination that the compositional region is adequately covered and the applicability of $\Delta \mathrm{G}_{\mathrm{P}}$ to the anticipated region can be technically defended. 
Objectives for the SB3 Phase 1 Variability Study are specified in Section 2.0. In Section 3.0, the inputs from which the anticipated SB3 compositional region and glass selection processes were based are discussed. Section 4.0 discusses the use of the Measurement Acceptance Region (MAR) selection criteria to establish the test matrix glasses. The fabrication and testing (both physical and chemical) of the SB3 Phase 1 Variability Study glasses are discussed in Section 5.0. Section 6.0 summarizes the results of the compositional analysis (target vs. measured compositions), the assessment of the applicability of $\Delta \mathrm{G}_{\mathrm{P}}$ to $\mathrm{SB} 3$, and the impact of redox on the durability response measure. Section 7.0 provides a summary of the Phase 1 test results.

Recommendations are presented in Section 8.0. 


\subsection{Objective}

The objective of this task is to assess the applicability of the current PCCS durability model $\left(\Delta \mathrm{G}_{\mathrm{P}}\right)$ to the anticipated compositional region of interest to SB3. Specifically, this study will demonstrate the applicability of durability predictions (as measured by the Product Consistency Test) for specific SB3 compositions.

These results will be used during the frit selection process for SB3. As discussed in Section 1.0, the frit selection process should not be made based on a single criterion but rather on a collection of criteria that provide insight into both the economics and processability of SB3. This work has been prepared to address technical issues discussed in Technical Task Request

HLW/DWPF/TTR-02-0027, Rev. 0 (Rios-Armstrong 2002b) and in accordance with the Task Technical and Quality Assurance Plan (Herman, Peeler, and Edwards 2002). 


\subsection{Compositional Basis for the Glass Selection Process}

To assess the applicability of the current durability model to the anticipated SB3 composition region one must first establish the SB3 composition region of interest. Composition regions of interest typically are developed around three primary factors: (1) sludge or waste stream composition(s), (2) frit composition(s), and (3) waste loading intervals of interest. These three primary inputs are briefly discussed below. It should be noted that the Phase 1 Variability Study will be based on nominal sludge compositions - composition variation around the nominal wash/decant sludge composition was not taken into account.

\subsection{Sludge Compositions}

Two primary inputs were used to define the SB3 sludge composition region of interest: (1) linear washing scenarios and (2) decant information from HLW PE - both based on Waste Characterization System (WCSystem) information. Sludge compositions that form the basis for the SB3 Phase 1 Variability Study glasses are presented in Sections 3.1.1 and 3.1.2.

\subsubsection{SB3 Compositions Based on Linear Washing Scenarios}

At the time this task was initiated, uncertainties associated with the actual quantity of sodium oxalate $\left(\mathrm{Na}_{2} \mathrm{C}_{2} \mathrm{O}_{4}\right)$ in Tank 7 and the fraction that would ultimately be transferred to SB3 given the various washing scenarios under consideration were being addressed. From a glass formulation perspective, the unknown quantity of $\mathrm{Na}_{2} \mathrm{O}$ resulting from the $\mathrm{Na}_{2} \mathrm{C}_{2} \mathrm{O}_{4}$ would have a major impact on the overall sludge composition and ultimately the frit development efforts. Peeler and Edwards (2002) performed initial assessments using compositions based on a linear washing scheme prior to receiving projected sludge compositions as a function of washing (or decant) from HLW PE (see Section 3.1.2). That assessment based on model predictions demonstrated the need to compositionally compensate (by adjusting the frit composition) for the varying $\mathrm{Na}_{2} \mathrm{O}$ concentrations present in the sludge as different washing schemes were considered.

Table 3-1 summarizes the nominal SB3 compositions for the linear washing scenarios (using the underlying assumptions as discussed by Peeler and Edwards (2002)). It also should be noted that these nominal SB3 compositions do not account for the potential introduction of a monosodium titanate (MST) stream (e.g., $\mathrm{TiO}_{2}$ is not present in the projected sludge compositions). Based on HLW System Plan (WSRC 2001), it is anticipated that a limited volume of MST will be blended into SB3. However, given the current uncertainties of if, when, and/or how (e.g., blended over the entire SB3 campaign or spiked into a limited portion of the SB3 campaign) that stream would be blended with SB3; its contribution was not accounted for in the initial assessments by Peeler and Edwards (2002). The contribution of this secondary stream is accounted for in the decant information provided by Elder (see Section 3.1.2). ${ }^{5}$

\footnotetext{
${ }^{5}$ Personal communication with H.H. Elder via email dated 7/30/02. Appendix A provides the data transmitted in the personal communication (elemental wt $\%$, calcine basis).
} 
Table 3-1. Nominal Compositions of SB3 as a Function of Linear Washing Scenario (wt\%, oxide basis).

\begin{tabular}{|c|c|c|c|c|c|}
\hline Oxide & $0 \%$ Washed & $25 \%$ Washed & $50 \%$ Washed & $75 \%$ Washed & $100 \%$ Washed \\
\hline $\mathrm{Ag}_{2} \mathrm{O}$ & 0.000 & 0.000 & 0.000 & 0.000 & 0.001 \\
\hline $\mathrm{Al}_{2} \mathrm{O}_{3}$ & 13.140 & 14.110 & 15.230 & 16.540 & 18.100 \\
\hline $\mathrm{AmO}_{2}$ & 0.003 & 0.003 & 0.003 & 0.004 & 0.004 \\
\hline $\mathrm{BaO}$ & 0.182 & 0.195 & 0.211 & 0.229 & 0.250 \\
\hline $\mathrm{CaO}$ & 2.610 & 2.800 & 3.030 & 3.290 & 3.600 \\
\hline $\mathrm{Ce}_{2} \mathrm{O}_{3}$ & 0.258 & 0.277 & 0.298 & 0.324 & 0.355 \\
\hline $\mathrm{Cm}_{2} \mathrm{O}_{3}$ & 0.000 & 0.000 & 0.000 & 0.000 & 0.001 \\
\hline $\mathrm{Cr}_{2} \mathrm{O}_{3}$ & 0.272 & 0.292 & 0.315 & 0.342 & 0.374 \\
\hline $\mathrm{CuO}$ & 0.144 & 0.154 & 0.167 & 0.181 & 0.198 \\
\hline $\mathrm{Eu}_{2} \mathrm{O}_{3}$ & 0.003 & 0.004 & 0.004 & 0.004 & 0.005 \\
\hline $\mathrm{Fe}_{2} \mathrm{O}_{3}$ & 29.230 & 31.380 & 33.870 & 36.800 & 40.270 \\
\hline $\mathrm{Gd}_{2} \mathrm{O}_{3}$ & 0.037 & 0.040 & 0.043 & 0.046 & 0.051 \\
\hline $\mathrm{K}_{2} \mathrm{O}$ & 0.313 & 0.336 & 0.362 & 0.393 & 0.431 \\
\hline $\mathrm{La}_{2} \mathrm{O}_{3}$ & 0.149 & 0.160 & 0.173 & 0.188 & 0.206 \\
\hline $\mathrm{Li}_{2} \mathrm{O}$ & 0.002 & 0.002 & 0.003 & 0.003 & 0.003 \\
\hline $\mathrm{MgO}$ & 0.137 & 0.147 & 0.158 & 0.172 & 0.188 \\
\hline $\mathrm{MnO}$ & 5.210 & 5.590 & 6.040 & 6.560 & 7.180 \\
\hline $\mathrm{Na}_{2} \mathrm{O}$ & 35.130 & 30.360 & 24.830 & 18.340 & 10.630 \\
\hline $\mathrm{Nd}_{2} \mathrm{O}_{3}$ & 0.495 & 0.531 & 0.573 & 0.623 & 0.682 \\
\hline $\mathrm{NiO}$ & 1.170 & 1.260 & 1.360 & 1.470 & 1.610 \\
\hline $\mathrm{PbO}$ & 0.220 & 0.236 & 0.254 & 0.276 & 0.276 \\
\hline $\mathrm{PdO}$ & 0.027 & 0.029 & 0.031 & 0.033 & 0.037 \\
\hline $\mathrm{Pr}_{2} \mathrm{O}_{3}$ & 0.135 & 0.145 & 0.157 & 0.170 & 0.186 \\
\hline $\mathrm{PuO}_{2}$ & 0.038 & 0.041 & 0.044 & 0.048 & 0.052 \\
\hline $\mathrm{RhO}_{2}$ & 0.056 & 0.061 & 0.065 & 0.071 & 0.078 \\
\hline $\mathrm{RuO}_{2}$ & 0.202 & 0.217 & 0.234 & 0.254 & 0.278 \\
\hline $\mathrm{SiO}_{2}$ & 2.430 & 2.610 & 2.810 & 3.050 & 3.340 \\
\hline $\mathrm{Sm}_{2} \mathrm{O}_{3}$ & 0.073 & 0.078 & 0.084 & 0.091 & 0.100 \\
\hline $\mathrm{ThO}_{2}$ & 0.104 & 0.112 & 0.120 & 0.131 & 0.143 \\
\hline $\mathrm{TiO}_{2}$ & 0.000 & 0.000 & 0.000 & 0.000 & 0.000 \\
\hline $\mathrm{U}_{3} \mathrm{O}_{8}$ & 7.390 & 7.940 & 8.570 & 9.310 & 10.190 \\
\hline $\mathrm{ZnO}$ & 0.299 & 0.320 & 0.345 & 0.375 & 0.411 \\
\hline $\mathrm{ZrO}_{2}$ & 0.540 & 0.579 & 0.625 & 0.679 & 0.743 \\
\hline Sum & 100.00 & 100.00 & 100.00 & 100.00 & 100.00 \\
\hline
\end{tabular}

\subsubsection{SB3 Composition as a Function of Decant}

As previously stated, assessments were initially performed using the linear washing schemes prior to receiving projected sludge compositions as a function of washing (or decant) from HLW PE. Elder provided the compositional estimates of SB3 as a function of decant (see Appendix A). The elemental concentrations provided by Elder were converted to an oxide basis (by multiplying by the appropriate gravimetric factor) and these data are presented in Table 3-2. 
General observations of composition as a function of washing indicate that the $\mathrm{Na}_{2} \mathrm{O}$ concentrations decrease with increased washing (or decanting). Initially, the $\mathrm{Na}_{2} \mathrm{O}$ concentration decreases relatively quickly with the differences becoming smaller as the decant number increases. All other components trend in the opposite direction - increased concentration as the decant number increases. Although this trend was expected, the $\mathrm{Na}_{2} \mathrm{O}$ concentration for the minimum washed sludge (Decant \#5) is lower than that associated with the nominal $0 \%$ linear washed case (see Table 3-1), 31.205 versus $35.13 \mathrm{wt} \%$, respectively. This difference is based on the assumptions made in the initial compositional projections given no washing models were available at the time. Communications with HLW PE indicated that a minimum wash/decant sequence must be performed in order to transfer Tank 7 . This fact was not originally considered when the $0 \%$ linear washed case was established as a viable compositional endpoint.

It should also be noted that the compositional projections provided by Elder include significant quantities of $\mathrm{TiO}_{2}$ ranging from $2.154 \%$ to $2.627 \%$ in Decant \#5 to Decant \#15, respectively. The $\mathrm{TiO}_{2}$ results from the introduction of the MST stream. $\mathrm{TiO}_{2}$ was considered as a major oxide when these sludges were evaluated by Peeler and Edwards (2002).

Table 3-2. Projected SB3 Compositions as a Function of Decant (oxide basis, wt\%).

\begin{tabular}{|c|c|c|c|c|c|c|c|c|c|c|c|}
\hline Oxide & $\begin{array}{c}\text { Decant } \\
\# 5\end{array}$ & $\begin{array}{c}\text { Decant } \\
\# 6\end{array}$ & $\begin{array}{c}\text { Decant } \\
\# 7\end{array}$ & $\begin{array}{c}\text { Decant } \\
\# 8\end{array}$ & $\begin{array}{c}\text { Decant } \\
\# 9\end{array}$ & $\begin{array}{c}\text { Decant } \\
\# 10\end{array}$ & $\begin{array}{c}\text { Decant } \\
\# 11\end{array}$ & $\begin{array}{c}\text { Decant } \\
\# 12\end{array}$ & $\begin{array}{c}\text { Decant } \\
\# 13\end{array}$ & $\begin{array}{c}\text { Decant } \\
\# 14\end{array}$ & $\begin{array}{c}\text { Decant } \\
\# 15\end{array}$ \\
\hline $\mathrm{Al}_{2} \mathrm{O}_{3}$ & 13.844 & 14.348 & 14.777 & 15.139 & 15.467 & 15.743 & 16.006 & 16.253 & 16.458 & \begin{tabular}{|l|}
16.669 \\
\end{tabular} & 16.886 \\
\hline $\mathrm{BaO}$ & 0.192 & 0.198 & 0.204 & 0.209 & 0.214 & 0.218 & 0.221 & 0.225 & 0.228 & 0.231 & 0.234 \\
\hline $\mathrm{CaO}$ & 2.755 & 2.855 & 2.940 & 3.012 & 3.077 & 3.132 & 3.185 & 3.234 & 3.274 & 3.316 & 3.360 \\
\hline $\mathrm{Ce}_{2} \mathrm{O}_{3}$ & 0.267 & 0.277 & 0.285 & 0.292 & 0.298 & 0.304 & 0.309 & 0.313 & 0.317 & 0.321 & 0.326 \\
\hline $\mathrm{Cr}_{2} \mathrm{O}_{3}$ & 0.283 & 0.293 & 0.302 & 0.310 & 0.316 & 0.322 & 0.327 & 0.332 & 0.337 & 0.341 & 0.345 \\
\hline $\mathrm{CuO}$ & 0.152 & 0.157 & 0.162 & 0.166 & 0.169 & 0.172 & 0.175 & 0.178 & 0.180 & 0.183 & 0.185 \\
\hline $\mathrm{Fe}_{2} \mathrm{O}_{3}$ & 30.784 & 31.905 & 32.858 & 33.664 & 34.392 & 35.007 & 35.591 & 36.141 & 36.596 & 37.065 & 37.549 \\
\hline $\mathrm{K}_{2} \mathrm{O}$ & 0.329 & 0.341 & 0.352 & 0.360 & 0.368 & 0.375 & 0.381 & 0.387 & 0.392 & 0.397 & 0.402 \\
\hline $\mathrm{La}_{2} \mathrm{O}_{3}$ & 0.176 & 0.182 & 0.187 & 0.192 & 0.196 & 0.200 & 0.203 & 0.206 & 0.209 & 0.211 & 0.214 \\
\hline $\mathrm{MgO}$ & 0.144 & 0.150 & 0.154 & 0.158 & 0.161 & 0.164 & 0.167 & 0.169 & 0.172 & 0.174 & 0.176 \\
\hline $\mathrm{MnO}$ & 5.491 & 5.690 & 5.860 & 6.004 & 6.134 & 6.244 & 6.348 & 6.446 & 6.527 & 6.611 & 6.697 \\
\hline $\mathrm{Na}_{2} \mathrm{O}$ & 31.205 & 28.701 & 26.571 & 24.770 & 23.142 & 21.770 & 20.464 & 19.235 & 18.219 & 17.170 & 16.089 \\
\hline $\mathrm{NiO}$ & 1.231 & 1.276 & 1.314 & 1.346 & 1.375 & 1.400 & 1.423 & 1.445 & 1.463 & 1.482 & 1.501 \\
\hline $\mathrm{PbO}$ & 0.230 & 0.238 & 0.245 & 0.251 & 0.257 & 0.261 & 0.265 & 0.270 & 0.273 & 0.276 & 0.280 \\
\hline $\mathrm{SiO}_{2}$ & 1.603 & 1.661 & 1.711 & 1.753 & 1.791 & 1.823 & 1.853 & 1.882 & 1.906 & 1.930 & 1.955 \\
\hline $\mathrm{ThO}_{2}$ & 0.110 & 0.114 & 0.117 & 0.120 & 0.122 & 0.125 & 0.127 & 0.129 & 0.130 & 0.132 & 0.134 \\
\hline $\mathrm{TiO}_{2}$ & 2.154 & 2.232 & 2.299 & 2.356 & 2.407 & 2.450 & 2.490 & 2.529 & 2.561 & 2.594 & 2.627 \\
\hline $\mathrm{U}_{3} \mathrm{O}_{8}$ & 7.792 & 8.075 & 8.316 & 8.520 & 8.705 & 8.860 & 9.008 & 9.147 & 9.262 & 9.381 & 9.504 \\
\hline $\mathrm{ZnO}$ & 0.315 & 0.326 & 0.336 & 0.344 & 0.351 & 0.358 & 0.364 & 0.369 & 0.374 & 0.379 & 0.384 \\
\hline $\mathrm{ZrO}_{2}$ & 0.568 & 0.589 & 0.607 & 0.622 & 0.635 & 0.646 & 0.657 & 0.667 & 0.676 & 0.684 & 0.693 \\
\hline Sum & 99.624 & 9.610 & 99.598 & 99588 & 99579 & 99572 & 99565 & 99558 & 9553 & 9547 & \\
\hline
\end{tabular}

Based on input from DWPF and HLW PE personnel, the SB3 Technical team decided to narrow the focus to span the range bounded by Decant \#5 through Decant \#9. The latter decision was made based on the lack of identifying any significant technical issues during the initial stages of the SB3 flowsheet development activities that would require significant sludge washing. Washing the sludge less does have advantages in terms of significant time and labor savings in the pretreatment/retrieval operation. A reduced washing campaign would also result in less water being sent to the evaporators. Conversely, targeting a more washed sludge would reduce the total 
number of canisters produced by some incremental amount and may minimize components that have potentially negative impacts to either $\mathrm{WL}$ or processability (such as $\mathrm{SO}_{4}$ ). A cost-benefit analysis should be performed to fully understand the advantages and disadvantages of selecting a targeted washing scenario. Even though the SB3 team did not have access to a detailed costbenefit analysis, they elected to narrow the decant/washing focus to span an interval covered by Decant \#5 through Decant \#9.

Although primarily interested in the decant information (given a more solid technical basis due to compositional projections being based on blending and washing models), the Phase 1 Variability Study also included glass compositions based on the linear washed sludge. The specific (and nominal) compositions used were based on the $0 \%, 25 \%$, and $50 \%$ linear washed projections as shown in Table 3-1. The linear washing compositions extend the overall SB3 compositional region of interest in Phase 1 relative to the sole use of Decants \#5 and \#9 - supporting the global Phase 1 strategy as discussed in Section 1.0.

\subsection{Frit Compositions}

Although Elder (2002) has established Frit 202 and Frit 320 as "baseline" frits for specific SB3 decant compositions, Peeler and Edwards (2002) developed a series of frits that provided attractive PCCS operational windows (based on model predictions) for the linear washing and decant compositions. Implementation or use of these alternative frit compositions may provide benefit in terms of maximizing waste throughput for SB3. Table 3-3 summarizes the frit compositions of interest for SB3. ${ }^{6}$

Based on the assessments performed by Peeler and Edwards (2002), nine alternative (400 series) and two existing frits (Frit 202 and Frit 320) were classified as primary candidates (shaded in gray in Table 3-3) for the specific washing scenarios of interest. This classification was based on the response of each frit/sludge combination (for both nominal and nominal $\pm 10 \%$ variation based sludges) for the model-based assessments. Model-based predictions indicated these frits would have large PCCS windows at attractive WLs and are robust to compositional variation for specific washing scenarios.

6 During the frit development process, an alphanumeric nomenclature or system was used to identify specific frit compositions. These alphanumeric identifiers have been converted to a numerical series (the 400 series). Appendix B provides the conversion chart. 
Table 3-3. Nominal Compositions of Candidate Frits (in wt\% on an oxide basis). (primary frit candidates shaded in gray)

\begin{tabular}{||c|c|c|c|c|c|c|c||}
\hline \hline Frit ID & $\mathrm{Al}_{2} \mathrm{O}_{3}$ & $\mathrm{~B}_{2} \mathrm{O}_{3}$ & $\mathrm{Li}_{2} \mathrm{O}$ & $\mathrm{Na}_{2} \mathrm{O}$ & $\mathrm{SiO}_{2}$ & $\mathrm{MgO}$ & Total \\
\hline Frit 202 & - & 8 & 7 & 6 & 77 & 2 & 100 \\
\hline Frit 320 & - & 8 & 8 & 12 & 72 & - & 100 \\
\hline Frit 400 & - & 20 & - & - & 80 & - & 100 \\
\hline Frit 401 & - & 30 & - & - & 70 & - & 100 \\
\hline Frit 402 & - & 12 & - & - & 88 & - & 100 \\
\hline Frit 403 & - & 15 & 3 & - & 82 & - & 100 \\
\hline Frit 404 & - & 12 & 3 & - & 85 & - & 100 \\
\hline Frit 405 & - & 20 & 5 & - & 75 & - & 100 \\
\hline Frit 406 & - & 15 & 5 & - & 80 & - & 100 \\
\hline Frit 407 & - & 8 & - & 6 & 86 & - & 100 \\
\hline Frit 408 & - & 8 & 2 & 6 & 84 & - & 100 \\
\hline Frit 409 & - & 8 & 4 & 6 & 82 & - & 100 \\
\hline Frit 410 & - & 8 & 5 & 5 & 82 & - & 100 \\
\hline Frit 411 & 1 & 8 & 5 & 12 & 74 & - & 100 \\
\hline Frit 412 & - & 8 & 5 & 12 & 75 & - & 100 \\
\hline Frit 413 & - & 9 & 5 & 15 & 71 & - & 100 \\
\hline Frit 414 & - & 8 & 6 & 14 & 72 & - & 100 \\
\hline Frit 415 & - & 10 & 5 & 15 & 70 & - & 100 \\
\hline Frit 416 & 1 & 8 & 8 & 11 & 72 & - & 100 \\
\hline Frit 417 & - & 8 & 8 & 11 & 73 & - & 100 \\
\hline Frit 418 & - & 8 & 8 & 8 & 76 & - & 100 \\
\hline Frit 419 & - & 8 & 8 & 15 & 69 & - & 100 \\
\hline Frit 420 & 1 & 8 & 5 & 7 & 79 & - & 100 \\
\hline Frit 421 & 1 & 10 & 5 & 7 & 77 & - & 100 \\
\hline Frit 422 & - & 8 & 8 & 3 & 81 & - & 100 \\
\hline Frit 423 & - & 10 & 8 & 3 & 79 & - & 100 \\
\hline
\end{tabular}

\subsection{Waste Loading}

Given definition of both sludge and frit compositions of interest, the last criterion needed prior to establishing specific SB3 Phase 1 glass compositions is a targeted WL or WL interval.

Ultimately, the DWPF will utilize PCCS to determine the acceptability of each batch of SME product before processing in the melter. PCCS acceptability is determined by imposing several constraints on the measured composition of samples from the SME batch. The PCCS constraints are related to process or product properties, which are derived directly from the SME

measurements or indirectly from them via model predictions. These predictions are generated using property- composition models (e.g., the durability $-\Delta \mathrm{G}_{\mathrm{P}}$ correlation). In addition, PCCS takes into account modeling, analytic, and measurement uncertainties as part of the acceptability determination. The uncertainties are accounted for in two steps. The first is the uncertainty due to the property model, which when accounted for provides the Property Acceptability Region (PAR). The second is the uncertainty due to sampling and analytical (grouped under the heading of measurement). This uncertainty is accounted for, when necessary, in addition to the property uncertainty, and the resulting (more restrictive) region defines the MAR. The baseline document guiding the use of these models is Revision 4 of "SME Acceptability Determination for DWPF Process Control" by Brown, Postles, and Edwards (2002). Ultimately, DWPF will utilize the PCCS MAR criteria to determine the acceptability of each batch of SME product before processing in the melter. 


\subsection{Selecting the Glasses for the SB3 Phase 1 Study}

With respect to the SB3 frit development effort, Peeler and Edwards (2002) utilized the PAR criteria to provide a preliminary assessment of the performance of candidate frits over the WL interval of $25-60 \%$. The authors also assessed the impact of applying the MAR criteria on the projected PAR operational windows over this WL range for select frit/sludge combinations. One purpose of that effort was to assure that a relatively large operational window provided by a PAR assessment was not reduced, via the MAR assessment, to an unmanageable interval (i.e., in terms of minimizing DWPF flexibility to the point of inoperability). A second benefit of the MAR assessment is that it provided the basis for the WL intervals used in this study. More specifically, the glass selection process for the Phase 1 Variability Study used the MAR criteria to establish upper and lower WL bounds for specific frit/sludge systems of interest.

The property predictions used to define the upper and lower WL for each system included durability (Product Consistency Test [PCT] [ASTM 1998] response in terms of $\Delta \mathrm{G}_{\mathrm{P}}$ ), viscosity at $1150^{\circ} \mathrm{C}\left(\eta_{1150^{\circ}} \mathrm{C}\right)$, liquidus temperature $\left(\mathrm{T}_{\mathrm{L}}\right)^{7}$, homogeneity, and $\mathrm{Al}_{2} \mathrm{O}_{3}$ and alkali concentrations. Jantzen et al. (1995) and Brown et al. (2001) provide a more detailed discussion on the development of these models.

Appendices $\mathrm{C} 1$ and $\mathrm{C} 2$ provide assessments of the projected operational windows for various sludge (Decant \#5, Decant \#9 in Appendix C1 and 0\% linear wash, 25\% linear wash, and 50\% linear wash in Appendix C2) and frit combinations based on the MAR criteria. ${ }^{8}$ The columns identified as Frit-Decant and Frit-Washing in these appendices indicate the specific frit/sludge combination being evaluated. The "\% WL" column provides the WL. The next five columns labeled as "Durability", "T $\mathrm{T}_{\mathrm{L}}$ ", "Visc", "Frit", and "Homog", represent the MAR determinations (in terms of pass or fail) for durability, liquidus temperature, viscosity, low/high frit, and homogeneity, respectively. A "-" entry in a specific cell under these columns indicates that the predicted property satisfies the MAR. For "durability", " $\mathrm{T}_{\mathrm{L}}$ ", and "homog", a "NO" in the respective cell indicates that the predicted property fails the MAR and that particular frit/sludge combination is not acceptable at that WL. For "visc" and "Frit", if "high" or "low" is shown, this is an indication that the predicted property fails the MAR and on which side (high or low). The last 7 columns of these appendices provide property values of interest. For example, Table 4-1 summarizes the MAR assessment for Frit 202 and Decant \#5 over the 25 - 60\% WL interval. At $25 \% \mathrm{WL}$, predictions of high frit and an inhomogeneous product restrict processing. Predictions of homogeneity still restrict processing up through $32 \% \mathrm{WL}$. At $38 \% \mathrm{WL}$, model predictions indicate that durability will be unsatisfactory. All properties are satisfied (at the MAR) for WLs of $33-37 \% \mathrm{WL}$; hence the projected operational window for this particular nominal sludge and frit combination as shown in Table 4-2.

7 Liquidus temperature was assessed using the revised $\mathrm{T}_{\mathrm{L}}$ model as described by Brown et al. (2001).

8 These MAR assessments were made utilizing the same approach as that used in developing the test cases supporting the implementation of Version 4.2 of DWPF's PCCS system. The approach and resulting test cases are provided in Edwards, T. B., "Revision of DWPF's PCCS: An Overview of the Modifications and Associated Test Cases (U)," SRT-SCS-2002-00032, May 24, 2002. Appendix C2 also includes a 75\% washed case with Frit 320. 
Table 4-1. MAR Assessment for Frit 202 and Decant \#5. (from Appendix C1)

\begin{tabular}{|c|c|c|c|c|c|c|c|c|c|c|c|c|c|}
\hline \multirow[b]{2}{*}{ Frit-Decant } & \multirow[b]{2}{*}{$\% \mathrm{WL}$} & \multicolumn{5}{|c|}{ "MAR Assessment } & \multicolumn{7}{|c|}{ Property Values } \\
\hline & & Durability & $\mathbf{T}_{\mathrm{L}}$ & Visc & Frit & Homog & $\overline{\mathrm{Al}_{2} \mathrm{O}_{3}}$ & Alkali & $\overline{T_{L}}$ & Visc & $\Delta \mathbf{G}_{\mathbf{P}}$ & Homog & Frit \\
\hline 202-Decant \#5 & 25 & - & - & - & High & $\mathrm{NO}$ & 3.46 & 17.61 & 750.1 & 81.02 & -10.646 & 198.96 & 81.76 \\
\hline 202-Decant \#5 & 26 & - & - & - & - & $\mathrm{NO}$ & 3.60 & \begin{tabular}{|l|}
17.79 \\
\end{tabular} & 760.4 & 76.74 & -10.788 & 200.63 & 81.11 \\
\hline 202-Decant \#5 & 27 & - & - & - & - & $\mathrm{NO}$ & 3.74 & 17.98 & 770.1 & 72.58 & -10.929 & 202.31 & 80.46 \\
\hline 202-Decant \#5 & 28 & - & - & - & - & $\mathrm{NO}$ & 3.88 & 18.16 & 779.6 & 68.53 & -11.071 & 203.98 & 79.81 \\
\hline 202-Decant \#5 & 29 & - & - & - & - & $\mathrm{NO}$ & 4.01 & 18.35 & 788.9 & 64.62 & -11.212 & 205.65 & 79.16 \\
\hline 202-Decant \#5 & 30 & - & - & - & - & $\mathrm{NO}$ & 4.15 & 18.53 & 797.8 & 60.82 & -11.354 & 207.33 & 78.51 \\
\hline 202-Decant \#5 & 31 & - & - & - & - & $\mathrm{NO}$ & 4.29 & \begin{tabular}{|l|}
18.72 \\
\end{tabular} & 806.5 & 57.15 & -11.496 & 209.00 & 77.86 \\
\hline 202-Decant \#5 & 32 & - & - & - & - & $\mathrm{NO}$ & 4.43 & \begin{tabular}{|l|}
18.90 \\
\end{tabular} & 814.9 & 53.61 & -11.637 & 210.67 & 77.21 \\
\hline 202-Decant \#5 & 33 & - & - & - & - & - & 4.57 & \begin{tabular}{|l|}
19.09 \\
\end{tabular} & 823.0 & 50.20 & -11.779 & 212.34 & 76.56 \\
\hline 202-Decant \#5 & 34 & - & - & - & - & - & 4.71 & \begin{tabular}{|l|}
19.27 \\
\end{tabular} & 830.9 & 46.91 & -11.921 & 214.02 & 75.92 \\
\hline 202-Decant \#5 & 35 & - & - & - & - & - & 4.85 & 19.45 & 838.6 & 43.75 & -12.062 & 215.69 & 75.27 \\
\hline 202-Decant \#5 & 36 & - & - & - & - & - & 4.98 & \begin{tabular}{|l|}
19.64 \\
\end{tabular} & 846.1 & 40.71 & -12.204 & 217.36 & 74.62 \\
\hline 202-Decant \#5 & 37 & - & - & - & - & - & 5.12 & 19.82 & 853.4 & 37.80 & -12.345 & 219.03 & 73.97 \\
\hline 202-Decant \#5 & 38 & $\mathrm{NO}$ & - & - & - & - & 5.26 & 20.01 & 860.5 & 35.02 & -12.487 & 220.71 & 73.32 \\
\hline 202-Decant \#5 & 39 & $\mathrm{NO}$ & - & - & - & - & 5.40 & 20.19 & 867.3 & 32.37 & -12.629 & 222.38 & 72.67 \\
\hline 202-Decant \#5 & 40 & $\mathrm{NO}$ & - & - & - & - & 5.54 & 20.38 & 874.1 & 29.84 & -12.770 & 224.05 & 72.02 \\
\hline 202-Decant \#5 & 41 & $\mathrm{NO}$ & - & - & - & - & 5.68 & 20.56 & 880.6 & 27.43 & -12.912 & 225.73 & 71.37 \\
\hline 202-Decant \#5 & 42 & $\mathrm{NO}$ & - & - & - & - & 5.81 & 20.75 & 886.9 & 25.15 & -13.053 & 227.40 & 70.72 \\
\hline 202-Decant \#5 & 43 & $\mathrm{NO}$ & - & Low & - & - & 5.95 & 20.93 & 893.1 & 22.99 & -13.195 & 229.07 & 70.07 \\
\hline 202-Decant \#5 & 44 & $\mathrm{NO}$ & - & Low & - & - & 6.09 & 21.11 & 899.2 & 20.96 & -13.336 & 230.74 & 69.42 \\
\hline 202-Decant \#5 & 45 & $\mathrm{NO}$ & - & Low & - & - & 6.23 & 21.30 & 905.1 & 19.04 & -13.478 & 232.42 & 68.77 \\
\hline 202-Decant \#5 & 46 & $\mathrm{NO}$ & - & Low & - & - & 6.37 & 21.48 & 910.8 & 17.23 & -13.620 & 234.09 & 68.12 \\
\hline 202-Decant \#5 & 47 & $\mathrm{NO}$ & - & Low & - & - & 6.51 & 21.67 & 916.5 & 15.54 & -13.761 & 235.76 & 67.47 \\
\hline 202-Decant \#5 & 48 & $\mathrm{NO}$ & - & Low & - & - & 6.65 & 21.85 & 921.9 & 13.97 & -13.903 & 237.44 & 66.82 \\
\hline 202-Decant \#5 & 49 & $\mathrm{NO}$ & - & Low & - & - & 6.78 & \begin{tabular}{|l|}
22.04 \\
\end{tabular} & 927.3 & 12.50 & -14.045 & 239.11 & 66.17 \\
\hline 202-Decant \#5 & 50 & $\mathrm{NO}$ & - & Low & - & - & 6.92 & 22.22 & 932.5 & 11.14 & -14.186 & 240.78 & 65.52 \\
\hline 202-Decant \#5 & 51 & $\mathrm{NO}$ & - & Low & - & - & 7.06 & \begin{tabular}{|l|}
22.41 \\
\end{tabular} & 937.6 & 9.88 & -14.328 & 242.45 & 64.87 \\
\hline 202-Decant \#5 & 52 & $\mathrm{NO}$ & - & Low & - & - & 7.20 & 22.59 & 942.6 & 8.73 & -14.469 & 244.13 & 64.22 \\
\hline 202-Decant \#5 & 53 & $\mathrm{NO}$ & - & Low & - & - & 7.34 & 22.77 & 947.5 & 7.67 & -14.611 & 245.80 & 63.57 \\
\hline 202-Decant \#5 & 54 & $\mathrm{NO}$ & - & Low & - & - & 7.48 & 22.96 & 952.3 & 6.70 & -14.753 & 247.47 & 62.92 \\
\hline 202-Decant \#5 & 55 & $\mathrm{NO}$ & - & Low & - & - & 7.61 & 23.14 & 956.9 & 5.82 & -14.894 & 249.14 & 62.27 \\
\hline 202-Decant \#5 & 56 & $\mathrm{NO}$ & - & Low & - & - & 7.75 & 23.33 & 961.5 & 5.02 & -15.036 & 250.82 & 61.62 \\
\hline 202-Decant \#5 & 57 & $\mathrm{NO}$ & - & Low & - & - & 7.89 & 23.51 & 965.9 & 4.31 & -15.177 & 252.49 & 60.98 \\
\hline 202-Decant \#5 & 58 & $\mathrm{NO}$ & - & Low & - & - & 8.03 & 23.70 & 970.3 & 3.67 & -15.319 & 254.16 & 60.33 \\
\hline 202-Decant \#5 & 59 & $\mathrm{NO}$ & - & Low & - & - & 8.17 & 23.88 & 974.6 & 3.11 & -15.461 & 255.83 & 59.68 \\
\hline 202-Decant \#5 & 60 & $\mathrm{NO}$ & - & Low & - & - & 8.31 & 24.06 & 978.8 & 2.60 & -15.602 & 257.51 & 59.03 \\
\hline
\end{tabular}

Table 4-2 summarizes the MAR assessments for the specific frit/sludge combinations of interest. Also listed in Table 4-2 is the property limiting both the upper and lower WLs. For example, for the Decant \#5 / Frit 202 system, predictions of inhomogeneity limit WLs below 33\% while durability predictions limit access to WLs exceeding $37 \%$.

Given two WLs for the twenty specific frit/sludge combinations of interest (see Table 4-2), a total of 40 glasses were identified. It should be noted that the TTR (Rios-Armstrong 2002b) provided direction to fabricate and test a few glasses outside the acceptable region. This was taken into consideration during the glass selection process. Two SB3 glasses were selected even though 
they would be classified as unacceptable based on model predictions at the MAR. Specifically, SB3-13 (Frit 411 and 50\% linear wash sludge at 25\% WL) and SB3-35 (Frit 411 and Decant \#9 at $25 \% \mathrm{WL}$ ). The MAR predictions (see Appendices $\mathrm{C} 1$ and $\mathrm{C} 2$ ) indicate that both glasses are inhomogeneous at 25\% WL - yet they were included in the SB3 Phase 1 test matrix. For these two systems, glasses at $26 \% \mathrm{WL}$ are predicted to be homogeneous which is reflected in the acceptable PCCS operating window as 26-39 and 26-45\% WL for SB3-13 and SB3-35, respectively.

Herman, Peeler, and Edwards (2002) indicated that Frit 320 would also be evaluated in the Phase 1 study given its classification as a "baseline" frit by Elder (2002). Based on the paper study assessment (Peeler and Edwards 2002), Frit 320 is a viable frit for the more advanced washed sludges (e.g., 75\% linear washing case, Decant \#12 and above). Combining Frit 320 with less washed sludges is typically prohibited by predictions of durability or low viscosity (a direct result of the high alkali concentrations of both the sludge and frit). Given the emphasis on the "lesser" washed sludges and the direction set by the SB3 Technical team, use of Frit 320 would be prohibited for the nominal sludges (Decant \#5 and Decant \#9) being considered. To assess the applicability of the durability model to a Frit 320-based system, two glasses were added to the SB3 Phase 1 test matrix. SB3-41 and SB3-42 are Frit 320-based glasses with the 75\% linear washed sludge at 26 and $41 \% \mathrm{WL}$, respectively. It should be noted that the upper and lower WLs for these two glasses are not based on the MAR criteria but on the less restrictive PAR. Peeler and Edwards (2002) indicate that the WL interval of interest for this system (nominal Frit 320 / $75 \%$ linear washing - see Appendix B of that report for details) is $26-41 \%$. Predictions of homogeneity and low viscosity restrict access to lower and upper WLs respectively. They also indicate that PCCS operational windows based on the PAR tend to reduce in size once the MAR criteria are applied (given the additional uncertainties due to sampling and analytical). ${ }^{9}$ Given the upper WL for this Frit 320 system is low viscosity limited at the PAR, once MAR uncertainties are included, one would expect the upper WL limit to be less than 41\%. The MAR assessments for this system are shown in Appendix C2 and are repeated in Table 4-3. As seen there, the projected operational window for this frit/sludge combination is $26-39 \% \mathrm{WL}$. Therefore, fabrication and testing of SB3-42 (41\% WL) should be "unacceptable" based on MAR criteria.

\footnotetext{
9 Peeler and Edwards (2002) demonstrated that for $\mathrm{T}_{\mathrm{L}}$-limited systems the use of the $1010^{\circ} \mathrm{C}$ PAR criterion for $\mathrm{T}_{\mathrm{L}}$ was "conservative." That is, for systems determined to be $\mathrm{T}_{\mathrm{L}}$-limited based upon a PAR criterion of $1010^{\circ} \mathrm{C}$ for $\mathrm{T}_{\mathrm{L}}$, application of the MAR did not reduce the size of the DWPF operational window.
} 
Table 4-2. Summary of Waste Loading Ranges for Select Frit/Sludge Combinations Using the MAR Criteria.

\begin{tabular}{|c|c|c|c|c|c|c|c|c|c|c|c|}
\hline & & Frit 202 & Frit 409 & Frit 410 & Frit 420 & Frit 421 & Frit 422 & Frit 423 & Frit 411 & Frit 405 & Frit 406 \\
\hline \multirow{3}{*}{ Decant \#5 } & WL (nominal) & $33-37$ & $34-48$ & $33-48$ & $30-43$ & $30-41$ & $31-45$ & $31-43$ & - & - & - \\
\hline & Lower limit & Homog & High Vis & High Vis & Homog & Homog & Homog & Homog & - & - & - \\
\hline & Upper limit & PCT & PCT & PCT & PCT & PCT & Low Vis & Low Vis & - & - & - \\
\hline \multirow[t]{3}{*}{ Decant \#9 } & WL (nominal) & $29-45$ & - & - & $35-47$ & $32-47$ & $31-46$ & $28-46$ & $26-45$ & - & - \\
\hline & Lower limit & Homog & - & - & High Vis & High Vis & High Vis & $\begin{array}{c}\text { High Vis/ } \\
\text { Homog }\end{array}$ & Homog & - & - \\
\hline & Upper limit & $\mathrm{T}_{\mathrm{L}}$ & - & - & $\mathrm{T}_{\mathrm{L}}$ & $\mathrm{T}_{\mathrm{L}}$ & $\mathrm{T}_{\mathrm{L}}$ & $\mathrm{T}_{\mathrm{L}}$ & Low Vis & - & - \\
\hline & & & & & & & & & & & \\
\hline \multirow[t]{3}{*}{$0 \%$ Wash } & WL (nominal) & - & - & - & - & - & - & - & - & $32-43$ & $32-46$ \\
\hline & Lower limit & - & - & - & - & - & - & - & - & Homog & $\begin{array}{c}\text { High Vis/ } \\
\text { Homog }\end{array}$ \\
\hline & Upper limit & - & - & - & - & - & - & - & - & Low Vis & PCT \\
\hline \multirow[t]{3}{*}{$25 \%$ Wash } & WL (nominal) & $31-38$ & - & - & - & - & - & - & - & $30-46$ & $35-50$ \\
\hline & Lower limit & Homog & - & - & - & - & - & - & - & Homog & High Vis \\
\hline & Upper limit & PCT & - & - & - & - & - & - & - & Low Vis & Low Vis \\
\hline \multirow[t]{3}{*}{$50 \%$ Wash } & WL (nominal) & $29-46$ & - & - & - & - & - & - & $26-39$ & - & - \\
\hline & Lower limit & Homog & - & - & - & - & - & - & Homog & - & - \\
\hline & Upper limit & $\mathrm{T}_{\mathrm{L}}$ & - & - & - & - & - & - & PCT & - & - \\
\hline
\end{tabular}


Immobilization Technology Section

WSRC-TR-2002-00549

Savannah River Technology Center

Rev. 0

Westinghouse Savannah River Company

Table 4-3. MAR Assessment for Frit 320 and $75 \%$ Linear Washed Sludge. (from Appendix C2)

\begin{tabular}{|c|c|c|c|c|c|c|c|c|c|c|c|c|c|}
\hline & & \multicolumn{5}{|c|}{ MAR Assessments } & \multicolumn{7}{|c|}{ Property Values } \\
\hline Frit-Washing & $\% \mathrm{WL}$ & Durability & $\mathbf{T}_{\mathrm{L}}$ & Visc & Frit & Homog & $\mathbf{A l}_{2} \mathbf{O}_{3}$ & Alkali & $T_{L}$ & Visc & $\Delta \mathbf{G}_{\mathbf{P}}$ & Homog & Frit \\
\hline 320-75\% Washed & 25 & - & - & - & - & $\mathrm{NO}$ & 4.13 & 19.68 & 735.9 & 45.57 & -12.230 & 210.56 & 80.45 \\
\hline 320-75\% Washed & 26 & - & 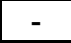 & - & - & 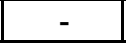 & 4.30 & 19.67 & 751.7 & 43.97 & -12.197 & 212.56 & 79.66 \\
\hline $320-75 \%$ Washed & 27 & - & - & - & - & - & 4.47 & \begin{tabular}{|c|}
19.66 \\
\end{tabular} & 767.0 & 42.39 & -12.163 & 214.57 & 78.88 \\
\hline $320-75 \%$ Washed & 28 & - & - & - & - & - & 4.63 & \begin{tabular}{|c|}
19.65 \\
\end{tabular} & 782.2 & 40.82 & -12.130 & 216.58 & 78.10 \\
\hline $320-75 \%$ Washed & 29 & - & - & - & - & - & 4.80 & 19.63 & 796.9 & 39.27 & -12.097 & 218.59 & 77.32 \\
\hline $320-75 \%$ Washed & 30 & - & - & - & - & - & 4.96 & \begin{tabular}{|c|}
19.62 \\
\end{tabular} & 811.3 & 37.75 & -12.064 & 220.60 & 76.54 \\
\hline $320-75 \%$ Washed & 31 & - & - & - & - & - & 5.13 & \begin{tabular}{|l|}
19.61 \\
\end{tabular} & 825.5 & 36.24 & -12.030 & 222.61 & 75.75 \\
\hline $320-75 \%$ Washed & 32 & - & - & - & - & - & 5.29 & 19.60 & 839.4 & 34.75 & -11.997 & 224.62 & 74.97 \\
\hline 320-75\% Washed & 33 & - & - & - & - & - & 5.46 & $\mid 19.58$ & 853.0 & 33.29 & -11.964 & 226.62 & 74.19 \\
\hline 320-75\% Washed & 34 & - & - & - & - & - & 5.62 & 19.57 & 866.3 & 31.85 & -11.931 & 228.63 & 73.41 \\
\hline 320-75\% Washed & 35 & - & - & - & - & - & 5.79 & 19.56 & 879.4 & 30.43 & -11.897 & 230.64 & 72.63 \\
\hline $320-75 \%$ Washed & 36 & - & - & - & - & - & 5.95 & \begin{tabular}{|l|}
19.55 \\
\end{tabular} & 892.3 & 29.03 & -11.864 & 232.65 & 71.84 \\
\hline $320-75 \%$ Washed & 37 & - & - & - & - & - & 6.12 & \begin{tabular}{|l|}
19.53 \\
\end{tabular} & 904.8 & 27.67 & -11.831 & 234.66 & 71.06 \\
\hline $320-75 \%$ Washed & 38 & - & - & 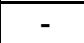 & - & - & 6.29 & 19.52 & 917.2 & 26.32 & -11.797 & 236.67 & 70.28 \\
\hline $320-75 \%$ Washed & 39 & - & - & - & - & - & 6.45 & $\mid 19.51$ & 929.4 & 25.01 & -11.764 & 238.67 & 69.50 \\
\hline $320-75 \%$ Washed & 40 & - & - & Low & - & - & 6.62 & \begin{tabular}{|l|}
19.49 \\
\end{tabular} & 941.3 & 23.72 & -11.731 & 240.68 & 68.71 \\
\hline $320-75 \%$ Washed & 41 & - & - & \begin{tabular}{|l|} 
Low \\
\end{tabular} & - & 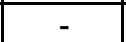 & 6.78 & \begin{tabular}{|l|}
19.48 \\
\end{tabular} & 953.0 & 22.46 & \begin{tabular}{|l|}
-11.698 \\
\end{tabular} & 242.69 & 67.93 \\
\hline 320-75\% Washed & 42 & - & - & Low & - & - & 6.95 & $\mid 19.47$ & 964.5 & 21.24 & -11.664 & 244.70 & 67.15 \\
\hline 320-75\% Washed & 43 & - & - & Low & - & - & 7.11 & \begin{tabular}{|l|}
19.46 \\
\end{tabular} & 975.9 & 20.04 & -11.631 & 246.71 & 66.37 \\
\hline 320-75\% Washed & 44 & - & - & Low & - & - & 7.28 & 19.44 & 987.0 & 18.87 & -11.598 & 248.72 & 65.59 \\
\hline 320-75\% Washed & 45 & - & - & Low & - & - & 7.44 & \begin{tabular}{|c|}
19.43 \\
\end{tabular} & 997.9 & 17.73 & -11.565 & 250.72 & 64.80 \\
\hline $320-75 \%$ Washed & 46 & - & - & Low & - & - & 7.61 & 19.42 & 1008.6 & 16.63 & -11.532 & 252.73 & 64.02 \\
\hline $320-75 \%$ Washed & 47 & - & $\mathrm{NO}$ & Low & - & - & 7.77 & 19.41 & 1019.2 & 15.56 & -11.498 & 254.74 & 63.24 \\
\hline 320-75\% Washed & 48 & - & $\mathrm{NO}$ & Low & - & - & 7.94 & \begin{tabular}{|c|}
19.39 \\
\end{tabular} & 1029.6 & 14.53 & -11.465 & 256.75 & 62.46 \\
\hline 320-75\% Washed & 49 & - & $\mathrm{NO}$ & Low & - & - & 8.10 & \begin{tabular}{|l|l|}
19.38 \\
\end{tabular} & 1039.8 & 13.53 & -11.432 & 258.76 & 61.68 \\
\hline $320-75 \%$ Washed & 50 & - & $\mathrm{NO}$ & \begin{tabular}{|l|} 
Low \\
\end{tabular} & - & - & 8.27 & $\mid 19.37$ & 1049.8 & 12.57 & \begin{tabular}{|l|}
-11.398 \\
\end{tabular} & 260.77 & 60.89 \\
\hline 320-75\% Washed & 51 & - & $\mathrm{NO}$ & Low & - & - & 8.44 & $\mid 19.36$ & 1059.7 & 11.64 & -11.365 & 262.77 & 60.11 \\
\hline $320-75 \%$ Washed & 52 & - & $\mathrm{NO}$ & Low & - & - & 8.60 & \begin{tabular}{|l|}
19.34 \\
\end{tabular} & 1069.4 & 10.75 & -11.332 & 264.78 & 59.33 \\
\hline $320-75 \%$ Washed & 53 & - & $\mathrm{NO}$ & Low & - & - & 8.77 & \begin{tabular}{|l|}
19.33 \\
\end{tabular} & 1079.1 & 9.90 & -11.299 & 266.79 & 58.55 \\
\hline 320-75\% Washed & 54 & - & $\mathrm{NO}$ & Low & - & - & 8.93 & $\mid 19.32$ & 1088.5 & 9.08 & -11.265 & 268.80 & 57.76 \\
\hline $320-75 \%$ Washed & 55 & - & $\mathrm{NO}$ & Low & - & - & 9.10 & \begin{tabular}{|l|}
19.30 \\
\end{tabular} & 1097.7 & 8.31 & -11.232 & 270.81 & 56.98 \\
\hline 320-75\% Washed & 56 & - & $\mathrm{NO}$ & Low & - & - & 9.26 & \begin{tabular}{|c|}
19.29 \\
\end{tabular} & 1106.9 & 7.57 & -11.199 & 272.82 & 56.20 \\
\hline 320-75\% Washed & 57 & - & $\mathrm{NO}$ & Low & - & - & 9.43 & \begin{tabular}{|l|}
19.28 \\
\end{tabular} & 1115.9 & 6.87 & $\begin{array}{l}-11.165 \\
\end{array}$ & 274.82 & 55.42 \\
\hline $320-75 \%$ Washed & 58 & - & $\mathrm{NO}$ & Low & - & 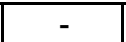 & 9.59 & \begin{tabular}{|l|}
19.27 \\
\end{tabular} & 1124.7 & 6.21 & -11.132 & 276.83 & 54.64 \\
\hline $320-75 \%$ Washed & 59 & - & $\mathrm{NO}$ & \begin{tabular}{|l|} 
Low \\
\end{tabular} & - & 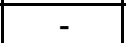 & 9.76 & 19.25 & 1133.4 & 5.59 & \begin{tabular}{|l|}
-11.099 \\
\end{tabular} & 278.84 & 53.85 \\
\hline $320-75 \%$ Washed & 60 & - & NO & Low & - & - & 9.92 & \begin{tabular}{|l|}
19.24 \\
\end{tabular} & 1142.0 & 5.00 & -11.066 & 280.85 & 53.07 \\
\hline
\end{tabular}

Table 4-4 summarizes the basis for the forty-two SB3 Phase 1 glass compositions. Table 4-5 summarizes the targeted compositions. The target compositions shown are a result of combining the nominal sludge (see Table 3-1 and Table 3-2) and frit compositions (see Table 3-3) at the selected upper and lower WLs. 
Table 4-4. SB3 Phase 1 Glass Identifiers and Compositional Basis.

\begin{tabular}{|c|c|c|}
\hline Glass Identification & Basis & Property Constraint $^{10}$ \\
\hline SB3-1 & Frit 405, 0\% Wash, 32\% WL & Homogeneity \\
\hline SB3-2 & Frit 405, 0\% Wash, 43\% WL & Low Viscosity \\
\hline SB3-3 & Frit 406, 0\% Wash, 32\% WL & High Viscosity / Homogeneous \\
\hline SB3-4 & Frit 406, 0\% Wash, 46\% WL & PCT \\
\hline SB3-5 & Frit 202, 25\% Wash, 31\% WL & Homogeneity \\
\hline SB3-6 & Frit 202, 25\% Wash, 38\% WL & PCT \\
\hline SB3-7 & Frit $405,25 \%$ Wash, $30 \%$ WL & Homogeneity \\
\hline SB3-8 & Frit $405,25 \%$ Wash, $46 \%$ WL & Low Viscosity \\
\hline SB3-9 & Frit 406, 25\% Wash, 35\% WL & High Viscosity \\
\hline SB3-10 & Frit 406, 25\% Wash, 50\% WL & Low Viscosity \\
\hline SB3-11 & Frit 202, 50\% Wash, 29\% WL & Homogeneity \\
\hline SB3-12 & Frit $202,50 \%$ Wash, $46 \%$ WL & $\mathrm{T}_{\mathrm{L}}$ \\
\hline SB3-13 & Frit $411,50 \%$ Wash, $25 \%$ WL & Homogeneity \\
\hline SB3-14 & Frit $411,50 \%$ Wash, 39\% WL & PCT \\
\hline SB3-15 & Frit 202, Decant \#5, 33\% WL & Homogeneity \\
\hline SB3-16 & Frit 202, Decant \#5, 37\% WL & PCT \\
\hline SB3-17 & Frit 420, Decant \#5, 30\% WL & Homogeneity \\
\hline SB3-18 & Frit 420, Decant \#5, 43\% WL & PCT \\
\hline SB3-19 & Frit 421, Decant \#5, 30\% WL & Homogeneity \\
\hline SB3-20 & Frit 421, Decant \#5, 41\% WL & PCT \\
\hline SB3-21 & Frit 409, Decant \#5, 34\% WL & High Viscosity \\
\hline SB3-22 & Frit 409, Decant \#5, 48\% WL & PCT \\
\hline SB3-23 & Frit 410, Decant \#5, 33\% WL & High Viscosity \\
\hline SB3-24 & Frit 410, Decant \#5, 48\% WL & PCT \\
\hline SB3-25 & Frit 422, Decant \#5, 31\% WL & Homogeneity \\
\hline SB3-26 & Frit 422, Decant \#5, 45\% WL & Low Viscosity \\
\hline SB3-27 & Frit 423, Decant \#5, 31\% WL & Homogeneity \\
\hline SB3-28 & Frit 423, Decant \#5, 43\% WL & Low Viscosity \\
\hline SB3-29 & Frit 202, Decant \#9, 29\% WL & Homogeneity \\
\hline SB3-30 & Frit 202, Decant \#9, 45\% WL & $\mathrm{T}_{\mathrm{L}}$ \\
\hline SB3-31 & Frit 420, Decant \#9, 35\% WL & High Viscosity \\
\hline SB3-32 & Frit 420, Decant \#9, 47\% WL & $\mathrm{T}_{\mathrm{L}}$ \\
\hline SB3-33 & Frit 421, Decant \#9, 32\% WL & High Viscosity \\
\hline SB3-34 & Frit 421, Decant \#9, 47\% WL & $\mathrm{T}_{\mathrm{L}}$ \\
\hline SB3-35 & Frit 411, Decant \#9, 25\% WL & Homogeneity \\
\hline SB3-36 & Frit 411, Decant \#9, 45\% WL & Low Viscosity \\
\hline SB3-37 & Frit 422, Decant \#9, 31\% WL & High Viscosity \\
\hline SB3-38 & Frit 422, Decant $\# 9,46 \% \mathrm{WL}$ & $\mathrm{T}_{\mathrm{L}}$ \\
\hline SB3-39 & Frit 423, Decant $\# 9,28 \% \mathrm{WL}$ & High Viscosity / Homogeneous \\
\hline SB3-40 & Frit 423, Decant $\# 9,46 \% \mathrm{WL}$ & $\mathrm{T}_{\mathrm{L}}$ \\
\hline SB3-41 & Frit $320,75 \%$ Wash, $26 \%$ WL & Homogeneity \\
\hline SB3-42 & Frit 320, 75\% Wash, $41 \%$ WL & Low Viscosity \\
\hline
\end{tabular}

${ }^{10}$ The property listed is the constraint that restricts access to higher (for the maximum WL) or lower (for the minimum WL) waste loadings (from Table 4-2). 
Table 4-5. Target Glass Compositions for the Forty-Two SB3 Phase 1 Variability Study Glasses. (in wt $\%$ on an calcined oxide basis)

\begin{tabular}{|c|c|c|c|c|c|c|c|c|c|c|}
\hline Oxide & "SB3-1 & "SB3-2 & "SB3-3 & "SB3-4 & SB3-5 & SB3-6 & "SB3-7 & SB3-8 & "SB3-9 & $\overline{\text { SB3-10 }}$ \\
\hline $\mathrm{Al}_{2} \mathrm{O}_{3}$ & 4.250 & 5.711 & 4.250 & 6.109 & 4.424 & 5.423 & 4.281 & 6.564 & 4.994 & 7.135 \\
\hline $\mathrm{B}_{2} \mathrm{O}_{3}$ & 13.600 & 11.400 & 10.200 & 8.100 & 5.520 & 4.960 & 14.000 & 10.8 & 9.750 & 7.500 \\
\hline $\mathrm{CaO}$ & 0.845 & 1.136 & 0.845 & 1.215 & 0.880 & 1.078 & 0.851 & 1.305 & 0.993 & 1.419 \\
\hline $\mathrm{Cr}_{2} \mathrm{O}_{3}$ & 0.088 & 0.118 & 0.088 & 0.126 & 0.091 & 0.112 & 0.088 & 0.136 & 0.103 & 0.147 \\
\hline $\mathrm{Fe}_{2} \mathrm{O}_{3}$ & 9.455 & 12.705 & 9.455 & 13.591 & 9.841 & 12.063 & 9.523 & 14.603 & 11.111 & 15.872 \\
\hline $\mathrm{K}_{2} \mathrm{O}$ & 0.101 & 0.136 & 0.101 & 0.145 & 0.105 & 0.129 & 0.102 & 0.156 & 0.119 & 0.170 \\
\hline $\mathrm{Li}_{2} \mathrm{O}$ & 3.401 & 2.851 & 3.401 & 2.701 & 4.831 & 4.341 & 3.501 & 2.701 & 3.251 & 2.501 \\
\hline $\mathrm{MnO}$ & 1.685 & 2.264 & 1.685 & 2.422 & 1.754 & 2.150 & 1.697 & 2.602 & 1.980 & 2.829 \\
\hline $\mathrm{Na}_{2} \mathrm{O}$ & 11.364 & 15.270 & 11.364 & 16.335 & 13.661 & 15.390 & 9.213 & 14.127 & 10.749 & 15.356 \\
\hline $\mathrm{NiO}$ & 0.378 & 0.508 & 0.378 & 0.544 & 0.394 & 0.483 & 0.381 & 0.584 & 0.445 & 0.635 \\
\hline $\mathrm{SiO}_{2}$ & 51.785 & 43.805 & 55.185 & 44.328 & 53.947 & 48.742 & 53.291 & 41.712 & 52.922 & 41.318 \\
\hline $\mathrm{ThO}_{2}$ & 0.034 & 0.045 & 0.034 & 0.048 & 0.035 & 0.043 & 0.034 & 0.052 & 0.039 & 0.056 \\
\hline $\mathrm{TiO}_{2}$ & 0.000 & 0.000 & 0.000 & 0.000 & 0.000 & 0.000 & 0.000 & 0.000 & 0.000 & 0.000 \\
\hline $\mathrm{U}_{3} \mathrm{O}_{8}$ & 2.392 & 3.214 & 2.392 & 3.438 & 2.490 & 3.052 & 2.409 & 3.694 & 2.811 & 4.016 \\
\hline $\mathrm{ZnO}$ & 0.097 & 0.130 & 0.097 & 0.139 & 0.100 & 0.123 & 0.097 & 0.149 & 0.113 & 0.162 \\
\hline $\mathrm{ZrO}_{2}$ & 0.174 & 0.234 & 0.174 & 0.251 & 0.182 & 0.223 & 0.176 & 0.269 & 0.205 & 0.293 \\
\hline $\mathrm{BaO}$ & 0.059 & 0.079 & 0.059 & 0.084 & 0.061 & 0.075 & 0.059 & 0.091 & 0.069 & 0.099 \\
\hline $\mathrm{Ce}_{2} \mathrm{O}_{3}$ & 0.083 & 0.112 & 0.083 & 0.120 & 0.087 & 0.106 & 0.084 & 0.129 & 0.098 & 0.140 \\
\hline $\mathrm{CuO}$ & 0.047 & 0.063 & 0.047 & 0.067 & 0.048 & 0.059 & 0.047 & 0.072 & 0.055 & 0.078 \\
\hline $\mathrm{La}_{2} \mathrm{O}_{3}$ & 0.048 & 0.065 & 0.048 & 0.069 & 0.050 & 0.062 & 0.049 & 0.075 & 0.057 & 0.081 \\
\hline $\mathrm{MgO}$ & 0.044 & 0.060 & 0.044 & 0.064 & 1.426 & 1.296 & 0.045 & 0.068 & 0.052 & 0.074 \\
\hline $\mathrm{PbO}$ & 0.071 & 0.095 & 0.071 & 0.102 & 0.074 & 0.091 & 0.072 & 0.11 & 0.083 & 0.119 \\
\hline Total & 100.00 & 100.00 & 100.00 & 100.00 & 100.00 & 100.00 & 100.00 & 100.00 & 100.00 & 100.00 \\
\hline
\end{tabular}


Table 4-5. Target Glass Compositions for the Forty-Two SB3 Phase 1 Variability Study Glasses. (continued) (in wt \% on an calcined oxide basis)

\begin{tabular}{|c|c|c|c|c|c|c|c|c|c|c|}
\hline Oxide & SB3-11 & SB3-12 & SB3-13 & SB3-14 & SB3-15 & SB3-16 & SB3-17 & SB3-18 & SB3-19 & SB3-20 \\
\hline $\mathrm{Al}_{2} \mathrm{O}_{3}$ & 4.471 & 7.092 & 4.604 & 6.623 & 4.586 & 5.142 & 4.869 & 6.546 & 4.869 & 6.288 \\
\hline $\mathrm{B}_{2} \mathrm{O}_{3}$ & 5.680 & 4.320 & 6.000 & 4.880 & 5.360 & 5.040 & 5.600 & 4.560 & 7.000 & 5.900 \\
\hline $\mathrm{CaO}$ & 0.889 & 1.410 & 0.766 & 1.196 & 0.912 & 1.023 & 0.829 & 1.189 & 0.829 & 1.134 \\
\hline $\mathrm{Cr}_{2} \mathrm{O}_{3}$ & 0.092 & 0.147 & 0.080 & 0.124 & 0.094 & 0.105 & 0.085 & 0.122 & 0.085 & 0.117 \\
\hline $\mathrm{Fe}_{2} \mathrm{O}_{3}$ & 9.946 & 15.777 & 8.575 & 13.376 & 10.197 & 11.433 & 9.270 & 13.287 & 9.270 & 12.669 \\
\hline $\mathrm{K}_{2} \mathrm{O}$ & 0.106 & 0.169 & 0.092 & 0.143 & 0.109 & 0.122 & 0.099 & 0.142 & 0.099 & 0.136 \\
\hline $\mathrm{Li}_{2} \mathrm{O}$ & 4.971 & 3.781 & 3.751 & 3.051 & 4.690 & 4.410 & 3.500 & 2.850 & 3.500 & 2.950 \\
\hline $\mathrm{MnO}$ & 1.773 & 2.812 & 1.528 & 2.384 & 1.819 & 2.039 & 1.653 & 2.370 & 1.653 & 2.260 \\
\hline $\mathrm{Na}_{2} \mathrm{O}$ & 11.550 & 14.804 & 15.285 & 17.124 & 14.357 & 15.370 & 14.297 & 17.459 & 14.297 & 16.973 \\
\hline $\mathrm{NiO}$ & 0.398 & 0.631 & 0.343 & 0.535 & 0.408 & 0.457 & 0.371 & 0.531 & 0.371 & 0.507 \\
\hline $\mathrm{SiO}_{2}$ & 55.496 & 42.890 & 56.212 & 46.251 & 52.121 & 49.105 & 55.783 & 45.722 & 54.383 & 46.090 \\
\hline $\mathrm{ThO}_{2}$ & 0.035 & 0.056 & 0.030 & 0.048 & 0.036 & 0.041 & 0.033 & 0.047 & 0.033 & 0.045 \\
\hline $\mathrm{TiO}_{2}$ & 0.000 & 0.000 & 0.000 & 0.000 & 0.714 & 0.800 & 0.649 & 0.930 & 0.649 & 0.887 \\
\hline $\mathrm{U}_{3} \mathrm{O}_{8}$ & 2.516 & 3.991 & 2.169 & 3.384 & 2.581 & 2.894 & 2.346 & 3.363 & 2.346 & 3.207 \\
\hline $\mathrm{ZnO}$ & 0.102 & 0.161 & 0.088 & 0.137 & 0.104 & 0.117 & 0.095 & 0.136 & 0.095 & 0.129 \\
\hline $\mathrm{ZrO}_{2}$ & 0.184 & 0.291 & 0.158 & 0.247 & 0.188 & 0.211 & 0.171 & 0.245 & 0.171 & 0.234 \\
\hline $\mathrm{BaO}$ & 0.062 & 0.098 & 0.053 & 0.083 & 0.063 & 0.071 & 0.058 & 0.083 & 0.058 & 0.079 \\
\hline $\mathrm{Ce}_{2} \mathrm{O}_{3}$ & 0.088 & 0.139 & 0.076 & 0.118 & 0.088 & 0.099 & 0.080 & 0.115 & 0.080 & 0.110 \\
\hline $\mathrm{CuO}$ & 0.049 & 0.078 & 0.042 & 0.066 & 0.050 & 0.056 & 0.046 & 0.065 & 0.046 & 0.062 \\
\hline $\mathrm{La}_{2} \mathrm{O}_{3}$ & 0.051 & 0.081 & 0.044 & 0.068 & 0.058 & 0.065 & 0.053 & 0.076 & 0.053 & 0.072 \\
\hline $\mathrm{MgO}$ & 1.467 & 1.154 & 0.040 & 0.063 & 1.388 & 1.314 & 0.043 & 0.062 & 0.043 & 0.059 \\
\hline $\mathrm{PbO}$ & 0.075 & 0.118 & 0.064 & 0.100 & 0.076 & 0.085 & 0.069 & 0.099 & 0.069 & 0.094 \\
\hline Total & 100.00 & 100.00 & 100.00 & 100.00 & 100.00 & 100.00 & 100.00 & 100.00 & 100.00 & 100.00 \\
\hline
\end{tabular}


Table 4-5. Target Glass Compositions for the Forty-Two SB3 Phase 1 Variability Study Glasses. (continued) (in wt \% on an calcined oxide basis)

\begin{tabular}{|c|c|c|c|c|c|c|c|c|c|c|}
\hline Oxide & SB3-21 & SB3-22 & SB3-23 & SB3-24 & SB3-25 & SB3-26 & SB3-27 & SB3-28 & SB3-29 & SB3-30 \\
\hline $\mathrm{Al}_{2} \mathrm{O}_{3}$ & 4.725 & 6.670 & 4.586 & 6.670 & 4.308 & 6.253 & 4.308 & 5.976 & 4.504 & 6.990 \\
\hline $\mathrm{B}_{2} \mathrm{O}_{3}$ & 5.280 & 4.160 & 5.360 & 4.160 & 5.520 & 4.400 & 6.900 & 5.700 & 5.680 & 4.400 \\
\hline $\mathrm{CaO}$ & 0.940 & 1.327 & 0.912 & 1.327 & 0.857 & 1.244 & 0.857 & 1.189 & 0.896 & 1.391 \\
\hline $\mathrm{Cr}_{2} \mathrm{O}_{3}$ & 0.097 & 0.136 & 0.094 & 0.136 & 0.088 & 0.128 & 0.088 & 0.122 & 0.092 & 0.143 \\
\hline $\mathrm{Fe}_{2} \mathrm{O}_{3}$ & 10.506 & 14.832 & 10.197 & 14.832 & 9.579 & 13.905 & 9.579 & 13.287 & 10.016 & 15.542 \\
\hline $\mathrm{K}_{2} \mathrm{O}$ & 0.112 & 0.159 & 0.109 & 0.159 & 0.103 & 0.149 & 0.103 & 0.142 & 0.107 & 0.166 \\
\hline $\mathrm{Li}_{2} \mathrm{O}$ & 2.640 & 2.080 & 3.350 & 2.600 & 5.520 & 4.400 & 5.520 & 4.560 & 4.970 & 3.850 \\
\hline $\mathrm{MnO}$ & 1.874 & 2.645 & 1.819 & 2.645 & 1.709 & 2.480 & 1.709 & 2.370 & 1.786 & 2.772 \\
\hline $\mathrm{Na}_{2} \mathrm{O}$ & 14.610 & 18.155 & 13.687 & 17.635 & 11.780 & 15.745 & 11.780 & 15.179 & 11.000 & 13.758 \\
\hline $\mathrm{NiO}$ & 0.420 & 0.593 & 0.408 & 0.593 & 0.383 & 0.556 & 0.383 & 0.531 & 0.401 & 0.621 \\
\hline $\mathrm{SiO}_{2}$ & 54.667 & 43.412 & 55.471 & 43.412 & 56.389 & 45.274 & 55.009 & 45.722 & 55.192 & 43.159 \\
\hline $\mathrm{ThO}_{2}$ & 0.037 & 0.053 & 0.036 & 0.053 & 0.034 & 0.049 & 0.034 & 0.047 & 0.036 & 0.055 \\
\hline $\mathrm{TiO}_{2}$ & 0.735 & 1.038 & 0.714 & 1.038 & 0.670 & 0.973 & 0.670 & 0.930 & 0.701 & 1.088 \\
\hline $\mathrm{U}_{3} \mathrm{O}_{8}$ & 2.659 & 3.754 & 2.581 & 3.754 & 2.425 & 3.519 & 2.425 & 3.363 & 2.535 & 3.934 \\
\hline $\mathrm{ZnO}$ & 0.107 & 0.152 & 0.104 & 0.152 & 0.098 & 0.142 & 0.098 & 0.136 & 0.102 & 0.159 \\
\hline $\mathrm{ZrO}_{2}$ & 0.194 & 0.274 & 0.188 & 0.274 & 0.177 & 0.257 & 0.177 & 0.245 & 0.185 & 0.287 \\
\hline $\mathrm{BaO}$ & 0.065 & 0.092 & 0.063 & 0.092 & 0.060 & 0.087 & 0.060 & 0.083 & 0.062 & 0.097 \\
\hline $\mathrm{Ce}_{2} \mathrm{O}_{3}$ & 0.091 & 0.129 & 0.088 & 0.129 & 0.083 & 0.121 & 0.083 & 0.115 & 0.087 & 0.135 \\
\hline $\mathrm{CuO}$ & 0.052 & 0.073 & 0.050 & 0.073 & 0.047 & 0.069 & 0.047 & 0.065 & 0.049 & 0.077 \\
\hline $\mathrm{La}_{2} \mathrm{O}_{3}$ & 0.060 & 0.085 & 0.058 & 0.085 & 0.055 & 0.079 & 0.055 & 0.076 & 0.057 & 0.089 \\
\hline $\mathrm{MgO}$ & 0.049 & 0.070 & 0.048 & 0.070 & 0.045 & 0.065 & 0.045 & 0.062 & 1.467 & 1.173 \\
\hline $\mathrm{PbO}$ & 0.078 & 0.111 & 0.076 & 0.111 & 0.071 & 0.104 & 0.071 & 0.099 & 0.075 & 0.116 \\
\hline Total & 100.00 & 100.00 & 100.00 & 100.00 & 100.00 & 100.00 & 100.00 & 100.00 & 100.00 & 100.00 \\
\hline
\end{tabular}


Table 4-5. Target Glass Compositions for the Forty-Two SB3 Phase 1 Variability Study Glasses. (continued) (in wt \% on an calcined oxide basis)

\begin{tabular}{|c|c|c|c|c|c|c|c|c|c|c|c|c|}
\hline Oxide & SB3-31 & SB3-32 & SB3-33 & SB3-34 & SB3-35 & SB3-36 & SB3-37 & SB3-38 & SB3-39 & SB3-40 & SB3-41 & SB3-42 \\
\hline $\mathrm{Al}_{2} \mathrm{O}_{3}$ & 6.086 & 7.830 & 5.650 & 7.830 & 4.633 & 7.540 & 4.815 & 7.145 & 4.349 & 7.145 & 4.359 & 6.874 \\
\hline $\mathrm{B}_{2} \mathrm{O}_{3}$ & 5.200 & 4.240 & 6.800 & 5.300 & 6.000 & 4.400 & 5.520 & 4.320 & 7.200 & 5.400 & 5.920 & 4.720 \\
\hline $\mathrm{CaO}$ & 1.082 & 1.452 & 0.989 & 1.452 & 0.773 & 1.391 & 0.958 & 1.422 & 0.865 & 1.422 & 0.867 & 1.367 \\
\hline $\mathrm{Cr}_{2} \mathrm{O}_{3}$ & 0.111 & 0.149 & 0.102 & 0.149 & 0.079 & 0.143 & 0.098 & 0.146 & 0.089 & 0.146 & 0.090 & 0.142 \\
\hline $\mathrm{Fe}_{2} \mathrm{O}_{3}$ & 12.088 & 16.233 & 11.052 & 16.233 & 8.634 & 15.542 & 10.707 & 15.887 & 9.671 & 15.887 & 9.697 & 15.292 \\
\hline $\mathrm{K}_{2} \mathrm{O}$ & 0.129 & 0.174 & 0.118 & 0.174 & 0.092 & 0.166 & 0.115 & 0.170 & 0.103 & 0.170 & 0.104 & 0.163 \\
\hline $\mathrm{Li}_{2} \mathrm{O}$ & 3.250 & 2.650 & 3.400 & 2.650 & 3.750 & 2.750 & 5.520 & 4.320 & 5.760 & 4.320 & 5.921 & 4.721 \\
\hline $\mathrm{MnO}$ & 2.156 & 2.895 & 1.971 & 2.895 & 1.540 & 2.772 & 1.910 & 2.834 & 1.725 & 2.834 & 1.728 & 2.725 \\
\hline $\mathrm{Na}_{2} \mathrm{O}$ & 12.684 & 14.633 & 12.197 & 14.633 & 14.810 & 17.058 & 9.274 & 12.310 & 8.667 & 12.310 & 13.714 & 14.702 \\
\hline $\mathrm{NiO}$ & 0.483 & 0.649 & 0.442 & 0.649 & 0.345 & 0.621 & 0.428 & 0.635 & 0.387 & 0.635 & 0.388 & 0.612 \\
\hline $\mathrm{SiO}_{2}$ & 51.979 & 42.715 & 52.936 & 41.655 & 55.950 & 41.509 & 56.448 & 44.567 & 57.384 & 43.487 & 54.085 & 43.750 \\
\hline $\mathrm{ThO}_{2}$ & 0.043 & 0.058 & 39 & 0.058 & 0.031 & 0.055 & 0.038 & 0.057 & 0.034 & 0.057 & 0.034 & 0.054 \\
\hline $\mathrm{TiO}_{2}$ & 0.846 & 1.136 & 0.773 & 1.136 & 0.604 & 1.088 & 0.749 & 1.112 & 0.677 & 1.112 & 0.000 & 0.000 \\
\hline $\mathrm{U}_{3} \mathrm{O}_{8}$ & 3.060 & 4.109 & 2.797 & 4.109 & 2.185 & 3.934 & 2.710 & 4.021 & 2.448 & 4.021 & 2.453 & 3.869 \\
\hline $\mathrm{ZnO}$ & 0.124 & 0.166 & 0.113 & 0.166 & 0.088 & 0.159 & 0.109 & 0.162 & 0.099 & 0.162 & 0.099 & 0.156 \\
\hline $\mathrm{ZrO}_{2}$ & 0.223 & 0.300 & 0.204 & 0.300 & 0.159 & 0.287 & 0.198 & 0.293 & 0.179 & 0.293 & 0.179 & 0.282 \\
\hline $\mathrm{BaO}$ & 0.075 & 0.101 & 0.069 & 0.101 & 0.054 & 0.097 & 0.067 & 0.099 & 0.060 & 0.099 & 0.060 & 0.095 \\
\hline $\mathrm{Ce}_{2} \mathrm{O}_{3}$ & 0.105 & 0.141 & 0.096 & 0.141 & 0.075 & 0.135 & 0.093 & 0.138 & 0.084 & 0.138 & 0.086 & 0.135 \\
\hline $\mathrm{CuO}$ & 0.060 & 0.080 & 0.054 & 0.080 & 0.043 & 0.077 & 0.053 & 0.078 & 0.048 & 0.078 & 0.048 & 0.075 \\
\hline $\mathrm{La}_{2} \mathrm{O}_{3}$ & 0.069 & 0.093 & 0.063 & 0.093 & 0.049 & 0.089 & 0.061 & 0.091 & 0.055 & 0.091 & 0.049 & 0.078 \\
\hline $\mathrm{MgO}$ & 0.057 & 0.076 & 0.052 & 0.076 & 0.040 & 0.073 & 0.050 & 0.074 & 0.045 & 0.074 & 0.045 & 0.072 \\
\hline $\mathrm{PbO}$ & 0.090 & 0.121 & 0.082 & 0.121 & 0.064 & 0.116 & 0.080 & 0.118 & 0.072 & 0.118 & 0.073 & 0.115 \\
\hline Total & 100.00 & 100.00 & 100.00 & 100.00 & 100.00 & 100.00 & 100.00 & 100.00 & 100.00 & 100.00 & 100.00 & 100.00 \\
\hline
\end{tabular}




\subsection{Experimental}

This section describes the experimental procedures used to fabricate and the analytical techniques used to physically and chemically characterize the SB3 Phase 1 Variability Study glasses.

\subsection{Glass Fabrication}

Table 4-5 identified the targeted compositions of the 42 SB3 glasses prepared for this study. Each glass was prepared from the proper proportions of reagent-grade metal oxides, carbonates, $\mathrm{H}_{3} \mathrm{BO}_{3}$, and salts in 150-g batches using SRTC technical procedure "Glass Batching - ITS-0001" (SRTC 2002a). Batch sheets were filled out as the materials were weighed. ${ }^{11}$ Once batched, the glasses were melted using SRTC technical procedure "Glass Melting - ITS-0003" (SRTC $2002 \mathrm{~b}$ ). In general, the raw materials were thoroughly mixed and placed into a $95 \%$ Platinum $/ 5 \%$ Gold 250-mL crucible. The batch was subsequently placed into a high-temperature furnace, and the temperature was increased at $\sim 8^{\circ} \mathrm{C} / \mathrm{min}$ until the target melt temperature $\left(1150^{\circ} \mathrm{C}\right)$ was reached. After an isothermal hold at $1150^{\circ} \mathrm{C}$ for $1.0 \mathrm{~h}$, the crucible was removed, and the glass was poured onto a clean stainless steel plate and allowed to air cool. Observations of the resulting pour patty and residual crucible glass were documented.

The pour patty and residual crucible glass were ground, and the crushed glass was subsequently transferred to its original $95 \%$ Platinum $/ 5 \%$ Gold $250-\mathrm{mL}$ crucible for a second melt at $1150^{\circ} \mathrm{C}$. After an isothermal hold at $1150^{\circ} \mathrm{C}$ for $1.0 \mathrm{~h}$, the crucible was removed, and the glass was poured onto a clean stainless steel plate and allowed to air cool. Observations of the resulting pour patty and residual crucible glass were documented. ${ }^{12}$ Approximately $140 \mathrm{~g}$ of glass was removed (poured) from the crucible while $\sim 10 \mathrm{~g}$ remained in the crucible along the walls. The pour patty was used as a sampling stock for the various heat treatments and property measurements (i.e., chemical composition and durability). Glasses were stored in marked containers (using the unique SB3 Phase 1 nomenclature as defined in Table 4-5).

To bound the effects of thermal history on the product performance, approximately $25 \mathrm{~g}$ of each SB3 glass were heat treated to simulate cooling along the centerline of a DWPF-type canister (Marra and Jantzen 1993). This cooling regime is commonly referred to as the centerline canister cooled (ccc) curve. This terminology will be used in this report to differentiate samples from different cooling regimes (quenched versus $\mathrm{ccc}$ ).

\subsection{Property Measurements}

This section provides a general discussion of the analysis of chemical compositions and the PCT for each SB3 Phase 1 glass.

\subsubsection{Chemical Composition Analysis}

To confirm that the "as-fabricated" glasses corresponded to the defined target compositions, a representative sample from each SB3 glass pour patty was submitted to the SRTC Mobile Laboratory (SRTC-ML) for chemical analysis. Edwards (see Appendix D) provided an analytical plan that accompanied these samples. This plan identified the cations to be analyzed and the dissolution techniques (i.e., sodium peroxide fusion $[\mathrm{PF}]$ and lithium-metaborate $[\mathrm{LM}]$ ) to be

11 Batch sheets can be found in WSRC-NB-2001-00060.

12 Observations of homogeneity were documented in WSRC-NB-2001-00060 for both the pour patty and the residual crucible glass. No visual signs of undissolved solids or compositional inhomogeneities were observed. 
used. Each glass was prepared in duplicate for the cation dissolution techniques (PF and LM). Concentrations (as mass \%) for the cations of interest were measured by inductively coupled plasma - atomic emission spectroscopy (ICP - AES). The analytical plan was developed in such a way as to provide the opportunity to evaluate potential sources of error. Glass standards were intermittently run to assess the performance of the ICP - AES over the course of these analyses and for potential bias-correction needs. The measurements were conducted to assess whether or not the targeted glass compositions were adequately met (see Section 6.1).

\subsubsection{Product Consistency Test}

The PCT was performed on each glass to assess chemical durability using technical procedure "Nuclear Waste Glass Product Consistency Test (PCT) Method - GTOP-3-025" (ASTM 1998). The PCT was conducted in triplicate for each SB3 glass (both quenched and ccc versions). Also included in this experimental test matrix were the Environmental Assessment (EA) glass (Jantzen et al. 1993), the Approved Reference Material (ARM-1) glass, and blanks from the sample cleaning batch. Samples were ground, washed, and prepared according to procedure. Fifteen $\mathrm{mL}$ of Type I American Society for Testing and Materials (ASTM) water were added to $1.5 \mathrm{~g}$ of glass in stainless steel vessels. The vessels were closed, sealed, and placed in an oven at $90 \pm 2{ }^{\circ} \mathrm{C}$ where the samples were maintained for 7 days. The resulting solutions (once cooled) were sampled (filtered and acidified), labeled (according to the analytical plan), and analyzed. Edwards provided analytical plans for the SRTC-ML analysis (see Appendices E, F, and G). Due to the large number of vessels and limited space in a single PCT oven, three groups of tests were initiated. Groups were primarily based on washing scheme (e.g., Group \#1 - SB3-1 through SB3-14 (both quenched and ccc) for the linear washing scenarios, Group \#2 - SB3-15 through SB3-28 (both quenched and ccc) for Decant \#5, and Group \#3 - SB3-29 through SB3-42 (both quenched and ccc) for Decant \#9) and each group contained the appropriate blanks and glass standards. ${ }^{13}$ The overall philosophy of these plans was to provide an opportunity to assess the consistency (repeatability) of the PCT and analytical procedures in the effort to evaluate chemical durability of the SB3 glasses. Normalized release rates were calculated based on targeted, measured, and bias-corrected compositions using the average of the logs of the leachate concentrations (see Section 6.2).

\subsection{Effect of Redox on PCT Response}

The forty-two Phase 1 Variability Study glasses were batched and melted under conditions that promote oxidizing conditions resulting in fully oxidized glasses. This technique has been utilized in previous DWPF variability studies (Peeler 1996a; Peeler 1996b; Edwards and Brown 1998; Harbour et al. 2000; and Herman et al. 2001) with technical justification given the oxidizing nature of the DWPF flowsheet (e.g., excessive nitric acid). In fact, Brown, Postles, and Edwards (2002) indicate that the majority of elements considered in PCCS possess only a single corresponding oxide as indicated by Schreiber and Hockman (1987) - with one exception copper. DWPF does not currently measure the redox ratio for their SME feed due to highly oxidizing nature of the DWPF melt (i.e., $\left.\left[\mathrm{Fe}^{3+}\right] \gg>\left[\mathrm{Fe}^{2+}\right]\right)$. Therefore, the ratio of $\mathrm{Fe}^{2+} / \mathrm{Fe}^{3+}$ has been assumed to be zero for previous DWPF use. More specifically, currently all Fe is converted to $\mathrm{Fe}_{2} \mathrm{O}_{3}$ for prediction considerations via PCCS.

Given the potential for the atypical components of coal and sodium oxalate (Goslen 1984; Fowler 1980 ) to be present in SB3 (and ultimately transferred to DWPF), redox control becomes a more critical issue. Herman (2002a) and Jantzen (2002) are performing specific studies to assess the impact of these components on the overall flowsheet with the objective of defining new acid

\footnotetext{
${ }^{13}$ Group \#3 also contained SB3-41 and SB3-42 based on Frit 320 and the 75\% linear washing scenario.
} 
addition and redox control strategies for this specific sludge batch if deemed necessary. Control of glass redox is critical to melter life and melter performance.

With a potential change or shift in acid addition or redox control strategies, previous assumptions regarding the impact (or lack thereof) of redox on the PCT response needed to be reassessed. This was specifically identified by Rios-Armstrong (2002b) in the TTR. In this study, two approaches were taken. The first is primarily based on experimental assessments of durability with glasses produced under conditions that bound the acceptable redox range for DWPF. The second approach is strictly based on model predictions of durability for the SB3 Phase 1 glasses considering potential redox impacts. The objective and basis for both approaches are discussed in detail below.

\subsubsection{Experimental Assessment of PCT as a Function of Redox}

Research is currently in progress to develop or augment the acid addition strategy and/or redox correlation for SB3 (Herman (2002a) and Jantzen (2002)). Preliminary studies (Herman 2002b) to support SB3 processing were performed with a Tank 8 simulant to determine the effects of sand, coal, and sodium oxalate on Sludge Receipt and Adjustment Tank (SRAT) and SME processing. SRAT and SME runs were performed with 75, 50, and 25\% of the anticipated levels of sodium oxalate, and the runs were identified as SB3-21, SB3-22, and SB3-23, respectively. Sand and coal were added at the anticipated or nominal levels for the sludge oxides (i.e., 1.12 $\mathrm{wt} \%$ for sand and $0.72 \mathrm{wt} \%$ for coal). The SRAT and SME cycles were performed per run plans written by Koopman (2002a, 2002b, 2002c) and prototyped DWPF SRAT and SME cycles including the addition of canister decontamination water during the SME cycle. The purpose of the runs was to refine the acid addition strategy for SB3 at the different sodium oxalate levels. The runs used the latest acid addition strategy and redox equations to target a $\mathrm{Fe}^{2+} / \mathrm{SFe}$ of 0.20 .

A summary of the test parameters is provided in Table 5-1. The acid addition amounts were based on the liters of starting feed (i.e., sludge slurry with sodium oxalate, trim chemicals, and any flush water - see Herman et al. 2002b). The "\% of Acid Stoichiometry" reflects the amount of acid added relative to the stoichiometric equivalent for the acid addition equation currently being used by DWPF (Koopman (2002a), Koopman (2002b), Koopman (2002c)). Both SB3-21 and SB3-23 contained the nominal levels of noble metals, while SB3-22 contained 10\% of the nominal level of noble metals (Peeler et al. (2002a)). ${ }^{14}$ The varying levels of noble metals required different amounts of acid to be added to meet SRAT processing acceptability (e.g., for nitrite destruction based on the demonstrated noble metals concentration and the nitrite destruction correlation). Based on the results of the testing, the acid addition amount used in Run SB3-21 was overly excessive resulting in the DWPF hydrogen limit to be exceeded. The nitrite was below $1000 \mathrm{mg} / \mathrm{kg}$ within the first 20 minutes of boiling; whereas, a lower level of acid addition may have taken the full boiling time. The other runs also had an overabundance of acid, but they did not result in the hydrogen limit being exceeded. Run SB3-22 had nitrite destroyed to less than $1000 \mathrm{mg} / \mathrm{kg}$ within the first 50 minutes of boiling, while nitrite was also below this level for Run SB3-23 within the first 20 minutes of boiling. The sludge compositions for each run were used to determine the proper frits to use in the SME cycle. Only frits readily available (Frits 202 and 320) were considered in the analyses. Based on this assessment, Frit 202 was selected for Run SB3-21, while Frit 320 was selected for the other runs. The frit selection results are slightly different than those that would be anticipated with actual SB3 simulant, because of the differences in composition of the Tank 8 and SB3 simulants. Different waste loading ranges were determined for each of the sodium oxalate cases/runs. However, to allow for a more equal

\footnotetext{
${ }^{14}$ Nominal concentrations of noble metals are: $\mathrm{Ag}=5.42 \mathrm{E}-04 \mathrm{wt} \% ; \mathrm{Pd}=2.76 \mathrm{E}-02 \mathrm{wt} \%, \mathrm{Rh}=5.11 \mathrm{E}-02 \mathrm{wt} \%$, and $\mathrm{Ru}$ $=1.83 \mathrm{E}-01 \mathrm{wt} \%$.
} 
comparison of the processes, $30 \mathrm{wt} \%$ waste loading was selected for all runs since it represented the midpoint in each frit/sludge system's acceptable waste loading range. The final calcined solids content of the SME products ranged from 40 to $44 \mathrm{wt} \%$. More details on the runs are given in Herman et al. (2003).

Table 5-1. SRAT/SME Parameters.

\begin{tabular}{||c|c|c|c|c|c||}
\hline \hline Run ID & $\begin{array}{c}\text { Noble Metals } \\
\text { Level }\end{array}$ & $\begin{array}{c}\text { Nitric Added } \\
\text { (moles/ L slurry) }\end{array}$ & $\begin{array}{c}\text { Formic Added } \\
\text { (moles/L slurry) }\end{array}$ & $\begin{array}{c}\% \text { of Acid } \\
\text { Stoichiometry }\end{array}$ & $\begin{array}{c}\text { Frit Type and } \\
\text { Amount }\end{array}$ \\
\hline SB3-21-SME & Nominal & 0.41 & 0.73 & $190 \%$ & $70 \mathrm{wt} \%$ Frit 202 \\
\hline SB3-22-SME & $10 \%$ of Nominal & 0.35 & 0.70 & $160 \%$ & $70 \mathrm{wt} \%$ Frit 320 \\
\hline SB3-23-SME & Nominal & 0.29 & 0.68 & $130 \%$ & $70 \mathrm{wt} \%$ Frit 320 \\
\hline
\end{tabular}

Given Herman et al. (2003) used similar nomenclatures for the SME products as were used to identify the SB3 Phase 1 Variability Study glasses, the suffix "-SME" has been added to avoid confusion. It should also be noted that the targeted compositions for SB3-21-SME and SB3-21 (Phase 1 glass) are not the same. The same can be said about SB3-22-SME and SB3-23-SME. As mentioned above, SB3-21-SME was produced with Frit 202 at $30 \mathrm{wt} \% \mathrm{WL}$; while SB3-22SME and SB3-23-SME were produced with Frit 320 at $30 \mathrm{wt} \%$ WL. One of the major differences between the SME products and SB3 Phase 1 glasses is the absence of $\mathrm{ThO}_{2}$ and $\mathrm{U}_{3} \mathrm{O}_{8}$ in the SME products as non-radioactive products are required for testing. ${ }^{15}$ Another difference alluded to above was the use of the Tank 8 simulant versus actual SB3 simulant. Tank 8 simulant contained twice as much $\mathrm{Ni}$, half as much $\mathrm{Mn}$, and slightly less Fe than its SB3 counterpart.

After characterization of the SME products were completed, they were subsequently dried, crushed, and then melted in the dry feed melt rate furnace (MRF). First, enough SME product for each run was measured out to produce approximately 500 grams of glass. The SME product was divided into 2 stainless-steel pans, placed in an oven with a setpoint of $110^{\circ} \mathrm{C}$ for drying (drying to completion), and then crushed and sieved (size $10 \mathrm{mesh}$ ). This sieved material was placed in a beaker for insertion into the MRF.

Lorier and McGrier (2002) provide a detailed discussion regarding the development, intended use, and operating procedures of the MRF. In general, the MRF was designed to mimic the heat transfer characteristics of a large-scale, joule-heated melter. That is, unidirectional heat transfer from the molten glass pool to the cold cap is simulated given this is the primary source of heat to convert the incoming melter feed to a glass product - thus controlling melt rate. The furnace inner chamber is approximately one cubic foot with 1925-watt plate heaters mounted on opposing walls. The insulation consists of approximately 6" of M-board on all sides of the furnace chamber. The top board has a 6" circular cutout through all layers of insulation to hold a 1200 $\mathrm{mL}$ stainless steel beaker and insulating sleeve in place. The tests were conducted with 6" deep stainless steel beakers inserted into the cutout so that the top flange of the beaker was just above the top of the furnace. The beaker bottom was approximately flush with the top of the inner furnace chamber. The furnace was heated to approximately $1150^{\circ} \mathrm{C}$ with the top opening covered with an insulating block. Once the furnace reached the setpoint temperature, the cover was removed and the beaker containing sufficient material to produce $\sim 500$ grams of glass was inserted.

\footnotetext{
${ }^{15}$ It should be reiterated that the Decant \#5 feed used to support the MRF testing program was non-radioactive (Lorier et al. 2003). The two radioactive components removed were $\mathrm{U}_{3} \mathrm{O}_{8}$ and $\mathrm{ThO}_{2}$ which constitute approximately 7.9 wt\% of the Decant \#5 sludge (see Table 3-2). There was no technical basis for spiking the feeds with a nonradioactive simulant. Therefore, the melt rates determined will be considered on a relative basis.
} 
The first run (SB3-21-SME) was performed for approximately 1 hour to assure complete conversion of the batch into a single-phase glass. Incomplete conversion could result in a biased glass sample from which the durability response would be measured. This is slightly longer than the standard 42 minutes typically used to assess melt rate (Lorier and McGrier (2002)) due to the fact that very few SB3 batches had resulted in complete conversion with this standard time. ${ }^{16}$ After 1 hour of melting, SB3-21-SME was completely converted to a single-phase glass and indications (based on thermocouple temperature review) were that the standard 42 minutes would have resulted in complete conversion. Thus, the subsequent two tests utilized or reverted to the 42-minute standard time, which allowed for direct melt rate comparisons between these tests and those being assessed for SB3 (Lorier et al. 2003). Differences in the rate of batch to glass conversion between the Tank 8 samples and the SB3 systems could be due to differences in sludge composition, acid addition strategy, or a combination of both.

It should be noted that the primary objective is not to assess or compare melt rates, but to produce glasses under conditions which mimic melter processing and result in bounding redox values over which PCT assessments can be made. Although the MRF is not a controlled atmosphere, partial oxidation of the melt could occur during the residence time.

After the allotted residence time, the samples were removed from the furnace and allowed to cool within the stainless beaker. Representative glass samples were then obtained and submitted for compositional and redox analysis. The PCT was performed on each Tank 8 glass (in triplicate) according to standard procedures (see Section 5.2.2 for more details).

\subsubsection{Redox Dependent Predictions}

To gain insight into the potential magnitude of the REDOX effect on PCT, predictions of durability for all 42 SB3 Phase 1 Variability Study glasses were made. For each glass, an assumption was made that the targeted $\mathrm{Fe}^{2+} / \Sigma \mathrm{Fe}$ would be 0.2 and controlled via an acid addition strategy below the upper redox acceptance limit as recommended by Schreiber and Hockman (1987) and Jantzen and Plodinec (1986). Based on this work, an acceptable iron redox $\left(\mathrm{Fe}^{2+} / \Sigma \mathrm{Fe}\right)$ range was recommended to be $0.09 \leq \mathrm{Fe}^{2+} / \Sigma \mathrm{Fe} \leq 0.33 .{ }^{17}$

Brown, Postles, and Edwards (2002) provided a revision of the SME acceptability decision criteria that included a redox dependent term for use with the durability model. ${ }^{18}$ More specifically, the model had adjustable redox term(s) that partitioned the redox of select species (e.g., $\mathrm{Fe}$ ) and assigned $\Delta \mathrm{G}_{\mathrm{i}}$ values accordingly per Jantzen et al. (1995). Given this model is available, one can easily predict the PCT response or $\Delta \mathrm{G}_{\mathrm{P}}$ values for the $42 \mathrm{SB} 3$ glasses as a function of redox. In this study, three key $\mathrm{Fe}^{2+} / \Sigma \mathrm{Fe}$ targets are assessed: (1) $\mathrm{Fe}^{2+} / \Sigma \mathrm{Fe}=0.0$ (fully oxidized), (2) $\mathrm{Fe}^{2+} / \Sigma \mathrm{Fe}=0.2$ (assumed target), and (3) $\mathrm{Fe}^{2+} / \Sigma \mathrm{Fe}=0.33$ (upper limit or bounding case). These predictions can then be used to ascertain the potential impact of redox on SME acceptability (e.g., relationship of $\Delta \mathrm{G}_{\mathrm{P}}$ to the DWPF SME acceptability criteria) and ultimately the projected operating window for the various frit/sludge systems of interest for SB3.

\footnotetext{
${ }^{16}$ Lorier et al. (2003) provide a detailed discussion on the study to assess melt rate as a function of frit composition for SB3.

${ }^{17}$ It should be noted that although a lower $\mathrm{Fe}^{2+} / \Sigma \mathrm{Fe}$ limit of 0.09 was recommended, this limit has not been implemented in DWPF.

${ }^{18}$ The modifications of the SME acceptability decision criteria necessary to reflect the effects of redox on the durability model were taken from Brown, K.G., "Changes Made to the DWPF PCCS Spreadsheet (U)," SRTC-GPD2002-00059, May 16, 2002.
} 


\subsection{Results and Discussion}

This section provides a detailed discussion of the chemical-composition measurements and an analysis of the PCT results.

\subsection{A Statistical Review of the Chemical Composition Measurements}

In this section, the measured versus targeted compositions of the 42 SB3 Phase 1 glasses (SB3-01 through SB3-42) are presented and compared. As stated earlier, the targeted compositions for these glasses are provided in Table 4-5. Chemical composition measurements for these glasses were conducted by the SRTC-ML following the analytical plan provided in Appendix D. Two dissolution methods were utilized in this plan: samples prepared by LM dissolution were used to measure elemental concentrations of barium $(\mathrm{Ba})$, calcium $(\mathrm{Ca})$, cerium $(\mathrm{Ce})$, chromium $(\mathrm{Cr})$, copper $(\mathrm{Cu})$, iron $(\mathrm{Fe})$, potassium $(\mathrm{K})$, lanthanum $(\mathrm{La})$, magnesium $(\mathrm{Mg})$, manganese $(\mathrm{Mn})$, sodium $(\mathrm{Na})$, nickel $(\mathrm{Ni})$, lead $(\mathrm{Pb})$, thorium $(\mathrm{Th})$, titanium $(\mathrm{Ti})$, uranium $(\mathrm{U})$, zinc $(\mathrm{Zn})$, and zirconium $(\mathrm{Zr})$ while samples prepared by $\mathrm{PF}$ dissolution were used to measure elemental concentrations of aluminum (Al), boron (B), lithium (Li), and silicon ( $\mathrm{Si}$ ). For each study glass, measurements were obtained from samples prepared in duplicate by each of these dissolution methods. All of the prepared samples were analyzed (twice for each element of interest) by ICP AES (with the instrumentation being re-calibrated between the duplicate analyses).

Table H.1 in Appendix H provides the elemental concentration measurements derived from the samples prepared using LM, and Table H.2 in Appendix H provides the measurements derived from the samples prepared using PF. Measurements for standards (Batch 1 and a uranium standard, $\mathrm{U}_{\text {std }}$, glass), that were included in the SRTC-ML analytical plans along with the SB3 glasses, are also provided in these two tables.

The elemental concentrations were converted to oxide concentrations by multiplying the values for each element by the gravimetric factor for the corresponding oxide. During this process, an elemental concentration that was determined to be below the detection limit of the analytical procedures used by the SRTC-ML was reduced to half of that detection limit as the oxide concentration was determined.

In the sections that follow, the analytical sequence of the measurements is explored, the measurements of the standards are investigated and used for bias correction, the measurements for each glass are reviewed, the average chemical compositions (measured and bias-corrected) for each glass are determined, and comparisons are made between these measurements and the targeted compositions for these glasses.

\subsubsection{Measurements in Analytical Sequence}

Exhibit H.1 in Appendix H provides plots of the measurements generated by the SRTC-ML for samples prepared using the LM method. These plots are in analytical sequence with different symbols and colors being used to represent each of the glasses (study glasses and standards) included in the analytical plan. Similar plots for samples prepared using the PF method are provided in Exhibit H.2 of Appendix H. These plots include all of the measurement data from Tables H.1 and H.2.

A review of these plots indicates no significant patterns or trends in the analytical process over the course of these measurements. No obvious outliers were evident in these chemical 
composition measurements with the exception of a pair of measurements (both from the same LM prep) on the minor components $\mathrm{Ce}_{2} \mathrm{O}_{3}, \mathrm{La}_{2} \mathrm{O}_{3}$, and $\mathrm{ZrO}_{2}$ for one of the $\mathrm{SB} 3$ glasses (SB3-29).

\subsubsection{Batch 1 and Uranium Standard Results}

In this section, the SRTC-ML measurements of the chemical compositions of the Batch 1 and $\mathrm{U}_{\text {std }}$ glasses are reviewed. These measurements are investigated across the ICP analytical blocks, and the results are used to bias correct the measurements for the SB3 Phase1 glasses.

Exhibit H.3 provides statistical analyses of the Batch 1 and $\mathrm{U}_{\text {std }}$ results generated by the LM prep method by analytical block. The results include an analysis of variance (ANOVA) investigation looking for statistically significant differences among the block means for each of the standards. The results from these statistical tests for the Batch 1 standard may be summarized as follows: the $\mathrm{BaO}, \mathrm{CaO}, \mathrm{Cr}_{2} \mathrm{O}_{3}, \mathrm{~K}_{2} \mathrm{O}, \mathrm{MgO}, \mathrm{Na}_{2} \mathrm{O}, \mathrm{NiO}, \mathrm{TiO}_{2}$, and $\mathrm{ZrO}_{2}$ measurements indicate a significant ICP calibration effect at the 5\% significance level. For $\mathrm{U}_{\text {std }}$, the $\mathrm{CaO}, \mathrm{Cr}_{2} \mathrm{O}_{3}, \mathrm{~K}_{2} \mathrm{O}, \mathrm{MgO}, \mathrm{MnO}$, $\mathrm{Na}_{2} \mathrm{O}, \mathrm{NiO}, \mathrm{ThO}_{2}$, and $\mathrm{TiO}_{2}$ measurements indicate a significant ICP calibration effect at the 5\% significance level. The reference values for the oxide concentrations of the standards are given in the header for each set of measurements in the exhibit.

Exhibit H.4 provides a similar set of analyses for the measurements derived from Batch 1 and $U_{\text {std }}$ samples prepared via the PF method. In this exhibit, there is no indication of a statistically significant (at the 5\% significance level) difference among the ICP analytical/calibration blocks for these data except for the $\mathrm{U}_{\text {std }} \mathrm{B}_{2} \mathrm{O}_{3}$ measurements.

Overall, these results suggest that it may be helpful to bias correct the oxide measurements of the SB3 Phase 1 glasses for the effect of the ICP calibration on each of the analytical blocks. The basis for this bias correction is presented as part of Exhibits H.3 and H.4 - the average measurements for Batch 1 for each ICP block for each oxide except for $\mathrm{U}_{3} \mathrm{O}_{8}$. For $\mathrm{U}_{3} \mathrm{O}_{8}$, the average measurements for $\mathrm{U}_{\text {std }}$ for each ICP block for $\mathrm{U}_{3} \mathrm{O}_{8}$ was used. That is, the Batch 1 results served as the basis for bias correcting all of the oxides (that were bias corrected) except $\mathrm{U}_{3} \mathrm{O}_{8}$. The $\mathrm{U}_{\text {std }}$ results were used to bias correct the $\mathrm{U}_{3} \mathrm{O}_{8}$ values. For the other oxides, the Batch 1 results were used to conduct the bias correction as long as the reference value for the oxide concentration in the Batch 1 glass was greater than or equal to $0.1 \mathrm{wt} \%$. Thus, applying this approach and based upon the information in the exhibits, the Batch 1 results were used to bias correct the $\mathrm{Al}_{2} \mathrm{O}_{3}, \mathrm{~B}_{2} \mathrm{O}_{3}, \mathrm{BaO}, \mathrm{CaO}, \mathrm{Cr}_{2} \mathrm{O}_{3}, \mathrm{CuO}, \mathrm{Fe}_{2} \mathrm{O}_{3}, \mathrm{Li}_{2} \mathrm{O}, \mathrm{K}_{2} \mathrm{O}, \mathrm{MgO}, \mathrm{MnO}, \mathrm{Na}_{2} \mathrm{O}, \mathrm{NiO}$, $\mathrm{SiO}_{2}$, and $\mathrm{TiO}_{2}$ measurements. No bias correction was conducted for $\mathrm{Ce}_{2} \mathrm{O}_{3}, \mathrm{La}_{2} \mathrm{O}_{3}, \mathrm{PbO}, \mathrm{ThO}_{2}$, $\mathrm{ZnO}$, or $\mathrm{ZrO}_{2}$.

The bias correction was conducted as follows. For each oxide, let $\overline{\mathrm{a}}_{\mathrm{ij}}$ be the average measurement for the $i^{\text {th }}$ oxide at analytical block $j$ for Batch 1 (or $\mathrm{U}_{\text {std }}$ for $\mathrm{U}_{3} \mathrm{O}_{8}$ ), and let $\mathrm{t}_{\mathrm{i}}$ be the reference value for the $\mathrm{i}^{\text {th }}$ oxide for Batch 1 (or for $\mathrm{U}_{\text {std }}$ if $\mathrm{U}_{3} \mathrm{O}_{8}$ ). (The averages and reference values are provided in Exhibits H.3 and H.4.) Let $\bar{c}_{\mathrm{ijk}}$ be the average measurement for the $\mathrm{i}^{\text {th }}$ oxide at analytical block $\mathrm{j}$ for the $\mathrm{k}^{\text {th }}$ glass. The bias adjustment was conducted as follows

$$
\bar{c}_{i j k} \bullet\left(1-\frac{\bar{a}_{i j}-t_{i}}{\bar{a}_{i j}}\right)=\bar{c}_{i j k} \cdot \frac{t_{i}}{\bar{a}_{i j}}
$$

Bias-corrected measurements are indicated by a "bc" suffix, and such adjustments were performed for all of the oxides of this study except for $\mathrm{Ce}_{2} \mathrm{O}_{3}, \mathrm{La}_{2} \mathrm{O}_{3}, \mathrm{PbO}, \mathrm{ThO}_{2}, \mathrm{ZnO}$, or $\mathrm{ZrO}_{2}$. 
Both measured and measured "bc" values are included in the discussion that follows. In these discussions bias-corrected values for $\mathrm{Ce}_{2} \mathrm{O}_{3}, \mathrm{La}_{2} \mathrm{O}_{3}, \mathrm{PbO}, \mathrm{ThO}_{2}, \mathrm{ZnO}$, or $\mathrm{ZrO}_{2}$ are included for completeness (e.g., to allow a sum of oxides to be computed for the bias-corrected results). These bias-corrected values are the same as the original $\mathrm{Ce}_{2} \mathrm{O}_{3}, \mathrm{La}_{2} \mathrm{O}_{3}, \mathrm{PbO}, \mathrm{ThO}_{2}, \mathrm{ZnO}$, or $\mathrm{ZrO}_{2}$ values (i.e., once again, no bias correction was performed for these oxides).

\subsubsection{Composition Measurements by Glass Number}

Exhibits H.5 and H.6 in Appendix H provide plots of the oxide concentration measurements by Glass ID \# (including both the Batch 1, labeled as glass number 100 and $\mathrm{U}_{\text {std }}$, labeled as glass number 101) for the measured and bias-corrected (bc) values for the LM and PF preparation methods, respectively. Different symbols and colors are used to represent the different glasses. These plots show the individual measurements across the duplicates of each preparation method and the two ICP calibrations for each glass.

A review of the plots presented in these exhibits reveals the repeatability of the four individual oxide values for each glass. As stated previously, one of the LM prepared samples of glass SB329 generated measurements on some of the minor components that were larger than the measurements generated by the other LM prepared sample. The $\mathrm{Cr}_{2} \mathrm{O}_{3}$ measurements for one of the SB3-39 prepared samples was larger than those from the other prepared sample. One $\mathrm{Fe}_{2} \mathrm{O}_{3}$ value for SB3-18 was somewhat higher than the other three values. One $\mathrm{TiO}_{2}$ value for SB3-17 was much lower than the other three values. Some scatter is seen in the $\mathrm{Al}_{2} \mathrm{O}_{3}$ values for glasses SB3-04 and SB3-30, in the $\mathrm{Li}_{2} \mathrm{O}$ values for SB3-04, and in the $\mathrm{SiO}_{2}$ values for SB3-04, SB3-05, and SB3-20.

More detailed discussions of the average, measured chemical compositions of the SB3 Phase 1 glasses are provided in the sections that follow.

\subsubsection{Measured versus Targeted Compositions}

The four measurements for each oxide for each glass (over both preparation methods) were averaged to determine a representative chemical composition for the glass. These determinations were conducted for the measured and bias-corrected data without excluding any of the measurements of Tables H.1 and H.2. A sum of oxides was also computed for each glass based upon both the measured and bias-corrected values. Exhibit H.7 in Appendix H provides plots for each glass for each oxide to help highlight the comparisons among the measured, bias-corrected, and targeted values.

Some observations from the plots of Exhibit $\mathrm{H} .7$ are offered. The $\mathrm{Al}_{2} \mathrm{O}_{3}$ measurement averages were above the targeted levels for several of the SB3 Phase 1 glasses. The $\mathrm{B}_{2} \mathrm{O}_{3}$ measurement averages followed their corresponding targeted levels very closely. The $\mathrm{Ce}_{2} \mathrm{O}_{3}$ and $\mathrm{La}_{2} \mathrm{O}_{3}$ measurement averages for SB3-29 were substantially above the targeted levels for this glass. This was due to the larger values generated for these oxides by one of the LM prepared samples (as previously discussed). The average $\mathrm{Fe}_{2} \mathrm{O}_{3}$ measurement for SB3-42 was substantially less than the targeted $\mathrm{Fe}_{2} \mathrm{O}_{3}$ value for this glass. The $\mathrm{Li}_{2} \mathrm{O}$ measurement average for SB3-42 was above the targeted value for this glass. The $\mathrm{MgO}$ measurement average for SB3-10 was above the targeted value for this glass. The $\mathrm{Na}_{2} \mathrm{O}$ measurement average for $\mathrm{SB} 3-32$ was above the targeted value for this glass. The $\mathrm{PbO}$ measurement averages were consistently below their targeted levels for the Phase 1 glasses. Bias correcting the $\mathrm{SiO}_{2}$ measurements appears to move the results farther away from the targeted levels. The $\mathrm{ThO}_{2}$ measurement averages were consistently above their targeted levels for this Phase 1 glasses. The $\mathrm{U}_{3} \mathrm{O}_{8}$ measurement averages followed their 
corresponding targeted levels very closely. The $\mathrm{ZnO}$ measurement averages were predominately below their targeted levels for these Phase 1 glasses. And, finally, the $\mathrm{ZrO}_{2}$ measurement average for SB3-38 was above it targeted level for this glass.

The review of these results prompted a request for the SRTC-ML to remeasure some of the prepared samples as well as to re-prep and measure new samples of SB3-29 and SB3-42. The results generated by the lab are presented in Table H.3 in Appendix H. As seen in this table, some of the re-measured values moved closer to their corresponding targets while others did not. The high $\mathrm{ThO}_{2}$ measurements for these glasses are thought to be due to an interference with iron and zirconium at the Th283 wavelength. Based upon all of the information available, it was decided that the original measurements (those of Tables H.1 and H.2) are to be used to provide the measured and measured bias-corrected views of the compositions of these SB3 Phase 1 glasses.

Table H.4 in Appendix H provides a summary of the average compositions as well as the targeted compositions and some associated differences and relative differences. Notice that the targeted sums of oxides for the glasses do not sum to $100 \%$ for the Batch $1 \mathrm{U}_{\text {std }}$ glasses due to an incomplete coverage of the oxides in these standard glasses. All of the sums of oxides (both measured and bias-corrected) fall within the interval of 95 to $105 \mathrm{wt} \%$ for the SB3 Phase 1 glasses with the exception of the bias-corrected measurements for SB3-04.

Entries in Table H.4 also show the relative differences between the measured or bias-corrected values and the targeted values. These differences are shaded when they are greater than or equal to 5\%. Overall, these comparisons between the measured and targeted compositions suggest that there were no significant problems in the batching or fabrication of the SB3 Phase 1 glasses other than those noted in Section 6.1.3.

\subsection{A Statistical Review of the PCT Measurements}

As discussed in Section 5.2.2, the PCTs were conducted in three groups and analytical plans for the three groups of PCTs, presented in Appendices E, F, and G, were provided to the SRTC-ML to support the measurement of the compositions of the solutions resulting from these PCTs. Samples of a multi-element, standard solution were also included in all of these analytical plans (as a check on the accuracy of the ICP - AES used for these measurements). In this and following sections, the measurements generated by the SRTC-ML for these PCTs are presented and reviewed.

Table I.1 provides the elemental leachate concentration measurements determined by the SRTCML for the solution samples generated by the PCTs. The PCT results for the centerline, canistercooled glasses are indicated by a "ccc" suffix. One of the quality control checkpoints for the PCT procedure is solution-weight loss over the course of the 7-day test. This criterion was satisfied for all of the PCT solutions generated for both the quenched and ccc versions for all the SB3 Phase 1 glasses. Any measurement in the as-reported columns of Table I.1 preceded by a "<" was below the reported corresponding elemental reported detection limit, and the measurement was replaced by $1 / 2$ of the detection limit in the last seven columns of the table. The values in the last seven columns were also adjusted for the dilution factors by multiplying the values in the asreceived columns by 1.6667 for the Phase 1 and ARM glasses and by 16.667 for the EA glass. Thus, the concentrations in the last seven columns reflect detection and dilution adjustments. Beyond these adjustments, no other modifications to these data were performed. 
In the sections that follow, the analytical sequence of the measurements is explored, the measurements of the standards are investigated and used to assess the overall accuracy of the ICP measurement process, the measurements for each glass are reviewed, the quenched versus centerline-cooled results are compared, the PCTs are normalized using the compositions (targeted, measured, and bias-corrected) presented in Table H.4, and the normalized PCTs are compared to durability predictions for these compositions generated from the current DWPF durability models as described by Brown, Postles, and Edwards (2002).

\subsubsection{PCT Measurements in Analytical Sequence}

Exhibit I.1 and I.2 provide plots of the SRTC-ML measurements in analytical sequence (over all three groups) with and without the EA results, respectively. Different colors are used for each glass with a plus being used to represent results from quenched glasses while an open circle represents ccc results. No problems are seen in these plots.

\subsubsection{Measurements of the Multi-Element Solution Standard}

Exhibit I.3 provides an analysis of the SRTC-ML measurements of the samples of the multielement solution standard by group by ICP analytical (or calibration) block for all three groups of PCTs. An ANOVA investigating for statistically significant differences among the block averages for these samples for each element of interest is included in these exhibits. The results indicate a statistically significant (at the 5\% level) difference among the average measurements over these blocks for all of the analytes considered in this study. However, no bias correction of the PCT results for the study glasses was conducted. This approach was taken since the triplicate PCTs for a single study glass were placed in different ICP blocks. Averaging the ppm's for each set of triplicates helps to minimize the impact of the ICP effects.

Table 6-1 summarizes the average measurements and the reference values for the 4 elements of primary interest. The results indicate consistent and accurate measurements from the SRTC-ML processes used to conduct these analyses. 
Table 6-1. Results from Samples of the Multi-Element Solution Standard.

\begin{tabular}{|c|c|c|c|c|c|}
\hline $\begin{array}{c}\text { PCT } \\
\text { Group }\end{array}$ & $\begin{array}{l}\text { Analytical } \\
\text { Block }\end{array}$ & $\begin{array}{c}\text { Avg } \\
\text { B (ppm) }\end{array}$ & $\begin{array}{c}\text { Avg } \\
\mathrm{Li}(\mathrm{ppm})\end{array}$ & $\begin{array}{c}\text { Avg } \\
\mathrm{Na}(\mathrm{ppm})\end{array}$ & $\begin{array}{c}\text { Avg } \\
\text { Si (ppm) }\end{array}$ \\
\hline \multirow{9}{*}{1} & 1 & 20.7 & 9.2 & 79.4 & 49.7 \\
\hline & 2 & 20.7 & 9.4 & 84.4 & 50.7 \\
\hline & 3 & 21.3 & 9.7 & 87.2 & 50.7 \\
\hline & 4 & 20.9 & 9.4 & 85.7 & 49.7 \\
\hline & 5 & 20.9 & 9.5 & 85.2 & 49.9 \\
\hline & 6 & 21.6 & 9.6 & 84.5 & 51.4 \\
\hline & Grand Average & 21.0 & 9.5 & 84.4 & 50.3 \\
\hline & Reference Value & 20.0 & 10.0 & 81.0 & 50.0 \\
\hline & $\%$ difference & $5.1 \%$ & $-5.2 \%$ & $4.2 \%$ & $0.7 \%$ \\
\hline \multirow{9}{*}{2} & 1 & 21.4 & 9.5 & 83.5 & 51.1 \\
\hline & 2 & 21.1 & 9.5 & 85.6 & 50.6 \\
\hline & 3 & 20.8 & 9.4 & 86.5 & 50.0 \\
\hline & 4 & 20.6 & 9.4 & 84.5 & 49.1 \\
\hline & 5 & 20.4 & 9.3 & 85.8 & 49.3 \\
\hline & 6 & 20.7 & 9.4 & 84.9 & 49.6 \\
\hline & Grand Average & 20.8 & 9.4 & 85.1 & 50.0 \\
\hline & Reference Value & 20.0 & 10.0 & 81.0 & 50.0 \\
\hline & $\%$ difference & $4.2 \%$ & $-5.8 \%$ & $5.1 \%$ & $-0.1 \%$ \\
\hline \multirow{9}{*}{3} & 1 & 20.9 & 9.5 & 84.8 & 49.6 \\
\hline & 2 & 20.8 & 9.5 & 86.2 & 49.9 \\
\hline & 3 & 20.6 & 9.4 & 85.3 & 49.8 \\
\hline & 4 & 20.8 & 9.5 & 86.7 & 49.4 \\
\hline & 5 & 20.8 & 9.4 & 86.1 & 49.9 \\
\hline & 6 & 20.5 & 9.3 & 83.5 & 49.0 \\
\hline & Grand Average & 20.7 & 9.4 & 85.4 & 49.6 \\
\hline & Reference Value & 20.0 & 10.0 & 81.0 & 50.0 \\
\hline & $\%$ difference & $3.6 \%$ & $-5.7 \%$ & $5.5 \%$ & $-0.8 \%$ \\
\hline
\end{tabular}

\subsubsection{Measurements for Glass ID Number}

Exhibits I.4 and I.5 provide plots of the leachate concentrations by type of sample with and without EA, respectively. These plots allow for the assessment of the repeatability of the measurements as well as preliminary comparisons between the quenched and ccc glasses. In these plots the quenched results are once again represented by a plus while the ccc results are represented by an open circle. For the primary elements $(\mathrm{B}, \mathrm{Li}, \mathrm{Na}$, and $\mathrm{Si}$ ), the results for SB342 show the most scatter among all of the glasses with the ccc results being higher than the quenched for $\mathrm{B}, \mathrm{Li}$, and $\mathrm{Na}$. No other problems are seen among these data.

\subsubsection{Quenched versus Centerline Canister Cooled}

Exhibits I.6 (in both ppm and log ppm for all glasses) and I.7 (in ppm and log ppm for all glasses except SB3-42) provide a closer look at the quenched versus centerline-cooled results for the SB3 Phase 1 glasses, including a statistical comparison of the average differences due to heat treatment for each primary element of interest. These paired-t statistical tests indicate a statistically significant (at the 5\% level) difference, on average, between the PCTs for the two heat treatments for $\mathrm{B}$ and $\mathrm{Li}$ in Exhibit I.6 over all of the data and for all elements except $\mathrm{Li}$ in 
Exhibit I.7 where the results for SB3-42 are excluded. Although statistically significant differences are observed, no practical implications are projected.

An additional look at the log [B (ppm)] values is provided in Figure 6-1, which provides a plot of the ccc versus quenched results for the SB3 Phase 1 glasses. These results were derived using all of the PCTs (i.e., all three groups). A statistical difference (at the 5\% significance level) is seen between the quenched and ccc average log [B (ppm)] results for these glasses. SB3-42 is labeled in this graph as well.

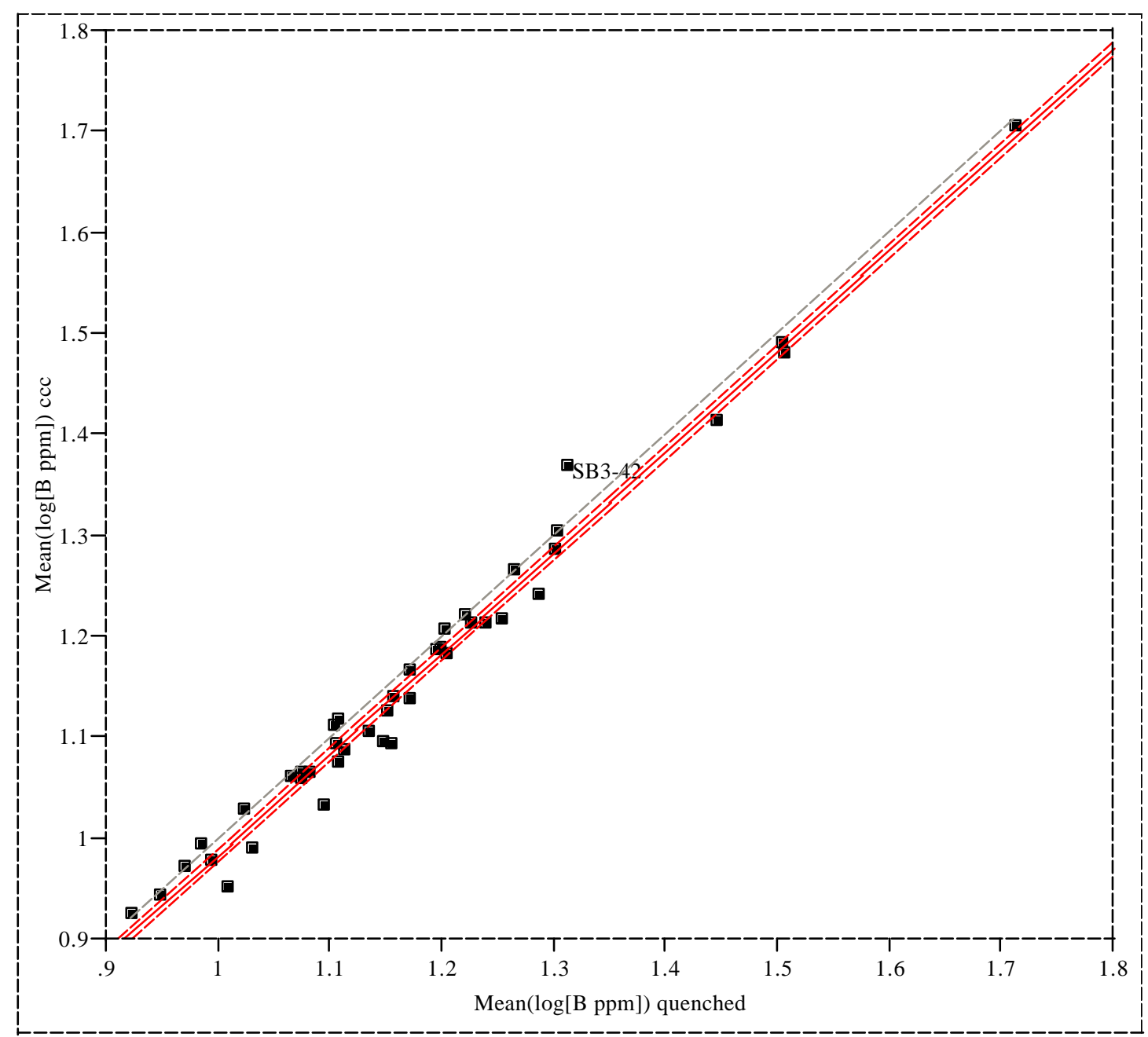

Figure 6-1 log [B (ppm)] for Quenched vs. CCC Glasses. 


\subsubsection{Normalized PCT Results}

PCT leachate concentrations are typically normalized using the cation composition (expressed as a weight percent) in the glass to obtain a grams-per-liter $(\mathrm{g} / \mathrm{L})$ leachate concentration. The normalization of the PCTs is usually conducted using the measured compositions of the glasses. This is the preferred normalization process for the PCTs. For completeness, the targeted cation and the bias-corrected cation compositions will also be used to conduct this normalization.

As is the usual convention, the common logarithm of the normalized PCT (normalized leachate, NL) for each element of interest will be determined and used for comparison. To accomplish this computation, one must

1. Determine the common logarithm of the elemental parts per million (ppm) leachate concentration for each of the triplicates and each of the elements of interest (these values are provided in Table H.1 of Appendix $\mathrm{H})$,

2. Average the common logarithms over the triplicates for each element of interest, and then

Normalizing Using Measured Composition (preferred method)

3. Subtract a quantity equal to 1 plus the common logarithm of the average cation measured concentration (expressed as a weight percent of the glass) from the average computed in step 2.

Or Normalizing Using Target Composition

3. Subtract a quantity equal to 1 plus the common logarithm of the target cation concentration (expressed as a weight percent of the glass) from the average computed in step 2.

Or Normalizing Using Measured Bias-Corrected Composition

3. Subtract a quantity equal to 1 plus the common logarithm of the measured bias-corrected cation concentration (expressed as a weight percent of the glass) from the average computed in step 2.

Exhibit I.8 provides scatter plots for these results and offers an opportunity to investigate the consistency in the leaching across the elements for the glasses of this study. All heat treatments (quenched and ccc versions) of the glasses and all compositional views (targeted, measured, and bias-corrected compositions) are represented in this set of plots. Consistency in the leaching (e.g., congruent dissolution) across the elements is typically demonstrated by a high degree of linear correlation among the values for pairs of these elements. A high degree of correlation is seen for these data for all pairs of the elements. The smallest correlation $(\sim 89 \%)$ is between $\mathrm{Na}$ and $\mathrm{Li}$.

\subsection{Applicability of $\Delta \mathbf{G}_{\mathrm{P}}$ to Projected SB3 Compositional Region}

As mentioned in Section 2.0, the objective of this study is to assess the applicability of the PCCS durability model $\left(\Delta \mathrm{G}_{\mathrm{P}}\right)$ to the anticipated compositional region of interest to SB3. Specifically, this study will demonstrate the applicability of durability predictions for specific SB3 compositions. 
Table 6-2 summarizes the PCT response for all 42 SB3 Phase 1 glasses. Normalized releases (in $\mathrm{g} / \mathrm{L}$ ) are provided for each glass based on three compositional views - target, measured, and measured bias-corrected. Normalized boron releases range from $0.499 \mathrm{~g} / \mathrm{L}$ for SB3-33 (most durable) to a high of $1.593 \mathrm{~g} / \mathrm{L}$ from SB3-42ccc (least durable). All glasses (both quenched and ccc) are acceptable being at least an order of magnitude more durable than the $16.695 \mathrm{~g} / \mathrm{L}$ limit for NL [B] as reported for EA (Jantzen et al. 1993). 
Table 6-2. PCT Results for the 42 SB3 Phase 1 Glasses.

\begin{tabular}{|c|c|c|c|c|c|}
\hline Glass ID/HT & Comp View & NL B $(g / L)$ & NL Li (g/L) & NL Na (g/L) & $\begin{array}{l}\mathrm{NL} \mathrm{Si}(\mathrm{g} / \mathrm{L}) \\
\end{array}$ \\
\hline SB3-01 & Targeted & 1.225 & 1.174 & 0.883 & 0.463 \\
\hline SB3-01 & Measured & 1.268 & 1.232 & 0.885 & 0.473 \\
\hline SB3-01 & Measured bc & 1.280 & 1.191 & 0.914 & 0.449 \\
\hline SB3-01\ccc & Targeted & 1.196 & 1.118 & 0.890 & 0.473 \\
\hline SB3-01\ccc & Measured & 1.239 & 1.173 & 0.893 & 0.483 \\
\hline SB3-01\ccc & Measured bc & 1.250 & 1.134 & 0.922 & 0.459 \\
\hline SB3-02 & Measured & 0.917 & 0.774 & 0.793 & 0.418 \\
\hline SB3-02 & Targeted & 0.907 & 0.760 & 0.826 & 0.415 \\
\hline SB3-02 & Measured bc & 0.934 & 0.750 & 0.848 & 0.388 \\
\hline SB3-02\ccc & Measured & 0.860 & 0.783 & 0.754 & 0.426 \\
\hline SB3-02\ccc & targeted & 0.850 & 0.769 & 0.785 & 0.423 \\
\hline SB3-02\ccc & Measured bc & 0.875 & 0.758 & 0.806 & 0.396 \\
\hline SB3-03 & Measured & 0.607 & 0.694 & 0.501 & 0.399 \\
\hline SB3-03 & targeted & 0.612 & 0.674 & 0.529 & 0.402 \\
\hline SB3-03 & Measured bc & 0.627 & 0.668 & 0.535 & 0.371 \\
\hline SB3-03\ccc & Measured & 0.545 & 0.623 & 0.477 & 0.383 \\
\hline SB3-03\ccc & targeted & 0.549 & 0.604 & 0.504 & 0.387 \\
\hline SB3-03\ccc & Measured bc & 0.563 & 0.599 & 0.510 & 0.356 \\
\hline \begin{tabular}{|l|} 
SB3-04 \\
\end{tabular} & Measured & 0.759 & 0.626 & 0.850 & 0.470 \\
\hline SB3-04 & targeted & 0.795 & 0.698 & 0.865 & 0.493 \\
\hline SB3-04 & Measured bc & 0.772 & 0.607 & 0.904 & 0.437 \\
\hline SB3-04\ccc & Measured & 0.731 & 0.657 & 0.792 & 0.456 \\
\hline SB3-04\ccc & targeted & 0.766 & 0.732 & 0.806 & 0.478 \\
\hline SB3-04\ccc & Measured bc & 0.744 & 0.636 & 0.843 & 0.424 \\
\hline SB3-05 & targeted & 0.935 & 0.921 & 1.025 & 0.607 \\
\hline SB3-05 & Measured & 0.911 & 0.963 & 1.003 & 0.626 \\
\hline SB3-05 & Measured bc & 0.939 & 0.930 & 1.044 & 0.599 \\
\hline SB3-05\ccc & targeted & 0.886 & 0.876 & 0.896 & 0.584 \\
\hline SB3-05\ccc & Measured & 0.863 & 0.917 & 0.877 & 0.602 \\
\hline SB3-05\ccc & Measured bc & 0.889 & 0.885 & 0.912 & 0.576 \\
\hline SB3-06 & targeted & 1.128 & 1.008 & 1.245 & 0.695 \\
\hline SB3-06 & Measured & 1.104 & 1.055 & 1.225 & 0.694 \\
\hline SB3-06 & Measured bc & 1.141 & 1.015 & 1.303 & 0.646 \\
\hline \begin{tabular}{|l} 
SB3-06\ccc \\
\end{tabular} & targeted & 1.056 & 0.961 & 1.093 & 0.650 \\
\hline SB3-06\ccc & Measured & 1.032 & 1.005 & 1.076 & 0.649 \\
\hline SB3-06\ccc & Measured bc & 1.067 & 0.967 & 1.144 & 0.604 \\
\hline SB3-07 & Measured & 0.741 & 0.837 & 0.507 & 0.381 \\
\hline SB3-07 & targeted & 0.736 & 0.822 & 0.516 & 0.381 \\
\hline SB3-07 & Measured bc & 0.754 & 0.810 & 0.528 & 0.354 \\
\hline SB3-07\ccc & Measured & 0.713 & 0.768 & 0.521 & 0.372 \\
\hline SB3-07\ccc & targeted & 0.709 & 0.755 & 0.530 & 0.371 \\
\hline SB3-07\ccc & Measured bc & 0.726 & 0.744 & 0.542 & 0.346 \\
\hline SB3-08 & targeted & 0.831 & 0.765 & 0.755 & 0.413 \\
\hline SB3-08 & Measured & 0.837 & 0.797 & 0.758 & 0.416 \\
\hline SB3-08 & Measured bc & 0.863 & 0.769 & 0.782 & 0.398 \\
\hline
\end{tabular}




\begin{tabular}{|c|c|c|c|c|c|}
\hline |Glass ID/HT & Comp View & $\overline{N L} \mathrm{~B}(\mathrm{~g} / \mathrm{L})$ & NL Li (g/L) & NL Na (g/L) & 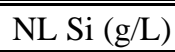 \\
\hline SB3-08\ccc & targeted & 0.770 & 0.748 & 0.720 & 0.399 \\
\hline SB3-08\ccc & Measured & 0.775 & 0.778 & 0.722 & 0.402 \\
\hline SB3-08\ccc & Measured bc & 0.799 & 0.751 & 0.746 & 0.385 \\
\hline $\mid$ SB3-09 & Measured & 0.520 & 0.660 & 0.442 & 0.369 \\
\hline SB3-09 & targeted & 0.518 & 0.632 & 0.466 & 0.370 \\
\hline $\mid$ SB3-09 & Measured bc & 0.537 & 0.635 & 0.473 & 0.343 \\
\hline SB3-09\сcc & Measured & 0.507 & 0.616 & 0.432 & 0.372 \\
\hline SB3-09\сcc & targeted & 0.505 & 0.591 & 0.456 & 0.373 \\
\hline SB3-09\сcc & Measured bc & 0.524 & 0.593 & 0.462 & 0.346 \\
\hline SB3-10 & Measured & 0.848 & 0.807 & 0.845 & 0.470 \\
\hline SB3-10 & targeted & 0.863 & 0.787 & 0.893 & 0.473 \\
\hline SB3-10 & Measured bc & 0.876 & 0.776 & 0.903 & 0.437 \\
\hline SB3-10\ccc & Measured & 0.845 & 0.838 & 0.803 & 0.487 \\
\hline SB3-10\ccc & targeted & 0.861 & 0.817 & 0.850 & 0.490 \\
\hline SB3-10\ccc & Measured bc & 0.873 & 0.806 & 0.859 & 0.453 \\
\hline SB3-11 & targeted & 0.814 & 0.842 & 0.805 & 0.547 \\
\hline SB3-11 & Measured & 0.810 & 0.862 & 0.795 & 0.553 \\
\hline SB3-11 & Measured bc & 0.818 & 0.833 & 0.827 & 0.525 \\
\hline SB3-11\ccc & targeted & 0.779 & 0.796 & 0.767 & 0.520 \\
\hline SB3-11\ccc & Measured & 0.775 & 0.815 & 0.757 & 0.525 \\
\hline $\mid \overline{S B 3-11 \backslash \mathrm{ccc}}$ & Measured bc & 0.783 & 0.788 & 0.788 & 0.499 \\
\hline SB3-12 & Measured & 1.281 & 1.070 & 1.213 & 0.675 \\
\hline SB3-12 & targeted & 1.256 & 0.999 & 1.229 & 0.653 \\
\hline SB3-12 & Measured bc & 1.304 & 1.036 & 1.262 & 0.627 \\
\hline SB3-12\ccc & Measured & 1.237 & 1.125 & 1.155 & 0.670 \\
\hline SB3-12lccc & targeted & 1.212 & 1.050 & 1.170 & 0.648 \\
\hline SB3-12\ccc & Measured bc & 1.259 & 1.089 & 1.202 & 0.623 \\
\hline SB3-13 & targeted & 0.798 & 0.831 & 1.014 & 0.596 \\
\hline SB3-13 & Measured & 0.819 & 0.868 & 1.009 & 0.605 \\
\hline SB3-13 & Measured bc & 0.827 & 0.839 & 1.073 & 0.575 \\
\hline SB3-13\ccc & targeted & 0.733 & 0.795 & 0.877 & 0.563 \\
\hline SB3-13\ccc & Measured & 0.753 & 0.831 & 0.872 & 0.572 \\
\hline SB3-13\сcс & Measured bc & 0.760 & 0.803 & 0.928 & 0.543 \\
\hline SB3-14 & Measured & 0.891 & 0.861 & 1.126 & 0.620 \\
\hline SB3-14 & targeted & 0.902 & 0.845 & 1.175 & 0.622 \\
\hline SB3-14 & Measured bc & 0.907 & 0.834 & 1.205 & 0.576 \\
\hline SB3-14lccc & Measured & 0.827 & 0.871 & 0.988 & 0.587 \\
\hline SB3-14lccc & targeted & 0.838 & 0.854 & 1.031 & 0.589 \\
\hline SB3-14lccc & Measured bc & 0.842 & 0.843 & 1.056 & 0.546 \\
\hline SB3-15 & Measured & 0.954 & 0.958 & 1.042 & 0.648 \\
\hline SB3-15 & targeted & 0.959 & 0.910 & 1.074 & 0.636 \\
\hline SB3-15 & Measured bc & 0.983 & 0.924 & 1.108 & 0.619 \\
\hline SB3-15\ccc & Measured & 0.961 & 0.987 & 0.982 & 0.638 \\
\hline SB3-15\ccc & targeted & 0.965 & 0.938 & 1.012 & 0.626 \\
\hline SB3-15\ccc & Measured bc & 0.990 & 0.952 & 1.045 & 0.610 \\
\hline SB3-16 & Measured & 1.067 & 1.001 & 1.168 & 0.693 \\
\hline SB3-16 & targeted & 1.062 & 0.954 & 1.198 & 0.676 \\
\hline SB3-16 & Measured bc & 1.086 & 0.969 & 1.242 & 0.644 \\
\hline
\end{tabular}




\begin{tabular}{|c|c|c|c|c|c|}
\hline Glass ID/HT & Comp View & NL B(g/L) & $\mid$ NL Li (g/L) & $\mathrm{NL} \mathrm{Na}(\mathrm{g} / \mathrm{L})$ & $\overline{N L ~ S i ~(g / L) ~}$ \\
\hline SB3-16lccc & Measured & 1.065 & 1.039 & 1.085 & 0.689 \\
\hline SB3-16\ccc & targeted & 1.059 & 0.990 & 1.114 & 0.672 \\
\hline SB3-16\ccc & Measured bc & 1.084 & 1.005 & 1.155 & 0.640 \\
\hline SB3-17 & targeted & 0.567 & 0.671 & 0.761 & 0.471 \\
\hline SB3-17 & Measured & 0.574 & 0.692 & 0.754 & 0.480 \\
\hline SB3-17 & Measured bc & 0.580 & 0.668 & 0.779 & 0.456 \\
\hline SB3-17\ccc & targeted & 0.545 & 0.647 & 0.689 & 0.447 \\
\hline SB3-17\ccc & Measured & 0.551 & 0.666 & 0.683 & 0.456 \\
\hline SB3-17\ccc & Measured bc & 0.557 & 0.644 & 0.705 & 0.433 \\
\hline SB3-18 & Measured & 0.838 & 0.832 & 1.093 & 0.598 \\
\hline SB3-18 & targeted & 0.840 & 0.789 & 1.135 & 0.596 \\
\hline SB3-18 & Measured bc & 0.866 & 0.801 & 1.128 & 0.556 \\
\hline SB3-18\ccc & Measured & 0.816 & 0.871 & 0.985 & 0.577 \\
\hline SB3-18\ccc & targeted & 0.818 & 0.826 & 1.023 & 0.575 \\
\hline SB3-18\ccc & Measured bc & 0.843 & 0.838 & 1.017 & 0.537 \\
\hline SB3-19 & targeted & 0.597 & 0.646 & 0.716 & 0.457 \\
\hline SB3-19 & Measured & 0.598 & 0.667 & 0.713 & 0.463 \\
\hline SB3-19 & Measured bc & 0.609 & 0.646 & 0.742 & 0.430 \\
\hline SB3-19\ccc & targeted & 0.561 & 0.622 & 0.655 & 0.448 \\
\hline SB3-19\ccc & Measured & 0.562 & 0.643 & 0.652 & 0.454 \\
\hline SB3-19\ccc & Measured bc & 0.572 & 0.623 & 0.678 & 0.422 \\
\hline SB3-20 & Measured & 0.773 & 0.759 & 1.022 & 0.547 \\
\hline SB3-20 & targeted & 0.774 & 0.738 & 1.031 & 0.538 \\
\hline SB3-20 & Measured bc & 0.787 & 0.735 & 1.087 & 0.508 \\
\hline SB3-20\ccc & Measured & 0.724 & 0.751 & 0.891 & 0.512 \\
\hline SB3-20\ccc & targeted & 0.725 & 0.731 & 0.899 & 0.504 \\
\hline SB3-20\ccc & Measured bc & 0.736 & 0.727 & 0.948 & 0.476 \\
\hline SB3-21 & Measured & 0.539 & 0.644 & 0.732 & 0.455 \\
\hline SB3-21 & targeted & 0.541 & 0.625 & 0.727 & 0.440 \\
\hline SB3-21 & Measured bc & 0.556 & 0.621 & 0.761 & 0.435 \\
\hline SB3-21\ccc & Measured & 0.530 & 0.632 & 0.659 & 0.435 \\
\hline SB3-21\ccc & targeted & 0.532 & 0.613 & 0.655 & 0.421 \\
\hline SB3-21\ccc & Measured bc & 0.546 & 0.609 & 0.686 & 0.416 \\
\hline \begin{tabular}{|l|} 
SB3-22 \\
\end{tabular} & Measured & 0.910 & 0.803 & 1.187 & 0.590 \\
\hline SB3-22 & targeted & 0.902 & 0.785 & 1.192 & 0.587 \\
\hline SB3-22 & Measured bc & 0.941 & 0.772 & 1.270 & 0.550 \\
\hline SB3-22\ccc & Measured & 0.897 & 0.870 & 1.113 & 0.584 \\
\hline SB3-22lccc & targeted & 0.888 & 0.850 & 1.118 & 0.581 \\
\hline SB3-22lccc & Measured bc & 0.927 & 0.837 & 1.191 & 0.544 \\
\hline SB3-23 & Measured & 0.557 & 0.708 & 0.719 & 0.462 \\
\hline SB3-23 & targeted & 0.560 & 0.669 & 0.728 & 0.454 \\
\hline SB3-23 & Measured bc & 0.575 & 0.683 & 0.748 & 0.442 \\
\hline SB3-23\ccc & Measured & 0.558 & 0.695 & 0.659 & 0.461 \\
\hline SB3-23\сcc & targeted & 0.560 & 0.657 & 0.667 & 0.454 \\
\hline SB3-23\lccc & Measured bc & 0.575 & 0.671 & 0.686 & 0.441 \\
\hline SB3-24 & Measured & 0.954 & 0.881 & 1.160 & 0.635 \\
\hline SB3-24 & targeted & 0.986 & 0.857 & 1.235 & 0.634 \\
\hline SB3-24 & Measured bc & 0.983 & 0.850 & 1.241 & 0.607 \\
\hline
\end{tabular}




\begin{tabular}{|c|c|c|c|c|c|}
\hline Glass ID/HT & Comp View & NL B(g/L) & NL Li (g/L) & $\mathrm{NL} \mathrm{Na}(\mathrm{g} / \mathrm{L})$ & NL Si $(\mathrm{g} / \mathrm{L})$ \\
\hline SB3-24lccc & Measured & 0.923 & 0.942 & 1.071 & 0.621 \\
\hline SB3-24\ccc & targeted & 0.954 & 0.917 & 1.140 & 0.621 \\
\hline SB3-24lccc & Measured bc & 0.952 & 0.909 & 1.146 & 0.594 \\
\hline SB3-25 & targeted & 0.704 & 0.849 & 0.819 & 0.562 \\
\hline SB3-25 & Measured & 0.704 & 0.872 & 0.810 & 0.570 \\
\hline SB3-25 & Measured bc & 0.716 & 0.844 & 0.836 & 0.530 \\
\hline SB3-25\ccc & targeted & 0.674 & 0.821 & 0.783 & 0.556 \\
\hline $\mid$ SB3-25\ccc & Measured & 0.673 & 0.843 & 0.774 & 0.563 \\
\hline SB3-25\ccc & Measured bc & 0.685 & 0.816 & 0.799 & 0.523 \\
\hline SB3-26 & Measured & 1.091 & 1.049 & 1.271 & 0.720 \\
\hline SB3-26 & targeted & 1.088 & 1.014 & 1.303 & 0.718 \\
\hline SB3-26 & Measured bc & 1.101 & 1.014 & 1.352 & 0.684 \\
\hline SB3-26\ccc & Measured & 1.070 & 1.122 & 1.172 & 0.701 \\
\hline SB3-26\ccc & targeted & 1.067 & 1.084 & 1.202 & 0.699 \\
\hline SB3-26lccc & Measured bc & 1.080 & 1.085 & 1.247 & 0.666 \\
\hline SB3-27 & Measured & 0.738 & 0.872 & 0.743 & 0.570 \\
\hline SB3-27 & targeted & 0.739 & 0.834 & 0.798 & 0.561 \\
\hline SB3-27 & Measured bc & 0.761 & 0.841 & 0.795 & 0.546 \\
\hline SB3-27\ccc & Measured & 0.717 & 0.856 & 0.715 & 0.556 \\
\hline SB3-27\lccc & targeted & 0.718 & 0.819 & 0.768 & 0.547 \\
\hline SB3-27\ccc & Measured bc & 0.739 & 0.826 & 0.765 & 0.532 \\
\hline SB3-28 & targeted & 1.015 & 0.973 & 1.253 & 0.669 \\
\hline \begin{tabular}{|l|} 
SB3-28 \\
\end{tabular} & Measured & 1.005 & 1.009 & 1.251 & 0.668 \\
\hline SB3-28 & Measured bc & 1.038 & 0.971 & 1.292 & 0.622 \\
\hline SB3-28\ccc & targeted & 0.928 & 0.944 & 1.045 & 0.616 \\
\hline SB3-28\ccc & Measured & 0.919 & 0.979 & 1.043 & 0.615 \\
\hline SB3-28\ccc & Measured bc & 0.950 & 0.942 & 1.077 & 0.573 \\
\hline \begin{tabular}{|l|} 
SB3-29 \\
\end{tabular} & targeted & 0.720 & 0.768 & 0.733 & 0.493 \\
\hline \begin{tabular}{|l|} 
SB3-29 \\
\end{tabular} & Measured & 0.719 & 0.804 & 0.727 & 0.504 \\
\hline \begin{tabular}{|l} 
SB3-29 \\
\end{tabular} & Measured bc & 0.742 & 0.775 & 0.750 & 0.482 \\
\hline SB3-29\ccc & targeted & 0.730 & 0.763 & 0.718 & 0.490 \\
\hline SB3-29\сcс & Measured & 0.730 & 0.799 & 0.711 & 0.500 \\
\hline SB3-29\ccc & Measured bc & 0.752 & 0.771 & 0.734 & 0.479 \\
\hline SB3-30 & Measured & 1.049 & 0.924 & 1.001 & 0.566 \\
\hline SB3-30 & targeted & 1.044 & 0.896 & 1.056 & 0.566 \\
\hline SB3-30 & Measured bc & 1.059 & 0.893 & 1.070 & 0.538 \\
\hline SB3-30\ccc & Measured & 0.909 & 0.885 & 0.913 & 0.557 \\
\hline SB3-30\ccc & targeted & 0.905 & 0.858 & 0.964 & 0.557 \\
\hline SB3-30\ccc & Measured bc & 0.918 & 0.855 & 0.977 & 0.529 \\
\hline SB3-31 & Measured & 0.510 & 0.664 & 0.595 & 0.407 \\
\hline SB3-31 & targeted & 0.518 & 0.640 & 0.619 & 0.404 \\
\hline SB3-31 & Measured bc & 0.526 & 0.641 & 0.619 & 0.389 \\
\hline SB3-31\ccc & Measured & 0.511 & 0.639 & 0.559 & 0.398 \\
\hline SB3-31 \ccc & targeted & 0.518 & 0.616 & 0.581 & 0.395 \\
\hline SB3-31\ccc & Measured bc & 0.526 & 0.617 & 0.582 & 0.381 \\
\hline SB3-32 & Measured & 0.762 & 0.768 & 0.769 & 0.472 \\
\hline SB3-32 & Measured bc & 0.776 & 0.743 & 0.793 & 0.439 \\
\hline SB3-32 & targeted & 0.775 & 0.748 & 0.883 & 0.464 \\
\hline
\end{tabular}




\begin{tabular}{|c|c|c|c|c|c|}
\hline Glass ID/HT & Comp View & NL B(g/L) & $\mid$ NL Li (g/L) & $\mathrm{NL} \mathrm{Na}(\mathrm{g} / \mathrm{L})$ & $\overline{N L ~ S i ~(g / L) ~}$ \\
\hline SB3-32\ccc & Measured & 0.664 & 0.778 & 0.715 & 0.470 \\
\hline SB3-32lccc & Measured bc & 0.676 & 0.753 & 0.739 & 0.437 \\
\hline SB3-32\ccc & targeted & 0.676 & 0.758 & 0.822 & 0.462 \\
\hline SB3-33 & Measured & 0.499 & 0.633 & 0.545 & 0.394 \\
\hline SB3-33 & targeted & 0.499 & 0.605 & 0.560 & 0.391 \\
\hline SB3-33 & Measured bc & 0.515 & 0.609 & 0.568 & 0.367 \\
\hline SB3-33\ccc & Measured & 0.503 & 0.629 & 0.535 & 0.396 \\
\hline SB3-33\ccc & targeted & 0.503 & 0.601 & 0.549 & 0.393 \\
\hline SB3-33\ccc & Measured bc & 0.520 & 0.605 & 0.557 & 0.368 \\
\hline SB3-34 & Measured & 0.736 & 0.754 & 0.824 & 0.450 \\
\hline SB3-34 & targeted & 0.756 & 0.737 & 0.847 & 0.448 \\
\hline SB3-34 & Measured bc & 0.758 & 0.728 & 0.858 & 0.430 \\
\hline SB3-34\ccc & Measured & 0.633 & 0.738 & 0.743 & 0.440 \\
\hline SB3-34\ccc & targeted & 0.651 & 0.721 & 0.763 & 0.438 \\
\hline SB3-34\ccc & Measured bc & 0.653 & 0.712 & 0.773 & 0.421 \\
\hline SB3-35 & Measured & 0.634 & 0.749 & 0.823 & 0.515 \\
\hline SB3-35 & targeted & 0.638 & 0.714 & 0.873 & 0.510 \\
\hline SB3-35 & Measured bc & 0.655 & 0.720 & 0.875 & 0.479 \\
\hline SB3-35\ccc & Measured & 0.608 & 0.737 & 0.740 & 0.495 \\
\hline SB3-35\ccc & targeted & 0.612 & 0.702 & 0.784 & 0.490 \\
\hline SB3-35\ccc & Measured bc & 0.628 & 0.709 & 0.787 & 0.460 \\
\hline SB3-36 & Measured & 0.911 & 0.829 & 1.085 & 0.565 \\
\hline SB3-36 & targeted & 0.939 & 0.815 & 1.127 & 0.572 \\
\hline SB3-36 & Measured bc & 0.942 & 0.798 & 1.154 & 0.526 \\
\hline SB3-36\ccc & Measured & 0.841 & 0.893 & 1.029 & 0.563 \\
\hline SB3-36\ccc & targeted & 0.866 & 0.878 & 1.069 & 0.569 \\
\hline SB3-36\ccc & Measured bc & 0.869 & 0.859 & 1.094 & 0.524 \\
\hline SB3-37 & Measured & 0.581 & 0.752 & 0.485 & 0.475 \\
\hline \begin{tabular}{|l|} 
SB3-37 \\
\end{tabular} & targeted & 0.563 & 0.719 & 0.517 & 0.462 \\
\hline SB3-37 & Measured bc & 0.586 & 0.727 & 0.519 & 0.451 \\
\hline SB3-37\ccc & Measured & 0.591 & 0.741 & 0.505 & 0.477 \\
\hline SB3-37\ccc & targeted & 0.573 & 0.709 & 0.539 & 0.465 \\
\hline SB3-37\ccc & Measured bc & 0.596 & 0.716 & 0.540 & 0.453 \\
\hline \begin{tabular}{|l|} 
SB3-38 \\
\end{tabular} & targeted & 0.801 & 0.819 & 0.843 & 0.505 \\
\hline SB3-38 & Measured & 0.808 & 0.852 & 0.822 & 0.511 \\
\hline SB3-38 & Measured bc & 0.816 & 0.824 & 0.849 & 0.485 \\
\hline SB3-38\ccc & targeted & 0.727 & 0.824 & 0.808 & 0.508 \\
\hline SB3-38\ccc & Measured & 0.733 & 0.857 & 0.788 & 0.514 \\
\hline SB3-38\ccc & Measured bc & 0.740 & 0.829 & 0.814 & 0.488 \\
\hline \begin{tabular}{|l|} 
SB3-39 \\
\end{tabular} & targeted & 0.572 & 0.723 & 0.489 & 0.468 \\
\hline SB3-39 & Measured & 0.571 & 0.752 & 0.478 & 0.474 \\
\hline $\begin{array}{l}\text { SB3-39 } \\
\end{array}$ & Measured bc & 0.590 & 0.724 & 0.498 & 0.442 \\
\hline SB3-39\ccc & targeted & 0.584 & 0.708 & 0.524 & 0.472 \\
\hline SB3-39\сcс & Measured & 0.583 & 0.737 & 0.513 & 0.478 \\
\hline SB3-39\ccc & Measured bc & 0.603 & 0.709 & 0.533 & 0.445 \\
\hline SB3-40 & Measured & 0.824 & 0.872 & 0.816 & 0.513 \\
\hline SB3-40 & targeted & 0.839 & 0.853 & 0.861 & 0.513 \\
\hline SB3-40 & Measured bc & 0.839 & 0.844 & 0.868 & 0.477 \\
\hline
\end{tabular}


Immobilization Technology Section

Westinghouse Savannah River Company

\begin{tabular}{|c|c|c|c|c|c|}
\hline Glass ID/HT & Comp View & $\mathrm{NL} \mathrm{B}(\mathrm{g} / \mathrm{L})$ & NL Li (g/L) & $\mathrm{NL} \mathrm{Na}(\mathrm{g} / \mathrm{L})$ & NL Si $(g / L)$ \\
\hline SB3-40\ccc & Measured & 0.726 & 0.863 & 0.776 & 0.511 \\
\hline SB3-40\ccc & targeted & 0.740 & 0.844 & 0.819 & 0.511 \\
\hline SB3-40\сcc & Measured bc & 0.739 & 0.836 & 0.826 & 0.475 \\
\hline SB3-41 & Measured & 1.012 & 1.133 & 1.197 & 0.735 \\
\hline SB3-41 & targeted & 1.000 & 1.105 & 1.241 & 0.729 \\
\hline SB3-41 & Measured bc & 1.021 & 1.095 & 1.273 & 0.698 \\
\hline SB3-41\ccc & Measured & 1.009 & 1.226 & 1.128 & 0.755 \\
\hline SB3-41\ccc & targeted & 0.997 & 1.196 & 1.170 & 0.749 \\
\hline SB3-41\ccc & Measured bc & 1.018 & 1.185 & 1.200 & 0.717 \\
\hline SB3-42 & Measured & 1.391 & 1.409 & 1.616 & 0.869 \\
\hline SB3-42 & Measured bc & 1.404 & 1.361 & 1.669 & 0.825 \\
\hline SB3-42 & targeted & 1.402 & 1.816 & 1.686 & 0.877 \\
\hline SB3-42\сcс & Measured & 1.578 & 2.279 & 1.616 & 1.130 \\
\hline SB3-42\сcc & Measured bc & 1.593 & 2.202 & 1.668 & 1.073 \\
\hline SB3-42/ccc & targeted & 1.591 & 2.938 & 1.685 & 1.141 \\
\hline
\end{tabular}


Figure 6-2 shows the measured $\log \mathrm{NL}[\mathrm{B}]$ versus $\Delta \mathrm{G}_{\mathrm{P}}$ for the $\mathrm{SB} 3$ Phase 1 glasses in an effort to assess predictability or applicability of $\Delta \mathrm{G}_{\mathrm{P}}$ to the anticipated compositional region of interest. In this report, predictability is based on the $95 \%$ two-sided confidence interval for an individual PCT response as generated by the Thermodynamic Hydration Energy Reaction Model $\left(\right.$ THERMO $\left.^{\mathrm{TM}}\right) \Delta \mathrm{G}_{\mathrm{P}}$ model (Jantzen et al. 1995). This definition is consistent with that used in recent variability studies (e.g., Harbour et al. 2000; Herman et al. 2001). A comparison is made of the actual leaching performance as determined by the PCT and the prediction limits for an individual glass generated by the THERMO ${ }^{\mathrm{TM}}$ model. The durability of a glass is considered predictable if its PCT response is within the $95 \%$ confidence interval.

In terms of predictability, 81 of 84 glasses (when considering both quenched and ccc) are predictable (i.e., lie within the upper and lower 95\% confidence interval) based on targeted compositions. The exceptions are: SB3-04ccc, SB3-20, and SB3-20ccc. These three glasses lie below the lower $95 \%$ confidence band.

When using the measured or measured-bias corrected compositional views to assess predictability, two glasses lie above and eleven glasses fall below the upper and lower $95 \%$ confidence bands respectively. Both quenched and ccc versions of SB3-07 (based on the measured-bias corrected compositional view) are located near a $\Delta \mathrm{G}_{\mathrm{P}}$ value of -7 and outside the upper 95\% confidence band in Figure 6-2. SB3-07 is a 30\% WL (lower limit) glass based on the 25\% washed sludge and Frit 405 (see Table 4-2). Historically, glasses observed in this region have been classified as "acceptable" in terms of DWPF processing (although unpredictable) given the positive $\Delta \mathrm{G}_{\mathrm{P}}$ value and the fact that the $\mathrm{NL}$ [B] release is well below the EA acceptance criteria. The same classification can be given to SB3-07. The NL [B] release for the quenched and ccc versions are $0.754 \mathrm{~g} / \mathrm{L}$ and $0.726 \mathrm{~g} / \mathrm{L}$, respectively - well below the EA limit.

The eleven glasses falling below the lower 95\% confidence band are: SB3-02ccc, SB3-04, SB304ccc, SB3-14, SB3-14ccc, SB3-18, SB3-18ccc, SB3-20, SB3-20ccc, SB3-24, and SB3-24ccc. (A total of six different targeted glass compositions). These glasses are classified as unpredictable and lie toward the more negative $\Delta \mathrm{G}_{\mathrm{P}}$ values (from approximately -12.5 to -13.5 $\mathrm{kcal} / \mathrm{mol}$ ). Although these glasses are unpredictable, their measured normalized boron releases are acceptable with the highest release (lowest durability) being $0.954 \mathrm{~g} / \mathrm{L}$ from SB3-24 (compared to the $16.695 \mathrm{~g} / \mathrm{L}$ for EA).

It should be noted that the $\Delta \mathrm{G}_{\mathrm{P}}$ model was developed for and is to be applied to homogeneous glasses (DWPF Waste Qualification Report [WQR] [Plodinec et al. 1995] and THERMO ${ }^{\text {TM }}$ [Jantzen et al. 1995]). Of these eleven glasses, six are ccc versions which may (or at least are more likely to) have resulted in an inhomogeneous glass (with respect to crystallization). ${ }^{19}$ Although X-ray diffraction (XRD) analysis was not performed on any glasses in this study, application to inhomogeneous glasses does have potential (and technical) merit, given that the impact of developing secondary phase(s) is minimal to the overall performance as discussed by Peeler et al. (2002b). In this report, it is assumed that the $\Delta \mathrm{G}_{\mathrm{P}}$ model applies to both quenched and ccc glasses.

\footnotetext{
${ }^{19}$ Amorphous phase separation is not considered to be an issue given predictions suggest that these glasses are predicted to be homogeneous.
} 


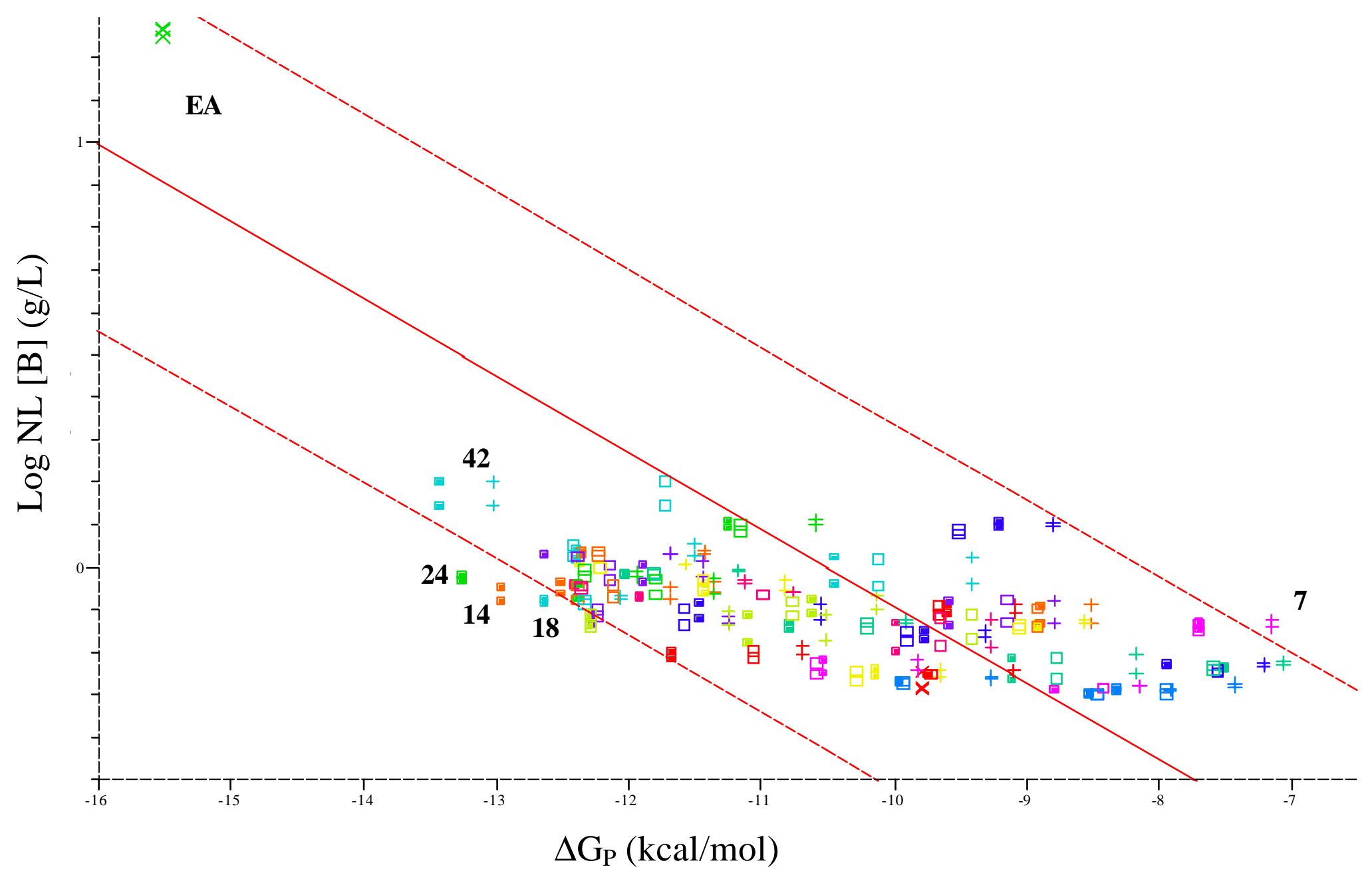

Figure 6-2. $\log$ NL [B] Versus $\Delta \mathbf{G}_{\mathrm{P}}$.

(both quenched and ccc glasses as well as the three compositional views are shown) 
From Table 4-2, all six of these glasses represent the upper WL limit as defined by the MAR acceptance criteria. Further, access to higher WLs for five of the six glass systems is limited by predictions of durability (as noted by PCT being listed in Table 4-2 for the upper WL limit). The exception being SB3-02ccc (based on Frit 405 and the 0\% linear washed sludge at 43\% WL) which would be limited by low viscosity at $44 \%$ WL and higher. However, at $45 \%$ WL predictions indicate that durability becomes an issue.

Although these glasses appear to be approaching a durability level in which acceptability is questionable (in fact restricted based on he current $\Delta \mathrm{G}_{\mathrm{P}}$ criterion), the measured durability response seems to indicate that higher WLs may be potentially achievable prior to reaching an unacceptable level. The results of these glasses are very similar to that observed during the development of Frit 320 for SB2 (Peeler et al. 2001). More specifically, Frit 304 was shown to have a higher melt rate for SB2 (at 25\% WL) compared to Frit 320. It was hypothesized that the increased alkali and/or lower $\mathrm{SiO}_{2}$ concentration in Frit 304 relative to Frit 320 was the primary mechanism for this improved melt rate. However, predictions of durability indicated that the glass would be unacceptable and thus this frit was not recommended by Lambert et al. (2001) for further testing. Formal measurement of the PCT response for this glass (both quenched and ccc versions) indicated that all reportable elemental normalized releases were less than $2 \mathrm{~g} / \mathrm{L}$. As with some of the SB3 glasses (see Figure 6-2), although measured releases are acceptable, predictions of durability limit access to higher waste loadings and potentially implementation of a frit that could improve melt rate and/or throughput.

In general, the SB3 glasses that challenge $\Delta \mathrm{G}_{\mathrm{P}}$ predictions are higher alkali glasses. SB3-16 and SB3-22 are also PCT limited but produce acceptable glasses in terms of durability. This provides an indication that the model, although conservative, may unduly constrain compositional regions that could yield higher melt rates or access to lower washed sludges. The potential implications for SB3 and the frit selection process is to avoid (or at least rank low) frits that are predicted to be PCT limited based solely on model predictions that also have narrow projected operational windows. One case of particular interest is the Frit 202 / Decant \#5 system. For that system, the PCCS operational window is relatively small but is limited on the upper WL side by predictions of durability. The question becomes: "How far can waste loading be increased before the PCT is measured to have an unacceptable behavior?".

Assuming all other factors equal (such as melt rate or tolerance to compositional variation), the frit selection process may minimize (or even eliminate) the potential use of Frit 406, Frit 410, Frit 411, Frit 420, and/or Frit 421 as primary candidates for SB3 based on this effect. However, as previously mentioned, the frit selection process should not be made based on a single criterion but on a collection of criteria that provide insight into the ease of processing SB3 while still making an acceptable glass. Peeler and Edwards (2002) provide more detailed assessments of the operational window size and the impact of projected compositional variation for these systems.

\section{4 “Unacceptable Glasses” Based on Model Predictions}

\subsubsection{Challenges to the Homogeneity Constraint (SB3-13 and SB3-35)}

Rios-Armstrong (2002b) provided direction to fabricate and test a few glasses outside the acceptable region. This was taken into consideration during the glass selection process with the selection of SB3-13 and SB3-35 (both Frit 411 based). The MAR predictions (see Appendices $\mathrm{C} 1$ and $\mathrm{C} 2$ ) indicate that both glasses are inhomogeneous at $25 \% \mathrm{WL}-$ yet they were included in the SB3 Phase 1 test matrix. 
The PCT responses from both SB3-13 and SB3-35 (quenched and ccc versions) were predictable as well as acceptable. Normalized boron releases for SB3-13 and SB3-13ccc are 0.798 and 0.733 $\mathrm{g} / \mathrm{L}$ (based on targeted composition), respectively. Normalized boron releases for SB3-35 and SB3-35ccc are 0.638 and $0.612 \mathrm{~g} / \mathrm{L}$ (based on targeted composition), respectively. These glasses challenge the use and/or application of the homogeneity constraint to SB3 given they produced both acceptable and predictable glasses. These results reinforce the recommendations made by Herman et al. (2002c) specific to SB3 with respect to applicability of the homogeneity constraint for SB3.

\subsubsection{Frit 320-Based Glasses}

Herman, Peeler, and Edwards (2002) indicated that Frit 320 would be evaluated in the Phase 1 study given its classification as a "baseline" frit by Elder (2002). As mentioned in Section 4.0, Frit 320 is a viable frit for the more advanced washed sludges (e.g., 75\% linear washing case, Decant \#12 and above). SB3-42 is a Frit 320-based glass with the 75\% linear washed sludge targeting a $41 \%$ WL. Higher WLs are prohibited by predictions of low viscosity based on the less restrictive PAR criteria. As discussed in Section 4.0, application of the MAR reduces the upper WL limit to 39\% (see Table 4-3) thus yielding the 41\% WL glass as "unacceptable". Predictions of homogeneity and low viscosity are still defining the projected operational window even at the MAR. It is noted that predictions of durability do not limit this system over the entire WL range of interest - therefore durability issues with this glass are not expected for a fully oxidized system.

Table 6-3 summarizes the elemental releases for SB3-42 (quenched and ccc). Although these glasses would be restricted from DWPF processing based on the MAR, their PCT responses are acceptable as well as predictable. The highest NL [B] release (or lowest durability) is $1.591 \mathrm{~g} / \mathrm{L}$ from SB3-42ccc (based on the targeted composition). The NL [B] for EA is $16.695 \mathrm{~g} / \mathrm{L}-$ more than an order of magnitude greater than the measured release of SB3-42ccc. Although this glass challenges the low viscosity constraint, the PCT response is predictable and acceptable and thus demonstrates the applicability of $\Delta \mathrm{G}_{\mathrm{P}}$ model in this compositional region. It should be noted that to obtain access to higher WLs for this particular system, the lower viscosity constraint would either have to be challenged or altered.

Table 6-3. Summary of PCT Results for SB3-42.

\begin{tabular}{||c|c|c|c|c|c||}
\hline \hline Glass ID/HT & Comp View & NL B(g/L) & NL Li (g/L) & NL Na (g/L) & NL Si (g/L) \\
\hline SB3-42 & Measured & 1.391 & 1.409 & 1.616 & 0.869 \\
\hline SB3-42 & Measured bc & 1.404 & 1.361 & 1.669 & 0.825 \\
\hline SB3-42 & Targeted & 1.402 & 1.816 & 1.686 & 0.877 \\
\hline SB3-42\ccc & Measured & 1.578 & 2.279 & 1.616 & 1.130 \\
\hline SB3-42\ccc & Measured bc & 1.593 & 2.202 & 1.668 & 1.073 \\
\hline SB3-42\ccc & Targeted & 1.591 & 2.938 & 1.685 & 1.141 \\
\hline \hline
\end{tabular}




\subsection{Experimental Assessment of PCT as a Function of Redox}

Glasses resulting from the MRF tests showed no visible signs of unreacted batch and appeared single-phased (crystallization or metallic species resulting from an overly reduced melt were not observed). Representative samples of each glass were submitted to the SRTC-ML for chemical analysis. The measured composition and redox of each Tank 8 based glass product are shown in Table 6-4. The measured redox values for the three glasses bound the iron redox $\left(\mathrm{Fe}^{2+} \sqrt{2 \mathrm{Fe}}\right)$ defined by Schreiber and Hockman (1987) of $0.09 \leq \mathrm{Fe}^{2+} / \Sigma \mathrm{Fe} \leq 0.33$. SB3-22-SME has a measured redox of 0.41 exceeding the upper 0.33 limit that minimizes the potential formation of nickel metal and/or nickel sulfide. SB3-23-SME has a measured redox of 0.04 which is lower than the 0.09 limit that is considered too oxidizing and can lead to foaming and increased refractory wear.

Table 6-4. Measured Compositions of the Tank 8 Glasses.

\begin{tabular}{|c|c|c|c|c|c|c|c|}
\hline Element & $\begin{array}{l}\text { SB3-21- } \\
\text { SME }\end{array}$ & $\begin{array}{l}\text { SB3-22- } \\
\text { SME }\end{array}$ & $\begin{array}{c}\text { SB3-23- } \\
\text { SME }\end{array}$ & " Oxide & $\begin{array}{l}\text { SB3-21- } \\
\text { SME }\end{array}$ & $\begin{array}{l}\text { SB3-22- } \\
\text { SME }\end{array}$ & $\begin{array}{c}\text { SB3-23- } \\
\text { SME }\end{array}$ \\
\hline $\mathrm{Al}$ & 2.81 & 3 & 3.12 & $\mathrm{Al}_{2} \mathrm{O}_{3}$ & 5.309 & 5.669 & 5.895 \\
\hline $\mathrm{B}$ & 1.65 & 1.55 & 1.59 & $\mathrm{~B}_{2} \mathrm{O}_{3}$ & 5.313 & 4.991 & 5.120 \\
\hline $\mathrm{Ba}$ & 0.048 & 0.064 & 0.067 & $\mathrm{BaO}$ & 0.054 & 0.071 & 0.075 \\
\hline $\mathrm{Ca}$ & 0.687 & 0.731 & 0.761 & $\mathrm{CaO}$ & 0.961 & 1.023 & 1.065 \\
\hline $\mathrm{Cr}$ & 0.064 & 0.07 & 0.073 & $\mathrm{Cr}_{2} \mathrm{O}_{3}$ & 0.094 & 0.102 & 0.107 \\
\hline$\overline{\mathrm{Cu}}$ & 0.031 & 0.039 & 0.04 & $\mathrm{CuO}$ & 0.039 & 0.049 & 0.050 \\
\hline $\mathrm{Fe}$ & 6.94 & 7.61 & 7.53 & $\mathrm{Fe}_{2} \mathrm{O}_{3}$ & 9.922 & 10.880 & 10.766 \\
\hline $\mathrm{Gd}$ & 0.004 & 0.004 & 0.005 & $\mathrm{Gd}_{2} \mathrm{O}_{3}$ & 0.005 & 0.005 & 0.006 \\
\hline $\mathrm{Li}$ & 2.14 & 2.36 & 2.44 & $\mathrm{Li}_{2} \mathrm{O}$ & 4.607 & 5.081 & 5.253 \\
\hline $\mathrm{Mg}$ & 0.859 & 0.01 & 0.016 & $\mathrm{MgO}$ & 1.424 & 0.017 & 0.027 \\
\hline $\mathrm{Mn}$ & 0.74 & 0.848 & 0.851 & $\mathrm{MnO}$ & 0.955 & 1.095 & 1.099 \\
\hline $\mathrm{Na}$ & 9.49 & 11.8 & 9.95 & $\mathrm{Na}_{2} \mathrm{O}$ & 12.793 & 15.906 & 13.413 \\
\hline $\mathrm{Ni}$ & 0.716 & 0.817 & 0.826 & $\mathrm{NiO}$ & 0.911 & 1.040 & 1.051 \\
\hline $\mathrm{P}$ & 0.027 & 0.026 & 0.017 & $\mathrm{P}_{2} \mathrm{O}_{5}$ & 0.062 & 0.060 & 0.039 \\
\hline $\mathrm{Pb}$ & 0.022 & 0.026 & 0.028 & $\mathrm{PbO}$ & 0.024 & 0.028 & 0.030 \\
\hline $\mathrm{Pd}$ & $<0.010$ & $<0.010$ & $<0.010$ & $\mathrm{PdO}$ & 0.006 & 0.006 & 0.006 \\
\hline $\mathrm{Rh}$ & 0.011 & $<0.010$ & 0.012 & $\mathrm{RhO}_{2}$ & 0.014 & 0.007 & 0.016 \\
\hline $\mathrm{Ru}$ & 0.017 & $<0.010$ & 0.02 & $\mathrm{RuO}_{2}$ & 0.022 & 0.007 & 0.026 \\
\hline $\mathrm{Si}$ & 25.1 & 23.4 & 24.1 & $\mathrm{SiO}_{2}$ & 53.696 & 50.060 & 51.557 \\
\hline $\mathrm{Sr}$ & 0.01 & 0.028 & 0.026 & $\mathrm{SrO}$ & 0.012 & 0.033 & 0.031 \\
\hline$\overline{Z n}$ & 0.053 & 0.076 & 0.085 & $\mathrm{ZnO}$ & 0.066 & 0.095 & 0.106 \\
\hline $\mathrm{Zr}$ & 0.135 & 0.144 & 0.145 & $\mathrm{ZrO}_{2}$ & 0.182 & 0.195 & 0.196 \\
\hline Total & & & & & 96.471 & 96.42 & 95.934 \\
\hline Redox & 0.1 & 0.41 & 0.04 & & & & \\
\hline $\begin{array}{c}\text { Noble Metals } \\
\text { Levels }\end{array}$ & Nominal & $\begin{array}{c}10 \% \text { of } \\
\text { Nominal }\end{array}$ & Nominal & & & & \\
\hline $\begin{array}{c}\text { Nitric Acid } \\
\text { (moles/L) }\end{array}$ & 0.41 & 0.35 & 0.29 & & & & \\
\hline $\begin{array}{c}\text { Formic Acid } \\
(\text { moles } / \mathrm{L})\end{array}$ & 0.73 & 0.70 & 0.68 & & & & \\
\hline $\begin{array}{c}\% \text { of Acid } \\
\text { Stoichiometry }\end{array}$ & $190 \%$ & $160 \%$ & $130 \%$ & & & & \\
\hline Sand & 1.12 & 1.12 & 1.12 & & & & \\
\hline Coal & 0.72 & 0.72 & 0.72 & & & & \\
\hline Oxalate $^{20}$ & $75 \%$ & $50 \%$ & $25 \%$ & & & & \\
\hline
\end{tabular}

\footnotetext{
${ }^{20}$ Oxalate levels shown are the $\%$ of the anticipated level (based on the 330,000 kgs as reported by Goslen).
} 
Representative samples of each glass were also subjected (in triplicate) to the PCT and the resulting solutions submitted to the SRTC-ML for analysis. Table 6-5 provides the measurements generated by the lab for these samples.

Table 6-5. PCTs (in ppm) for Tank 8 Glass Products and Standards.

\begin{tabular}{|c|c|c|c|c|c|c|c|c|c|c|c|c|}
\hline & \multicolumn{6}{|c|}{ As Reported by the SRTC-ML } & \multicolumn{6}{|c|}{ VValues (in ppm) After Adjusting for Dilution Factor } \\
\hline Sample & $\mathrm{Al}$ & $\mathrm{B}$ & $\mathrm{Fe}$ & $\mathrm{Li}$ & $\mathrm{Na}$ & $\mathrm{Si}$ & $\mathrm{Al}$ & $\mathrm{B}$ & $\mathrm{Fe}$ & $\mathrm{Li}$ & $\mathrm{Na}$ & $\mathrm{Si}$ \\
\hline Std1 & 4.36 & 21.0 & 4.05 & 9.51 & 85.3 & 49.3 & 4.36 & 21.0 & 4.05 & 9.51 & 85.3 & 49.3 \\
\hline Blk1 & 0.0045 & 0.056 & 0.002 & 0.100 & 0.05 & 0.0395 & 0.015 & 0.187 & 0.007 & 0.167 & 0.017 & 0.132 \\
\hline Blk2 & 0.0085 & 0.042 & 0.002 & 0.100 & 0.05 & 0.0395 & 0.028 & 0.140 & 0.007 & 0.167 & 0.017 & 0.132 \\
\hline Arm1 & 3.11 & 10.7 & $<0.004$ & 8.69 & 14.1 & 40.4 & 5.183 & 17.834 & 0.007 & 14.484 & 23.500 & 67.335 \\
\hline Arm2 & 3.10 & 11.1 & $<0.004$ & 8.61 & 14.0 & 40.0 & 5.167 & 18.500 & 0.007 & 14.350 & 23.334 & 66.668 \\
\hline Arm3 & 3.28 & 11.1 & $<0.004$ & 8.67 & 14.2 & 40.2 & 5.467 & 18.500 & 0.007 & 14.450 & \begin{tabular}{|l|}
23.667 \\
\end{tabular} & 67.001 \\
\hline SB3-21-1 & 6.92 & 9.21 & 4.90 & 12.7 & 57.3 & 90.6 & 11.534 & .350 & 8.167 & 21.167 & 95.502 & 151.003 \\
\hline SB3-21-2 & 23.0 & 9.16 & 4.05 & 12.3 & 54.9 & 87.1 & 38.334 & 15.267 & 6.750 & 20.500 & 91.502 & 145.170 \\
\hline SB3-21-3 & 6.10 & 9.03 & 4.88 & 12.6 & 55.4 & 94.9 & 10.167 & 15.050 & 8.133 & 21.000 & \begin{tabular}{|l|}
92.335 \\
\end{tabular} & 158.170 \\
\hline SB3-22-1 & 14.4 & 12.0 & 22.4 & 22.7 & 114 & 131 & 24.000 & 20.000 & 37.334 & 37.834 & 190.004 & 218.338 \\
\hline SB3-22-2 & 13.7 & 11.4 & 20.6 & 21.6 & 108 & 126 & 22.834 & 19.000 & 34.334 & 36.001 & 180.004 & 210.004 \\
\hline SB3-22-3 & 15.0 & 11.5 & 22.9 & 21.9 & 110 & 131 & 25.001 & 19.167 & 38.167 & 36.501 & 183.337 & 218.338 \\
\hline SB3-23-1 & 12.4 & 9.24 & 18.6 & 15.7 & 67.8 & 95.4 & 20.667 & 15.400 & 31.001 & 26.167 & 113.002 & 159.003 \\
\hline SB3-23-2 & 12.6 & 9.07 & 11.7 & 15.3 & 67.3 & 92.6 & 21.000 & 15.117 & 19.500 & 25.501 & 112.169 & 154.336 \\
\hline SB3-23-3 & 10.1 & 9.33 & 12.4 & 15.7 & 67.9 & 95.4 & 16.834 & 15.550 & 20.667 & 26.167 & 113.169 & 159.003 \\
\hline
\end{tabular}

Table 6-6 summarizes the PCT results (normalized based on measured compositions - see Table 6-4) for each glass product. Also listed in Table 6-6 are the predicted normalized releases for each glass product based on the measured compositions, measured REDOX values, and partitioning the Fe accordingly in the PCCS model.

Table 6-6. Redox and PCT Response of Tank 8 Glass Products.

\begin{tabular}{||c|c|c|c|c|c||}
\hline Measured & NL [B] g/L & NL [Li] g/L & NL [Na] g/L & NL [Si] g/L & $\mathrm{Fe}^{2+} / \Sigma \mathrm{Fe}$ \\
\hline SB3-21-SME & 0.923 & 0.981 & 0.981 & 0.609 & 0.1 \\
\hline SB3-22-SME & 1.251 & 1.559 & 1.563 & 0.921 & 0.41 \\
\hline SB3-23-SME & 0.966 & 1.069 & 1.134 & 0.658 & 0.04 \\
\hline & & & & & \\
\hline Predicted & NL [B] g/L & NL [Li] g/L & NL [Na] g/L & NL [Si] g/L & $\Delta G_{\mathrm{p}}$ \\
\hline SB3-21-SME & 0.848 & 0.870 & 0.846 & 0.437 & -10.1061 \\
\hline SB3-22-SME & 4.637 & 3.460 & 4.212 & 1.468 & -14.1839 \\
\hline SB3-23-SME & 1.050 & 1.035 & 1.035 & 0.509 & -10.6194 \\
\hline
\end{tabular}

Regardless of the measured redox, all three glasses were very acceptable with respect to the EA limits. Note that the $\Delta \mathrm{G}_{\mathrm{P}}$ of -14.1839 for SB3-22-SME would not be processable by PCCS (e.g., more negative than the SME acceptability limit). The highest measured release (or lowest durability - NL [B] of $1.251 \mathrm{~g} / \mathrm{L}$ ) is from the SB3-22-SME glass that is the most reduced. Following proposed theory, this glass would have a higher portion of reduced iron and thus a negative impact on durability would be projected (i.e., the $\Delta \mathrm{G}_{\mathrm{i}}$ value for $\mathrm{FeO}$ being more negative than that for $\mathrm{Fe}_{2} \mathrm{O}_{3}$ - see Jantzen et al. 1995). If one were to control the redox of this glass within the acceptable limits $\left(\mathrm{Fe}^{2+} / \Sigma \mathrm{Fe} \leq 0.33\right)$ thus yielding a more oxidized glass, one would anticipate the measured releases to decrease or durability to increase. 
SB3-22-SME and SB3-23-SME are both Frit 320 based glasses targeting 30\% WL. The main difference between these two glasses is the acid addition strategy, which is ultimately expressed through a significant difference in measured redox. Given the compositional measurements (see Table 6-4) provide a high degree of confidence that the two glasses are similar in composition, a basis for comparing the effect of redox on PCT response can be made. The redox of SB3-22SME and SB3-23-SME are 0.41 and 0.04, respectively - bounding the acceptable redox values as defined by Schreiber and Hockman (1987). The measured NL [B] for SB3-22-SME and SB3-23$\mathrm{SME}$ are $1.251 \mathrm{~g} / \mathrm{L}$ and $0.966 \mathrm{~g} / \mathrm{L}$, respectively. These data suggest that as the glass becomes more reduced the durability decreases - following theory. Although the normalized releases suggest a reduction in durability, the difference is only $0.285 \mathrm{~g} / \mathrm{L}$ over a very broad redox range. One could argue that the lower $\mathrm{Na}_{2} \mathrm{O}$ and/or slightly higher $\mathrm{SiO}_{2}$ or $\mathrm{Al}_{2} \mathrm{O}_{3}$ concentrations in $\mathrm{SB} 3$ 23-SME could be responsible for this difference.

Also shown in Table 6-6 are the predicted normalized releases for these glasses based on the measured compositions and redox values. For the more oxidized glasses (SB3-21-SME and SB323-SME), the predicted NL [B] are in good agreement with the measured release. The prediction associated with SB3-21-SME product appears to slightly underestimate the measured release while that for SB3-23-SME slightly over predicts. For SB3-22-SME (the most reduced glass), the predicted boron release is $4.637 \mathrm{~g} / \mathrm{L}$, while the measured release is $1.251 \mathrm{~g} / \mathrm{L}$.

These data (albeit limited) suggest that the model prediction is extremely conservative toward the more reduced end of the redox spectrum. The latter statement regarding model conservatism assumes that the predicting data fall directly on the prediction line without accounting for uncertainties in the prediction (e.g., the upper and lower confidence bands were not applied).

\subsection{Redox Dependent Predictions}

Given the redox dependent model is available and was proven effective (or at least conservative for a limited dataset) in Section 6.4, its application to the 42 SB3 Phase 1 glasses will provide insight into the effect of redox on predicted PCT and/or PCCS acceptability. Durability predictions for each targeted glass composition were performed assuming three redox conditions: (1) $\mathrm{Fe}^{2+} / \Sigma \mathrm{Fe}=0$ (fully oxidized), (2) $\mathrm{Fe}^{2+} / \Sigma \mathrm{Fe}=0.2$ (assumed target), and (3) $\mathrm{Fe}^{2+} / \Sigma \mathrm{Fe}=0.33$ (the upper acceptance limit). Given the trends observed with the SB3 SME glasses (see Section 6.4), one would expect that the predictions assuming a redox of 0.33 may be conservative (e.g., the predicted PCT response may be greater than the measured or actual release). It should be noted that the degree of conservatism observed with SB3-22-SME $\left(\mathrm{Fe}^{2+} / \Sigma \mathrm{Fe} \leq 0.41\right.$ with a predicted and measured NL [B] of $4.637 \mathrm{~g} / \mathrm{L}$ and $1.251 \mathrm{~g} / \mathrm{L}$, respectively) may not be as great given the targeted redox of 0.33 .

Table 6-7 shows the predictions of normalized releases for each SB3 Phase 1 glass at 0.0, 0.2 and $0.33 \mathrm{Fe}^{2+} / \Sigma \mathrm{Fe}$ ratios. Also included in Table 6-7 are the measured normalized releases for each glass which should be fully oxidized (assumed $\mathrm{Fe}^{2+} / \Sigma \mathrm{Fe}=0.0$ ) given the raw material selection, exclusion of oxalate and/or coal, and fabrication technique.

When comparing the quenched (measured) PCT releases with their counterpart "fully oxidized" predicted values, the model appears to, depending on the glass, either over or under predict the boron release. This reflects the fact that the model provides a measure of the PCT response in the absence of uncertainties. The uncertainties associated with the model are represented by the $95 \%$ confidence bands (as shown in Figure 6-2). Therefore, observing measured values both above and below the predicted line is expected. Historically (and supported by the Phase 1 data), there 
is evidence that glasses lying at the more positive $\Delta \mathrm{G}_{\mathrm{P}}$ values are typically under predicted by the model while those lying toward the more negative $\Delta \mathrm{G}_{\mathrm{P}}$ values are over predicted.

As the $\mathrm{Fe}^{2+} / \Sigma \mathrm{Fe}$ ratio shifts from 0 to 0.33 , Jantzen et al. (1995) indicate that the durability of the glass should decrease given the presence of $\mathrm{FeO}$ in the glass. This is reflected in the projected PCT responses. For example, consider SB3-01 and SB3-02 with predicted NL [B] releases of $0.66 \mathrm{~g} / \mathrm{L}$ and $1.96 \mathrm{~g} / \mathrm{L}$ (based on a fully oxidized system), respectively. The corresponding predicted NL [B] values for a $\mathrm{Fe}^{2+} / \Sigma \mathrm{Fe}$ ratio of 0.2 (for SB3-01 and SB3-02) increase to $0.88 \mathrm{~g} / \mathrm{L}$ and $2.86 \mathrm{~g} / \mathrm{L}$, respectively. The magnitude of the predicted boron release shift is dependent on the overall glass composition but is highly influenced by the percentage of Fe present.

Proceeding to the more reduced glasses $\left(\mathrm{Fe}^{2+} / \Sigma \mathrm{Fe}=0.33\right)$, predictions indicate that the normalized releases increase (i.e., durability decreases) relative to their more oxidized counterparts. The corresponding predicted NL [B] values for a $\mathrm{Fe}^{2+} / \Sigma \mathrm{Fe}$ ratio of 0.33 (for SB301 and SB3-02) increases to $1.06 \mathrm{~g} / \mathrm{L}$ and $3.66 \mathrm{~g} / \mathrm{L}$, respectively. The highest predicted NL [B] $(4.50 \mathrm{~g} / \mathrm{L})$ is from SB3-22 assuming a $\mathrm{Fe}^{2+} / \Sigma \mathrm{Fe}=0.33$. The more oxidizing SB3-22 glasses $\left(\mathrm{Fe}^{2+} / \Sigma \mathrm{Fe}\right.$ of 0.0 and 0.2$)$ have predicted $\mathrm{NL}[\mathrm{B}]$ of 2.17 and $3.37 \mathrm{~g} / \mathrm{L}$, respectively. The measured NL [B] of SB3-22 (assumed to be fully oxidized) is $0.902 \mathrm{~g} / \mathrm{L}-$ almost 2.5 times less than the predicted value of $2.17 \mathrm{~g} / \mathrm{L}$. Assuming the model becomes more conservative as the glass becomes more reduced, the measured normalized boron release for SB3-22 (with a Fe ${ }^{2+} / \Sigma \mathrm{Fe}$ $=0.33$ ) should be less than the predicted $4.50 \mathrm{~g} / \mathrm{L}$. Even if the model was not conservative, the predicted NL [B] of $4.50 \mathrm{~g} / \mathrm{L}$ is well below the EA acceptance criteria (ignoring uncertainties).

Applying the redox effect over a range from 0.00 (fully oxidized) to 0.33 (upper acceptance limit), the $\Delta \mathrm{G}_{\mathrm{P}}$ predictions indicate that the durability decreases as the glasses become more reduced and the maximum release (or lowest durability) is $4.50 \mathrm{~g} / \mathrm{L}$ which is still acceptable with respect to the EA criterion.

The issue of "acceptability" may give way to the issues of predictability or the classification of "processable". Acceptability being defined based on the measured normalized release compared to $\log \mathrm{NL}[\mathrm{B}]=1.0 \mathrm{~g} / \mathrm{L}-\mathrm{a}$ threshold established by Edwards and Brown (1998) in a review of data to establish alternative criteria for the homogeneity constraint. Predictability being based on the 95\% two-sided confidence interval for an individual PCT response as generated by the THERMO ${ }^{\mathrm{TM}} \Delta \mathrm{G}_{\mathrm{P}}$ model (Jantzen et al. 1995). "Processable" being determined by the relationship of the measured SME products' predicted $\Delta \mathrm{G}_{\mathrm{P}}$ to the SME acceptability $\Delta \mathrm{G}_{\mathrm{P}}$ criterion of $-12.7808 \mathrm{kcal} / \mathrm{mol}$ and ultimately the projected operating window. ${ }^{21}$

\footnotetext{
${ }^{21}$ This value is the SME Acceptability value at the PAR and will be used as a measure of acceptability in this section. The more restrictive MAR value is not used given it is depedent upon the glass composition and thus the value changes with each SB3 Phase 1 glass. It should be noted that a glass deemed unacceptable at the PAR would be classified as unacceptable at the MAR.
} 
Table 6-7. Summary of Predicted and Measured NL [B] Releases (in g/L) for the SB3 Phase 1 Glasses for Various Redox Values Based Upon Targeted Compositional View.

\begin{tabular}{|c|c|c|c|c|c|}
\hline \multirow[b]{2}{*}{$\begin{array}{c}\text { Glass } \\
\text { Identification }\end{array}$} & \multicolumn{3}{|c|}{ Predicted NL[B (g/L)] } & \multicolumn{2}{|c|}{ Measured PCT $^{22}$} \\
\hline & $\begin{array}{c}\mathrm{Fe}^{2+} / \Sigma \mathrm{Fe}=0.0 \\
\quad \text { (assumed) }\end{array}$ & $\begin{array}{l}\mathrm{Fe}^{2+} / \Sigma \mathrm{Fe}=0.2 \\
\text { (assumed target) }\end{array}$ & $\begin{array}{c}\mathrm{Fe}^{2+} / \Sigma \mathrm{Fe}=0.33 \\
\text { (upper limit) }\end{array}$ & Quenched & $\mathrm{CCC}$ \\
\hline SB3-1 & 0.66 & 0.88 & 1.06 & 1.225 & 1.196 \\
\hline SB3-2 & 1.96 & 2.86 & 3.66 & 0.907 & 0.850 \\
\hline SB3-3 & 0.49 & 0.65 & 0.78 & 0.612 & 0.549 \\
\hline SB3-4 & 2.06 & 3.09 & 4.02 & 0.795 & 0.766 \\
\hline SB3-5 & 1.48 & 1.98 & 2.40 & 0.935 & 0.886 \\
\hline SB3-6 & 2.22 & 3.19 & 4.03 & 1.128 & 1.056 \\
\hline SB3-7 & 0.31 & 0.41 & 0.50 & 0.736 & 0.709 \\
\hline SB3-8 & 1.11 & 1.72 & 2.29 & 0.831 & 0.770 \\
\hline SB3-9 & 0.35 & 0.48 & 0.60 & 0.518 & 0.505 \\
\hline SB3-10 & 1.22 & 1.96 & 2.67 & 0.863 & 0.861 \\
\hline SB3-11 & 0.70 & 0.95 & 1.15 & 0.814 & 0.779 \\
\hline SB3-12 & 1.31 & 2.10 & 2.85 & 1.256 & 1.212 \\
\hline SB3-13 & 1.57 & 2.03 & 2.39 & 0.798 & 0.733 \\
\hline SB3-14 & 2.19 & 3.27 & 4.24 & 0.902 & 0.838 \\
\hline SB3-15 & 1.73 & 2.34 & 2.86 & 0.959 & 0.965 \\
\hline SB3-16 & 2.19 & 3.09 & 3.85 & 1.062 & 1.059 \\
\hline SB3-17 & 0.91 & 1.20 & 1.44 & 0.567 & 0.545 \\
\hline SB3-18 & 2.15 & 3.20 & 4.14 & 0.840 & 0.818 \\
\hline SB3-19 & 1.03 & 1.36 & 1.63 & 0.597 & 0.561 \\
\hline SB3-20 & 2.10 & 3.06 & 3.92 & 0.774 & 0.725 \\
\hline SB3-21 & 0.79 & 1.08 & 1.32 & 0.541 & 0.532 \\
\hline SB3-22 & 2.17 & 3.37 & 4.50 & 0.902 & 0.888 \\
\hline SB3-23 & 0.72 & 0.98 & 1.19 & 0.560 & 0.560 \\
\hline SB3-24 & 2.14 & 3.34 & 4.45 & 0.986 & 0.954 \\
\hline SB3-25 & 0.78 & 1.04 & 1.25 & 0.704 & 0.674 \\
\hline SB3-26 & 2.05 & 3.11 & 4.08 & 1.088 & 1.067 \\
\hline SB3-27 & 0.88 & 1.17 & 1.41 & 0.739 & 0.718 \\
\hline SB3-28 & 1.98 & 2.95 & 3.82 & 1.015 & 0.928 \\
\hline SB3-29 & 0.55 & 0.74 & 0.89 & 0.720 & 0.730 \\
\hline SB3-30 & 0.85 & 1.36 & 1.84 & 1.044 & 0.905 \\
\hline SB3-31 & 0.42 & 0.60 & 0.76 & 0.518 & 0.518 \\
\hline SB3-32 & 0.64 & 1.03 & 1.42 & 0.775 & 0.676 \\
\hline SB3-33 & 0.43 & 0.60 & 0.74 & 0.499 & 0.503 \\
\hline SB3-34 & 0.70 & 1.14 & 1.56 & 0.756 & 0.651 \\
\hline SB3-35 & 1.26 & 1.63 & 1.93 & 0.638 & 0.612 \\
\hline SB3-36 & 1.71 & 2.73 & 3.69 & 0.939 & 0.866 \\
\hline SB3-37 & 0.29 & 0.40 & 0.50 & 0.563 & 0.573 \\
\hline SB3-38 & 0.52 & 0.83 & 1.13 & 0.801 & 0.727 \\
\hline SB3-39 & 0.30 & 0.40 & 0.48 & 0.572 & 0.584 \\
\hline SB3-40 & 0.57 & 0.92 & 1.25 & 0.839 & 0.740 \\
\hline SB3-41 & 2.04 & 2.73 & 3.29 & 1.000 & 0.997 \\
\hline SB3-42 & 1.66 & 2.62 & 3.53 & 1.402 & 1.591 \\
\hline
\end{tabular}

\footnotetext{
${ }^{22}$ Given the conditions under which the Phase 1 glasses were melted, these redox of these glasses should be considered fully oxidized.
} 
Hypothetically, consider a fully oxidized glass that is both predictable and processable. As the redox of this glass is shifted toward the more reduced state this glass could become unpredictable, unprocessable, both, or neither. Figure 6-3 illustrates this concept. Point A is a glass that falls with the $95 \%$ confidence bands (predictable) and has a predicted $\Delta \mathrm{G}_{\mathrm{P}}$ more positive than the $12.7808 \mathrm{kcal} / \mathrm{mol}$ PAR SME acceptance criterion. This glass would be deemed processable in DWPF. Point B is the same targeted glass composition with the exception that redox has shifted to a more reduced state and it has been assumed that there was no practical impact on the measured PCT response. Given the anticipated partitioning of both $\mathrm{FeO}$ and $\mathrm{Fe}_{2} \mathrm{O}_{3}$, this glass is now unpredictable (shifted outside the lower $95 \%$ confidence band) but would still be consider processable given its $\Delta \mathrm{G}_{\mathrm{P}}$ is more positive than the $-12.7808 \mathrm{kcal} / \mathrm{mol}$ acceptance criteria.

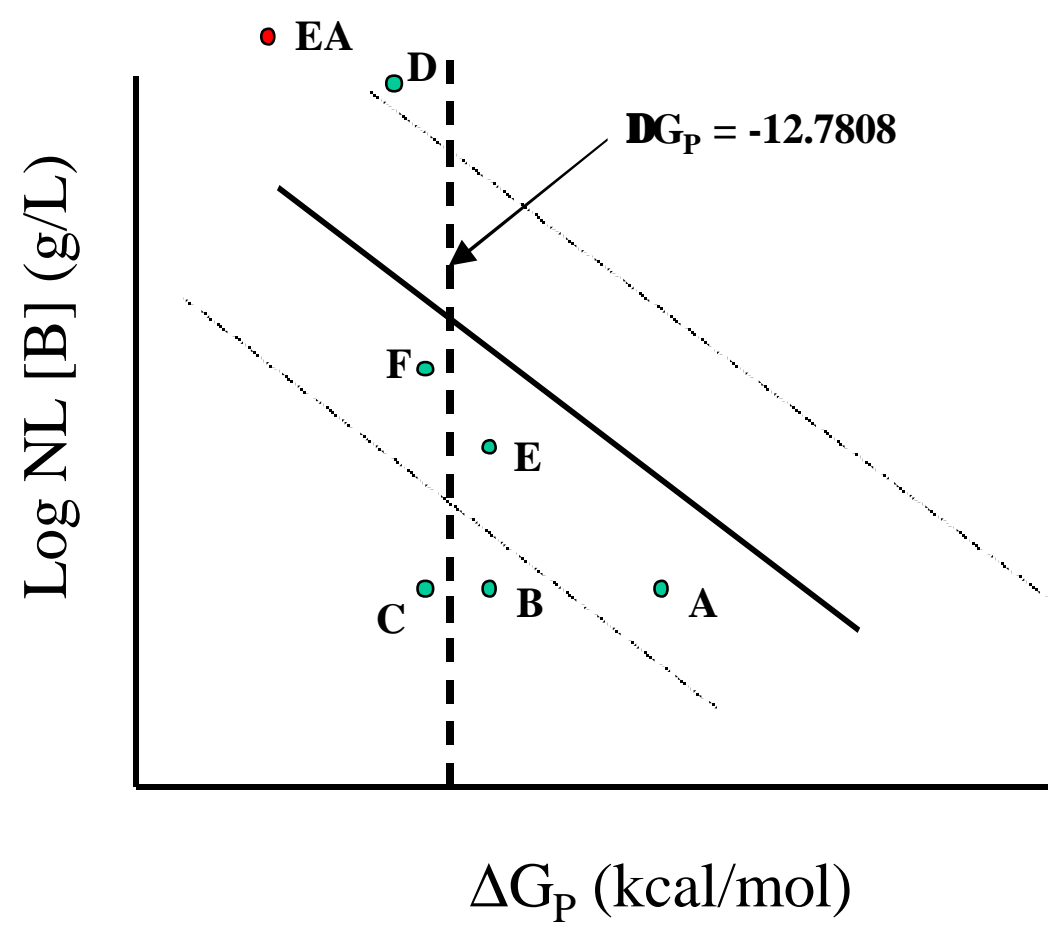

Figure 6-3. Schematic of $\Delta G_{P}$ vs. $\log$ NL [B] and Potential Prediction Scenarios.

The magnitude of the $\Delta \mathrm{G}_{\mathrm{P}}$ shift from Point $\mathrm{A}$ to Point $\mathrm{B}$ is solely dependent upon the application of the $\Delta \mathrm{G}_{\mathrm{i}}$ values for $\mathrm{Fe}_{2} \mathrm{O}_{3}$ and $\mathrm{FeO}$, the concentration of $\mathrm{Fe}$ in the glass, and the glass redox. Appendix $\mathrm{J}$ provides a matrix of the $\Delta \mathrm{G}_{\mathrm{P}}$ impact as a function of Fe concentration in glass and redox. For example, consider an oxidized glass composition with a Fe concentration of $7 \mathrm{wt} \%$ and a predicted $\Delta \mathrm{G}_{\mathrm{P}}$ value of $-10.0 \mathrm{kcal} / \mathrm{mol}$. If the redox of that glass were shifted to 0.2 $\left(\mathrm{Fe}^{2+} / \Sigma \mathrm{Fe}\right.$ ), the predicted impact or shift in terms of $\Delta \mathrm{G}_{\mathrm{P}}$ (strictly from partitioning and application of the $\Delta \mathrm{G}_{\mathrm{i}}$ values) would be $-0.7172 \mathrm{kcal} / \mathrm{mol}$ - resulting in a predicted value for the more reduced version of that same glass of $-10.7172 \mathrm{kcal} / \mathrm{mol}$ which would still be acceptable from a SME acceptability standpoint. Based on the values shown in Appendix J, the higher the Fe concentration in glass and the larger the redox difference, the higher the magnitude of the shift. Those glass systems whose $\Delta \mathrm{G}_{\mathrm{P}}$ value are already challenging the SME acceptability limit under oxidizing conditions, will have a high probability of being classified as "unacceptable" with a shift in redox. 
The theory supporting the redox term in $\Delta \mathrm{G}_{\mathrm{P}}$ implies that the overall glass composition does not play a role in determining the magnitude of the shift but is strictly a function of the Fe concentration. Feng et al. (1990) studied the effect of redox on durability for West Valley waste glasses. It was concluded that the redox effects on durability are strongly dependent on glass composition - not just the Fe concentration. More specifically, the leach rate differed by a factor of 12 from the most oxidized to the most reduced glass at low $\mathrm{Al}_{2} \mathrm{O}_{3}$ concentrations $(\sim 3.3 \mathrm{wt} \%)$, while the factor fell to below 2 for glass compositions with higher $\mathrm{Al}_{2} \mathrm{O}_{3}$ concentrations $(\sim 5.7$ $\mathrm{wt} \%$ and $\sim 9.9 \mathrm{wt} \%$ ). Therefore, the application of the $\Delta \mathrm{G}_{\mathrm{i}}$ values without consideration of the overall glass composition may lead to a more significant $\Delta \mathrm{G}_{\mathrm{P}}$ shift (larger magnitude) than is real. This latter statement being based on the assumption that the potential interactive effects of components such as $\mathrm{Al}_{2} \mathrm{O}_{3}$ have not been accounted for in the overall $\Delta \mathrm{G}_{\mathrm{P}}$ prediction with the redox term activated. This does not suggest that there is not an effect of redox on durability, but the predicted effect may be conservative without consideration of other possible interactive effects. The PCT response (in terms of log NL [B]) determines the magnitude of the shift on the $\mathrm{y}$-axis - this is a physical measurement; not theory-based.

If the $\mathrm{Fe}^{2+} / \Sigma \mathrm{Fe}$ ratio is increased (more reduced), there is a possibility that the glass would become both unpredictable and classified as "unprocessable" given the $\Delta \mathrm{G}_{\mathrm{P}}$ predicted value (again assuming no practical impact on the measured PCT). The fact that it is both unpredictable and unprocessable would be justified if the measured release challenged the EA acceptance criteria (Point D). If not, the conservatism in the model could limit access to both compositional and redox space that would produce an acceptable glass (Point C).

Points E and F conceptualize the option in which an increase in the measured NL [B] occurs as redox transitions from the oxidized to the reduced state. Both points are predictable (lie within the $95 \%$ confidence bands) but Point $\mathrm{E}$ is processable from a PCCS perspective while Point $\mathrm{F}$ is not.

Using the predicted $\Delta \mathrm{G}_{\mathrm{P}}$ values as a function of redox, the concept of acceptability (with respect to SME acceptability PAR criteria) can be applied to the SB3 Phase 1 glasses. Note, that the following discussion does not address the applicability of the model given no formal PCT measurements were taken for all 42 Phase 1 glasses under more reduced conditions. Tables 6-8, 6-9, and 6-10 provide a list of glasses whose predicted $\Delta \mathrm{G}_{\mathrm{P}}$ values are more negative than the $-12.7808 \mathrm{kcal} / \mathrm{mol}$ PAR criteria for $\mathrm{Fe}^{2+} / \Sigma \mathrm{Fe}$ ratios of $0.0,0.2$, and 0.33 , respectively. These glasses would ultimately be classified as "unprocessable" in DWPF. Based solely on the size of each table, a review indicates that as the glasses become more reduced the percentage of SB3 glasses that would be classified as unprocessable in DWPF increases. This trend is expected based on the theoretical nature of the $\Delta \mathrm{G}_{\mathrm{p}}$ model (i.e., the partitioning of $\mathrm{Fe}^{2+}$ and $\mathrm{Fe}^{3+}$ ).

Based on these predicted values and taking a conservative approach, the projected impact on the operating window could be quite large. The projected operational windows presented in Section 4.0 were based on fully oxidized systems. Assuming the impact of redox is adequately captured by the model, for systems that are PCT limited (see Table 4-3), as REDOX shifts toward the more reduced state, more of the operating window will be eliminated. Even for systems that were not PCT limited as defined in Section 4.0, if PCT is a "close second" with respect to failing the MAR criteria and the $\mathrm{Fe}^{2+} / \Sigma \mathrm{Fe}$ ratio is highly reduced (yet still $\leq 0.33$ ), the operating window could change. In both cases, the anticipated effect would be to restrict access to higher WLs - the degree of impact would vary depending upon the overall glass composition and the percent of $\mathrm{Fe}$ in the system. 
For example, consider Table 6-10 $\left(\mathrm{Fe}^{2+} / \Sigma \mathrm{Fe}=0.33\right)$. A review of this table in relation to the systems that are PCT limited (or limited by low viscosity with PCT a close second - e.g., see SB3-02 in Table 4-4) indicates that there are a number of systems which would be classified as unprocessable by the current PCCS models (for all three compositional views). These include: SB3-02, SB3-04, SB3-14, SB3-16, SB3-22, SB3-24, SB3-26, SB3-28, SB3-36, and SB3-42. Each of these glasses is based on the upper WL limit as defined by the MAR acceptance criteria (for a fully oxidized system). Once redox is accounted for in the prediction, these glasses would not be processable. It is interesting to note that the Frit 202-based, Decant \#5 glasses, SB3-15 (33\% WL) and SB3-16 (37\% WL), are classified as "unprocessable" (exception is the measured bias- corrected compositional view of SB3-15). Based on the assessment performed by Peeler and Edwards (2002), Frit 202 was a viable candidate for Decant \#5 although the operational window was rather small compared to the use of Frit 202 with more advanced washed sludges. Their assessment was based on a fully oxidized system. The impact of redox on the predicted PCT response for that system essentially closes any opportunity for processing that system when the $\mathrm{Fe}^{2+} / \Sigma \mathrm{Fe}$ approaches the upper limit of 0.33 . The potential implications to the SB3 frit selection process may be to avoid frits that drive the upper WLs to be PCT limited in an attempt to eliminate this issue.

The concern for the SB3 Frit Development team is the measured PCT responses (although limited) from the MRF glasses (see Section 6.4) as they compare to the predicted values. The predicted NL [B] for SB3-22-SME (with a measured $\mathrm{Fe}^{2+} / \Sigma \mathrm{Fe}=0.41$ ) was $4.637 \mathrm{~g} / \mathrm{L}$ while the measured NL [B] was $1.251 \mathrm{~g} / \mathrm{L}$. As previously discussed, these data suggest that the model may be conservative at the more reduced state in this compositional region. Assuming this is the case (and recognizing that the degree of conservatism may decrease as the system becomes more oxidized), one should question the extent to which the predictions (see Table 6-10 especially) are unduly constraining or restricting access to the SB3 composition region at the higher $\mathrm{Fe}^{2+} \Sigma \mathrm{Fe}$ values. Although these glasses may be classified as "unprocessable" and even "unpredictable", they may yield acceptable glasses if the PCT is formally measured. For example, the $\Delta \mathrm{G}_{\mathrm{P}}$ for SB3-22-SME at $\mathrm{Fe}^{2+} / \Sigma \mathrm{Fe}$ ratios of 0.2 and 0.33 place this glass on the "unprocessable" list (see Tables 6-9 and 6-10 respectively). However, even with a $\mathrm{Fe}^{2+} / \mathrm{LF}$ ratio of 0.41 , the measured $\mathrm{NL}[\mathrm{B}]$ was $1.251 \mathrm{~g} / \mathrm{L}-$ acceptable with respect to EA. Given theory suggests that as the glass becomes more oxidizing, durability should increase (forming more $\mathrm{Fe}_{2} \mathrm{O}_{3}$ ), and one would expect that the NL [B] for the 0.2 and 0.33 states would be less than the measured $1.251 \mathrm{~g} / \mathrm{L}$ for boron.

Given activation of the redox term in PCCS, the result may be to eliminate candidate frits, which produce acceptable PCT results, from further consideration that may have led to the possibility of higher melt rates or total waste throughput. This latter statement being based on the fact that those systems that challenge PCT predictions are anticipated to have higher melt rates given the higher alkali contents.

\section{Table 6-8. Predicted $\Delta \mathbf{G}_{\mathrm{P}}$ Values for $\mathrm{SB3}$ Glasses} Assuming $\mathrm{Fe}^{2+} / \Sigma \mathrm{Fe}=0.00$ (Fully Oxidized).

\begin{tabular}{||c|c|c||}
\hline Glass ID & Comp View & $\Delta \mathrm{G}_{\mathrm{p}}$ \\
\hline SB3-42 & Measured & -13.4256 \\
\hline SB3-24 & Measured & -13.2645 \\
\hline SB3-42 & Measured bc & -13.0332 \\
\hline SB3-14 & Measured & -12.9674 \\
\hline
\end{tabular}


Table 6-9 Predicted $\Delta G_{\mathrm{P}}$ Values for SB3 Glasses Assuming $\mathrm{Fe}^{2+} / \Sigma \mathrm{Fe}=0.2$ (assumed targeted value).

\begin{tabular}{||c|c|c|c|c|c|c||}
\hline Glass ID & Comp View & $\Delta \mathrm{G}_{\mathrm{p}}$ & & Glass ID & Comp View & $\Delta \mathrm{G}_{\mathrm{p}}$ \\
\hline SB3-42 & Targeted & -12.8181 & & SB3-06 & targeted & -13.2845 \\
\hline SB3-28 & Measured & -12.8956 & & SB3-18 & targeted & -13.2933 \\
\hline SB3-41 & Targeted & -12.9083 & & SB3-26 & Measured & -13.3296 \\
\hline SB3-36 & Targeted & -12.9084 & & SB3-14 & targeted & -13.3463 \\
\hline SB3-24 & Measured bc & -12.9720 & & SB3-22-SME & Measured & -13.3715 \\
\hline SB3-10 & Measured & -12.9956 & & SB3-24 & targeted & -13.3933 \\
\hline SB3-02 & targeted & -13.0231 & & SB3-02 & Measured & -13.4011 \\
\hline SB3-41 & Measured & -13.0675 & & SB3-22 & Measured & -13.4058 \\
\hline SB3-18 & Measured bc & -13.0814 & & SB3-16 & Measured & -13.4108 \\
\hline SB3-28 & targeted & -13.0976 & & SB3-22 & targeted & -13.4204 \\
\hline SB3-20 & targeted & -13.1890 & & SB3-36 & Measured & -13.4409 \\
\hline SB3-16 & targeted & -13.2065 & & SB3-18 & Measured & -13.6926 \\
\hline SB3-04 & targeted & -13.2073 & & SB3-14 & Measured & -13.8659 \\
\hline SB3-20 & Measured & -13.2156 & & SB3-42 & Measured bc & -13.9166 \\
\hline SB3-26 & targeted & -13.2271 & & SB3-24 & Measured & -14.2793 \\
\hline SB3-04 & Measured & -13.2404 & & SB3-42 & Measured & -14.3377 \\
\hline SB3-06 & Measured & -13.2521 & \multicolumn{3}{|l}{} \\
\hline
\end{tabular}

Table 6-10. Predicted $\Delta \mathbf{G}_{\mathbf{P}}$ Values for SB3 Glasses Assuming $\mathrm{Fe}^{2+} / \Sigma \mathrm{Fe}=0.33$ (Upper Acceptance Limit).

\begin{tabular}{||c|c|c|c|c|c|c||}
\hline Glass ID & Comp View & $\Delta \mathrm{GP}_{\mathrm{p}}$ & & Glass ID & Comp View & $\Delta \mathrm{G}_{\mathrm{p}}$ \\
\hline SB3-24 & Measured & -14.939 & & SB3-10 & Measured & -13.6939 \\
\hline SB3-42 & Measured & -14.9306 & & SB3-24 & Measured bc & -13.6416 \\
\hline SB3-42 & Measured bc & -14.4909 & & SB3-36 & targeted & -13.6324 \\
\hline SB3-14 & Measured & -14.45 & & SB3-02 & targeted & -13.6149 \\
\hline SB3-18 & Measured & -14.3751 & & SB3-28 & Measured & -13.5416 \\
\hline SB3-36 & Measured & -14.1306 & & SB3-42 & targeted & -13.5304 \\
\hline SB3-22 & Targeted & -14.1113 & & SB3-41 & Measured & -13.5222 \\
\hline SB3-24 & Targeted & -14.0842 & & SB3-41 & targeted & -13.36 \\
\hline SB3-22 & Measured & -14.058 & & SB3-15 & Measured & -13.2166 \\
\hline SB3-02 & Measured & -13.9759 & & SB3-14 & Measured bc & -13.2012 \\
\hline SB3-14 & Targeted & -13.9693 & & SB3-36 & Measured bc & -13.0905 \\
\hline SB3-26 & Measured & -13.9639 & & SB3-28 & Measured bc & -13.0339 \\
\hline SB3-16 & Measured & -13.9136 & & SB3-26 & Measured bc & -13.0314 \\
\hline SB3-18 & Targeted & -13.9122 & & SB3-15 & targeted & -13.022 \\
\hline SB3-22-SME & Measured & -13.8792 & & SB3-12 & targeted & -13.0166 \\
\hline SB3-26 & Targeted & -13.8748 & & SB3-32 & Measured & -13.005 \\
\hline SB3-04 & Measured & -13.8658 & & SB3-12 & Measured & -13.0037 \\
\hline SB3-06 & Targeted & -13.8464 & & SB3-16 & Measured bc & -12.9517 \\
\hline SB3-04 & Targeted & -13.8404 & & SB3-06 & Measured bc & -12.9052 \\
\hline SB3-16 & Targeted & -13.7391 & & SB3-10 & targeted & -12.862 \\
\hline SB3-28 & Targeted & -13.7165 & \multicolumn{3}{|c}{} \\
\hline \hline
\end{tabular}




\subsection{Summary}

The objective of this task was to assess the applicability of the current PCCS durability model $\left(\Delta \mathrm{G}_{\mathrm{P}}\right)$ to the anticipated compositional region of interest to SB3. Specifically, the focus of this study was to demonstrate the applicability of durability predictions (as measured by the Product Consistency Test) for specific SB3 compositions. The SB3 composition region of interest was defined based on three primary factors: (1) sludge or waste stream composition(s), (2) frit composition(s), and (3) waste loading interval of interest.

Two primary inputs were used to define the SB3 sludge composition region of interest: (1) linear washing scenarios and (2) decant information from HLW PE - both based on WCSystems information. Based on input from DWPF and HLW PE personnel, the SB3 Technical team decided to narrow the focus to span the interval bounded by Decant \#5 through Decant \#9. The latter decision was made based on the lack of any significant technical issues being identified during the initial stages of the SB3 flowsheet development activities. Although primarily interested in the decant information (given a more solid technical basis due to compositional projections being based on blending and washing models), the Phase 1 Variability Study also included glass compositions based on the linear washed sludge. The specific (and nominal) compositions used were based on the $0 \%, 25 \%$, and $50 \%$ linear washed projections. The linear washing compositions extend the overall SB3 compositional region of interest in Phase 1 relative to the sole use of Decants \#5 and \#9.

Based on the assessments performed by Peeler and Edwards (2002), nine alternative (400 series) and two existing frits (Frit 202 and Frit 320) were classified as primary candidates for the specific washing scenarios of interest. This classification was based on the response of each frit/sludge combination (for both nominal and nominal $\pm 10 \%$ variation based sludges) from the model-based assessments performed by Peeler and Edwards (2002). Model-based predictions indicated these frits would have large PCCS windows at attractive waste loadings and are robust to compositional variation for specific washing scenarios.

The glass selection process for the Phase 1 Variability Study used the MAR criteria to establish upper and lower WL bounds for specific frit/sludge systems of interest. The result was a 42-glass test matrix. This Phase 1 matrix assessed applicability of the $\Delta \mathrm{G}_{\mathrm{P}}$ model over a bounding or global composition region. The general composition region that was assessed was developed based on the Tank 7 compositional estimates or projections (provided by High Level Waste Process Engineering - see Appendix A), candidate frit composition, and a waste loading interval of interest. Due to the number of uncertainties in the SB3 integrated flowsheet, a more focused study may not be bounding in terms of compositional applicability. Phase 2 (if required) will be a more compositionally focused task. More specifically, as the washing scenario, acid addition and redox control strategies, and frit selection become more definitive, and the results of the Tank 7 sample analysis are received, Phase 2 may be performed to back fill any data gaps resulting from the Phase 1 analysis or gaps not covered by existing and qualified data.

The forty-two Phase 1 Variability Study glasses were batched and melted under conditions that promote oxidizing conditions. To confirm that the "as-fabricated" glasses corresponded to the defined target compositions, a representative sample from each SB3 glass pour patty was submitted to SRTC-ML for chemical analysis under the auspices of an analytical plan. Representative samples of each SB3 glass were subjected to the PCT (both quenched and ccc versions) to assess model applicability. 
Given the potential for the atypical components of coal and sodium oxalate to be present in SB3 (and ultimately transferred to DWPF), redox control becomes a more critical issue. With a potential change or shift in acid addition or redox control strategies, previous assumptions regarding the impact (or lack thereof) of redox on the PCT response needed to be assessed. In this study, two approaches were taken. The first is primarily based on experimental assessments of durability with glasses produced under conditions that bound the acceptable redox range for DWPF. The second approach is strictly based on model predictions of durability for the SB3 Phase 1 glasses considering potential redox impacts. More specifically, the impact of redox on $\Delta \mathrm{G}_{\mathrm{P}}$ and its associated limit of $-12.7808 \mathrm{kcal} / \mathrm{mol}$ was assessed which ultimately dictates the projected operational window. A summary of the Phase 1 Variability Study results is provided below.

\section{Compositional Analysis}

Comparisons were made with respect to target, measured, and measured-bias corrected compositional views for all 42 SB3 glasses. Overall, these comparisons suggest that there were no significant problems in the batching or fabrication of the SB3 Phase 1 Variability Study glasses.

\section{PCT Results}

A statistical comparison of the average differences due to heat treatment (quenched vs. ccc) for each primary element of interest was performed. These paired-t statistical tests indicate a statistically significant (at the 5\% level) difference, on average, between the PCTs for the two heat treatments for $\mathrm{B}$ and $\mathrm{Li}$ over all of the data and for all elements except $\mathrm{Li}$ where the results for SB3-42 are excluded. Although a statistically significant difference is observed, no practical implications are projected.

Normalized boron releases range from $0.499 \mathrm{~g} / \mathrm{L}$ for SB3-33 (most durable) to a high of 1.593 $\mathrm{g} / \mathrm{L}$ from SB3-42ccc (least durable). All glasses (both quenched and ccc) are acceptable being at least an order of magnitude more durable than the $16.695 \mathrm{~g} / \mathrm{L}$ value for NL [B] as reported for EA (Jantzen et al. 1993).

In terms of predictability, 81 of 84 glasses (when considering both quenched and ccc) are predictable (i.e., lie within the upper and lower 95\% confidence interval) based on targeted compositions. The exceptions are: SB3-04ccc, SB3-20, and SB3-20ccc. These three glasses lie below the lower 95\% confidence band, and thus, demonstrate the model's conservatism for these glass compositions.

When using the measured or measured-bias corrected compositional views to assess predictability, two glasses lie above and eleven glasses fall below the upper and lower $95 \%$ confidence bands respectively. Both quenched and ccc versions of SB3-07 (based on the measured-bias corrected compositional view) are located near a $\Delta \mathrm{G}_{\mathrm{P}}$ value of -7 and outside the upper 95\% confidence band in Figure 6-2. SB3-07 is a 30\% WL (lower limit) glass based on the 25\% washed sludge and Frit 405 (see Table 4-2). Historically, glasses observed in this region have been classified as "acceptable" in terms of DWPF processing (although unpredictable) given the more positive $\Delta \mathrm{G}_{\mathrm{P}}$ value and the fact that the NL [B] release is well below the EA acceptance criteria. The same classification can be given to SB3-07. The NL [B] release for the quenched and ccc versions are $0.754 \mathrm{~g} / \mathrm{L}$ and $0.726 \mathrm{~g} / \mathrm{L}$, respectively - well below the EA value. 
The eleven glasses falling outside the lower 95\% confidence band are: SB3-02ccc, SB3-04, SB304ccc, SB3-14, SB3-14ccc, SB3-18, SB3-18ccc, SB3-20, SB3-20ccc, SB3-24, and SB3-24ccc. (A total of six different targeted glass compositions.) These glasses are classified as unpredictable and lie toward the more negative $\Delta \mathrm{G}_{\mathrm{P}}$ values (from approximately -12.5 to -13.5 $\mathrm{kcal} / \mathrm{mol}$ ). Although these glasses are unpredictable, their measured normalized boron releases are very acceptable with the highest release (lowest durability) being $0.954 \mathrm{~g} / \mathrm{L}$ from SB3-24 (compared to the $16.695 \mathrm{~g} / \mathrm{L}$ for EA).

In general, the SB3 glasses that challenge $\Delta \mathrm{G}_{\mathrm{P}}$ predictions are higher alkali glasses. This provides an indication that the model, although conservative, may unduly constrain compositional regions that could yield higher melt rates or access to lower washed sludges. The potential implications for SB3 and the frit selection process are to avoid (or at least rank low) frits that are predicted to be PCT limited based solely on model predictions and that also have narrow projected operational windows. One case of particular interest is the Frit 202 / Decant \#5 system. For that system, the PCCS operational window is relatively small but is limited on the upper WL side by predictions of durability. The question becomes: "How far can waste loading be increased before the PCT is measured to have an unacceptable behavior?".

The experimental results demonstrated applicability (or conservatism) of the $\Delta \mathrm{G}_{\mathrm{P}}$ model for each of the 42 SB3 test matrix glasses when fabricated under oxidizing conditions. The PCT response for all glasses (both quenched and centerline cansiter cooled) were also acceptable being at least an order of magnitude more durable than the $16.695 \mathrm{~g} / \mathrm{L}$ value for normalized boron release as reported for the Environmental Assessment (EA) glass.

\section{Challenges to Homogeneity}

The PCT responses from both SB3-13 and SB3-35 (quenched and ccc versions) were predictable as well as acceptable even though these glasses were predicted to be inhomogeneous at the PAR. Normalized boron releases for SB3-13 and SB3-13ccc are 0.798 and $0.733 \mathrm{~g} / \mathrm{L}$ (based on targeted composition), respectively. Normalized boron releases for SB3-35 and SB3-35ccc are 0.638 and $0.612 \mathrm{~g} / \mathrm{L}$ (based on targeted composition), respectively. These glasses challenge the use of the homogeneity constraint but given they produced both acceptable and predictable glasses the results reinforce the recommendations made by Herman et al. (2002) specific to SB3 that support the elimination or replacement of the homogeneity constraint with proposed criteria for alumina and/or sum of alkali.

\section{Redox Effect on PCT: Measurements}

Regardless of the measured redox, all three SB3 MRF glasses are very acceptable with respect to the EA limits. The highest release (or lowest durability - NL [B] of $1.251 \mathrm{~g} / \mathrm{L}$ ) is from the SB3$22-\mathrm{SME}$ glass that is the most reduced $\left(\mathrm{Fe}^{2+} / \Sigma \mathrm{Fe}=0.41\right)$. Following proposed theory, this glass would have a higher portion of reduced iron, and thus a negative impact on durability would be projected (i.e., the $\Delta \mathrm{G}_{\mathrm{i}}$ value for $\mathrm{FeO}$ being more negative than that for $\mathrm{Fe}_{2} \mathrm{O}_{3}$ ). These data (albeit limited) suggest that the model prediction is extremely conservative toward the more reduced end of the redox spectrum. 


\section{Redox Effect on $\Delta \mathrm{G}_{\mathrm{p}}:$ Predictions}

The $\Delta \mathrm{G}_{\mathrm{P}}$ predictions indicate that the durability decreases as the glasses become more reduced. The maximum release (or lowest durability) predicted for the SB3 Phase 1 glasses is $4.499 \mathrm{~g} / \mathrm{L}$ which is still acceptable with respect to the EA criterion.

Based on these predicted values and taking a conservative approach (e.g., assuming the redox would be controlled closer to the more reduced state for SB3), the projected impact on the operating window could be quite large. The projected operational windows presented in Section 4.0 were based on a fully oxidized system. Assuming the impact of redox is adequately captured by the model, for systems that are PCT limited (see Table 4-3), as redox shifts toward the more reduced state, more of the operating window will be eliminated. Even for systems that were not PCT limited as defined in Section 4.0, if PCT is a "close second" with respect to failing the MAR criteria and the $\mathrm{Fe}^{2+} / \Sigma \mathrm{Fe}$ ratio is highly reduced (yet still within the acceptance limits), the operating window could change. In both cases, the anticipated effect would be to restrict access to higher WLs - the degree of impact would vary depending upon the overall glass composition and the percent of Fe in the system. The potential implications to the SB3 frit selection process may be to avoid frits that drive the upper WLs to be PCT limited in an attempt to eliminate this issue.

The concern for the SB3 Frit Development team is the measured PCT responses (albeit limited) as compared to predicted values from the MRF glasses (see Section 6.4). The predicted NL [B] for SB3-22-SME (with a measured $0.41 \mathrm{Fe}^{2+} \Sigma \mathrm{Fe}$ ratio) was $4.637 \mathrm{~g} / \mathrm{L}$ while the measured NL [B] was $1.251 \mathrm{~g} / \mathrm{L}$. As previously discussed, these data suggest that the model is conservative at the more reduced state. Assuming this is the case (and recognizing that the degree of conservatism may decrease as the system becomes more oxidized), one should question the extent to which the predictions (see Table 6-10 especially) are unduly constraining or restricting access to the SB3 composition region at the higher $\mathrm{Fe}^{2+} / \Sigma \mathrm{Fe}$ values. Although these glasses may be classified as "unprocessable" and even "unpredictable", they may yield acceptable glasses if the PCT is formally measured. For example, although the $\Delta \mathrm{G}_{\mathrm{P}}$ for SB3-22-SME was not predicted at a $\mathrm{Fe}^{2+} / \Sigma \mathrm{Fe}$ ratio $=0.41$, its corresponding values at 0.09 and 0.33 place this glass on the "unprocessable" list (see Tables 6-9 and 6-10 respectively). However, even with a $\mathrm{Fe}^{2+} / \Sigma \mathrm{Fe}$ ratio of 0.41 , the measured NL [B] was $1.251 \mathrm{~g} / \mathrm{L}$ - very acceptable with respect to EA. Given theory suggest that as the glass becomes more oxidizing, durability should increase (forming more $\mathrm{Fe}_{2} \mathrm{O}_{3}$ ), one would expect that the NL [B] for the 0.09 and 0.33 states would be less than the measured $1.251 \mathrm{~g} / \mathrm{L}$ for boron. 


\subsection{Recommendations}

With respect to the SB3 frit selection process, the key criteria or aspects that will provide the technical basis for selecting a frit include: (1) maximizing the projected operational window size (i.e., the waste loading interval) over the anticipated SB3 composition region, (2) providing a frit that is robust or insensitive to anticipated sludge composition variation, (3) improving or maintaining high waste loadings (WLs), (4) improving or maintaining high melt rates, and (5) providing a "frittable" additive or frit composition. The results of the Phase 1 Variability Study should be used in this process as well. Given the five key criteria can be competing, the basis for not only developing but ultimately selecting a frit for SB3 is complex. The selection process should not be made based on a single criterion but a collection of criteria that provide insight into the economics of processing SB3. A balanced approach should be utilized in both the development and selection.

Based on the Phase 1 Variability Study results, the following recommendations are made:

Derform a Phase 2 supplemental variability study based on the latest Tank 7 sample results to assess the applicability of the $\Delta \mathrm{G}_{\mathrm{P}}$ model while considering redox

The Phase 2 Variability Study would be comprised of a paper study assessment and an experimental study. The paper study assessment would include a review or comparison of the measured Tank 7 composition as it relates to the projected decant or washing scenarios used in the SB3 Frit Development program. Based on that comparison, primary frits would be identified (or those that have been identified would be confirmed to be applicable to Tank 7). Projected operational windows for the primary frits of interest would be determined based on PCCS models. An evaluation of the impact of the redox term associated with the current durability model will be made as upper and lower WLs are defined for specific glass forming systems of interest.

The objective of the experimental portion of the Phase 2 Variability Study will be to demonstrate the applicability of the $\Delta \mathrm{G}_{\mathrm{P}}$ for reduced glasses. The model-based predictions from the Phase 1 study indicated that activating the redox term in the durability model has potential negative impacts to the frit selection process. Although the $\Delta \mathrm{G}_{\mathrm{P}}$ model was applicable (or at least conservative) for the 42 Phase 1 glasses, the fact that they were made under oxidizing conditions did not allow for a complete experimental assessment of the impact of redox on PCT. The limited data that were presented indicated that the model may conservative for certain frit/sludge systems once redox is accounted for. To address this issue, it is recommended that the latest results from the Tank 7 samples be used to support this assessment. Specific frit/sludge systems will be selected based on the output of the Phase 2 paper study. Glasses will be produced targeting redox vaues of interest (input from the acid addition strategy team will be utilized to establish reasonable bounds) and PCT will be measured.

The results of the Phase 2 experimental study would also provide insight into the potential conservatism of the current $\Delta \mathrm{G}_{\mathrm{p}}$ model with the redox term activated. More specifically, the measured releases will be used to assess if the $\Delta \mathrm{G}_{\mathrm{i}}$ value for FeO shifts the $\Delta \mathrm{G}_{\mathrm{P}}$ value more negatively than necessary for this compositional region. 


\section{Decision on Phase 3 Variability Study}

The decision as to whether to perform a Phase 3 SB3 Variability Study would be based on two primary factors. First, an assessment of the SB3 qualification sample (as compared to the Tank 7 samples) may provide compositional justification to perform either a paper study assessment and/or an experimental study. The paper study assessment would provide assurance that the PCCS predictions allow for satisfactory processing windows for the specific frit and SB3 sludge combinations of interest. More specifically, as the washing scenario, acid addition and redox control strategies, and frit selection are established, and the results of the SB3 Tank 51 qualification sample analysis are received, Phase 3 may be performed to assess projected operational windows.

Initiating the experimental portion of the Phase 3 Study could be based on two issues: (1) to back fill any data gaps resulting from the Phase 1 or the Phase 2 analysis or gaps not covered by existing and qualified data, or (2) to provide data from which the effect of redox on PCT can be validated for the specific compositional region of interest (if not fully addressed in Phase 2). If model predictions remain extremely conservative, an alternative approach for predictions of durability may be needed specific to SB3. 


\subsection{References}

ASTM 1998. Standard Test Methods for Determining Chemical Durability of Nuclear Waste Glasses: The Product Consistency Test (PCT), ASTM C-1285-98, 1998.

Bronikowski, MG, MC Thompson, FR Graham, TL Fellinger, WR Wilmarth, and DT Hobbs, 2002. Technical Task and Quality Assurance Plan for Assessing Downstream Effects of Plutonium/Gadolinium in Sludge Washing and SRAT, WSRC-RP-2002-00178, Westinghouse Savannah River Company, Aiken, South Carolina.

Brown KG, CM Jantzen, and G Ritzhaupt. 2001. Relating Liquidus Temperature to Composition for Defense Waste Processing Facility (DWPF) Process Control, WSRC-TR-2001-00520, Westinghouse Savannah River Company, Aiken, South Carolina.

Brown, KG, RL Postles, and TB Edwards, 2002. SME Acceptability Determination for DWPF Process Control, WSRC-TR-95-0364, Revision 4, Westinghouse Savannah River Company, Aiken, South Carolina.

Brown, KG. 2002. Changes Made to the DWPF PCCS Spreadsheet, SRTC-GPD-2002-00059, Westinghouse Savannah River Company, Aiken, South Carolina.

Edwards TB, and KG Brown. 1998. Evaluating the Glasses Batched for the Tank 42 Variability Study (U), SRT-SCS-98-017, Revision 0, Westinghouse Savannah River Company, Aiken, South Carolina.

Edwards TB. 2002. Revision of DWPF's PCCS: An Overview of the Modifications and Assocaited Test Cases, SRTC-SCS-2002-00032, Westinghouse Savannah River Company, Aiken, South Carolina.

Elder, HH. 2002. Impact of Processing Americium and Curium in ESP, DWPF, and Saltstone, Savannah River Site, High Level Waste Process Engineering Position Paper, HLW-SDT-200100244, Rev. 1.

Fellinger, TL. 2002. Sludge Batch 3 - Overall Program View (U), WSRC-RP-2002-00353, Westinghouse Savannah River Company, Aiken, South Carolina.

Feng, X, IL Pegg, E Saad, S Cucinell, A Barkatt. 1990. Redox Effect on the Durability and Viscosity of Nuclear Waste Glasses, In Ceramic Transactions: Nuclear Waste Management III, Edited by G.B. Mellinger, Vol. 9, pp. 165-174. American Ceramic Society, Westerville, Ohio.

Fowler, JR. 1980. "Estimate of Maximum Amount of Reducing Agents and Sand in the SRP Waste for DWPF TDS”, Don't Say It Write Memo, September 9, 1980.

Goslen, AQ. 1984. "Estimated Sodium Oxalate in the Tank Farm”, March 22, 1984.

Harbour, JR, TB Edwards, and RJ Workman. 2000. Summary of Results for Macrobatch 3 Variability Study, WSRC-TR-2000-00351, Revision 0, Westinghouse Savannah River Company, Aiken, South Carolina. 
Westinghouse Savannah River Company

Herman, CC, TB Edwards, and DM Marsh. 2001. Summary of Results for the Expanded Macrobatch 3 Variability Study, WSRC-TR-2001-00511, Revision 0, Westinghouse Savannah River Company, Aiken, South Carolina.

Herman, CC, DK Peeler, and TB Edwards. 2002. Task Technical and QA Plan: Sludge Batch 3 Variability Studies with Simulants, WSRC-RP-2002-00386, Westinghouse Savannah River Company, Aiken, South Carolina.

Herman, CC, TL Fellinger, NE Bibler, and DC Koopman. 2002a. Scoping SRAT Runs with Simulated Sludge Batch 3, SRT-GPD-2002-00044, Westinghouse Savannah River Company, Aiken, South Carolina.

Herman, CC, TL Fellinger, NE Bibler, and DC Koopman. 2002b. Scoping SRAT Runs with Simulated Sludge Batch 3, SRT-GPD-2002-00044, Westinghouse Savannah River Company, Aiken, South Carolina.

Herman, CC, TB Edwards, DR Best, DM Marsh, and RJ Workman. 2002c. Reduction of Constraints: Phase 2 Experimental Assessment for Sludge-Only Processing, WSRC-TR-200200482, Rev. 0, Westinghouse Savannah River Company, Aiken, South Carolina.

Herman CC. 2002a. Task Technical \& QA Plan: Sludge Batch 3 Simulant Flowsheet Studies, WSRC-RP-2002-00563, Westinghouse Savannah River Company, Aiken, South Carolina.

Herman CC. 2002b. Task Technical \& QA Plan: Oxalate Studies for Sludge Batch 3 - SRAT Processing, WSRC-RP-2002-00320, Westinghouse Savannah River Company, Aiken, South Carolina.

Herman CC, TB Edwards, DR Best, JC George, DC Koopman, and MF Williams. 2003. Data Summary from SRAT Runs SB3-19 to SB3-24 to Evaluate Sodium Oxalate Addition Levels and SME Processing, SRT-GPD-2002-00200, January 3, 2003.

Jantzen CM and MJ Plodinec. 1986. Composition and Redox Control of Waste Glasses: Recommendation for Process Control Limit, DPST-86-773, E.I. duPont de Nemours and Company, Savannah River Laboratory, Aiken, South Carolina.

Jantzen CM, NE Bibler, DC Beam, CL Crawford, and MA Pickett. 1993. Characterization of the DWPF Environmental Assessment (EA) Glass Standard Reference Material (U), WSRC-TR92-346, Rev. 1, Westinghouse Savannah River Company, Aiken, SC.

Jantzen, CM, JB Pickett, KG Brown, TB Edwards, and DC Beam. 1995. Process/Product Models for the Defense Waste Processing Facility (DWPF): Part I. Predicting Glass Durability from Composition Using a Thermodynamic Hydration Energy Reaction Model (THERMO) (U), WSRC-TR-93-672, Revision 1, Volume 1, Westinghouse Savannah River Company, Aiken, South Carolina.

Jantzen, CM, RF Swingle, and FG Smith. 2002. Impact of Tank 19F Zeolite Mound on DWPF Vitrification of Sludge Batch 3, WSRC-TR-2002-00288, Revision 0, Westinghouse Savannah River Company, Aiken, South Carolina. 
Jantzen, CM. 2002. REDOX Studies and Modeling for DWPF Sludge Batch 3, WSRC-RP-200200341, Westinghouse Savannah River Company, Aiken, South Carolina.

Jilani, I. 2002. Evaluation of Impact of HLW of Using Gadolinium as a Neutron Poison for Plutonium, Technical Task Request, NMMD-HTS-2002-010, Westinghouse Savannah River Company, Aiken, South Carolina.

Koopman DC. 2002a. Sludge Batch 3 - Run 21, SRAT/SME with 75\% Residual Oxalate, SRTGPD-2002-00118, Westinghouse Savannah River Company, Aiken, South Carolina.

Koopman DC. 2002b. Sludge Batch 3 - Run 22, SRAT/SME with 50\% Residual Oxalate, SRTGPD-2002-00118, Westinghouse Savannah River Company, Aiken, South Carolina.

Koopman DC. 2002c. Sludge Batch 3 - Run 23, SRAT/SME with 25\% Residual Oxalate, SRTGPD-2002-00119, Westinghouse Savannah River Company, Aiken, South Carolina.

Lambert, DP, TH Lorier, DK Peeler, and ME Stone. 2001. Melt Rate Improvement for DWPF MB3: Summary and Recommendations, WSRC-TR-2001-00148, Westinghouse Savannah River Company, Aiken, South Carolina.

Lorier TH, DK Peeler, and DC Koopman. 2003. Sludge Batch 3 Melt Rate Assessment, WSRCTR-2003-00027, Revision 0, Westinghouse Savannah River Company, Aiken, South Carolina.

Lorier, TH and PL McGrier. 2002. Melt Rate Improvement for the DWPF: Higher Waste Loading Testing, WSRC-TR-2002-00344, Revision 0, Westinghouse Savannah River Company, Aiken, South Carolina.

Marra SL, and CM Jantzen. 1993. Characterization of Projected DWPF Glasses Heat Treated to Simulate Canister Centerline Cooling (U), WSRC-TR-92-142, Revision 1, Westinghouse Savannah River Company, Aiken, South Carolina.

Patel, PM. 2002. Evaluation of Impacts of Sludge Containing “Americium/Curium” on $\mathrm{H}_{2}$ Generation and Glass Quality, Technical Assistance Request, HLW/DWPF-TAR-02-0007, Westinghouse Savannah River Company, Aiken, South Carolina.

Peeler, DK. 1996a. Batch 1 Variability Study Using Twice Washed Tank 51 Sludge and Frit 200 $(U)$, WSRC-RP-96-0020, Revision 0, Westinghouse Savannah River Company, Aiken, South Carolina.

Peeler, DK. 1996b. Batch 1 Variability Study Using Twice Washed Tank 51 Sludge (U), WSRCRP-95-1045, Revision 1, Westinghouse Savannah River Company, Aiken, South Carolina.

Peeler, DK, TH Lorier, DF Bickford, DC Witt, TB Edwards, KG Brown, IA Reamer, RJ Workman, JD Vienna. 2001. Melt Rate Improvement for DWPF MB3: Frit Development and Model Assessment, WSRC-TR-2001-00131, Revision 0, Westinghouse Savannah River Company, Aiken, South Carolina. 
Peeler, DK, NE, Bibler, and TB Edwards. 2002a. An Assessment of the Impacts of Adding $\mathrm{Am} / \mathrm{Cm}$ and $\mathrm{Pu} / \mathrm{Gd}$ Waste Streams to Sludge Batch 3 (SB3) on DWPF $\mathrm{H}_{2}$ Generation Rates and Glass Properties, WSRC-TR-2002-00145, Revision 0, Westinghouse Savannah River Company, Aiken, South Carolina.

Peeler DK, KG Brown, TB Edwards, DR Best, RJ Workman, and IA Reamer. 2002b. Reduction of Constraints: Phase 1 Experimental Assessment of Centroid-Based Sludge-Only Glasses, WSRC-TR-2002-00120, Revision 0, Westinghouse Savannah River Company, Aiken, South Carolina.

Peeler DK and TB Edwards. 2002. Frit Development for Sludge Batch 3, WSRC-TR-200200491, Revision 0, Westinghouse Savannah River Company, Aiken, South Carolina.

Plodinec MJ, KG Brown, SL Marra, CM Jantzen, and TB Edwards. 1995. "Technical Bases for the DWPF Glass Product Control Program", In: Waste Qualification Report (WQR), WSRC-IM91-116-5, Revision 1, Volume 5, Aiken, South Carolina.

Rios-Armstrong, MA. 2002a. Technical Task Request, Sludge Batch 3 Carbon Content Analysis, HLW/DWPF/TTR-02-0017, Westinghouse Savannah River Company, Aiken, South Carolina.

Rios-Armstrong, MA. 2002b. Sludge Batch 3 Variability Studies with Simulants, HLW/DWPF/TTR-02-0027, Westinghouse Savannah River Company, Aiken, South Carolina.

Schreiber, HD and AL Hockman. 1987. "REDOX Chemistry in Candidate Glasses for Nuclear Waste Immobilization," J. Amer. Ceram. Soc., Volume 70, No. 8, pp. 591 - 594.

Savannah River Technology Center (SRTC). 2002a. "Batching Procedure," SRTC Procedure Manual, L29, ITS-0001, Westinghouse Savannah River Company, Aiken, SC.

Savannah River Technology Center (SRTC). 2002b. “Melting Procedure,” SRTC Procedure Manual, L29, ITS-0003, Westinghouse Savannah River Company, Aiken, SC.

Westinghouse Savannah River Company (WSRC). 2001. Savannah River Site High Level Waste System Plan (HLW), HLW-2001-00040, Revision 13, Aiken, South Carolina. 
Immobilization Technology Section

WSRC-TR-2002-00549

Savannah River Technology Center

Rev. 0

Westinghouse Savannah River Company

\section{Appendix A}

Nominal Decant Compositions

(Personal Communication with H.H. Elder) 
Immobilization Technology Section

WSRC-TR-2002-00491

Savannah River Technology Center

Rev. 0

Westinghouse Savannah River Company

\begin{tabular}{|c|c|c|c|c|c|c|c|c|c|c|c|}
\hline \multicolumn{12}{|c|}{ Elemental } \\
\hline wt. \% & Decant \#5 & Decant \#6 & Decant \#7 & Decant \#8 & Decant \#9 & Decant \#10 & Decant \#11 & Decant \#12 & Decant \#13 & Decant \#14 & Decant \#15 \\
\hline $\mathrm{Al}$ & 7.33 & 7.59 & 7.82 & 8.01 & 8.18 & 8.33 & 8.47 & 8.60 & 8.71 & 8.82 & 8.93 \\
\hline B & 0.00 & 0.00 & 0.00 & 0.00 & 0.00 & 0.00 & 0.00 & 0.00 & 0.00 & 0.00 & 0.00 \\
\hline $\mathrm{Ba}$ & 0.17 & 0.18 & 0.18 & 0.19 & 0.19 & 0.19 & 0.20 & 0.20 & 0.20 & 0.21 & 0.21 \\
\hline $\mathrm{Ca}$ & 1.97 & 2.04 & 2.10 & 2.15 & 2.20 & 2.24 & 2.28 & 2.31 & 2.34 & 2.37 & 2.40 \\
\hline $\mathrm{Ce}$ & 0.23 & 0.24 & 0.24 & 0.25 & 0.25 & 0.26 & 0.26 & 0.27 & 0.27 & 0.27 & 0.28 \\
\hline Cs & 0.00 & 0.00 & 0.00 & 0.00 & 0.00 & 0.00 & 0.00 & 0.00 & 0.00 & 0.00 & 0.00 \\
\hline $\mathrm{Cu}$ & 0.12 & 0.13 & 0.13 & 0.13 & 0.14 & 0.14 & 0.14 & 0.14 & 0.14 & 0.15 & 0.15 \\
\hline $\mathrm{Fe}$ & 21.53 & 22.31 & 22.98 & 23.54 & 24.05 & 24.48 & 24.89 & 25.27 & 25.59 & 25.92 & 26.26 \\
\hline $\mathrm{K}$ & 0.27 & 0.28 & 0.29 & 0.30 & 0.31 & 0.31 & 0.32 & 0.32 & 0.33 & 0.33 & 0.33 \\
\hline $\mathrm{La}$ & 0.15 & 0.16 & 0.16 & 0.16 & 0.17 & 0.17 & 0.17 & 0.18 & 0.18 & 0.18 & 0.18 \\
\hline $\mathrm{Li}$ & 0.00 & 0.00 & 0.00 & 0.00 & 0.00 & 0.00 & 0.00 & 0.00 & 0.00 & 0.00 & 0.00 \\
\hline $\mathrm{Mg}$ & 0.09 & 0.09 & 0.09 & 0.10 & 0.10 & 0.10 & 0.10 & 0.10 & 0.10 & 0.10 & 0.11 \\
\hline Mo & 0.00 & 0.00 & 0.00 & 0.00 & 0.00 & 0.00 & 0.00 & 0.00 & 0.00 & 0.00 & 0.00 \\
\hline $\mathrm{Na}$ & 23.15 & 21.29 & 19.71 & 18.38 & 17.17 & 16.15 & 15.18 & 14.27 & 13.52 & 12.74 & 11.94 \\
\hline $\mathrm{Nb}$ & 0.00 & 0.00 & 0.00 & 0.00 & 0.00 & 0.00 & 0.00 & 0.00 & 0.00 & 0.00 & 0.00 \\
\hline $\mathrm{Ni}$ & 0.97 & 1.00 & 1.03 & 1.06 & 1.08 & 1.10 & 1.12 & 1.14 & 1.15 & 1.16 & 1.18 \\
\hline $\mathrm{Pb}$ & 0.21 & 0.22 & 0.23 & 0.23 & 0.24 & 0.24 & 0.25 & 0.25 & 0.25 & 0.26 & 0.26 \\
\hline $\mathrm{Si}$ & 0.75 & 0.78 & 0.80 & 0.82 & 0.84 & 0.85 & 0.87 & 0.88 & 0.89 & 0.90 & 0.91 \\
\hline Th & 0.10 & 0.10 & 0.10 & 0.11 & 0.11 & 0.11 & 0.11 & 0.11 & 0.11 & 0.12 & 0.12 \\
\hline $\mathrm{Ti}$ & 1.29 & 1.34 & 1.38 & 1.41 & 1.44 & 1.47 & 1.49 & 1.52 & 1.54 & 1.55 & 1.58 \\
\hline $\mathrm{U}$ & 6.61 & 6.85 & 7.05 & 7.23 & 7.38 & 7.51 & 7.64 & 7.76 & 7.86 & 7.96 & 8.06 \\
\hline $\mathrm{Y}$ & 0.00 & 0.00 & 0.00 & 0.00 & 0.00 & 0.00 & 0.00 & 0.00 & 0.00 & 0.00 & 0.00 \\
\hline $\mathrm{Zn}$ & 0.25 & 0.26 & 0.27 & 0.28 & 0.28 & 0.29 & 0.29 & 0.30 & 0.30 & 0.30 & 0.31 \\
\hline $\mathrm{Zr}$ & 0.44 & 0.45 & 0.46 & 0.48 & 0.49 & 0.49 & 0.50 & 0.51 & 0.52 & 0.52 & 0.53 \\
\hline
\end{tabular}


Immobilization Technology Section

WSRC-TR-2002-00549

Savannah River Technology Center

Rev. 0

Westinghouse Savannah River Company

\section{Appendix B}

Conversion Table of Frits Nomenclatures 
Immobilization Technology Section

Rev. 0

Westinghouse Savannah River Company

\begin{tabular}{||c|c||}
\hline Numeric & Alphanumeric \\
\hline 400 & $\mathrm{~A}$ \\
\hline 401 & $\mathrm{~B}$ \\
\hline 402 & $\mathrm{E}$ \\
\hline 403 & $\mathrm{C}$ \\
\hline 404 & $\mathrm{D}$ \\
\hline 405 & $\mathrm{O}$ \\
\hline 406 & $\mathrm{P}$ \\
\hline 407 & $\mathrm{Q}$ \\
\hline 408 & $\mathrm{~F}$ \\
\hline 409 & $\mathrm{~S}$ \\
\hline 410 & $\mathrm{~T}$ \\
\hline 411 & $\mathrm{G}$ \\
\hline 412 & $\mathrm{I}$ \\
\hline 413 & $\mathrm{H}$ \\
\hline 414 & $\mathrm{~K}$ \\
\hline 415 & $\mathrm{~L}$ \\
\hline 416 & $320-\mathrm{M}$ \\
\hline 417 & $\mathrm{M}$ \\
\hline 418 & $\mathrm{~N}$ \\
\hline 419 & $\mathrm{R}$ \\
\hline 420 & $202-\mathrm{M}$ \\
\hline 421 & $202-\mathrm{M} 2$ \\
\hline 422 & $\mathrm{U}$ \\
\hline 423 & $\mathrm{U} 2$ \\
\hline
\end{tabular}


Immobilization Technology Section

WSRC-TR-2002-00549

Savannah River Technology Center

Rev. 0

Westinghouse Savannah River Company

\section{Appendix C1}

MAR Calculations for Various Frit/Decant Combinations 
Immobilization Technology Section

WSRC-TR-2002-00549

Savannah River Technology Center

Rev. 0

Westinghouse Savannah River Company

\begin{tabular}{|c|c|c|c|c|c|c|c|c|c|c|c|c|c|}
\hline & & \multicolumn{5}{|c|}{ "MAR Assessments } & \multicolumn{7}{|c|}{ 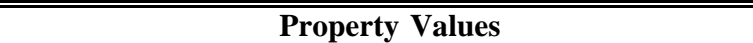 } \\
\hline Frit-Decant & $\% \mathrm{WL}$ & Durability & $\mathbf{T}_{\mathbf{L}}$ & $\begin{array}{l}\text { Visc } \\
\end{array}$ & Frit & \begin{tabular}{|l|} 
Homog \\
\end{tabular} & $\mathbf{A l}_{2} \mathbf{O}_{3}$ & alkali & $\mathbf{T}_{\mathbf{L}}$ & Visc & $\Delta \mathbf{G}_{\mathbf{P}}$ & \begin{tabular}{|l|} 
Homog \\
\end{tabular} & Frit \\
\hline 202-Decant \#5 & 25 & - & - & - & \begin{tabular}{|l|} 
High \\
\end{tabular} & $\mathrm{NO}$ & 3.46 & 17.61 & 750.1 & 81.02 & \begin{tabular}{|l|}
-10.646 \\
\end{tabular} & \begin{tabular}{|l|}
198.96 \\
\end{tabular} & 81.76 \\
\hline 202-Decant \#5 & 26 & - & - & - & 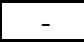 & $\mathrm{NO}$ & 3.60 & \begin{tabular}{|l|}
17.79 \\
\end{tabular} & 760.4 & 76.74 & \begin{tabular}{|l|}
-10.788 \\
\end{tabular} & 200.63 & 81.11 \\
\hline 202-Decant \#5 & 27 & - & - & - & - & $\mathrm{NO}$ & 3.74 & \begin{tabular}{|l|}
17.98 \\
\end{tabular} & 770.1 & \begin{tabular}{|l|}
72.58 \\
\end{tabular} & \begin{tabular}{|l|}
-10.929 \\
\end{tabular} & \begin{tabular}{|l|}
202.31 \\
\end{tabular} & 80.46 \\
\hline 202-Decant \#5 & 28 & - & - & - & - & $\mathrm{NO}$ & 3.88 & \begin{tabular}{|l|}
18.16 \\
\end{tabular} & 779.6 & 68.53 & \begin{tabular}{|l|}
-11.071 \\
\end{tabular} & \begin{tabular}{|l|}
203.98 \\
\end{tabular} & 79.81 \\
\hline 202-Decant \#5 & 29 & - & - & - & - & $\mathrm{NO}$ & 4.01 & 18.35 & 788.9 & 64.62 & \begin{tabular}{|l|}
-11.212 \\
\end{tabular} & 205.65 & 79.16 \\
\hline 202-Decant \#5 & 30 & - & - & - & 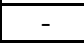 & $\mathrm{NO}$ & 4.15 & 18.53 & 797.8 & \begin{tabular}{|l|}
60.82 \\
\end{tabular} & \begin{tabular}{|l|}
-11.354 \\
\end{tabular} & \begin{tabular}{|l|}
207.33 \\
\end{tabular} & 78.51 \\
\hline 202-Decant \#5 & 31 & - & - & - & - & $\mathrm{NO}$ & 4.29 & 18.72 & 806.5 & 57.15 & \begin{tabular}{|l|}
-11.496 \\
\end{tabular} & 209.00 & 77.86 \\
\hline 202-Decant \#5 & 32 & - & - & - & - & $\mathrm{NO}$ & 4.43 & 18.90 & 814.9 & 53.61 & \begin{tabular}{|l|}
-11.637 \\
\end{tabular} & 210.67 & 77.21 \\
\hline 202-Decant \#5 & 33 & - & - & - & - & ${ }^{2}$ & 4.57 & \begin{tabular}{|l|}
19.09 \\
\end{tabular} & 823.0 & 50.20 & \begin{tabular}{|l|}
-11.779 \\
\end{tabular} & \begin{tabular}{|l|}
212.34 \\
\end{tabular} & 76.56 \\
\hline 202-Decant \#5 & 34 & - & - & - & - & - & 4.71 & \begin{tabular}{|l|}
19.27 \\
\end{tabular} & 830.9 & 46.91 & \begin{tabular}{|l|}
-11.921 \\
\end{tabular} & 214.02 & 75.92 \\
\hline 202-Decant \#5 & 35 & - & - & - & - & - & 4.85 & 19.45 & 838.6 & \begin{tabular}{|l|}
43.75 \\
\end{tabular} & \begin{tabular}{|l|}
-12.062 \\
\end{tabular} & \begin{tabular}{|l|}
215.69 \\
\end{tabular} & 75.27 \\
\hline 202-Decant \#5 & 36 & - & - & - & - & - & 4.98 & \begin{tabular}{|l|l|}
19.64 \\
\end{tabular} & 846.1 & 40.71 & \begin{tabular}{|l|}
-12.204 \\
\end{tabular} & 217.36 & 74.62 \\
\hline 202-Decant \#5 & 37 & - & - & - & - & - & 5.12 & \begin{tabular}{|l|}
19.82 \\
\end{tabular} & 853.4 & 37.80 & \begin{tabular}{|l|}
-12.345 \\
\end{tabular} & 219.03 & 73.97 \\
\hline 202-Decant \#5 & 38 & $\mathrm{NO}$ & - & - & - & - & 5.26 & \begin{tabular}{|l|}
20.01 \\
\end{tabular} & 860.5 & 35.02 & \begin{tabular}{|l|}
-12.487 \\
\end{tabular} & 2220.71 & 73.32 \\
\hline 202-Decant \#5 & 39 & $\mathrm{NO}$ & - & - & - & - & 5.40 & \begin{tabular}{|l|}
20.19 \\
\end{tabular} & 867.3 & \begin{tabular}{|l|}
32.37 \\
\end{tabular} & \begin{tabular}{|l|}
-12.629 \\
\end{tabular} & 222.38 & 72.67 \\
\hline 202-Decant \#5 & 40 & $\mathrm{NO}$ & - & - & - & - & 5.54 & 20.38 & 874.1 & \begin{tabular}{|l|}
29.84 \\
\end{tabular} & \begin{tabular}{|l|}
-12.770 \\
\end{tabular} & 224.05 & 72.02 \\
\hline 202-Decant \#5 & 41 & $\mathrm{NO}$ & - & - & - & - & 5.68 & \begin{tabular}{|l|}
20.56 \\
\end{tabular} & 880.6 & \begin{tabular}{|l|}
27.43 \\
\end{tabular} & \begin{tabular}{|l|}
-12.912 \\
\end{tabular} & 225.73 & 71.37 \\
\hline 202-Decant \#5 & 42 & $\mathrm{NO}$ & - & - & - & - & 5.81 & 20.75 & 886.9 & 25.15 & \begin{tabular}{|l|}
-13.053 \\
\end{tabular} & \begin{tabular}{|l|}
227.40 \\
\end{tabular} & 70.72 \\
\hline 202-Decant \#5 & 43 & $\mathrm{NO}$ & - & Low & - & - & 5.95 & \begin{tabular}{|l|}
20.93 \\
\end{tabular} & 893.1 & 22.99 & \begin{tabular}{|l|}
-13.195 \\
\end{tabular} & \begin{tabular}{|l|}
229.07 \\
\end{tabular} & 70.07 \\
\hline 202-Decant \#5 & 44 & $\mathrm{NO}$ & - & Low & - & - & 6.09 & 21.11 & 899.2 & 20.96 & \begin{tabular}{|l|}
-13.336 \\
\end{tabular} & \begin{tabular}{|l|}
230.74 \\
\end{tabular} & 69.42 \\
\hline 202-Decant \#5 & 45 & $\mathrm{NO}$ & - & Low & - & - & 6.23 & 21.30 & 905.1 & \begin{tabular}{|l|}
19.04 \\
\end{tabular} & \begin{tabular}{|l|}
-13.478 \\
\end{tabular} & \begin{tabular}{|l|}
232.42 \\
\end{tabular} & 68.77 \\
\hline 202-Decant \#5 & 46 & $\mathrm{NO}$ & - & Low & - & - & 6.37 & \begin{tabular}{|l|}
21.48 \\
\end{tabular} & 910.8 & 17.23 & \begin{tabular}{|l|}
-13.620 \\
\end{tabular} & \begin{tabular}{|l|}
234.09 \\
\end{tabular} & 68.12 \\
\hline 202-Decant \#5 & 47 & $\mathrm{NO}$ & - & Low & - & - & 6.51 & \begin{tabular}{|l|}
21.67 \\
\end{tabular} & 916.5 & 15.54 & \begin{tabular}{|l|}
-13.761 \\
\end{tabular} & 235.76 & 67.47 \\
\hline 202-Decant \#5 & 48 & $\mathrm{NO}$ & - & Low & - & - & 6.65 & 21.85 & 921.9 & 13.97 & \begin{tabular}{|l|}
-13.903 \\
\end{tabular} & 237.44 & 66.82 \\
\hline 202-Decant \#5 & 49 & $\mathrm{NO}$ & - & Low & - & - & 6.78 & 22.04 & 927.3 & 12.50 & \begin{tabular}{|l|}
-14.045 \\
\end{tabular} & 239.11 & 66.17 \\
\hline 202-Decant \#5 & 50 & $\mathrm{NO}$ & - & Low & - & - & 6.92 & 22.22 & 932.5 & 11.14 & \begin{tabular}{|l|}
-14.186 \\
\end{tabular} & 240.78 & 65.52 \\
\hline 202-Decant \#5 & 51 & $\mathrm{NO}$ & - & Low & - & - & 7.06 & 22.41 & 937.6 & 9.88 & \begin{tabular}{|l|}
-14.328 \\
\end{tabular} & 242.45 & 64.87 \\
\hline 202-Decant \#5 & 52 & $\mathrm{NO}$ & - & Low & - & - & 7.20 & 22.59 & 942.6 & 8.73 & \begin{tabular}{|l|}
-14.469 \\
\end{tabular} & 244.13 & 64.22 \\
\hline 202-Decant \#5 & 53 & $\mathrm{NO}$ & - & Low & - & - & 7.34 & 22.77 & 947.5 & 7.67 & \begin{tabular}{|l|}
-14.611 \\
\end{tabular} & 245.80 & 63.57 \\
\hline 202-Decant \#5 & 54 & $\mathrm{NO}$ & - & Low & - & - & 7.48 & 22.96 & 952.3 & 6.70 & \begin{tabular}{|l|}
-14.753 \\
\end{tabular} & 247.47 & 62.92 \\
\hline 202-Decant \#5 & 55 & $\mathrm{NO}$ & - & Low & - & - & 7.61 & 23.14 & 956.9 & 5.82 & \begin{tabular}{|l|}
-14.894 \\
\end{tabular} & 249.14 & 62.27 \\
\hline 202-Decant \#5 & 56 & $\mathrm{NO}$ & - & Low & - & - & 7.75 & 23.33 & 961.5 & 5.02 & \begin{tabular}{|l|}
-15.036 \\
\end{tabular} & 250.82 & 61.62 \\
\hline 202-Decant \#5 & 57 & $\mathrm{NO}$ & - & Low & - & - & 7.89 & 23.51 & 965.9 & 4.31 & \begin{tabular}{|l|}
-15.177 \\
\end{tabular} & 252.49 & 60.98 \\
\hline 202-Decant \#5 & 58 & $\mathrm{NO}$ & - & Low & - & - & 8.03 & 23.70 & 970.3 & 3.67 & \begin{tabular}{|l|}
-15.319 \\
\end{tabular} & 254.16 & 60.33 \\
\hline 202-Decant \#5 & 59 & $\mathrm{NO}$ & - & Low & 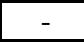 & - & 8.17 & \begin{tabular}{|l|}
23.88 \\
\end{tabular} & 974.6 & 3.11 & \begin{tabular}{|l|}
-15.461 \\
\end{tabular} & 255.83 & 59.68 \\
\hline 202-Decant \#5 & 60 & $\mathrm{NO}$ & - & Low & - & - & 8.31 & 24.06 & 978.8 & 2.60 & \begin{tabular}{|l|}
-15.602 \\
\end{tabular} & 257.51 & 59.03 \\
\hline 202-M-Decant \#5 & 25 & - & - & High & \begin{tabular}{|l|} 
High \\
\end{tabular} & $\mathrm{NO}$ & 4.21 & 16.86 & 731.6 & 121.22 & \begin{tabular}{|l|}
-9.459 \\
\end{tabular} & 204.40 & 82.51 \\
\hline 202-M-Decant \#5 & 26 & - & - & High & High & $\mathrm{NO}$ & 4.34 & 17.05 & 742.3 & 114.94 & -9.616 & 206.00 & 81.85 \\
\hline 202-M-Decant \#5 & 27 & - & - & High & 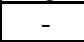 & $\mathrm{NO}$ & 4.47 & 17.25 & 752.6 & 108.85 & -9.774 & 207.60 & 81.19 \\
\hline 202-M-Decant \#5 & 28 & - & - & High & - & $\mathrm{NO}$ & 4.60 & 17.44 & 762.6 & 102.92 & -9.931 & 209.20 & 80.53 \\
\hline 202-M-Decant \#5 & 29 & - & - & & 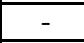 & $\mathrm{NO}$ & 4.72 & \begin{tabular}{|l|l|}
17.64 \\
\end{tabular} & 772.3 & \begin{tabular}{|l|}
97.16 \\
\end{tabular} & \begin{tabular}{|l|}
-10.089 \\
\end{tabular} & \begin{tabular}{|l|}
210.80 \\
\end{tabular} & 79.87 \\
\hline 202-M-Decant \#5 & 30 & - & - & - & - & - & 4.85 & 17.83 & 781.6 & \begin{tabular}{|l|}
91.58 \\
\end{tabular} & \begin{tabular}{|l|}
-10.246 \\
\end{tabular} & 212.40 & 79.21 \\
\hline 202-M-Decant \#5 & 31 & - & - & - & - & - & 4.98 & 18.03 & 790.7 & \begin{tabular}{|l|}
86.18 \\
\end{tabular} & \begin{tabular}{|l|}
-10.403 \\
\end{tabular} & 214.00 & 78.55 \\
\hline 202-M-Decant \#5 & 32 & - & - & - & - & - & 5.11 & \begin{tabular}{|l|}
18.22 \\
\end{tabular} & 799.5 & \begin{tabular}{|l|}
80.95 \\
\end{tabular} & \begin{tabular}{|l|}
-10.561 \\
\end{tabular} & \begin{tabular}{|l|}
215.60 \\
\end{tabular} & 77.89 \\
\hline $202-N$ & 33 & - & - & - & - & - & 5.24 & 18.42 & 808.1 & \begin{tabular}{|l|}
75.91 \\
\end{tabular} & \begin{tabular}{|l|}
-10.718 \\
\end{tabular} & 217.20 & 77.23 \\
\hline 202-M-Decant \#5 & 34 & - & - & - & 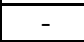 & - & 5.37 & \begin{tabular}{|l|}
18.61 \\
\end{tabular} & 816.4 & \begin{tabular}{|l|}
71.04 \\
\end{tabular} & \begin{tabular}{|l|}
-10.876 \\
\end{tabular} & 218.80 & 76.57 \\
\hline 202-M-Decant \#5 & 35 & - & - & - & - & - & 5.50 & 18.80 & 824.5 & \begin{tabular}{|l|}
66.35 \\
\end{tabular} & \begin{tabular}{|l|}
-11.033 \\
\end{tabular} & 220.40 & 75.92 \\
\hline 202-M-Decant \#5 & 36 & - & - & - & - & - & 5.62 & \begin{tabular}{|l|}
19.00 \\
\end{tabular} & 832.3 & \begin{tabular}{|l|}
61.85 \\
\end{tabular} & \begin{tabular}{|l|}
-11.191 \\
\end{tabular} & 222.00 & 75.26 \\
\hline 202-M-Decant \#5 & 37 & - & - & - & - & - & 5.75 & \begin{tabular}{|l|}
19.19 \\
\end{tabular} & 840.0 & 57.53 & \begin{tabular}{|l|}
-11.348 \\
\end{tabular} & 223.60 & 74.60 \\
\hline $202-\mathrm{M}$ & 38 & - & - & - & - & - & 5.88 & \begin{tabular}{|l|}
19.39 \\
\end{tabular} & 847.4 & 53.39 & \begin{tabular}{|l|}
-11.505 \\
\end{tabular} & 225.20 & 73.94 \\
\hline 202-M-Decant \#5 & 39 & - & - & - & - & - & 6.01 & \begin{tabular}{|l|}
19.58 \\
\end{tabular} & 854.6 & \begin{tabular}{|l|}
49.43 \\
\end{tabular} & \begin{tabular}{|l|}
-11.663 \\
\end{tabular} & \begin{tabular}{|l|}
226.80 \\
\end{tabular} & 73.28 \\
\hline 202-M-Decant \#5 & 40 & - & - & - & - & - & 6.14 & \begin{tabular}{|l|}
19.78 \\
\end{tabular} & 861.7 & 45.65 & \begin{tabular}{|l|}
-11.820 \\
\end{tabular} & 228.40 & 72.62 \\
\hline
\end{tabular}




\begin{tabular}{|c|c|c|c|c|c|c|c|c|c|c|c|c|c|}
\hline \multirow[b]{2}{*}{ Frit-Decant } & \multirow[b]{2}{*}{$\% \mathrm{WL}$} & \multicolumn{5}{|c|}{ MAR Assessments } & \multicolumn{7}{|c|}{ Property Values } \\
\hline & & Durability & $T_{L}$ & Visc & Frit & Homog & $\mathrm{Al}_{2} \mathrm{O}_{3}$ & alkali & $T_{L}$ & Visc & $\Delta \mathbf{G}_{\mathbf{P}}$ & Homog & Frit \\
\hline 202-M-Decant \#5 & 41 & - & - & - & - & - & 6.27 & 19.97 & 868.5 & 42.05 & \begin{tabular}{|l|}
-11.978 \\
\end{tabular} & 230.00 & 71.96 \\
\hline 202-M-Decant \#5 & 42 & - & - & - & - & - & 6.39 & 20.17 & 875.1 & 38.63 & \begin{tabular}{|l|}
-12.135 \\
\end{tabular} & 231.60 & 71.30 \\
\hline 202-M-Decant \#5 & 43 & - & - & - & - & - & 6.52 & 20.36 & 881.7 & 35.38 & \begin{tabular}{|l|}
-12.293 \\
\end{tabular} & 233.20 & 70.64 \\
\hline 202-M-Decant \#5 & 44 & $\mathrm{NO}$ & - & - & - & - & 6.65 & 20.55 & 888.0 & 32.31 & \begin{tabular}{|l|}
-12.450 \\
\end{tabular} & 234.80 & 69.98 \\
\hline 202-M-Decant \#5 & 45 & $\mathrm{NO}$ & - & - & - & - & 6.78 & 20.75 & 894.2 & 29.41 & \begin{tabular}{|l|}
-12.608 \\
\end{tabular} & 236.41 & 69.32 \\
\hline 202-M-Decant \#5 & 46 & $\mathrm{NO}$ & - & 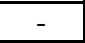 & 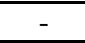 & - & 6.91 & 20.94 & 900.2 & 26.68 & \begin{tabular}{|l|}
-12.765 \\
\end{tabular} & 238.00 & 68.66 \\
\hline 202-M-Decant \#5 & 47 & $\mathrm{NO}$ & - & Low & - & - & 7.04 & 21.14 & 906.1 & 24.12 & \begin{tabular}{|l|}
-12.922 \\
\end{tabular} & 239.61 & 68.00 \\
\hline 202-M-Decant \#5 & 48 & $\mathrm{NO}$ & - & Low & - & - & 7.17 & 21.33 & 911.9 & 21.73 & \begin{tabular}{|l|}
-13.080 \\
\end{tabular} & 241.21 & 67.34 \\
\hline 202-M-Decant \#5 & 49 & $\mathrm{NO}$ & - & Low & - & - & 7.29 & 21.53 & 917.5 & 19.49 & \begin{tabular}{|l|}
-13.237 \\
\end{tabular} & 242.80 & 66.68 \\
\hline 202-M-Decant \#5 & 50 & $\mathrm{NO}$ & - & Low & 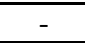 & - & 7.42 & 21.72 & 923.0 & 17.41 & \begin{tabular}{|l|}
-13.395 \\
\end{tabular} & 244.41 & 66.02 \\
\hline 202-M-Decant \#5 & 51 & $\mathrm{NO}$ & - & Low & - & - & 7.55 & 21.92 & 928.4 & 15.49 & \begin{tabular}{|l|}
-13.552 \\
\end{tabular} & 246.01 & 65.36 \\
\hline 202-M-Decant \#5 & 52 & $\mathrm{NO}$ & - & Low & - & - & 7.68 & 22.11 & 933.6 & 13.71 & \begin{tabular}{|l|}
-13.709 \\
\end{tabular} & 247.61 & 64.70 \\
\hline 202-M-Decant \#5 & 53 & $\mathrm{NO}$ & - & Low & 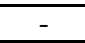 & - & 7.81 & 22.30 & 938.7 & 12.08 & \begin{tabular}{|l|}
-13.867 \\
\end{tabular} & 249.21 & 64.04 \\
\hline 202-M-Decant \#5 & 54 & $\mathrm{NO}$ & - & Low & 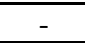 & - & 7.94 & 22.50 & 943.8 & 10.59 & \begin{tabular}{|l|}
-14.024 \\
\end{tabular} & 250.81 & 63.38 \\
\hline 202-M-Decant \#5 & 55 & $\mathrm{NO}$ & - & Low & - & - & 8.06 & 22.69 & 948.7 & 9.22 & \begin{tabular}{|l|}
-14.182 \\
\end{tabular} & 252.41 & 62.72 \\
\hline 202-M-Decant \#5 & 56 & $\mathrm{NO}$ & - & Low & - & - & 8.19 & 22.89 & 953.5 & 7.99 & \begin{tabular}{|l|}
-14.339 \\
\end{tabular} & 254.01 & 62.06 \\
\hline 202-M-D & 57 & $\mathrm{NO}$ & - & Low & - & - & 8.32 & 23.08 & 958.2 & 6.88 & \begin{tabular}{|l|}
-14.497 \\
\end{tabular} & \begin{tabular}{|l|}
255.61 \\
\end{tabular} & 61.41 \\
\hline $202-\mathrm{M}-\mathrm{I}$ & 58 & $\mathrm{NO}$ & - & Low & - & - & 8.45 & 23.28 & 962.8 & 5.88 & \begin{tabular}{|l|}
-14.654 \\
\end{tabular} & 257.21 & 60.75 \\
\hline 202-M-De & 59 & $\mathrm{NO}$ & - & Low & - & - & 8.58 & 23.47 & 967.3 & 4.99 & \begin{tabular}{|l|}
-14.811 \\
\end{tabular} & 258.81 & 60.09 \\
\hline 202-M-Decant \#5 & 60 & $\mathrm{NO}$ & - & Low & - & - & 8.71 & 23.66 & 971.7 & 4.20 & \begin{tabular}{|l|}
-14.969 \\
\end{tabular} & 260.41 & 59.43 \\
\hline 202-M2-Decant \#5 & 25 & - & - & High & High & $\mathrm{NO}$ & 4.21 & 16.86 & 733.4 & 104.37 & \begin{tabular}{|l|}
-9.785 \\
\end{tabular} & 204.40 & 82.51 \\
\hline $202-\mathrm{M}$ & 26 & - & - & - & High & $\mathrm{NO}$ & 4.34 & 17.05 & 744.2 & 98.84 & 38 & 206.00 & 81.85 \\
\hline 202-M2-Decant \#5 & 27 & - & - & - & 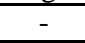 & $\mathrm{NO}$ & 4.47 & 17.25 & 754.4 & 93.47 & \begin{tabular}{|l|}
-10.091 \\
\end{tabular} & 207.60 & 81.19 \\
\hline 202-M2-Decant \#5 & 28 & - & - & - & - & $\mathrm{NO}$ & 4.60 & 17.44 & 764.4 & 88.26 & \begin{tabular}{|l|}
-10.244 \\
\end{tabular} & 209.20 & 80.53 \\
\hline 202-M2-Decant \#5 & 29 & - & - & - & - & $\mathrm{NO}$ & 4.72 & 17.64 & 774.0 & 83.20 & \begin{tabular}{|l|}
-10.397 \\
\end{tabular} & 210.80 & 79.87 \\
\hline $202-\mathrm{N}$ & 30 & - & - & - & - & - & 4.85 & 17.83 & 783.4 & 78.31 & \begin{tabular}{|l|}
-10.550 \\
\end{tabular} & 212.40 & 79.21 \\
\hline $202-\mathrm{N}$ & 31 & - & - & - & 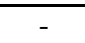 & - & 4.98 & 18.03 & 792.5 & 73.58 & \begin{tabular}{|l|}
-10.703 \\
\end{tabular} & 214.00 & 78.55 \\
\hline 202-M2-Decant \#5 & 32 & - & - & - & - & - & 5.11 & 18.22 & 801.3 & 69.01 & \begin{tabular}{|l|}
-10.856 \\
\end{tabular} & 215.60 & 77.89 \\
\hline 202-M2-Decant \#5 & 33 & - & - & - & - & - & 5.24 & 18.42 & 809.8 & 64.61 & \begin{tabular}{|l|}
-11.009 \\
\end{tabular} & 217.20 & 77.23 \\
\hline $202-\mathrm{N}$ & 34 & - & - & - & - & - & 5.37 & 18.61 & 818.0 & 60.37 & \begin{tabular}{|l|}
-11.162 \\
\end{tabular} & 218.80 & 76.58 \\
\hline 202-M2-D & 35 & - & - & - & - & - & 5.50 & 18.80 & 826.1 & 56.29 & \begin{tabular}{|l|}
-11.316 \\
\end{tabular} & 220.40 & 75.92 \\
\hline 202-M2-Decant \#5 & 36 & - & - & - & - & - & 5.62 & 19.00 & 834.0 & 52.38 & \begin{tabular}{|l|}
-11.469 \\
\end{tabular} & 222.00 & 75.26 \\
\hline 202-M2-Decant \#5 & 37 & - & - & - & - & - & 5.75 & 19.19 & 841.6 & 48.64 & \begin{tabular}{|l|}
-11.622 \\
\end{tabular} & 223.60 & 74.60 \\
\hline $202-\mathrm{N}$ & 38 & - & - & - & - & - & 5.88 & 19.39 & 848.9 & 45.05 & 775 & 225.20 & 73.94 \\
\hline 202-M2-I & 39 & - & - & - & - & - & 6.01 & 19.58 & 856.1 & 41.63 & \begin{tabular}{|l|}
-11.928 \\
\end{tabular} & 226.80 & 73.28 \\
\hline 202-M2-Decant \#5 & 40 & - & - & - & - & - & 6.14 & 19.78 & 863.2 & 38.37 & \begin{tabular}{|l|}
-12.081 \\
\end{tabular} & 228.40 & 72.62 \\
\hline 202-M2-Decant \#5 & 41 & - & - & - & - & - & 6.27 & 19.97 & 869.9 & 35.27 & \begin{tabular}{|l|}
-12.234 \\
\end{tabular} & 230.00 & 71.96 \\
\hline 202-M2-Decant \#5 & 42 & $\mathrm{NO}$ & - & - & 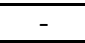 & - & 6.39 & 20.17 & 876.6 & 32.34 & \begin{tabular}{|l|}
-12.387 \\
\end{tabular} & 231.60 & 71.30 \\
\hline $202-\mathrm{M}$ & 43 & & - & - & - & - & 6.52 & 20.36 & 883.1 & 29.56 & \begin{tabular}{|l|}
-12.540 \\
\end{tabular} & 233.20 & 70.64 \\
\hline 202-M2-Decant \#5 & 44 & $\bar{N}$ & - & - & - & - & 6.65 & 20.55 & 889.4 & 26.93 & \begin{tabular}{|l|}
-12.693 \\
\end{tabular} & 234.80 & 69.98 \\
\hline 202-M2-Decant \#5 & 45 & $\mathrm{NO}$ & - & - & - & - & 6.78 & 20.75 & 895.6 & 24.46 & \begin{tabular}{|l|}
-12.846 \\
\end{tabular} & 236.41 & 69.32 \\
\hline 202-M2-Decant \#5 & 46 & $\mathrm{NO}$ & - & Low & - & - & 6.91 & 20.94 & 901.6 & 22.14 & \begin{tabular}{|l|}
-12.999 \\
\end{tabular} & 238.00 & 68.66 \\
\hline 202-M2-Decant \#5 & 47 & $\mathrm{NO}$ & - & Low & - & 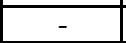 & 7.04 & 21.14 & 907.4 & 19.96 & \begin{tabular}{|l|}
-13.153 \\
\end{tabular} & 239.61 & 68.00 \\
\hline 202-M2-Decant \#5 & 48 & 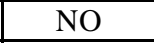 & - & Low & - & - & 7.17 & 21.33 & 913.2 & 17.94 & \begin{tabular}{|l|}
-13.306 \\
\end{tabular} & 241.21 & 67.34 \\
\hline 202-M2-Decant \#5 & 49 & $\mathrm{NO}$ & - & Low & - & - & 7.29 & 21.53 & 918.8 & 16.05 & \begin{tabular}{|l|}
-13.459 \\
\end{tabular} & 242.80 & 66.68 \\
\hline 202-M2-Decant \#5 & 50 & $\mathrm{NO}$ & - & Low & - & - & 7.42 & \begin{tabular}{|l|}
21.72 \\
\end{tabular} & 924.2 & 14.30 & \begin{tabular}{|l|}
-13.612 \\
\end{tabular} & 244.41 & 66.02 \\
\hline -Decant \#5 & 51 & $\mathrm{NO}$ & - & Low & $\sigma_{1}$ & - & 7.55 & 21.92 & 929.6 & 12.68 & \begin{tabular}{|l|}
-13.765 \\
\end{tabular} & 246.01 & 65.36 \\
\hline $202-$ & 52 & & - & Low & - & - & 7.68 & 22.11 & 934.8 & 11.20 & \begin{tabular}{|l|}
-13.918 \\
\end{tabular} & \begin{tabular}{|l|}
247.61 \\
\end{tabular} & 64.70 \\
\hline 202-M2-Decant \#5 & 53 & PO & - & Low & - & - & 7.81 & 22.30 & 939.9 & 9.83 & \begin{tabular}{|l|}
-14.071 \\
\end{tabular} & 249.21 & 64.04 \\
\hline 202-M2-Decant \#5 & 54 & $\mathrm{NO}$ & - & Low & - & - & 7.94 & 22.50 & 944.9 & 8.59 & \begin{tabular}{|l|}
-14.224 \\
\end{tabular} & 250.80 & 63.38 \\
\hline 202-M2-Decant \#5 & 55 & $\mathrm{NO}$ & - & Low & - & - & 8.06 & 22.69 & 949.7 & 7.46 & \begin{tabular}{|l|}
-14.377 \\
\end{tabular} & 252.41 & 62.72 \\
\hline 202-M2-Decant \#5 & 56 & $\mathrm{NO}$ & - & Low & - & - & 8.19 & 22.89 & 954.6 & 6.44 & \begin{tabular}{|l|}
-14.530 \\
\end{tabular} & 254.01 & 62.06 \\
\hline 202-M2-Decant \#5 & 57 & $\mathrm{NC}$ & - & Low & - & - & 8.32 & 23.08 & 959.2 & 5.52 & \begin{tabular}{|l|}
-14.683 \\
\end{tabular} & 255.61 & 61.40 \\
\hline 202-M2-Decant \#5 & 58 & $\mathrm{NO}$ & - & Low & - & - & 8.45 & 23.28 & 963.8 & 4.70 & \begin{tabular}{|l|}
-14.836 \\
\end{tabular} & 257.21 & 60.75 \\
\hline
\end{tabular}


Immobilization Technology Section

WSRC-TR-2002-00549

Savannah River Technology Center

Rev. 0

Westinghouse Savannah River Company

\begin{tabular}{|c|c|c|c|c|c|c|c|c|c|c|c|c|c|}
\hline & & \multicolumn{5}{|c|}{ "MAR Assessments } & \multicolumn{7}{|c|}{ Property Values } \\
\hline Frit-Decant & $\% \mathrm{WL}$ & Durability & $\mathbf{T}_{\mathrm{L}}$ & Visc & Frit & Homog & $\mathrm{Al}_{2} \mathrm{O}_{3}$ & alkali & $\mathbf{T}_{\mathrm{L}}$ & Visc & $\Delta \mathbf{G}_{\mathbf{P}}$ & Homog & Frit \\
\hline 202-M2-Decant \#5 & 59 & $\mathrm{NO}$ & - & Low & - & - & 8.58 & 23.47 & 968.3 & 3.98 & -14.990 & 258.81 & 60.09 \\
\hline 202-M2-Decant \#5 & 60 & $\mathrm{NO}$ & - & Low & - & - & 8.71 & 23.66 & 972.7 & 3.34 & -15.143 & 260.41 & 59.43 \\
\hline S-Decant \#5 & 25 & - & & High & High & $\mathrm{NO}$ & 3.46 & 15.36 & 735.3 & 164.40 & -8.338 & 201.37 & 83.26 \\
\hline S-Decant \#5 & 26 & - & - & High & High & $\mathrm{NO}$ & 3.60 & 15.57 & 746.5 & 156.18 & -8.511 & 203.01 & 82.59 \\
\hline S-Decant \#5 & 27 & - & - & High & High & $\mathrm{NO}$ & 3.74 & 15.79 & 757.2 & 148.17 & -8.683 & 204.65 & 81.92 \\
\hline S-Decant \#5 & 28 & - & - & High & - & $\mathrm{NO}$ & 3.88 & 16.00 & 767.6 & 140.37 & -8.856 & 206.29 & 81.25 \\
\hline S-Decant \#5 & 29 & - & - & High & - & $\mathrm{NO}$ & 4.01 & 16.22 & 777.6 & 132.78 & -9.028 & 207.93 & 80.58 \\
\hline S-Decant \#5 & 30 & - & - & High & - & $\mathrm{NO}$ & 4.15 & 16.43 & 787.3 & 125.41 & -9.200 & 209.57 & 79.91 \\
\hline S-Decant \#5 & 31 & - & - & High & - & - & 4.29 & 16.65 & 796.6 & 118.25 & -9.373 & 211.21 & 79.24 \\
\hline S-Decant \#5 & 32 & - & - & High & - & - & 4.43 & 16.86 & 805.7 & 111.32 & -9.545 & 212.85 & 78.57 \\
\hline S-Decant \#5 & 33 & - & - & High & - & - & 4.57 & 17.08 & 814.4 & 104.61 & -9.717 & 214.49 & 77.90 \\
\hline S-Decant \#5 & 34 & - & - & - & - & - & 4.71 & 17.29 & 822.8 & 98.13 & -9.890 & 216.13 & 77.24 \\
\hline S-Decant \#5 & 35 & - & - & - & - & - & 4.85 & \begin{tabular}{|l|}
17.50 \\
\end{tabular} & 831.1 & 91.87 & -10.062 & 217.77 & 76.57 \\
\hline S-Decant \#5 & 36 & - & - & - & - & - & 4.98 & 17.72 & 839.1 & 85.84 & -10.235 & 219.41 & 75.90 \\
\hline S-Decant \#5 & 37 & - & - & - & - & - & 5.12 & 17.93 & 846.8 & 80.04 & -10.407 & 221.05 & 75.23 \\
\hline S-Decant \#5 & 38 & - & - & - & - & - & 5.26 & 18.15 & 854.3 & 74.46 & -10.579 & 222.69 & 74.56 \\
\hline S-Decant \#5 & 39 & - & - & - & - & - & 5.40 & 18.36 & 861.5 & 69.12 & -10.752 & 224.34 & 73.89 \\
\hline S-Decant \#5 & 40 & - & - & - & - & - & 5.54 & 18.58 & 868.7 & 64.01 & -10.924 & 225.98 & 73.22 \\
\hline S-Decant \#5 & 41 & - & - & - & - & - & 5.68 & 18.79 & 875.5 & 59.12 & -11.096 & 227.62 & 72.55 \\
\hline S-Decant \#5 & 42 & - & - & - & - & - & 5.81 & 19.01 & 882.2 & 54.47 & -11.269 & 229.26 & 71.88 \\
\hline S-Decant \#5 & 43 & - & - & - & - & - & 5.95 & 19.22 & 888.7 & 50.04 & -11.441 & 230.90 & 71.21 \\
\hline S-Decant \#5 & 44 & - & - & - & - & - & 6.09 & 19.43 & 895.0 & 45.83 & -11.614 & 232.54 & 70.54 \\
\hline S-Decant \#5 & 45 & - & - & - & - & - & 6.23 & 19.65 & 901.2 & 41.86 & -11.786 & 234.18 & 69.87 \\
\hline S-Decant \#5 & 46 & - & - & - & - & - & 6.37 & 19.86 & 907.2 & 38.10 & -11.958 & 235.82 & 69.20 \\
\hline S-Decant \#5 & 47 & - & - & - & - & - & 6.51 & 20.08 & 913.1 & 34.56 & -12.131 & 237.46 & 68.53 \\
\hline S-Decant \#5 & 48 & - & - & - & - & - & 6.65 & 20.29 & 918.8 & 31.24 & -12.303 & 239.10 & 67.86 \\
\hline S-Decant \#5 & 49 & $\mathrm{NO}$ & - & - & - & - & 6.78 & 20.51 & 924.3 & 28.13 & -12.475 & 240.74 & 67.19 \\
\hline S-Decant \#5 & 50 & $\mathrm{NO}$ & - & - & - & - & 6.92 & 20.72 & 929.7 & 25.22 & -12.648 & 242.38 & 66.52 \\
\hline S-Decant \#5 & 51 & $\mathrm{NO}$ & - & Low & - & - & 7.06 & 20.94 & 935.0 & 22.52 & \begin{tabular}{|l|}
-12.820 \\
\end{tabular} & 244.03 & 65.85 \\
\hline S-Decant \#5 & 52 & $\mathrm{NO}$ & - & Low & - & - & 7.20 & 21.15 & 940.2 & 20.02 & -12.992 & 245.67 & 65.18 \\
\hline S-Decant \#5 & 53 & $\mathrm{NO}$ & - & Low & - & - & 7.34 & 21.36 & 945.2 & 17.71 & -13.165 & 247.31 & 64.51 \\
\hline S-Decant \#5 & 54 & $\mathrm{NO}$ & - & Low & - & - & 7.48 & 21.58 & 950.2 & 15.59 & -13.337 & 248.94 & 63.84 \\
\hline S-Decant \#5 & 55 & $\mathrm{NO}$ & - & Low & - & - & 7.61 & 21.79 & 954.9 & 13.65 & -13.510 & 250.59 & 63.17 \\
\hline S-Decant \#5 & 56 & $\mathrm{NO}$ & - & Low & - & - & 7.75 & 22.01 & 959.7 & 11.88 & -13.682 & 252.23 & 62.50 \\
\hline S-Decant \#5 & 57 & $\mathrm{NO}$ & - & Low & - & - & 7.89 & 22.22 & 964.2 & 10.27 & -13.854 & 253.87 & 61.84 \\
\hline S-Decant \#5 & 58 & $\mathrm{NO}$ & - & Low & - & - & 8.03 & 22.44 & 968.7 & 8.83 & -14.027 & 255.51 & 61.17 \\
\hline S-Decant \#5 & 59 & $\mathrm{NO}$ & - & Low & - & - & 8.17 & 22.65 & 973.1 & 7.53 & -14.199 & 257.15 & 60.50 \\
\hline S-Decant \#5 & 60 & NO & - & Low & - & - & 8.31 & 22.86 & 977.4 & 6.38 & \begin{tabular}{|l|}
-14.371 \\
\end{tabular} & 258.79 & 59.83 \\
\hline T-Decant \#5 & 25 & - & - & High & High & $\mathrm{NO}$ & 3.46 & 15.36 & 735.7 & 150.39 & -8.299 & 201.37 & 83.26 \\
\hline T-Decant \#5 & 26 & - & - & High & High & $\mathrm{NO}$ & 3.60 & 15.57 & 747.0 & 142.86 & -8.472 & 203.01 & 82.59 \\
\hline T-Decant \#5 & 27 & - & - & High & High & $\mathrm{NO}$ & 3.74 & 15.79 & 757.7 & 135.54 & -8.645 & 204.65 & 81.92 \\
\hline T-Decant \#5 & 28 & - & - & High & - & $\mathrm{NO}$ & 3.88 & 16.00 & 768.1 & 128.41 & -8.818 & 206.29 & 81.25 \\
\hline T-Decant \#5 & 29 & - & - & High & - & $\mathrm{NO}$ & 4.01 & 16.22 & 778.1 & 121.47 & -8.991 & 207.93 & 80.58 \\
\hline T-Decant \#5 & 30 & - & - & High & - & $\mathrm{NO}$ & 4.15 & 16.43 & 787.8 & 114.73 & -9.164 & 209.57 & 79.91 \\
\hline T-Decant \#5 & 31 & - & - & High & - & - & 4.29 & 16.65 & 797.1 & 108.19 & -9.337 & 211.21 & 79.24 \\
\hline T-Decant \#5 & 32 & - & - & High & - & - & 4.43 & 16.86 & 806.2 & 101.85 & -9.510 & 212.85 & 78.57 \\
\hline T-Decant \#5 & 33 & - & - & - & - & - & 4.57 & 17.08 & 814.9 & 95.72 & -9.683 & 214.49 & 77.90 \\
\hline T-Decant \#5 & 34 & - & - & - & - & - & 4.71 & \begin{tabular}{|l|}
17.29 \\
\end{tabular} & 823.4 & 89.79 & -9.855 & 216.13 & 77.23 \\
\hline T-Decant \#5 & 35 & - & - & - & - & - & 4.85 & 17.50 & 831.7 & 84.06 & -10.028 & 217.77 & 76.57 \\
\hline T-Decant \#5 & 36 & - & - & - & - & - & 4.98 & 17.72 & 839.6 & 78.55 & -10.201 & 219.41 & 75.90 \\
\hline T-Decant \#5 & 37 & - & - & - & - & - & 5.12 & 17.93 & 847.4 & 73.24 & -10.374 & 221.05 & 75.23 \\
\hline T-Decant \#5 & 38 & - & - & - & - & - & 5.26 & 18.15 & 854.9 & 68.15 & -10.547 & 222.69 & 74.56 \\
\hline T-Decant \#5 & 39 & - & - & - & - & - & 5.40 & 18.36 & 862.1 & 63.26 & -10.720 & 224.34 & 73.89 \\
\hline T-Decant \#5 & 40 & - & - & - & - & - & 5.54 & 18.58 & 869.2 & 58.58 & -10.893 & 225.98 & 73.22 \\
\hline
\end{tabular}


Immobilization Technology Section

WSRC-TR-2002-00549

Savannah River Technology Center

Rev. 0

Westinghouse Savannah River Company

\begin{tabular}{|c|c|c|c|c|c|c|c|c|c|c|c|c|c|}
\hline \multirow[b]{2}{*}{ Frit-Decant } & \multirow[b]{2}{*}{$\% \mathrm{WL}$} & \multicolumn{5}{|c|}{ "MAR Assessments } & \multicolumn{7}{|c|}{ 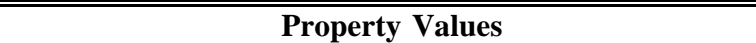 } \\
\hline & & Durability & $T_{L}$ & Visc & Frit & \begin{tabular}{|l|} 
Homog \\
\end{tabular} & $\mathbf{A l}_{2} \mathbf{O}_{3}$ & alkali & $\mathbf{T}_{\mathrm{L}}$ & Visc & $\Delta \mathbf{G}_{\mathbf{P}}$ & Homog & Frit \\
\hline T-Decant \#5 & 41 & - & - & - & - & - & 5.68 & \begin{tabular}{|l|}
18.79 \\
\end{tabular} & 876.0 & 54.11 & \begin{tabular}{|l|}
-11.066 \\
\end{tabular} & 227.62 & 72.55 \\
\hline T-Decant \#5 & 42 & - & - & - & - & - & 5.81 & \begin{tabular}{|l|}
19.01 \\
\end{tabular} & 882.7 & 49.85 & \begin{tabular}{|l|}
-11.238 \\
\end{tabular} & 229.26 & 71.88 \\
\hline T-Decant \#5 & 43 & - & - & - & - & - & 5.95 & \begin{tabular}{|l|}
19.22 \\
\end{tabular} & 889.2 & 45.80 & \begin{tabular}{|l|}
-11.412 \\
\end{tabular} & 230.90 & 71.21 \\
\hline T-Decant \#5 & 44 & - & - & - & - & -- & 6.09 & \begin{tabular}{|c|}
19.43 \\
\end{tabular} & 895.6 & 41.96 & \begin{tabular}{|l|}
-11.584 \\
\end{tabular} & 232.54 & 70.54 \\
\hline T-Decant \#5 & 45 & - & - & - & - & - & 6.23 & 19.65 & 901.7 & 38.32 & \begin{tabular}{|l|}
-11.757 \\
\end{tabular} & 234.18 & 69.87 \\
\hline T-Decant \#5 & 46 & - & - & - & - & - & 6.37 & \begin{tabular}{|l|}
19.86 \\
\end{tabular} & 907.7 & 34.88 & \begin{tabular}{|l|}
-11.930 \\
\end{tabular} & 235.82 & 69.20 \\
\hline T-Decant \#5 & 47 & - & - & & - & - & 6.51 & 20.08 & 913.6 & 31.64 & \begin{tabular}{|l|}
-12.103 \\
\end{tabular} & 237.46 & 68.53 \\
\hline T-Decant \#5 & 48 & - & - & & - & - & 6.65 & 20.29 & 919.3 & 28.60 & \begin{tabular}{|l|}
-12.276 \\
\end{tabular} & 239.10 & 67.86 \\
\hline T-Decant \#5 & 49 & $\mathrm{NO}$ & - & & - & - & 6.78 & 20.51 & 924.8 & 25.75 & \begin{tabular}{|l|}
-12.449 \\
\end{tabular} & 240.74 & 67.19 \\
\hline T-Decant \#5 & 50 & $\mathrm{NO}$ & - & Low & - & - & 6.92 & 20.72 & 930.2 & 23.10 & \begin{tabular}{|l|}
-12.622 \\
\end{tabular} & 242.38 & 66.52 \\
\hline T-Decant \#5 & 51 & $\mathrm{NO}$ & - & Low & - & - & 7.06 & \begin{tabular}{|l|}
20.94 \\
\end{tabular} & 935.5 & 20.63 & \begin{tabular}{|l|}
-12.795 \\
\end{tabular} & 244.03 & 65.85 \\
\hline T-Decant \#5 & 52 & $\mathrm{NO}$ & - & Low & - & - & 7.20 & 21.15 & 940.7 & 18.34 & \begin{tabular}{|l|}
-12.967 \\
\end{tabular} & 245.67 & 65.18 \\
\hline T-Decant \#5 & 53 & $\mathrm{NO}$ & - & Low & - & - & 7.34 & 21.36 & 945.7 & 16.22 & \begin{tabular}{|l|}
-13.140 \\
\end{tabular} & 247.31 & 64.51 \\
\hline T-Decant \#5 & 54 & $\mathrm{NO}$ & - & Low & - & - & 7.48 & 21.58 & 950.7 & 14.28 & \begin{tabular}{|l|}
-13.313 \\
\end{tabular} & \begin{tabular}{|l|}
248.94 \\
\end{tabular} & 63.84 \\
\hline T-Decant \#5 & 55 & $\mathrm{NO}$ & - & Low & - & - & 7.61 & 21.79 & 955.4 & 12.50 & \begin{tabular}{|l|}
-13.486 \\
\end{tabular} & \begin{tabular}{|l|}
250.59 \\
\end{tabular} & 63.17 \\
\hline T-Decant \#5 & 56 & $\mathrm{NO}$ & - & Low & - & - & 7.75 & \begin{tabular}{|l|}
22.01 \\
\end{tabular} & 960.2 & 10.88 & \begin{tabular}{|l|}
-13.659 \\
\end{tabular} & \begin{tabular}{|l|}
252.23 \\
\end{tabular} & 62.50 \\
\hline T-Decant \#5 & 57 & $\mathrm{NO}$ & - & Low & - & - & 7.89 & 22.22 & 964.7 & 9.41 & \begin{tabular}{|l|}
-13.832 \\
\end{tabular} & \begin{tabular}{|l|}
253.87 \\
\end{tabular} & 61.84 \\
\hline T-Decant \#5 & 58 & $\mathrm{NO}$ & - & Low & - & - & 8.03 & 22.44 & 969.2 & 8.09 & \begin{tabular}{|l|}
-14.005 \\
\end{tabular} & \begin{tabular}{|l|}
255.51 \\
\end{tabular} & 61.17 \\
\hline T-Decant \#5 & 59 & $\mathrm{NO}$ & - & Low & - & - & 8.17 & 22.65 & 973.6 & 6.90 & \begin{tabular}{|l|}
-14.178 \\
\end{tabular} & \begin{tabular}{|l|}
257.15 \\
\end{tabular} & 60.50 \\
\hline T-Decant \#5 & 60 & $\mathrm{NO}$ & - & Low & 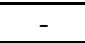 & - & 8.31 & 22.86 & 977.9 & 5.85 & \begin{tabular}{|l|}
-14.351 \\
\end{tabular} & \begin{tabular}{|l|}
258.79 \\
\end{tabular} & 59.83 \\
\hline U-Decant \#5 & 25 & - & - & High & High & $\mathrm{NO}$ & 3.46 & \begin{tabular}{|l|l|}
16.11 \\
\end{tabular} & 723.3 & 102.24 & -8.876 & \begin{tabular}{|l|}
201.37 \\
\end{tabular} & 83.26 \\
\hline U-Decant \#5 & 26 & - & - & - & High & $\mathrm{NO}$ & 3.60 & 16.31 & 734.8 & 97.07 & \begin{tabular}{|l|}
-9.041 \\
\end{tabular} & 203.01 & 82.59 \\
\hline U-Decant \#5 & 27 & - & - & - & High & $\mathrm{NO}$ & 3.74 & 16.52 & 745.7 & 92.06 & -9.206 & 204.65 & 81.92 \\
\hline U-Decant \#5 & 28 & - & - & - & - & $\mathrm{NO}$ & 3.88 & \begin{tabular}{|l|}
16.72 \\
\end{tabular} & 756.4 & 87.17 & -9.371 & 206.29 & 81.25 \\
\hline U-Decant \#5 & 29 & - & - & - & - & $\mathrm{NO}$ & 4.01 & \begin{tabular}{|l|}
16.93 \\
\end{tabular} & 766.6 & 82.41 & -9.536 & \begin{tabular}{|l|}
207.93 \\
\end{tabular} & 80.58 \\
\hline U-Decant \#5 & 30 & - & - & - & - & $\mathrm{NO}$ & 4.15 & 17.13 & 776.5 & $\begin{array}{l}77.79 \\
\end{array}$ & -9.702 & 209.57 & 79.91 \\
\hline U-Decant \#5 & 31 & - & - & - & - & - & 4.29 & \begin{tabular}{|l|l|}
17.34 \\
\end{tabular} & 786.1 & 73.32 & \begin{tabular}{|l|}
-9.867 \\
\end{tabular} & 211.21 & 79.24 \\
\hline U-Decant \#5 & 32 & - & - & - & - & - & 4.43 & 17.54 & 795.4 & 68.98 & \begin{tabular}{|l|}
-10.032 \\
\end{tabular} & 212.85 & 78.57 \\
\hline U-Decant \#5 & 33 & - & - & - & - & - & 4.57 & 17.75 & 804.4 & \begin{tabular}{|l|}
64.79 \\
\end{tabular} & \begin{tabular}{|l|}
-10.197 \\
\end{tabular} & \begin{tabular}{|l|}
214.49 \\
\end{tabular} & 77.90 \\
\hline U-Decant \#5 & 34 & - & - & - & - & - & 4.71 & 17.95 & 813.1 & 60.74 & \begin{tabular}{|l|}
-10.362 \\
\end{tabular} & \begin{tabular}{|l|}
216.13 \\
\end{tabular} & 77.23 \\
\hline U-Decant \#5 & 35 & - & - & - & - & - & 4.85 & \begin{tabular}{|l|}
18.15 \\
\end{tabular} & 821.6 & 56.83 & \begin{tabular}{|l|}
-10.528 \\
\end{tabular} & \begin{tabular}{|l|}
217.77 \\
\end{tabular} & 76.57 \\
\hline U-Decant \#5 & 36 & - & - & - & - & - & 4.98 & \begin{tabular}{|l|}
18.36 \\
\end{tabular} & 829.8 & 53.07 & \begin{tabular}{|l|}
-10.693 \\
\end{tabular} & \begin{tabular}{|l|}
219.42 \\
\end{tabular} & 75.90 \\
\hline U-Decant \#5 & 37 & - & - & - & - & - & 5.12 & \begin{tabular}{|l|}
18.56 \\
\end{tabular} & 837.8 & $\begin{array}{l}49.45 \\
\end{array}$ & \begin{tabular}{|l|}
-10.858 \\
\end{tabular} & 221.05 & 75.23 \\
\hline U-Decant \#5 & 38 & - & - & - & - & - & 5.26 & \begin{tabular}{|l|l|}
18.77 \\
\end{tabular} & 845.5 & 45.98 & \begin{tabular}{|l|}
-11.023 \\
\end{tabular} & \begin{tabular}{|l|}
222.69 \\
\end{tabular} & 74.56 \\
\hline U-Decant \#5 & 39 & - & - & - & - & - & 5.40 & \begin{tabular}{|l|}
18.97 \\
\end{tabular} & 853.0 & 42.65 & \begin{tabular}{|l|}
-11.189 \\
\end{tabular} & 224.34 & 73.89 \\
\hline U-Decant \#5 & 40 & - & - & - & - & - & 5.54 & \begin{tabular}{|l|l|}
19.18 \\
\end{tabular} & 860.4 & 39.47 & \begin{tabular}{|l|}
-11.354 \\
\end{tabular} & 225.98 & 73.22 \\
\hline U-Decant \#5 & 41 & - & - & - & - & - & 5.68 & \begin{tabular}{|l|l|}
19.38 \\
\end{tabular} & 867.4 & 36.43 & \begin{tabular}{|l|}
-11.519 \\
\end{tabular} & 227.62 & 72.55 \\
\hline U-Decant \#5 & 42 & - & - & - & - & - & 5.81 & \begin{tabular}{|l|}
19.59 \\
\end{tabular} & 874.3 & 33.54 & \begin{tabular}{|l|}
-11.684 \\
\end{tabular} & 229.26 & 71.88 \\
\hline U-Decant \#5 & 43 & - & - & - & - & - & 5.95 & \begin{tabular}{|l|}
19.79 \\
\end{tabular} & 881.0 & 30.79 & \begin{tabular}{|l|}
-11.849 \\
\end{tabular} & 230.90 & 71.21 \\
\hline U-Decant \#5 & 44 & - & - & - & - & - & 6.09 & \begin{tabular}{|l|}
19.99 \\
\end{tabular} & 887.6 & 28.18 & \begin{tabular}{|l|}
-12.014 \\
\end{tabular} & 232.54 & 70.54 \\
\hline U-Decant \#5 & 45 & - & - & - & - & - & 6.23 & 20.20 & 894.0 & 25.71 & \begin{tabular}{|l|}
-12.180 \\
\end{tabular} & 234.18 & 69.87 \\
\hline U-Decant \#5 & 46 & 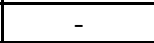 & - & Low & - & - & 6.37 & 20.40 & 900.2 & 23.39 & \begin{tabular}{|l|}
-12.345 \\
\end{tabular} & 235.82 & 69.20 \\
\hline U-Decant \#5 & 47 & $\mathrm{NO}$ & - & Low & - & - & 6.51 & 20.61 & 906.2 & 21.20 & \begin{tabular}{|l|}
-12.510 \\
\end{tabular} & 237.46 & 68.53 \\
\hline U-Decant \#5 & 48 & $\mathrm{NO}$ & - & Low & - & - & 6.65 & \begin{tabular}{|l|}
20.81 \\
\end{tabular} & 912.2 & 19.14 & \begin{tabular}{|l|}
-12.675 \\
\end{tabular} & 239.10 & 67.86 \\
\hline U-Decant \#5 & 49 & $\mathrm{NO}$ & - & Low & - & - & 6.78 & 21.02 & 917.9 & 17.22 & \begin{tabular}{|l|}
-12.841 \\
\end{tabular} & \begin{tabular}{|l|}
240.74 \\
\end{tabular} & 67.19 \\
\hline U-Decant \#5 & 50 & $\mathrm{NO}$ & - & Low & - & - & 6.92 & \begin{tabular}{|l|}
21.22 \\
\end{tabular} & 923.5 & 15.43 & \begin{tabular}{|l|}
-13.006 \\
\end{tabular} & 242.38 & 66.52 \\
\hline U-Decant \#5 & 51 & $\mathrm{NO}$ & - & Low & - & - & 7.06 & \begin{tabular}{|l|}
21.43 \\
\end{tabular} & 929.0 & 13.76 & \begin{tabular}{|l|}
-13.171 \\
\end{tabular} & 244.03 & 65.85 \\
\hline U-Decant \#5 & 52 & $\mathrm{NO}$ & - & Low & - & - & 7.20 & \begin{tabular}{|l|}
21.63 \\
\end{tabular} & 934.4 & 12.22 & \begin{tabular}{|l|}
-13.336 \\
\end{tabular} & \begin{tabular}{|l|}
245.67 \\
\end{tabular} & 65.18 \\
\hline U-Decant \#5 & 53 & $\mathrm{NO}$ & - & Low & - & - & 7.34 & \begin{tabular}{|l|}
21.83 \\
\end{tabular} & 939.5 & 10.80 & \begin{tabular}{|l|}
-13.501 \\
\end{tabular} & \begin{tabular}{|l|}
247.31 \\
\end{tabular} & 64.51 \\
\hline U-Decant \#5 & 54 & $\mathrm{NO}$ & - & Low & - & - & 7.48 & 22.04 & 944.7 & 9.50 & \begin{tabular}{|l|}
-13.666 \\
\end{tabular} & 248.94 & 63.84 \\
\hline U-Decant \#5 & 55 & $\mathrm{NO}$ & - & Low & - & - & 7.61 & \begin{tabular}{|l|}
22.24 \\
\end{tabular} & 949.6 & 8.30 & \begin{tabular}{|l|}
-13.832 \\
\end{tabular} & 250.59 & 63.17 \\
\hline U-Decant \#5 & 56 & $\mathrm{NO}$ & - & Low & 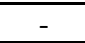 & - & 7.75 & 22.45 & 954.6 & 7.22 & \begin{tabular}{|l|}
-13.997 \\
\end{tabular} & 252.23 & 62.50 \\
\hline U-Decant \#5 & 57 & $\mathrm{NO}$ & - & Low & - & - & 7.89 & 22.65 & 959.3 & 6.23 & \begin{tabular}{|l|}
-14.162 \\
\end{tabular} & \begin{tabular}{|l|}
253.87 \\
\end{tabular} & 61.84 \\
\hline U-Decant \#5 & 58 & $\mathrm{NO}$ & - & Low & - & - & 8.03 & 22.86 & 963.9 & 5.35 & \begin{tabular}{|l|}
-14.327 \\
\end{tabular} & 255.51 & 61.17 \\
\hline
\end{tabular}


Immobilization Technology Section

WSRC-TR-2002-00549

Savannah River Technology Center

Rev. 0

Westinghouse Savannah River Company

\begin{tabular}{|c|c|c|c|c|c|c|c|c|c|c|c|c|c|}
\hline \multirow[b]{2}{*}{ Frit-Decant } & \multirow[b]{2}{*}{$\% \mathrm{WL}$} & \multicolumn{5}{|c|}{ "MAR Assessments } & \multicolumn{7}{|c|}{ 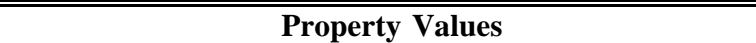 } \\
\hline & & Durability & $\mathbf{T}_{\mathrm{L}}$ & Visc & Frit & Homog & $\mathrm{Al}_{2} \mathrm{O}_{3}$ & alkali & $\mathbf{T}_{L}$ & Visc & $\Delta \mathbf{G}_{\mathbf{P}}$ & Homog & Frit \\
\hline U-Decant \#5 & 59 & $\mathrm{NO}$ & - & Low & - & - & 8.17 & 23.06 & 968.5 & 4.56 & -14.493 & 257.15 & 60.50 \\
\hline U-Decant \#5 & 60 & $\mathrm{NO}$ & - & Low & - & - & 8.31 & 23.26 & 973.0 & 3.86 & -14.658 & 258.79 & 59.83 \\
\hline U2-Decant \#5 & 25 & - & - & - & High & $\mathrm{NO}$ & 3.46 & 16.11 & 725.1 & 87.98 & \begin{tabular}{|l|}
-9.201 \\
\end{tabular} & \begin{tabular}{|l|}
201.37 \\
\end{tabular} & 83.26 \\
\hline U2-Decant \#5 & 26 & - & - & - & High & $\mathrm{NO}$ & 3.60 & \begin{tabular}{|l|}
16.31 \\
\end{tabular} & 736.5 & 83.44 & -9.362 & \begin{tabular}{|l|}
203.01 \\
\end{tabular} & 82.59 \\
\hline U2-Decant \#5 & 27 & - & - & - & High & $\mathrm{NO}$ & 3.74 & 16.52 & 747.5 & 79.02 & -9.523 & 204.65 & 81.92 \\
\hline U2-Decant \#5 & 28 & - & - & - & - & $\mathrm{NO}$ & 3.88 & 16.72 & 758.1 & 74.73 & -9.684 & 206.29 & 81.25 \\
\hline U2-Decant \#5 & 29 & - & - & - & - & $\mathrm{NO}$ & 4.01 & 16.93 & 768.3 & 70.56 & -9.845 & 207.93 & 80.58 \\
\hline U2-Decant \#5 & 30 & - & - & - & - & $\mathrm{NO}$ & 4.15 & 17.13 & 778.2 & $\begin{array}{ll}66.51 \\
\end{array}$ & -10.006 & \begin{tabular}{|l|}
209.57 \\
\end{tabular} & 79.91 \\
\hline U2-Decant \#5 & 31 & - & - & - & - & - & 4.29 & 17.34 & 787.8 & 62.59 & -10.167 & 211.21 & 79.24 \\
\hline U2-Decant \#5 & 32 & - & - & - & - & - & 4.43 & 17.54 & 797.1 & 58.81 & -10.327 & 212.85 & 78.57 \\
\hline U2-Decant \#5 & 33 & - & - & - & - & - & 4.57 & 17.75 & 806.0 & 55.15 & -10.488 & \begin{tabular}{|l|}
214.49 \\
\end{tabular} & 77.90 \\
\hline U2-Decant \#5 & 34 & - & - & - & - & - & 4.71 & 17.95 & 814.7 & 51.62 & -10.649 & 216.13 & 77.23 \\
\hline U2-Decant \#5 & 35 & - & - & - & - & - & 4.85 & 18.15 & 823.2 & 48.23 & -10.810 & \begin{tabular}{|l|}
217.77 \\
\end{tabular} & 76.57 \\
\hline U2-Decant \#5 & 36 & - & - & - & - & - & 4.98 & 18.36 & 831.4 & 44.96 & -10.971 & 219.42 & 75.90 \\
\hline U2-Decant \#5 & 37 & - & - & - & - & - & 5.12 & 18.56 & 839.3 & 41.82 & -11.132 & 221.05 & 75.23 \\
\hline U2-Decant \#5 & 38 & - & - & - & - & - & 5.26 & \begin{tabular}{|l|l|}
18.77 \\
\end{tabular} & 847.0 & 38.82 & -11.293 & \begin{tabular}{|l|}
222.69 \\
\end{tabular} & 74.56 \\
\hline U2-Decant \#5 & 39 & - & - & - & - & - & 5.40 & \begin{tabular}{|l|}
18.97 \\
\end{tabular} & 854.5 & 35.95 & -11.454 & \begin{tabular}{|l|}
224.34 \\
\end{tabular} & 73.89 \\
\hline U2-Decant \#5 & 40 & - & - & - & - & - & 5.54 & 19.18 & 861.8 & 33.20 & \begin{tabular}{|l|}
-11.614 \\
\end{tabular} & 225.98 & 73.22 \\
\hline U2-Decant \#5 & 41 & - & - & - & - & - & 5.68 & 19.38 & 868.8 & 30.59 & -11.775 & 227.62 & 72.55 \\
\hline U2-Decant \#5 & 42 & - & - & - & - & - & 5.81 & 19.59 & 875.7 & 28.10 & -11.936 & \begin{tabular}{|l|}
229.26 \\
\end{tabular} & 71.88 \\
\hline U2-Decant \#5 & 43 & - & - & - & - & - & 5.95 & 19.79 & 882.4 & 25.75 & -12.097 & 230.90 & 71.21 \\
\hline U2-Decant \#5 & 44 & - & - & Low & - & - & 6.09 & \begin{tabular}{|l|}
19.99 \\
\end{tabular} & 889.0 & 23.52 & -12.258 & 232.54 & 70.54 \\
\hline U2-Decant \#5 & 45 & $\mathrm{NO}$ & - & Low & - & - & 6.23 & 20.20 & 895.3 & 21.41 & -12.419 & 234.18 & 69.87 \\
\hline U2-Decant \#5 & 46 & $\mathrm{NO}$ & - & Low & - & - & 6.37 & 20.40 & 901.5 & 19.43 & -12.579 & 235.82 & 69.20 \\
\hline U2-Decant \#5 & 47 & $\mathrm{NO}$ & - & Low & - & - & 6.51 & \begin{tabular}{|l|}
20.61 \\
\end{tabular} & 907.5 & 17.57 & -12.740 & 237.46 & 68.53 \\
\hline U2-Decant \#5 & 48 & $\mathrm{NO}$ & - & Low & - & - & 6.65 & 20.81 & 913.4 & 15.83 & -12.901 & \begin{tabular}{|l|}
239.10 \\
\end{tabular} & 67.86 \\
\hline U2-Decant \#5 & 49 & $\mathrm{NO}$ & - & Low & - & - & 6.78 & 21.02 & 919.1 & 14.20 & -13.062 & \begin{tabular}{|l|}
240.74 \\
\end{tabular} & 67.19 \\
\hline U2-Decant \#5 & 50 & $\mathrm{NO}$ & - & Low & - & - & 6.92 & 21.22 & 924.7 & 12.69 & -13.223 & \begin{tabular}{|l|}
242.38 \\
\end{tabular} & 66.52 \\
\hline U2-Decant \#5 & 51 & $\mathrm{NO}$ & - & Low & - & - & 7.06 & 21.43 & 930.2 & $\begin{array}{l}11.29 \\
\end{array}$ & -13.384 & 244.03 & 65.85 \\
\hline U2-Decant \#5 & 52 & $\mathrm{NO}$ & - & Low & - & - & 7.20 & 21.63 & 935.5 & 10.00 & -13.545 & \begin{tabular}{|l|}
245.67 \\
\end{tabular} & 65.18 \\
\hline U2-Decant \#5 & 53 & $\mathrm{NO}$ & - & Low & - & - & 7.34 & 21.83 & 940.7 & 8.81 & -13.706 & \begin{tabular}{|l|}
247.31 \\
\end{tabular} & 64.51 \\
\hline U2-Decant \#5 & 54 & $\mathrm{NO}$ & - & Low & - & - & 7.48 & 22.04 & 945.8 & 7.72 & -13.866 & \begin{tabular}{|l|}
248.94 \\
\end{tabular} & 63.84 \\
\hline U2-Decant \#5 & 55 & $\mathrm{NO}$ & - & Low & 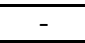 & - & 7.61 & 22.24 & 950.7 & 6.73 & \begin{tabular}{|l|}
-14.027 \\
\end{tabular} & \begin{tabular}{|l|}
250.59 \\
\end{tabular} & 63.17 \\
\hline $\mathrm{U} 2-\mathrm{De}$ & 56 & $\mathrm{NO}$ & - & Low & - & - & 7.75 & \begin{tabular}{|l|}
22.45 \\
\end{tabular} & 955.6 & 5.83 & \begin{tabular}{|l|l|}
-14.188 \\
\end{tabular} & \begin{tabular}{|l|}
252.23 \\
\end{tabular} & 62.50 \\
\hline U2-Decant \#5 & 57 & $\mathrm{NO}$ & - & Low & - & - & 7.89 & 22.65 & 960.3 & 5.02 & -14.349 & \begin{tabular}{|l|}
253.87 \\
\end{tabular} & 61.84 \\
\hline U2-Decant \#5 & 58 & $\mathrm{NO}$ & - & Low & - & - & 8.03 & 22.86 & 964.9 & 4.30 & -14.510 & \begin{tabular}{|l|}
255.51 \\
\end{tabular} & 61.17 \\
\hline U2-Decant \#5 & 59 & $\mathrm{NO}$ & - & Low & - & - & 8.17 & 23.06 & 969.5 & 3.65 & -14.671 & 257.15 & 60.50 \\
\hline U2-Decant \#5 & 60 & $\mathrm{NO}$ & - & Low & - & - & 8.31 & 23.26 & 973.9 & 3.07 & -14.832 & \begin{tabular}{|l|}
258.79 \\
\end{tabular} & 59.83 \\
\hline 202-Decant \#9 & 25 & - & - & High & - & $\mathrm{NO}$ & 3.87 & 15.60 & 827.0 & 101.68 & -8.759 & \begin{tabular}{|l|}
203.77 \\
\end{tabular} & 79.80 \\
\hline 202-Decant \#9 & 26 & - & - & - & - & $\mathrm{NO}$ & 4.02 & 15.71 & 839.3 & 97.48 & -8.826 & 205.63 & 79.07 \\
\hline 202-Decant \#9 & 27 & - & - & - & - & $\mathrm{NO}$ & 4.18 & 15.81 & 851.1 & 93.36 & -8.892 & \begin{tabular}{|l|}
207.50 \\
\end{tabular} & 78.34 \\
\hline 202-Decant \#9 & 28 & - & - & - & - & $\mathrm{NO}$ & 4.33 & \begin{tabular}{|l|}
15.91 \\
\end{tabular} & 862.6 & 89.30 & -8.958 & \begin{tabular}{|l|}
209.36 \\
\end{tabular} & 77.62 \\
\hline $202-\mathrm{D}$ & 29 & - & - & - & - & - & 4.49 & 16.02 & 873.8 & 85.32 & -9.024 & 211.23 & 76.89 \\
\hline 202-Decant \#9 & 30 & - & - & - & - & - & 4.64 & 16.12 & 884.6 & 81.41 & -9.090 & \begin{tabular}{|l|}
213.09 \\
\end{tabular} & 76.16 \\
\hline 202-Decant \#9 & 31 & - & - & - & - & - & 4.79 & 16.23 & 895.1 & 77.58 & -9.156 & \begin{tabular}{|l|}
214.96 \\
\end{tabular} & 75.43 \\
\hline 202-Decant \#9 & 32 & - & - & - & - & - & 4.95 & 16.33 & 905.3 & 73.83 & -9.222 & \begin{tabular}{|l|}
216.82 \\
\end{tabular} & 74.70 \\
\hline 202-Decant \#9 & 33 & - & - & - & - & - & 5.10 & 16.43 & 915.3 & 70.16 & -9.288 & \begin{tabular}{|l|}
218.69 \\
\end{tabular} & 73.97 \\
\hline 202-Decant \#9 & 34 & 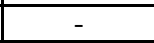 & - & - & - & - & 5.26 & 16.54 & 925.0 & 66.57 & -9.355 & 220.55 & 73.25 \\
\hline 202-Decant \#9 & 35 & - & - & - & - & - & 5.41 & 16.64 & 934.4 & 63.07 & -9.421 & 222.42 & 72.52 \\
\hline 202-Decant \#9 & 36 & - & - & - & - & - & 5.57 & \begin{tabular}{|l|l|}
16.75 \\
\end{tabular} & 943.5 & 59.66 & -9.487 & \begin{tabular}{|l|}
224.28 \\
\end{tabular} & 71.79 \\
\hline 202-Decant \#9 & 37 & - & - & - & - & - & 5.72 & 16.85 & 952.4 & 56.33 & -9.553 & 226.15 & 71.06 \\
\hline 202-Decant \#9 & 38 & - & - & - & - & - & 5.88 & 16.95 & 961.1 & 53.10 & -9.619 & \begin{tabular}{|l|}
228.02 \\
\end{tabular} & 70.33 \\
\hline 202-Decant \#9 & 39 & - & - & - & 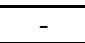 & - & 6.03 & 17.06 & 969.5 & 49.95 & -9.685 & \begin{tabular}{|l|}
229.88 \\
\end{tabular} & 69.61 \\
\hline 202-Decant \#9 & 40 & - & - & - & - & - & 6.19 & 17.16 & 977.8 & 46.90 & -9.751 & 231.74 & 68.88 \\
\hline
\end{tabular}


Immobilization Technology Section

WSRC-TR-2002-00549

Savannah River Technology Center

Rev. 0

Westinghouse Savannah River Company

\begin{tabular}{|c|c|c|c|c|c|c|c|c|c|c|c|c|c|}
\hline & & \multicolumn{5}{|c|}{ "MAR Assessments } & \multicolumn{7}{|c|}{ 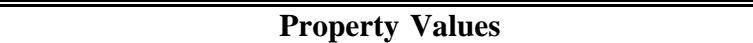 } \\
\hline Frit-Decant & $\% \mathrm{WL}$ & Durability & $\mathbf{T}_{\mathrm{L}}$ & \begin{tabular}{|l|} 
Visc \\
\end{tabular} & Frit & Homog & $\mathrm{Al}_{2} \mathrm{O}_{3}$ & alkali & $\mathbf{T}_{\mathrm{L}}$ & Visc & $\Delta \mathbf{G}_{\mathbf{P}}$ & \begin{tabular}{|l|} 
Homog \\
\end{tabular} & Frit \\
\hline 202-Decant \#9 & 41 & - & - & - & - & - & 6.34 & 17.27 & 985.8 & 43.95 & -9.818 & 233.61 & 68.15 \\
\hline 202-Decant \#9 & 42 & - & - & - & - & - & 6.50 & \begin{tabular}{|l|}
17.37 \\
\end{tabular} & \begin{tabular}{|l|}
993.7 \\
\end{tabular} & 41.09 & -9.884 & 235.47 & 67.42 \\
\hline 202-Decant \#9 & 43 & - & - & - & - & - & 6.65 & 17.47 & \begin{tabular}{|l|}
1001.3 \\
\end{tabular} & 38.34 & -9.950 & \begin{tabular}{|l|}
237.34 \\
\end{tabular} & 66.69 \\
\hline 202-Decant \#9 & 44 & - & - & - & - & - & 6.81 & 17.58 & \begin{tabular}{|l|}
1008.7 \\
\end{tabular} & 35.68 & -10.016 & 239.20 & 65.97 \\
\hline 202-Decant \#9 & 45 & - & - & - & 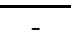 & - & 6.96 & \begin{tabular}{|l|}
17.68 \\
\end{tabular} & \begin{tabular}{|l|}
1016.1 \\
\end{tabular} & 33.12 & -10.082 & 241.07 & 65.24 \\
\hline 202-Decant \#9 & 46 & - & $\mathrm{NO}$ & - & - & - & 7.11 & 17.79 & \begin{tabular}{|l|}
1023.1 \\
\end{tabular} & 30.67 & -10.148 & 242.93 & 64.51 \\
\hline 202-Decant \#9 & 47 & - & NO & - & - & - & 7.27 & 17.89 & \begin{tabular}{|l|}
1030.1 \\
\end{tabular} & 28.32 & -10.214 & 244.80 & 63.78 \\
\hline 202-Decant \#9 & 48 & - & $\mathrm{NO}$ & - & - & - & 7.42 & \begin{tabular}{|l|}
18.00 \\
\end{tabular} & \begin{tabular}{|l|}
1036.9 \\
\end{tabular} & 26.07 & -10.281 & \begin{tabular}{|l|}
246.67 \\
\end{tabular} & 63.05 \\
\hline 202-Decant \#9 & 49 & - & NO & \begin{tabular}{|l|} 
Low \\
\end{tabular} & - & - & 7.58 & 18.10 & 1043.5 & 23.93 & -10.346 & 248.53 & 62.33 \\
\hline 202-Decant \#9 & 50 & - & NO & \begin{tabular}{|l|} 
Low \\
\end{tabular} & - & - & 7.73 & 18.20 & \begin{tabular}{|l|}
1050.0 \\
\end{tabular} & 21.89 & -10.413 & \begin{tabular}{|l|}
250.39 \\
\end{tabular} & 61.60 \\
\hline 202-Decant \#9 & 51 & - & NO & \begin{tabular}{|l|} 
Low \\
\end{tabular} & - & - & 7.89 & 18.31 & 1056.3 & 19.96 & -10.479 & 252.26 & 60.87 \\
\hline 202-Decant \#9 & 52 & - & NO & \begin{tabular}{|l|} 
Low \\
\end{tabular} & - & - & 8.04 & 18.41 & 1062.5 & 18.13 & -10.545 & 254.13 & 60.14 \\
\hline 202-Decant \#9 & 53 & - & NO & \begin{tabular}{|l|} 
Low \\
\end{tabular} & - & - & 8.20 & 18.52 & \begin{tabular}{|l|}
1068.6 \\
\end{tabular} & 16.40 & -10.611 & 255.99 & 59.41 \\
\hline 202-Decant \#9 & 54 & - & NO & \begin{tabular}{|l|} 
Low \\
\end{tabular} & - & - & 8.35 & 18.62 & \begin{tabular}{|l|}
1074.5 \\
\end{tabular} & 14.78 & -10.677 & 257.86 & 58.69 \\
\hline 202-Decant \#9 & 55 & - & NO & \begin{tabular}{|l|} 
Low \\
\end{tabular} & - & - & 8.51 & 18.72 & \begin{tabular}{|l|}
1080.3 \\
\end{tabular} & 13.26 & -10.743 & 259.72 & 57.96 \\
\hline 202-Decant \#9 & 56 & - & NO & Low & - & - & 8.66 & \begin{tabular}{|l|}
18.83 \\
\end{tabular} & 1086.0 & 11.84 & -10.810 & \begin{tabular}{|l|}
261.58 \\
\end{tabular} & 57.23 \\
\hline 202-Decant \#9 & 57 & - & \begin{tabular}{|l|}
$\mathrm{NO}$ \\
\end{tabular} & \begin{tabular}{|l|} 
Low \\
\end{tabular} & - & - & 8.82 & \begin{tabular}{|l|}
18.93 \\
\end{tabular} & \begin{tabular}{|l|}
1091.5 \\
\end{tabular} & 10.52 & -10.876 & 263.45 & 56.50 \\
\hline 202-Decant \#9 & 58 & - & NO & \begin{tabular}{|l|} 
Low \\
\end{tabular} & 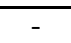 & - & 8.97 & 19.04 & \begin{tabular}{|l|}
1097.0 \\
\end{tabular} & 9.29 & -10.942 & 265.32 & 55.77 \\
\hline 202-Decant \#9 & 59 & - & NO & Low & - & - & 9.13 & \begin{tabular}{|l|}
19.14 \\
\end{tabular} & 1102.4 & 8.17 & -11.008 & 267.18 & 55.05 \\
\hline $202-\mathrm{D}$ & 60 & - & $\mathrm{NO}$ & \begin{tabular}{|l|} 
Low \\
\end{tabular} & - & - & 9.28 & \begin{tabular}{|l|}
19.24 \\
\end{tabular} & \begin{tabular}{|l|}
1107.6 \\
\end{tabular} & 7.13 & -11.074 & 269.04 & 54.32 \\
\hline 202-M-Decant \#9 & 25 & 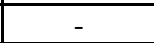 & - & \begin{tabular}{|l|} 
High \\
\end{tabular} & - & $\mathrm{NO}$ & 4.62 & 14.85 & 805.7 & \begin{tabular}{|l|}
151.21 \\
\end{tabular} & -7.572 & 209.21 & 80.55 \\
\hline 202-M-Decant \#9 & 26 & - & - & \begin{tabular}{|l|} 
High \\
\end{tabular} & - & - & 4.76 & 14.97 & \begin{tabular}{|l|}
818.4 \\
\end{tabular} & \begin{tabular}{|l|}
145.10 \\
\end{tabular} & -7.654 & 211.00 & 79.81 \\
\hline 202-M-Decant \#9 & 27 & - & - & \begin{tabular}{|l|} 
High \\
\end{tabular} & - & - & 4.91 & 15.08 & 830.7 & \begin{tabular}{|l|}
139.08 \\
\end{tabular} & -7.736 & 212.79 & 79.07 \\
\hline 202-M-Decant \#9 & 28 & - & - & \begin{tabular}{|l|} 
High \\
\end{tabular} & - & - & 5.05 & 15.19 & 842.6 & 133.17 & -7.818 & 214.58 & 78.34 \\
\hline $202-\mathrm{M}-\mathrm{D}$ & 29 & - & - & High & - & - & 5.20 & 15.31 & \begin{tabular}{|l|}
854.2 \\
\end{tabular} & \begin{tabular}{|l|}
127.35 \\
\end{tabular} & -7.900 & 216.38 & 77.60 \\
\hline 202-M-Decant \#9 & 30 & - & - & \begin{tabular}{|l|} 
High \\
\end{tabular} & - & - & 5.34 & 15.42 & \begin{tabular}{|l|}
865.4 \\
\end{tabular} & 121.65 & -7.982 & 218.17 & 76.86 \\
\hline 202-M-Decant \#9 & 31 & - & - & High & - & - & 5.48 & 15.54 & 876.3 & \begin{tabular}{|l|}
116.03 \\
\end{tabular} & -8.064 & 219.96 & 76.12 \\
\hline 202-M-Decant \#9 & 32 & - & - & \begin{tabular}{|l|} 
High \\
\end{tabular} & - & - & 5.63 & 15.65 & 886.9 & \begin{tabular}{|l|}
110.54 \\
\end{tabular} & -8.146 & 221.75 & 75.38 \\
\hline 202-M-De & 33 & - & - & High & - & - & 5.77 & \begin{tabular}{|l|}
15.76 \\
\end{tabular} & \begin{tabular}{|l|}
897.2 \\
\end{tabular} & \begin{tabular}{|l|}
105.16 \\
\end{tabular} & -8.228 & 223.55 & 74.64 \\
\hline 202-M-Decant \#9 & 34 & - & - & High & - & - & 5.92 & 15.88 & \begin{tabular}{|l|}
907.3 \\
\end{tabular} & \begin{tabular}{|l|}
99.89 \\
\end{tabular} & -8.310 & 225.34 & 73.91 \\
\hline 202-M-Decant \#9 & 35 & - & - & - & - & - & 6.06 & 15.99 & 917.0 & \begin{tabular}{|l|}
94.74 \\
\end{tabular} & -8.392 & 227.13 & 73.17 \\
\hline 202-M-Decant \#9 & 36 & - & - & - & 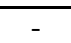 & - & 6.21 & $\mid 16.11$ & 926.5 & \begin{tabular}{|l|}
89.73 \\
\end{tabular} & -8.474 & 228.93 & 72.43 \\
\hline $202-\mathrm{N}$ & 37 & - & - & - & - & - & 6.35 & 16.22 & \begin{tabular}{|l|}
935.8 \\
\end{tabular} & \begin{tabular}{|l|}
84.82 \\
\end{tabular} & -8.556 & 230.72 & 71.69 \\
\hline 202-M-Decant \#9 & 38 & - & - & - & - & - & 6.50 & 16.33 & 944.8 & 80.05 & -8.638 & 232.51 & 70.95 \\
\hline 202-M-Decant \#9 & 39 & - & - & - & - & - & 6.64 & 16.45 & 953.6 & 75.41 & -8.720 & 234.30 & 70.22 \\
\hline 202-M-Decant \#9 & 40 & - & - & - & - & - & 6.79 & 16.56 & \begin{tabular}{|l|}
962.2 \\
\end{tabular} & \begin{tabular}{|l|}
70.90 \\
\end{tabular} & -8.801 & 236.09 & 69.48 \\
\hline $202-\mathrm{N}$ & 41 & - & - & - & 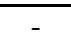 & - & 6.93 & 16.68 & 970.5 & \begin{tabular}{|l|}
66.52 \\
\end{tabular} & 84 & 237.89 & 68.74 \\
\hline $202-\mathrm{M}$ & 42 & - & - & - & - & - & 7.08 & \begin{tabular}{|l|}
16.79 \\
\end{tabular} & \begin{tabular}{|l|}
978.7 \\
\end{tabular} & \begin{tabular}{|l|}
62.29 \\
\end{tabular} & -8.966 & \begin{tabular}{|l|}
239.68 \\
\end{tabular} & 68.00 \\
\hline 202-M-Decant \#9 & 43 & - & - & - & - & - & 7.22 & 16.90 & \begin{tabular}{|l|}
986.6 \\
\end{tabular} & \begin{tabular}{|l|}
58.19 \\
\end{tabular} & -9.048 & 241.47 & 67.26 \\
\hline 202-M-Decant \#9 & 44 & - & - & - & - & - & 7.37 & 17.02 & 994.4 & \begin{tabular}{|l|}
54.25 \\
\end{tabular} & -9.129 & 243.26 & 66.53 \\
\hline 202-M-Decant \#9 & 45 & - & - & - & - & - & 7.51 & 17.13 & 1002.0 & 50.44 & -9.211 & 245.06 & 65.79 \\
\hline $202-\mathrm{N}$ & 46 & - & - & - & - & - & 7.65 & 17.25 & \begin{tabular}{|l|}
1009.4 \\
\end{tabular} & 46.77 & & 246.85 & 65.05 \\
\hline 202-M-D & 47 & - & - & - & - & - & 7.80 & 17.36 & \begin{tabular}{|l|}
1016.6 \\
\end{tabular} & 43.26 & -9.375 & 248.64 & 64.31 \\
\hline 202-M-Decant \#9 & 48 & - & NO & - & - & - & 7.94 & \begin{tabular}{|l|}
17.47 \\
\end{tabular} & 1023.7 & \begin{tabular}{|l|}
39.89 \\
\end{tabular} & -9.457 & 250.44 & 63.57 \\
\hline 202-M-Decant \#9 & 49 & - & NO & - & - & - & 8.09 & 17.59 & \begin{tabular}{|l|}
1030.7 \\
\end{tabular} & 36.68 & -9.539 & 252.23 & 62.84 \\
\hline 202-M-Decant \#9 & 50 & - & $\mathrm{NO}$ & - & - & - & 8.23 & 17.70 & \begin{tabular}{|l|}
1037.5 \\
\end{tabular} & 33.62 & -9.621 & 254.02 & 62.10 \\
\hline 202 & 51 & - & NO & - & - & - & 8.38 & 17.82 & 1044.1 & 30.71 & & 255.81 & 61.36 \\
\hline 202-M-Decant \#9 & 52 & - & NO & - & - & - & 8.52 & \begin{tabular}{|l|}
17.93 \\
\end{tabular} & \begin{tabular}{|l|}
1050.6 \\
\end{tabular} & \begin{tabular}{|l|}
27.94 \\
\end{tabular} & -9.785 & 257.61 & 60.62 \\
\hline 202-M-Decant \#9 & 53 & - & NO & - & - & - & 8.67 & 18.05 & \begin{tabular}{|l|}
1056.9 \\
\end{tabular} & 25.34 & -9.867 & 259.40 & 59.88 \\
\hline 202-M-Decant \#9 & 54 & - & NO & \begin{tabular}{|l|} 
Low \\
\end{tabular} & - & - & 8.81 & \begin{tabular}{|c|}
18.16 \\
\end{tabular} & \begin{tabular}{|l|}
1063.2 \\
\end{tabular} & 22.88 & -9.949 & 261.19 & 59.15 \\
\hline 202-M-Decant \#9 & 55 & - & NO & \begin{tabular}{|l|} 
Low \\
\end{tabular} & - & - & 8.96 & 18.27 & \begin{tabular}{|l|}
1069.3 \\
\end{tabular} & 20.57 & -10.031 & 262.98 & 58.41 \\
\hline $202-\mathrm{M}$ & 56 & - & NO & \begin{tabular}{|l|} 
Low \\
\end{tabular} & & - & 9.10 & \begin{tabular}{|l|}
18.39 \\
\end{tabular} & 1075.2 & 18.41 & -10.113 & 264.78 & 57.67 \\
\hline 202-M-Decant \#9 & 57 & - & NO & \begin{tabular}{|l|} 
Low \\
\end{tabular} & - & - & 9.25 & 18.50 & \begin{tabular}{|l|}
1081.1 \\
\end{tabular} & 16.39 & -10.195 & 266.57 & 56.93 \\
\hline 202-M-Decant \#9 & 58 & - & NO & \begin{tabular}{|l|} 
Low \\
\end{tabular} & - & - & 9.39 & 18.62 & \begin{tabular}{|l|}
1086.8 \\
\end{tabular} & 14.52 & -10.277 & 268.36 & 56.19 \\
\hline
\end{tabular}


Immobilization Technology Section

WSRC-TR-2002-00549

Savannah River Technology Center

Rev. 0

Westinghouse Savannah River Company

\begin{tabular}{|c|c|c|c|c|c|c|c|c|c|c|c|c|c|}
\hline & & \multicolumn{5}{|c|}{ "MAR Assessments } & \multicolumn{7}{|c|}{ 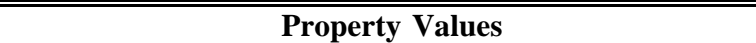 } \\
\hline Frit-Decant & $\% \mathrm{WL}$ & Durability & $\mathbf{T}_{L}$ & \begin{tabular}{|l|} 
Visc \\
\end{tabular} & Frit & \begin{tabular}{|l|} 
Homog \\
\end{tabular} & $\mathbf{A l}_{2} \mathbf{O}_{3}$ & alkali & $T_{L}$ & Visc & $\Delta \mathbf{G}_{\mathbf{P}}$ & Homog & Frit \\
\hline 202-M-Decant \#9 & 59 & - & \begin{tabular}{|l|}
$\mathrm{NO}$ \\
\end{tabular} & \begin{tabular}{|l|} 
Low \\
\end{tabular} & - & - & 9.54 & 18.73 & \begin{tabular}{|l|}
1092.4 \\
\end{tabular} & 12.79 & \begin{tabular}{|l|}
-10.359 \\
\end{tabular} & 270.15 & 55.46 \\
\hline 202-M-Decant \#9 & 60 & - & $\mathrm{NO}$ & \begin{tabular}{|l|} 
Low \\
\end{tabular} & - & - & 9.68 & \begin{tabular}{|l|}
18.84 \\
\end{tabular} & \begin{tabular}{|l|}
1097.9 \\
\end{tabular} & 11.20 & \begin{tabular}{|l|}
-10.441 \\
\end{tabular} & 271.95 & 54.72 \\
\hline 202-M2-Decant \#9 & 25 & - & - & High & - & $\mathrm{NO}$ & 4.62 & 14.85 & \begin{tabular}{|l|}
808.0 \\
\end{tabular} & 130.95 & -7.898 & \begin{tabular}{|l|}
209.21 \\
\end{tabular} & 80.55 \\
\hline 202-M2-Decant \#9 & 26 & - & - & High & - & - & 4.76 & \begin{tabular}{|l|}
14.97 \\
\end{tabular} & 820.7 & 125.54 & -7.976 & 211.00 & 79.81 \\
\hline 202-M2-Decant \#9 & 27 & - & - & \begin{tabular}{|l|} 
High \\
\end{tabular} & 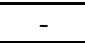 & - & 4.91 & 15.08 & \begin{tabular}{|l|}
832.9 \\
\end{tabular} & 120.21 & -8.053 & 212.79 & 79.07 \\
\hline 202-M2-Decant \#9 & 28 & - & - & \begin{tabular}{|l|} 
High \\
\end{tabular} & - & - & 5.05 & 15.19 & \begin{tabular}{|l|}
844.9 \\
\end{tabular} & \begin{tabular}{|l|}
114.97 \\
\end{tabular} & -8.131 & 214.58 & 78.34 \\
\hline 202-M2-Decant \#9 & 29 & - & - & \begin{tabular}{|l|} 
High \\
\end{tabular} & - & - & 5.20 & 15.31 & \begin{tabular}{|l|}
856.4 \\
\end{tabular} & \begin{tabular}{|l|}
109.84 \\
\end{tabular} & -8.209 & 216.38 & 77.60 \\
\hline 202-M2-Decant \#9 & 30 & - & - & \begin{tabular}{|l|} 
High \\
\end{tabular} & - & - & 5.34 & 15.42 & \begin{tabular}{|l|}
867.6 \\
\end{tabular} & \begin{tabular}{|l|}
104.80 \\
\end{tabular} & -8.286 & \begin{tabular}{|l|}
218.17 \\
\end{tabular} & 76.86 \\
\hline 202-M2-Decant \#9 & 31 & - & - & \begin{tabular}{|l|} 
High \\
\end{tabular} & 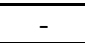 & - & 5.48 & \begin{tabular}{|l|}
15.54 \\
\end{tabular} & \begin{tabular}{|l|}
878.5 \\
\end{tabular} & \begin{tabular}{|l|}
99.85 \\
\end{tabular} & -8.364 & 219.96 & 76.12 \\
\hline 202-M2-Decant \#9 & 32 & - & - & - & - & - & 5.63 & 15.65 & 889.1 & 95.01 & -8.441 & 221.76 & 75.38 \\
\hline 202-M2-Decant \#9 & 33 & - & - & - & - & - & 5.77 & \begin{tabular}{|l|}
15.76 \\
\end{tabular} & \begin{tabular}{|l|}
899.4 \\
\end{tabular} & \begin{tabular}{|l|}
90.28 \\
\end{tabular} & -8.519 & 223.55 & 74.64 \\
\hline 202-M2-Decant \#9 & 34 & - & - & - & - & - & 5.92 & \begin{tabular}{|l|}
15.88 \\
\end{tabular} & \begin{tabular}{|l|}
909.4 \\
\end{tabular} & 85.65 & -8.597 & 225.34 & 73.91 \\
\hline 202-M2-Decant \#9 & 35 & - & - & - & 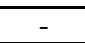 & - & 6.06 & 15.99 & 919.1 & 81.13 & -8.674 & 227.13 & 73.17 \\
\hline 202-M2-Decant \#9 & 36 & - & - & - & - & - & 6.21 & 16.11 & \begin{tabular}{|l|}
928.5 \\
\end{tabular} & 76.73 & -8.752 & 228.93 & 72.43 \\
\hline 202-M2-Decant \#9 & 37 & - & - & - & - & - & 6.35 & 16.22 & 937.8 & \begin{tabular}{|l|}
72.44 \\
\end{tabular} & -8.829 & 230.72 & 71.69 \\
\hline 202-M2-Decant \#9 & 38 & - & - & - & - & - & 6.50 & \begin{tabular}{|l|}
16.33 \\
\end{tabular} & 946.8 & 68.27 & -8.907 & 232.51 & 70.95 \\
\hline $202-\mathrm{M}_{2}^{2}$ & 39 & - & - & - & - & - & 6.64 & 16.45 & 955.5 & 64.22 & -8.985 & 234.30 & 70.22 \\
\hline 202-M2-Decant \#9 & 40 & - & - & - & - & - & 6.79 & \begin{tabular}{|l|}
16.56 \\
\end{tabular} & 964.1 & \begin{tabular}{|l|}
60.29 \\
\end{tabular} & -9.062 & \begin{tabular}{|l|}
236.09 \\
\end{tabular} & 69.48 \\
\hline 202-M2-Decant \#9 & 41 & - & - & - & - & - & 6.93 & \begin{tabular}{|l|}
16.68 \\
\end{tabular} & 972.4 & 56.48 & -9.140 & 237.89 & 68.74 \\
\hline 202-M2-Decant \#9 & 42 & - & - & - & - & - & 7.08 & \begin{tabular}{|l|}
16.79 \\
\end{tabular} & 980.5 & 52.81 & -9.218 & 239.68 & 68.00 \\
\hline $202-\mathrm{M}$ & 43 & - & - & - & - & - & 7.22 & 16.90 & \begin{tabular}{|l|}
988.4 \\
\end{tabular} & 49.25 & -9.295 & 241.47 & 67.26 \\
\hline 202-M2-Decant \#9 & 44 & - & - & - & - & - & 7.37 & 17.02 & 996.2 & 45.83 & -9.373 & 243.26 & 66.53 \\
\hline 202-M2-Decant \#9 & 45 & - & - & - & - & - & 7.51 & 17.13 & \begin{tabular}{|l|}
1003.8 \\
\end{tabular} & 42.54 & -9.450 & 245.06 & 65.79 \\
\hline 202-M2-Decant \#9 & 46 & - & - & - & - & - & 7.65 & 17.25 & 1011.1 & 39.38 & -9.528 & 246.85 & 65.05 \\
\hline 202-M2 & 47 & - & - & - & - & - & 7.80 & 17.36 & 1018.3 & 36.36 & -9.606 & \begin{tabular}{|l|}
248.64 \\
\end{tabular} & 64.31 \\
\hline $202-\mathrm{M} 2-\mathrm{I}$ & 48 & - & $\mathrm{NO}$ & - & - & - & 7.94 & 17.47 & 1025.4 & 33.46 & -9.683 & 250.44 & 63.57 \\
\hline 202-M2-Decant \#9 & 49 & - & $\mathrm{NO}$ & - & - & - & 8.09 & 17.59 & 1032.3 & 30.71 & -9.761 & 252.23 & 62.84 \\
\hline 202-M2-Decant \#9 & 50 & - & NO & - & - & - & 8.23 & 17.70 & \begin{tabular}{|l|}
1039.1 \\
\end{tabular} & 28.09 & -9.838 & 254.02 & 62.10 \\
\hline 202-M2-Decant \#9 & 51 & - & $\mathrm{NO}$ & - & - & - & 8.38 & 17.82 & 1045.7 & 25.60 & -9.916 & 255.81 & 61.36 \\
\hline 202-M2 & 52 & - & NO & Low & - & - & 8.52 & 17.93 & \begin{tabular}{|l|}
1052.1 \\
\end{tabular} & 23.24 & -9.994 & 257.61 & 60.62 \\
\hline 202-M2-Decant \#9 & 53 & - & NO & \begin{tabular}{|l|} 
Low \\
\end{tabular} & - & - & 8.67 & 18.05 & \begin{tabular}{|l|}
1058.4 \\
\end{tabular} & 21.03 & \begin{tabular}{|l|}
-10.071 \\
\end{tabular} & 259.40 & 59.88 \\
\hline 202-M2-Decant \#9 & 54 & - & $\mathrm{NO}$ & Low & - & - & 8.81 & \begin{tabular}{|l|}
18.16 \\
\end{tabular} & \begin{tabular}{|l|}
1064.6 \\
\end{tabular} & 18.94 & \begin{tabular}{|l|}
-10.149 \\
\end{tabular} & 261.19 & 59.15 \\
\hline 202-M2-Decant \#9 & 55 & - & $\mathrm{NO}$ & \begin{tabular}{|l|} 
Low \\
\end{tabular} & 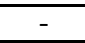 & - & 8.96 & \begin{tabular}{|l|}
18.27 \\
\end{tabular} & \begin{tabular}{|l|}
1070.7 \\
\end{tabular} & 16.99 & \begin{tabular}{|l|}
-10.227 \\
\end{tabular} & \begin{tabular}{|l|}
262.98 \\
\end{tabular} & 58.41 \\
\hline $202-\mathrm{M}$ & 56 & - & $\mathrm{NO}$ & Low & - & - & 9.10 & \begin{tabular}{|l|}
18.39 \\
\end{tabular} & \begin{tabular}{|l|}
1076.6 \\
\end{tabular} & 15.16 & \begin{tabular}{|l|}
-10.304 \\
\end{tabular} & \begin{tabular}{|l|}
264.78 \\
\end{tabular} & 57.67 \\
\hline 202-M2-Decant \#9 & 57 & - & $\mathrm{NO}$ & Low & - & - & 9.25 & 18.50 & 1082.4 & 13.47 & \begin{tabular}{|l|}
-10.382 \\
\end{tabular} & 266.57 & 56.93 \\
\hline 202-M2-Decant \#9 & 58 & - & $\mathrm{NO}$ & Low & - & - & 9.39 & 18.62 & 1088.1 & 11.90 & \begin{tabular}{|l|}
-10.459 \\
\end{tabular} & \begin{tabular}{|l|}
268.36 \\
\end{tabular} & 56.19 \\
\hline 202-M2-Decant \#9 & 59 & - & $\mathrm{NO}$ & Low & - & - & 9.54 & \begin{tabular}{|l|}
18.73 \\
\end{tabular} & 1093.7 & 10.45 & \begin{tabular}{|l|}
-10.537 \\
\end{tabular} & 270.15 & 55.46 \\
\hline 202-M2-Decant \#9 & 60 & - & $\mathrm{NO}$ & \begin{tabular}{|l|} 
Low \\
\end{tabular} & - & - & 9.68 & \begin{tabular}{|l|}
18.84 \\
\end{tabular} & \begin{tabular}{|l|}
1099.2 \\
\end{tabular} & 9.12 & \begin{tabular}{|l|}
-10.615 \\
\end{tabular} & 271.95 & 54.72 \\
\hline G-Decant \#9 & 25 & - & - & - & - & $\mathrm{NO}$ & 4.62 & 18.60 & \begin{tabular}{|l|}
745.2 \\
\end{tabular} & 80.63 & \begin{tabular}{|l|}
-11.037 \\
\end{tabular} & 209.21 & 80.55 \\
\hline G-Decant \#9 & 26 & 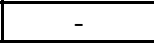 & - & - & - & - & 4.76 & 18.67 & \begin{tabular}{|l|}
758.3 \\
\end{tabular} & 77.18 & \begin{tabular}{|l|}
-11.073 \\
\end{tabular} & 211.00 & 79.81 \\
\hline G-Decant \#9 & 27 & - & - & - & - & - & 4.91 & 18.73 & \begin{tabular}{|l|}
771.0 \\
\end{tabular} & \begin{tabular}{|l|}
73.80 \\
\end{tabular} & \begin{tabular}{|l|}
-11.109 \\
\end{tabular} & \begin{tabular}{|l|}
212.79 \\
\end{tabular} & 79.07 \\
\hline G-Decant \#9 & 28 & - & - & - & - & - & 5.05 & \begin{tabular}{|l|}
18.79 \\
\end{tabular} & \begin{tabular}{|l|}
783.4 \\
\end{tabular} & \begin{tabular}{|l|}
70.47 \\
\end{tabular} & \begin{tabular}{|l|}
-11.144 \\
\end{tabular} & 214.58 & 78.34 \\
\hline G-De & 29 & - & - & - & - & - & 5.20 & 18.86 & 795.5 & \begin{tabular}{|l|}
67.21 \\
\end{tabular} & \begin{tabular}{|l|}
-11.180 \\
\end{tabular} & 216.38 & 77.60 \\
\hline G-Decant \#9 & 30 & 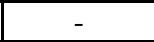 & - & - & - & - & 5.34 & \begin{tabular}{|l|}
18.92 \\
\end{tabular} & 807.3 & 64.03 & \begin{tabular}{|l|}
-11.216 \\
\end{tabular} & 218.17 & 76.86 \\
\hline G-Decant \#9 & 31 & - & - & - & - & - & 5.48 & \begin{tabular}{|l|}
18.99 \\
\end{tabular} & \begin{tabular}{|l|}
818.8 \\
\end{tabular} & 60.90 & \begin{tabular}{|l|}
-11.252 \\
\end{tabular} & \begin{tabular}{|l|}
219.96 \\
\end{tabular} & 76.12 \\
\hline G-Decant \#9 & 32 & - & - & - & - & - & 5.63 & 19.05 & \begin{tabular}{|l|}
830.1 \\
\end{tabular} & \begin{tabular}{|l|}
57.85 \\
\end{tabular} & \begin{tabular}{|l|}
-11.287 \\
\end{tabular} & 221.75 & 75.38 \\
\hline G-De & 33 & - & - & - & - & - & 5.77 & \begin{tabular}{|l|l|}
19.11 \\
\end{tabular} & 841.1 & \begin{tabular}{|l|}
54.87 \\
\end{tabular} & \begin{tabular}{|l|}
-11.323 \\
\end{tabular} & 223.55 & 74.64 \\
\hline te \#9 & 34 & - & - & - & - & - & 5.92 & \begin{tabular}{|l|l|}
19.18 \\
\end{tabular} & 851.8 & 51.96 & \begin{tabular}{|l|}
-11.359 \\
\end{tabular} & 225.34 & 73.91 \\
\hline G-Decant \#9 & 35 & - & - & - & - & - & 6.06 & \begin{tabular}{|l|l|}
19.24 \\
\end{tabular} & \begin{tabular}{|l|}
862.3 \\
\end{tabular} & \begin{tabular}{|l|}
49.12 \\
\end{tabular} & \begin{tabular}{|l|}
-11.395 \\
\end{tabular} & \begin{tabular}{|l|}
227.13 \\
\end{tabular} & 73.17 \\
\hline G-Decant \#9 & 36 & - & - & - & - & - & 6.21 & \begin{tabular}{|l|}
19.31 \\
\end{tabular} & 872.5 & \begin{tabular}{|l|}
46.37 \\
\end{tabular} & \begin{tabular}{|l|}
-11.430 \\
\end{tabular} & 228.93 & 72.43 \\
\hline G-Decant \#9 & 37 & - & - & - & - & - & 6.35 & \begin{tabular}{|l|l|}
19.37 \\
\end{tabular} & \begin{tabular}{|l|}
882.6 \\
\end{tabular} & 43.69 & \begin{tabular}{|l|}
-11.466 \\
\end{tabular} & 230.72 & 71.69 \\
\hline G-Decant \#9 & 38 & - & - & - & - & - & 6.50 & \begin{tabular}{|l|l|}
19.43 \\
\end{tabular} & 892.4 & 41.09 & \begin{tabular}{|l|}
-11.502 \\
\end{tabular} & 232.51 & 70.95 \\
\hline$\# 9$ & 39 & - & - & - & 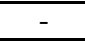 & - & 6.64 & \begin{tabular}{|l|}
19.50 \\
\end{tabular} & \begin{tabular}{|l|}
902.0 \\
\end{tabular} & 38.57 & \begin{tabular}{|l|}
-11.538 \\
\end{tabular} & 234.30 & 70.22 \\
\hline G-Decant \#9 & 40 & - & - & - & - & - & 6.79 & \begin{tabular}{|l|}
19.56 \\
\end{tabular} & \begin{tabular}{|l|}
911.4 \\
\end{tabular} & 36.13 & \begin{tabular}{|l|}
-11.574 \\
\end{tabular} & 236.09 & 69.48 \\
\hline
\end{tabular}


Immobilization Technology Section

WSRC-TR-2002-00549

Savannah River Technology Center

Rev. 0

Westinghouse Savannah River Company

\begin{tabular}{|c|c|c|c|c|c|c|c|c|c|c|c|c|c|}
\hline \multirow[b]{2}{*}{ Frit-Decant } & \multirow[b]{2}{*}{$\% \mathrm{WL}$} & \multicolumn{5}{|c|}{ "MAR Assessments } & \multicolumn{7}{|c|}{ Property Values } \\
\hline & & Durability & $\mathbf{T}_{\mathrm{L}}$ & Visc & Frit & Homog & $\mathbf{A l}_{2} \mathrm{O}_{3}$ & alkali & $\mathbf{T}_{\mathrm{L}}$ & Visc & $\Delta \mathbf{G}_{\mathbf{P}}$ & Homog & Frit \\
\hline G-Decant \#9 & 41 & - & - & - & - & - & 6.93 & 19.63 & 920.5 & 33.77 & -11.609 & 237.89 & 68.74 \\
\hline G-Decant \#9 & 42 & - & - & - & - & - & 7.08 & 19.69 & 929.5 & 31.50 & -11.645 & 239.68 & 68.00 \\
\hline G-Decant \#9 & 43 & - & - & - & - & - & 7.22 & 19.75 & 938.3 & 29.31 & -11.681 & 241.47 & 67.26 \\
\hline G-Decant \#9 & 44 & - & - & - & - & - & 7.37 & 19.82 & 947.0 & 27.21 & -11.717 & 243.26 & 66.53 \\
\hline G-Decant \#9 & 45 & - & - & - & - & - & 7.51 & 19.88 & 955.5 & 25.19 & -11.752 & 245.06 & 65.79 \\
\hline G-Decant \#9 & 46 & - & - & Low & - & - & 7.65 & 19.95 & 963.8 & 23.26 & \begin{tabular}{|l|}
-11.788 \\
\end{tabular} & 246.85 & 65.05 \\
\hline G-Decant \#9 & 47 & - & - & Low & - & - & 7.80 & 20.01 & 971.9 & 21.41 & -11.824 & 248.64 & 64.31 \\
\hline G-Decant \#9 & 48 & - & - & Low & - & - & 7.94 & 20.07 & 979.9 & 19.65 & -11.860 & 250.44 & 63.57 \\
\hline G-Decant \#9 & 49 & - & - & Low & - & - & 8.09 & 20.14 & 987.7 & 17.98 & -11.895 & 252.23 & 62.84 \\
\hline G-Decant \#9 & 50 & - & - & Low & - & - & 8.23 & 20.20 & 995.4 & 16.40 & -11.931 & 254.02 & 62.10 \\
\hline G-Decant \#9 & 51 & - & - & Low & - & - & 8.38 & 20.27 & 1002.9 & 14.90 & -11.967 & 255.81 & 61.36 \\
\hline G-Decant \#9 & 52 & - & - & Low & - & - & 8.52 & 20.33 & 1010.3 & 13.48 & -12.003 & 257.61 & 60.62 \\
\hline G-Decant \#9 & 53 & - & - & Low & - & - & 8.67 & 20.40 & 1017.6 & 12.16 & -12.038 & 259.40 & 59.88 \\
\hline G-Decant \#9 & 54 & - & $\mathrm{NO}$ & Low & - & - & 8.81 & 20.46 & 1024.7 & 10.91 & -12.074 & 261.19 & 59.15 \\
\hline G-Decant \#9 & 55 & - & $\mathrm{NO}$ & Low & - & - & 8.96 & 20.52 & 1031.7 & 9.75 & -12.110 & 262.98 & 58.41 \\
\hline G-Decant \#9 & 56 & - & NO & Low & - & - & 9.10 & 20.59 & 1038.6 & 8.67 & -12.146 & 264.78 & 57.67 \\
\hline G-Decant \#9 & 57 & - & $\mathrm{NO}$ & Low & - & - & 9.25 & 20.65 & 1045.3 & 7.67 & -12.181 & 266.57 & 56.93 \\
\hline G-Decant \#9 & 58 & - & $\mathrm{NO}$ & Low & - & - & 9.39 & 20.72 & 1052.0 & 6.75 & -12.217 & 268.36 & 56.19 \\
\hline G-Decant \#9 & 59 & - & $\mathrm{NO}$ & Low & - & - & 9.54 & 20.78 & 1058.5 & 5.90 & -12.253 & 270.15 & 55.46 \\
\hline G-Decant \#9 & 60 & - & $\mathrm{NO}$ & Low & - & - & 9.68 & 20.84 & 1064.9 & 5.13 & -12.289 & 271.95 & 54.72 \\
\hline U-Decant \#9 & 25 & - & - & High & - & $\mathrm{NO}$ & 3.87 & 14.10 & 801.9 & 126.86 & -6.989 & 206.17 & 81.30 \\
\hline U-Decant \#9 & 26 & - & - & High & - & $\mathrm{NO}$ & 4.02 & 14.23 & 815.4 & 121.86 & -7.079 & 208.01 & 80.55 \\
\hline U-Decant \#9 & 27 & - & - & High & - & $\mathrm{NO}$ & 4.18 & 14.35 & 828.4 & 116.94 & -7.168 & 209.84 & 79.80 \\
\hline U-Decant \#9 & 28 & - & - & High & - & - & 4.33 & 14.47 & 841.0 & 112.09 & -7.258 & 211.67 & 79.06 \\
\hline U-Decant \#9 & 29 & - & - & High & - & - & 4.49 & 14.60 & 853.2 & 107.33 & -7.348 & 213.51 & 78.31 \\
\hline U-Decant \#9 & 30 & - & - & High & - & - & 4.64 & 14.72 & 865.0 & 102.63 & -7.438 & 215.34 & 77.56 \\
\hline U-Decant \#9 & 31 & - & - & - & - & - & 4.79 & 14.85 & 876.4 & 98.02 & -7.528 & 217.17 & 76.81 \\
\hline U-Decant \#9 & 32 & - & - & - & - & - & 4.95 & 14.97 & 887.5 & 93.50 & -7.617 & 219.00 & 76.06 \\
\hline U-Decant \#9 & 33 & - & - & - & - & - & 5.10 & 15.09 & 898.3 & 89.07 & -7.707 & 220.84 & 75.31 \\
\hline U-Decant \#9 & 34 & - & - & - & - & - & 5.26 & 15.22 & 908.7 & 84.72 & -7.797 & 222.67 & 74.57 \\
\hline U-Decant \#9 & 35 & - & - & - & - & - & 5.41 & 15.34 & 918.8 & 80.46 & -7.886 & 224.50 & 73.82 \\
\hline U-Decant \#9 & 36 & - & - & - & - & - & 5.57 & 15.47 & 928.7 & 76.30 & -7.976 & 226.34 & 73.07 \\
\hline U-Decant \#9 & 37 & - & - & - & - & - & 5.72 & 15.59 & 938.3 & 72.24 & -8.066 & 228.17 & 72.32 \\
\hline U-Decant \#9 & 38 & - & - & - & - & - & 5.88 & 15.71 & 947.6 & 68.28 & -8.155 & 230.00 & 71.57 \\
\hline U-Decant \#9 & 39 & - & - & - & - & - & 6.03 & 15.84 & 956.6 & 64.42 & -8.245 & 231.83 & 70.83 \\
\hline U-Decant \#9 & 40 & - & - & - & - & - & 6.19 & 15.96 & 965.5 & 60.66 & -8.335 & 233.67 & 70.08 \\
\hline U-Decant \#9 & 41 & - & - & - & - & - & 6.34 & 16.09 & 974.0 & 57.02 & -8.425 & 235.50 & 69.33 \\
\hline U-Decant \#9 & 42 & - & - & - & - & - & 6.50 & 16.21 & 982.4 & 53.48 & -8.514 & 237.33 & 68.58 \\
\hline U-Decant \#9 & 43 & - & - & - & - & - & 6.65 & 16.33 & 990.5 & 50.05 & -8.604 & 239.17 & 67.83 \\
\hline U-Decant \#9 & 44 & - & - & - & - & - & 6.81 & 16.46 & 998.5 & 46.73 & -8.694 & 241.00 & 67.09 \\
\hline U-Decant \#9 & 45 & - & - & - & - & - & 6.96 & 16.58 & 1006.2 & 43.54 & -8.784 & 242.83 & 66.34 \\
\hline U-Decant \#9 & 46 & - & - & - & - & - & 7.11 & 16.71 & 1013.7 & 40.45 & -8.873 & 244.67 & 65.59 \\
\hline U-Decant \#9 & 47 & - & NO & - & - & - & 7.27 & 16.83 & 1021.1 & 37.49 & -8.963 & 246.50 & 64.84 \\
\hline U-Decant \#9 & 48 & - & NO & - & - & - & 7.42 & 16.95 & 1028.3 & 34.64 & -9.053 & 248.33 & 64.09 \\
\hline U-Decant \#9 & 49 & - & NO & - & - & - & 7.58 & 17.08 & 1035.3 & 31.92 & -9.143 & 250.17 & 63.35 \\
\hline U-Decant \#9 & 50 & - & NO & - & - & - & 7.73 & 17.20 & 1042.2 & 29.32 & -9.232 & 252.00 & 62.60 \\
\hline U-Decant \#9 & 51 & - & NO & - & - & - & 7.89 & 17.33 & 1048.8 & 26.84 & -9.322 & 253.83 & 61.85 \\
\hline U-Decant \#9 & 52 & - & $\mathrm{NO}$ & Low & - & - & 8.04 & 17.45 & 1055.3 & 24.49 & -9.412 & 255.67 & 61.10 \\
\hline U-Decant \#9 & 53 & - & $\mathrm{NO}$ & Low & - & - & 8.20 & 17.58 & 1061.8 & 22.26 & -9.502 & 257.50 & 60.35 \\
\hline U-Decant \#9 & 54 & - & $\mathrm{NO}$ & Low & - & - & 8.35 & 17.70 & 1068.0 & 20.15 & -9.591 & 259.33 & 59.61 \\
\hline U-Decant \#9 & 55 & - & $\mathrm{NO}$ & Low & - & - & 8.51 & 17.82 & 1074.1 & 18.16 & -9.681 & 261.16 & 58.86 \\
\hline U-Decant \#9 & 56 & - & $\mathrm{NO}$ & Low & - & - & 8.66 & 17.95 & 1080.1 & 16.30 & \begin{tabular}{|l|}
-9.771 \\
\end{tabular} & 263.00 & 58.11 \\
\hline U-Decant \#9 & 57 & - & NO & Low & - & - & 8.82 & 18.07 & 1085.9 & 14.56 & -9.860 & 264.83 & 57.36 \\
\hline U-Decant \#9 & 58 & - & $\mathrm{NO}$ & Low & - & - & 8.97 & 18.20 & 1091.6 & 12.94 & -9.950 & 266.66 & 56.61 \\
\hline
\end{tabular}


Immobilization Technology Section

WSRC-TR-2002-00549

Savannah River Technology Center

Rev. 0

Westinghouse Savannah River Company

\begin{tabular}{|c|c|c|c|c|c|c|c|c|c|c|c|c|c|}
\hline \multirow[b]{2}{*}{ Frit-Decant } & \multirow[b]{2}{*}{$\% \mathrm{WL}$} & \multicolumn{5}{|c|}{ "MAR Assessments } & \multicolumn{7}{|c|}{ Property Values } \\
\hline & & Durability & $\mathbf{T}_{\mathbf{L}}$ & Visc & Frit & Homog & $\mathbf{A l}_{2} \mathbf{O}_{3}$ & alkali & $\mathbf{T}_{\mathrm{L}}$ & Visc & $\Delta \mathbf{G}_{\mathbf{P}}$ & Homog & Frit \\
\hline U-Decant \#9 & 59 & - & $\mathrm{NO}$ & Low & - & - & 9.13 & 18.32 & 1097.2 & 11.43 & -10.040 & 268.49 & 55.87 \\
\hline U-Decant \#9 & 60 & - & NO & Low & - & - & 9.28 & 18.44 & 1102.7 & 10.05 & -10.130 & 270.33 & 55.12 \\
\hline U2-Decant \#9 & 25 & - & - & High & - & $\mathrm{NO}$ & 3.87 & 14.10 & 804.1 & 109.77 & -7.315 & 206.17 & 81.30 \\
\hline U2-Decant \#9 & 26 & - & - & High & - & $\mathrm{NO}$ & 4.02 & 14.23 & 817.6 & 105.35 & -7.400 & 208.01 & 80.55 \\
\hline U2-Decant \#9 & 27 & - & - & High & - & $\mathrm{NO}$ & 4.18 & 14.35 & 830.6 & 101.00 & -7.486 & 209.84 & 79.80 \\
\hline U2-Decant \#9 & 28 & - & - & - & - & - & 4.33 & 14.47 & 843.2 & 96.72 & -7.571 & 211.67 & 79.06 \\
\hline U2-Decant \#9 & 29 & - & - & - & - & - & 4.49 & 14.60 & 855.4 & 92.51 & -7.656 & 213.51 & 78.31 \\
\hline U2-Decant \#9 & 30 & - & - & - & - & - & 4.64 & 14.72 & 867.1 & 88.37 & -7.742 & 215.34 & 77.56 \\
\hline U2-Decant \#9 & 31 & - & - & - & - & - & 4.79 & 14.85 & 878.5 & 84.31 & -7.827 & 217.17 & 76.81 \\
\hline U2-Decant \#9 & 32 & - & - & - & - & - & 4.95 & 14.97 & 889.6 & 80.33 & -7.912 & 219.00 & 76.06 \\
\hline U2-Decant \#9 & 33 & - & - & - & - & - & 5.10 & 15.09 & 900.3 & 76.43 & -7.998 & 220.84 & 75.31 \\
\hline U2-Decant \#9 & 34 & - & - & - & - & - & 5.26 & 15.22 & 910.8 & 72.62 & -8.083 & 222.67 & 74.57 \\
\hline U2-Decant \#9 & 35 & - & - & - & - & - & 5.41 & 15.34 & 920.9 & 68.89 & -8.169 & 224.50 & 73.82 \\
\hline U2-Decant \#9 & 36 & - & - & - & - & - & 5.57 & 15.47 & 930.7 & 65.24 & -8.254 & 226.34 & 73.07 \\
\hline U2-Decant \#9 & 37 & - & - & - & - & - & 5.72 & 15.59 & 940.2 & 61.69 & -8.340 & 228.17 & 72.32 \\
\hline U2-Decant \#9 & 38 & - & - & - & - & - & 5.88 & 15.71 & 949.5 & 58.23 & -8.425 & 230.00 & 71.57 \\
\hline U2-Decant \#9 & 39 & - & - & - & - & - & 6.03 & 15.84 & 958.5 & 54.87 & -8.510 & 231.84 & 70.83 \\
\hline U2-Decant \#9 & 40 & - & - & - & - & - & 6.19 & 15.96 & 967.4 & 51.59 & -8.596 & 233.67 & 70.08 \\
\hline U2-Decant \#9 & 41 & - & - & - & - & - & 6.34 & 16.09 & 975.9 & 48.42 & -8.681 & 235.50 & 69.33 \\
\hline U2-Decant \#9 & 42 & - & - & - & - & - & 6.50 & 16.21 & 984.2 & 45.35 & -8.766 & 237.33 & 68.58 \\
\hline U2-Decant \#9 & 43 & - & - & - & - & - & 6.65 & 16.33 & 992.3 & 42.38 & -8.852 & 239.17 & 67.83 \\
\hline U2-Decant \#9 & 44 & - & - & - & - & - & 6.81 & 16.46 & 1000.2 & 39.51 & -8.937 & 241.00 & 67.09 \\
\hline U2-Decant \#9 & 45 & - & - & - & - & - & 6.96 & 16.58 & 1008.0 & 36.74 & -9.022 & 242.83 & 66.34 \\
\hline U2-Decant \#9 & 46 & - & - & - & - & - & 7.11 & 16.71 & 1015.4 & 34.08 & -9.108 & 244.67 & 65.59 \\
\hline U2-Decant \#9 & 47 & - & NO & - & - & - & 7.27 & 16.83 & 1022.8 & 31.53 & -9.193 & 246.50 & 64.84 \\
\hline U2-Decant \#9 & 48 & - & $\mathrm{NO}$ & - & - & - & 7.42 & 16.95 & 1029.9 & 29.08 & -9.279 & 248.33 & 64.09 \\
\hline U2-Decant \#9 & 49 & - & NO & - & - & - & 7.58 & 17.08 & 1036.9 & 26.75 & -9.364 & 250.17 & 63.35 \\
\hline U2-Decant \#9 & 50 & - & $\mathrm{NO}$ & Low & - & - & 7.73 & 17.20 & 1043.8 & 24.52 & -9.450 & 252.00 & 62.60 \\
\hline U2-Decant \#9 & 51 & - & $\mathrm{NO}$ & Low & - & - & 7.89 & 17.33 & 1050.4 & 22.40 & -9.535 & 253.83 & 61.85 \\
\hline U2-Decant \#9 & 52 & - & $\mathrm{NO}$ & Low & - & - & 8.04 & 17.45 & 1056.9 & 20.40 & -9.620 & 255.67 & 61.10 \\
\hline U2-Decant \#9 & 53 & - & $\mathrm{NO}$ & Low & - & - & 8.20 & 17.58 & 1063.2 & 18.50 & -9.706 & 257.50 & 60.35 \\
\hline U2-Decant \#9 & 54 & - & $\mathrm{NO}$ & Low & - & - & 8.35 & 17.70 & 1069.5 & 16.71 & -9.791 & 259.33 & 59.61 \\
\hline U2-Decant \#9 & 55 & - & $\mathrm{NO}$ & Low & - & - & 8.51 & 17.82 & 1075.5 & 15.03 & -9.877 & 261.16 & 58.86 \\
\hline U2-Decant \#9 & 56 & - & $\mathrm{NO}$ & Low & - & - & 8.66 & 17.95 & 1081.4 & 13.45 & -9.962 & 263.00 & 58.11 \\
\hline U2-Decant \#9 & 57 & - & $\mathrm{NO}$ & Low & - & - & 8.82 & 18.07 & 1087.2 & 11.98 & -10.047 & 264.83 & 57.36 \\
\hline U2-Decant \#9 & 58 & - & $\mathrm{NO}$ & Low & - & - & 8.97 & 18.20 & 1092.9 & 10.62 & -10.133 & 266.66 & 56.61 \\
\hline U2-Decant \#9 & 59 & - & $\mathrm{NO}$ & Low & - & - & 9.13 & 18.32 & 1098.5 & 9.36 & -10.218 & 268.49 & 55.87 \\
\hline U2-Decant \#9 & 60 & - & NO & Low & - & - & 9.28 & 18.44 & 1103.9 & 8.20 & -10.304 & 270.33 & 55.12 \\
\hline
\end{tabular}


Immobilization Technology Section

WSRC-TR-2002-00549

Savannah River Technology Center

Rev. 0

Westinghouse Savannah River Company

\section{Appendix C2}

MAR Calculations for Various Frit/Washing Combinations 


\begin{tabular}{|c|c|c|c|c|c|c|c|c|c|c|c|c|c|}
\hline & & \multicolumn{5}{|c|}{ MAR Assessment } & \multicolumn{7}{|c|}{ Property Values } \\
\hline Frit/Washing & $\% \mathrm{WL}$ & Durability & $\mathbf{T}_{\mathbf{L}}$ & Visc & Frit & Homog & $\mathbf{A l}_{2} \mathbf{O}_{3}$ & alkali & $\mathbf{T}_{\mathbf{L}}$ & Visc & $\Delta \mathbf{G p}$ & Homog & Frit \\
\hline 202-Washing @ 0\% & 25 & - & - & - & High & $\mathrm{NO}$ & 3.29 & 18.61 & 715.9 & 73.29 & -11.666 & 197.82 & 82.97 \\
\hline 202-Washing @ 0\% & 26 & - & - & - & High & $\mathrm{NO}$ & 3.42 & 18.84 & 725.4 & 69.05 & -11.848 & 199.45 & 82.37 \\
\hline 202-Washing @ 0\% & 27 & - & - & - & High & $\mathrm{NO}$ & 3.55 & 19.06 & 734.5 & 64.96 & -12.030 & 201.08 & 81.77 \\
\hline 202-Washing @ 0\% & 28 & - & - & - & - & $\mathrm{NO}$ & 3.68 & 19.28 & 743.4 & 61.01 & -12.213 & \begin{tabular}{|l|}
202.71 \\
\end{tabular} & 81.17 \\
\hline 202-Washing @ 0\% & 29 & $\mathrm{NO}$ & - & - & - & $\mathrm{NO}$ & 3.81 & 19.51 & 752.0 & 57.21 & -12.395 & 204.33 & 80.56 \\
\hline 202-Washing @ 0\% & 30 & NO & - & - & - & $\mathrm{NO}$ & 3.94 & 19.73 & 760.4 & \begin{tabular}{|l|}
53.55 \\
\end{tabular} & -12.578 & 205.96 & 79.96 \\
\hline 202-Washing @ 0\% & 31 & $\mathrm{NO}$ & - & - & - & $\mathrm{NO}$ & 4.07 & 19.96 & 768.5 & \begin{tabular}{|l|}
50.03 \\
\end{tabular} & -12.760 & \begin{tabular}{|l|}
207.59 \\
\end{tabular} & 79.36 \\
\hline 202-Washing @ 0\% & 32 & $\mathrm{NO}$ & - & - & - & $\mathrm{NO}$ & 4.20 & 20.18 & 776.4 & 46.65 & -12.942 & 209.22 & 78.76 \\
\hline 202-Washing @ 0\% & 33 & $\mathrm{NO}$ & - & - & - & $\mathrm{NO}$ & 4.34 & 20.41 & 784.0 & 43.42 & -13.125 & 210.84 & 78.16 \\
\hline 202-Washing @ 0\% & 34 & $\mathrm{NO}$ & - & - & - & - & 4.47 & 20.63 & 791.4 & 40.32 & -13.307 & \begin{tabular}{|l|}
212.47 \\
\end{tabular} & 77.56 \\
\hline 202-Washing @ 0\% & 35 & $\mathrm{NO}$ & - & - & - & - & 4.60 & 20.86 & 798.6 & 37.37 & -13.490 & 214.10 & 76.96 \\
\hline 202-Washing @ 0\% & 36 & $\mathrm{NO}$ & - & - & - & - & 4.73 & 21.08 & 805.7 & 34.55 & -13.672 & 215.72 & 76.36 \\
\hline 202-Washing @ 0\% & 37 & $\mathrm{NO}$ & - & - & - & - & 4.86 & 21.30 & 812.5 & \begin{tabular}{|l|}
31.87 \\
\end{tabular} & -13.854 & 217.35 & 75.75 \\
\hline 202-Washing @ 0\% & 38 & $\mathrm{NO}$ & - & - & - & - & 4.99 & 21.53 & 819.1 & 29.32 & -14.037 & \begin{tabular}{|l|}
218.98 \\
\end{tabular} & 75.15 \\
\hline 202-Washing @ 0\% & 39 & $\mathrm{NO}$ & - & - & - & - & 5.12 & 21.75 & 825.6 & 26.91 & -14.219 & 220.61 & 74.55 \\
\hline 202-Washing @ 0\% & 40 & $\mathrm{NO}$ & - & - & - & - & 5.26 & 21.98 & 831.9 & 24.63 & -14.401 & 222.23 & 73.95 \\
\hline 202-Washing @ 0\% & 41 & $\mathrm{NO}$ & - & Low & - & - & 5.39 & 22.20 & 838.0 & 22.48 & -14.584 & \begin{tabular}{|l|}
223.86 \\
\end{tabular} & 73.35 \\
\hline 202-Washing @ 0\% & 42 & $\mathrm{NO}$ & - & Low & - & - & 5.52 & 22.43 & 844.0 & 20.46 & -14.766 & 225.49 & 72.75 \\
\hline 202-Washing @ 0\% & 43 & $\mathrm{NO}$ & - & Low & - & - & 5.65 & 22.65 & 849.8 & 18.56 & -14.948 & \begin{tabular}{|l|}
227.11 \\
\end{tabular} & 72.15 \\
\hline 202-Washing @ 0\% & 44 & $\mathrm{NO}$ & - & Low & - & - & 5.78 & 22.88 & 855.5 & 16.78 & \begin{tabular}{|l|}
-15.131 \\
\end{tabular} & 228.74 & 71.54 \\
\hline 202-Washing @ 0\% & 45 & $\mathrm{NO}$ & - & Low & - & - & 5.91 & 23.10 & 861.0 & 15.12 & -15.313 & \begin{tabular}{|l|}
230.37 \\
\end{tabular} & 70.94 \\
\hline 202-Washing @ 0\% & 46 & $\mathrm{NO}$ & - & Low & - & - & 6.04 & 23.32 & 866.4 & \begin{tabular}{|l|}
13.57 \\
\end{tabular} & -15.496 & 232.00 & 70.34 \\
\hline 202-Washing @ 0\% & 47 & $\mathrm{NO}$ & - & Low & - & - & 6.18 & 23.55 & 871.7 & 12.14 & -15.678 & 233.62 & 69.74 \\
\hline 202-Washing @ 0\% & 48 & $\mathrm{NO}$ & - & Low & - & - & 6.31 & 23.77 & 876.9 & \begin{tabular}{|l|}
10.81 \\
\end{tabular} & -15.860 & 235.25 & 69.14 \\
\hline 202-Washing @ 0\% & 49 & $\mathrm{NO}$ & - & Low & - & - & 6.44 & 24.00 & 881.9 & 9.59 & -16.043 & 236.88 & 68.54 \\
\hline 202-Washing @ 0\% & 50 & $\mathrm{NO}$ & - & Low & - & - & 6.57 & 24.22 & 886.8 & 8.47 & -16.225 & 238.51 & 67.94 \\
\hline 202- Washing @ 0\% & 51 & $\mathrm{NO}$ & - & Low & - & - & 6.70 & 24.45 & 891.6 & 7.44 & -16.407 & 240.13 & 67.34 \\
\hline 202-Washing @ 0\% & 52 & $\mathrm{NO}$ & - & Low & - & - & 6.83 & 24.67 & 896.3 & 6.50 & -16.590 & 241.76 & 66.73 \\
\hline 202-Washing @ 0\% & 53 & $\mathrm{NO}$ & - & Low & - & - & 6.96 & 24.90 & 900.9 & 5.66 & -16.772 & 243.39 & 66.13 \\
\hline 202-Washing @ 0\% & 54 & $\mathrm{NO}$ & - & Low & - & - & 7.10 & 25.12 & 905.4 & 4.89 & -16.955 & 245.01 & 65.53 \\
\hline 202-Washing @ 0\% & 55 & $\mathrm{NO}$ & - & Low & - & - & 7.23 & 25.34 & 909.8 & 4.20 & -17.137 & 246.64 & 64.93 \\
\hline 202-Washing @ 0\% & 56 & $\mathrm{NO}$ & - & Low & - & - & 7.36 & 25.57 & 914.1 & 3.59 & -17.319 & 248.27 & 64.33 \\
\hline 202-Washing @ 0\% & 57 & $\mathrm{NO}$ & - & Low & - & - & 7.49 & 25.79 & 918.3 & 3.05 & -17.502 & 249.90 & 63.73 \\
\hline 202-Washing @ 0\% & 58 & $\mathrm{NO}$ & - & Low & - & - & 7.62 & 26.02 & 922.5 & 2.56 & -17.684 & 251.52 & 63.13 \\
\hline 202-Washing @ 0\% & 59 & $\mathrm{NO}$ & - & Low & - & - & 7.75 & 26.24 & 926.5 & 2.14 & -17.867 & 253.15 & 62.53 \\
\hline 202-Washing @ 0\% & 60 & $\mathrm{NO}$ & - & Low & - & - & 7.88 & 26.47 & 930.5 & 1.78 & -18.049 & \begin{tabular}{|l|}
254.78 \\
\end{tabular} & 61.93 \\
\hline O- Washing @ 0\% & 25 & - & - & High & High & $\mathrm{NO}$ & 3.29 & $12.61 \mid$ & 781.4 & 103.84 & -7.809 & 200.23 & 84.47 \\
\hline O- Washing@0\% & 26 & - & - & - & High & $\mathrm{NO}$ & 3.42 & 12.92 & 790.7 & 97.66 & -8.043 & 201.83 & 83.85 \\
\hline O- Washing @ 0\% & 27 & - & - & - & High & $\mathrm{NO}$ & 3.55 & 13.22 & 799.6 & 91.71 & -8.277 & 203.42 & 83.23 \\
\hline O- Washing @ 0\% & 28 & - & - & - & High & $\mathrm{NO}$ & 3.68 & 13.52 & 808.1 & 85.98 & -8.510 & 205.02 & 82.60 \\
\hline O- Washing @ 0\% & 29 & - & - & - & High & $\mathrm{NO}$ & 3.81 & 13.83 & 816.2 & 80.46 & -8.744 & 206.61 & 81.98 \\
\hline O- Washing @ 0\% & 30 & - & - & - & - & $\mathrm{NO}$ & 3.94 & 14.13 & 824.0 & 75.17 & -8.978 & \begin{tabular}{|l|}
208.21 \\
\end{tabular} & 81.36 \\
\hline O- Washing @ 0\% & 31 & - & - & - & - & $\mathrm{NO}$ & 4.07 & 14.44 & 831.5 & 70.09 & -9.212 & 209.80 & 80.74 \\
\hline O- Washing @ 0\% & 32 & - & - & - & - & - & 4.20 & 14.74 & 838.6 & 65.22 & -9.446 & 211.40 & 80.12 \\
\hline O- Washing @ 0\% & 33 & - & - & - & - & - & 4.34 & 15.05 & 845.5 & 60.57 & -9.680 & 212.99 & 79.50 \\
\hline O- Washing @ 0\% & 34 & - & - & - & - & - & 4.47 & 15.35 & 852.1 & 56.13 & -9.913 & \begin{tabular}{|l|}
214.59 \\
\end{tabular} & 78.88 \\
\hline O- Washing @ 0\% & 35 & - & - & - & - & - & 4.60 & 15.66 & 858.5 & 51.90 & -10.147 & \begin{tabular}{|l|}
216.18 \\
\end{tabular} & 78.26 \\
\hline O- Washing @ 0\% & 36 & - & - & - & - & - & 4.73 & 15.96 & 864.7 & 47.88 & -10.381 & 217.78 & 77.64 \\
\hline O- Washing @ 0\% & 37 & - & - & - & - & - & 4.86 & 16.26 & 870.6 & 44.06 & -10.615 & \begin{tabular}{|l|}
219.37 \\
\end{tabular} & 77.01 \\
\hline O- Washing @ 0\% & 38 & - & - & - & - & - & 4.99 & 16.57 & 876.3 & 40.44 & -10.849 & 220.97 & 76.39 \\
\hline O-Washing @0\% & 39 & - & - & - & - & - & 5.12 & 16.87 & 881.7 & 37.02 & -11.082 & 222.56 & 75.77 \\
\hline O- Washing @ 0\% & 40 & - & - & - & - & - & 5.26 & 17.18 & 887.1 & 33.80 & -11.316 & 224.16 & 75.15 \\
\hline O- Washing @0\% & 41 & - & - & - & - & - & 5.39 & 17.48 & 892.2 & 30.77 & -11.550 & 225.75 & 74.53 \\
\hline
\end{tabular}


Immobilization Technology Section

WSRC-TR-2002-00549

Savannah River Technology Center

Rev. 0

Westinghouse Savannah River Company

\begin{tabular}{|c|c|c|c|c|c|c|c|c|c|c|c|c|c|}
\hline & & \multicolumn{5}{|c|}{ MAR Assessment } & \multicolumn{7}{|c|}{ Property Values } \\
\hline Frit/Washing & $\% \mathrm{WL}$ & Durability & $\mathbf{T}_{\mathbf{L}}$ & Visc & Frit & Homog & $\mathbf{A l}_{2} \mathbf{O}_{3}$ & alkali & $\mathbf{T}_{\mathrm{L}}$ & Visc & $\Delta \mathbf{G p}$ & Homog & Frit \\
\hline O- Washing @ 0\% & 42 & - & - & - & - & - & 5.52 & 17.79 & 897.2 & 27.92 & -11.784 & 227.35 & 73.91 \\
\hline O- Washing @0\% & 43 & - & - & - & - & - & 5.65 & 18.09 & 902.0 & 25.26 & -12.018 & 228.94 & 73.29 \\
\hline O- Washing @ 0\% & 44 & - & - & Low & - & - & 5.78 & 18.40 & 906.6 & 22.77 & -12.251 & 230.54 & 72.67 \\
\hline O- Washing @ 0\% & 45 & $\mathrm{NO}$ & - & Low & - & - & 5.91 & 18.70 & 911.1 & 20.45 & -12.485 & 232.13 & 72.04 \\
\hline O- Washing @ 0\% & 46 & $\mathrm{NO}$ & - & Low & - & - & 6.04 & 19.00 & 915.4 & 18.30 & \begin{tabular}{|l|}
-12.719 \\
\end{tabular} & \begin{tabular}{|l|}
233.73 \\
\end{tabular} & 71.42 \\
\hline O- Washing @ 0\% & 47 & $\mathrm{NO}$ & - & Low & - & - & 6.18 & 19.31 & 919.7 & 16.32 & \begin{tabular}{|l|}
-12.953 \\
\end{tabular} & \begin{tabular}{|l|}
235.32 \\
\end{tabular} & 70.80 \\
\hline O- Washing @ 0\% & 48 & $\mathrm{NO}$ & - & Low & - & - & 6.31 & 19.61 & 923.8 & 14.49 & \begin{tabular}{|l|}
-13.186 \\
\end{tabular} & \begin{tabular}{|l|}
236.92 \\
\end{tabular} & 70.18 \\
\hline O- Washing @ 0\% & 49 & $\mathrm{NO}$ & - & Low & - & - & 6.44 & 19.92 & 927.8 & 12.80 & -13.420 & 238.52 & 69.56 \\
\hline O-Washing @ 0\% & 50 & $\mathrm{NO}$ & - & Low & - & - & 6.57 & 20.22 & 931.6 & 11.26 & -13.654 & \begin{tabular}{|l|}
240.11 \\
\end{tabular} & 68.94 \\
\hline O-Washing @ 0\% & 51 & $\mathrm{NO}$ & - & Low & - & - & 6.70 & 20.53 & 935.4 & 9.86 & -13.888 & \begin{tabular}{|l|}
241.71 \\
\end{tabular} & 68.32 \\
\hline O-Washing @ 0\% & 52 & $\mathrm{NO}$ & - & Low & - & - & 6.83 & 20.83 & 939.0 & 8.59 & \begin{tabular}{|l|}
-14.122 \\
\end{tabular} & \begin{tabular}{|l|}
243.30 \\
\end{tabular} & 67.70 \\
\hline O- Washing @ 0\% & 53 & $\mathrm{NO}$ & - & Low & - & - & 6.96 & 21.14 & 942.6 & 7.44 & -14.356 & 244.89 & 67.07 \\
\hline O- Washing @ 0\% & 54 & $\mathrm{NO}$ & - & Low & - & - & 7.10 & 21.44 & 946.0 & 6.41 & \begin{tabular}{|l|}
-14.589 \\
\end{tabular} & \begin{tabular}{|l|}
246.49 \\
\end{tabular} & 66.45 \\
\hline O- Washing @ 0\% & 55 & $\mathrm{NO}$ & - & Low & - & - & 7.23 & 21.74 & 949.4 & 5.48 & -14.823 & 248.09 & 65.83 \\
\hline O- Washing @ 0\% & 56 & $\mathrm{NO}$ & - & Low & - & - & 7.36 & 22.05 & 952.6 & 4.66 & -15.057 & 249.68 & 65.21 \\
\hline O- Washing @ 0\% & 57 & $\mathrm{NO}$ & - & Low & - & - & 7.49 & 22.35 & 955.8 & 3.94 & -15.291 & 251.28 & 64.59 \\
\hline O- Washing @ 0\% & 58 & $\mathrm{NO}$ & - & Low & - & - & 7.62 & 22.66 & 958.9 & 3.30 & -15.525 & 252.87 & 63.97 \\
\hline O-Washing @ 0\% & 59 & $\mathrm{NO}$ & - & Low & - & - & 7.75 & 22.96 & 961.9 & 2.74 & -15.758 & \begin{tabular}{|l|}
254.47 \\
\end{tabular} & 63.35 \\
\hline O- Washing @ 0\% & 60 & $\mathrm{NO}$ & - & Low & - & - & 7.88 & 23.27 & 964.8 & 2.26 & -15.992 & 256.06 & 62.72 \\
\hline P- Wash & 25 & - & - & High & High & $\mathrm{NO}$ & 3.29 & 12.61 & 775.1 & 150.10 & \begin{tabular}{|l|}
-6.995 \\
\end{tabular} & 200.23 & 84.47 \\
\hline P-Washing @ 0\% & 26 & - & - & High & High & $\mathrm{NO}$ & 3.42 & 12.92 & 784.6 & 141.69 & -7.239 & \begin{tabular}{|l|}
201.83 \\
\end{tabular} & 83.85 \\
\hline $\mathrm{P}-\mathrm{Was}$ & 27 & - & - & High & High & $\mathrm{NO}$ & 3.55 & 13.22 & 793.5 & 133.54 & 484 & \begin{tabular}{|l|}
203.42 \\
\end{tabular} & 83.23 \\
\hline P-Washing @ 0\% & 28 & - & - & High & High & $\mathrm{NO}$ & 3.68 & 13.52 & 802.2 & 125.67 & -7.728 & 205.01 & 82.60 \\
\hline P-Washing @ 0\% & 29 & - & - & High & High & $\mathrm{NO}$ & 3.81 & 13.83 & 810.4 & 118.06 & -7.973 & \begin{tabular}{|l|}
206.61 \\
\end{tabular} & 81.98 \\
\hline P-Washing @ 0\% & 30 & - & - & High & - & $\mathrm{NO}$ & 3.94 & 14.13 & 818.3 & 110.73 & -8.218 & 208.21 & 81.36 \\
\hline P-Washing @ 0\% & 31 & - & - & High & - & $\mathrm{NO}$ & 4.07 & 14.44 & 826.0 & 103.67 & -8.463 & 209.80 & 80.74 \\
\hline P- Wash & 32 & - & - & - & - & - & 4.20 & 14.74 & 833.2 & 96.87 & -8.707 & 211.40 & 80.12 \\
\hline P- Wash & 33 & - & - & - & - & - & 4.34 & 15.05 & 840.3 & 90.35 & 52 & \begin{tabular}{|l|}
212.99 \\
\end{tabular} & 79.50 \\
\hline P-Wash & 34 & - & - & - & - & - & 4.47 & 15.35 & 847.0 & 84.10 & -9.196 & \begin{tabular}{|l|}
214.59 \\
\end{tabular} & 78.88 \\
\hline P- Wasl & 35 & - & - & - & - & - & 4.60 & 15.66 & 853.5 & 78.12 & -9.441 & 216.18 & 78.26 \\
\hline P-Washing @ 0\% & 36 & - & - & - & - & - & 4.73 & 15.96 & 859.8 & 72.40 & -9.686 & \begin{tabular}{|l|}
217.78 \\
\end{tabular} & 77.64 \\
\hline P-Washing @ 0\% & 37 & - & - & - & - & - & 4.86 & 16.26 & 865.8 & 66.95 & -9.931 & 219.37 & 77.01 \\
\hline P-Washing @ 0\% & 38 & - & - & - & - & - & 4.99 & 16.57 & 871.6 & 61.75 & -10.175 & 220.97 & 76.39 \\
\hline P-Washing @ 0\% & 39 & - & - & - & - & - & 5.12 & 16.87 & 877.2 & 56.82 & -10.420 & 222.56 & 75.77 \\
\hline @ $0 \%$ & 40 & - & - & - & - & - & 5.26 & 17.18 & 882.7 & 52.15 & \begin{tabular}{|l|}
-10.664 \\
\end{tabular} & \begin{tabular}{|l|}
224.16 \\
\end{tabular} & 75.15 \\
\hline $\mathrm{P}-\mathrm{Wa}$ & 41 & - & - & - & - & - & 5.39 & 17.48 & 887.9 & 47.72 & 909 & 75 & 74.53 \\
\hline @ $0 \%$ & 42 & - & - & - & - & - & 5.52 & 17.79 & 893.0 & 43.55 & \begin{tabular}{|l|}
-11.154 \\
\end{tabular} & \begin{tabular}{|l|}
227.35 \\
\end{tabular} & 73.91 \\
\hline P-Washing @ 0\% & 43 & - & - & - & - & - & 5.65 & 18.09 & 897.9 & 39.62 & \begin{tabular}{|l|}
-11.398 \\
\end{tabular} & \begin{tabular}{|l|}
228.94 \\
\end{tabular} & 73.29 \\
\hline P-Washing @ 0\% & 44 & - & - & - & - & - & 5.78 & 18.40 & 902.6 & 35.93 & -11.643 & 230.54 & 72.66 \\
\hline P-Washi & 45 & - & - & - & - & - & 5.91 & 18.70 & 907.3 & 32.47 & \begin{tabular}{|l|}
-11.888 \\
\end{tabular} & 232.13 & 72.04 \\
\hline P-Washing @ 0\% & 46 & - & - & - & - & - & 6.04 & 19.00 & 911.7 & 29.24 & -12.133 & 233.73 & 71.42 \\
\hline P-Was & 47 & & - & - & - & - & 6.18 & 19.31 & 916.0 & 26.24 & -12.377 & 235.32 & 70.80 \\
\hline P-Washing @ 0\% & 48 & $\mathrm{NO}$ & - & Low & - & - & 6.31 & 19.61 & 920.3 & 23.45 & \begin{tabular}{|l|}
-12.622 \\
\end{tabular} & \begin{tabular}{|l|}
236.92 \\
\end{tabular} & 70.18 \\
\hline P-Washing @ 0\% & 49 & $\mathrm{NO}$ & - & Low & - & - & 6.44 & 19.92 & 924.3 & 20.87 & -12.866 & 238.52 & 69.56 \\
\hline P-Washing @ 0\% & 50 & $\mathrm{NO}$ & - & Low & - & - & 6.57 & 20.22 & 928.3 & 18.50 & -13.111 & \begin{tabular}{|l|}
240.11 \\
\end{tabular} & 68.94 \\
\hline P-Washing @ 0\% & 51 & $\mathrm{NO}$ & - & Low & - & - & 6.70 & 20.53 & 932.1 & 16.31 & -13.356 & \begin{tabular}{|l|}
241.71 \\
\end{tabular} & 68.32 \\
\hline P-Was & 52 & $\mathrm{NO}$ & - & Low & - & - & 6.83 & 20.83 & 935.9 & 14.32 & -13.600 & 243.30 & 67.70 \\
\hline P-Washing @ 0\% & 53 & $\mathrm{NO}$ & - & Low & - & - & 6.96 & 21.14 & 939.5 & 12.50 & -13.845 & \begin{tabular}{|l|}
244.89 \\
\end{tabular} & 67.07 \\
\hline P-Washing @ 0\% & 54 & $\mathrm{NO}$ & - & Low & - & - & 7.10 & 21.44 & 943.0 & 10.86 & -14.090 & 246.49 & 66.45 \\
\hline P-Washing @ 0\% & 55 & $\mathrm{NO}$ & - & Low & - & - & 7.23 & 21.74 & 946.5 & 9.37 & -14.335 & \begin{tabular}{|l|}
248.09 \\
\end{tabular} & 65.83 \\
\hline P-Washing @ 0\% & 56 & $\mathrm{NO}$ & - & Low & - & - & 7.36 & 22.05 & 949.8 & 8.04 & \begin{tabular}{|l|}
-14.579 \\
\end{tabular} & \begin{tabular}{|l|}
249.68 \\
\end{tabular} & 65.21 \\
\hline P-Washing @ 0\% & 57 & $\mathrm{NO}$ & - & Low & - & - & 7.49 & 22.35 & 953.1 & 6.85 & \begin{tabular}{|l|}
-14.824 \\
\end{tabular} & \begin{tabular}{|l|}
251.28 \\
\end{tabular} & 64.59 \\
\hline P-Washing @ 0\% & 58 & $\mathrm{NO}$ & - & Low & - & - & 7.62 & 22.66 & 956.3 & 5.80 & -15.068 & \begin{tabular}{|l|}
252.87 \\
\end{tabular} & 63.97 \\
\hline P-Washing @ 0\% & 59 & $\mathrm{NO}$ & - & Low & - & - & 7.75 & 22.96 & 959.3 & 4.87 & -15.313 & \begin{tabular}{|l|}
254.47 \\
\end{tabular} & 63.35 \\
\hline
\end{tabular}


Immobilization Technology Section

WSRC-TR-2002-00549

Savannah River Technology Center

Rev. 0

Westinghouse Savannah River Company

\begin{tabular}{|c|c|c|c|c|c|c|c|c|c|c|c|c|c|}
\hline & & \multicolumn{5}{|c|}{ "MAR Assessment } & \multicolumn{7}{|c|}{ Property Values } \\
\hline Frit/Washing & $\% \mathrm{WL}$ & Durability & $\mathbf{T}_{\mathrm{L}}$ & Visc & Frit & Homog & $\mathrm{Al}_{2} \mathrm{O}_{3}$ & alkali & $\mathbf{T}_{\mathrm{L}}$ & Visc & $\Delta \mathbf{G p}$ & Homog & Frit \\
\hline P-Washing @0\% & 60 & $\mathrm{NO}$ & - & Low & - & - & 7.88 & 23.27 & 962.4 & 4.06 & -15.558 & 256.06 & 62.72 \\
\hline 202-Washing @ 25\% & 25 & - & - & - & High & $\mathrm{NO}$ & 3.53 & 17.42 & 761.3 & 83.86 & -10.556 & 200.76 & 81.83 \\
\hline 202-Washing @ 25\% & 26 & - & - & - & 2 & $\mathrm{NO}$ & 3.67 & 17.60 & 772.0 & 79.59 & -10.694 & 202.51 & 81.18 \\
\hline 202-Washing @ 25\% & 27 & - & - & - & - & $\mathrm{NO}$ & 3.81 & 17.78 & 782.4 & 75.43 & -10.832 & 204.25 & 80.53 \\
\hline 202-Washing @ 25\% & 28 & - & - & - & - & $\mathrm{NO}$ & 3.95 & 17.96 & 792.5 & 71.39 & -10.970 & 205.99 & 79.89 \\
\hline 202-Washing @ 25\% & 29 & - & - & - & - & $\mathrm{NO}$ & 4.09 & 18.13 & 802.2 & 67.47 & -11.108 & 207.74 & 79.24 \\
\hline 202-Washing @ 25\% & 30 & - & - & - & - & $\mathrm{NO}$ & 4.23 & 18.31 & 811.7 & 63.66 & -11.246 & 209.48 & 78.59 \\
\hline 202-Washing @ 25\% & 31 & - & - & - & - & - & 4.37 & 18.49 & 820.9 & 59.97 & -11.384 & 211.23 & 77.95 \\
\hline 202-Washing @ 25\% & 32 & - & - & - & 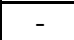 & - & 4.52 & 18.66 & 829.9 & 56.40 & -11.522 & 212.97 & 77.30 \\
\hline 202-Washing @ 25\% & 33 & - & - & - & - & - & 4.66 & 18.84 & 838.6 & 52.96 & -11.660 & 214.72 & 76.65 \\
\hline 202-Washing @25\% & 34 & - & - & - & - & - & 4.80 & 19.02 & 847.0 & 49.63 & -11.798 & 216.46 & 76.00 \\
\hline 202-Washing @ 25\% & 35 & - & - & - & - & - & 4.94 & 19.19 & 855.3 & 46.42 & -11.936 & 218.21 & 75.36 \\
\hline 202-Washing @ 25\% & 36 & - & - & - & 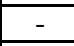 & - & 5.08 & 19.37 & 863.2 & 43.34 & -12.074 & 219.95 & 74.71 \\
\hline 202-Washing @ 25\% & 37 & - & - & - & - & - & 5.22 & 19.55 & 871.0 & 40.37 & -12.212 & 221.70 & 74.06 \\
\hline 202-Washing @ 25\% & 38 & - & - & - & - & - & 5.36 & 19.73 & 878.7 & 37.53 & -12.350 & 223.44 & 73.42 \\
\hline 202-Washing @ 25\% & 39 & $\mathrm{NO}$ & - & - & - & - & 5.50 & 19.90 & 886.1 & 34.81 & -12.488 & 225.19 & 72.77 \\
\hline 202-Washing@25\% & 40 & $\mathrm{NO}$ & - & - & - & - & 5.64 & 20.08 & 893.2 & 32.21 & -12.626 & 226.93 & 72.12 \\
\hline 202-Washing @ 25\% & 41 & $\mathrm{NO}$ & - & - & - & - & 5.79 & 20.26 & 900.3 & 29.73 & -12.764 & 228.68 & 71.48 \\
\hline 202-Washing @ 25\% & 42 & $\mathrm{NO}$ & - & - & - & - & 5.93 & 20.43 & 907.1 & 27.37 & -12.902 & 230.42 & 70.83 \\
\hline ing @ 25\% & 43 & $\mathrm{NO}$ & - & - & - & - & 6.07 & 20.61 & 913.8 & 25.13 & 040 & 232.17 & 70.18 \\
\hline 202-Washing @ 25\% & 44 & $\mathrm{NO}$ & - & Low & - & - & 6.21 & 20.79 & 920.3 & 23.00 & -13.178 & 233.91 & 69.54 \\
\hline g@25\% & 45 & $\mathrm{NO}$ & - & Low & 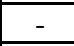 & - & 6.35 & 20.96 & 926.7 & 21.00 & 316 & 235.66 & 68.89 \\
\hline 202-Washing @ 25\% & 46 & $\mathrm{NO}$ & - & Low & - & - & 6.49 & 21.14 & 932.9 & 19.10 & -13.454 & 237.40 & 68.24 \\
\hline 202-Washing @ 25\% & 47 & $\mathrm{NO}$ & - & Low & - & - & 6.63 & 21.32 & 939.0 & 17.32 & -13.592 & 239.14 & 67.59 \\
\hline 202-Washing @ 25\% & 48 & $\mathrm{NO}$ & - & Low & - & - & 6.77 & 21.50 & 944.9 & 15.65 & -13.730 & 240.89 & 66.95 \\
\hline 202-Washing @ 25\% & 49 & $\mathrm{NO}$ & - & Low & - & - & 6.91 & 21.67 & 950.8 & 14.09 & -13.868 & 242.63 & 66.30 \\
\hline g@25\% & 50 & $\mathrm{NO}$ & - & Low & - & - & 7.06 & 21.85 & 956.4 & 12.63 & -14.006 & 244.38 & 65.65 \\
\hline 202-Washing @ 25\% & 51 & $\mathrm{NO}$ & - & Low & - & - & 7.20 & 22.03 & 962.0 & 11.28 & -14.144 & 246.12 & 65.01 \\
\hline g@25\% & 52 & $\mathrm{NO}$ & - & $\mathrm{Lo}$ & - & - & 7.34 & 22.20 & 967.4 & 10.03 & 282 & 247.87 & 64.36 \\
\hline 202-Washing @ 25\% & 53 & $\mathrm{NO}$ & - & Low & 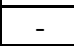 & - & 7.48 & 22.38 & 972.7 & 8.88 & 420 & 249.61 & 63.71 \\
\hline Ig@25\% & 54 & $\mathrm{NO}$ & - & Low & - & - & 7.62 & 22.56 & 977.9 & 7.82 & -14.558 & 251.36 & 63.07 \\
\hline g@25\% & 55 & $\mathrm{NO}$ & - & Low & 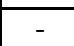 & - & 7.76 & 22.73 & 983.0 & 6.85 & -14.696 & 253.10 & 62.42 \\
\hline 202-Washing @ 25\% & 56 & $\mathrm{NO}$ & - & Low & - & - & 7.90 & 22.91 & 988.0 & 5.96 & -14.834 & 254.85 & 61.77 \\
\hline 202-Washing @ 25\% & 57 & $\mathrm{NO}$ & - & Low & - & - & 8.04 & 23.09 & 992.9 & 5.16 & -14.972 & 256.59 & 61.13 \\
\hline g@ $25 \%$ & 58 & $\mathrm{NO}$ & - & Low & - & - & 8.18 & 23.26 & 997.7 & 4.44 & -15.110 & 258.34 & 60.48 \\
\hline 202-Washing @ 25\% & 59 & $\mathrm{NO}$ & - & Low & - & - & 8.32 & 23.44 & 1002.3 & 3.80 & -15.248 & 260.08 & 59.83 \\
\hline g@25\% & 60 & $\mathrm{NO}$ & - & & 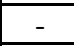 & - & 8.47 & 23.62 & 1006.9 & 3.22 & 386 & 261.83 & 59.18 \\
\hline ig@ $25 \%$ & 25 & - & - & High & Hign & $\mathrm{NO}$ & 3.53 & 11.42 & 837.3 & 119.20 & -6.700 & \begin{tabular}{|l|}
203.17 \\
\end{tabular} & 83.33 \\
\hline O-Washing @ 25\% & 26 & - & - & High & High & $\mathrm{NO}$ & 3.67 & 11.68 & 847.8 & 112.95 & -6.889 & 204.88 & 82.66 \\
\hline O-Washing @ 25\% & 27 & - & - & High & High & $\mathrm{NO}$ & 3.81 & 11.94 & 857.8 & 106.88 & -7.078 & 206.59 & 81.99 \\
\hline O-Washing @ 25\% & 28 & - & - & High & - & $\mathrm{NO}$ & 3.95 & 12.20 & 867.4 & 100.99 & -7.268 & 208.30 & 81.33 \\
\hline @ 25\% & 29 & - & - & - & - & $\mathrm{NO}$ & 4.09 & 12.45 & 876.6 & 95.27 & -7 & 210.02 & 80.66 \\
\hline O-Washing @ 25\% & 30 & - & - & - & - & - & 4.23 & 12.71 & 885.4 & 89.74 & -7.647 & 211.73 & 79.99 \\
\hline O-Washing @ 25\% & 31 & - & - & - & 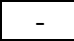 & - & 4.37 & 12.97 & 893.9 & 84.39 & -7.836 & 213.44 & 79.33 \\
\hline O-Washing @ 25\% & 32 & - & - & - & - & - & 4.52 & 13.22 & 902.0 & 79.22 & -8.026 & 215.15 & 78.66 \\
\hline O-Washing @ 25\% & 33 & - & - & - & - & - & 4.66 & 13.48 & 909.9 & 74.23 & -8.215 & \begin{tabular}{|l|}
216.87 \\
\end{tabular} & 77.99 \\
\hline g@25\% & 34 & - & - & - & - & - & 4.80 & 13.74 & 917.4 & 69.43 & 04 & 218.58 & 77.32 \\
\hline O-Washing @ 25\% & 35 & - & - & - & - & - & 4.94 & 13.99 & \begin{tabular}{|l|}
924.7 \\
\end{tabular} & 64.82 & -8.594 & 220.29 & 76.66 \\
\hline O-Washing @ 25\% & 36 & - & - & - & - & - & 5.08 & 14.25 & 931.6 & 60.39 & -8.783 & 222.00 & 75.99 \\
\hline O-Washing @ 25\% & 37 & - & - & - & - & - & 5.22 & 14.51 & 938.4 & 56.14 & -8.973 & 223.72 & 75.32 \\
\hline O-Washing@ @25\% & 38 & - & - & - & - & - & 5.36 & 14.77 & 944.9 & 52.07 & -9.162 & 225.43 & 74.66 \\
\hline O-Washing@25\% & 39 & - & - & - & - & - & 5.50 & 15.02 & 951.2 & 48.19 & -9.351 & 227.14 & 73.99 \\
\hline @ 25\% & 40 & - & - & - & - & - & 5.64 & 15.28 & 957.3 & 44.48 & -9.541 & 228.85 & 73.32 \\
\hline O-Washing @ 25\% & 41 & - & - & - & - & - & 5.79 & 15.54 & 963.1 & 40.96 & -9.730 & 230.57 & 72.66 \\
\hline
\end{tabular}




\begin{tabular}{|c|c|c|c|c|c|c|c|c|c|c|c|c|c|}
\hline & & \multicolumn{5}{|c|}{ "MAR Assessment } & \multicolumn{7}{|c|}{ Property Values } \\
\hline Frit/Washing & $\% \mathrm{WL}$ & Durability & $\mathbf{T}_{\mathrm{L}}$ & Visc & Frit & Homog & $\mathrm{Al}_{2} \mathrm{O}_{3}$ & alkali & $\mathbf{T}_{\mathrm{L}}$ & Visc & $\Delta \mathbf{G p}$ & Homog & Frit \\
\hline O-Washing @ 25\% & 42 & - & - & - & - & - & 5.93 & 15.79 & 968.8 & 37.61 & -9.920 & 232.28 & 71.99 \\
\hline O-Washing @ 25\% & 43 & - & - & - & - & - & 6.07 & 16.05 & 974.3 & 34.45 & -10.109 & 233.99 & 71.32 \\
\hline O-Washing @ 25\% & 44 & - & - & - & - & - & 6.21 & 16.31 & 979.7 & 31.45 & -10.299 & 235.70 & 70.66 \\
\hline O-Washing @ 25\% & 45 & - & - & - & - & - & 6.35 & 16.56 & 984.9 & 28.63 & -10.488 & 237.42 & 69.99 \\
\hline O-Washing @ 25\% & 46 & - & - & - & - & - & 6.49 & 16.82 & 989.9 & 25.97 & -10.677 & 239.13 & 69.32 \\
\hline O-Washing @ 25\% & 47 & - & - & Low & - & - & 6.63 & 17.08 & 994.7 & 23.48 & -10.867 & 240.84 & 68.65 \\
\hline O-Washing @ 25\% & 48 & - & - & Low & - & - & 6.77 & 17.34 & 999.5 & 21.16 & -11.056 & 242.56 & 67.99 \\
\hline O-Washing @ 25\% & 49 & - & - & Low & - & - & 6.91 & 17.59 & 1004.0 & 18.99 & -11.246 & 244.27 & 67.32 \\
\hline O-Washing @ 25\% & 50 & - & - & Low & - & - & 7.06 & 17.85 & 1008.5 & 16.97 & -11.435 & 245.98 & 66.65 \\
\hline O-Washing @ 25\% & 51 & - & - & Low & - & - & 7.20 & 18.11 & 1012.8 & 15.10 & -11.625 & 247.69 & 65.99 \\
\hline O-Washing @ 25\% & 52 & - & $\mathrm{NO}$ & Low & - & - & 7.34 & 18.36 & 1017.0 & 13.38 & -11.814 & 249.41 & 65.32 \\
\hline O-Washing @ 25\% & 53 & - & $\mathrm{NO}$ & Low & - & - & 7.48 & 18.62 & 1021.1 & 11.80 & -12.003 & 251.12 & 64.65 \\
\hline O-Washing @ 25\% & 54 & - & $\mathrm{NO}$ & Low & - & - & 7.62 & 18.88 & 1025.1 & 10.35 & -12.193 & 252.83 & 63.99 \\
\hline O-Washing @ 25\% & 55 & $\mathrm{NO}$ & $\mathrm{NO}$ & Low & - & - & 7.76 & 19.13 & 1029.0 & 9.03 & -12.382 & 254.55 & 63.32 \\
\hline O-Washing @ 25\% & 56 & $\mathrm{NO}$ & $\mathrm{NO}$ & Low & - & - & 7.90 & 19.39 & 1032.8 & 7.84 & -12.572 & 256.26 & 62.65 \\
\hline O-Washing @ 25\% & 57 & $\mathrm{NO}$ & $\mathrm{NO}$ & Low & - & - & 8.04 & 19.65 & 1036.4 & 6.76 & -12.761 & 257.97 & 61.99 \\
\hline O-Washing @ 25\% & 58 & $\mathrm{NO}$ & $\mathrm{NO}$ & Low & - & - & 8.18 & 19.90 & 1040.0 & 5.79 & -12.950 & 259.68 & 61.32 \\
\hline O-Washing @ 25\% & 59 & $\mathrm{NO}$ & $\mathrm{NO}$ & Low & - & - & 8.32 & 20.16 & 1043.5 & 4.93 & -13.140 & 261.40 & 60.65 \\
\hline O-Washing @ 25\% & 60 & $\mathrm{NO}$ & $\mathrm{NO}$ & Low & - & - & 8.47 & 20.42 & 1046.9 & 4.16 & -13.329 & 263.11 & 59.98 \\
\hline @ 25\% & 25 & - & - & High & High & $\mathrm{NO}$ & 3.53 & 11.42 & 830.0 & 170.80 & & 17 & 83.33 \\
\hline P-Washing @ 25\% & 26 & - & - & High & High & $\mathrm{NO}$ & 3.67 & 11.68 & 840.6 & 162.35 & -6.085 & 204.88 & 82.66 \\
\hline @ 25\% & 27 & - & - & High & Hig & $\mathrm{NO}$ & 3.81 & 11.94 & 850.8 & 154.12 & & 206.59 & 81.99 \\
\hline P-Washing@25\% & 28 & - & - & High & - & $\mathrm{NO}$ & 3.95 & 12.20 & 860.5 & 146.10 & -6.486 & 208.30 & 81.33 \\
\hline P-Washing @25\% & 29 & - & - & High & - & $\mathrm{NO}$ & 4.09 & 12.45 & 869.9 & 138.29 & -6.686 & 210.02 & 80.66 \\
\hline P-Washing @25\% & 30 & - & - & High & - & - & 4.23 & 12.71 & 878.8 & 130.71 & -6.886 & 211.73 & 79.99 \\
\hline P-Washing @ 25\% & 31 & - & - & High & - & - & 4.37 & 12.97 & 887.4 & 123.35 & -7.087 & 213.44 & 79.33 \\
\hline g@25\% & 32 & - & - & High & - & - & 4.52 & 13.22 & 895.7 & 116.22 & 287 & 215.15 & 78.66 \\
\hline P-Washing @ 25\% & 33 & - & - & High & 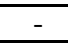 & - & 4.66 & 13.48 & 903.7 & 109.31 & -7.487 & 216.87 & 77.99 \\
\hline P-Washing @ 25\% & 34 & - & - & High & - & - & 4.80 & 13.74 & 911.4 & 102.64 & -7.688 & \begin{tabular}{|l|}
218.58 \\
\end{tabular} & 77.32 \\
\hline P-Washing @ 25\% & 35 & - & - & - & 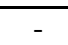 & - & 4.94 & 13.99 & 918.8 & 96.19 & -7.888 & 220.29 & 76.66 \\
\hline P-Washing@25\% & 36 & - & - & - & - & - & 5.08 & 14.25 & 925.8 & 89.98 & -8.088 & 222.00 & 75.99 \\
\hline P-Washing @ 25\% & 37 & - & - & - & - & - & 5.22 & 14.51 & 932.7 & 83.99 & -8.288 & 223.72 & 75.32 \\
\hline P-Washing @ 25\% & 38 & - & - & - & - & - & 5.36 & 14.77 & 939.4 & 78.24 & -8.489 & 225.43 & 74.66 \\
\hline P-Washing @ 25\% & 39 & - & - & - & - & - & 5.50 & 15.02 & 945.8 & 72.72 & -8.689 & 227.14 & 73.99 \\
\hline ng @ 25\% & 40 & - & - & - & - & - & 5.64 & 15.28 & 952.0 & 67.44 & 89 & 228.86 & 73.32 \\
\hline ng@ $95 \%$ & 41 & - & - & - & - & - & 5.79 & 15.54 & 958.0 & 2.39 & & 57 & 72.66 \\
\hline P-Washing @ 25\% & 42 & - & - & - & 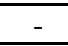 & - & 5.93 & 15.79 & 963.8 & 57.57 & -9.290 & 232.28 & 71.99 \\
\hline P-Washing @ 25\% & 43 & - & - & - & - & - & 6.07 & 16.05 & 969.5 & 52.98 & -9.490 & 233.99 & 71.32 \\
\hline P-Washing @ 25\% & 44 & - & - & - & - & - & 6.21 & 16.31 & 974.9 & 48.63 & -9.690 & 235.70 & 70.66 \\
\hline P-Washing @ 25\% & 45 & - & - & - & - & - & 6.35 & 16.56 & 980.2 & 44.50 & -9.891 & 237.42 & 69.99 \\
\hline P-Washing @25\% & 46 & - & - & - & - & - & 6.49 & 16.82 & 985.4 & 40.59 & -10.091 & 239.13 & 69.32 \\
\hline g $@ 25 \%$ & 47 & - & - & - & 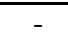 & - & 6.63 & 17.08 & 990.4 & 36.91 & -10.291 & 240.84 & 68.65 \\
\hline P-Washing @ 25\% & 48 & - & - & - & - & - & 6.77 & 17.34 & 995.2 & 33.44 & -10.492 & 242.56 & 67.99 \\
\hline P-Washing @25\% & 49 & - & - & - & - & - & 6.91 & 17.59 & 999.9 & 30.19 & -10.692 & 244.27 & 67.32 \\
\hline P-Washing @ 25\% & 50 & - & - & - & - & - & 7.06 & 17.85 & 1004.5 & 27.16 & -10.892 & 245.98 & 66.65 \\
\hline P-Washing @ 25\% & 51 & - & - & Low & - & - & 7.20 & 18.11 & 1008.9 & 24.32 & -11.092 & 247.70 & 65.99 \\
\hline g@25\% & 52 & - & - & Low & - & - & 7.34 & 18.36 & 1013.2 & 21.70 & -11.293 & 249.41 & 65.32 \\
\hline P-Washing @ 25\% & 53 & - & $\mathrm{NO}$ & Low & - & - & 7.48 & 18.62 & 1017.4 & 19.26 & -11.493 & 251.12 & 64.65 \\
\hline P-Washing @ 25\% & 54 & - & $\mathrm{NO}$ & Low & - & - & 7.62 & 18.88 & 1021.5 & 17.02 & -11.693 & 252.83 & 63.99 \\
\hline P-Washing @ 25\% & 55 & - & $\mathrm{NO}$ & Low & - & - & 7.76 & 19.13 & 1025.5 & 14.96 & -11.893 & 254.55 & 63.32 \\
\hline P-Washing@25\% & 56 & - & $\mathrm{NO}$ & Low & - & - & 7.90 & 19.39 & 1029.4 & 13.08 & -12.094 & 256.26 & 62.65 \\
\hline P-Washing@25\% & 57 & - & $\mathrm{NO}$ & Low & - & - & 8.04 & 19.65 & 1033.2 & 11.37 & -12.294 & 257.97 & 61.99 \\
\hline P-Washing@25\% & 58 & $\mathrm{NO}$ & $\mathrm{NO}$ & Low & - & - & 8.18 & 19.90 & 1036.9 & 9.83 & -12.494 & 259.68 & 61.32 \\
\hline P-Washing @ 25\% & 59 & $\mathrm{NO}$ & $\mathrm{NO}$ & Low & - & - & 8.32 & 20.16 & 1040.4 & 8.43 & -12.695 & 261.40 & 60.65 \\
\hline
\end{tabular}




\begin{tabular}{|c|c|c|c|c|c|c|c|c|c|c|c|c|c|}
\hline & & \multicolumn{5}{|c|}{ "MAR Assessment } & \multicolumn{7}{|c|}{ Property Values } \\
\hline Frit/Washing & $\% \mathrm{WL}$ & Durability & $\mathbf{T}_{\mathrm{L}}$ & Visc & Frit & Homog & $\mathrm{Al}_{2} \mathrm{O}_{3}$ & alkali & $\mathbf{T}_{\mathrm{L}}$ & Visc & $\Delta \mathbf{G p}$ & Homog & Frit \\
\hline P-Washing @ 25\% & 60 & $\mathrm{NO}$ & $\mathrm{NO}$ & Low & - & - & 8.47 & 20.42 & 1043.9 & 7.19 & -12.895 & 263.11 & 59.98 \\
\hline 202-Washing @ 50\% & 25 & - & - & - & - & $\mathrm{NO}$ & 3.81 & 16.05 & 815.4 & 98.00 & -9.272 & 204.16 & 80.50 \\
\hline 202-Washing @ 50\% & 26 & - & - & - & - & $\mathrm{NO}$ & 3.96 & 16.17 & 827.7 & 93.78 & -9.358 & 206.04 & 79.80 \\
\hline 202-Washing @ 50\% & 27 & - & - & - & - & $\mathrm{NO}$ & 4.11 & 16.29 & 839.6 & 89.65 & -9.445 & 207.92 & 79.10 \\
\hline 202-Washing @ 50\% & 28 & - & - & - & - & $\mathrm{NO}$ & 4.26 & 16.41 & 851.1 & 85.60 & \begin{tabular}{|l|}
-9.532 \\
\end{tabular} & \begin{tabular}{|l|}
209.81 \\
\end{tabular} & 78.40 \\
\hline 202-Washing @ 50\% & 29 & - & - & - & - & - & 4.42 & 16.54 & 862.3 & 81.63 & \begin{tabular}{|l|}
-9.618 \\
\end{tabular} & \begin{tabular}{|l|}
211.69 \\
\end{tabular} & 77.70 \\
\hline 202-Washing @ 50\% & 30 & - & - & - & - & - & 4.57 & 16.66 & 873.2 & 77.75 & -9.705 & 213.57 & 77.00 \\
\hline 202-Washing @ 50\% & 31 & - & - & - & - & - & 4.72 & 16.78 & 883.8 & 73.95 & -9.792 & 215.45 & 76.30 \\
\hline 202-Washing @ 50\% & 32 & - & - & - & - & - & 4.87 & 16.90 & 894.1 & 70.24 & -9.878 & 217.33 & 75.60 \\
\hline 202-Washing @ 50\% & 33 & - & - & - & - & - & 5.03 & 17.02 & 904.1 & 66.61 & -9.965 & 219.21 & 74.90 \\
\hline 202-Washing @ 50\% & 34 & - & - & - & - & - & 5.18 & 17.15 & 913.9 & 63.08 & -10.051 & 221.09 & 74.20 \\
\hline 202-Washing @ 50\% & 35 & - & - & - & - & - & 5.33 & 17.27 & 923.4 & 59.64 & -10.138 & 222.97 & 73.50 \\
\hline 202-Washing @ 50\% & 36 & - & - & - & - & - & 5.48 & 17.39 & 932.6 & 56.30 & -10.225 & \begin{tabular}{|l|}
224.85 \\
\end{tabular} & 72.80 \\
\hline 202-Washing @ 50\% & 37 & - & - & - & - & - & 5.64 & 17.51 & 941.7 & 53.05 & -10.311 & 226.73 & 72.10 \\
\hline 202-Washing @ 50\% & 38 & - & - & - & - & - & 5.79 & 17.63 & 950.5 & 49.90 & -10.398 & 228.61 & 71.40 \\
\hline 202-Washing @ 50\% & 39 & - & - & - & - & - & 5.94 & 17.76 & 959.1 & 46.85 & -10.485 & 230.50 & 70.70 \\
\hline 202-Washing@50\% & 40 & - & - & - & - & - & 6.09 & 17.88 & 967.5 & 43.89 & -10.571 & 232.37 & 70.00 \\
\hline 202-Washing @ 50\% & 41 & - & - & - & - & - & 6.24 & 18.00 & 975.6 & 41.04 & -10.658 & 234.26 & 69.30 \\
\hline 202-Washing @ 50\% & 42 & - & - & - & - & - & 6.40 & 18.12 & 983.6 & 38.29 & -10.745 & 236.14 & 68.60 \\
\hline g@ $@ 50 \%$ & 43 & - & - & - & - & - & 6.55 & 18.24 & 991.4 & 35.64 & -10.831 & 238.02 & 67.90 \\
\hline 202-Washing @ 50\% & 44 & - & - & - & - & - & 6.70 & 18.37 & 999.0 & 33.10 & -10.918 & 239.90 & 67.20 \\
\hline g@ 50\% & 45 & - & - & - & - & - & 6.85 & 18.49 & 1006.4 & 30.66 & -11.004 & \begin{tabular}{|l|}
241.78 \\
\end{tabular} & 66.50 \\
\hline 202-Washing @ 50\% & 46 & - & - & - & - & - & 7.01 & 18.61 & 1013.7 & 28.33 & -11.091 & 243.66 & 65.80 \\
\hline 202-Washing @ 50\% & 47 & - & $\mathrm{NO}$ & - & - & - & 7.16 & 18.73 & 1020.8 & 26.10 & -11.178 & 245.54 & 65.10 \\
\hline 202-Washing @ 50\% & 48 & - & $\mathrm{NO}$ & Low & - & - & 7.31 & 18.85 & 1027.7 & 23.97 & -11.264 & 247.42 & 64.40 \\
\hline $\mathrm{g} @ 50 \%$ & 49 & - & $\mathrm{NO}$ & Low & - & - & 7.46 & 18.98 & 1034.5 & 21.95 & -11.351 & 249.30 & 63.70 \\
\hline g@ $50 \%$ & 50 & - & $\mathrm{NO}$ & Low & - & - & 7.62 & 19.10 & 1041.1 & 20.04 & -11.438 & 251.18 & 63.00 \\
\hline 202-Wash & 51 & - & $\mathrm{NO}$ & Low & - & - & 7.77 & 19.22 & 1047.6 & 18.22 & -11.524 & 253.06 & 62.30 \\
\hline @ $50 \%$ & 52 & - & $\mathrm{NO}$ & & - & - & 7.92 & 19.34 & 1054.0 & 16.52 & \begin{tabular}{|l|}
-11.611 \\
\end{tabular} & \begin{tabular}{|l|}
254.95 \\
\end{tabular} & 61.60 \\
\hline g@ @ $50 \%$ & 53 & - & $\mathrm{NO}$ & Low & - & - & 8.07 & 19.46 & 1060.3 & 14.91 & -11.697 & \begin{tabular}{|l|}
256.83 \\
\end{tabular} & 60.90 \\
\hline g@ $@ 50 \%$ & 54 & - & $\mathrm{NO}$ & Low & - & - & 8.22 & 19.58 & 1066.3 & 13.40 & \begin{tabular}{|l|}
-11.784 \\
\end{tabular} & \begin{tabular}{|l|}
258.71 \\
\end{tabular} & 60.20 \\
\hline g@ $@ 50 \%$ & 55 & - & $\mathrm{NO}$ & Low & - & - & 8.38 & 19.71 & 1072.3 & 12.00 & -11.871 & 260.59 & 59.50 \\
\hline g@ $050 \%$ & 56 & - & $\mathrm{NO}$ & Low & - & - & 8.53 & 19.83 & 1078.2 & 10.69 & -11.957 & \begin{tabular}{|l|}
262.47 \\
\end{tabular} & 58.80 \\
\hline g@ @ 50\% & 57 & - & $\mathrm{NO}$ & Low & - & - & 8.68 & 19.95 & 1083.9 & 9.48 & -12.044 & 264.35 & 58.10 \\
\hline g@ $@ 50 \%$ & 58 & - & $\mathrm{NO}$ & Low & - & - & 8.83 & 20.07 & 1089.5 & 8.36 & -12.131 & \begin{tabular}{|l|}
266.23 \\
\end{tabular} & 57.40 \\
\hline 202-Washing @ 50\% & 59 & - & $\mathrm{NO}$ & Low & - & - & 8.99 & 20.19 & 1095.0 & 7.33 & -12.217 & \begin{tabular}{|l|}
268.11 \\
\end{tabular} & 56.70 \\
\hline g@ $@ 50 \%$ & 60 & - & $\mathrm{NO}$ & Low & - & - & 9.14 & 20.32 & 1100.4 & 6.39 & -12.304 & 269.99 & 56.00 \\
\hline g@ $@ 0 \%$ & 25 & - & - & - & - & $\mathrm{NO}$ & 4.56 & 19.05 & 733.5 & 77.68 & -11.549 & \begin{tabular}{|l|}
209.60 \\
\end{tabular} & 81.25 \\
\hline G-Washing @ 50\% & 26 & - & - & - & - & - & 4.70 & 19.13 & 746.6 & 74.22 & -11.606 & 211.41 & 80.54 \\
\hline 1g@50\% & 27 & - & - & - & - & - & 4.84 & 19.21 & 759.3 & 70.83 & -11.662 & 213.22 & 79.83 \\
\hline G-Washing @ 50\% & 28 & - & - & - & - & - & 4.98 & 19.29 & 771.8 & 67.52 & -11.718 & 215.03 & 79.12 \\
\hline @ 50\% & 29 & - & - & - & - & - & 5.13 & 19.38 & 783.9 & 64.28 & -11.774 & 216.84 & 78.41 \\
\hline G-Washing @ 50\% & 30 & - & - & - & - & - & 5.27 & 19.46 & 795.7 & 61.12 & -11.831 & \begin{tabular}{|l|}
218.64 \\
\end{tabular} & 77.70 \\
\hline G-Washing @ 50\% & 31 & - & - & - & - & - & 5.41 & 19.54 & 807.2 & 58.02 & -11.887 & 220.45 & 76.99 \\
\hline G-Washing @ 50\% & 32 & - & - & - & - & - & 5.55 & 19.62 & 818.6 & 55.01 & -11.943 & \begin{tabular}{|l|}
222.26 \\
\end{tabular} & 76.28 \\
\hline g@ $@ 50 \%$ & 33 & - & - & - & - & - & 5.70 & 19.70 & 829.6 & 52.07 & -12.000 & \begin{tabular}{|l|}
224.07 \\
\end{tabular} & 75.57 \\
\hline @ $90 \%$ & 34 & - & - & - & - & - & 5.84 & 19.79 & 840.4 & 49.21 & -12.056 & \begin{tabular}{|l|}
225.88 \\
\end{tabular} & 74.86 \\
\hline G-Washing @ 50\% & 35 & - & - & - & - & - & 5.98 & 19.87 & 850.9 & 46.44 & -12.112 & \begin{tabular}{|l|}
227.68 \\
\end{tabular} & 74.15 \\
\hline G-Washing @ 50\% & 36 & - & - & - & - & - & 6.12 & 19.95 & 861.2 & 43.74 & -12.168 & \begin{tabular}{|l|}
229.49 \\
\end{tabular} & 73.44 \\
\hline G-Washing @ 50\% & 37 & - & - & - & - & - & 6.27 & 20.03 & 871.3 & 41.13 & -12.225 & 231.30 & 72.73 \\
\hline G-Washing @ 50\% & 38 & - & - & - & - & - & 6.41 & 20.11 & 881.3 & 38.61 & -12.281 & \begin{tabular}{|l|}
233.11 \\
\end{tabular} & 72.02 \\
\hline G-Washing @ 50\% & 39 & - & - & - & - & - & 6.55 & 20.20 & 890.9 & 36.16 & -12.337 & \begin{tabular}{|l|}
234.92 \\
\end{tabular} & 71.31 \\
\hline @ 50\% & 40 & $\mathrm{NO}$ & - & - & - & - & 6.69 & 20.28 & 900.5 & 33.80 & -12.393 & \begin{tabular}{|l|}
236.73 \\
\end{tabular} & 70.60 \\
\hline G-Washing @ 50\% & 41 & $\mathrm{NO}$ & - & - & - & - & 6.83 & 20.36 & 909.8 & 31.53 & -12.450 & \begin{tabular}{|l|}
238.54 \\
\end{tabular} & 69.89 \\
\hline
\end{tabular}


Immobilization Technology Section

WSRC-TR-2002-00549

Savannah River Technology Center

Rev. 0

Westinghouse Savannah River Company

\begin{tabular}{|c|c|c|c|c|c|c|c|c|c|c|c|c|c|}
\hline & & \multicolumn{5}{|c|}{ "MAR Assessment } & \multicolumn{7}{|c|}{ Property Values } \\
\hline Frit/Washing & $\% \mathrm{WL}$ & Durability & $\mathbf{T}_{\mathrm{L}}$ & Visc & Frit & Homog & $\mathrm{Al}_{2} \mathrm{O}_{3}$ & alkali & $\mathbf{T}_{L}$ & Visc & $\Delta \mathbf{G p}$ & Homog & Frit \\
\hline G-Washing @ 50\% & 42 & $\mathrm{NO}$ & - & - & - & - & 6.98 & 20.44 & 918.9 & 29.35 & -12.506 & 240.34 & 69.18 \\
\hline G-Washing @ 50\% & 43 & $\mathrm{NO}$ & - & - & - & - & 7.12 & 20.52 & 927.8 & 27.25 & -12.562 & 242.15 & 68.47 \\
\hline G-Washing @ 50\% & 44 & $\mathrm{NO}$ & - & - & - & - & 7.26 & 20.61 & 936.6 & 25.24 & -12.618 & 243.96 & 67.76 \\
\hline G-Washing @ 50\% & 45 & $\mathrm{NO}$ & - & Low & - & - & 7.40 & 20.69 & 945.2 & 23.31 & -12.675 & 245.77 & 67.05 \\
\hline G-Washing @ 50\% & 46 & $\mathrm{NO}$ & - & Low & - & - & 7.55 & 20.77 & 953.6 & 21.48 & -12.731 & 247.58 & 66.34 \\
\hline G-Washing @ 50\% & 47 & $\mathrm{NO}$ & - & Low & - & - & 7.69 & 20.85 & 961.9 & 19.73 & -12.787 & 249.39 & 65.63 \\
\hline G-Washing @ 50\% & 48 & $\mathrm{NO}$ & - & Low & - & - & 7.83 & 20.93 & 970.0 & 18.07 & -12.843 & 251.19 & 64.92 \\
\hline G-Washing @ 50\% & 49 & $\mathrm{NO}$ & - & Low & - & - & 7.97 & 21.02 & \begin{tabular}{|l|}
978.0 \\
\end{tabular} & 16.50 & -12.900 & 253.00 & 64.21 \\
\hline G-Washing @ 50\% & 50 & $\mathrm{NO}$ & - & Low & - & - & 8.12 & 21.10 & 985.8 & 15.01 & -12.956 & 254.81 & 63.50 \\
\hline G-Washing @ 50\% & 51 & $\mathrm{NO}$ & - & Low & - & - & 8.26 & 21.18 & 993.5 & 13.61 & -13.012 & 256.62 & 62.79 \\
\hline G-Washing @ 50\% & 52 & $\mathrm{NO}$ & - & Low & - & - & 8.40 & 21.26 & 1001.1 & 12.29 & -13.068 & 258.43 & 62.08 \\
\hline G-Washing @ 50\% & 53 & $\mathrm{NO}$ & - & Low & - & - & 8.54 & 21.34 & 1008.5 & 11.06 & -13.125 & 260.24 & 61.37 \\
\hline G-Washing @ 50\% & 54 & $\mathrm{NO}$ & - & Low & - & - & 8.68 & 21.42 & 1015.8 & 9.91 & -13.181 & 262.04 & 60.66 \\
\hline G-Washing @ 50\% & 55 & $\mathrm{NO}$ & $\mathrm{NO}$ & Low & - & - & 8.83 & 21.51 & 1023.0 & 8.83 & -13.237 & 263.85 & 59.95 \\
\hline G-Washing @ 50\% & 56 & $\mathrm{NO}$ & $\mathrm{NO}$ & Low & - & - & 8.97 & 21.59 & 1030.0 & 7.84 & -13.293 & 265.66 & 59.24 \\
\hline G-Washing @ 50\% & 57 & $\mathrm{NO}$ & NO & Low & - & - & 9.11 & 21.67 & 1036.9 & 6.92 & -13.350 & 267.47 & 58.53 \\
\hline G-Washing @ 50\% & 58 & $\mathrm{NO}$ & $\mathrm{NO}$ & Low & - & - & 9.25 & 21.75 & 1043.7 & 6.08 & -13.406 & 269.28 & 57.82 \\
\hline G-Washing @ 50\% & 59 & $\mathrm{NO}$ & $\mathrm{NO}$ & Low & - & - & 9.40 & 21.83 & 1050.5 & 5.31 & -13.462 & \begin{tabular}{|l|}
271.09 \\
\end{tabular} & 57.11 \\
\hline G-Washing @ 50\% & 60 & $\mathrm{NO}$ & $\mathrm{NO}$ & Low & - & - & 9.54 & 21.92 & 1057.0 & 4.61 & -13.519 & 272.89 & 56.40 \\
\hline $320-75 \%$ Washed & 25 & - & - & - & - & $\mathrm{NO}$ & 4.13 & 19.68 & 735.9 & 45.57 & -12.230 & 210.56 & 80.45 \\
\hline $320-75 \%$ Washed & 26 & - & - & - & - & - & 4.30 & 19.67 & 751.7 & 43.97 & -12.197 & 212.56 & 79.66 \\
\hline $320-75 \%$ Washed & 27 & - & - & - & - & - & 4.47 & 19.66 & 767.0 & 42.39 & -12.163 & 214.57 & 78.88 \\
\hline $320-75 \%$ Washed & 28 & - & - & - & - & - & 4.63 & 19.65 & 782.2 & 40.82 & -12.130 & 216.58 & 78.10 \\
\hline $320-75 \%$ Washed & 29 & - & - & - & - & - & 4.80 & 19.63 & 796.9 & 39.27 & -12.097 & 218.59 & 77.32 \\
\hline $320-75 \%$ Washed & 30 & - & - & - & - & - & 4.96 & 19.62 & 811.3 & 37.75 & -12.064 & 220.60 & 76.54 \\
\hline $320-75 \%$ Washed & 31 & - & - & - & - & - & 5.13 & 19.61 & 825.5 & 36.24 & -12.030 & 222.61 & 75.75 \\
\hline $320-75 \%$ Washed & 32 & - & - & - & - & - & 5.29 & 19.60 & 839.4 & 34.75 & -11.997 & 224.62 & 74.97 \\
\hline $320-75 \%$ Washed & 33 & - & - & - & - & - & 5.46 & 19.58 & 853.0 & 33.29 & -11.964 & 226.62 & 74.19 \\
\hline $320-75 \%$ Washed & 34 & - & - & - & - & - & 5.62 & 19.57 & 866.3 & 31.85 & -11.931 & 228.63 & 73.41 \\
\hline $320-75 \%$ Washed & 35 & - & - & - & - & - & 5.79 & 19.56 & 879.4 & 30.43 & -11.897 & 230.64 & 72.63 \\
\hline $320-75 \%$ Washed & 36 & - & - & - & - & - & 5.95 & 19.55 & 892.3 & 29.03 & -11.864 & 232.65 & 71.84 \\
\hline $320-75 \%$ Washed & 37 & - & - & - & - & - & 6.12 & 19.53 & 904.8 & 27.67 & -11.831 & 234.66 & 71.06 \\
\hline $320-75 \%$ Washed & 38 & - & - & - & - & - & 6.29 & 19.52 & \begin{tabular}{|l|}
917.2 \\
\end{tabular} & 26.32 & -11.797 & 236.67 & 70.28 \\
\hline $320-75 \%$ Washed & 39 & - & - & - & - & - & 6.45 & 19.51 & 929.4 & 25.01 & -11.764 & 238.67 & 69.50 \\
\hline $320-75 \%$ Washed & 40 & - & - & Low & - & - & 6.62 & 19.49 & 941.3 & 23.72 & -11.731 & 240.68 & 68.71 \\
\hline $320-75 \%$ Washed & 41 & - & - & Low & - & - & 6.78 & 19.48 & 953.0 & 22.46 & -11.698 & 242.69 & 67.93 \\
\hline $320-75 \%$ Washed & 42 & - & - & Low & - & - & 6.95 & 19.47 & 964.5 & 21.24 & -11.664 & 244.70 & 67.15 \\
\hline $320-75 \%$ Washed & 43 & - & - & Low & - & - & 7.11 & 19.46 & 975.9 & 20.04 & -11.631 & 246.71 & 66.37 \\
\hline $320-75 \%$ Washed & 44 & - & - & Low & - & - & 7.28 & 19.44 & 987.0 & 18.87 & -11.598 & 248.72 & 65.59 \\
\hline $320-75 \%$ Washed & 45 & - & - & Low & - & - & 7.44 & 19.43 & 997.9 & 17.73 & -11.565 & 250.72 & 64.80 \\
\hline $320-75 \%$ Washed & 46 & - & - & Low & - & - & 7.61 & 19.42 & 1008.6 & 16.63 & -11.532 & 252.73 & 64.02 \\
\hline $320-75 \%$ Washed & 47 & - & $\mathrm{NO}$ & Low & - & - & 7.77 & 19.41 & 1019.2 & 15.56 & -11.498 & 254.74 & 63.24 \\
\hline $320-75 \%$ Washed & 48 & - & $\mathrm{NO}$ & Low & - & - & 7.94 & 19.39 & 1029.6 & 14.53 & -11.465 & 256.75 & 62.46 \\
\hline $320-75 \%$ Washed & 49 & - & $\mathrm{NO}$ & Low & - & - & 8.10 & 19.38 & 1039.8 & 13.53 & -11.432 & 258.76 & 61.68 \\
\hline $320-75 \%$ Washed & 50 & - & $\mathrm{NO}$ & Low & - & - & 8.27 & 19.37 & $\mid 1049.8$ & 12.57 & $\mid-11.398$ & 260.77 & 60.89 \\
\hline $320-75 \%$ Washed & 51 & - & $\mathrm{NO}$ & Low & - & - & 8.44 & 19.36 & 1059.7 & 11.64 & -11.365 & 262.77 & 60.11 \\
\hline $320-75 \%$ Washed & 52 & - & NO & Low & - & - & 8.60 & 19.34 & 1069.4 & 10.75 & -11.332 & 264.78 & 59.33 \\
\hline $320-75 \%$ Washed & 53 & - & $\mathrm{NO}$ & Low & - & - & 8.77 & 19.33 & 1079.1 & 9.90 & -11.299 & 266.79 & 58.55 \\
\hline $320-75 \%$ Washed & 54 & - & $\mathrm{NO}$ & Low & - & - & 8.93 & 19.32 & 1088.5 & 9.08 & -11.265 & 268.80 & 57.76 \\
\hline $320-75 \%$ Washed & 55 & - & NO & Low & - & - & 9.10 & 19.30 & 1097.7 & 8.31 & -11.232 & 270.81 & 56.98 \\
\hline $320-75 \%$ Washed & 56 & - & $\mathrm{NO}$ & Low & - & - & 9.26 & 19.29 & 1106.9 & 7.57 & -11.199 & 272.82 & 56.20 \\
\hline
\end{tabular}


Immobilization Technology Section

Westinghouse Savannah River Company

\begin{tabular}{|c|c|c|c|c|c|c|c|c|c|c|c|c|c|}
\hline & & \multicolumn{5}{|c|}{ "MAR Assessment } & \multicolumn{7}{|c|}{ Property Values } \\
\hline Frit/Washing & $\% \mathrm{WL}$ & Durability & $\mathbf{T}_{\mathrm{L}}$ & Visc & Frit & Homog & $\mathrm{Al}_{2} \mathrm{O}_{3}$ & alkali & $\mathbf{T}_{\mathrm{L}}$ & Visc & $\Delta \mathbf{G p}$ & Homog & Frit \\
\hline $320-75 \%$ Washed & 57 & - & $\mathrm{NO}$ & Low & - & - & 9.43 & \begin{tabular}{|l|}
19.28 \\
\end{tabular} & 1115.9 & 6.87 & -11.165 & 274.82 & 55.42 \\
\hline $320-75 \%$ Washed & 58 & - & $\mathrm{NO}$ & Low & - & - & 9.59 & \begin{tabular}{|l|}
19.27 \\
\end{tabular} & 1124.7 & 6.21 & \begin{tabular}{|l|}
-11.132 \\
\end{tabular} & 276.83 & 54.64 \\
\hline $320-75 \%$ Washed & 59 & - & $\mathrm{NO}$ & Low & - & - & 9.76 & 19.25 & 1133.4 & 5.59 & -11.099 & 278.84 & 53.85 \\
\hline $320-75 \%$ Washed & 60 & - & NO & Low & - & - & 9.92 & 19.24 & 1142.0 & 5.00 & -11.066 & 280.85 & 53.07 \\
\hline
\end{tabular}


Immobilization Technology Section

WSRC-TR-2002-00549

Savannah River Technology Center

Rev. 0

Westinghouse Savannah River Company

\section{Appendix D}

Chemical Composition Analytical Plan 
September 16, 2002

To: C. C. Herman, 773-43A

D. K. Peeler, 999-W

cc: $\quad$ D. R. Best, 786-1A (wo)

S. P. Harris, 773-42A (es)

I. A. Reamer, 773-A

S. L. Marra, 999-W (es)

P. A. Toole, 786-1A (wo)

D. J. Pittman, 786-1A (wo)

R. C. Tuckfield, 773-42A

R. J. Workman, 999-1W

From: $\quad$ T. B. Edwards, 773-42A (5-5148)

Statistical Consulting Section

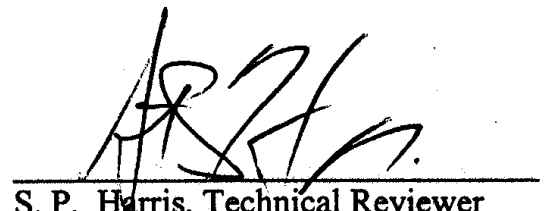

S. P. Hirris, Technical Reviewe

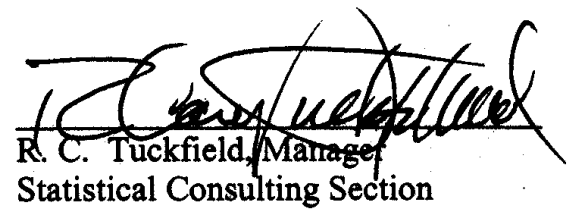

wo - without glass identifiers es - executive summary only

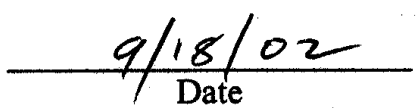

$9 / 18 / 02$

\section{An Analytical Plan for Measuring the ChemicAl COMPOSITIONS OF PHASE 1 GLASSES FROM THE SB3 VARIABILITY STUDY (U)}




\subsection{EXECUTIVE SUMMARY}

A study is being conducted by the Savannah River Technology Center (SRTC) for the Defense Waste Processing Facility (DWPF) that involves investigating the glass region anticipated for the processing of sludge batch 3 (SB3). Forty-two (42) glass compositions were selected for batching and testing to support the first phase of this effort.

The chemical compositions of the 42 glasses from Phase 1 of this study are to be determined by the Savannah River Technology Center - Mobile Laboratory (SRTC-ML). This memorandum provides an analytical plan to direct and support these measurements at the SRTC-ML. 


\subsection{INTRODUCTION}

A glass variability study for Sludge Batch 3 (SB3) is being conducted for the Defense Waste Processing Facility (DWPF) by the Savannah River Technology Center (SRTC) [1]. Forty-two (42) glass compositions were selected for batching and testing to support Phase 1 of this effort.

The chemical compositions of the 42 glasses from this study are to be determined by the Savannah River Technology Center - Mobile Laboratory (SRTC-ML). This memorandum provides an analytical plan in support of [2] to direct and support these measurements at the SRTC-ML.

\subsection{Analytical Plan}

The analytical procedures used by the SRTC-ML to determine cation concentrations for a glass sample include steps for sample preparation and for instrument calibration. Each glass is to be prepared in duplicate by each of two dissolution methods: lithium metaborate (LM) and sodium peroxide fusion $(\mathrm{PF})$.

The primary measurements of interest are to be acquired as follows: the samples prepared by lithium metaborate $(\mathrm{LM})$ are to be measured for barium $(\mathrm{Ba})$, calcium $(\mathrm{Ca})$, cerium $(\mathrm{Ce})$, chromium $(\mathrm{Cr})$, copper $(\mathrm{Cu})$, iron $(\mathrm{Fe})$, potassium $(\mathrm{K})$, lanthanum $(\mathrm{La})$, magnesium $(\mathrm{Mg})$, manganese $(\mathrm{Mn})$, sodium $(\mathrm{Na})$, nickel $(\mathrm{Ni})$, lead $(\mathrm{Pb})$, silicon $(\mathrm{Si})$, thorium $(\mathrm{Th})$, titanium $(\mathrm{Ti})$, uranium $(\mathrm{U})$, zinc $(\mathrm{Zn})$, and zirconium $(\mathrm{Zr})$ concentrations. Samples prepared by sodium peroxide (PF) are to be measured for aluminum (Al), boron (B) and lithium (Li). Samples dissolved by either of these two preparation methods are to be measured using Inductively Coupled Plasma - Atomic Emission Spectrometry (ICP-AES). It should be noted that some of these elements are minor components and may be below detection limits for most, if not all, of the study glasses.

Randomizing the preparation steps and blocking and randomizing the measurements for the ICPAES are of primary concern in the development of this analytical plan. The sources of uncertainty for the analytical procedure used by the SRTC-ML to determine the cation concentrations for the submitted glass samples primarily involve the dissolution step in the preparation of the sample and the calibrations of the ICP-AES.

Samples of two standard glasses will be included in the analytical plan to provide an opportunity for checking the performance of the instrumentation over the course of the analyses and for potential bias correction. Specifically, several samples of Waste Compliance Plan (WCP) Batch $1(\mathrm{BCH})$ [3] and a glass containing uranium (UST) are included in this analytical plan. The reference compositions of these glasses are provided in Table 1. These standards will be referred to using the short identifier provided in Table 1 in the remainder of this memo. 
Table 1: Oxide Compositions of WCP Batch 1 (BCH) and Uranium Standard (UST) Glasses (wt\%).

\begin{tabular}{|ccc|}
\hline Oxide/ & $\begin{array}{c}\text { BCH } \\
(\mathbf{w t} \%)\end{array}$ & $\begin{array}{c}\text { US T } \\
\text { Anion }\end{array}$ \\
$\mathrm{Al}_{2} \mathrm{O}_{3}$ & 4.877 & 4.1 \\
$\mathrm{~B}_{2} \mathrm{O}_{3}$ & 7.777 & 9.209 \\
$\mathrm{BaO}$ & 0.151 & 0.00 \\
$\mathrm{CaO}$ & 1.220 & 1.301 \\
$\mathrm{CdO}$ & 0.00 & 0.00 \\
$\mathrm{Cl}$ & 0.00 & 0.00 \\
$\mathrm{Cr}_{2} \mathrm{O}_{3}$ & 0.107 & 0.00 \\
$\mathrm{Cs} \mathrm{O}_{2} \mathrm{O}$ & 0.060 & 0.00 \\
$\mathrm{CuO}$ & 0.399 & 0.00 \\
$\mathrm{~F}$ & 0.00 & 0.00 \\
$\mathrm{Fe}_{2} \mathrm{O}_{3}$ & 12.839 & 13.196 \\
$\mathrm{~K}_{2} \mathrm{O}$ & 3.327 & 2.999 \\
$\mathrm{Li}_{2} \mathrm{O}$ & 4.429 & 3.057 \\
$\mathrm{MgO}^{\mathrm{MnO}}$ & 1.419 & 1.21 \\
$\mathrm{MnO}$ & 1.726 & 2.892 \\
$\mathrm{NoO}_{3}$ & 0.00 & 0.00 \\
$\mathrm{Na}_{2} \mathrm{O}$ & 9.003 & 11.795 \\
$\mathrm{Nd}_{2} \mathrm{O}_{3}$ & 0.147 & 0.00 \\
$\mathrm{NiO}$ & 0.751 & 1.12 \\
$\mathrm{P}_{2} \mathrm{O}_{5}$ & 0.00 & 0.00 \\
$\mathrm{PbO}_{\mathrm{RuO}}$ & 0.00 & 0.00 \\
$\mathrm{SiO}_{2}$ & 0.0214 & 0.00 \\
$\mathrm{SnO}_{2}$ & 50.22 & 45.353 \\
$\mathrm{SO}_{3}$ & 0.00 & 0.00 \\
$\mathrm{TiO}_{2}$ & 0.00 & 0.00 \\
$\mathrm{U}_{3} \mathrm{O}_{8}$ & 0.677 & 1.049 \\
$\mathrm{ZrO}_{2}$ & 0.00 & 2.406 \\
& 0.098 & 0.00 \\
\hline
\end{tabular}

Each glass sample submitted to the SRTC-ML will be prepared in duplicate by the LM and PF dissolution methods. Each sample prepared using LM or PF will be read twice by ICP-AES, with the instrument being calibrated before each of these two sets of readings. This will lead to four measurements for each cation of interest for each submitted glass.

Table 2 presents identifying codes, $\mathrm{k} 01$ through $\mathrm{k} 42$, for the 42 glasses batched for Phase 1 of this variability study. The table provides a naming convention that is to be used in analyzing the glasses and reporting the measurements of their compositions. ${ }^{23}$

23 Renaming these samples helps to ensure that they will be processed as blind samples within the SRTC-ML. Table 2 is not shown in its entirety in those copies going to the SRTC-ML. 
Table 2: Identifiers to Establish Blind Samples for the SRTC-ML.

\begin{tabular}{|c|c|c|c|c|c|}
\hline Glass ID & Sample ID & Glass ID & Sample ID & Glass ID & Sample ID \\
\hline SB3-01 & $\mathrm{k} 41$ & SB3-15 & k37 & SB3-29 & k11 \\
\hline SB3-02 & k32 & SB3-16 & $\mathrm{k} 27$ & SB3-30 & k31 \\
\hline SB3-03 & k13 & SB3-17 & k01 & SB3-31 & k08 \\
\hline SB3-04 & $\mathrm{k} 22$ & SB3-18 & k04 & SB3-32 & k36 \\
\hline SB3-05 & k35 & SB3-19 & k18 & SB3-33 & k16 \\
\hline SB3-06 & $\mathrm{k} 02$ & SB3-20 & $\mathrm{k} 42$ & SB3-34 & k09 \\
\hline SB3-07 & $\mathrm{k} 38$ & SB3-21 & $\mathrm{k} 25$ & SB3-35 & k07 \\
\hline SB3-08 & $\mathrm{k} 30$ & SB3-22 & $\mathrm{k} 12$ & SB3-36 & k05 \\
\hline SB3-09 & $\mathrm{k} 24$ & SB3-23 & k17 & SB3-37 & $\mathrm{k} 10$ \\
\hline SB3-10 & $\mathrm{k} 21$ & SB3-24 & $\mathrm{k} 23$ & SB3-38 & $\mathrm{k} 03$ \\
\hline SB3-11 & $\mathrm{k} 28$ & SB3-25 & k39 & SB3-39 & $\mathrm{k} 26$ \\
\hline SB3-12 & k14 & SB3-26 & $\mathrm{k} 40$ & SB3-40 & k19 \\
\hline SB3-13 & k33 & SB3-27 & k06 & SB3-41 & $\mathrm{k} 15$ \\
\hline SB3-14 & k34 & SB3-28 & $\mathrm{k} 29$ & SB3-42 & $\mathrm{k} 20$ \\
\hline
\end{tabular}

\subsection{PREPARATION OF THE SAMPLES}

Each of the 42 glasses included in this analytical plan is to be prepared in duplicate by the LM and PF dissolution method. Thus, the total number of prepared glass samples is determined by $42 \cdot 2 \cdot 2=168$, not including the samples of the BCH and UST glass standards that are to be prepared.

Tables 3a-3b provide blocking and (random) sequencing schema for conducting the preparation steps of the analytical procedures. Four blocks of preparation work are provided for each preparation method to facilitate the scheduling of activities by work shift. The identifier for each of the prepared samples indicates the sample identifier (ID), preparation method, and duplicate number. 
Immobilization Technology Section

WSRC-TR-2002-00549

Savannah River Technology Center

Rev. 0

Westinghouse Savannah River Company

Table 3a: LM (Lithium Metaborate) Preparation Blocks

\begin{tabular}{|cccc|}
\hline 1 & $\mathbf{2}$ & $\mathbf{3}$ & $\mathbf{4}$ \\
k20LM1 & k13LM1 & k42LM1 & k35LM1 \\
k08LM1 & k09LM1 & k03LM1 & k30LM1 \\
k24LM1 & k40LM1 & k34LM1 & k04LM1 \\
k29LM1 & k22LM1 & k25LM1 & k15LM1 \\
k24LM2 & k09LM2 & k03LM2 & k27LM1 \\
k01LM1 & k16LM1 & k34LM2 & k30LM2 \\
k08LM2 & k02LM1 & k18LM1 & k15LM2 \\
k17LM1 & k33LM1 & k25LM2 & k35LM2 \\
k17LM2 & k13LM2 & k18LM2 & k23LM1 \\
k01LM2 & k02LM2 & k42LM2 & k04LM2 \\
k29LM2 & k40LM2 & k26LM1 & k23LM2 \\
k32LM1 & k39LM1 & k28LM1 & k05LM1 \\
k20LM2 & k39LM2 & k12LM1 & k07LM1 \\
k32LM2 & k22LM2 & k26LM2 & k19LM1 \\
k41LM1 & k33LM2 & k36LM1 & k05LM2 \\
k21LM1 & k16LM2 & k31LM1 & k27LM2 \\
k38LM1 & k10LM1 & k11LM1 & k06LM1 \\
k37LM1 & k14LM1 & k11LM2 & k19LM2 \\
k38LM2 & k10LM2 & k12LM2 & k06LM2 \\
k41LM2 & k14LM2 & k28LM2 & k07LM2 \\
k37LM2 & & k31LM2 & \\
k21LM2 & & k36LM2 & \\
\hline
\end{tabular}


Table 3b: PF (Peroxide Fusion) Preparation Blocks

\begin{tabular}{|cccc|}
\hline 1 & $\mathbf{2}$ & $\mathbf{3}$ & $\mathbf{4}$ \\
k35PF1 & k27PF1 & k12PF1 & k07PF1 \\
k28PF1 & k04PF1 & k23PF1 & k15PF1 \\
k21PF1 & k14PF1 & k29PF1 & k05PF1 \\
k41PF1 & k27PF2 & k29PF2 & k26PF1 \\
k41PF2 & k14PF2 & k11PF1 & k09PF1 \\
k13PF1 & k18PF1 & k12PF2 & k19PF1 \\
k28PF2 & k04PF2 & k08PF1 & k03PF1 \\
k13PF2 & k34PF1 & k39PF1 & k10PF1 \\
k22PF1 & k37PF1 & k11PF2 & k16PF1 \\
k35PF2 & k01PF1 & k23PF2 & k07PF2 \\
k22PF2 & k33PF1 & k36PF1 & k16PF2 \\
k38PF1 & k37PF2 & k06PF1 & k19PF2 \\
k02PF1 & k01PF2 & k40PF1 & k09PF2 \\
k21PF2 & k42PF1 & k39PF2 & k05PF2 \\
k24PF1 & k42PF2 & k08PF2 & k26PF2 \\
k32PF1 & k25PF1 & k31PF1 & k15PF2 \\
k02PF2 & k34PF2 & k17PF1 & k20PF1 \\
k24PF2 & k18PF2 & k36PF2 & k03PF2 \\
k38PF2 & k33PF2 & k31PF2 & k10PF2 \\
k30PF1 & k25PF2 & k06PF2 & k20PF2 \\
k32PF2 & & k40PF2 & \\
k30PF2 & & k17PF2 & \\
\hline
\end{tabular}

\subsection{ICP-AES Calibration Blocks}

The glass samples prepared by LM and PF dissolution methods are to be analyzed using ICPAES instrumentation calibrated for the particular preparation method. After the initial set of cation concentration measurements, the ICP-AES instrumentation is to be recalibrated and a second set of concentration measurements for the cations determined.

Randomized plans for measuring cation concentrations in the LM-prepared and PF-prepared samples are provided in Tables 4 and 5, respectively. The cations to be measured are specified in the header of each of these tables. In these tables, the sample identifiers for the 42 study glasses have been modified by the addition of a suffix (a " 1 " or a " 2 ") to indicate whether the measurement was made during the first or second (respectively) ICP-AES calibration group. The identifiers for the $\mathrm{BCH}$ and UST samples have been modified to indicate that each of these prepared samples is to be read 3 times (mirrored in the corresponding suffix of 1, 2, or 3 ) per calibration block. 
Table 4: ICP-AES Blocks and Calibration Groups for Samples Prepared Using LM (Used to Measure Elemental Ba, Ca, Ce, Cr, Cu, Fe, K, La, Mg, Mn, Na, Ni, Pb, Si, Th, Ti, $\mathrm{U}, \mathrm{Zn}$, and $\mathrm{Zr}$ )

\begin{tabular}{|c|c|c|c|c|c|c|c|}
\hline \multicolumn{2}{|c|}{ ICP-AES Block 1} & \multicolumn{2}{|c|}{ ICP-AES Block 2} & \multicolumn{2}{|c|}{ ICP-AES Block 3} & \multicolumn{2}{|c|}{ ICP-AES Block 4} \\
\hline $\begin{array}{c}\text { Calibration } \\
1\end{array}$ & $\begin{array}{c}\text { Calibration } \\
2\end{array}$ & $\begin{array}{c}\text { Calibration } \\
1\end{array}$ & $\begin{array}{c}\text { Calibration } \\
2\end{array}$ & $\begin{array}{c}\text { Calibration } \\
1\end{array}$ & $\begin{array}{c}\text { Calibration } \\
2\end{array}$ & $\begin{array}{c}\text { Calibration } \\
1\end{array}$ & $\begin{array}{c}\text { Calibration } \\
2\end{array}$ \\
\hline BCHLM111 & BCHLM121 & BCHLM 211 & BCHLM 221 & BCHLM311 & BCHLM321 & BCHLM411 & BCHLM 421 \\
\hline USTLM111 & USTLM121 & USTLM211 & USTLM221 & USTLM311 & USTLM321 & USTLM411 & USTLM421 \\
\hline k09LM21 & k25LM22 & k33LM21 & k37LM22 & k29LM11 & k04LM12 & k10LM11 & k34LM12 \\
\hline k16LM21 & k09LM12 & k42LM11 & k22LM12 & k29LM21 & k36LM12 & k12LM11 & k24LM12 \\
\hline k14LM11 & k16LM22 & k40LM11 & k07LM22 & k11LM21 & k36LM22 & k32LM21 & k06LM12 \\
\hline k35LM21 & k18LM12 & k02LM11 & k22LM22 & k36LM11 & k04LM22 & k31LM21 & k31LM22 \\
\hline k35LM11 & $\mathrm{k} 1$ & k02LM21 & & k30LM11 & & k23LM21 & k13LM12 \\
\hline k18LM11 & k38LM12 & k22LM11 & k27LM22 & k30LM21 & k11LM22 & k34LM11 & k12LM22 \\
\hline k18LM21 & k38LM22 & k15LM21 & k27LM12 & k41LM11 & k29LM12 & k31LM11 & k10LM12 \\
\hline k28LM21 & k09LM22 & k37LM11 & k33LM12 & k01LM21 & k41LM22 & k34LM21 & k10LM22 \\
\hline k08LM21 & k25LM12 & k05LM21 & k42LM12 & k41LM21 & k03LM22 & k32LM11 & k24LM22 \\
\hline k08LM11 & $\mathrm{k} 35$ & $\mathrm{k} 07 \mathrm{LN}$ & & $\mathrm{k} 01 \mathrm{LM}$ & $\mathrm{k} 20$ & k10LM21 & k21LM12 \\
\hline k14LM21 & $\mathrm{k} 18 \mathrm{LI}$ & k19LM11 & $\mathrm{k} 15 \mathrm{~L}$ & BCHLM312 & BCHLM322 & BCHLM412 & BCHLM 422 \\
\hline BCHLM112 & BCHLM122 & BCHLM 212 & BCHLM 222 & USTLM312 & USTLM 322 & USTLM 412 & USTLM422 \\
\hline USTLM112 & USTLM122 & USTLM212 & USTLM222 & k39LM11 & k39LM22 & k24LM21 & k13LM22 \\
\hline k16LM11 & k26LM22 & k15LM11 & & k39LM21 & LM22 & k24LM11 & k34LM22 \\
\hline k25LM21 & k14LM12 & $\mathrm{k} 22 \mathrm{LM} 21$ & k33LM22 & k03LM21 & k03LM12 & k21LM21 & k32LM12 \\
\hline k25LM11 & k28LN & k27LM11 & 12 & k11LM11 & k11LM12 & k12LM21 & k06LM22 \\
\hline k09LM11 & k14LM22 & $\mathrm{k} 37 \mathrm{LM}$ & k19LI & k03LM11 & k01LM12 & k23LM11 & k32LM22 \\
\hline k38LM11 & k08LM22 & k42LM21 & k40LM12 & k20LM21 & k30LM12 & k13LM11 & k23LM22 \\
\hline k28LM11 & & k27LM21 & & k04LM11 & k01LM22 & k06LM21 & k12LM12 \\
\hline k38LM21 & k08LM12 & k19LM21 & k07LM12 & k20LM11 & k39LM12 & k06LM11 & k23LM12 \\
\hline k17LM11 & k16LM12 & k40LM21 & & k36LM21 & k30LM22 & k21LM11 & k21LM22 \\
\hline k26LM11 & k28LM12 & k05LM11 & k05LM12 & k04LM21 & k29LM22 & k13LM21 & k31LM12 \\
\hline k17LM21 & k26LM12 & k07LM21 & k15LM12 & BCHLM313 & BCHLM323 & BCHLM413 & BCHLM 423 \\
\hline k26LM21 & k17LM22 & k33LM11 & k19LM22 & USTLM313 & USTLM 323 & USTLM413 & USTLM423 \\
\hline BCHLM113 & BCHLM123 & BCHLM 213 & BCHLM 223 & & & & \\
\hline USTLM113 & USTLM 123 & USTLM213 & USTLM223 & & & & \\
\hline
\end{tabular}




\section{Table 5: ICP-AES Blocks and Calibration Groups for Samples Prepared Using PF (Used to Measure Elemental Al, B, and Li)}

\begin{tabular}{|c|c|c|c|c|c|c|c|}
\hline \multicolumn{2}{|c|}{ ICP-AES Block 1} & \multicolumn{2}{|c|}{ ICP-AES Block 2} & \multicolumn{2}{|c|}{ ICP-AES Block 3} & \multicolumn{2}{|c|}{ ICP-AES Block 4} \\
\hline $\begin{array}{c}\text { Calibration } \\
1\end{array}$ & $\begin{array}{c}\text { Calibration } \\
2\end{array}$ & $\begin{array}{c}\text { Calibration } \\
1\end{array}$ & $\begin{array}{c}\text { Calibration } \\
2\end{array}$ & $\begin{array}{c}\text { Calibration } \\
1\end{array}$ & $\begin{array}{c}\text { Calibration } \\
2\end{array}$ & $\begin{array}{c}\text { Calibration } \\
1\end{array}$ & $\begin{array}{c}\text { Calibration } \\
2\end{array}$ \\
\hline BCHPF111 & BCHPF121 & BCHPF211 & BCHPF221 & BCHPF311 & BCHPF321 & BCHPF411 & BCHPF421 \\
\hline USTPF111 & USTPF121 & USTPF211 & USTPF221 & USTPF311 & USTPF321 & USTPF411 & USTPF421 \\
\hline k24PF21 & k02PF12 & k18PF21 & k14PF22 & k06PF11 & k09PF22 & k40PF11 & k20PF22 \\
\hline k04PF21 & $\mathrm{k} 21 \mathrm{PF} 22$ & k36PF21 & k39PF22 & k09PF21 & k17PF22 & k10PF21 & k31PF12 \\
\hline k26PF21 & k05PF22 & k32PF21 & $\mathrm{k} 22 \mathrm{PF} 22$ & k17PF21 & $\mathrm{k} 25 \mathrm{PF} 22$ & $\mathrm{k} 28 \mathrm{PF} 11$ & $\mathrm{k} 41 \mathrm{PF} 22$ \\
\hline k04PF11 & $\mathrm{k} 04 \mathrm{PF} 22$ & k38PF11 & $\mathrm{k} 42 \mathrm{PF} 12$ & k37PF11 & k08PF22 & k41PF11 & k33PF22 \\
\hline k05PF11 & k26PF22 & k27PF11 & k14PF12 & k08PF11 & k06PF22 & k28PF21 & k03PF22 \\
\hline $\mathrm{k} 21 \mathrm{PF} 11$ & $\mathrm{k} 24 \mathrm{PF} 22$ & k22PF11 & k39PF12 & k30PF21 & k11PF12 & k40PF21 & $\mathrm{k} 41 \mathrm{PF} 12$ \\
\hline $\mathrm{k} 26 \mathrm{PF} 11$ & k29PF22 & k39 & F 22 & $\mathrm{k} 25 \mathrm{PF}$ & k35PF22 & $\mathrm{k} 31 \mathrm{P}$ & PF22 \\
\hline $\mathrm{k} 16 \mathrm{PF} 21$ & $\mathrm{k} 26 \mathrm{PF} 12$ & $\mathrm{k} 22 \mathrm{PF} 21$ & $\mathrm{k} 42 \mathrm{PF} 22$ & $\mathrm{k} 11 \mathrm{PF} 11$ & $\mathrm{k} 30 \mathrm{PF} 12$ & k01PF11 & $\mathrm{k} 10 \mathrm{PF} 12$ \\
\hline k07PF11 & $\mathrm{k} 24 \mathrm{PF} 12$ & k14PF11 & k32PF12 & k25PF11 & k23PF12 & k41PF21 & k28PF22 \\
\hline k16PF11 & k13PF12 & k34PF11 & $\mathrm{k} 32 \mathrm{PF} 22$ & k17PF11 & k30PF22 & k20PF21 & k40PF12 \\
\hline $\mathrm{k} 12 \mathrm{PF} 11$ & k13PF22 & k39PF11 & k38PF12 & BCHPF312 & BCHPF322 & BCHPF412 & BCHPF422 \\
\hline BCHPF112 & BCHPF122 & $\mathrm{BCHPF}^{\prime}$ & BCHPF222 & USTPF & USTPF322 & USTPF & USTPF422 \\
\hline USTPF112 & USTPF122 & USTPF 212 & USTPF222 & $\mathrm{k} 23 \mathrm{PF} 21$ & $\mathrm{k} 35 \mathrm{PF} 12$ & k03PF21 & k31PF22 \\
\hline k02PF11 & k07PF22 & $\mathrm{k} 36 \mathrm{PF} 11$ & $\mathrm{k} 34 \mathrm{PF} 22$ & k30PF11 & k37PF12 & k03PF11 & k03PF12 \\
\hline k29PF11 & k02PF22 & k27PF21 & k22PF12 & k23PF11 & k17PF12 & k01PF21 & k20PF12 \\
\hline k13PF21 & $\mathrm{k} 21 \mathrm{PF} 12$ & k14PF21 & k19PF22 & k35PF11 & k37PF22 & k33PF21 & k01PF12 \\
\hline k21PF21 & k07PF12 & k38PF21 & PF12 & k37PF21 & k11PF22 & $\mathrm{k} 15 \mathrm{PI}$ & k15PF22 \\
\hline $\mathrm{k} 05 \mathrm{PF} 21$ & k16PF12 & k32PF11 & k36PF12 & k06PF21 & k09PF12 & k10PF11 & $\mathrm{k} 40 \mathrm{PF} 22$ \\
\hline k13PF11 & k04PF12 & k19PF11 & $\mathrm{k} 27 \mathrm{PF} 12$ & $\mathrm{k} 35 \mathrm{PF} 21$ & k06PF12 & $\mathrm{k} 15 \mathrm{PF} 21$ & $\mathrm{k} 15 \mathrm{PF} 12$ \\
\hline k07PF21 & $\mathrm{k} 16 \mathrm{PF} 22$ & k19PF21 & k38PF22 & k11PF21 & k23PF22 & k20PF11 & k01PF22 \\
\hline k02PF21 & k05PF12 & k42PF11 & k27PF22 & k08PF21 & k08PF12 & k31PF21 & k33PF12 \\
\hline $\mathrm{k} 24 \mathrm{PF} 11$ & $\mathrm{k} 12 \mathrm{PF} 12$ & k18PF11 & k36PF22 & k09PF11 & $\mathrm{k} 25 \mathrm{PF} 12$ & k33PF11 & k28PF12 \\
\hline $\mathrm{k} 12 \mathrm{PF} 21$ & $\mathrm{k} 12 \mathrm{PF} 22$ & $\mathrm{k} 42 \mathrm{PF} 21$ & k34PF12 & BCHPF313 & BCHPF323 & BCHPF413 & BCHPF423 \\
\hline k29PF21 & k29PF12 & $\mathrm{k} 34 \mathrm{PF} 21$ & $\mathrm{k} 18 \mathrm{PF} 12$ & USTPF313 & USTPF323 & USTPF413 & USTPF423 \\
\hline BCHPF113 & BCHPF123 & BCHPF213 & BCHPF223 & & & & \\
\hline USTPF113 & USTPF123 & USTPF213 & USTPF223 & & & & \\
\hline
\end{tabular}

\subsection{Concluding Comments}

In summary, this analytical plan identifies several ICP-AES calibration blocks in Tables $4-5$ as well as eight preparation blocks in Tables 3a-3b for use by the SRTC-ML. The sequencing of the activities associated with each of the steps in the analytical procedures has been randomized. The size of each of the blocks was selected so that it could be completed in a single work shift.

If a problem is discovered while measuring samples in a calibration block, the instrument should be re-calibrated and the block of samples re-measured in its entirety. If for some reason the measurements are not conducted in the sequences presented in this report, a record should be made of the actual order used along with any explanative comments.

The analytical plan indicated in the preceding tables should be modified by the personnel of SRTC-ML to include any calibration check standards and/or other standards that are part of their routine operating procedures. It is also recommended that the solutions resulting from each of the prepared samples be archived for some period, considering the "shelf-life" of the solutions, in case questions arise during data analysis. This would allow for the solutions to be rerun without additional preparations, thus minimizing cost. 


\subsection{REFERENCES}

[1] Herman, C. C., D. K. Peeler, and T. B. Edwards, “Task Technical/Quality Assurance Plan: Sludge Batch 3 Variability Studies with Simulants,” WSRC-RP-2002-00386, Revision 0, July 11, 2002.

[2] Peeler, D. K., C. C. Herman, and T. B. Edwards, "Analytical Study Plan: Sludge Batch 3 Variability Studies with Simulants," WSRC-RP-2002-00394, Revision 0, July 11, 2002.

[3] Jantzen, C. M., J. B. Pickett, K. G. Brown, T. B. Edwards, and D. C. Beam, "Process/Product Models for the Defense Waste Processing Facility (DWPF): Part I. Predicting Glass Durability from Composition Using a Thermodynamic Hydration Energy Reaction Model $\left(\right.$ THERMO $^{\mathrm{TM}}$ ) (U)," WSRC-TR-93-673, Rev. 1, Volume 2, Table B.1, pp. B.9, 1995. 
Immobilization Technology Section

WSRC-TR-2002-00549

Savannah River Technology Center

Rev. 0

Westinghouse Savannah River Company

\section{Appendix E}

PCT Analytical Plan for Set \#1

SB3-1 through SB3-14 
September 23, 2002

To: C. C. Herman, 773-43A

D. K. Peeler, 999-W

cc: $\quad$ R.A. Baker, 773-42A

I. A. Reamer, 773-A

D. R. Best, 786-1A (wo)

S. L. Marra, 999-W (es)

P. A. Toole, 786-1A (wo)

D. J. Pittman, 786-1A (wo)

R. C. Tuckfield, 773-42A

R. J. Workman, 999-1W

From: $\quad$ T. B. Edwards, 773-42A (5-5148)

Statistical Consulting Section

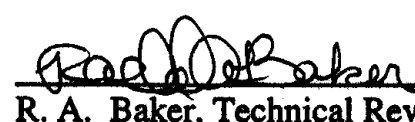

R. A. Baker, Technical Reviewer

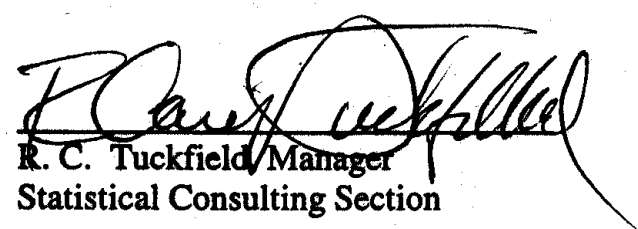

wo - without glass identifiers

es - executive summary only

\section{An Analytical Plan for Measuring the FIRST SET OF PCT SOLUTIONS FROM THE PHASE 1 GLASSES FOR THE SB3 VARIABILITY STUDY (U)}




\subsection{EXECUTIVE SuMmaRY}

A study is being conducted by the Savannah River Technology Center (SRTC) for the Defense Waste Processing Facility (DWPF) that involves investigating the glass region anticipated for the processing of Sludge Batch 3 (SB3). Forty-two (42) glass compositions were selected for batching and testing to support the first phase of this effort.

Fourteen (14) of the 42 study glasses are the focus of this analytical plan; they form the first set of glasses that are to be subjected to the Product Consistency Test, or PCT. The glasses were cooled both by quenching and centerline canister cooling, and the durabilities of the resulting twentyeight glasses are to be measured in triplicate using the PCT, the requirements of which are described in ASTM C1285-97 (Method A).

The Savannah River Technology Center-Mobile Laboratory (SRTC-ML) is to be used to measure elemental concentrations of the resulting leachate solutions from the PCTs. This memorandum provides an analytical plan for the SRTC-ML to follow in measuring the compositions of the leachate solutions resulting from the PCT procedures for the glasses. 


\subsection{INTRODUCTION}

A glass variability study for Sludge Batch 3 (SB3) is being conducted for the Defense Waste Processing Facility (DWPF) by the Savannah River Technology Center (SRTC) [1]. Forty-two (42) glass compositions were selected for batching and testing to support Phase 1 of this effort.

Fourteen (14) of the 42 study glasses are the focus of this analytical plan; they form the first set of glasses that are to be subjected to the Product Consistency Test, or PCT. The glasses were cooled both by quenching and by centerline canister cooling, and the durabilities of the resulting twentyeight glasses are to be measured in triplicate using the PCT, the requirements of which are described in ASTM C1285-97 (Method A) [2].

The identifiers for the first set of study glasses are presented in Table 1. The centerline canister cooled glasses are denoted by a "ccc" suffix.

Table 1: Identifiers for the First Set of Study Glasses

\begin{tabular}{|c|c|c|c|}
\hline Quenched & $\begin{array}{c}\text { Centerline } \\
\text { Canister } \\
\text { Cooled }\end{array}$ & Quenched & $\begin{array}{c}\text { Centerline } \\
\text { Canister } \\
\text { Cooled }\end{array}$ \\
\hline SB3-01 & SB3-01ccc & SB3-08 & SB3-08ccc \\
\hline SB3-02 & SB3-02ccc & SB3-09 & SB3-09ccc \\
\hline SB3-03 & SB3-03ccc & SB3-10 & SB3-10ccc \\
\hline SB3-04 & SB3-04ccc & SB3-11 & SB3-11ccc \\
\hline SB3-05 & SB3-05ccc & SB3-12 & SB3-12ccc \\
\hline SB3-06 & SB3-06ccc & SB3-13 & SB3-13ccc \\
\hline SB3-07 & SB3-07ccc & SB3-14 & SB3-14ccc \\
\hline
\end{tabular}

This memorandum is in support of the Analytical Study Plan [3] and provides an analytical plan for the Savannah River Technology Center's Mobile Laboratory (SRTC-ML) to follow in measuring the compositions of the PCT leachate solutions for these glasses.

\subsection{DISCUSSION}

The quenched and centerline canister cooled versions of the study glasses are to be subjected to the PCT. The 2 different thermal histories for each of the 14 glasses lead to 28 glasses that are to be measured (in triplicate) using the PCT. In addition to those for the study glasses, triplicate PCTs are to be conducted on a sample of the Approved Reference Material (ARM) glass and a sample of the Environmental Assessment (EA) glass. Two reagent blank samples are also to be included in these tests. This results in 92 sample solutions being required to complete these PCTs.

The leachates from these tests will be diluted by adding $4 \mathrm{~mL}$ of $0.4 \mathrm{M} \mathrm{HNO}_{3}$ to $6 \mathrm{~mL}$ of the leachate (a 6:10 volume to volume, v:v, dilution) before being submitted to the SRTC-ML. The EA leachates will be further diluted $(1: 10 \mathrm{v}: \mathrm{v})$ with deionized water prior to submission to the SRTC-ML in order to prevent problems with the nebulizer.

Table 2 presents identifying codes, $\mathrm{j} 01$ through $\mathrm{j} 92$, for the individual solutions required for the PCTs of the study glasses and of the standards (EA, ARM, and blanks). This provides a naming 
convention that is to be used by the SRTC-ML in analyzing the solutions and reporting the relevant concentration measurements. ${ }^{24}$

Table 2: Identifiers for the PCT Solutions

\begin{tabular}{|c|c|c|c|c|c|c|c|}
\hline $\begin{array}{l}\text { Original } \\
\text { Sample }\end{array}$ & $\begin{array}{l}\text { Solution } \\
\text { Identifier }\end{array}$ & $\begin{array}{l}\text { Original } \\
\text { Sample }\end{array}$ & $\begin{array}{l}\text { Solution } \\
\text { Identifier }\end{array}$ & $\begin{array}{l}\text { Original } \\
\text { Sample }\end{array}$ & $\begin{array}{l}\text { Solution } \\
\text { Identifier }\end{array}$ & $\begin{array}{l}\text { Original } \\
\text { Sample }\end{array}$ & $\begin{array}{l}\text { Solution } \\
\text { Identifier }\end{array}$ \\
\hline SB3-01 & j61 & SB3-05 & j41 & SB3-09 & j90 & SB3-13 & j32 \\
\hline SB3-01 & j18 & SB3-05 & j19 & SB3-09 & j92 & SB3-13 & j39 \\
\hline SB3-01 & j30 & SB3-05 & j33 & SB3-09 & j02 & SB3-13 & $\mathrm{j} 47$ \\
\hline SB3-01ccc & j28 & SB3-05ccc & j31 & SB3-09ccc & j71 & SB3-13ccc & j57 \\
\hline SB3-01ccc & j73 & SB3-05ccc & j10 & SB3-09ccc & j56 & SB3-13ccc & $j 70$ \\
\hline SB3-01ccc & j89 & SB3-05ccc & j86 & SB3-09ccc & $\mathrm{j} 45$ & SB3-13ccc & j22 \\
\hline SB3-02 & j05 & SB3-06 & j50 & SB3-10 & j37 & SB3-14 & $\mathrm{j} 53$ \\
\hline SB3-02 & j03 & SB3-06 & j24 & SB3-10 & $\mathrm{j} 65$ & SB3-14 & j78 \\
\hline SB3-02 & j64 & SB3-06 & j46 & SB3-10 & j29 & SB3-14 & j58 \\
\hline SB3-02ccc & j11 & SB3-06ccc & j01 & SB3-10ccc & j16 & SB3-14ccc & j51 \\
\hline SB3-02ccc & j68 & SB3-06ccc & j77 & SB3-10ccc & $\mathrm{j} 54$ & SB3-14ccc & j09 \\
\hline SB3-02ccc & j08 & SB3-06ccc & j76 & SB3-10ccc & j79 & SB3-14ccc & j63 \\
\hline SB3-03 & j88 & SB3-07 & $\mathrm{j} 42$ & SB3-11 & $\mathrm{j} 62$ & ARM & j60 \\
\hline SB3-03 & j34 & SB3-07 & j06 & SB3-11 & $\mathrm{j} 40$ & ARM & j67 \\
\hline SB3-03 & j04 & SB3-07 & j21 & SB3-11 & j38 & ARM & j66 \\
\hline SB3-03ccc & j23 & SB3-07ccc & j36 & SB3-11ccc & j17 & EA & $\mathrm{j} 52$ \\
\hline SB3-03ccc & j43 & SB3-07ccc & j83 & SB3-11ccc & j12 & EA & j55 \\
\hline SB3-03ccc & j49 & SB3-07ccc & j35 & SB3-11ccc & $\mathrm{j} 59$ & EA & j44 \\
\hline SB3-04 & j75 & SB3-08 & j25 & SB3-12 & j74 & blank & j80 \\
\hline SB3-04 & j72 & SB3-08 & j85 & SB3-12 & j81 & blank & j82 \\
\hline SB3-04 & j69 & SB3-08 & j15 & SB3-12 & j07 & & \\
\hline SB3-04ccc & j13 & SB3-08ccc & j48 & SB3-12ccc & j26 & & \\
\hline SB3-04ccc & j87 & SB3-08ccc & j14 & SB3-12ccc & j91 & & \\
\hline SB3-04ccc & $\mathrm{j} 27$ & SB3-08ccc & j84 & SB3-12ccc & j20 & & \\
\hline
\end{tabular}

\subsection{Analytical Plan}

The analytical plan for the SRTC-ML is provided in this section. Each of the solution samples submitted to the SRTC-ML is to be analyzed only once for each of the following: aluminum, $(\mathrm{Al})$, boron $(\mathrm{B})$, iron $(\mathrm{Fe})$, lithium $(\mathrm{Li})$, sodium $(\mathrm{Na})$, silicon $(\mathrm{Si})$, and uranium $(\mathrm{U})$. The measurements are to be made in parts per million (ppm). The analytical procedure used by the SRTC-ML to determine the concentrations utilizes an Inductively Coupled Plasma - Atomic Emission Spectrometer (ICP-AES). The PCT solutions (as identified in Table 2) are grouped in six ICP-AES blocks for processing by the SRTC-ML in Table 3. Each block requires a different calibration of the ICP-AES. 
Table 3: ICP-AES Calibration Blocks for Leachate Measurements.

\begin{tabular}{|cccccc|}
\hline Block 1 & Block 2 & Block 3 & Block 4 & Block 5 & Block 6 \\
std-b1-1 & std-b2-1 & std-b3-1 & std-b4-1 & std-b5-1 & std-b6-1 \\
$\mathrm{j} 48$ & $\mathrm{j} 65$ & $\mathrm{j} 29$ & $\mathrm{j} 01$ & $\mathrm{j} 82$ & $\mathrm{j} 20$ \\
$\mathrm{j} 41$ & $\mathrm{j} 78$ & $\mathrm{j} 58$ & $\mathrm{j} 60$ & $\mathrm{j} 55$ & $\mathrm{j} 66$ \\
$\mathrm{j} 51$ & $\mathrm{j} 85$ & $\mathrm{j} 86$ & $\mathrm{j} 05$ & $\mathrm{j} 68$ & $\mathrm{j} 44$ \\
$\mathrm{j} 23$ & $\mathrm{j} 34$ & $\mathrm{j} 49$ & $\mathrm{j} 50$ & $\mathrm{j} 73$ & $\mathrm{j} 21$ \\
$\mathrm{j} 32$ & $\mathrm{j} 87$ & $\mathrm{j} 22$ & $\mathrm{j} 52$ & $\mathrm{j} 92$ & $\mathrm{j} 35$ \\
$\mathrm{j} 37$ & $\mathrm{j} 14$ & $\mathrm{j} 84$ & $\mathrm{j} 36$ & $\mathrm{j} 91$ & $\mathrm{j} 64$ \\
$\mathrm{j} 17$ & $\mathrm{j} 70$ & $\mathrm{j} 15$ & $\mathrm{j} 42$ & $\mathrm{j} 18$ & $\mathrm{j} 89$ \\
$\mathrm{j} 53$ & $\mathrm{j} 10$ & $\mathrm{j} 59$ & $\mathrm{j} 28$ & $\mathrm{j} 06$ & $\mathrm{j} 08$ \\
$\mathrm{std}-\mathrm{b} 1-2$ & $\mathrm{std}-\mathrm{b} 2-2$ & $\mathrm{std}-\mathrm{b} 3-2$ & $\mathrm{std}-\mathrm{b} 4-2$ & $\mathrm{std}-\mathrm{b} 5-2$ & $\mathrm{std}-\mathrm{b} 6-2$ \\
$\mathrm{j} 31$ & $\mathrm{j} 39$ & $\mathrm{j} 04$ & $\mathrm{j} 74$ & $\mathrm{j} 03$ & $\mathrm{j} 07$ \\
$\mathrm{j} 13$ & $\mathrm{j} 43$ & $\mathrm{j} 63$ & $\mathrm{j} 90$ & $\mathrm{j} 77$ & $\mathrm{j} 76$ \\
$\mathrm{j} 88$ & $\mathrm{j} 54$ & $\mathrm{j} 33$ & $\mathrm{j} 80$ & $\mathrm{j} 83$ & $\mathrm{j} 46$ \\
$\mathrm{j} 25$ & $\mathrm{j} 40$ & $\mathrm{j} 79$ & $\mathrm{j} 26$ & $\mathrm{j} 81$ & $\mathrm{j} 30$ \\
$\mathrm{j} 75$ & $\mathrm{j} 09$ & $\mathrm{j} 69$ & $\mathrm{j} 61$ & $\mathrm{j} 24$ & $\mathrm{j} 02$ \\
$\mathrm{j} 57$ & $\mathrm{j} 12$ & $\mathrm{j} 47$ & $\mathrm{j} 71$ & $\mathrm{j} 56$ & $\mathrm{j} 45$ \\
$\mathrm{j} 16$ & $\mathrm{j} 72$ & $\mathrm{j} 27$ & $\mathrm{j} 11$ & $\mathrm{j} 67$ & $\mathrm{std}-\mathrm{b} 6-3$ \\
$\mathrm{j} 62$ & $\mathrm{j} 19$ & $\mathrm{j} 38$ & $\mathrm{std}-\mathrm{b} 4-3$ & $\mathrm{std}-\mathrm{b} 5-3$ & \\
std-b1-3 & std-b2-3 & std-b3-3 & & & \\
\hline
\end{tabular}

A multi-element solution standard (denoted by "std-bi-j" where $i=1$ to 6 represents the block number and $\mathrm{j}=1,2$, and 3 represents the position in the block) was added at the beginning, middle, and end of each of the nine blocks. This standard may be useful in checking and correcting for bias in the concentration measurements arising from the ICP calibrations.

\subsection{SUMMARY}

In summary, this analytical plan provides identifiers for the PCT solutions in Table 2 and six ICPAES calibration blocks in Table 3 for the SRTC-ML to use in conducting the aluminum, (Al), boron $(B)$, iron $(\mathrm{Fe})$, lithium $(\mathrm{Li})$, sodium $(\mathrm{Na})$, silicon $(\mathrm{Si})$, and uranium $(\mathrm{U})$ concentration measurements for this PCT study. The sequencing of the activities associated with each of the steps in the analytical procedure has been randomized. The size of the blocks was selected so that the block could be completed in a single work shift. If for some reason the measurements are not conducted in the sequence presented in this memorandum, the actual order should be recorded along with any explanative comments.

The analytical plan indicated in the preceding tables should be modified by the personnel of the SRTC-ML to include any calibration check standards and/or other standards that are part of their standard operating procedures. 


\subsection{REFERENCE}

[1] Herman, C. C., D. K. Peeler, and T. B. Edwards, "Task Technical/Quality Assurance Plan: Sludge Batch 3 Variability Studies with Simulants,” WSRC-RP-2002-00386, Revision 0, July 11, 2002.

[2] ASTM C1285-97, "Standard Test Methods for Determining Chemical Durability of Nuclear Waste Glasses: The Product Consistency Test (PCT)," 1997.

[3] Peeler, D. K., C. C. Herman, and T. B. Edwards, "Analytical Study Plan: Sludge Batch 3 Variability Studies with Simulants,” WSRC-RP-2002-00394, Revision 0, July 11, 2002. 
Immobilization Technology Section

WSRC-TR-2002-00549

Savannah River Technology Center

Rev. 0

Westinghouse Savannah River Company

\section{Appendix F}

PCT Analytical Plan for Set \#2 SB3-15 through SB3-28 
September 30, 2002

To: $\quad$ C. C. Herman, 773-43A

D. K. Peeler, 999-W

cc: $\quad$ R. A. Baker, 773-42A

I. A. Reamer, 773-A

D. R. Best, 786-1A (wo)

P. A. Toole, 786-1A (wo)

S. L. Marra, 999-W (es)

R. C. Tuckfield, 773-42A

D. J. Pittman, 786-1A (wo)

R. J. Workman, 999-1W

From: T. B. Edwards, 773-42A (5-5148)

Statistical Consulting Section

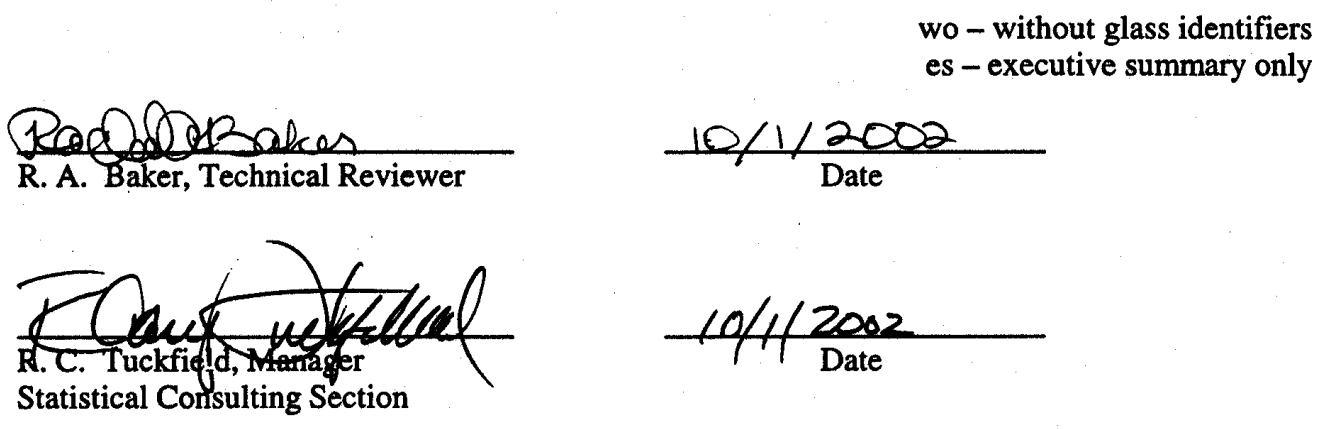

\section{An AnAlytical Plan for MEASURing the SECOND SET OF PCT SOLUTIONS FROM THE PhASE 1 GLASSES FOR THE SB3 VARIABILITY STUDY (U)}




\subsection{EXECUTIVE SUMMARY}

A study is being conducted by the Savannah River Technology Center (SRTC) for the Defense Waste Processing Facility (DWPF) that involves investigating the glass region anticipated for the processing of Sludge Batch 3 (SB3). Forty-two (42) glass compositions were selected for batching and testing to support the first phase of this effort.

Fourteen (14) of the 42 study glasses are the focus of this analytical plan; they form the second set of glasses that are to be subjected to the Product Consistency Test, or PCT. The glasses were cooled both by quenching and centerline canister cooling, and the durabilities of the resulting twenty-eight glasses are to be measured in triplicate using the PCT, the requirements of which are described in ASTM C1285-97 (Method A).

The Savannah River Technology Center-Mobile Laboratory (SRTC-ML) is to be used to measure elemental concentrations of the resulting leachate solutions from the PCTs. This memorandum provides an analytical plan for the SRTC-ML to follow in measuring the compositions of the leachate solutions resulting from the PCT procedures for the glasses. 


\subsection{INTRODUCTION}

A glass variability study for Sludge Batch 3 (SB3) is being conducted for the Defense Waste Processing Facility (DWPF) by the Savannah River Technology Center (SRTC) [1]. Forty-two (42) glass compositions were selected for batching and testing to support Phase 1 of this effort.

Fourteen (14) of the 42 study glasses are the focus of this analytical plan; they form the second set of glasses that are to be subjected to the Product Consistency Test, or PCT. The glasses were cooled both by quenching and by centerline canister cooling, and the durabilities of the resulting twenty-eight glasses are to be measured in triplicate using the PCT, the requirements of which are described in ASTM C1285-97 (Method A) [2].

The identifiers for the second set of study glasses are presented in Table 1. The centerline canister cooled glasses are denoted by a "ccc" suffix.

Table 1: Identifiers for the Second Set of Study Glasses

\begin{tabular}{|c|c|c|c|}
\hline Quenched & $\begin{array}{c}\text { Centerline } \\
\text { Canister } \\
\text { Cooled }\end{array}$ & Quenched & $\begin{array}{c}\text { Centerline } \\
\text { Canister } \\
\text { Cooled }\end{array}$ \\
\hline SB3-15 & SB3-15ccc & SB3-22 & SB3-22ccc \\
\hline SB3-16 & SB3-16ccc & SB3-23 & SB3-23ccc \\
\hline SB3-17 & SB3-17ccc & SB3-24 & SB3-24ccc \\
\hline SB3-18 & SB3-18ccc & SB3-25 & SB3-25ccc \\
\hline SB3-19 & SB3-19ccc & SB3-26 & SB3-26ccc \\
\hline SB3-20 & SB3-20ccc & SB3-27 & SB3-27ccc \\
\hline SB3-21 & SB3-21ccc & SB3-28 & SB3-28ccc \\
\hline
\end{tabular}

This memorandum is in support of the Analytical Study Plan [3] and provides an analytical plan for the Savannah River Technology Center's Mobile Laboratory (SRTC-ML) to follow in measuring the compositions of the PCT leachate solutions for these glasses.

\subsection{DisCUSSION}

The quenched and centerline canister cooled versions of the study glasses are to be subjected to the PCT. The 2 different thermal histories for each of the 14 glasses lead to 28 glasses that are to be measured (in triplicate) using the PCT. In addition to those for the study glasses, triplicate PCTs are to be conducted on a sample of the Approved Reference Material (ARM) glass and a sample of the Environmental Assessment (EA) glass. Two reagent blank samples are also to be included in these tests. This results in 92 sample solutions being required to complete these PCTs.

The leachates from these tests will be diluted by adding $4 \mathrm{~mL}$ of $0.4 \mathrm{M} \mathrm{HNO}_{3}$ to $6 \mathrm{~mL}$ of the leachate (a 6:10 volume to volume, v:v, dilution) before being submitted to the SRTC-ML. The EA leachates will be further diluted $(1: 10 \mathrm{v}: \mathrm{v})$ with deionized water prior to submission to the SRTC-ML in order to prevent problems with the nebulizer.

Table 2 presents identifying codes, m01 through m92, for the individual solutions required for the PCTs of the study glasses and of the standards (EA, ARM, and blanks). This provides a naming 
convention that is to be used by the SRTC-ML in analyzing the solutions and reporting the relevant concentration measurements. ${ }^{25}$

Table 2: Identifiers for the PCT Solutions

\begin{tabular}{|c|c|c|c|c|c|c|c|}
\hline $\begin{array}{l}\text { Original } \\
\text { Sample }\end{array}$ & $\begin{array}{l}\text { Solution } \\
\text { Identifier }\end{array}$ & $\begin{array}{l}\text { Original } \\
\text { Sample }\end{array}$ & $\begin{array}{l}\text { Solution } \\
\text { Identifier }\end{array}$ & $\begin{array}{l}\text { Original } \\
\text { Sample }\end{array}$ & $\begin{array}{l}\text { Solution } \\
\text { Identifier }\end{array}$ & $\begin{array}{l}\text { Original } \\
\text { Sample }\end{array}$ & $\begin{array}{l}\text { Solution } \\
\text { Identifier }\end{array}$ \\
\hline SB3-15 & m52 & SB3-19 & $\mathrm{m} 79$ & SB3-23 & $\mathrm{m} 03$ & SB3-27 & $\mathrm{m} 21$ \\
\hline SB3-15 & $\mathrm{m} 13$ & SB3-19 & $\mathrm{m} 12$ & SB3-23 & $\mathrm{m} 31$ & SB3-27 & $\mathrm{m} 46$ \\
\hline SB3-15 & m51 & SB3-19 & $\mathrm{m} 81$ & SB3-23 & m92 & SB3-27 & $\mathrm{m} 70$ \\
\hline SB3-15ccc & m59 & SB3-19ccc & $\mathrm{m} 48$ & SB3-23ccc & $\mathrm{m} 86$ & SB3-27ccc & $\mathrm{m} 14$ \\
\hline SB3-15ccc & m87 & SB3-19ccc & $\mathrm{m} 73$ & SB3-23ccc & m55 & SB3-27ccc & m53 \\
\hline SB3-15ccc & $\mathrm{m} 69$ & SB3-19ccc & $\mathrm{m} 84$ & SB3-23ccc & $\mathrm{m} 50$ & SB3-27ccc & $\mathrm{m} 29$ \\
\hline SB3-16 & m77 & SB3-20 & $\mathrm{m} 18$ & SB3-24 & $\mathrm{m} 44$ & SB3-28 & $\mathrm{m} 60$ \\
\hline SB3-16 & m58 & SB3-20 & $\mathrm{m} 25$ & SB3-24 & $\mathrm{m} 01$ & SB3-28 & m56 \\
\hline SB3-16 & m64 & SB3-20 & $\mathrm{m} 80$ & SB3-24 & $\mathrm{m} 57$ & SB3-28 & $\mathrm{m} 38$ \\
\hline SB3-16ccc & $\mathrm{m} 28$ & SB3-20ccc & $\mathrm{m} 11$ & SB3-24ccc & $\mathrm{m} 08$ & SB3-28ccc & m33 \\
\hline SB3-16ccc & $\mathrm{m} 06$ & SB3-20ccc & m34 & SB3-24ccc & $\mathrm{m} 47$ & SB3-28ccc & m39 \\
\hline SB3-16ccc & $\mathrm{m} 30$ & SB3-20ccc & m61 & SB3-24ccc & $\mathrm{m} 26$ & SB3-28ccc & $\mathrm{m} 17$ \\
\hline SB3-17 & m75 & SB3-21 & $\mathrm{m} 62$ & SB3-25 & $\mathrm{m} 05$ & ARM & $\mathrm{m} 78$ \\
\hline SB3-17 & $\mathrm{m} 02$ & SB3-21 & $\mathrm{m} 23$ & SB3-25 & $\mathrm{m} 32$ & ARM & m63 \\
\hline SB3-17 & $\mathrm{m} 45$ & SB3-21 & m66 & SB3-25 & $\mathrm{m} 20$ & ARM & $\mathrm{m} 49$ \\
\hline SB3-17ccc & $\mathrm{m} 89$ & SB3-21ccc & $\mathrm{m} 76$ & SB3-25ccc & $\mathrm{m} 27$ & EA & $\mathrm{m} 43$ \\
\hline SB3-17ccc & $\mathrm{m} 88$ & SB3-21ccc & $\mathrm{m} 65$ & SB3-25ccc & $\mathrm{m} 37$ & EA & m67 \\
\hline SB3-17ccc & $\mathrm{m} 40$ & SB3-21ccc & $\mathrm{m} 85$ & SB3-25ccc & $\mathrm{m} 72$ & EA & m91 \\
\hline SB3-18 & $\mathrm{m} 24$ & SB3-22 & $\mathrm{m} 16$ & SB3-26 & $\mathrm{m} 36$ & blank & $\mathrm{m} 35$ \\
\hline SB3-18 & $\mathrm{m} 74$ & SB3-22 & $\mathrm{m} 71$ & SB3-26 & $\mathrm{m} 10$ & blank & $\mathrm{m} 42$ \\
\hline SB3-18 & $\mathrm{m} 54$ & SB3-22 & m19 & SB3-26 & $\mathrm{m} 82$ & & \\
\hline SB3-18ccc & $\mathrm{m} 68$ & SB3-22ccc & $\mathrm{m} 22$ & SB3-26ccc & $\mathrm{m} 90$ & & \\
\hline SB3-18ccc & $\mathrm{m} 41$ & SB3-22ccc & $\mathrm{m} 07$ & SB3-26ccc & $\mathrm{m} 15$ & & \\
\hline SB3-18ccc & $\mathrm{m} 83$ & SB3-22ccc & $\mathrm{m} 04$ & SB3-26ccc & m09 & & \\
\hline
\end{tabular}

\subsection{Analytical Plan}

The analytical plan for the SRTC-ML is provided in this section. Each of the solution samples submitted to the SRTC-ML is to be analyzed only once for each of the following: aluminum (Al), boron $(\mathrm{B})$, iron $(\mathrm{Fe})$, lithium $(\mathrm{Li})$, sodium $(\mathrm{Na})$, silicon $(\mathrm{Si})$, and uranium $(\mathrm{U})$. The measurements are to be made in parts per million ( $\mathrm{ppm}$ ). The analytical procedure used by the SRTC-ML to determine the concentrations utilizes an Inductively Coupled Plasma - Atomic Emission Spectrometer (ICP-AES). The PCT solutions (as identified in Table 2) are grouped in six ICPAES blocks for processing by the SRTC-ML in Table 3. Each block requires a different calibration of the ICP-AES. 
Table 3: ICP-AES Calibration Blocks for Leachate Measurements.

\begin{tabular}{|cccccc|}
\hline Block 1 & Block 2 & Block 3 & Block 4 & Block 5 & Block 6 \\
std-b1-1 & std-b2-1 & std-b3-1 & std-b4-1 & std-b5-1 & std-b6-1 \\
m03 & $\mathrm{m} 01$ & $\mathrm{~m} 81$ & $\mathrm{~m} 43$ & $\mathrm{~m} 32$ & $\mathrm{~m} 40$ \\
$\mathrm{~m} 90$ & $\mathrm{~m} 87$ & $\mathrm{~m} 29$ & $\mathrm{~m} 76$ & $\mathrm{~m} 02$ & $\mathrm{~m} 20$ \\
$\mathrm{~m} 68$ & $\mathrm{~m} 58$ & $\mathrm{~m} 83$ & $\mathrm{~m} 18$ & $\mathrm{~m} 42$ & $\mathrm{~m} 66$ \\
$\mathrm{~m} 44$ & $\mathrm{~m} 12$ & $\mathrm{~m} 54$ & $\mathrm{~m} 60$ & $\mathrm{~m} 23$ & $\mathrm{~m} 80$ \\
$\mathrm{~m} 24$ & $\mathrm{~m} 10$ & $\mathrm{~m} 50$ & $\mathrm{~m} 22$ & $\mathrm{~m} 25$ & $\mathrm{~m} 19$ \\
$\mathrm{~m} 21$ & $\mathrm{~m} 15$ & $\mathrm{~m} 64$ & $\mathrm{~m} 75$ & $\mathrm{~m} 88$ & $\mathrm{~m} 17$ \\
$\mathrm{~m} 14$ & $\mathrm{~m} 06$ & $\mathrm{~m} 69$ & $\mathrm{~m} 89$ & $\mathrm{~m} 37$ & $\mathrm{~m} 91$ \\
$\mathrm{~m} 28$ & $\mathrm{~m} 74$ & $\mathrm{~m} 26$ & $\mathrm{~m} 27$ & $\mathrm{~m} 34$ & $\mathrm{~m} 45$ \\
std-b1-2 & std-b2-2 & std-b3-2 & std-b4-2 & std-b5-2 & std-b6-2 \\
$\mathrm{m} 36$ & $\mathrm{~m} 55$ & $\mathrm{~m} 51$ & $\mathrm{~m} 16$ & $\mathrm{~m} 63$ & $\mathrm{~m} 04$ \\
$\mathrm{~m} 77$ & $\mathrm{~m} 53$ & $\mathrm{~m} 09$ & $\mathrm{~m} 05$ & $\mathrm{~m} 65$ & $\mathrm{~m} 61$ \\
$\mathrm{~m} 52$ & $\mathrm{~m} 31$ & $\mathrm{~m} 57$ & $\mathrm{~m} 33$ & $\mathrm{~m} 71$ & $\mathrm{~m} 38$ \\
$\mathrm{~m} 08$ & $\mathrm{~m} 13$ & $\mathrm{~m} 70$ & $\mathrm{~m} 62$ & $\mathrm{~m} 56$ & $\mathrm{~m} 85$ \\
$\mathrm{~m} 48$ & $\mathrm{~m} 73$ & $\mathrm{~m} 92$ & $\mathrm{~m} 11$ & $\mathrm{~m} 07$ & $\mathrm{~m} 72$ \\
$\mathrm{~m} 79$ & $\mathrm{~m} 47$ & $\mathrm{~m} 82$ & $\mathrm{~m} 35$ & $\mathrm{~m} 67$ & $\mathrm{~m} 49$ \\
$\mathrm{~m} 59$ & $\mathrm{~m} 41$ & $\mathrm{~m} 30$ & $\mathrm{~m} 78$ & $\mathrm{~m} 39$ & std-b6-3 \\
$\mathrm{m} 86$ & $\mathrm{~m} 46$ & $\mathrm{~m} 84$ & $\mathrm{std}-\mathrm{b} 4-3$ & $\mathrm{std}-\mathrm{b} 5-3$ & \\
std-b1-3 & std-b2-3 & std-b3-3 & & & \\
\hline
\end{tabular}

A multi-element solution standard (denoted by "std-bi-j" where $\mathrm{i}=1$ to 6 represents the block number and $\mathrm{j}=1,2$, and 3 represents the position in the block) was added at the beginning, middle, and end of each of the nine blocks. This standard may be useful in checking and correcting for bias in the concentration measurements arising from the ICP-AES calibrations.

\subsection{SUMMARY}

In summary, this analytical plan provides identifiers for the PCT solutions in Table 2 and six ICPAES calibration blocks in Table 3 for the SRTC-ML to use in conducting the aluminum (Al), boron $(\mathrm{B})$, iron $(\mathrm{Fe})$, lithium $(\mathrm{Li})$, sodium $(\mathrm{Na})$, silicon $(\mathrm{Si})$, and uranium $(\mathrm{U})$ concentration measurements for this PCT study. The sequencing of the activities associated with each of the steps in the analytical procedure has been randomized. The size of the blocks was selected so that the block could be completed in a single work shift. If for some reason the measurements are not conducted in the sequence presented in this memorandum, the actual order should be recorded along with any explanative comments.

The analytical plan indicated in the preceding tables should be modified by the personnel of the SRTC-ML to include any calibration check standards and/or other standards that are part of their standard operating procedures. 


\subsection{REFERENCES}

[1] Herman, C. C., D. K. Peeler, and T. B. Edwards, "Task Technical/Quality Assurance Plan: Sludge Batch 3 Variability Studies with Simulants," WSRC-RP-2002-00386, Revision 0, July 11, 2002.

[2] ASTM C1285-97, "Standard Test Methods for Determining Chemical Durability of Nucle ar Waste Glasses: The Product Consistency Test (PCT)," 1997.

[3] Peeler, D. K., C. C. Herman, and T. B. Edwards, "Analytical Study Plan: Sludge Batch 3 Variability Studies with Simulants,” WSRC-RP-2002-00394, Revision 0, July 11, 2002. 
Immobilization Technology Section

WSRC-TR-2002-00549

Savannah River Technology Center

Rev. 0

Westinghouse Savannah River Company

\section{Appendix G}

PCT Analytical Plan for Set \#3

SB3-29 through SB3-42 
October 7, 2002

To: C. C. Herman, 773-43A

D. K. Peeler, 999-W

cc: $\quad$ R. A. Baker, 773-42A

I. A. Reamer, 773-A

D. R. Best, 786-1A (wo)

P. A. Toole, 786-1A (wo)

S. L. Marra, 999-W (es)

R. C. Tuckfield, 773-42A

D. J. Pittman, 786-1A (wo)

R. J. Workman, 999-1W

From: T. B. Edwards, 773-42A (5-5148)

Statistical Consulting Section

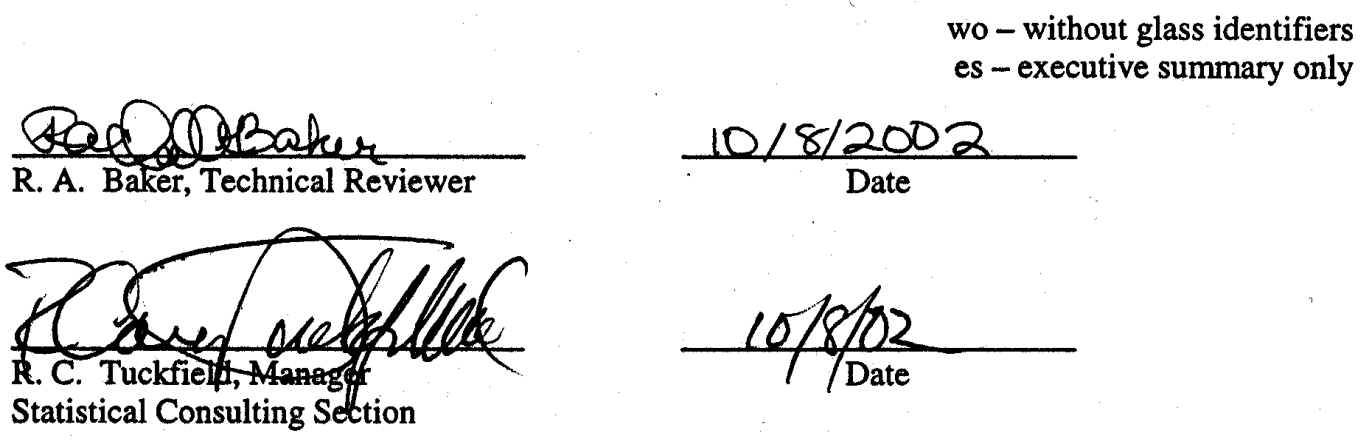

\section{An Analytical Plan For MEASURING the THIRD SET OF PCT SOLUTIONS FROM THE PhASE 1 Glasses FOR THE SB3 VARIABILITY STUDY (U)}




\subsection{EXECUTIVE SUMMARY}

A study is being conducted by the Savannah River Technology Center (SRTC) for the Defense Waste Processing Facility (DWPF) that involves investigating the glass region anticipated for the processing of Sludge Batch 3 (SB3). Forty-two (42) glass compositions were selected for batching and testing to support the first phase of this effort.

Fourteen (14) of the 42 study glasses are the focus of this analytical plan; they form the third set of glasses that are to be subjected to the Product Consistency Test, or PCT. The glasses were cooled both by quenching and centerline canister cooling, and the durabilities of the resulting twenty-eight glasses are to be measured in triplicate using the PCT, the requirements of which are described in ASTM C1285-97 (Method A).

The Savannah River Technology Center-Mobile Laboratory (SRTC-ML) is to be used to measure elemental concentrations of the resulting leachate solutions from the PCTs. This memorandum provides an analytical plan for the SRTC-ML to follow in measuring the compositions of the leachate solutions resulting from the PCT procedures for the glasses. 


\subsection{INTRODUCTION}

A glass variability study for Sludge Batch 3 (SB3) is being conducted for the Defense Waste Processing Facility (DWPF) by the Savannah River Technology Center (SRTC) [1]. Forty-two (42) glass compositions were selected for batching and testing to support Phase 1 of this effort.

Fourteen (14) of the 42 study glasses are the focus of this analytical plan; they form the third set of glasses that are to be subjected to the Product Consistency Test, or PCT. The glasses were cooled both by quenching and by centerline canister cooling, and the durabilities of the resulting twenty-eight glasses are to be measured in triplicate using the PCT, the requirements of which are described in ASTM C1285-97 (Method A) [2].

The identifiers for the third set of study glasses are presented in Table 1. The centerline canister cooled glasses are denoted by a "ccc" suffix.

Table 1: Identifiers for the Third Set of Study Glasses

\begin{tabular}{|c|c|c|c|}
\hline Quenched & $\begin{array}{c}\text { Centerline } \\
\text { Canister } \\
\text { Cooled }\end{array}$ & Quenched & $\begin{array}{c}\text { Centerline } \\
\text { Canister } \\
\text { Cooled }\end{array}$ \\
\hline SB3-29 & SB3-29ccc & SB3-36 & SB3-36ccc \\
\hline SB3-30 & SB3-30ccc & SB3-37 & SB3-37ccc \\
\hline SB3-31 & SB3-31ccc & SB3-38 & SB3-38ccc \\
\hline SB3-32 & SB3-32ccc & SB3-39 & SB3-39ccc \\
\hline SB3-33 & SB3-33ccc & SB3-40 & SB3-40ccc \\
\hline SB3-34 & SB3-34ccc & SB3-41 & SB3-41ccc \\
\hline SB3-35 & SB3-35ccc & SB3-42 & SB3-42ccc \\
\hline
\end{tabular}

This memorandum is in support of the Analytical Study Plan [3] and provides an analytical plan for the Savannah River Technology Center's Mobile Laboratory (SRTC-ML) to follow in measuring the compositions of the PCT leachate solutions for these glasses.

\subsection{DisCUSSION}

The quenched and centerline canister cooled versions of the study glasses are to be subjected to the PCT. The 2 different thermal histories for each of the 14 glasses lead to 28 glasses that are to be measured (in triplicate) using the PCT. In addition to those for the study glasses, triplicate PCTs are to be conducted on a sample of the Approved Reference Material (ARM) glass and a sample of the Environmental Assessment (EA) glass. Two reagent blank samples are also to be included in these tests. This results in 92 sample solutions being required to complete these PCTs.

The leachates from these tests will be diluted by adding $4 \mathrm{~mL}$ of $0.4 \mathrm{M} \mathrm{HNO}_{3}$ to $6 \mathrm{~mL}$ of the leachate (a 6:10 volume to volume, v:v, dilution) before being submitted to the SRTC-ML. The EA leachates will be further diluted $(1: 10 \mathrm{v}: \mathrm{v})$ with deionized water prior to submission to the SRTC-ML in order to prevent problems with the nebulizer.

Table 2 presents identifying codes, n01 through n92, for the individual solutions required for the PCTs of the study glasses and of the standards (EA, ARM, and blanks). This provides a naming 
convention that is to be used by the SRTC-ML in analyzing the solutions and reporting the relevant concentration measurements. ${ }^{26}$

Table 2: Identifiers for the PCT Solutions

\begin{tabular}{|c|c|c|c|c|c|c|c|}
\hline $\begin{array}{l}\text { Original } \\
\text { Sample }\end{array}$ & $\begin{array}{l}\text { Solution } \\
\text { Identifier }\end{array}$ & $\begin{array}{l}\text { Original } \\
\text { Sample }\end{array}$ & $\begin{array}{l}\text { Solution } \\
\text { Identifier }\end{array}$ & $\begin{array}{l}\text { Original } \\
\text { Sample }\end{array}$ & $\begin{array}{l}\text { Solution } \\
\text { Identifier }\end{array}$ & $\begin{array}{l}\text { Original } \\
\text { Sample }\end{array}$ & $\begin{array}{l}\text { Solution } \\
\text { Identifier }\end{array}$ \\
\hline SB3-29 & n47 & SB3-33 & n70 & SB3-37 & n35 & SB3-41 & n91 \\
\hline SB3-29 & n62 & SB3-33 & n24 & SB3-37 & n30 & SB3-41 & n33 \\
\hline SB3-29 & n72 & SB3-33 & n41 & SB3-37 & n22 & SB3-41 & n65 \\
\hline SB3-29ccc & n32 & SB3-33ccc & n27 & SB3-37ccc & n87 & SB3-41ccc & n29 \\
\hline SB3-29ccc & n84 & SB3-33ccc & $\mathrm{n} 25$ & SB3-37ccc & n02 & SB3-41ccc & n82 \\
\hline SB3-29ccc & n80 & SB3-33ccc & n79 & SB3-37ccc & n14 & SB3-41ccc & n31 \\
\hline SB3-30 & n58 & SB3-34 & n59 & SB3-38 & n07 & SB3-42 & n48 \\
\hline SB3-30 & n54 & SB3-34 & n43 & SB3-38 & n15 & SB3-42 & n55 \\
\hline SB3-30 & n52 & SB3-34 & n16 & SB3-38 & n56 & SB3-42 & n73 \\
\hline SB3-30ccc & n20 & SB3-34ccc & $\mathrm{n} 45$ & SB3-38ccc & n09 & SB3-42ccc & $\mathrm{n} 01$ \\
\hline SB3-30ccc & n36 & SB3-34ccc & n78 & SB3-38ccc & n67 & SB3-42ccc & n04 \\
\hline SB3-30ccc & $\mathrm{n} 12$ & SB3-34ccc & n86 & SB3-38ccc & n08 & SB3-42ccc & n75 \\
\hline SB3-31 & n63 & SB3-35 & $\mathrm{n} 42$ & SB3-39 & n03 & ARM & n37 \\
\hline SB3-31 & n34 & SB3-35 & n19 & SB3-39 & n74 & ARM & n05 \\
\hline SB3-31 & $\mathrm{n} 40$ & SB3-35 & n66 & SB3-39 & n49 & ARM & n51 \\
\hline SB3-31ccc & n61 & SB3-35ccc & n76 & SB3-39ccc & n90 & EA & n13 \\
\hline SB3-31 ccc & n89 & SB3-35ccc & $\mathrm{n} 11$ & SB3-39ccc & n17 & EA & $\mathrm{n} 10$ \\
\hline SB3-31ccc & $\mathrm{n} 85$ & SB3-35ccc & $\mathrm{n} 44$ & SB3-39ccc & n38 & EA & n39 \\
\hline SB3-32 & n53 & SB3-36 & n71 & SB3-40 & $\mathrm{n} 23$ & blank & $\mathrm{n} 50$ \\
\hline SB3-32 & n57 & SB3-36 & n83 & SB3-40 & n18 & blank & n88 \\
\hline SB3-32 & $\mathrm{n} 46$ & SB3-36 & n68 & SB3-40 & n64 & & \\
\hline SB3-32ccc & n77 & SB3-36ccc & n60 & SB3-40ccc & n26 & & \\
\hline SB3-32ccc & n69 & SB3-36ccc & $\mathrm{n} 28$ & SB3-40ccc & $\mathrm{n} 21$ & & \\
\hline SB3-32ccc & n92 & SB3-36ccc & n06 & SB3-40ccc & $\mathrm{n} 81$ & & \\
\hline
\end{tabular}

\subsection{Analytical Plan}

The analytical plan for the SRTC-ML is provided in this section. Each of the solution samples submitted to the SRTC-ML is to be analyzed only once for each of the following: aluminum (Al), boron $(\mathrm{B})$, iron $(\mathrm{Fe})$, lithium $(\mathrm{Li})$, sodium $(\mathrm{Na})$, silicon $(\mathrm{Si})$, and uranium $(\mathrm{U})$. The measurements are to be made in parts per million (ppm). The analytical procedure used by the SRTC-ML to determine the concentrations utilizes an Inductively Coupled Plasma - Atomic Emission Spectrometer (ICP-AES). The PCT solutions (as identified in Table 2) are grouped in six ICPAES blocks for processing by the SRTC-ML in Table 3. Each block requires a different calibration of the ICP-AES. 
Table 3: ICP-AES Calibration Blocks for Leachate Measurements.

\begin{tabular}{|cccccc|}
\hline Block 1 & Block 2 & Block 3 & Block 4 & Block 5 & Block 6 \\
std-b1-1 & std-b2-1 & std-b3-1 & std-b4-1 & std-b5-1 & std-b6-1 \\
n87 & $\mathrm{n} 57$ & $\mathrm{n} 80$ & $\mathrm{n} 76$ & $\mathrm{n} 55$ & $\mathrm{n} 65$ \\
$\mathrm{n} 61$ & $\mathrm{n} 83$ & $\mathrm{n} 16$ & $\mathrm{n} 70$ & $\mathrm{n} 15$ & $\mathrm{n} 49$ \\
$\mathrm{n} 45$ & $\mathrm{n} 30$ & $\mathrm{n} 64$ & $\mathrm{n} 42$ & $\mathrm{n} 67$ & $\mathrm{n} 12$ \\
$\mathrm{n} 47$ & $\mathrm{n} 43$ & $\mathrm{n} 92$ & $\mathrm{n} 27$ & $\mathrm{n} 17$ & $\mathrm{n} 08$ \\
$\mathrm{n} 59$ & $\mathrm{n} 10$ & $\mathrm{n} 06$ & $\mathrm{n} 01$ & $\mathrm{n} 54$ & $\mathrm{n} 75$ \\
$\mathrm{n} 26$ & $\mathrm{n} 05$ & $\mathrm{n} 86$ & $\mathrm{n} 48$ & $\mathrm{n} 36$ & $\mathrm{n} 56$ \\
$\mathrm{n} 63$ & $\mathrm{n} 21$ & $\mathrm{n} 46$ & $\mathrm{n} 20$ & $\mathrm{n} 25$ & $\mathrm{n} 31$ \\
$\mathrm{n} 60$ & $\mathrm{n} 18$ & $\mathrm{n} 40$ & $\mathrm{n} 90$ & $\mathrm{n} 88$ & $\mathrm{n} 52$ \\
std-b1-2 & $\mathrm{std}-\mathrm{b} 2-2$ & $\mathrm{std}-\mathrm{b} 3-2$ & $\mathrm{std}-\mathrm{b} 4-2$ & $\mathrm{std}-\mathrm{b} 5-2$ & $\mathrm{std}-\mathrm{b} 6-2$ \\
$\mathrm{n} 53$ & $\mathrm{n} 89$ & $\mathrm{n} 39$ & $\mathrm{n} 29$ & $\mathrm{n} 04$ & $\mathrm{n} 79$ \\
$\mathrm{n} 77$ & $\mathrm{n} 84$ & $\mathrm{n} 68$ & $\mathrm{n} 07$ & $\mathrm{n} 24$ & $\mathrm{n} 38$ \\
$\mathrm{n} 13$ & $\mathrm{n} 34$ & $\mathrm{n} 85$ & $\mathrm{n} 09$ & $\mathrm{n} 11$ & $\mathrm{n} 66$ \\
$\mathrm{n} 32$ & $\mathrm{n} 28$ & $\mathrm{n} 72$ & $\mathrm{n} 50$ & $\mathrm{n} 33$ & $\mathrm{n} 73$ \\
$\mathrm{n} 37$ & $\mathrm{n} 02$ & $\mathrm{n} 81$ & $\mathrm{n} 03$ & $\mathrm{n} 82$ & $\mathrm{n} 44$ \\
$\mathrm{n} 35$ & $\mathrm{n} 62$ & $\mathrm{n} 22$ & $\mathrm{n} 91$ & $\mathrm{n} 19$ & $\mathrm{n} 41$ \\
$\mathrm{n} 71$ & $\mathrm{n} 78$ & $\mathrm{n} 14$ & $\mathrm{n} 58$ & $\mathrm{n} 74$ & $\mathrm{std}-\mathrm{b} 6-3$ \\
$\mathrm{n} 23$ & $\mathrm{n} 69$ & $\mathrm{n} 51$ & $\mathrm{std}-\mathrm{b} 4-3$ & $\mathrm{std}-\mathrm{b} 5-3$ & \\
std-b1-3 & $\mathrm{std}-\mathrm{b} 2-3$ & $\mathrm{std}-\mathrm{b} 3-3$ & & & \\
\hline
\end{tabular}

A multi-element solution standard (denoted by "std-bi-j" where $\mathrm{i}=1$ to 6 represents the block number and $\mathrm{j}=1,2$, and 3 represents the position in the block) was added at the beginning, middle, and end of each of the nine blocks. This standard may be useful in checking and correcting for bias in the concentration measurements arising from the ICP-AES calibrations.

\subsection{SUMMARY}

In summary, this analytical plan provides identifiers for the PCT solutions in Table 2 and six ICPAES calibration blocks in Table 3 for the SRTC-ML to use in conducting the aluminum (Al), boron $(\mathrm{B})$, iron $(\mathrm{Fe})$, lithium $(\mathrm{Li})$, sodium $(\mathrm{Na})$, silicon $(\mathrm{Si})$, and uranium $(\mathrm{U})$ concentration measurements for this PCT study. The sequencing of the activities associated with each of the steps in the analytical procedure has been randomized. The size of the blocks was selected so that the block could be completed in a single work shift. If for some reason the measurements are not conducted in the sequence presented in this memorandum, the actual order should be recorded along with any explanative comments.

The analytical plan indicated in the preceding tables should be modified by the personnel of the SRTC-ML to include any calibration check standards and/or other standards that are part of their standard operating procedures. 


\subsection{REFERENCES}

[1] Herman, C. C., D. K. Peeler, and T. B. Edwards, "Task Technical/Quality Assurance Plan: Sludge Batch 3 Variability Studies with Simulants," WSRC-RP-2002-00386, Revision 0, July 11, 2002.

[2] ASTM C1285-97, "Standard Test Methods for Determining Chemical Durability of Nuclear Waste Glasses: The Product Consistency Test (PCT)," 1997.

[3] Peeler, D. K., C. C. Herman, and T. B. Edwards, "Analytical Study Plan: Sludge Batch 3 Variability Studies with Simulants,” WSRC-RP-2002-00394, Revision 0, July 11, 2002. 
Immobilization Technology Section

WSRC-TR-2002-00549

Savannah River Technology Center

Rev. 0

Westinghouse Savannah River Company

\section{Appendix H}

Tables and Exhibits Supporting the Statistical Analysis of the Measured Chemical Compositions of the

Study Glasses 
Table H.1: Measured Elemental Concentrations (wt\%) for the SB3 Phase 1 Glasses Prepared Using Lithium Metaborate

\begin{tabular}{|c|c|c|c|c|c|c|c|c|c|c|c|c|c|c|c|c|c|c|c|c|c|c|}
\hline $\begin{array}{c}\text { Glass } \\
\text { ID }\end{array}$ & $\begin{array}{c}\text { SRTC-ML } \\
\text { ID }\end{array}$ & Block & $\begin{array}{c}\text { Sub- } \\
\text { Block }\end{array}$ & $\begin{array}{l}\text { nalytical } \\
\text { iequence }\end{array}$ & $\mathrm{Ba}$ & $\mathrm{Ca}$ & $\mathrm{Ce}$ & $\mathrm{Cr}$ & $\mathrm{Cu}$ & $\mathrm{Fe}$ & $\mathrm{K}$ & $\mathrm{La}$ & $\mathrm{Mg}$ & $\mathrm{Mn}$ & $\mathrm{Na}$ & $\mathrm{Ni}$ & $\mathrm{Pb}$ & Th & $\mathrm{Ti}$ & U & $\mathrm{Zn}$ & $\mathrm{Zr}$ \\
\hline Batch 1 & BCHLM111 & $\overline{1}$ & $\overline{1}$ & $\overline{1}$ & 0.123 & 0.949 & 0.010 & .068 & 0.297 & .47 & 3.05 & $<0.010$ & 0.846 & 1.27 & 6.87 & 0.533 & 0.010 & $<0.010$ & 0.382 & 60.100 & 60.010 & 0.061 \\
\hline U std & USTLM111 & 1 & 1 & 2 & .010 & 936 & $<0.010$ & 160 & .002 & .52 & & & 0.706 & 2.01 & 8.23 & & 0.010 & & & & 0.010 & $<0.010$ \\
\hline 33-34 & k09LM21 & 1 & 1 & 3 & 079 & & & 086 & 063 & 0.4 & & 63 & 0.021 & 06 & 11.0 & & & & & & & 97 \\
\hline $33-33$ & k16LM21 & 1 & 1 & 4 & 0.052 & 0.737 & 0.079 & 0.054 & 0.051 & 7.54 & 0.060 & 0.041 & 0.007 & 1.52 & 9.28 & 0.330 & 0.069 & 0.102 & 0.442 & 2.32 & .082 & 0.139 \\
\hline $33-12$ & k14LM11 & 1 & 1 & 5 & 0.076 & 1.070 & 0.116 & 0.084 & 0.060 & 10.1 & 0.128 & 0.053 & 0.674 & 2.01 & 10.9 & 0.404 & 0.099 & 0.149 & $<0.010$ & 3.32 & .114 & 0.194 \\
\hline $33-05$ & k35LM21 & 1 & -5 & 6 & 0.045 & 0.710 & 0.072 & 0.053 & 0.036 & 7.05 & 0.078 & & 0.830 & .29 & 10.1 & & & & $<0.010$ & & & 0.123 \\
\hline $3-05$ & k35LM11 & 1 & 1 & 7 & 45 & 657 & 0.070 & .052 & .036 & .85 & 0.049 & 0. & 0.856 & 1.32 & 9.91 & 0.288 & 0.061 & 0.090 & $<0.010$ & & .070 & 0.120 \\
\hline & k18LM11 & 1 & 1 & 8 & 40 & 722 & 0.068 & 078 & .035 & .99 & 0.051 & & 0.003 & 1.20 & 10.4 & & 51 & & & & & .111 \\
\hline $3-19$ & $\mathrm{k} 18 \mathrm{LM} 21$ & 1 & 1 & 9 & 0.041 & .645 & 0.068 & 0.043 & 0.041 & 6.17 & 0.054 & 0.034 & 0.004 & 1.23 & 10.4 & 0.265 & 0.060 & 0.085 & & 1. & .063 & 0.117 \\
\hline & k28LM21 & 1 & 1 & 10 & & & 0.106 & & & .42 & & & & 1.30 & & & & & & & & 0.131 \\
\hline-31 & k08LM21 & 1 & 1 & 11 & 0 & 818 & 0.0 & 57 & 16 & .79 & 0.088 & 0. & 11 & 1.63 & 9.28 & 0.350 & 0.077 & 0. & 0 & 2.73 & 39 & 0.151 \\
\hline & k08I & 1 & 1 & 12 & & & 0.0 & 57 & 46 & 24 & 0.096 & & & 61 & 9.71 & & & & & & & \\
\hline-12 & k14LM21 & 1 & 1 & & & 1.079 & 0.1 & 091 & 0.061 & 然 & 0.129 & & & .13 & 10.8 & & & & & & & 03 \\
\hline tch 1 & BCHL & 1 & 1 & 14 & & 26 & $<0$. & 71 & & .06 & & & & 1 & 6.76 & & & & & & & 0.061 \\
\hline std & USTI & 1 & 1 & 15 & .010 & 953 & $<0.010$ & 167 & 0.003 & 9.11 & 2.50 & $<0.010$ & 0.743 & .11 & 8.30 & 0.801 & $<0.010$ & 0.060 & 0.5 & & $<0.010$ & $<0.010$ \\
\hline $3-33$ & 111 & 1 & 1 & 1 & & 742 & 0.078 & 056 & 042 & .52 & 0.062 & & 0.008 & .55 & 8.81 & 0.336 & & & & & & 0.142 \\
\hline & $\mathrm{k} 25 \mathrm{~L}$ & 1 & 1 & & & 5 & 0.074 & .050 & 0.043 & 670 & 0.077 & & & 1.31 & 10.3 & & & & & & & \\
\hline-21 & $\mathrm{k} 25 \mathrm{~L}$ & 1 & 1 & 18 & & $J$ & 0.0 & 2 & & 7.14 & 0.067 & & & 1.40 & 10.6 & & & & & & & 0.131 \\
\hline $3-34$ & k09LM11 & 1 & 1 & 15 & 0.089 & 1.095 & 0.120 & 0.092 & 0.063 & 11.8 & 0.131 & 0.065 & 0.024 & 2.18 & 10.8 & 0.451 & 0.095 & 0. & & 3.46 & .129 & 0.209 \\
\hline-07 & & 1 & 1 & 2 & & 670 & 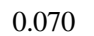 & 053 & 036 & .65 & 0.0 & & & 1.28 & 6.71 & & & & & & & \\
\hline & & 1 & 1 & & & & 0.070 & & & 7 & & & & & 8.30 & & & & & & & 25 \\
\hline-07 & k38LM21 & 1 & 1 & 22 & & 7 & 0. & 9 & 0. & 2 & 0.0 & & 0 & 0 & 6.76 & 0.2 & & & & & 78 & 16 \\
\hline-23 & k17LM11 & 1 & 1 & 2 & & 718 & $0.0^{7}$ & .043 & 0.042 & .04 & 0.066 & 0. & & 1.41 & 9.92 & 0. & & & & & 81 & 26 \\
\hline-39 & 111 & 1 & 1 & & & 665 & & 175 & & 721 & & & & 3 & 6.22 & & & & & & & 94 \\
\hline & & 1 & 1 & & & & & & & 9 & & & & & 9.98 & & & & & & & \\
\hline-39 & k26LM21 & 1 & 1 & & & 33 & 0 & 0 & 0. & 7.21 & 0.060 & & 2 & 2 & 6.51 & 0. & 77 & & & & 0.078 & 0.124 \\
\hline ch 1 & 113 & 1 & 1 & & & & & & & & & & & & 6.70 & & & & & & & \\
\hline & 18 & 1 & 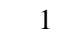 & 28 & & & & & & & & & & & 8.42 & & & & & & & $<0.010$ \\
\hline tch 1 & 21 & & 2 & & & & & & & & & & & & 7.32 & & & & & & & \\
\hline & USTLI & 1 & 2 & 2 & & 50 & $<0$. & 3 & 0.008 & 9.92 & 52 & & 0. & 2.07 & 9.61 & 0.779 & $<0$ & 0 & & 1.96 & $<0.010$ & $<0.010$ \\
\hline-21 & $\mathrm{k} 25 \mathrm{LM} 22$ & 1 & 2 & 3 & & & & & & 2 & 4 & & & & 10.8 & & & & & & & \\
\hline & & 1 & 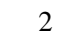 & -2 & & 77 & & & & 11.4 & & & & & 11.4 & & & & & & & \\
\hline & & & & & & & & & & 7.80 & & & & & 9.62 & & & & & & & \\
\hline & M12 & 1 & 2 & 6 & & & & & & 6.25 & & & & & 10.8 & & & & & 1.9 & & 26 \\
\hline-23 & k17LM12 & 1 & 2 & 1 & & 2 & & 4 & 0 & 6.84 & 0.066 & & & 1.43 & 10.5 & 0.301 & & & & & & 136 \\
\hline & & 1 & 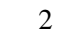 & 8 & & & & & & 6.36 & 0.0 & & & 1. & 7.18 & & & & & & & 31 \\
\hline & & & 2 & 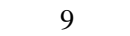 & & & & & & & & & & & & & & & & & & \\
\hline & LM22 & 1 & 2 & 10 & & & & & & 5 & & & & 2.15 & 11.4 & & & & & 3.37 & 23 & 213 \\
\hline SB3-21 & k25LM12 & 1 & 2 & 11 & 0.054 & 0.756 & 0.078 & 0.055 & 0.045 & 6.95 & 0.068 & 0.044 & 0.009 & 1.47 & 11.4 & 0.308 & 0.066 & 0.097 & 0.414 & 2.25 & 0.082 & 0.142 \\
\hline
\end{tabular}


Table H.1: Measured Elemental Concentrations (wt\%) for the SB3 Phase 1 Glasses Prepared Using Lithium Metaborate (continued)

\begin{tabular}{|c|c|c|c|c|c|c|c|c|c|c|c|c|c|c|c|c|c|c|c|c|c|c|}
\hline $\begin{array}{c}\text { Glass } \\
\text { ID }\end{array}$ & $\begin{array}{c}\text { SRTC-ML } \\
\text { ID }\end{array}$ & Block & $\begin{array}{c}\text { Sub- } \\
\text { Block }\end{array}$ & $\begin{array}{l}\text { nalytical } \\
\text { iequence }\end{array}$ & $\mathrm{Ba}$ & $\mathrm{Ca}$ & $\mathrm{Ce}$ & $\mathrm{Cr}$ & $\mathrm{Cu}$ & $\mathrm{Fe}$ & $\mathrm{K}$ & $\mathrm{La}$ & $\mathrm{Mg}$ & $\mathrm{Mn}$ & $\mathrm{Na}$ & $\mathrm{Ni}$ & $\mathrm{Pb}$ & Th & $\mathrm{Ti}$ & U & $\mathrm{Zn}$ & $\mathrm{Zr}$ \\
\hline SB3-05 & k35LM12 & $\overline{1}$ & $\overline{2}$ & 12 & 0.048 & 0.671 & 0.073 & .055 & 0.039 & 6.85 & 0.052 & 0.034 & 0.869 & 1.38 & 10.5 & 0.293 & 0.071 & 0.094 & $<0.010$ & 2.18 & 0.071 & 0.133 \\
\hline 33-19 & k18LM22 & 1 & 2 & & & 633 & & .045 & & .44 & 0.057 & & & 1.28 & 11.0 & & & & & & .062 & 130 \\
\hline $\operatorname{tch} 1$ & BCHLM122 & 1 & 2 & 14 & 124 & 960 & .010 & & & 27 & 13 & .010 & & 29 & 6.99 & 26 & 10 & & & & & 972 \\
\hline std & USTLM122 & 1 & 2 & 15 & $<0.010$ & 0.964 & $<0.010$ & 155 & 0.006 & 8.96 & 2.58 & $<0.010$ & 0.667 & 1.97 & 8.58 & 0.745 & $<0.010$ & 0.060 & 0.538 & 1.99 & $<0.010$ & $<0.010$ \\
\hline 33-39 & k26LM22 & 1 & 2 & 16 & .044 & 0.701 & 0.070 & 0.076 & 0.043 & 6.88 & 0.065 & 0.039 & 0.001 & 1.24 & 6.96 & 0.257 & 0.059 & 0.088 & 0.370 & 2.04 & 0.065 & 0.130 \\
\hline $33-12$ & $\mathrm{k} 14 \mathrm{LM} 12$ & 1 & 2 & 17 & 077 & 1.099 & 0.119 & 0.086 & 0.064 & 10.4 & 0.135 & & 0.661 & 2.04 & 11.3 & & & & & 3.43 & & 0.207 \\
\hline $3-11$ & k28LM22 & 1 & 2 & 8 & 1.044 & .704 & $0.0 / 3$ & .058 & 0.040 & .66 & 0.072 & 0.034 & 0.809 & 1.28 & 9.00 & 0.267 & 0.064 & 0.086 & 10 & 2.05 & .057 & 0.130 \\
\hline & k14LM22 & 1 & 2 & 19 & 076 & 098 & 0.122 & 087 & 0.063 & 0.4 & 0.137 & & 55 & 203 & 11.5 & 12 & & & & & & 0.210 \\
\hline $3-31$ & k08LM22 & 1 & 2 & 20 & 0.057 & 0.842 & 0.091 & 0.055 & 0.047 & 8.02 & 0.096 & 0.047 & 0.010 & 1.58 & 9.84 & 0.330 & & 0.116 & & 2.78 & .076 & 0.158 \\
\hline $3-05$ & k35LM22 & 1 & $2+2$ & 21 & & 0.712 & 0.074 & 0.054 & & .18 & 0.067 & & & .31 & 10.9 & & & & & & & 34 \\
\hline $3-31$ & k08LM12 & 1 & 2 & 22 & (7) & 56 & 0.0 & 57 & 0.049 & .25 & 0.103 & $0 .($ & 0 & 99 & 10.3 & 0. & 0. & 0. & & 2 & 84 & 0.158 \\
\hline-33 & k16 & 1 & 2 & 23 & & & 0.0 & 56 & 44 & .43 & 0.065 & & 09 & .57 & 9.43 & & & & & & & \\
\hline-11 & k28LM12 & 1 & 2 & & & 0.673 & 0.0 & 066 & 8 & 56 & 0.054 & & & .35 & 8.94 & & & & & & & \\
\hline $3-39$ & $\mathrm{k} 26 \mathrm{~L}$ & 1 & 2 & & & 54 & 0.0 & .173 & & 6.97 & 0.056 & & & 1.32 & 6.60 & & & & & & & 203 \\
\hline $3-23$ & k17LM22 & 1 & 2 & 2 & 0 & 732 & 0.0 & .044 & 0.045 & 6.93 & 0.079 & $0 .($ & 0.007 & 1.40 & 10.7 & 0.297 & 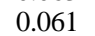 & $0 .($ & & 2. & 0.085 & 0.130 \\
\hline $\operatorname{tch} 1$ & 1123 & 1 & 2 & 2 & & 941 & $<0.010$ & 068 & 303 & .16 & 3.14 & & 0.822 & 1.27 & 7.06 & 0.524 & & & & & & 0.071 \\
\hline & USTI M123 & 1 & 2 & 0 & & & & 163 & & 15 & 250 & & & & & & & & & & & $<0.010$ \\
\hline $\operatorname{atch} 1$ & BCHL & 2 & & & & 40 & $<0$. & 68 & & .81 & 5.1 & & 0.806 & & 7.03 & & & & & & & 70 \\
\hline std & USTLM211 & 2 & . & 2 & 0.010 & 960 & $<0.010$ & .155 & 0.009 & 8.71 & 2.54 & $<0.010$ & 0.673 & 1.94 & 8.62 & 0.744 & $<0.010$ & 0.061 & 0. & 1. & $<0.010$ & $<0.010$ \\
\hline $3-13$ & 21 & 2 & 1 & 3 & & 579 & & .045 & 039 & 5.64 & 0.043 & & 0 & 1.1 & 11.4 & & & & & & & \\
\hline & 111 & 2 & 1 & 4 & & & & & & .90 & 0.095 & & & & 13.0 & & & & & & & \\
\hline-26 & LM11 & 2 & 1 & 5 & & 6 & 0 & 0 & 8 & .21 & 0.131 & & & 1 & 12.1 & 0. & & & & & 91 & 63 \\
\hline-06 & $\mathrm{k} 02 \mathrm{~L}$ & 2 & 1 & ( & & 828 & . & 073 & & .17 & & & & & 11.8 & & & & & & & 49 \\
\hline-06 & $\mathrm{k} 02 \mathrm{~L}$ & & 1 & 7 & & & & 71 & & .17 & 0.090 & & & 1. & 11.7 & & & & & & & 47 \\
\hline & & 2 & 1 & $\varepsilon$ & & & & & & 0 & & & & & 12.6 & & & & & & & \\
\hline-41 & k15LM21 & 2 & 1 & 9 & & 4 & 0.0 & .054 & 0.040 & 6.64 & 0.060 & 0. & 0. & 1. & 10.9 & 0.264 & & 0 & & & 63 & 0.123 \\
\hline-15 & & & 1 & & & & & & & 7 & & & & & 10.9 & & & & & & & \\
\hline & & 2 & 1 & & & & & & & 10.1 & & & & & 13.1 & & & & & & & \\
\hline & & 2 & 1 & & & & & & & 56 & & & & & 11.8 & & & & & & & \\
\hline-40 & LM11 & 2 & 1 & & & 9 & 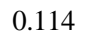 & 4 & 0. & 10.5 & 0.138 & & & 1. & 9.72 & 0.404 & 0. & & & & 11 & 0.194 \\
\hline ch 1 & HLM212 & & 1 & & & & & & & 6 & & & & & 7.39 & & & & & & & \\
\hline & 1212 & 2 & 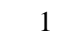 & & & & & & & & & & & & 9.31 & & & & & & & 010 \\
\hline $3-41$ & & & 1 & & & & & & & & & & & & 10.6 & & & & & & & \\
\hline & 121 & 2 & 1 & & & & & & & 5 & & & & & 12.7 & & & & & 0 & & \\
\hline-16 & k27LM11 & 2 & 1 & 1 & & 8 & & 3 & 0. & 7.56 & 0. & & & 1.45 & 12.0 & 0.307 & & & & & & 45 \\
\hline & 121 & 2 & 1 & 1 & & 7 & & & & 7.00 & & & & & 11.2 & 0. & & & & & & \\
\hline & & & 1 & & & & & & & 8. & & & & & 13.1 & & & & & & & \\
\hline & (1) & 2 & 1 & & & & & & & 7.37 & & & & 1.45 & 12.0 & & & & & 2.34 & 78 & .138 \\
\hline SB3-40 & k19LM21 & 2 & 1 & 22 & 0.077 & 1.090 & 0.114 & 0.099 & 0.066 & 10.1 & 0.149 & 0.065 & 0.019 & 1.96 & 9.81 & 0.389 & 0.093 & 0.145 & 0.621 & 3.24 & 0.123 & 0.192 \\
\hline
\end{tabular}


Table H.1: Measured Elemental Concentrations (wt\%) for the SB3 Phase 1 Glasses Prepared Using Lithium Metaborate (continued)

\begin{tabular}{|c|c|c|c|c|c|c|c|c|c|c|c|c|c|c|c|c|c|c|c|c|c|c|}
\hline $\begin{array}{c}\text { Glass } \\
\text { ID }\end{array}$ & $\begin{array}{l}\text { SRTC-ML } \\
\text { ID }\end{array}$ & Block & $\begin{array}{c}\text { Sub- } \\
\text { Block }\end{array}$ & $\begin{array}{l}\text { Analytical } \\
\text { Sequence }\end{array}$ & $\mathrm{Ba}$ & $\mathrm{Ca}$ & $\mathrm{Ce}$ & $\mathrm{Cr}$ & $\mathrm{Cu}$ & $\mathrm{Fe}$ & $\mathrm{K}$ & $\mathrm{La}$ & $\mathrm{Mg}$ & $\mathrm{Mn}$ & $\mathrm{Na}$ & $\mathrm{Ni}$ & $\mathrm{Pb}$ & Th & $\mathrm{Ti}$ & $\mathrm{U}$ & $\mathrm{Zn}$ & $\mathrm{Zr}$ \\
\hline SB3-26 & k40LM21 & 2 & 1 & 23 & 0.067 & 0.976 & 0.101 & 0.083 & 0.062 & 9.05 & 0.114 & 0.056 & 0.011 & 1.75 & 12.3 & 0.370 & 0.073 & 0.122 & 0.542 & 2.90 & 0.098 & 0.170 \\
\hline SB3-36 & k05LM11 & 2 & 1 & 24 & 0.074 & 1.034 & 0.107 & 0.090 & 0.062 & 9.82 & 0.125 & 0.061 & 0.016 & 1.90 & 13.6 & 0.404 & 0.091 & 0.139 & 0.593 & 3.17 & 0.102 & 0.188 \\
\hline SB3-35 & k07LM21 & 2 & 1 & 25 & 0.040 & 0.585 & 0.062 & 0.046 & 0.036 & 5.95 & 0.047 & 0.035 & 0.004 & 1.13 & 11.9 & 0.242 & 0.053 & 0.081 & 0.317 & 1.85 & 0.065 & 0.107 \\
\hline SB3-13 & k33LM11 & 2 & 1 & 26 & 0.040 & 0.564 & 0.063 & 0.045 & 0.035 & 5.79 & 0.038 & 0.031 & 0.011 & 1.15 & 11.8 & 0.250 & 0.052 & 0.082 & 0.014 & 1.85 & 0.060 & 0.108 \\
\hline Batch 1 & BCHLM213 & 2 & 1 & 27 & 0.118 & 0.949 & $<0.010$ & 0.067 & 0.300 & 8.96 & 3.14 & $<0.010$ & 0.788 & 1.20 & 7.09 & 0.509 & $<0.010$ & $<0.010$ & 0.371 & $<0.100$ & $<0.010$ & 0.063 \\
\hline U std & USTLM213 & 2 & 1 & 28 & $<0.010$ & 0.958 & $<0.010$ & 0.152 & 0.006 & 9.56 & 2.56 & $<0.010$ & 0.658 & 1.90 & 9.18 & 0.733 & $<0.010$ & 0.060 & 0.533 & 1.93 & $<0.010$ & 0.003 \\
\hline Batch 1 & BCHLM221 & 2 & 2 & 1 & 0.124 & 0.935 & $<0.010$ & 0.070 & 0.301 & 9.10 & 3.07 & $<0.010$ & 0.834 & 1.27 & 7.00 & 0.526 & $<0.010$ & $<0.010$ & 0.374 & $<0.100$ & $<0.010$ & 0.055 \\
\hline U std & USTLM221 & 2 & 2 & 2 & $<0.010$ & 0.952 & $<0.010$ & 0.162 & 0.008 & 8.97 & 2.51 & $<0.010$ & 0.708 & 2.03 & 8.55 & 0.772 & $<0.010$ & 0.060 & 0.546 & 1.96 & $<0.010$ & $<0.010$ \\
\hline SB3-15 & k37LM22 & 2 & 2 & 3 & 0.049 & 0.706 & 0.072 & 0.066 & 0.040 & 6.87 & 0.072 & 0.039 & 0.803 & 1.34 & 11.0 & 0.279 & 0.061 & 0.092 & 0.391 & 2.15 & 0.067 & 0.140 \\
\hline 33-04 & k22LM12 & 2 & 2 & 4 & 0.069 & 0.927 & 0.098 & 0.085 & 0.056 & 9.32 & 0.115 & 0.048 & 0.011 & 1.75 & 12.1 & 0.366 & 0.077 & 0.123 & $<0.010$ & 2.78 & 0.100 & 0.154 \\
\hline $3-35$ & k07LM22 & 2 & 2 & 5 & 0.043 & 0.568 & 0.063 & 0.049 & 0.034 & 6.09 & 0.047 & 0.034 & 0.005 & 1.19 & 11.5 & 0.251 & 0.055 & 0.082 & 0.324 & 1.88 & 0.066 & 0.101 \\
\hline SB3-04 & k22LM22 & 2 & 2 & 6 & 0.070 & 0.929 & 0.100 & 0.083 & 0.055 & 9.59 & 0.112 & 0.049 & 0.012 & 1.78 & 11.9 & 0.368 & 0.080 & 0.124 & $<0.010$ & 2.82 & 0.097 & 0.157 \\
\hline $3-06$ & k02LM22 & 2 & 2 & 7 & 0.062 & 0.819 & 0.090 & 0.076 & 0.048 & 8.30 & 0.085 & 0.045 & 0.780 & 1.63 & 11.4 & 0.347 & 0.079 & 0.113 & $<0.010$ & 2.58 & 0.089 & 0.144 \\
\hline $3-16$ & k27LM22 & 2 & 2 & 8 & 0.054 & 0.783 & 0.085 & 0.070 & 0.048 & 7.59 & 0.090 & 0.047 & 0.763 & 1.50 & 11.3 & 0.312 & 0.067 & 0.103 & 0.425 & 2.38 & 0.077 & 0.134 \\
\hline $3-16$ & k27LM12 & 2 & 2 & 9 & 0.054 & 0.767 & 0.094 & 0.075 & 0.048 & 7.68 & 0.081 & 0.057 & 0.759 & 1.50 & 11.5 & 0.312 & 0.070 & 0.103 & 0.428 & 2.37 & 0.079 & 0.139 \\
\hline SB3-13 & k33LM12 & 2 & 2 & 10 & 0.041 & 0.559 & 0.063 & 0.047 & 0.034 & 5.85 & 0.037 & 0.031 & 0.011 & 1.19 & 11.2 & 0.255 & 0.054 & 0.083 & 0.012 & 1.90 & 0.059 & 0.102 \\
\hline $3-20$ & k42LM12 & 2 & 2 & 11 & 0.064 & 0.845 & 0.089 & 0.083 & 0.054 & 8.99 & 0.092 & 0.052 & 0.011 & 1.69 & 12.4 & 0.362 & 0.078 & 0 . & 0. & 2.76 & 0.099 & 0.150 \\
\hline $3-20$ & $\mathrm{k} 42 \mathrm{LM} 22$ & 2 & 2 & 12 & 0.063 & 0.856 & 0.087 & 0.078 & 0.050 & 8.89 & 0.092 & 0.052 & 0.009 & 1.66 & 12.3 & 0.358 & 0.077 & 0.117 & 0.506 & 2.73 & 0.087 & 0.152 \\
\hline SB3-41 & k15LM22 & 2 & 2 & 13 & 0.046 & 0.659 & 0.070 & 0.057 & 0.038 & 6.95 & 0.059 & 0.033 & 0.001 & 1.27 & 10.3 & 0.268 & 0.059 & 0.087 & $<0.010$ & 2.06 & 0.062 & 0.116 \\
\hline Batch 1 & BCHLM222 & 2 & 2 & 14 & 0.121 & 0.928 & $<0.010$ & 0.069 & 0.298 & 9.21 & 3.05 & $<0.010$ & 0.814 & 1.24 & 6.84 & 0.513 & $<0.010$ & $<0.010$ & 0.372 & $<0.100$ & $<0.010$ & 0.056 \\
\hline U std & USTLM222 & 2 & 2 & 15 & $<0.010$ & 0.945 & $<0.010$ & 0.155 & 0.004 & 9.37 & 2.51 & $<0.010$ & 0.682 & 1.96 & 8.38 & 0.747 & $<0.010$ & 0.059 & 0.536 & 1.96 & $<0.010$ & $<0.010$ \\
\hline SB3-36 & k05LM22 & 2 & 2 & 16 & 0.074 & 1.041 & 0.105 & 0.091 & 0.060 & 10.7 & 0.131 & 0.060 & 0.014 & 1.90 & 12.6 & 0.397 & 0.090 & 0.136 & 0.593 & 3.17 & 0.098 & 0.175 \\
\hline $3-13$ & k33LM22 & 2 & 2 & 17 & 0.039 & 0.572 & 0.060 & 0.045 & 0.036 & 6.00 & 0.044 & 0.029 & 0.011 & 1.15 & 11.2 & 0.244 & 0.050 & 0.079 & 0.013 & 1.84 & 0.053 & 0.102 \\
\hline SB3-06 & k02LM12 & 2 & 2 & 18 & 0.059 & 0.804 & 0.084 & 0.074 & 0.050 & 8.72 & 0.083 & 0.044 & 0.753 & 1.57 & 11.5 & 0.341 & 0.076 & 0.111 & $<0.010$ & 2.57 & 0.084 & 0.140 \\
\hline $3-40$ & k19LM12 & 2 & 2 & 19 & 0.074 & 1.062 & 0.110 & 0.082 & 0.060 & 11.2 & 0.137 & $0 .($ & 0.016 & 1.95 & 9.41 & 0.395 & 0.091 & 0. & 0.621 & 3.27 & 0.102 & 0.186 \\
\hline $3-26$ & k40LM12 & 2 & 2 & 20 & 0.064 & 0.972 & 0.100 & 0.082 & 0.056 & 9.84 & 0.127 & 0.053 & 0.009 & 1.70 & 11.7 & 0.355 & 0.071 & 0.113 & 0.520 & 2.78 & 0.090 & 0.156 \\
\hline SB3-26 & k40LM22 & 2 & 2 & 21 & 0.067 & 0.960 & 0.100 & 0.084 & 0.059 & 10.0 & 0.114 & 0.055 & 0.010 & 1.78 & 11.8 & 0.375 & 0.073 & 0.119 & 0.541 & 2.89 & 0.093 & 0.164 \\
\hline & k07LM12 & 2 & 2 & 22 & 0.040 & 0.591 & 0.062 & 0.047 & 0.033 & 6.12 & 0.056 & 0.033 & $<0.001$ & 1.14 & 11.4 & 0.233 & & & & 1.78 & & 0.095 \\
\hline & k37LM12 & 2 & 2 & 23 & 0.050 & 0.694 & 0.070 & 0.061 & 0.042 & 7.34 & 0.070 & 0.039 & 0.820 & 1.35 & 10.8 & 0.286 & $0 .($ & 0.0 & 0.399 & 2.13 & 0.074 & 0.118 \\
\hline SB3-36 & k05LM12 & 2 & 2 & 24 & 0.077 & 1.027 & 0.106 & 0.094 & 0.060 & 10.8 & 0.125 & 0.061 & 0.016 & 1.97 & 13.3 & 0.418 & 0.095 & 0.140 & 0.603 & 3.17 & 0.104 & 0.182 \\
\hline SB3-41 & k15LM12 & 2 & 2 & 25 & 0.046 & 0.658 & 0.068 & 0.062 & 0.039 & 7.10 & 0.058 & 0.033 & 0.001 & 1.26 & 10.4 & 0.264 & 0.057 & 0.085 & $<0.010$ & 2.00 & 0.063 & 0.117 \\
\hline SB3-40 & k19LM22 & 2 & 2 & 26 & 0.078 & 1.091 & 0.114 & 0.103 & 0.064 & 10.9 & 0.153 & & 0.019 & 2.02 & 9.59 & 0.393 & & & & & & 0.185 \\
\hline $\operatorname{tch} 1$ & BCHLM223 & 2 & 2 & 27 & 0126 & 0.915 & $<0.010$ & 0.070 & 0.297 & 9.29 & 3.02 & & & 1.27 & 7.27 & 0.528 & & & 0.379 & $<0.100$ & $<0.010$ & 0.055 \\
\hline U std & USTLM223 & 2 & 2 & 28 & $<0.010$ & 0.929 & $<0.010$ & 0.163 & 0.004 & 9.59 & 2.47 & $<0.010$ & 0.710 & 2.04 & 8.90 & 0.771 & $<0.010$ & 0.059 & 0.547 & 1.96 & $<0.010$ & $<0.010$ \\
\hline Batch 1 & BCHLM311 & 3 & 1 & 1 & 0.119 & 0.951 & $<0.010$ & 0.065 & 0.302 & 8.93 & 3.08 & $<0.010$ & 0.833 & 1.27 & 7.00 & 0.533 & $<0.010$ & $<0.010$ & 0.384 & $<0.100$ & $<0.010$ & 0.054 \\
\hline & USTLM311 & 3 & 1 & 2 & $<0.010$ & 0.956 & $<0.010$ & 0.156 & 0.008 & 8.98 & 2.53 & & 0.689 & 2.01 & 8.53 & 0.761 & $<0.010$ & & & 1.99 & $<0.010$ & $<0.010$ \\
\hline SB3-28 & k29LM11 & 3 & 1 & 3 & 0.057 & 0.918 & 0.093 & 0.078 & 0.052 & 9.34 & 0.114 & & 0.008 & 177 & 5 & & 0.077 & & 0.522 & 2.79 & 0.085 & 0.154 \\
\hline SB3-28 & k29LM21 & 3 & 1 & 4 & 0.057 & 0.900 & 0.091 & 0.076 & 0.052 & 9.34 & 0.107 & 0.052 & 0.009 & 1.72 & 11.3 & 0.363 & 0.077 & 0.117 & 0.525 & 2.80 & 0.085 & 0.154 \\
\hline SB3-29 & k11LM21 & 3 & 1 & 5 & 0.040 & 0.692 & 0.598 & 0.032 & 0.041 & 7.36 & 0.061 & 0.603 & 0.841 & 1.32 & 8.31 & 0.284 & 0.083 & 0.090 & 0.406 & 2.06 & 0.061 & 0.238 \\
\hline
\end{tabular}


Table H.1: Measured Elemental Concentrations (wt\%) for the SB3 Phase 1 Glasses Prepared Using Lithium Metaborate (continued)

\begin{tabular}{|c|c|c|c|c|c|c|c|c|c|c|c|c|c|c|c|c|c|c|c|c|c|c|}
\hline $\begin{array}{c}\text { Glass } \\
\text { ID }\end{array}$ & $\begin{array}{c}\text { SRTC-ML } \\
\text { ID }\end{array}$ & Block & $\begin{array}{c}\text { Sub- } \\
\text { Block }\end{array}$ & $\begin{array}{l}\text { Analytical } \\
\text { Sequence }\end{array}$ & $\mathrm{Ba}$ & $\mathrm{Ca}$ & $\mathrm{Ce}$ & $\mathrm{Cr}$ & $\mathrm{Cu}$ & $\mathrm{Fe}$ & $\mathrm{K}$ & $\mathrm{La}$ & $\mathrm{Mg}$ & $\mathrm{Mn}$ & $\mathrm{Na}$ & $\mathrm{Ni}$ & $\mathrm{Pb}$ & Th & $\mathrm{Ti}$ & U & $\mathrm{Zn}$ & $\mathrm{Zr}$ \\
\hline$\overline{\text { SB3-32 }}$ & k36LM11 & 3 & 1 & 6 & 0.075 & 1.151 & 0.114 & 0.080 & 0.066 & 11.0 & 0.153 & 0.065 & 0.017 & 2.05 & 11.4 & 0.417 & 0.094 & 0.146 & 0.648 & 3.43 & 0.111 & 0.190 \\
\hline SB3-08 & k30LM11 & 3 & 1 & 7 & 0.067 & 0.998 & 0.101 & 0.118 & 0.062 & 10.1 & 0.106 & 0.052 & 0.015 & 1.90 & 10.5 & 0.352 & 0.096 & 0.132 & $<0.010$ & 3.16 & 0.107 & 0.168 \\
\hline SB3-08 & k30LM21 & 3 & 1 & 8 & 0.065 & 0.991 & 0.099 & 0.134 & 0.058 & 10.3 & 0.116 & 0.051 & 0.013 & 1.88 & 10.6 & 0.383 & 0.090 & 0.129 & $<0.010$ & 3.05 & 0.095 & 0.169 \\
\hline SB3-01 & k41LM11 & 3 & 1 & 9 & 0.040 & 0.647 & 0.062 & 0.045 & 0.042 & 6.75 & 0.059 & 0.031 & 0.002 & 1.22 & 8.58 & 0.264 & 0.055 & 0.083 & $<0.010$ & 2.03 & 0.062 & 0.108 \\
\hline SB3-17 & k01LM21 & 3 & 1 & 10 & 0.031 & 0.640 & 0.061 & 0.046 & 0.038 & 7.02 & 0.060 & 0.035 & $<0.001$ & 1.20 & 10.2 & 0.251 & 0.051 & 0.082 & 0.339 & 1.98 & 0.051 & 0.109 \\
\hline $3-01$ & k41LM21 & 3 & 1 & 11 & 0.039 & 0.656 & 0.061 & 0.043 & 0.038 & 6.65 & 0.065 & 0.030 & 0.001 & 1.19 & 8.59 & 0.255 & 0.053 & 0.081 & $<0.010$ & 1.97 & 0.056 & 0.100 \\
\hline SB3-17 & k01LM11 & 3 & 1 & 12 & 0.035 & 0.635 & 0.060 & 0.045 & 0.036 & 6.67 & 0.061 & 0.033 & $<0.001$ & 1.18 & 10.9 & 0.253 & 0.053 & 0.081 & 0.337 & 1.96 & 0.050 & 0.108 \\
\hline Batch 1 & BCHLM312 & 3 & 1 & 13 & 0.107 & 0.963 & $<0.010$ & 0.060 & 0.302 & 9.48 & 3.14 & $<0.010$ & 0.761 & 1.17 & 6.88 & 0.493 & $<0.010$ & $<0.010$ & 0.362 & $<0.100$ & $<0.010$ & 0.048 \\
\hline U std & USTLM312 & 3 & 1 & 14 & $<0.010$ & 0.963 & $<0.010$ & 0.143 & 0.005 & 9.41 & 2.56 & $<0.010$ & 0.632 & 1.87 & 8.52 & 0.721 & $<0.010$ & 0.055 & 0.519 & 1.93 & $<0.010$ & $<0.010$ \\
\hline SB3-25 & k39LM11 & 3 & 1 & 15 & 0.038 & 0.667 & 0.063 & 0.051 & 0.038 & 7.05 & 0.067 & 0.036 & $<0.001$ & 1.19 & 9.07 & 0.264 & 0.054 & 0.082 & 0.369 & 2.01 & 0.050 & 0.109 \\
\hline SB3-25 & k39LM21 & 3 & 1 & 16 & 0.036 & 0.659 & 0.064 & 0.051 & 0.040 & 6.92 & 0.066 & 0.035 & $<0.001$ & 1.18 & 8.99 & 0.257 & 0.051 & 0.080 & 0.366 & 2.00 & 0.055 & 0.106 \\
\hline SB3-38 & k03LM21 & 3 & 1 & 17 & 0.067 & 1.101 & 0.112 & 0.054 & 0.061 & 11.1 & 0.149 & 0.059 & 0.013 & 1.91 & 9.60 & 0.391 & 0.085 & 0.139 & 0.610 & 3.26 & 0.094 & 0.285 \\
\hline SB3-29 & k11LM11 & 3 & 1 & 18 & 0.036 & 0.693 & 0.066 & 0.028 & 0.041 & 7.24 & 0.067 & 0.036 & 0.782 & 1.23 & 8.32 & 0.268 & 0.054 & 0.084 & 0.392 & 2.06 & 0.058 & 0.113 \\
\hline SB3-38 & k03LM11 & 3 & 1 & 19 & 0.067 & 1.083 & 0.112 & 0.050 & 0.061 & 11.0 & 0.143 & 0.058 & 0.012 & 1.91 & 9.53 & 0.396 & 0.086 & 0.139 & 0.609 & 3.27 & 0.095 & 0.285 \\
\hline SB3-42 & k20LM21 & 3 & 1 & 20 & 0.065 & 1.069 & 0.105 & 0.089 & 0.061 & 8.63 & 0.134 & 0.050 & 0.017 & 1.87 & 11.6 & 0.409 & 0.090 & 0.135 & $<0.010$ & 3.20 & 0.096 & 0.174 \\
\hline SB3-18 & k04LM11 & 3 & 1 & 21 & 0.052 & 0.919 & 0.090 & 0.078 & 0.052 & 9.29 & 0.113 & 0.047 & 0.008 & 1.59 & 13.0 & 0.339 & 0.073 & 0.112 & 0.496 & 2.68 & 0.081 & 0.150 \\
\hline SB3-42 & k20LM11 & 3 & 1 & 22 & 0.061 & 1.070 & 0.105 & 0.090 & 0.065 & 9.01 & 0.135 & 0.051 & 0.018 & 1.90 & 11.4 & 0.410 & 0.091 & 0.133 & $<0.010$ & 3.21 & 0.098 & 0.175 \\
\hline 3-32 & k36LM21 & 3 & 1 & 23 & 0.069 & 1.229 & 0.115 & 0.070 & 0.066 & 11.2 & 0.176 & 0.060 & 0.016 & 1.91 & 13.7 & 0.384 & 0.083 & 0.139 & 0.618 & 3.32 & 0.098 & 0.181 \\
\hline SB3-18 & k04LM21 & 3 & 1 & 24 & 0.051 & 0.921 & 0.089 & 0.070 & 0.051 & 9.95 & 0.105 & 0.047 & 0.005 & 1.59 & 13.8 & 0.346 & 0.073 & 0.113 & 0.504 & 2.73 & 0.077 & 0.150 \\
\hline Batch 1 & BCHLM313 & 3 & 1 & 25 & 0107 & 0.942 & $<0.010$ & 0.059 & 0.295 & 9.16 & 3.11 & $<0.010$ & 0.764 & 1.17 & 7.08 & 0.495 & $<0.010$ & $<0.010$ & 0.366 & $<0.100$ & $<0.010$ & 0.051 \\
\hline $\mathrm{U}$ std & USTLM313 & 3 & 1 & 26 & $<0.010$ & 0.953 & $<0.010$ & 0.145 & 0.007 & 9.14 & 2.55 & $<0.010$ & 0.650 & 1.89 & 8.67 & 0.732 & $<0.010$ & 0.054 & 0.525 & 1.92 & $<0.010$ & $<0.010$ \\
\hline Batch 1 & BCHLM321 & 3 & 2 & 1 & 0.121 & 0.933 & $<0.010$ & 0.065 & 0.301 & 8.93 & 3.08 & $<0.010$ & 0.830 & 1.27 & 6.76 & 0.524 & $<0.010$ & $<0.010$ & 0.378 & $<0.100$ & $<0.010$ & 0.061 \\
\hline U std & USTLM321 & 3 & 2 & 2 & $<0.010$ & 0.963 & $<0.010$ & 0.154 & 0.007 & 9.10 & 2.55 & $<0.010$ & 0.683 & 2.01 & 8.39 & 0.754 & $<0.010$ & 0.057 & 0.544 & 2.00 & $<0.010$ & $<0.010$ \\
\hline SB3-18 & k04LM12 & 3 & 2 & 3 & 0.060 & 0.912 & 0.093 & 0.083 & 0.053 & 9.65 & 0.108 & 0.050 & 0.008 & 1.69 & 12.6 & 0.355 & & & & & & 0.157 \\
\hline 3-32 & k36LM12 & 3 & 2 & 4 & 0.079 & 1.152 & 0.118 & 0.081 & 0.066 & 11.2 & 0.153 & 0.066 & 0.015 & 2.08 & 11.4 & 0.419 & 0.090 & 0.148 & 0.651 & 3.49 & 0.110 & 0.194 \\
\hline SB3-32 & k36LM22 & 3 & 2 & 5 & 0.077 & 1.243 & 0.118 & 0.074 & 0.068 & 11.5 & 0.172 & 0.064 & 0.016 & 2.03 & 13.4 & 0.398 & 0.087 & 0.145 & 0.642 & 3.47 & 0.105 & 0.191 \\
\hline SB3-18 & k04LM22 & 3 & 2 & 6 & 0.057 & 0.938 & 0.091 & 0.073 & 0.051 & 12.1 & 0.103 & 0.049 & 0.004 & 1.66 & 14.4 & 0.353 & 0.073 & 0.118 & 0.517 & 2.84 & 0.081 & 0.157 \\
\hline 33-01 & k41LM12 & 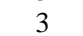 & 2 & 7 & 0.043 & 0.649 & 0.065 & 0.046 & 0.042 & 7.16 & 0.056 & 0.031 & $<0.001$ & 1.22 & 8.25 & 0.260 & 0.053 & 0.083 & $<0.010$ & & 0.060 & 0.108 \\
\hline $3-29$ & k11LM22 & 3 & 2 & 8 & 0.042 & 0.704 & 0.606 & 0.033 & 0.041 & 8.59 & 0.060 & 0.607 & 0.826 & 1.31 & 8.11 & 0.278 & 0.077 & 0.089 & 0.400 & 2.06 & 0.056 & 0.240 \\
\hline SB3-28 & k29LM12 & 3 & 2 & 9 & 0.058 & 0.942 & 0.091 & 0.076 & 0.051 & 10.2 & 0.117 & 0.051 & 0.004 & 1.65 & 11.1 & 0.345 & 0.071 & 0.114 & 0.513 & 2.76 & 0.078 & 0.152 \\
\hline SB3-01 & k41LM22 & 3 & 2 & 10 & 0.042 & 0.657 & 0.065 & 0.044 & 0.037 & 7.40 & 0.061 & 0.030 & $<0.001$ & 1.19 & 8.21 & 0.253 & 0.049 & 0.080 & $<0.010$ & 1.99 & 0.054 & 0.101 \\
\hline $33-38$ & k03LM22 & 2 & 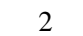 & 11 & 0.073 & 1.091 & 0.116 & 0.057 & 0.061 & 12.1 & 0.144 & & 0.012 & 1.98 & 9.08 & 0.396 & & & & 3.32 & & 0.291 \\
\hline $3-42$ & k20LM12 & 3 & 2 & 12 & 0.069 & 1.080 & 0.1 & 0.091 & 0.063 & 8.75 & 0.130 & 0.052 & 0. & 1.91 & 11.3 & 0.407 & 0.088 & 0.134 & $<0.010$ & 3.25 & 0.095 & 0.177 \\
\hline Batch 1 & BCHLM322 & 3 & 2 & 13 & 0.114 & 0.966 & $<0.010$ & 0.062 & 0.299 & 9.47 & 3.12 & $<0.010$ & 0.787 & 1.20 & 6.85 & 0.504 & $<0.010$ & $<0.010$ & 0.368 & $<0.100$ & $<0.010$ & 0.051 \\
\hline U std & USTLM322 & 3 & 2 & 14 & $<0.010$ & 0.948 & $<0.010$ & 0.148 & 0.005 & 9.76 & 2.53 & $<0.010$ & 0.662 & 1.91 & 8.18 & 0.730 & $<0.010$ & 0.055 & 0.529 & 1.94 & $<0.010$ & $<0.010$ \\
\hline $33-25$ & k39LM22 & 3 & 2 & 15 & 0.042 & 0.662 & 0.067 & 0.054 & & 7.70 & 0.061 & 0.036 & $<0.001$ & 1.22 & 8.61 & 0.262 & & 0.080 & 0.370 & 2.02 & & 0.109 \\
\hline $3-42$ & k20LM22 & 3 & 2 & 16 & 0.070 & 1.067 & 0.108 & 0.092 & 0.060 & 9.22 & 0.131 & 0.051 & 0.015 & 1.92 & 11.2 & 0.417 & 0.088 & 0.136 & $<0.010$ & 3.24 & 0.099 & 0.176 \\
\hline SB3-38 & k03LM12 & 3 & 2 & 17 & 0.071 & 1.090 & 0.116 & 0.051 & 0.061 & 11.6 & 0.140 & 0.059 & 0.010 & 1.94 & 9.24 & 0.396 & 0.086 & 0.142 & 0.608 & 3.28 & 0.095 & 0.288 \\
\hline SB3-29 & k11LM12 & 3 & 2 & 18 & 0.040 & 0.679 & 0.071 & 0.029 & 0.040 & 8.21 & 0.062 & 0.036 & 0.800 & 1.26 & 8.19 & 0.268 & 0.051 & 0.086 & 0.394 & 2.08 & 0.059 & 0.115 \\
\hline
\end{tabular}


Table H.1: Measured Elemental Concentrations (wt\%) for the SB3 Phase 1 Glasses Prepared Using Lithium Metaborate (continued)

\begin{tabular}{|c|c|c|c|c|c|c|c|c|c|c|c|c|c|c|c|c|c|c|c|c|c|c|}
\hline $\begin{array}{c}\text { Glass } \\
\text { ID }\end{array}$ & $\begin{array}{l}\text { SRTC-ML } \\
\text { ID }\end{array}$ & Block & $\begin{array}{c}\text { Sub- } \\
\text { Block }\end{array}$ & $\begin{array}{l}\text { Analytical } \\
\text { Sequence }\end{array}$ & $\mathrm{Ba}$ & $\mathrm{Ca}$ & $\mathrm{Ce}$ & $\mathrm{Cr}$ & $\mathrm{Cu}$ & $\mathrm{Fe}$ & $\mathrm{K}$ & $\mathrm{La}$ & $\mathrm{Mg}$ & $\mathrm{Mn}$ & $\mathrm{Na}$ & $\mathrm{Ni}$ & $\mathrm{Pb}$ & Th & $\mathrm{Ti}$ & U & $\mathrm{Zn}$ & $\mathrm{Zr}$ \\
\hline SB3-17 & k01LM12 & 3 & 2 & 19 & 0.038 & 0.627 & 0.066 & 0.045 & 0.035 & 7.34 & 0.057 & 0.033 & $<0.001$ & 1.17 & 10.7 & 0.247 & 0.050 & 0.080 & 0.331 & 1.92 & 0.048 & 0.106 \\
\hline SB3-08 & k30LM12 & 3 & 2 & 20 & 0.067 & 0.989 & 0.104 & 0.115 & 0.060 & 10.7 & 0.104 & 0.051 & 0.011 & 1.85 & 10.4 & 0.343 & 0.091 & 0.128 & $<0.010$ & 3.08 & 0.102 & 0.164 \\
\hline $3-17$ & k01LM22 & 3 & 2 & 21 & 0.030 & 0.645 & 0.061 & 0.047 & 0.040 & 7.31 & 0.058 & 0.037 & $<0.001$ & 1.21 & 11.0 & 0.249 & 0.052 & 0.079 & $<0.010$ & 1.90 & 0.049 & 0.106 \\
\hline SB3-25 & k39LM12 & 3 & 2 & 22 & 0.041 & 0.654 & 0.068 & 0.052 & 0.038 & 7.17 & 0.062 & 0.036 & $<0.001$ & 1.20 & 8.70 & 0.263 & 0.050 & 0.080 & 0.369 & 2.02 & 0.051 & 0.111 \\
\hline B3-08 & k30LM22 & 3 & 2 & 23 & 0.065 & 1.005 & 0.102 & 0.132 & 0.057 & 10.8 & 0.116 & 0.050 & 0.009 & 1.84 & 10.3 & 0.376 & 0.084 & 0.127 & $<0.010$ & 3.02 & 0.090 & 0.166 \\
\hline SB3-28 & k29LM22 & 3 & 2 & 24 & 0.055 & 0.912 & 0.092 & 0.071 & 0.051 & 9.92 & 0.112 & 0.049 & 0.003 & 1.60 & 11.2 & 0.341 & 0.069 & 0.112 & 0.502 & 2.74 & 0.073 & 0.149 \\
\hline Batch 1 & BCHLM323 & 3 & 2 & 25 & 0.108 & 0.919 & $<0.010$ & 0.059 & 0.292 & 9.66 & 3.09 & $<0.010$ & 0.755 & 1.17 & 6.80 & 0.487 & $<0.010$ & $<0.010$ & 0.360 & $<0.100$ & $<0.010$ & 0.051 \\
\hline $\mathrm{U}$ std & USTLM323 & 3 & 2 & 26 & $<0.010$ & 0.950 & $<0.010$ & 0.143 & 0.005 & 9.49 & 2.55 & $<0.010$ & 0.634 & 1.86 & 8.32 & 0.715 & $<0.010$ & 0.053 & 0.516 & 1.92 & $<0.010$ & $<0.010$ \\
\hline Batch 1 & BCHLM411 & 4 & 1 & 1 & 0.122 & 0.913 & $<0.010$ & 0.067 & 0.304 & 8.22 & 3.08 & $<0.010$ & 0.847 & 1.27 & 7.10 & 0.534 & $<0.010$ & $<0.010$ & 0.384 & $<0.100$ & $<0.010$ & 0.066 \\
\hline U std & USTLM411 & 4 & 1 & 2 & $<0.010$ & 0.945 & $<0.010$ & 0.162 & $<0.010$ & 9.14 & 2.53 & $<0.010$ & 0.721 & 2.01 & 8.81 & 0.785 & $<0.010$ & 0.064 & 0.556 & 1.98 & $<0.010$ & $<0.010$ \\
\hline SB3-37 & k10LM11 & 4 & 1 & 3 & 0.049 & 0.728 & 0.081 & 0.059 & 0.041 & 6.89 & 0.082 & 0.042 & 0.012 & 1.42 & 7.39 & 0.308 & 0.062 & 0.108 & 0.433 & 2. & 0.075 & 0.133 \\
\hline $3-22$ & k12LM11 & 4 & 1 & 4 & 0.069 & 0.982 & 0.102 & 0.094 & 0.055 & 9.40 & 0.118 & 0.057 & 0.025 & 1.87 & 13.3 & 0.400 & 0.085 & .151 & 0.584 & 3. & & 0.177 \\
\hline $33-02$ & k32LM21 & 4 & 1 & 5 & 0.061 & 0.831 & 0.102 & 0.061 & 0.047 & 8.33 & 0.089 & 0.061 & 0.018 & 1.63 & 11.5 & 0.333 & 0.076 & 26 & $<0.010$ & 2.53 & 0.093 & 0.150 \\
\hline $33-30$ & k31LM21 & 4 & 1 & 6 & 0.077 & 1.02 & 0.107 & 0.085 & 0.061 & 10.6 & 0.118 & 0.065 & 0.712 & 2.06 & 10.8 & 0.430 & 0.099 & 0.162 & 0.638 & 3.33 & 0.114 & 0.197 \\
\hline $3-24$ & k23LM21 & 4 & 1 & 7 & 0.072 & 0.985 & 0.104 & 0.084 & 0.056 & 9.64 & 0.118 & 0.059 & 0.024 & 1.92 & 13.8 & 0.414 & 0.091 & 0.154 & 0.596 & 3.11 & 0.103 & 0.181 \\
\hline $3-14$ & k34LM11 & 4 & 1 & 8 & 0.064 & 0.886 & 0.092 & 0.089 & 0.051 & 8.47 & 0.115 & 0.046 & 0.021 & 1.75 & 13.4 & 0.380 & 0.079 & 0.137 & $<0.010$ & 2.80 & 0.099 & 0.156 \\
\hline $33-30$ & k31LM11 & 4 & 1 & 9 & 0.077 & 1.01 & 0.108 & 0.084 & 0.058 & 10.2 & 0.124 & 0.065 & 0.712 & 2.03 & 10.9 & 0.413 & 0.099 & & 0.640 & 3.28 & & 0.198 \\
\hline $3-14$ & k34LM21 & 4 & 1 & 10 & 0.065 & 0.912 & 0.093 & 0.098 & 0.051 & 8.68 & 0.121 & 0.046 & 0.020 & 1.74 & 13.3 & 0.377 & 0.080 & 38 & $<0$ & 2.80 & 0.095 & 0.176 \\
\hline 3-02 & k32LM11 & 4 & 1 & 11 & 0.065 & 0.859 & 0.089 & 0.066 & 0.050 & 8.54 & 0.095 & 0.046 & 0.019 & 1.70 & 12.2 & 0.368 & 0.076 & 0.130 & $<0.010$ & 2.67 & 0.097 & 0.147 \\
\hline $33-37$ & k10LM21 & 4 & 1 & 12 & 0.048 & 0.717 & 0.080 & 0.058 & 0.042 & 7.05 & 0.077 & 0.042 & 0.012 & 1.42 & 7.32 & 0.306 & 0.063 & 0.107 & 0.431 & 2.27 & 0.074 & 0.130 \\
\hline Batch 1 & BCHLM412 & 4 & 1 & 13 & 0.120 & 0.933 & $<0.010$ & 0.067 & 0.305 & 8.70 & 3.09 & $<0.010$ & 0.834 & 1.25 & 7.27 & 0.530 & $<0.010$ & $<0.010$ & 31 & $<0.100$ & $<0.010$ & 0.057 \\
\hline U std & USTLM412 & 4 & 1 & 14 & $<0.010$ & 0.921 & $<0.010$ & 0.159 & $<0.010$ & 8.45 & 2.55 & $<0.010$ & 0.701 & 1.96 & 8.85 & 0.763 & $<0.010$ & 65 & 0. & 1.96 & $<0.010$ & $<0.010$ \\
\hline SB3-09 & k24LM21 & 4 & 1 & 15 & 0.051 & 0.741 & 0.083 & 0.071 & 0.046 & 6.68 & 0.080 & 0.038 & 0.011 & 1.44 & 8.46 & 0.318 & 0.066 & 0.115 & 0.002 & 2.35 & 0.074 & 0.135 \\
\hline 3-09 & k24LM11 & 4 & 1 & 16 & 0.053 & 0.738 & 0.175 & 0.060 & 0.042 & 6.78 & 0.086 & 0.145 & 0.010 & 1.40 & 8.46 & 0.309 & 0.068 & 0.115 & 0.001 & 2. & 0.073 & 0.155 \\
\hline & k21LM21 & 4 & 1 & 1 & & 1.0 & 0.116 & 0.092 & $0 .($ & 9.74 & 46 & & & & 12.1 & & & & & 3. & & 93 \\
\hline $3-22$ & $\mathrm{k} 12 \mathrm{LM} 21$ & 4 & 1 & 18 & 0.069 & 0.979 & 0.108 & 0.096 & 0.054 & 9.57 & 0.125 & 0.058 & 0.024 & 1.86 & 13.8 & 0.395 & 0.087 & 0.151 & 0.580 & 3.03 & 0.098 & 0.180 \\
\hline $3-24$ & k23LM11 & 4 & 1 & 19 & 0.069 & 0.997 & 0.112 & 0.081 & 0.059 & 9.48 & 0.144 & 0.057 & 0.024 & 1.85 & 14.3 & 0.391 & 0.086 & 0.146 & 0.581 & 3. & 0.103 & 0.171 \\
\hline $3-03$ & k13LM11 & 4 & 1 & 20 & 0.044 & 0.611 & 0.074 & 0.067 & 0.037 & 5.71 & 0.063 & 0.030 & 0.006 & 1.19 & 9.02 & 0.256 & 0.053 & 0.093 & 1 & 1. & 0.061 & 0.114 \\
\hline $3-27$ & k06LM21 & 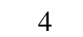 & 1 & 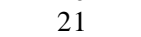 & 0.041 & 0.641 & 0.071 & 0.050 & 0.038 & 6.17 & 0.067 & 0.036 & 0.007 & 1.21 & 9.57 & 0.260 & & & & 1. & & 0.108 \\
\hline $3-27$ & k06LM11 & 4 & 1 & 22 & 0.042 & 0.651 & 0.075 & 0.079 & 0.046 & 5.93 & 0.076 & 0.036 & 0.005 & 1.22 & 9.50 & 0.261 & 0.050 & 0.092 & 0.366 & 1.94 & 0.055 & 0.117 \\
\hline $3-10$ & k21LM11 & 4 & 1 & 2 & 0.083 & 1.09 & 0.127 & 0.102 & 0.065 & 10.6 & 0.145 & 0.060 & 0.734 & 2.11 & 12.4 & 0.459 & 0.100 & 0.177 & 0.000 & 3.47 & 0.118 & 0.210 \\
\hline SB3-03 & k13LM21 & 4 & 1 & 24 & 0.048 & 0.616 & 0.073 & 0.078 & 0.035 & 6.19 & 0.054 & & & 1.24 & 8.98 & 0.281 & & & & 1.98 & & 0.120 \\
\hline Batch 1 & BCHLM413 & ( & 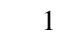 & & & 0.912 & $<0.010$ & 0.068 & & 8.88 & 3.16 & & & 1.80 & 7.28 & 0.533 & & & & $<0.100$ & & \\
\hline $\mathrm{U}$ std & USTLM413 & 4 & 1 & 26 & $<0.010$ & 0.927 & 0.010 & 0.163 & 0.010 & 8.74 & 2.58 & 10 & 0.715 & 1.97 & 8.90 & 0.781 & $<0.010$ & 0.066 & 0.551 & 1.97 & $<0.010$ & $<0.010$ \\
\hline Batch 1 & BCHLM421 & 4 & 2 & 1 & 0.123 & 0.907 & $<0.010$ & 0.068 & 0.300 & 9.10 & 3.08 & $<0.010$ & 0.840 & 1.30 & 6.97 & 0.528 & $<0.010$ & $<0.010$ & 0.384 & $<0.100$ & $<0.010$ & 0.061 \\
\hline U std & USTLM421 & 4 & 2 & 2 & $<0.010$ & 0.928 & $<0.010$ & 0.161 & $<0.010$ & 9.05 & 2.51 & $<0.010$ & 0.707 & 2.06 & 8.69 & 0.771 & $<0.010$ & 0.062 & & 1.91 & $<0.010$ & $<0.010$ \\
\hline SB3-14 & k34LM12 & . & - & - & $0 .($ & 0.902 & & 0.088 & 0.047 & 8.94 & 0.120 & & & & 13.23 & & & & & 2.76 & & 0.157 \\
\hline-09 & k24LM12 & 4 & 2 & 4 & 0.052 & 0.739 & 0.087 & 0.059 & 0.039 & 7.16 & 0.090 & 0.040 & 0.014 & 1.47 & 8.28 & 0.309 & 0.066 & 0.111 & 0.000 & 2.24 & 0.078 & 0.130 \\
\hline SB3-27 & k06LM12 & 4 & 2 & 5 & 0.045 & 0.660 & 0.070 & 0.078 & 0.043 & 6.43 & 0.080 & 0.036 & 0.009 & 1.27 & 9.19 & 0.260 & 0.050 & 0.090 & 0.366 & 1.89 & 0.066 & 0.113 \\
\hline
\end{tabular}


Table H.1: Measured Elemental Concentrations (wt\%) for the SB3 Phase 1 Glasses Prepared Using Lithium Metaborate (continued)

\begin{tabular}{|c|c|c|c|c|c|c|c|c|c|c|c|c|c|c|c|c|c|c|c|c|c|c|}
\hline $\begin{array}{l}\text { Glass } \\
\text { ID }\end{array}$ & $\begin{array}{l}\text { SRTC-ML } \\
\text { ID }\end{array}$ & Block & $\begin{array}{c}\text { Sub- } \\
\text { Block }\end{array}$ & $\begin{array}{l}\text { Analytical } \\
\text { Sequence }\end{array}$ & $\mathrm{Ba}$ & $\mathrm{Ca}$ & $\mathrm{Ce}$ & $\mathrm{Cr}$ & $\mathrm{Cu}$ & $\mathrm{Fe}$ & $\mathrm{K}$ & $\mathrm{La}$ & $\mathrm{Mg}$ & $\mathrm{Mn}$ & $\mathrm{Na}$ & $\mathrm{Ni}$ & $\mathrm{Pb}$ & Th & $\mathrm{Ti}$ & U & $\mathrm{Zn}$ & $\mathrm{Zr}$ \\
\hline SB3-30 & k31LM22 & 4 & 2 & 6 & 0.080 & 1.01 & 0.114 & 0.086 & 0.058 & 11.0 & 0.120 & 0.066 & 0.720 & 2.16 & 10.7 & 0.432 & 0.099 & 0.164 & 0.649 & 3.21 & 0.122 & 0.194 \\
\hline SB3-03 & k13LM12 & 4 & 2 & 7 & 0.047 & 0.611 & 0.071 & 0.068 & 0.032 & 6.20 & 0.065 & 0.030 & 0.009 & 1.28 & 8.73 & 0.261 & 0.053 & 0.090 & 0.001 & 1.84 & 0.065 & 0.110 \\
\hline SB3-22 & k12LM22 & 4 & 2 & 8 & 0.073 & 0.988 & 0.106 & 0.097 & 0.051 & 10.1 & 0.125 & 0.058 & 0.028 & 1.97 & 13.7 & 0.400 & 0.089 & 0.151 & 0.585 & 2.93 & 0.110 & 0.177 \\
\hline SB3-37 & k10LM12 & 4 & 2 & 9 & 0.050 & 0.727 & 0.082 & 0.059 & 0.039 & 7.48 & 0.086 & 0.042 & 0.015 & 1.48 & 7.35 & 0.303 & 0.063 & 0.107 & 0.433 & 2.17 & 0.081 & 0.128 \\
\hline SB3-37 & k10LM22 & 4 & 2 & 10 & 0.050 & 0.715 & 0.078 & 0.058 & 0.039 & 7.32 & 0.080 & 0.042 & 0.015 & 1.49 & 7.30 & 0.306 & 0.065 & 0.107 & 0.433 & 2.18 & 0.079 & 0.128 \\
\hline SB3-09 & k24LM22 & 4 & 2 & 11 & 0.052 & 0.722 & 0.083 & 0.070 & 0.043 & 7.23 & 0.082 & 0.038 & 0.014 & 1.52 & 8.44 & 0.312 & 0.066 & 0.112 & 0.001 & 2.24 & 0.079 & 0.130 \\
\hline SB3-10 & k21LM12 & 4 & 2 & 12 & 0.084 & 1.09 & 0.124 & 0.101 & 0.062 & 11.1 & 0.147 & 0.059 & 0.733 & 2.19 & 12.0 & 0.457 & 0.102 & 0.172 & 0.000 & 3.31 & 0.125 & 0.206 \\
\hline Batch 1 & BCHLM422 & 4 & 2 & 13 & 0.125 & 0.915 & $<0.010$ & 0.069 & 0.300 & 9.06 & 3.03 & $<0.010$ & 0.858 & 1.32 & 7.12 & 0.544 & $<0.010$ & $<0.010$ & 0.382 & $<0.100$ & $<0.010$ & 0.054 \\
\hline U std & USTLM422 & 4 & 2 & 14 & $<0.010$ & 0.936 & $<0.010$ & 0.163 & $<0.010$ & 9.94 & 2.53 & $<0.010$ & 0.714 & 2.10 & 9.02 & 0.783 & $<0.010$ & 0.064 & 0.557 & 1.89 & $<0.010$ & $<0.010$ \\
\hline SB3-03 & k13LM22 & 4 & 2 & 15 & 0.049 & 0.614 & 0.070 & 0.077 & 0.033 & 6.56 & 0.058 & 0.032 & 0.010 & 1.34 & 8.92 & 0.275 & 0.061 & 0.095 & 0.001 & 1.91 & 0.067 & 0.114 \\
\hline SB3-14 & k34LM22 & 4 & 2 & 16 & 0.067 & 0.925 & 0.098 & 0.098 & 0.048 & 8.99 & 0.128 & 0.046 & 0.024 & 1.75 & 13.1 & 0.375 & 0.080 & 0.138 & $<0.010$ & 2.67 & 0.102 & 0.175 \\
\hline SB3-02 & k32LM12 & 4 & 2 & 17 & 0.067 & 0.849 & 0.094 & 0.067 & 0.048 & 8.92 & 0.100 & 0.047 & 0.023 & 1.68 & 12.1 & 0.371 & 0.080 & 0.128 & $<0.010$ & 2.53 & 0.105 & 0.151 \\
\hline SB3-27 & k06LM22 & 4 & 2 & 18 & 0.044 & 0.648 & 0.069 & 0.051 & 0.035 & 6.59 & 0.073 & 0.037 & 0.011 & 1.22 & 9.30 & 0.266 & 0.052 & 0.095 & 0.368 & 1.86 & 0.069 & 0.109 \\
\hline SB3-02 & k32LM22 & 4 & 2 & 19 & 0.065 & 0.816 & 0.097 & 0.063 & 0.045 & 8.73 & 0.097 & 0.053 & 0.022 & 1.60 & 11.4 & 0.344 & 0.080 & 0.125 & $<0.010$ & 2.42 & 0.101 & 0.147 \\
\hline SB3-24 & k23LM22 & 4 & 2 & 20 & 0.076 & 0.995 & 0.107 & 0.087 & 0.054 & 10.3 & 0.127 & 0.061 & 0.028 & 1.95 & 13.6 & 0.422 & 0.093 & 0.154 & 0.609 & 2.95 & 0.114 & 0.183 \\
\hline SB3-22 & k12LM12 & 4 & 2 & 21 & 0.072 & 0.983 & 0.105 & 0.095 & 0.052 & 10.1 & 0.126 & 0.058 & 0.028 & 1.92 & 13.3 & 0.401 & 0.091 & 0.150 & 0.585 & 2.89 & 0.109 & 0.177 \\
\hline SB3-24 & k23LM12 & 4 & 2 & 22 & 0.073 & 1.02 & 0.109 & 0.083 & 0.056 & 10.2 & 0.144 & 0.059 & 0.028 & 1.98 & 14.0 & 0.399 & 0.089 & 0.148 & 0.590 & 2.90 & 0.111 & 0.175 \\
\hline SB3-10 & k21LM22 & 4 & 2 & 23 & 0.078 & 1.05 & 0.118 & 0.094 & 0.057 & 10.5 & 0.148 & 0.054 & 0.676 & 2.15 & 11.7 & 0.423 & 0.094 & 0.161 & $<0.010$ & 3.13 & 0.114 & 0.192 \\
\hline SB3-30 & k31LM12 & 4 & 2 & 24 & 0.078 & 1.01 & 0.113 & 0.083 & 0.055 & 11.0 & 0.127 & 0.065 & 0.704 & 2.19 & 10.7 & 0.408 & 0.096 & 0.161 & 0.642 & 3.16 & 0.113 & 0.194 \\
\hline Batch 1 & BCHLM423 & 4 & 2 & 25 & 0.131 & 0.910 & $<0.010$ & 0.074 & 0.308 & 9.11 & 3.08 & $<0.010$ & 0.899 & 1.40 & 7.12 & 0.577 & $<0.010$ & $<0.010$ & 0.406 & $<0.100$ & $<0.010$ & 0.058 \\
\hline U std & USTLM423 & 4 & 2 & 26 & $<0.010$ & 0.917 & $<0.010$ & 0.163 & $<0.010$ & 9.52 & 2.50 & $<0.010$ & 0.713 & 2.19 & 8.79 & 0.779 & $<0.010$ & 0.064 & 0.553 & 1.91 & $<0.010$ & $<0.010$ \\
\hline
\end{tabular}


Immobilization Technology Section

WSRC-TR-2002-00549

Savannah River Technology Center

Rev. 0

Westinghouse Savannah River Company

Table H.2: Measured Elemental Concentrations (wt\%) for the SB3 Phase 1 Glasses Prepared Using Peroxide Fusion

\begin{tabular}{|c|c|c|c|c|c|c|c|c|}
\hline $\begin{array}{c}\text { Glass } \\
\text { ID }\end{array}$ & $\begin{array}{c}\text { SRTC-ML } \\
\text { ID }\end{array}$ & Block & $\begin{array}{l}\text { Sub- } \\
\text { Block }\end{array}$ & $\begin{array}{l}\text { Analytical } \\
\text { Sequence }\end{array}$ & Al & B & $\mathbf{L i}$ & $\mathbf{S i}$ \\
\hline Batch 1 & BCHPF111 & 1 & 1 & 1 & 2.4524 & 2.6452 & 1.9939 & 22.031 \\
\hline $\mathrm{U}$ std & USTPF111 & 1 & 1 & 2 & 1.87 & 2.81 & 1.28 & 22.1 \\
\hline SB3-09 & $\mathrm{k} 24 \mathrm{PF} 21$ & 1 & 1 & 3 & 2.70 & 3.04 & 1.44 & 24.7 \\
\hline SB3-18 & k04PF21 & 1 & 1 & 4 & 3.36 & 1.46 & 1.26 & 21.4 \\
\hline SB3-39 & k26PF21 & 1 & 1 & 5 & 2.29 & 2.27 & 2.55 & 26.4 \\
\hline SB3-18 & k04PF11 & 1 & 1 & 6 & 3.45 & 1.45 & 1.28 & 21.6 \\
\hline SB3-36 & k05PF11 & 1 & 1 & 7 & 3.83 & 1.43 & 1.26 & 19.7 \\
\hline SB3-10 & k21PF11 & 1 & 1 & 8 & 3.69 & 2.37 & 1.13 & 19.3 \\
\hline SB3-39 & $\mathrm{k} 26 \mathrm{PF} 11$ & 1 & 1 & 9 & 2.29 & 2.25 & 2.55 & 26.5 \\
\hline SB3-33 & k16PF21 & 1 & 1 & 10 & 3.02 & 2.16 & 1.53 & 24.9 \\
\hline SB3-35 & k07PF11 & 1 & 1 & 11 & 2.49 & 1.87 & 1.64 & 25.6 \\
\hline SB3-33 & $\mathrm{k} 16 \mathrm{PF} 11$ & 1 & 1 & 12 & 3.14 & 2.12 & 1.50 & 24.3 \\
\hline SB3-22 & $\mathrm{k} 12 \mathrm{PF} 11$ & 1 & 1 & 13 & 3.47 & 1.29 & 0.947 & 20.1 \\
\hline Batch 1 & BCHPF112 & 1 & 1 & 14 & 2.51 & 2.46 & 1.98 & 22.2 \\
\hline $\mathrm{U}$ std & USTPF112 & 1 & 1 & 15 & 1.86 & 2.79 & 1.25 & 22.3 \\
\hline SB3-06 & k02PF11 & 1 & 1 & 16 & 2.87 & 1.59 & 1.92 & 22.9 \\
\hline SB3-28 & k29PF11 & 1 & 1 & 17 & 3.12 & 1.81 & 2.03 & 21.5 \\
\hline SB3-03 & k13PF21 & 1 & 1 & 18 & 2.31 & 3.15 & 1.48 & 25.7 \\
\hline SB3-10 & k21PF21 & 1 & 1 & 19 & 3.78 & 2.41 & 1.14 & 19.5 \\
\hline SB3-36 & k05PF21 & 1 & 1 & 20 & 3.83 & 1.44 & 1.27 & 19.9 \\
\hline SB3-03 & k13PF11 & 1 & 1 & 21 & 2.31 & 3.19 & 1.51 & 25.9 \\
\hline SB3-35 & k07PF21 & 1 & 1 & 22 & 2.48 & 1.90 & 1.68 & 26.0 \\
\hline SB3-06 & k02PF21 & 1 & 1 & 23 & 3.02 & 1.63 & 1.99 & 23.6 \\
\hline SB3-09 & $\mathrm{k} 24 \mathrm{PF} 11$ & 1 & 1 & 24 & 2.72 & 3.09 & 1.46 & 25.3 \\
\hline SB3-22 & k12PF21 & 1 & 1 & 25 & 3.45 & 1.33 & 0.97 & 20.6 \\
\hline SB3-28 & k29PF21 & 1 & 1 & 26 & 3.24 & 1.85 & 2.09 & 21.9 \\
\hline Batch 1 & BCHPF113 & 1 & 1 & 27 & 2.54 & 2.52 & 2.01 & 22.0 \\
\hline $\mathrm{U}$ std & USTPF113 & 1 & 1 & 28 & 1.96 & 2.90 & 1.33 & 23.6 \\
\hline Batch 1 & BCHPF121 & 1 & 2 & 1 & 2.51 & 2.50 & 1.98 & 21.5 \\
\hline U std & USTPF121 & 1 & 2 & 2 & 1.97 & 2.73 & 1.27 & 21.9 \\
\hline SB3-06 & k02PF12 & 1 & 2 & 3 & 2.85 & 1.53 & 1.90 & 22.2 \\
\hline SB3-10 & $\mathrm{k} 21 \mathrm{PF} 22$ & 1 & 2 & 4 & 3.95 & 2.41 & 1.16 & 19.8 \\
\hline SB3-36 & k05PF22 & 1 & 2 & 5 & 3.85 & 1.38 & 1.25 & 19.3 \\
\hline SB3-18 & k04PF22 & 1 & 2 & 6 & 3.39 & 1.38 & 1.24 & 20.9 \\
\hline SB3-39 & k26PF22 & 1 & 2 & 7 & 2.36 & 2.21 & 2.55 & 26.3 \\
\hline SB3-09 & k24PF22 & 1 & 2 & 8 & 2.84 & 2.98 & 1.46 & 24.7 \\
\hline SB3-28 & k29PF22 & 1 & 2 & 9 & 3.23 & 1.76 & 2.05 & 21.0 \\
\hline SB3-39 & k26PF12 & 1 & 2 & 10 & 2.53 & 2.23 & 2.63 & 26.7 \\
\hline SB3-09 & $\mathrm{k} 24 \mathrm{PF} 12$ & 1 & 2 & 11 & 2.68 & 2.95 & 1.43 & 24.5 \\
\hline SB3-03 & k13PF12 & 1 & 2 & 12 & 2.50 & 3.20 & 1.55 & 26.0 \\
\hline SB3-03 & k13PF22 & 1 & 2 & 13 & 2.56 & 3.23 & 1.59 & 26.6 \\
\hline Batch 1 & BCHPF122 & 1 & 2 & 14 & 2.94 & 2.43 & 1.96 & 21.5 \\
\hline $\mathrm{U}$ std & USTPF122 & 1 & 2 & 15 & 2.00 & 2.77 & 1.25 & 22.2 \\
\hline SB3-35 & k07PF22 & 1 & 2 & 16 & 2.46 & 1.83 & 1.62 & 25.5 \\
\hline SB3-06 & k02PF22 & 1 & 2 & 17 & 2.96 & 1.55 & 1.90 & 22.6 \\
\hline SB3-10 & $\mathrm{k} 21 \mathrm{PF} 12$ & 1 & 2 & 18 & 3.74 & 2.30 & 1.10 & 19.2 \\
\hline SB3-35 & k07PF12 & 1 & 2 & 19 & 2.58 & 1.90 & 1.70 & 26.5 \\
\hline SB3-33 & $\mathrm{k} 16 \mathrm{PF} 12$ & 1 & 2 & 20 & 3.44 & 2.09 & 1.50 & 24.6 \\
\hline SB3-18 & k04PF12 & 1 & 2 & 21 & 3.47 & 1.39 & 1.24 & 21.3 \\
\hline SB3-33 & k16PF22 & 1 & 2 & 22 & 3.05 & 2.08 & 1.51 & 24.5 \\
\hline SB3-36 & k05PF12 & 1 & 2 & 23 & 3.89 & 1.38 & 1.24 & 19.6 \\
\hline SB3-22 & k12PF12 & 1 & 2 & 24 & 3.54 & 1.25 & 0.929 & 19.9 \\
\hline SB3-22 & k12PF22 & 1 & 2 & 25 & 3.68 & 1.25 & 0.931 & 20.1 \\
\hline SB3-28 & k29PF12 & 1 & 2 & 26 & 3.20 & 1.73 & 2.00 & 21.2 \\
\hline Batch 1 & BCHPF123 & 1 & 2 & 27 & 2.54 & 2.42 & 1.95 & 21.9 \\
\hline $\mathrm{U}$ std & USTPF123 & 1 & 2 & 28 & 1.95 & 2.72 & 1.27 & 22.6 \\
\hline Batch 1 & BCHPF211 & 2 & 1 & 1 & 2.49 & 2.52 & 1.98 & 21.8 \\
\hline U std & USTPF211 & 2 & 1 & 2 & 1.91 & 2.74 & 1.27 & 21.8 \\
\hline SB3-19 & $\mathrm{k} 18 \mathrm{PF} 21$ & 2 & 1 & 3 & 2.70 & 2.20 & 1.58 & 25.3 \\
\hline SB3-32 & k36PF21 & 2 & 1 & 4 & 4.25 & 1.34 & 1.18 & 19.4 \\
\hline SB3-02 & k32PF21 & 2 & 1 & 5 & 3.16 & 3.49 & 1.31 & 20.5 \\
\hline SB3-07 & k38PF11 & 2 & 1 & 6 & 2.48 & 4.48 & 1.67 & 25.7 \\
\hline
\end{tabular}


Immobilization Technology Section

WSRC-TR-2002-00549

Savannah River Technology Center

Rev. 0

Westinghouse Savannah River Company

Table H.2: Measured Elemental Concentrations (wt\%)

for the SB3 Phase 1 Glasses Prepared Using Peroxide Fusion (continued)

\begin{tabular}{|c|c|c|c|c|c|c|c|c|}
\hline $\begin{array}{c}\text { Glass } \\
\text { ID }\end{array}$ & $\begin{array}{c}\text { SRTC-ML } \\
\text { ID }\end{array}$ & Block & $\begin{array}{l}\text { Sub- } \\
\text { Block }\end{array}$ & $\begin{array}{l}\text { Analytical } \\
\text { Sequence }\end{array}$ & Al & B & $\mathbf{L i}$ & $\mathbf{S i}$ \\
\hline SB3-16 & k27PF11 & 2 & 1 & 7 & 2.80 & 1.59 & 1.99 & 22.7 \\
\hline SB3-04 & k22PF11 & 2 & 1 & 8 & 4.21 & 2.83 & 1.65 & 23.2 \\
\hline SB3-25 & k39PF21 & 2 & 1 & 9 & 2.45 & 1.77 & 2.59 & 26.5 \\
\hline SB3-04 & k22PF21 & 2 & 1 & 10 & 3.29 & 2.53 & 1.23 & 20.9 \\
\hline SB3-12 & k14PF11 & 2 & 1 & 11 & 3.82 & 1.35 & 1.72 & 20.1 \\
\hline SB3-14 & k34PF11 & 2 & 1 & 12 & 3.57 & 1.51 & 1.39 & 21.7 \\
\hline SB3-25 & k39PF11 & 2 & 1 & 13 & 2.43 & 1.73 & 2.56 & 26.6 \\
\hline Batch 1 & BCHPF212 & 2 & 1 & 14 & 2.52 & 2.42 & 1.99 & 22.0 \\
\hline U std & USTPF212 & 2 & 1 & 15 & 2.01 & 2.78 & 1.28 & 22.1 \\
\hline SB3-32 & k36PF11 & 2 & 1 & 16 & 4.64 & 1.39 & 1.25 & 20.3 \\
\hline SB3-16 & k27PF21 & 2 & 1 & 17 & 2.80 & 1.56 & 1.97 & 22.5 \\
\hline SB3-12 & k14PF21 & 2 & 1 & 18 & 3.76 & 1.30 & 1.63 & 19.6 \\
\hline SB3-07 & k38PF21 & 2 & 1 & 19 & 2.40 & 4.35 & 1.61 & 25.2 \\
\hline SB3-02 & k32PF11 & 2 & 1 & 20 & 3.10 & 3.61 & 1.32 & 20.9 \\
\hline SB3-40 & k19PF11 & 2 & 1 & 21 & 4.04 & 1.76 & 2.04 & 20.9 \\
\hline SB3-40 & k19PF21 & 2 & 1 & 22 & 3.92 & 1.73 & 2.00 & 20.7 \\
\hline SB3-20 & k42PF11 & 2 & 1 & 23 & 3.56 & 1.92 & 1.40 & 22.5 \\
\hline SB3-19 & k18PF11 & 2 & 1 & 24 & 2.75 & 2.23 & 1.63 & 25.9 \\
\hline SB3-20 & k42PF21 & 2 & 1 & 25 & 3.35 & 1.82 & 1.33 & 21.0 \\
\hline SB3-14 & k34PF21 & 2 & 1 & 26 & 3.75 & 1.59 & 1.45 & 22.6 \\
\hline Batch 1 & BCHPF213 & 2 & 1 & 27 & 2.63 & 2.45 & 2.02 & 21.9 \\
\hline U std & USTPF213 & 2 & 1 & 28 & 1.94 & 2.70 & 1.26 & 22.3 \\
\hline Batch 1 & BCHPF221 & 2 & 2 & 1 & 2.49 & 2.48 & 1.98 & 21.9 \\
\hline U std & USTPF221 & 2 & 2 & 2 & 1.92 & 2.70 & 1.24 & 21.5 \\
\hline SB3-12 & k14PF22 & 2 & 2 & 3 & 3.62 & 1.29 & 1.56 & 18.7 \\
\hline SB3-25 & k39PF22 & 2 & 2 & 4 & 2.28 & 1.68 & 2.40 & 25.4 \\
\hline SB3-04 & k22PF22 & 2 & 2 & 5 & 3.17 & 2.47 & 1.18 & 20.3 \\
\hline SB3-20 & k42PF12 & 2 & 2 & 6 & 3.32 & 1.80 & 1.30 & 20.8 \\
\hline SB3-12 & k14PF12 & 2 & 2 & 7 & 3.69 & 1.32 & 1.65 & 19.2 \\
\hline SB3-25 & k39PF12 & 2 & 2 & 8 & 2.32 & 1.68 & 2.44 & 25.5 \\
\hline SB3-19 & k18PF22 & 2 & 2 & 9 & 2.58 & 2.10 & 1.51 & 24.4 \\
\hline SB3-20 & k42PF22 & 2 & 2 & 10 & 3.28 & 1.80 & 1.30 & 20.5 \\
\hline SB3-02 & k32PF12 & 2 & 2 & 11 & 2.98 & 3.47 & 1.28 & 20.0 \\
\hline SB3-02 & k32PF22 & 2 & 2 & 12 & 3.22 & 3.43 & 1.29 & 20.0 \\
\hline SB3-07 & k38PF12 & 2 & 2 & 13 & 2.33 & 4.24 & 1.57 & 24.3 \\
\hline Batch 1 & BCHPF222 & 2 & 2 & 14 & 2.54 & 2.44 & 1.98 & 21.5 \\
\hline U std & USTPF222 & 2 & 2 & 15 & 2.00 & 2.78 & 1.27 & 21.9 \\
\hline SB3-14 & k34PF22 & 2 & 2 & 16 & 3.51 & 1.54 & 1.36 & 21.2 \\
\hline SB3-04 & k22PF12 & 2 & 2 & 17 & 4.01 & 2.71 & 1.53 & 22.5 \\
\hline SB3-40 & k19PF22 & 2 & 2 & 18 & 3.75 & 1.69 & 1.92 & 20.0 \\
\hline SB3-40 & k19PF12 & 2 & 2 & 19 & 3.75 & 1.65 & 1.89 & 19.7 \\
\hline SB3-32 & k36PF12 & 2 & 2 & 20 & 4.48 & 1.31 & 1.19 & 19.4 \\
\hline SB3-16 & k27PF12 & 2 & 2 & 21 & 2.71 & 1.52 & 1.93 & 22.1 \\
\hline SB3-07 & k38PF22 & 2 & 2 & 22 & 2.31 & 4.21 & 1.54 & 24.3 \\
\hline SB3-16 & k27PF22 & 2 & 2 & 23 & 2.72 & 1.56 & 1.92 & 22.2 \\
\hline SB3-32 & k36PF22 & 2 & 2 & 24 & 4.25 & 1.32 & 1.18 & 19.4 \\
\hline SB3-14 & k34PF12 & 2 & 2 & 25 & 3.50 & 1.50 & 1.36 & 21.2 \\
\hline SB3-19 & k18PF12 & 2 & 2 & 26 & 2.64 & 2.15 & 1.57 & 24.8 \\
\hline Batch 1 & BCHPF223 & 2 & 2 & 27 & 2.59 & 2.44 & 2.00 & 21.8 \\
\hline U std & USTPF223 & 2 & 2 & 28 & 1.93 & 2.71 & 1.27 & 22.2 \\
\hline Batch 1 & BCHPF311 & 3 & 1 & 1 & 2.48 & 2.57 & 1.98 & 22.4 \\
\hline U std & USTPF311 & 3 & 1 & 2 & 2.04 & 2.80 & 1.27 & 22.2 \\
\hline SB3-27 & k06PF11 & 3 & 1 & 3 & 2.34 & 2.16 & 2.43 & 25.1 \\
\hline SB3-34 & k09PF21 & 3 & 1 & 4 & 4.14 & 1.70 & 1.18 & 19.2 \\
\hline SB3-23 & k17PF21 & 3 & 1 & 5 & 2.55 & 1.68 & 1.48 & 25.5 \\
\hline SB3-15 & k37PF11 & 3 & 1 & 6 & 2.40 & 1.64 & 2.05 & 23.3 \\
\hline SB3-31 & k08PF11 & 3 & 1 & 7 & 3.30 & 1.63 & 1.44 & 23.9 \\
\hline SB3-08 & k30PF21 & 3 & 1 & 8 & 3.30 & 3.30 & 1.18 & 18.9 \\
\hline SB3-21 & k25PF21 & 3 & 1 & 9 & 2.48 & 1.64 & 1.17 & 24.6 \\
\hline SB3-29 & k11PF11 & 3 & 1 & 10 & 2.34 & 1.76 & 2.18 & 25.0 \\
\hline SB3-21 & k25PF11 & 3 & 1 & 11 & 2.47 & 1.62 & 1.18 & 24.2 \\
\hline SB3-23 & k17PF11 & 3 & 1 & 12 & 2.45 & 1.64 & 1.45 & 25.0 \\
\hline
\end{tabular}


Immobilization Technology Section

WSRC-TR-2002-00549

Savannah River Technology Center

Rev. 0

Westinghouse Savannah River Company

Table H.2: Measured Elemental Concentrations (wt\%)

for the SB3 Phase 1 Glasses Prepared Using Peroxide Fusion (continued)

\begin{tabular}{|c|c|c|c|c|c|c|c|c|}
\hline $\begin{array}{c}\text { Glass } \\
\text { ID }\end{array}$ & $\begin{array}{c}\text { SRTC-ML } \\
\text { ID }\end{array}$ & Block & $\begin{array}{l}\text { Sub- } \\
\text { Block }\end{array}$ & $\begin{array}{l}\text { Analytical } \\
\text { Sequence }\end{array}$ & Al & B & $\mathbf{L i}$ & $\mathbf{S i}$ \\
\hline Batch 1 & BCHPF312 & 3 & 1 & 13 & 2.46 & 2.44 & 1.96 & 21.5 \\
\hline U std & USTPF312 & 3 & 1 & 14 & 1.97 & 2.79 & 1.26 & 21.9 \\
\hline SB3-24 & k23PF21 & 3 & 1 & 15 & 3.51 & 1.34 & 1.17 & 20.0 \\
\hline SB3-08 & k30PF11 & 3 & 1 & 16 & 3.36 & 3.34 & 1.21 & 19.3 \\
\hline SB3-24 & k23PF11 & 3 & 1 & 17 & 3.58 & 1.33 & 1.16 & 20.0 \\
\hline SB3-05 & k35PF11 & 3 & 1 & 18 & 2.64 & 1.63 & 2.00 & 23.0 \\
\hline SB3-15 & k37PF21 & 3 & 1 & 19 & 2.45 & 1.68 & 2.09 & 23.9 \\
\hline SB3-27 & k06PF21 & 3 & 1 & 20 & 2.33 & 2.14 & 2.47 & 25.1 \\
\hline SB3-05 & k35PF21 & 3 & 1 & 21 & 2.54 & 1.88 & 2.30 & 25.6 \\
\hline SB3-29 & k11PF21 & 3 & 1 & 22 & 2.42 & 1.75 & 2.21 & 25.0 \\
\hline SB3-31 & k08PF21 & 3 & 1 & 23 & 3.22 & 1.63 & 1.45 & 23.8 \\
\hline SB3-34 & k09PF11 & 3 & 1 & 24 & 4.19 & 1.69 & 1.22 & 19.6 \\
\hline Batch 1 & BCHPF313 & 3 & 1 & 25 & 2.45 & 2.51 & 2.00 & 23.0 \\
\hline U std & USTPF313 & 3 & 1 & 26 & 1.98 & 2.83 & 1.31 & 22.7 \\
\hline Batch 1 & BCHPF321 & 3 & 2 & 1 & 2.42 & 2.45 & 1.97 & 22.6 \\
\hline U std & USTPF321 & 3 & 2 & 2 & 1.92 & 2.78 & 1.29 & 22.1 \\
\hline SB3-34 & k09PF22 & 3 & 2 & 3 & 4.04 & 1.69 & 1.19 & 19.1 \\
\hline SB3-23 & k17PF22 & 3 & 2 & 4 & 2.48 & 1.68 & 1.48 & 25.7 \\
\hline SB3-21 & $\mathrm{k} 25 \mathrm{PF} 22$ & 3 & 2 & 5 & 2.53 & 1.65 & 1.20 & 25.0 \\
\hline SB3-31 & k08PF22 & 3 & 2 & 6 & 3.18 & 1.62 & 1.44 & 23.9 \\
\hline SB3-27 & k06PF22 & 3 & 2 & 7 & 2.29 & 2.11 & 2.43 & 25.1 \\
\hline SB3-29 & k11PF12 & 3 & 2 & 8 & 2.38 & 1.77 & 2.21 & 25.4 \\
\hline SB3-05 & k35PF22 & 3 & 2 & 9 & 2.53 & 1.85 & 2.27 & 25.6 \\
\hline SB3-08 & k30PF12 & 3 & 2 & 10 & 3.37 & 3.34 & 1.22 & 19.7 \\
\hline SB3-24 & $\mathrm{k} 23 \mathrm{PF} 12$ & 3 & 2 & 11 & 3.63 & 1.33 & 1.18 & 20.3 \\
\hline SB3-08 & k30PF22 & 3 & 2 & 12 & 3.36 & 3.35 & 1.21 & 19.5 \\
\hline Batch 1 & BCHPF322 & 3 & 2 & 13 & 2.47 & 2.48 & 1.99 & 22.8 \\
\hline U std & USTPF322 & 3 & 2 & 14 & 2.10 & 2.81 & 1.28 & 22.3 \\
\hline SB3-05 & k35PF12 & 3 & 2 & 15 & 2.71 & 1.68 & 2.01 & 23.6 \\
\hline SB3-15 & k37PF12 & 3 & 2 & 16 & 2.44 & 1.67 & 2.07 & 23.9 \\
\hline SB3-23 & k17PF12 & 3 & 2 & 17 & 2.51 & 1.69 & 1.47 & 25.8 \\
\hline SB3-15 & k37PF22 & 3 & 2 & 18 & 2.47 & 1.70 & 2.07 & 24.6 \\
\hline SB3-29 & k11PF22 & 3 & 2 & 19 & 2.47 & 1.78 & 2.22 & 25.7 \\
\hline SB3-34 & k09PF12 & 3 & 2 & 20 & 4.21 & 1.69 & 1.22 & 19.7 \\
\hline SB3-27 & k06PF12 & 3 & 2 & 21 & 2.41 & 2.18 & 2.48 & 25.9 \\
\hline SB3-24 & k23PF22 & 3 & 2 & 22 & 3.54 & 1.34 & 1.19 & 20.8 \\
\hline SB3-31 & k08PF12 & 3 & 2 & 23 & 3.42 & 1.67 & 1.49 & 24.8 \\
\hline SB3-21 & k25PF12 & 3 & 2 & 24 & 2.57 & 1.67 & 1.21 & 25.1 \\
\hline Batch 1 & BCHPF323 & 3 & 2 & 25 & 2.53 & 2.49 & 2.01 & 22.4 \\
\hline U std & USTPF323 & 3 & 2 & 26 & 2.05 & 2.83 & 1.32 & 23.1 \\
\hline Batch 1 & BCHPF411 & 4 & 1 & 1 & 2.47 & 2.53 & 1.97 & 22.5 \\
\hline U std & USTPF411 & 4 & 1 & 2 & 2.08 & 2.72 & 1.27 & 22.1 \\
\hline SB3-26 & k40PF11 & 4 & 1 & 3 & 3.50 & 1.41 & 2.00 & 21.2 \\
\hline SB3-37 & k10PF21 & 4 & 1 & 4 & 2.59 & 1.67 & 2.43 & 25.5 \\
\hline SB3-11 & k28PF11 & 4 & 1 & 5 & 2.57 & 1.77 & 2.21 & 25.7 \\
\hline SB3-01 & k41PF11 & 4 & 1 & 6 & 2.32 & 4.10 & 1.50 & 23.7 \\
\hline SB3-11 & k28PF21 & 4 & 1 & 7 & 2.51 & 1.79 & 2.27 & 25.3 \\
\hline SB3-26 & k40PF21 & 4 & 1 & 8 & 3.32 & 1.34 & 1.93 & 20.7 \\
\hline SB3-30 & k31PF11 & 4 & 1 & 9 & 3.77 & 1.35 & 1.72 & 20.1 \\
\hline SB3-17 & k01PF11 & 4 & 1 & 10 & 2.77 & 1.71 & 1.57 & 24.9 \\
\hline SB3-01 & k41PF21 & 4 & 1 & 11 & 2.29 & 4.08 & 1.49 & 23.7 \\
\hline SB3-42 & k20PF21 & 4 & 1 & 12 & 3.72 & 1.47 & 2.78 & 20.4 \\
\hline Batch 1 & BCHPF412 & 4 & 1 & 13 & 2.49 & 2.42 & 1.98 & 22.6 \\
\hline U std & USTPF412 & 4 & 1 & 14 & 2.04 & 2.78 & 1.29 & 21.9 \\
\hline SB3-38 & k03PF21 & 4 & 1 & 15 & 3.89 & 1.38 & 1.93 & 20.9 \\
\hline SB3-38 & k03PF11 & 4 & 1 & 16 & 3.80 & 1.31 & 1.90 & 20.2 \\
\hline SB3-17 & k01PF21 & 4 & 1 & 17 & 2.71 & 1.73 & 1.57 & 26.0 \\
\hline SB3-13 & k33PF21 & 4 & 1 & 18 & 2.54 & 1.82 & 1.66 & 25.7 \\
\hline SB3-41 & k15PF11 & 4 & 1 & 19 & 2.40 & 1.82 & 2.68 & 24.9 \\
\hline SB3-37 & k10PF11 & 4 & 1 & 20 & 2.65 & 1.67 & 2.47 & 25.8 \\
\hline SB3-41 & k15PF21 & 4 & 1 & 21 & 2.50 & 1.82 & 2.68 & 24.9 \\
\hline SB3-42 & k20PF11 & 4 & 1 & 22 & 3.80 & 1.48 & 2.89 & 20.8 \\
\hline
\end{tabular}


Immobilization Technology Section

WSRC-TR-2002-00549

Savannah River Technology Center

Rev. 0

Westinghouse Savannah River Company

Table H.2: Measured Elemental Concentrations (wt\%)

for the SB3 Phase 1 Glasses Prepared Using Peroxide Fusion (continued)

\begin{tabular}{|c|c|c|c|c|c|c|c|c|}
\hline $\begin{array}{c}\text { Glass } \\
\text { ID }\end{array}$ & $\begin{array}{c}\text { SRTC-ML } \\
\text { ID }\end{array}$ & Block & $\begin{array}{l}\text { Sub- } \\
\text { Block }\end{array}$ & $\begin{array}{l}\text { Analytical } \\
\text { Sequence }\end{array}$ & Al & B & $\mathbf{L i}$ & Si \\
\hline SB3-30 & k31PF21 & 4 & 1 & 23 & 4.36 & 1.36 & 1.76 & 20.2 \\
\hline SB3-13 & k33PF11 & 4 & 1 & 24 & 2.54 & 1.81 & 1.68 & 26.0 \\
\hline Batch 1 & BCHPF413 & 4 & 1 & 25 & 2.57 & 2.34 & 1.99 & 21.2 \\
\hline U std & USTPF413 & 4 & 1 & 26 & 1.94 & 2.62 & 1.27 & 21.6 \\
\hline Batch 1 & BCHPF421 & 4 & 2 & 1 & 2.49 & 2.51 & 1.99 & 22.3 \\
\hline U std & USTPF421 & 4 & 2 & 2 & 1.96 & 2.73 & 1.29 & 22.1 \\
\hline SB3-42 & k20PF22 & 4 & 2 & 3 & 3.71 & 1.49 & 2.80 & 20.4 \\
\hline SB3-30 & k31PF12 & 4 & 2 & 4 & 3.70 & 1.34 & 1.72 & 19.8 \\
\hline SB3-01 & k41PF22 & 4 & 2 & 5 & 2.31 & 4.09 & 1.51 & 23.7 \\
\hline SB3-13 & k33PF22 & 4 & 2 & 6 & 2.50 & 1.82 & 1.66 & 25.5 \\
\hline SB3-38 & k03PF22 & 4 & 2 & 7 & 3.86 & 1.34 & 1.97 & 20.7 \\
\hline SB3-01 & k41PF12 & 4 & 2 & 8 & 2.30 & 4.05 & 1.52 & 23.8 \\
\hline SB3-37 & k10PF22 & 4 & 2 & 9 & 2.60 & 1.66 & 2.47 & 25.8 \\
\hline SB3-37 & k10PF12 & 4 & 2 & 10 & 2.62 & 1.65 & 2.44 & 25.7 \\
\hline SB3-11 & k28PF22 & 4 & 2 & 11 & 2.51 & 1.76 & 2.28 & 25.5 \\
\hline SB3-26 & k40PF12 & 4 & 2 & 12 & 3.47 & 1.36 & 2.01 & 21.3 \\
\hline Batch 1 & BCHPF422 & 4 & 2 & 13 & 2.50 & 2.38 & 1.99 & 22.7 \\
\hline U std & USTPF422 & 4 & 2 & 14 & 1.99 & 2.75 & 1.29 & 22.3 \\
\hline SB3-30 & k31PF22 & 4 & 2 & 15 & 4.28 & 1.39 & 1.74 & 20.5 \\
\hline SB3-38 & k03PF12 & 4 & 2 & 16 & 3.77 & 1.29 & 1.91 & 20.5 \\
\hline SB3-42 & k20PF12 & 4 & 2 & 17 & 3.71 & 1.47 & 2.84 & 21.0 \\
\hline SB3-17 & k01PF12 & 4 & 2 & 18 & 2.81 & 1.71 & 1.60 & 25.3 \\
\hline SB3-41 & k15PF22 & 4 & 2 & 19 & 2.52 & 1.82 & 2.69 & 25.4 \\
\hline SB3-26 & k40PF22 & 4 & 2 & 20 & 3.36 & 1.34 & 1.96 & 21.2 \\
\hline SB3-41 & k15PF12 & 4 & 2 & 21 & 2.41 & 1.81 & 2.68 & 25.2 \\
\hline SB3-17 & k01PF22 & 4 & 2 & 22 & 2.71 & 1.72 & 1.57 & 26.2 \\
\hline SB3-13 & k33PF12 & 4 & 2 & 23 & 2.53 & 1.81 & 1.67 & 26.3 \\
\hline SB3-11 & k28PF12 & 4 & 2 & 24 & 2.63 & 1.77 & 2.26 & 26.3 \\
\hline Batch 1 & BCHPF423 & 4 & 2 & 25 & 2.53 & 2.45 & 2.01 & 22.5 \\
\hline U std & USTPF423 & 4 & 2 & 26 & 2.05 & 2.76 & 1.33 & 22.9 \\
\hline
\end{tabular}


Table H.3: Re-Measurements Generated by the SRTC-ML (wt\%)

\begin{tabular}{||c|c|c|c|c||}
\hline Glass & \multicolumn{3}{|c|}{ Element } & Oxide \\
\hline & & Remeasured \#1 & Remeasured \#2 & Average \\
\hline SB3-4 & $\mathrm{Al}$ & 3.29 & 3.29 & 6.22 \\
\hline SB3-8 & $\mathrm{Cr}$ & 0.134 & 0.154 & 0.21 \\
\hline SB3-9 & $\mathrm{Fe}$ & 7.19 & 7.34 & 10.39 \\
\hline SB3-10 & $\mathrm{Mg}$ & 0.797 & 0.751 & 1.28 \\
\hline SB3-14 & $\mathrm{Cr}$ & 0.095 & 0.095 & 0.139 \\
\hline SB3-17 & $\mathrm{Ti}$ & 0.367 & 0.348 & 0.60 \\
\hline SB3-18 & $\mathrm{Fe}$ & 8.06 & 8.24 & 11.65 \\
\hline SB3-21 & $\mathrm{Cr}$ & 0.055 & 0.051 & 0.08 \\
\hline SB3-21 & $\mathrm{Fe}$ & 7.02 & 6.57 & 9.72 \\
\hline SB3-23 & $\mathrm{Cr}$ & 0.050 & 0.051 & 0.07 \\
\hline SB3-29 & $\mathrm{La}$ & 0.040 & 0.041 & 0.047 \\
\hline SB3-29 & $\mathrm{Ce}$ & 0.071 & 0.071 & 0.09 \\
\hline SB3-29 & $\mathrm{Cr}$ & 0.037 & 0.037 & 0.054 \\
\hline SB3-30 & $\mathrm{Al}$ & 3.80 & 3.76 & 7.14 \\
\hline SB3-31 & $\mathrm{Cr}$ & 0.058 & 0.059 & 0.09 \\
\hline SB3-32 & $\mathrm{Al}$ & 4.39 & 4.25 & 8.16 \\
\hline SB3-32 & $\mathrm{Ca}$ & 1.15 & 1.13 & 1.60 \\
\hline SB3-32 & $\mathrm{Na}$ & 12.4 & 11.4 & 16.07 \\
\hline SB3-38 & $\mathrm{Zr}$ & 0.330 & 0.323 & 0.441 \\
\hline SB3-39 & $\mathrm{Cr}$ & 0.065 & 0.066 & 0.10 \\
\hline SB3-42 & $\mathrm{Fe}$ & 7.91 & 7.93 & 11.33 \\
\hline SB3-42 & $\mathrm{Li}$ & 2.80 & 2.84 & 6.06 \\
\hline \hline
\end{tabular}


Table H.4: Average Measured and Bias-Corrected Chemical Compositions Versus Targeted Compositions by Oxide by Glass Number

(Batch 1: 100; 101-U std)

\begin{tabular}{|c|c|c|c|c|c|c|c|c|c|}
\hline Glass \# & Glass ID & Oxide & $\begin{array}{c}\text { Measured } \\
(\mathbf{w t} \%)\end{array}$ & $\begin{array}{c}\text { Measured } \\
\text { Bias-Corrected } \\
(w t \%)\end{array}$ & $\begin{array}{c}\text { Targeted } \\
\text { (wt\%) }\end{array}$ & $\begin{array}{c}\text { Diff of } \\
\text { Measured }\end{array}$ & $\begin{array}{c}\text { Diff of } \\
\text { Meas BC }\end{array}$ & $\begin{array}{l}\% \text { Diff of } \\
\text { Measured }\end{array}$ & $\begin{array}{l}\% \text { Diff of } \\
\text { Meas BC }\end{array}$ \\
\hline 1 & SB3-01 & $\mathrm{A} 12 \mathrm{O} 3(\mathrm{wt} \%)$ & 4.3553 & 4.4817 & 4.2500 & 0.1053 & 0.2317 & $2.5 \%$ & $5.5 \%$ \\
\hline 1 & SB3-01 & $\mathrm{B} 2 \mathrm{O} 3(\mathrm{wt} \%)$ & 13.1372 & 13.0133 & 13.6000 & -0.4628 & -0.5867 & $-3.4 \%$ & $-4.3 \%$ \\
\hline 1 & SB3-01 & $\mathrm{BaO}(\mathrm{wt} \%)$ & 0.0458 & 0.0549 & 0.0590 & -0.0132 & -0.0041 & $-22.4 \%$ & $-6.9 \%$ \\
\hline 1 & SB3-01 & $\mathrm{CaO}(\mathrm{wt} \%)$ & 0.9126 & 0.8415 & 0.8450 & 0.0676 & -0.0035 & $8.0 \%$ & $-0.4 \%$ \\
\hline 1 & SB3-01 & $\mathrm{Ce} 2 \mathrm{O} 3(\mathrm{wt} \%)$ & 0.0741 & 0.0741 & 0.0830 & -0.0089 & -0.0089 & $-10.7 \%$ & $-10.7 \%$ \\
\hline 1 & SB3-01 & $\mathrm{Cr} 2 \mathrm{O} 3(\mathrm{wt} \%)$ & 0.0650 & 0.0772 & 0.0880 & -0.0230 & -0.0108 & $-26.1 \%$ & $-12.3 \%$ \\
\hline 1 & SB3-01 & $\mathrm{CuO}(\mathrm{wt} \%)$ & 0.0498 & 0.0531 & 0.0470 & 0.0028 & 0.0061 & $5.9 \%$ & $13.0 \%$ \\
\hline 1 & SB3-01 & $\mathrm{Fe} 2 \mathrm{O} 3(\mathrm{wt} \%)$ & 9.9936 & 9.6767 & 9.4550 & 0.5386 & 0.2217 & $5.7 \%$ & $2.3 \%$ \\
\hline 1 & SB3-01 & $\mathrm{K} 2 \mathrm{O}(\mathrm{wt} \%)$ & 0.0726 & 0.0646 & 0.1010 & -0.0284 & -0.0364 & $-28.1 \%$ & $-36.1 \%$ \\
\hline 1 & SB3-01 & $\mathrm{La} 2 \mathrm{O} 3($ wt $\%)$ & 0.0358 & 0.0358 & 0.0480 & -0.0122 & -0.0122 & $-25.5 \%$ & $-25.5 \%$ \\
\hline 1 & SB3-01 & $\mathrm{Li} 2 \mathrm{O}(\mathrm{wt} \%)$ & 3.2401 & 3.3523 & 3.4010 & -0.1609 & -0.0487 & $-4.7 \%$ & $-1.4 \%$ \\
\hline 1 & SB3-01 & $\mathrm{MgO}(\mathrm{wt} \%)$ & 0.0017 & 0.0018 & 0.0440 & -0.0423 & -0.0422 & $-96.2 \%$ & $-95.9 \%$ \\
\hline 1 & SB3-01 & $\mathrm{MnO}(\mathrm{wt} \%)$ & 1.5559 & 1.7213 & 1.6850 & -0.1291 & 0.0363 & $-7.7 \%$ & $2.2 \%$ \\
\hline 1 & SB3-01 & $\mathrm{Na} 2 \mathrm{O}(\mathrm{wt} \%)$ & 11.3333 & 10.9768 & 11.3640 & -0.0307 & -0.3872 & $-0.3 \%$ & $-3.4 \%$ \\
\hline 1 & SB3-01 & $\mathrm{NiO}(\mathrm{wt} \%)$ & 0.3283 & 0.3829 & 0.3780 & -0.0497 & 0.0049 & $-13.1 \%$ & $1.3 \%$ \\
\hline 1 & SB3-01 & $\mathrm{PbO}(\mathrm{wt} \%)$ & 0.0566 & 0.0566 & 0.0710 & -0.0144 & -0.0144 & $-20.3 \%$ & $-20.3 \%$ \\
\hline 1 & SB3-01 & $\mathrm{SiO} 2(\mathrm{wt} \%)$ & 50.7549 & 53.4329 & 51.7850 & -1.0301 & 1.6479 & $-2.0 \%$ & $3.2 \%$ \\
\hline 1 & SB3-01 & ThO2 (wt $\%)$ & 0.0930 & 0.0930 & 0.0340 & 0.0590 & 0.0590 & $173.6 \%$ & $173.6 \%$ \\
\hline 1 & SB3-01 & $\mathrm{TiO} 2(\mathrm{wt} \%)$ & 0.0083 & 0.0092 & 0.0000 & 0.0083 & 0.0092 & & \\
\hline 1 & SB3-01 & $\mathrm{U} 3 \mathrm{O} 8(\mathrm{wt} \%)$ & 2.3613 & 2.4708 & 2.3920 & -0.0307 & 0.0788 & $-1.3 \%$ & $3.3 \%$ \\
\hline 1 & SB3-01 & $\mathrm{ZnO}(\mathrm{wt} \%)$ & 0.0722 & 0.0722 & 0.0970 & -0.0248 & -0.0248 & $-25.6 \%$ & $-25.6 \%$ \\
\hline 1 & SB3-01 & $\mathrm{ZrO} 2(\mathrm{wt} \%)$ & 0.1408 & 0.1408 & 0.1740 & -0.0332 & -0.0332 & $-19.1 \%$ & $-19.1 \%$ \\
\hline 1 & SB3-01 & Sum of Oxides & 98.6882 & 101.0834 & 100.0010 & -1.3128 & 1.0824 & $-1.3 \%$ & $1.1 \%$ \\
\hline 2 & SB3-02 & $\mathrm{A} 12 \mathrm{O} 3(\mathrm{wt} \%)$ & 5.8858 & 5.9732 & 5.7110 & 0.1748 & 0.2622 & $3.1 \%$ & $4.6 \%$ \\
\hline 2 & SB3-02 & $\mathrm{B} 2 \mathrm{O} 3(\mathrm{wt} \%)$ & 11.2697 & 11.0721 & 11.4000 & -0.1304 & -0.3279 & $-1.1 \%$ & $-2.9 \%$ \\
\hline 2 & SB3-02 & $\mathrm{BaO}(\mathrm{wt} \%)$ & 0.0720 & 0.0786 & 0.0790 & -0.0070 & -0.0004 & $-8.8 \%$ & $-0.4 \%$ \\
\hline 2 & SB3-02 & $\mathrm{CaO}(\mathrm{wt} \%)$ & 1.1736 & 1.1183 & 1.1360 & 0.0376 & -0.0177 & $3.3 \%$ & $-1.6 \%$ \\
\hline 2 & SB3-02 & $\mathrm{Ce} 2 \mathrm{O} 3(\mathrm{wt} \%)$ & 0.1119 & 0.1119 & 0.1120 & -0.0001 & -0.0001 & $-0.1 \%$ & $-0.1 \%$ \\
\hline 2 & SB3-02 & $\mathrm{Cr} 2 \mathrm{O} 3(\mathrm{wt} \%)$ & 0.0939 & 0.0999 & 0.1180 & -0.0241 & -0.0181 & $-20.4 \%$ & $-15.3 \%$ \\
\hline 2 & SB3-02 & $\mathrm{CuO}(\mathrm{wt} \%)$ & 0.0595 & 0.0624 & 0.0630 & -0.0035 & -0.0006 & $-5.6 \%$ & $-1.0 \%$ \\
\hline 2 & SB3-02 & $\mathrm{Fe} 2 \mathrm{O} 3(\mathrm{wt} \%)$ & 12.3383 & 12.5287 & 12.7050 & -0.3667 & -0.1763 & $-2.9 \%$ & $-1.4 \%$ \\
\hline 2 & SB3-02 & $\mathrm{K} 2 \mathrm{O}(\mathrm{wt} \%)$ & 0.1147 & 0.1027 & 0.1360 & -0.0213 & -0.0333 & $-15.6 \%$ & $-24.5 \%$ \\
\hline 2 & SB3-02 & $\mathrm{La} 2 \mathrm{O} 3$ (wt $\%)$ & 0.0607 & 0.0607 & 0.0650 & -0.0043 & -0.0043 & $-6.6 \%$ & $-6.6 \%$ \\
\hline 2 & SB3-02 & $\mathrm{Li} 2 \mathrm{O}(\mathrm{wt} \%)$ & 2.7988 & 2.8908 & 2.8510 & -0.0522 & 0.0398 & $-1.8 \%$ & $1.4 \%$ \\
\hline 2 & SB3-02 & $\mathrm{MgO}(\mathrm{wt} \%)$ & 0.0340 & 0.0340 & 0.0600 & -0.0260 & -0.0260 & $-43.3 \%$ & $-43.3 \%$ \\
\hline 2 & SB3-02 & $\mathrm{MnO}(\mathrm{wt} \%)$ & 2.1337 & 2.0541 & 2.2640 & -0.1303 & -0.2099 & $-5.8 \%$ & $-9.3 \%$ \\
\hline 2 & SB3-02 & $\mathrm{Na} 2 \mathrm{O}(\mathrm{wt} \%)$ & 15.9064 & 14.8729 & 15.2700 & 0.6364 & -0.3971 & $4.2 \%$ & $-2.6 \%$ \\
\hline 2 & SB3-02 & $\mathrm{NiO}(\mathrm{wt} \%)$ & 0.4505 & 0.4915 & 0.5080 & -0.0575 & -0.0165 & $-11.3 \%$ & $-3.3 \%$ \\
\hline 2 & SB3-02 & $\mathrm{PbO}(\mathrm{wt} \%)$ & 0.0840 & 0.0840 & 0.0950 & -0.0110 & -0.0110 & $-11.6 \%$ & $-11.6 \%$ \\
\hline 2 & SB3-02 & $\mathrm{SiO} 2(\mathrm{wt} \%)$ & 43.5348 & 46.8415 & 43.8050 & -0.2702 & 3.0365 & $-0.6 \%$ & $6.9 \%$ \\
\hline 2 & SB3-02 & ThO2 (wt $\%)$ & 0.1448 & 0.1448 & 0.0450 & 0.0998 & 0.0998 & $221.8 \%$ & $221.8 \%$ \\
\hline 2 & SB3-02 & $\mathrm{TiO} 2$ (wt $\%)$ & 0.0083 & 0.0088 & 0.0000 & 0.0083 & 0.0088 & & \\
\hline 2 & SB3-02 & $\mathrm{U} 3 \mathrm{O} 8(\mathrm{wt} \%)$ & 2.9922 & 3.1520 & 3.2140 & -0.2218 & -0.0620 & $-6.9 \%$ & $-1.9 \%$ \\
\hline 2 & SB3-02 & $\mathrm{ZnO}(\mathrm{wt} \%)$ & 0.1232 & 0.1232 & 0.1300 & -0.0068 & -0.0068 & $-5.2 \%$ & $-5.2 \%$ \\
\hline 2 & SB3-02 & $\mathrm{ZrO} 2(\mathrm{wt} \%)$ & 0.2009 & 0.2009 & 0.2340 & -0.0331 & -0.0331 & $-14.1 \%$ & $-14.1 \%$ \\
\hline 2 & SB3-02 & Sum of Oxides & 99.5916 & 102.1069 & 100.0010 & -0.4094 & 2.1059 & $-0.4 \%$ & $2.1 \%$ \\
\hline 3 & SB3-03 & $\mathrm{Al} 2 \mathrm{O} 3$ (wt $\%$ ) & 4.5726 & 4.5689 & 4.2500 & 0.3226 & 0.3189 & $7.6 \%$ & $7.5 \%$ \\
\hline 3 & SB3-03 & $\mathrm{B} 2 \mathrm{O} 3(\mathrm{wt} \%)$ & 10.2795 & 9.9523 & 10.2000 & 0.0795 & -0.2477 & $0.8 \%$ & $-2.4 \%$ \\
\hline 3 & SB3-03 & $\mathrm{BaO}(\mathrm{wt} \%)$ & 0.0525 & 0.0573 & 0.0590 & -0.0065 & -0.0017 & $-11.1 \%$ & $-2.9 \%$ \\
\hline 3 & SB3-03 & $\mathrm{CaO}(\mathrm{wt} \%)$ & 0.8577 & 0.8174 & 0.8450 & 0.0127 & -0.0276 & $1.5 \%$ & $-3.3 \%$ \\
\hline 3 & SB3-03 & $\mathrm{Ce} 2 \mathrm{O} 3(\mathrm{wt} \%)$ & 0.0843 & 0.0843 & 0.0830 & 0.0013 & 0.0013 & $1.6 \%$ & $1.6 \%$ \\
\hline 3 & SB3-03 & $\mathrm{Cr} 2 \mathrm{O} 3(\mathrm{wt} \%)$ & 0.1060 & 0.1128 & 0.0880 & 0.0180 & 0.0248 & $20.4 \%$ & $28.1 \%$ \\
\hline 3 & SB3-03 & $\mathrm{CuO}(\mathrm{wt} \%)$ & 0.0429 & 0.0450 & 0.0470 & -0.0041 & -0.0020 & $-8.8 \%$ & $-4.3 \%$ \\
\hline
\end{tabular}


Table H.4: Average Measured and Bias-Corrected Chemical Compositions Versus Targeted Compositions by Oxide by Glass Number (continued)

(Batch 1: 100; 101-U std)

\begin{tabular}{|c|c|c|c|c|c|c|c|c|c|}
\hline Glass \# & Glass ID & Oxide & $\begin{array}{c}\text { Measured } \\
(\mathrm{wt} \%)\end{array}$ & $\begin{array}{c}\text { Measured } \\
\text { Bias-Corrected } \\
(w \mathbf{w})\end{array}$ & $\begin{array}{c}\text { Targeted } \\
(\mathrm{wt} \%)\end{array}$ & $\begin{array}{c}\text { Diff of } \\
\text { Measured }\end{array}$ & $\begin{array}{c}\text { Diff of } \\
\text { Meas BC }\end{array}$ & $\begin{array}{c}\% \text { Diff of } \\
\text { Measured }\end{array}$ & $\begin{array}{l}\% \text { Diff of } \\
\text { Meas BC }\end{array}$ \\
\hline 3 & SB3-03 & $\mathrm{Fe} 2 \mathrm{O} 3(\mathrm{wt} \%)$ & 8.8141 & 8.9471 & 9.4550 & -0.6409 & -0.5079 & $-6.8 \%$ & $-5.4 \%$ \\
\hline 3 & SB3-03 & $\mathrm{K} 2 \mathrm{O}(\mathrm{wt} \%)$ & 0.0723 & 0.0647 & 0.1010 & -0.0287 & -0.0363 & $-28.4 \%$ & $-36.0 \%$ \\
\hline 3 & SB3-03 & $\mathrm{La} 2 \mathrm{O} 3(\mathrm{wt} \%)$ & 0.0367 & 0.0367 & 0.0480 & -0.0114 & -0.0114 & $-23.6 \%$ & $-23.6 \%$ \\
\hline 3 & SB3-03 & $\mathrm{Li} 2 \mathrm{O}(\mathrm{wt} \%)$ & 3.2993 & 3.4306 & 3.4010 & -0.1017 & 0.0296 & $-3.0 \%$ & $0.9 \%$ \\
\hline 3 & SB3-03 & $\mathrm{MgO}(\mathrm{wt} \%)$ & 0.0137 & 0.0137 & 0.0440 & -0.0303 & -0.0303 & $-68.9 \%$ & $-68.9 \%$ \\
\hline 3 & SB3-03 & $\mathrm{MnO}(\mathrm{wt} \%)$ & 1.6301 & 1.5718 & 1.6850 & -0.0549 & -0.1132 & $-3.3 \%$ & $-6.7 \%$ \\
\hline 3 & SB3-03 & $\mathrm{Na} 2 \mathrm{O}(\mathrm{wt} \%)$ & 12.0141 & 11.2328 & 11.3640 & 0.6500 & -0.1312 & $5.7 \%$ & $-1.2 \%$ \\
\hline 3 & SB3-03 & $\mathrm{NiO}(\mathrm{wt} \%)$ & 0.3413 & 0.3725 & 0.3780 & -0.0367 & -0.0055 & $-9.7 \%$ & $-1.5 \%$ \\
\hline 3 & SB3-03 & $\mathrm{PbO}(\mathrm{wt} \%)$ & 0.0606 & 0.0606 & 0.0710 & -0.0104 & -0.0104 & $-14.7 \%$ & $-14.7 \%$ \\
\hline 3 & SB3-03 & $\mathrm{SiO} 2(\mathrm{wt} \%)$ & 55.7288 & 59.8711 & 55.1850 & 0.5438 & 4.6861 & $1.0 \%$ & $8.5 \%$ \\
\hline 3 & SB3-03 & ThO2 (wt\%) & 0.1072 & 0.1072 & 0.0340 & 0.0732 & 0.0732 & $215.4 \%$ & $215.4 \%$ \\
\hline 3 & SB3-03 & $\mathrm{TiO} 2(\mathrm{wt} \%)$ & 0.0017 & 0.0018 & 0.0000 & 0.0017 & 0.0018 & & \\
\hline 3 & SB3-03 & $\mathrm{U} 3 \mathrm{O} 8(\mathrm{wt} \%)$ & 2.2464 & 2.3667 & 2.3920 & -0.1456 & -0.0253 & $-6.1 \%$ & $-1.1 \%$ \\
\hline 3 & SB3-03 & $\mathrm{ZnO}(\mathrm{wt} \%)$ & 0.0790 & 0.0790 & 0.0970 & -0.0180 & -0.0180 & $-18.5 \%$ & $-18.5 \%$ \\
\hline 3 & SB3-03 & $\mathrm{ZrO} 2(\mathrm{wt} \%)$ & 0.1547 & 0.1547 & 0.1740 & -0.0193 & -0.0193 & $-11.1 \%$ & $-11.1 \%$ \\
\hline 3 & SB3-03 & Sum of Oxides & 100.5954 & 103.9489 & 100.0010 & 0.5944 & 3.9479 & $0.6 \%$ & $3.9 \%$ \\
\hline 4 & SB3-04 & $\mathrm{Al} 2 \mathrm{O} 3(\mathrm{wt} \%)$ & 6.9345 & 7.0373 & 6.1090 & 0.8255 & 0.9283 & $13.5 \%$ & $15.2 \%$ \\
\hline 4 & SB3-04 & B2O3 (wt\%) & 8.4844 & 8.3356 & 8.1000 & 0.3844 & 0.2356 & $4.7 \%$ & $2.9 \%$ \\
\hline 4 & SB3-04 & $\mathrm{BaO}(\mathrm{wt} \%)$ & 0.0756 & 0.0840 & 0.0840 & -0.0084 & 0.0000 & $-9.9 \%$ & $-0.1 \%$ \\
\hline 4 & SB3-04 & $\mathrm{CaO}(\mathrm{wt} \%)$ & 1.3104 & 1.2256 & 1.2150 & 0.0954 & 0.0106 & $7.8 \%$ & $0.9 \%$ \\
\hline 4 & SB3-04 & $\mathrm{Ce} 2 \mathrm{O} 3(\mathrm{wt} \%)$ & 0.1154 & 0.1154 & 0.1200 & -0.0046 & -0.0046 & $-3.9 \%$ & $-3.9 \%$ \\
\hline 4 & SB3-04 & $\mathrm{Cr} 2 \mathrm{O} 3(\mathrm{wt} \%)$ & 0.1206 & 0.1282 & 0.1260 & -0.0054 & 0.0022 & $-4.3 \%$ & $1.8 \%$ \\
\hline 4 & SB3-04 & $\mathrm{CuO}(\mathrm{wt} \%)$ & 0.0701 & 0.0747 & 0.0670 & 0.0031 & 0.0077 & $4.6 \%$ & $11.5 \%$ \\
\hline 4 & SB3-04 & $\mathrm{Fe} 2 \mathrm{O} 3(\mathrm{wt} \%)$ & 13.4249 & 13.2909 & 13.5910 & -0.1661 & -0.3001 & $-1.2 \%$ & $-2.2 \%$ \\
\hline 4 & SB3-04 & $\mathrm{K} 2 \mathrm{O}(\mathrm{wt} \%)$ & 0.1376 & 0.1234 & 0.1450 & -0.0074 & -0.0216 & $-5.1 \%$ & $-14.9 \%$ \\
\hline 4 & SB3-04 & $\mathrm{La} 2 \mathrm{O} 3$ (wt\%) & 0.0569 & 0.0569 & 0.0690 & -0.0121 & -0.0121 & $-17.6 \%$ & $-17.6 \%$ \\
\hline 4 & SB3-04 & $\mathrm{Li} 2 \mathrm{O}(\mathrm{wt} \%)$ & 3.0087 & 3.1075 & 2.7010 & 0.3077 & 0.4065 & $11.4 \%$ & $15.0 \%$ \\
\hline 4 & SB3-04 & $\mathrm{MgO}(\mathrm{wt} \%)$ & 0.0195 & 0.0203 & 0.0640 & -0.0445 & -0.0437 & $-69.6 \%$ & $-68.2 \%$ \\
\hline 4 & SB3-04 & $\mathrm{MnO}(\mathrm{wt} \%)$ & 2.2305 & 2.4044 & 2.4220 & -0.1915 & -0.0176 & $-7.9 \%$ & $-0.7 \%$ \\
\hline 4 & SB3-04 & $\mathrm{Na} 2 \mathrm{O}(\mathrm{wt} \%)$ & 16.6141 & 15.6186 & 16.3350 & 0.2791 & -0.7164 & $1.7 \%$ & $-4.4 \%$ \\
\hline 4 & SB3-04 & $\mathrm{NiO}(\mathrm{wt} \%)$ & 0.4616 & 0.5252 & 0.5440 & -0.0824 & -0.0188 & $-15.1 \%$ & $-3.5 \%$ \\
\hline 4 & SB3-04 & $\mathrm{PbO}(\mathrm{wt} \%)$ & 0.0840 & 0.0840 & 0.1020 & -0.0180 & -0.0180 & $-17.6 \%$ & $-17.6 \%$ \\
\hline 4 & SB3-04 & $\mathrm{SiO} 2(\mathrm{wt} \%)$ & 46.4763 & 50.0069 & 44.3280 & 2.1483 & 5.6789 & $4.8 \%$ & $12.8 \%$ \\
\hline 4 & SB3-04 & ThO2 (wt $\%)$ & 0.1397 & 0.1397 & 0.0480 & 0.0917 & 0.0917 & $191.0 \%$ & $191.0 \%$ \\
\hline 4 & SB3-04 & $\mathrm{TiO} 2(\mathrm{wt} \%)$ & 0.0083 & 0.0090 & 0.0000 & 0.0083 & 0.0090 & & \\
\hline 4 & SB3-04 & U3O8 (wt\%) & 3.2664 & 3.4177 & 3.4380 & -0.1716 & -0.0203 & $-5.0 \%$ & $-0.6 \%$ \\
\hline 4 & SB3-04 & $\mathrm{ZnO}(\mathrm{wt} \%)$ & 0.1226 & 0.1226 & 0.1390 & -0.0164 & -0.0164 & $-11.8 \%$ & $-11.8 \%$ \\
\hline 4 & SB3-04 & $\mathrm{ZrO} 2(\mathrm{wt} \%)$ & 0.2138 & 0.2138 & 0.2510 & -0.0372 & -0.0372 & $-14.8 \%$ & $-14.8 \%$ \\
\hline 4 & SB3-04 & Sum of Oxides & 103.3758 & 106.1417 & 99.9980 & 3.3778 & 6.1437 & $3.4 \%$ & $6.1 \%$ \\
\hline 5 & SB3-05 & $\mathrm{Al} 2 \mathrm{O} 3(\mathrm{wt} \%)$ & 4.9221 & 5.1470 & 4.4240 & 0.4981 & 0.7230 & $11.3 \%$ & $16.3 \%$ \\
\hline 5 & SB3-05 & $\mathrm{B} 2 \mathrm{O} 3(\mathrm{wt} \%)$ & 5.6670 & 5.4973 & 5.5200 & 0.1470 & -0.0227 & $2.7 \%$ & $-0.4 \%$ \\
\hline 5 & SB3-05 & $\mathrm{BaO}(\mathrm{wt} \%)$ & 0.0514 & 0.0555 & 0.0610 & -0.0096 & -0.0055 & $-15.8 \%$ & $-9.0 \%$ \\
\hline 5 & SB3-05 & $\mathrm{CaO}(\mathrm{wt} \%)$ & 0.9620 & 0.8887 & 0.8800 & 0.0820 & 0.0087 & $9.3 \%$ & $1.0 \%$ \\
\hline 5 & SB3-05 & $\mathrm{Ce} 2 \mathrm{O} 3(\mathrm{wt} \%)$ & 0.0846 & 0.0846 & 0.0870 & -0.0024 & -0.0024 & $-2.7 \%$ & $-2.7 \%$ \\
\hline 5 & SB3-05 & $\mathrm{Cr} 2 \mathrm{O} 3(\mathrm{wt} \%)$ & 0.0782 & 0.0822 & 0.0910 & -0.0128 & -0.0088 & $-14.1 \%$ & $-9.7 \%$ \\
\hline 5 & SB3-05 & $\mathrm{CuO}(\mathrm{wt} \%)$ & 0.0469 & 0.0497 & 0.0480 & -0.0011 & 0.0017 & $-2.2 \%$ & $3.5 \%$ \\
\hline 5 & SB3-05 & $\mathrm{Fe} 2 \mathrm{O} 3(\mathrm{wt} \%)$ & 9.9829 & 9.9095 & 9.8410 & 0.1419 & 0.0685 & $1.4 \%$ & $0.7 \%$ \\
\hline 5 & SB3-05 & $\mathrm{K} 2 \mathrm{O}(\mathrm{wt} \%)$ & 0.0741 & 0.0663 & 0.1050 & -0.0309 & -0.0387 & $-29.4 \%$ & $-36.9 \%$ \\
\hline 5 & SB3-05 & $\mathrm{La} 2 \mathrm{O} 3(\mathrm{wt} \%)$ & 0.0372 & 0.0372 & 0.0500 & -0.0128 & -0.0128 & $-25.5 \%$ & $-25.5 \%$ \\
\hline 5 & SB3-05 & $\mathrm{Li} 2 \mathrm{O}(\mathrm{wt} \%)$ & 4.6180 & 4.7861 & 4.8310 & -0.2130 & -0.0449 & $-4.4 \%$ & $-0.9 \%$ \\
\hline 5 & SB3-05 & $\mathrm{MgO}(\mathrm{wt} \%)$ & 1.4028 & 1.4117 & 1.4260 & -0.0232 & -0.0143 & $-1.6 \%$ & $-1.0 \%$ \\
\hline 5 & SB3-05 & $\mathrm{MnO}(\mathrm{wt} \%)$ & 1.7108 & 1.7708 & 1.7540 & -0.0432 & 0.0168 & $-2.5 \%$ & $1.0 \%$ \\
\hline 5 & SB3-05 & $\mathrm{Na} 2 \mathrm{O}(\mathrm{wt} \%)$ & 13.9552 & 13.4077 & 13.6610 & 0.2942 & -0.2533 & $2.2 \%$ & $-1.9 \%$ \\
\hline
\end{tabular}


Table H.4: Average Measured and Bias-Corrected Chemical Compositions Versus Targeted Compositions by Oxide by Glass Number (continued)

(Batch 1: 100; 101-U std)

\begin{tabular}{|c|c|c|c|c|c|c|c|c|c|}
\hline Glass \# & Glass ID & Oxide & $\begin{array}{c}\text { Measured } \\
(\mathbf{w t} \%)\end{array}$ & $\begin{array}{c}\text { Measured } \\
\text { Bias-Corrected } \\
(w t \%)\end{array}$ & $\begin{array}{c}\text { Targeted } \\
\text { (wt\%) }\end{array}$ & $\begin{array}{c}\text { Diff of } \\
\text { Measured }\end{array}$ & $\begin{array}{c}\text { Diff of } \\
\text { Meas BC }\end{array}$ & $\begin{array}{l}\% \text { Diff of } \\
\text { Measured }\end{array}$ & $\begin{array}{l}\% \text { Diff of } \\
\text { Meas BC }\end{array}$ \\
\hline 5 & SB3-05 & $\mathrm{NiO}(\mathrm{wt} \%)$ & 0.3620 & 0.3997 & 0.3940 & -0.0320 & 0.0057 & $-8.1 \%$ & $1.5 \%$ \\
\hline 5 & SB3-05 & $\mathrm{PbO}(\mathrm{wt} \%)$ & 0.0692 & 0.0692 & 0.0740 & -0.0048 & -0.0048 & $-6.5 \%$ & $-6.5 \%$ \\
\hline 5 & SB3-05 & $\mathrm{SiO} 2(\mathrm{wt} \%)$ & 52.3059 & 54.6941 & 53.9470 & -1.6411 & 0.7471 & $-3.0 \%$ & $1.4 \%$ \\
\hline 5 & SB3-05 & $\mathrm{ThO} 2(\mathrm{wt} \%)$ & 0.1038 & 0.1038 & 0.0350 & 0.0688 & 0.0688 & $196.7 \%$ & $196.7 \%$ \\
\hline 5 & SB3-05 & $\mathrm{TiO} 2(\mathrm{wt} \%)$ & 0.0083 & 0.0088 & 0.0000 & 0.0083 & 0.0088 & & \\
\hline 5 & SB3-05 & $\mathrm{U} 3 \mathrm{O} 8(\mathrm{wt} \%)$ & 2.4822 & 2.5752 & 2.4900 & -0.0078 & 0.0852 & $-0.3 \%$ & $3.4 \%$ \\
\hline 5 & SB3-05 & $\mathrm{ZnO}(\mathrm{wt} \%)$ & 0.0856 & 0.0856 & 0.1000 & -0.0144 & -0.0144 & $-14.4 \%$ & $-14.4 \%$ \\
\hline 5 & SB3-05 & $\mathrm{ZrO} 2(\mathrm{wt} \%)$ & 0.1722 & 0.1722 & 0.1820 & -0.0098 & -0.0098 & $-5.4 \%$ & $-5.4 \%$ \\
\hline 5 & SB3-05 & Sum of Oxides & 99.1825 & 101.3029 & 100.0010 & -0.8185 & 1.3019 & $-0.8 \%$ & $1.3 \%$ \\
\hline 6 & SB3-06 & $\mathrm{Al} 2 \mathrm{O} 3(\mathrm{wt} \%)$ & 5.5268 & 5.5314 & 5.4230 & 0.1038 & 0.1084 & $1.9 \%$ & $2.0 \%$ \\
\hline 6 & SB3-06 & $\mathrm{B} 2 \mathrm{O} 3(\mathrm{wt} \%)$ & 5.0713 & 4.9073 & 4.9600 & 0.1113 & -0.0527 & $2.2 \%$ & $-1.1 \%$ \\
\hline 6 & SB3-06 & $\mathrm{BaO}(\mathrm{wt} \%)$ & 0.0656 & 0.0728 & 0.0750 & -0.0094 & -0.0022 & $-12.5 \%$ & $-2.9 \%$ \\
\hline 6 & SB3-06 & $\mathrm{CaO}(\mathrm{wt} \%)$ & 1.1554 & 1.0806 & 1.0780 & 0.0774 & 0.0026 & $7.2 \%$ & $0.2 \%$ \\
\hline 6 & SB3-06 & $\mathrm{Ce} 2 \mathrm{O} 3(\mathrm{wt} \%)$ & 0.1019 & 0.1019 & 0.1060 & -0.0041 & -0.0041 & $-3.9 \%$ & $-3.9 \%$ \\
\hline 6 & SB3-06 & $\mathrm{Cr} 2 \mathrm{O} 3(\mathrm{wt} \%)$ & 0.1074 & 0.1142 & 0.1120 & -0.0046 & 0.0022 & $-4.1 \%$ & $2.0 \%$ \\
\hline 6 & SB3-06 & $\mathrm{CuO}(\mathrm{wt} \%)$ & 0.0626 & 0.0667 & 0.0590 & 0.0036 & 0.0077 & $6.1 \%$ & $13.1 \%$ \\
\hline 6 & SB3-06 & $\mathrm{Fe} 2 \mathrm{O} 3(\mathrm{wt} \%)$ & 11.9237 & 11.8025 & 12.0630 & -0.1393 & -0.2605 & $-1.2 \%$ & $-2.2 \%$ \\
\hline 6 & SB3-06 & $\mathrm{K} 2 \mathrm{O}(\mathrm{wt} \%)$ & 0.1027 & 0.0921 & 0.1290 & -0.0263 & -0.0369 & $-20.4 \%$ & $-28.6 \%$ \\
\hline 6 & SB3-06 & $\mathrm{La} 2 \mathrm{O} 3(\mathrm{wt} \%)$ & 0.0522 & 0.0522 & 0.0620 & -0.0098 & -0.0098 & $-15.8 \%$ & $-15.8 \%$ \\
\hline 6 & SB3-06 & $\mathrm{Li} 2 \mathrm{O}(\mathrm{wt} \%)$ & 4.1497 & 4.3136 & 4.3410 & -0.1913 & -0.0274 & $-4.4 \%$ & $-0.6 \%$ \\
\hline 6 & SB3-06 & $\mathrm{MgO}(\mathrm{wt} \%)$ & 1.2374 & 1.2906 & 1.2960 & -0.0586 & -0.0054 & $-4.5 \%$ & $-0.4 \%$ \\
\hline 6 & SB3-06 & $\mathrm{MnO}(\mathrm{wt} \%)$ & 2.0143 & 2.1711 & 2.1500 & -0.1357 & 0.0211 & $-6.3 \%$ & $1.0 \%$ \\
\hline 6 & SB3-06 & $\mathrm{Na} 2 \mathrm{O}(\mathrm{wt} \%)$ & 15.6368 & 14.7017 & 15.3900 & 0.2468 & -0.6883 & $1.6 \%$ & $-4.5 \%$ \\
\hline 6 & SB3-06 & $\mathrm{NiO}(\mathrm{wt} \%)$ & 0.4288 & 0.4879 & 0.4830 & -0.0542 & 0.0049 & $-11.2 \%$ & $1.0 \%$ \\
\hline 6 & SB3-06 & $\mathrm{PbO}(\mathrm{wt} \%)$ & 0.0811 & 0.0811 & 0.0910 & -0.0099 & -0.0099 & $-10.9 \%$ & $-10.9 \%$ \\
\hline 6 & SB3-06 & $\mathrm{SiO} 2(\mathrm{wt} \%)$ & 48.8295 & 52.4440 & 48.7420 & 0.0875 & 3.7020 & $0.2 \%$ & $7.6 \%$ \\
\hline 6 & SB3-06 & ThO2 (wt\%) & 0.1274 & 0.1274 & 0.0430 & 0.0844 & 0.0844 & $196.4 \%$ & $196.4 \%$ \\
\hline 6 & SB3-06 & $\mathrm{TiO} 2(\mathrm{wt} \%)$ & 0.0083 & 0.0090 & 0.0000 & 0.0083 & 0.0090 & & \\
\hline 6 & SB3-06 & U3O8 (wt\%) & 3.0188 & 3.1586 & 3.0520 & -0.0332 & 0.1066 & $-1.1 \%$ & $3.5 \%$ \\
\hline 6 & SB3-06 & $\mathrm{ZnO}(\mathrm{wt} \%)$ & 0.1061 & 0.1061 & 0.1230 & -0.0169 & -0.0169 & $-13.7 \%$ & $-13.7 \%$ \\
\hline 6 & SB3-06 & $\mathrm{ZrO} 2(\mathrm{wt} \%)$ & 0.1959 & 0.1959 & 0.2230 & -0.0271 & -0.0271 & $-12.2 \%$ & $-12.2 \%$ \\
\hline 6 & SB3-06 & Sum of Oxides & 100.0037 & 102.9088 & 100.0010 & 0.0027 & 2.9078 & $0.0 \%$ & $2.9 \%$ \\
\hline 7 & SB3-07 & $\mathrm{Al} 2 \mathrm{O} 3(\mathrm{wt} \%)$ & 4.4970 & 4.5637 & 4.2810 & 0.2160 & 0.2827 & $5.0 \%$ & $6.6 \%$ \\
\hline 7 & SB3-07 & $\mathrm{B} 2 \mathrm{O} 3(\mathrm{wt} \%)$ & 13.9100 & 13.6659 & 14.0000 & -0.0900 & -0.3341 & $-0.6 \%$ & $-2.4 \%$ \\
\hline 7 & SB3-07 & $\mathrm{BaO}(\mathrm{wt} \%)$ & 0.0497 & 0.0537 & 0.0590 & -0.0093 & -0.0053 & $-15.8 \%$ & $-9.0 \%$ \\
\hline 7 & SB3-07 & $\mathrm{CaO}(\mathrm{wt} \%)$ & 0.9336 & 0.8625 & 0.8510 & 0.0826 & 0.0115 & $9.7 \%$ & $1.4 \%$ \\
\hline 7 & SB3-07 & $\mathrm{Ce} 2 \mathrm{O} 3(\mathrm{wt} \%)$ & 0.0811 & 0.0811 & 0.0840 & -0.0029 & -0.0029 & $-3.4 \%$ & $-3.4 \%$ \\
\hline 7 & SB3-07 & $\mathrm{Cr} 2 \mathrm{O} 3(\mathrm{wt} \%)$ & 0.0826 & 0.0868 & 0.0880 & -0.0054 & -0.0012 & $-6.2 \%$ & $-1.4 \%$ \\
\hline 7 & SB3-07 & $\mathrm{CuO}(\mathrm{wt} \%)$ & 0.0466 & 0.0493 & 0.0470 & -0.0004 & 0.0023 & $-0.8 \%$ & $5.0 \%$ \\
\hline 7 & SB3-07 & $\mathrm{Fe} 2 \mathrm{O} 3(\mathrm{wt} \%)$ & 9.3073 & 9.2396 & 9.5230 & -0.2157 & -0.2834 & $-2.3 \%$ & $-3.0 \%$ \\
\hline 7 & SB3-07 & $\mathrm{K} 2 \mathrm{O}(\mathrm{wt} \%)$ & 0.0783 & 0.0700 & 0.1020 & -0.0237 & -0.0320 & $-23.2 \%$ & $-31.4 \%$ \\
\hline 7 & SB3-07 & $\mathrm{La} 2 \mathrm{O} 3(\mathrm{wt} \%)$ & 0.0381 & 0.0381 & 0.0490 & -0.0109 & -0.0109 & $-22.2 \%$ & $-22.2 \%$ \\
\hline 7 & SB3-07 & $\mathrm{Li} 2 \mathrm{O}(\mathrm{wt} \%)$ & 3.4393 & 3.5523 & 3.5010 & -0.0617 & 0.0513 & $-1.8 \%$ & $1.5 \%$ \\
\hline 7 & SB3-07 & $\mathrm{MgO}(\mathrm{wt} \%)$ & 0.0120 & 0.0121 & 0.0450 & -0.0330 & -0.0329 & $-73.3 \%$ & $-73.1 \%$ \\
\hline 7 & SB3-07 & $\mathrm{MnO}(\mathrm{wt} \%)$ & 1.6850 & 1.7440 & 1.6970 & -0.0120 & 0.0470 & $-0.7 \%$ & $2.8 \%$ \\
\hline 7 & SB3-07 & $\mathrm{Na} 2 \mathrm{O}(\mathrm{wt} \%)$ & 9.3720 & 9.0048 & 9.2130 & 0.1590 & -0.2082 & $1.7 \%$ & $-2.3 \%$ \\
\hline 7 & SB3-07 & $\mathrm{NiO}(\mathrm{wt} \%)$ & 0.3611 & 0.3987 & 0.3810 & -0.0199 & 0.0177 & $-5.2 \%$ & $4.6 \%$ \\
\hline 7 & SB3-07 & $\mathrm{PbO}(\mathrm{wt} \%)$ & 0.0665 & 0.0665 & 0.0720 & -0.0055 & -0.0055 & $-7.6 \%$ & $-7.6 \%$ \\
\hline 7 & SB3-07 & $\mathrm{SiO} 2(\mathrm{wt} \%)$ & 53.2151 & 57.2558 & 53.2910 & -0.0759 & 3.9648 & $-0.1 \%$ & $7.4 \%$ \\
\hline 7 & SB3-07 & ThO2 (wt $\%)$ & 0.0984 & 0.0984 & 0.0340 & 0.0644 & 0.0644 & $189.5 \%$ & $189.5 \%$ \\
\hline 7 & SB3-07 & $\mathrm{TiO} 2(\mathrm{wt} \%)$ & 0.0050 & 0.0053 & 0.0000 & 0.0050 & 0.0053 & & \\
\hline 7 & SB3-07 & $\mathrm{U} 3 \mathrm{O} 8$ (wt $\%)$ & 2.3761 & 2.4651 & 2.4090 & -0.0329 & 0.0561 & $-1.4 \%$ & $2.3 \%$ \\
\hline 7 & SB3-07 & $\mathrm{ZnO}(\mathrm{wt} \%)$ & 0.0890 & 0.0890 & 0.0970 & -0.0080 & -0.0080 & $-8.2 \%$ & $-8.2 \%$ \\
\hline
\end{tabular}


Table H.4: Average Measured and Bias-Corrected Chemical Compositions Versus Targeted Compositions by Oxide by Glass Number (continued)

(Batch 1: 100; 101-U std)

\begin{tabular}{|c|c|c|c|c|c|c|c|c|c|}
\hline Glass \# & Glass ID & Oxide & $\begin{array}{c}\text { Measured } \\
(\mathbf{w t} \%)\end{array}$ & $\begin{array}{c}\text { Measured } \\
\text { Bias-Corrected } \\
(w t \%)\end{array}$ & $\begin{array}{c}\text { Targeted } \\
(\text { wt } \%)\end{array}$ & $\begin{array}{c}\text { Diff of } \\
\text { Measured }\end{array}$ & $\begin{array}{c}\text { Diff of } \\
\text { Meas BC }\end{array}$ & $\begin{array}{l}\% \text { Diff of } \\
\text { Measured }\end{array}$ & $\begin{array}{l}\% \text { Diff of } \\
\text { Meas BC }\end{array}$ \\
\hline 7 & SB3-07 & $\mathrm{ZrO} 2(\mathrm{wt} \%)$ & 0.1672 & 0.1672 & 0.1760 & -0.0088 & -0.0088 & $-5.0 \%$ & $-5.0 \%$ \\
\hline 7 & SB3-07 & Sum of Oxides & 99.9110 & 103.5698 & 100.0000 & -0.0890 & 3.5698 & $-0.1 \%$ & $3.6 \%$ \\
\hline 8 & SB3-08 & $\mathrm{Al} 2 \mathrm{O} 3(\mathrm{wt} \%)$ & 6.3251 & 6.6140 & 6.5640 & -0.2389 & 0.0500 & $-3.6 \%$ & $0.8 \%$ \\
\hline 8 & SB3-08 & $\mathrm{B} 2 \mathrm{O} 3(\mathrm{wt} \%)$ & 10.7303 & 10.4091 & 10.8000 & -0.0697 & -0.3909 & $-0.6 \%$ & $-3.6 \%$ \\
\hline 8 & SB3-08 & $\mathrm{BaO}(\mathrm{wt} \%)$ & 0.0737 & 0.0885 & 0.0910 & -0.0173 & -0.0025 & $-19.0 \%$ & $-2.8 \%$ \\
\hline 8 & SB3-08 & $\mathrm{CaO}(\mathrm{wt} \%)$ & 1.3933 & 1.2847 & 1.3050 & 0.0883 & -0.0203 & $6.8 \%$ & $-1.6 \%$ \\
\hline 8 & SB3-08 & $\mathrm{Ce} 2 \mathrm{O} 3(\mathrm{wt} \%)$ & 0.1189 & 0.1189 & 0.1290 & -0.0101 & -0.0101 & $-7.8 \%$ & $-7.8 \%$ \\
\hline 8 & SB3-08 & $\mathrm{Cr} 2 \mathrm{O} 3(\mathrm{wt} \%)$ & 0.1823 & 0.2165 & 0.1360 & 0.0463 & 0.0805 & $34.1 \%$ & $59.2 \%$ \\
\hline 8 & SB3-08 & $\mathrm{CuO}(\mathrm{wt} \%)$ & 0.0742 & 0.0792 & 0.0720 & 0.0022 & 0.0072 & $3.0 \%$ & $10.0 \%$ \\
\hline 8 & SB3-08 & $\mathrm{Fe} 2 \mathrm{O} 3(\mathrm{wt} \%)$ & 14.9761 & 14.5031 & 14.6030 & 0.3731 & -0.0999 & $2.6 \%$ & $-0.7 \%$ \\
\hline 8 & SB3-08 & $\mathrm{K} 2 \mathrm{O}(\mathrm{wt} \%)$ & 0.1331 & 0.1185 & 0.1560 & -0.0229 & -0.0375 & $-14.7 \%$ & $-24.1 \%$ \\
\hline 8 & SB3-08 & $\mathrm{La} 2 \mathrm{O} 3(\mathrm{wt} \%)$ & 0.0598 & 0.0598 & 0.0750 & -0.0152 & -0.0152 & $-20.2 \%$ & $-20.2 \%$ \\
\hline 8 & SB3-08 & $\mathrm{Li} 2 \mathrm{O}(\mathrm{wt} \%)$ & 2.5942 & 2.6886 & 2.7010 & -0.1068 & -0.0124 & $-4.0 \%$ & $-0.5 \%$ \\
\hline 8 & SB3-08 & $\mathrm{MgO}(\mathrm{wt} \%)$ & 0.0199 & 0.0216 & 0.0680 & -0.0481 & -0.0464 & $-70.7 \%$ & $-68.2 \%$ \\
\hline 8 & SB3-08 & $\mathrm{MnO}(\mathrm{wt} \%)$ & 2.4113 & 2.6677 & 2.6020 & -0.1907 & 0.0657 & $-7.3 \%$ & $2.5 \%$ \\
\hline 8 & SB3-08 & $\mathrm{Na} 2 \mathrm{O}(\mathrm{wt} \%)$ & 14.0866 & 13.6455 & 14.1270 & -0.0404 & -0.4815 & $-0.3 \%$ & $-3.4 \%$ \\
\hline 8 & SB3-08 & $\mathrm{NiO}(\mathrm{wt} \%)$ & 0.4626 & 0.5395 & 0.5840 & -0.1214 & -0.0445 & $-20.8 \%$ & $-7.6 \%$ \\
\hline 8 & SB3-08 & $\mathrm{PbO}(\mathrm{wt} \%)$ & 0.0972 & 0.0972 & 0.1100 & -0.0128 & -0.0128 & $-11.6 \%$ & $-11.6 \%$ \\
\hline 8 & SB3-08 & $\mathrm{SiO} 2(\mathrm{wt} \%)$ & 41.3955 & 43.2836 & 41.7120 & -0.3165 & 1.5716 & $-0.8 \%$ & $3.8 \%$ \\
\hline 8 & SB3-08 & ThO2 (wt $\%)$ & 0.1468 & 0.1468 & 0.0520 & 0.0948 & 0.0948 & $182.3 \%$ & $182.3 \%$ \\
\hline 8 & SB3-08 & $\mathrm{TiO} 2(\mathrm{wt} \%)$ & 0.0083 & 0.0092 & 0.0000 & 0.0083 & 0.0092 & & \\
\hline 8 & SB3-08 & $\mathrm{U} 3 \mathrm{O} 8(\mathrm{wt} \%)$ & 3.6290 & 3.7972 & 3.6940 & -0.0650 & 0.1032 & $-1.8 \%$ & $2.8 \%$ \\
\hline 8 & SB3-08 & $\mathrm{ZnO}(\mathrm{wt} \%)$ & 0.1226 & 0.1226 & 0.1490 & -0.0264 & -0.0264 & $-17.7 \%$ & $-17.7 \%$ \\
\hline 8 & SB3-08 & $\mathrm{ZrO} 2(\mathrm{wt} \%)$ & 0.2252 & 0.2252 & 0.2690 & -0.0438 & -0.0438 & $-16.3 \%$ & $-16.3 \%$ \\
\hline 8 & SB3-08 & Sum of Oxides & 99.2660 & 100.7370 & 99.9990 & -0.7330 & 0.7380 & $-0.7 \%$ & $0.7 \%$ \\
\hline 9 & SB3-09 & $\mathrm{A} 12 \mathrm{O} 3(\mathrm{wt} \%)$ & 5.1678 & 5.1695 & 4.9940 & 0.1738 & 0.1755 & $3.5 \%$ & $3.5 \%$ \\
\hline 9 & SB3-09 & $\mathrm{B} 2 \mathrm{O} 3(\mathrm{wt} \%)$ & 9.7080 & 9.3949 & 9.7500 & -0.0420 & -0.3551 & $-0.4 \%$ & $-3.6 \%$ \\
\hline 9 & SB3-09 & $\mathrm{BaO}(\mathrm{wt} \%)$ & 0.0581 & 0.0634 & 0.0690 & -0.0109 & -0.0056 & $-15.9 \%$ & $-8.1 \%$ \\
\hline 9 & SB3-09 & $\mathrm{CaO}(\mathrm{wt} \%)$ & 1.0284 & 0.9800 & 0.9930 & 0.0354 & -0.0130 & $3.6 \%$ & $-1.3 \%$ \\
\hline 9 & SB3-09 & $\mathrm{Ce} 2 \mathrm{O} 3(\mathrm{wt} \%)$ & 0.1253 & 0.1253 & 0.0980 & 0.0273 & 0.0273 & $27.9 \%$ & $27.9 \%$ \\
\hline 9 & SB3-09 & $\mathrm{Cr} 2 \mathrm{O} 3(\mathrm{wt} \%)$ & 0.0950 & 0.1011 & 0.1030 & -0.0080 & -0.0019 & $-7.8 \%$ & $-1.8 \%$ \\
\hline 9 & SB3-09 & $\mathrm{CuO}(\mathrm{wt} \%)$ & 0.0532 & 0.0558 & 0.0550 & -0.0018 & 0.0008 & $-3.3 \%$ & $1.5 \%$ \\
\hline 9 & SB3-09 & $\mathrm{Fe} 2 \mathrm{O} 3$ (wt $\%)$ & 9.9543 & 10.1049 & 11.1110 & -1.1567 & -1.0061 & $-10.4 \%$ & $-9.1 \%$ \\
\hline 9 & SB3-09 & $\mathrm{K} 2 \mathrm{O}(\mathrm{wt} \%)$ & 0.1018 & 0.0911 & 0.1190 & -0.0172 & -0.0279 & $-14.5 \%$ & $-23.4 \%$ \\
\hline 9 & SB3-09 & $\mathrm{La} 2 \mathrm{O} 3$ (wt $\%$ ) & 0.0765 & 0.0765 & 0.0570 & 0.0195 & 0.0195 & $34.3 \%$ & $34.3 \%$ \\
\hline 9 & SB3-09 & $\mathrm{Li} 2 \mathrm{O}(\mathrm{wt} \%)$ & 3.1163 & 3.2397 & 3.2510 & -0.1347 & -0.0113 & $-4.1 \%$ & $-0.3 \%$ \\
\hline 9 & SB3-09 & $\mathrm{MgO}(\mathrm{wt} \%)$ & 0.0203 & 0.0203 & 0.0520 & -0.0317 & -0.0317 & $-60.9 \%$ & $-60.9 \%$ \\
\hline 9 & SB3-09 & $\mathrm{MnO}(\mathrm{wt} \%)$ & 1.8819 & 1.8138 & 1.9800 & -0.0981 & -0.1662 & $-5.0 \%$ & $-8.4 \%$ \\
\hline 9 & SB3-09 & $\mathrm{Na} 2 \mathrm{O}(\mathrm{wt} \%)$ & 11.3367 & 10.5999 & 10.7490 & 0.5877 & -0.1491 & $5.5 \%$ & $-1.4 \%$ \\
\hline 9 & SB3-09 & $\mathrm{NiO}(\mathrm{wt} \%)$ & 0.3970 & 0.4333 & 0.4450 & -0.0480 & -0.0117 & $-10.8 \%$ & $-2.6 \%$ \\
\hline 9 & SB3-09 & $\mathrm{PbO}(\mathrm{wt} \%)$ & 0.0716 & 0.0716 & 0.0830 & -0.0114 & -0.0114 & $-13.7 \%$ & $-13.7 \%$ \\
\hline 9 & SB3-09 & $\mathrm{SiO} 2(\mathrm{wt} \%)$ & 53.0546 & 56.9880 & 52.9220 & 0.1326 & 4.0660 & $0.3 \%$ & $7.7 \%$ \\
\hline 9 & SB3-09 & ThO2 (wt $\%)$ & 0.1289 & 0.1289 & 0.0390 & 0.0899 & 0.0899 & $230.4 \%$ & $230.4 \%$ \\
\hline 9 & SB3-09 & $\mathrm{TiO} 2(\mathrm{wt} \%)$ & 0.0017 & 0.0018 & 0.0000 & 0.0017 & 0.0018 & & \\
\hline 9 & SB3-09 & $\mathrm{U} 3 \mathrm{O} 8(\mathrm{wt} \%)$ & 2.6886 & 2.8325 & 2.8110 & -0.1224 & 0.0215 & $-4.4 \%$ & $0.8 \%$ \\
\hline 9 & SB3-09 & $\mathrm{ZnO}(\mathrm{wt} \%)$ & 0.0946 & 0.0946 & 0.1130 & -0.0184 & -0.0184 & $-16.3 \%$ & $-16.3 \%$ \\
\hline 9 & SB3-09 & $\mathrm{ZrO} 2(\mathrm{wt} \%)$ & 0.1857 & 0.1857 & 0.2050 & -0.0193 & -0.0193 & $-9.4 \%$ & $-9.4 \%$ \\
\hline 9 & SB3-09 & Sum of Oxides & 99.3464 & 102.5727 & 99.9990 & -0.6526 & 2.5737 & $-0.7 \%$ & $2.6 \%$ \\
\hline 10 & SB3-10 & $\mathrm{A} 12 \mathrm{O} 3(\mathrm{wt} \%)$ & 7.1612 & 7.1624 & 7.1350 & 0.0262 & 0.0274 & $0.4 \%$ & $0.4 \%$ \\
\hline 10 & SB3-10 & $\mathrm{B} 2 \mathrm{O} 3(\mathrm{wt} \%)$ & 7.6392 & 7.3941 & 7.5000 & 0.1392 & -0.1059 & $1.9 \%$ & $-1.4 \%$ \\
\hline 10 & SB3-10 & $\mathrm{BaO}(\mathrm{wt} \%)$ & 0.0893 & 0.0976 & 0.0990 & -0.0097 & -0.0014 & $-9.8 \%$ & $-1.4 \%$ \\
\hline 10 & SB3-10 & $\mathrm{CaO}(\mathrm{wt} \%)$ & 1.4936 & 1.4234 & 1.4190 & 0.0746 & 0.0044 & $5.3 \%$ & $0.3 \%$ \\
\hline 10 & SB3-10 & $\mathrm{Ce} 2 \mathrm{O} 3(\mathrm{wt} \%)$ & 0.1420 & 0.1420 & 0.1400 & 0.0020 & 0.0020 & $1.4 \%$ & $1.4 \%$ \\
\hline
\end{tabular}


Table H.4: Average Measured and Bias-Corrected Chemical Compositions Versus Targeted Compositions by Oxide by Glass Number (continued)

(Batch 1: 100; 101-U std)

\begin{tabular}{|c|c|c|c|c|c|c|c|c|c|}
\hline Glass \# & Glass ID & Oxide & $\begin{array}{c}\text { Measured } \\
(\mathrm{wt} \%)\end{array}$ & $\begin{array}{c}\text { Measured } \\
\text { Bias-Corrected } \\
(\mathrm{wt} \%)\end{array}$ & $\begin{array}{c}\text { Targeted } \\
(\text { wt } \%)\end{array}$ & $\begin{array}{c}\text { Diff of } \\
\text { Measured }\end{array}$ & $\begin{array}{c}\text { Diff of } \\
\text { Meas BC }\end{array}$ & $\begin{array}{l}\% \text { Diff of } \\
\text { Measured }\end{array}$ & $\begin{array}{l}\% \text { Diff of } \\
\text { Meas BC }\end{array}$ \\
\hline 10 & SB3-10 & $\mathrm{Cr} 2 \mathrm{O} 3(\mathrm{wt} \%)$ & 0.1421 & 0.1512 & 0.1470 & -0.0049 & 0.0042 & $-3.3 \%$ & $2.9 \%$ \\
\hline 10 & SB3-10 & $\mathrm{CuO}(\mathrm{wt} \%)$ & 0.0764 & 0.0801 & 0.0780 & -0.0016 & 0.0021 & $-2.1 \%$ & $2.7 \%$ \\
\hline 10 & SB3-10 & $\mathrm{Fe} 2 \mathrm{O} 3(\mathrm{wt} \%)$ & 14.9904 & 15.2186 & 15.8720 & -0.8816 & -0.6534 & $-5.6 \%$ & $-4.1 \%$ \\
\hline 10 & SB3-10 & $\mathrm{K} 2 \mathrm{O}(\mathrm{wt} \%)$ & 0.1765 & 0.1579 & 0.1700 & 0.0065 & -0.0121 & $3.8 \%$ & $-7.1 \%$ \\
\hline 10 & SB3-10 & $\mathrm{La} 2 \mathrm{O} 3(\mathrm{wt} \%)$ & 0.0666 & 0.0666 & 0.0810 & -0.0144 & -0.0144 & $-17.8 \%$ & $-17.8 \%$ \\
\hline 10 & SB3-10 & $\mathrm{Li} 2 \mathrm{O}(\mathrm{wt} \%)$ & 2.4382 & 2.5347 & 2.5010 & -0.0628 & 0.0337 & $-2.5 \%$ & $1.3 \%$ \\
\hline 10 & SB3-10 & $\mathrm{MgO}(\mathrm{wt} \%)$ & 1.1694 & 1.1723 & 0.0740 & 1.0954 & 1.0983 & $1480.2 \%$ & $1484.2 \%$ \\
\hline 10 & SB3-10 & $\mathrm{MnO}(\mathrm{wt} \%)$ & 2.7277 & 2.6291 & 2.8290 & -0.1013 & -0.1999 & $-3.6 \%$ & $-7.1 \%$ \\
\hline 10 & SB3-10 & $\mathrm{Na} 2 \mathrm{O}(\mathrm{wt} \%)$ & 16.2434 & 15.1861 & 15.3560 & 0.8874 & -0.1699 & $5.8 \%$ & $-1.1 \%$ \\
\hline 10 & SB3-10 & $\mathrm{NiO}(\mathrm{wt} \%)$ & 0.5599 & 0.6110 & 0.6350 & -0.0751 & -0.0240 & $-11.8 \%$ & $-3.8 \%$ \\
\hline 10 & SB3-10 & $\mathrm{PbO}(\mathrm{wt} \%)$ & 0.1050 & 0.1050 & 0.1190 & -0.0140 & -0.0140 & $-11.7 \%$ & $-11.7 \%$ \\
\hline 10 & SB3-10 & $\mathrm{SiO} 2(\mathrm{wt} \%)$ & 41.6094 & 44.6991 & 41.3180 & 0.2914 & 3.3811 & $0.7 \%$ & $8.2 \%$ \\
\hline 10 & SB3-10 & ThO2 (wt $\%)$ & 0.1909 & 0.1909 & 0.0560 & 0.1349 & 0.1349 & $240.9 \%$ & $240.9 \%$ \\
\hline 10 & SB3-10 & $\mathrm{TiO} 2(\mathrm{wt} \%)$ & 0.0042 & 0.0044 & 0.0000 & 0.0042 & 0.0044 & & \\
\hline 10 & SB3-10 & $\mathrm{U} 3 \mathrm{O} 8(\mathrm{wt} \%)$ & 3.8766 & 4.0840 & 4.0160 & -0.1394 & 0.0680 & $-3.5 \%$ & $1.7 \%$ \\
\hline 10 & SB3-10 & $\mathrm{ZnO}(\mathrm{wt} \%)$ & 0.1444 & 0.1444 & 0.1620 & -0.0176 & -0.0176 & $-10.9 \%$ & $-10.9 \%$ \\
\hline 10 & SB3-10 & $\mathrm{ZrO} 2(\mathrm{wt} \%)$ & 0.2705 & 0.2705 & 0.2930 & -0.0225 & -0.0225 & $-7.7 \%$ & $-7.7 \%$ \\
\hline 10 & SB3-10 & Sum of Oxides & 101.3168 & 103.5251 & 100.0000 & 1.3168 & 3.5251 & $1.3 \%$ & $3.5 \%$ \\
\hline 11 & SB3-11 & $\mathrm{Al} 2 \mathrm{O} 3(\mathrm{wt} \%)$ & 4.8277 & 4.9678 & 4.4710 & 0.3567 & 0.4968 & $8.0 \%$ & $11.1 \%$ \\
\hline 11 & SB3-11 & $\mathrm{B} 2 \mathrm{O} 3(\mathrm{wt} \%)$ & 5.7073 & 5.6535 & 5.6800 & 0.0273 & -0.0265 & $0.5 \%$ & $-0.5 \%$ \\
\hline 11 & SB3-11 & $\mathrm{BaO}(\mathrm{wt} \%)$ & 0.0514 & 0.0555 & 0.0620 & -0.0106 & -0.0065 & $-17.2 \%$ & $-10.5 \%$ \\
\hline 11 & SB3-11 & $\mathrm{CaO}(\mathrm{wt} \%)$ & 0.9654 & 0.8919 & 0.8890 & 0.0764 & 0.0029 & $8.6 \%$ & $0.3 \%$ \\
\hline 11 & SB3-11 & $\mathrm{Ce} 2 \mathrm{O} 3(\mathrm{wt} \%)$ & 0.0940 & 0.0940 & 0.0880 & 0.0060 & 0.0060 & $6.8 \%$ & $6.8 \%$ \\
\hline 11 & SB3-11 & $\mathrm{Cr} 2 \mathrm{O} 3(\mathrm{wt} \%)$ & 0.0914 & 0.0960 & 0.0920 & -0.0006 & 0.0040 & $-0.7 \%$ & $4.3 \%$ \\
\hline 11 & SB3-11 & $\mathrm{CuO}(\mathrm{wt} \%)$ & 0.0482 & 0.0510 & 0.0490 & -0.0008 & 0.0020 & $-1.6 \%$ & $4.1 \%$ \\
\hline 11 & SB3-11 & $\mathrm{Fe} 2 \mathrm{O} 3(\mathrm{wt} \%)$ & 9.4289 & 9.3596 & 9.9460 & -0.5171 & -0.5864 & $-5.2 \%$ & $-5.9 \%$ \\
\hline 11 & SB3-11 & $\mathrm{K} 2 \mathrm{O}(\mathrm{wt} \%)$ & 0.0738 & 0.0660 & 0.1060 & -0.0322 & -0.0400 & $-30.4 \%$ & $-37.8 \%$ \\
\hline 11 & SB3-11 & La2O3 (wt \%) & 0.0498 & 0.0498 & 0.0510 & -0.0012 & -0.0012 & $-2.3 \%$ & $-2.3 \%$ \\
\hline 11 & SB3-11 & $\mathrm{Li} 2 \mathrm{O}(\mathrm{wt} \%)$ & 4.8548 & 5.0229 & 4.9710 & -0.1162 & 0.0519 & $-2.3 \%$ & $1.0 \%$ \\
\hline 11 & SB3-11 & $\mathrm{MgO}(\mathrm{wt} \%)$ & 1.4239 & 1.4321 & 1.4670 & -0.0431 & -0.0349 & $-2.9 \%$ & $-2.4 \%$ \\
\hline 11 & SB3-11 & $\mathrm{MnO}(\mathrm{wt} \%)$ & 1.7076 & 1.7672 & 1.7730 & -0.0654 & -0.0058 & $-3.7 \%$ & $-0.3 \%$ \\
\hline 11 & SB3-11 & $\mathrm{Na} 2 \mathrm{O}(\mathrm{wt} \%)$ & 11.6973 & 11.2383 & 11.5500 & 0.1473 & -0.3117 & $1.3 \%$ & $-2.7 \%$ \\
\hline 11 & SB3-11 & $\mathrm{NiO}(\mathrm{wt} \%)$ & 0.3598 & 0.3971 & 0.3980 & -0.0382 & -0.0009 & $-9.6 \%$ & $-0.2 \%$ \\
\hline 11 & SB3-11 & $\mathrm{PbO}(\mathrm{wt} \%)$ & 0.0700 & 0.0700 & 0.0750 & -0.0050 & -0.0050 & $-6.6 \%$ & $-6.6 \%$ \\
\hline 11 & SB3-11 & $\mathrm{SiO} 2(\mathrm{wt} \%)$ & 54.9800 & 57.8775 & 55.4960 & -0.5160 & 2.3815 & $-0.9 \%$ & $4.3 \%$ \\
\hline 11 & SB3-11 & ThO2 (wt $\%)$ & 0.1004 & 0.1004 & 0.0350 & 0.0654 & 0.0654 & $186.9 \%$ & $186.9 \%$ \\
\hline 11 & SB3-11 & $\mathrm{TiO} 2(\mathrm{wt} \%)$ & 0.0083 & 0.0088 & 0.0000 & 0.0083 & 0.0088 & & \\
\hline 11 & SB3-11 & U3O8 (wt\%) & 2.4233 & 2.5140 & 2.5160 & -0.0927 & -0.0020 & $-3.7 \%$ & $-0.1 \%$ \\
\hline 11 & SB3-11 & $\mathrm{ZnO}(\mathrm{wt} \%)$ & 0.0831 & 0.0831 & 0.1020 & -0.0189 & -0.0189 & $-18.5 \%$ & $-18.5 \%$ \\
\hline 11 & SB3-11 & $\mathrm{ZrO} 2(\mathrm{wt} \%)$ & 0.1756 & 0.1756 & 0.1840 & -0.0084 & -0.0084 & $-4.6 \%$ & $-4.6 \%$ \\
\hline 11 & SB3-11 & Sum of Oxides & 99.2219 & 101.9723 & 100.0010 & -0.7791 & 1.9713 & $-0.8 \%$ & $2.0 \%$ \\
\hline 12 & SB3-12 & $\mathrm{A} 12 \mathrm{O} 3(\mathrm{wt} \%)$ & 7.0337 & 7.1380 & 7.0920 & -0.0583 & 0.0460 & $-0.8 \%$ & $0.6 \%$ \\
\hline 12 & SB3-12 & $\mathrm{B} 2 \mathrm{O} 3(\mathrm{wt} \%)$ & 4.2342 & 4.1600 & 4.3200 & -0.0858 & -0.1600 & $-2.0 \%$ & $-3.7 \%$ \\
\hline 12 & SB3-12 & $\mathrm{BaO}(\mathrm{wt} \%)$ & 0.0865 & 0.0935 & 0.0980 & -0.0115 & -0.0045 & $-11.7 \%$ & $-4.6 \%$ \\
\hline 12 & SB3-12 & $\mathrm{CaO}(\mathrm{wt} \%)$ & 1.5202 & 1.4044 & 1.4100 & 0.1102 & -0.0056 & $7.8 \%$ & $-0.4 \%$ \\
\hline 12 & SB3-12 & $\mathrm{Ce} 2 \mathrm{O} 3(\mathrm{wt} \%)$ & 0.1388 & 0.1388 & 0.1390 & -0.0002 & -0.0002 & $-0.1 \%$ & $-0.1 \%$ \\
\hline 12 & SB3-12 & $\mathrm{Cr} 2 \mathrm{O} 3(\mathrm{wt} \%)$ & 0.1272 & 0.1336 & 0.1470 & -0.0198 & -0.0134 & $-13.5 \%$ & $-9.1 \%$ \\
\hline 12 & SB3-12 & $\mathrm{CuO}(\mathrm{wt} \%)$ & 0.0776 & 0.0821 & 0.0780 & -0.0004 & 0.0041 & $-0.5 \%$ & $5.3 \%$ \\
\hline 12 & SB3-12 & $\mathrm{Fe} 2 \mathrm{O} 3(\mathrm{wt} \%)$ & 14.7617 & 14.6530 & 15.7770 & -1.0153 & -1.1240 & $-6.4 \%$ & $-7.1 \%$ \\
\hline 12 & SB3-12 & $\mathrm{K} 2 \mathrm{O}(\mathrm{wt} \%)$ & 0.1593 & 0.1424 & 0.1690 & -0.0097 & -0.0266 & $-5.7 \%$ & $-15.7 \%$ \\
\hline 12 & SB3-12 & $\mathrm{La} 2 \mathrm{O} 3(\mathrm{wt} \%)$ & 0.0654 & 0.0654 & 0.0810 & -0.0156 & -0.0156 & $-19.3 \%$ & $-19.3 \%$ \\
\hline 12 & SB3-12 & $\mathrm{Li} 2 \mathrm{O}(\mathrm{wt} \%)$ & 3.5308 & 3.6468 & 3.7810 & -0.2502 & -0.1342 & $-6.6 \%$ & $-3.5 \%$ \\
\hline 12 & SB3-12 & $\mathrm{MgO}(\mathrm{wt} \%)$ & 1.1209 & 1.1273 & 1.1540 & -0.0331 & -0.0267 & $-2.9 \%$ & $-2.3 \%$ \\
\hline
\end{tabular}


Table H.4: Average Measured and Bias-Corrected Chemical Compositions Versus Targeted Compositions by Oxide by Glass Number (continued)

(Batch 1: 100; 101-U std)

\begin{tabular}{|c|c|c|c|c|c|c|c|c|c|}
\hline Glass \# & Glass ID & Oxide & $\begin{array}{c}\text { Measured } \\
(\mathrm{wt} \%)\end{array}$ & $\begin{array}{c}\text { Measured } \\
\text { Bias-Corrected } \\
(w t \%)\end{array}$ & $\begin{array}{c}\text { Targeted } \\
\text { (wt\%) }\end{array}$ & $\begin{array}{c}\text { Diff of } \\
\text { Measured }\end{array}$ & $\begin{array}{c}\text { Diff of } \\
\text { Meas BC }\end{array}$ & $\begin{array}{l}\% \text { Diff of } \\
\text { Measured }\end{array}$ & $\begin{array}{l}\% \text { Diff of } \\
\text { Meas BC }\end{array}$ \\
\hline 12 & SB3-12 & $\mathrm{MnO}(\mathrm{wt} \%)$ & 2.6502 & 2.7426 & 2.8120 & -0.1618 & -0.0694 & $-5.8 \%$ & $-2.5 \%$ \\
\hline 12 & SB3-12 & $\mathrm{Na} 2 \mathrm{O}(\mathrm{wt} \%)$ & 14.9965 & 14.4114 & 14.8040 & 0.1925 & -0.3926 & $1.3 \%$ & $-2.7 \%$ \\
\hline 12 & SB3-12 & $\mathrm{NiO}(\mathrm{wt} \%)$ & 0.5281 & 0.5829 & 0.6310 & -0.1029 & -0.0481 & $-16.3 \%$ & $-7.6 \%$ \\
\hline 12 & SB3-12 & $\mathrm{PbO}(\mathrm{wt} \%)$ & 0.1091 & 0.1091 & 0.1180 & -0.0089 & -0.0089 & $-7.6 \%$ & $-7.6 \%$ \\
\hline 12 & SB3-12 & $\mathrm{SiO} 2(\mathrm{wt} \%)$ & 41.5024 & 44.6537 & 42.8900 & -1.3876 & 1.7637 & $-3.2 \%$ & $4.1 \%$ \\
\hline 12 & SB3-12 & ThO2 (wt $\%)$ & 0.1715 & 0.1715 & 0.0560 & 0.1155 & 0.1155 & $206.3 \%$ & $206.3 \%$ \\
\hline 12 & SB3-12 & $\mathrm{TiO} 2(\mathrm{wt} \%)$ & 0.0071 & 0.0075 & 0.0000 & 0.0071 & 0.0075 & & \\
\hline 12 & SB3-12 & $\mathrm{U} 3 \mathrm{O} 8(\mathrm{wt} \%)$ & 3.9651 & 4.1136 & 3.9910 & -0.0259 & 0.1226 & $-0.6 \%$ & $3.1 \%$ \\
\hline 12 & SB3-12 & $\mathrm{ZnO}(\mathrm{wt} \%)$ & 0.1447 & 0.1447 & 0.1610 & -0.0163 & -0.0163 & $-10.1 \%$ & $-10.1 \%$ \\
\hline 12 & SB3-12 & $\mathrm{ZrO} 2(\mathrm{wt} \%)$ & 0.2749 & 0.2749 & 0.2910 & -0.0161 & -0.0161 & $-5.5 \%$ & $-5.5 \%$ \\
\hline 12 & SB3-12 & Sum of Oxides & 97.2057 & 99.9972 & 100.0000 & -2.7943 & -0.0028 & $-2.8 \%$ & $0.0 \%$ \\
\hline 13 & SB3-13 & $\mathrm{Al} 2 \mathrm{O} 3$ (wt\%) & 4.7757 & 4.9143 & 4.6040 & 0.1717 & 0.3103 & $3.7 \%$ & $6.7 \%$ \\
\hline 13 & SB3-13 & $\mathrm{B} 2 \mathrm{O} 3(\mathrm{wt} \%)$ & 5.8441 & 5.7890 & 6.0000 & -0.1559 & -0.2110 & $-2.6 \%$ & $-3.5 \%$ \\
\hline 13 & SB3-13 & $\mathrm{BaO}(\mathrm{wt} \%)$ & 0.0444 & 0.0493 & 0.0530 & -0.0086 & -0.0037 & $-16.3 \%$ & $-7.0 \%$ \\
\hline 13 & SB3-13 & $\mathrm{CaO}(\mathrm{wt} \%)$ & 0.7954 & 0.7440 & 0.7660 & 0.0294 & -0.0220 & $3.8 \%$ & $-2.9 \%$ \\
\hline 13 & SB3-13 & $\mathrm{Ce} 2 \mathrm{O} 3(\mathrm{wt} \%)$ & 0.0729 & 0.0729 & 0.0760 & -0.0031 & -0.0031 & $-4.1 \%$ & $-4.1 \%$ \\
\hline 13 & SB3-13 & $\mathrm{Cr} 2 \mathrm{O} 3(\mathrm{wt} \%)$ & 0.0665 & 0.0707 & 0.0800 & -0.0135 & -0.0093 & $-16.9 \%$ & $-11.6 \%$ \\
\hline 13 & SB3-13 & $\mathrm{CuO}(\mathrm{wt} \%)$ & 0.0451 & 0.0480 & 0.0420 & 0.0031 & 0.0060 & $7.3 \%$ & $14.4 \%$ \\
\hline 13 & SB3-13 & $\mathrm{Fe} 2 \mathrm{O} 3(\mathrm{wt} \%)$ & 8.3209 & 8.2365 & 8.5750 & -0.2541 & -0.3385 & $-3.0 \%$ & $-3.9 \%$ \\
\hline 13 & SB3-13 & $\mathrm{K} 2 \mathrm{O}(\mathrm{wt} \%)$ & 0.0488 & 0.0438 & 0.0920 & -0.0432 & -0.0482 & $-47.0 \%$ & $-52.4 \%$ \\
\hline 13 & SB3-13 & La2O3 (wt \%) & 0.0358 & 0.0358 & 0.0440 & -0.0082 & -0.0082 & $-18.7 \%$ & $-18.7 \%$ \\
\hline 13 & SB3-13 & $\mathrm{Li} 2 \mathrm{O}(\mathrm{wt} \%)$ & 3.5900 & 3.7144 & 3.7510 & -0.1610 & -0.0366 & $-4.3 \%$ & $-1.0 \%$ \\
\hline 13 & SB3-13 & $\mathrm{MgO}(\mathrm{wt} \%)$ & 0.0187 & 0.0195 & 0.0400 & -0.0213 & -0.0205 & $-53.4 \%$ & $-51.3 \%$ \\
\hline 13 & SB3-13 & $\mathrm{MnO}(\mathrm{wt} \%)$ & 1.4913 & 1.6078 & 1.5280 & -0.0367 & 0.0798 & $-2.4 \%$ & $5.2 \%$ \\
\hline 13 & SB3-13 & $\mathrm{Na} 2 \mathrm{O}(\mathrm{wt} \%)$ & 15.3672 & 14.4476 & 15.2850 & 0.0822 & -0.8374 & $0.5 \%$ & $-5.5 \%$ \\
\hline 13 & SB3-13 & $\mathrm{NiO}(\mathrm{wt} \%)$ & 0.3162 & 0.3598 & 0.3430 & -0.0268 & 0.0168 & $-7.8 \%$ & $4.9 \%$ \\
\hline 13 & SB3-13 & $\mathrm{PbO}(\mathrm{wt} \%)$ & 0.0557 & 0.0557 & 0.0640 & -0.0083 & -0.0083 & $-12.9 \%$ & $-12.9 \%$ \\
\hline 13 & SB3-13 & $\mathrm{SiO} 2(\mathrm{wt} \%)$ & 55.3544 & 58.2751 & 56.2120 & -0.8576 & 2.0631 & $-1.5 \%$ & $3.7 \%$ \\
\hline 13 & SB3-13 & ThO2 (wt\%) & 0.0927 & 0.0927 & 0.0300 & 0.0627 & 0.0627 & $209.1 \%$ & $209.1 \%$ \\
\hline 13 & SB3-13 & $\mathrm{TiO} 2(\mathrm{wt} \%)$ & 0.0225 & 0.0244 & 0.0000 & 0.0225 & 0.0244 & & \\
\hline 13 & SB3-13 & $\mathrm{U} 3 \mathrm{O} 8(\mathrm{wt} \%)$ & 2.1963 & 2.2981 & 2.1690 & 0.0273 & 0.1291 & $1.3 \%$ & $5.9 \%$ \\
\hline 13 & SB3-13 & $\mathrm{ZnO}(\mathrm{wt} \%)$ & 0.0716 & 0.0716 & 0.0880 & -0.0164 & -0.0164 & $-18.7 \%$ & $-18.7 \%$ \\
\hline 13 & SB3-13 & $\mathrm{ZrO} 2(\mathrm{wt} \%)$ & 0.1425 & 0.1425 & 0.1580 & -0.0155 & -0.0155 & $-9.8 \%$ & $-9.8 \%$ \\
\hline 13 & SB3-13 & Sum of Oxides & 98.7686 & 101.1135 & 100.0000 & -1.2314 & 1.1135 & $-1.2 \%$ & $1.1 \%$ \\
\hline 14 & SB3-14 & $\mathrm{Al} 2 \mathrm{O} 3(\mathrm{wt} \%)$ & 6.7691 & 6.8695 & 6.6230 & 0.1461 & 0.2465 & $2.2 \%$ & $3.7 \%$ \\
\hline 14 & SB3-14 & $\mathrm{B} 2 \mathrm{O} 3(\mathrm{wt} \%)$ & 4.9425 & 4.8559 & 4.8800 & 0.0625 & -0.0241 & $1.3 \%$ & $-0.5 \%$ \\
\hline 14 & SB3-14 & $\mathrm{BaO}(\mathrm{wt} \%)$ & 0.0729 & 0.0796 & 0.0830 & -0.0101 & -0.0034 & $-12.2 \%$ & $-4.1 \%$ \\
\hline 14 & SB3-14 & $\mathrm{CaO}(\mathrm{wt} \%)$ & 1.2680 & 1.2084 & 1.1960 & 0.0720 & 0.0124 & $6.0 \%$ & $1.0 \%$ \\
\hline 14 & SB3-14 & $\mathrm{Ce} 2 \mathrm{O} 3(\mathrm{wt} \%)$ & 0.1110 & 0.1110 & 0.1180 & -0.0070 & -0.0070 & $-5.9 \%$ & $-5.9 \%$ \\
\hline 14 & SB3-14 & $\mathrm{Cr} 2 \mathrm{O} 3(\mathrm{wt} \%)$ & 0.1363 & 0.1450 & 0.1240 & 0.0123 & 0.0210 & $9.9 \%$ & $17.0 \%$ \\
\hline 14 & SB3-14 & $\mathrm{CuO}(\mathrm{wt} \%)$ & 0.0617 & 0.0647 & 0.0660 & -0.0043 & -0.0013 & $-6.6 \%$ & $-2.0 \%$ \\
\hline 14 & SB3-14 & $\mathrm{Fe} 2 \mathrm{O} 3(\mathrm{wt} \%)$ & 12.5385 & 12.7321 & 13.3760 & -0.8375 & -0.6439 & $-6.3 \%$ & $-4.8 \%$ \\
\hline 14 & SB3-14 & $\mathrm{K} 2 \mathrm{O}(\mathrm{wt} \%)$ & 0.1458 & 0.1305 & 0.1430 & 0.0028 & -0.0125 & $1.9 \%$ & $-8.8 \%$ \\
\hline 14 & SB3-14 & $\mathrm{La} 2 \mathrm{O} 3(\mathrm{wt} \%)$ & 0.0537 & 0.0537 & 0.0680 & -0.0143 & -0.0143 & $-21.1 \%$ & $-21.1 \%$ \\
\hline 14 & SB3-14 & Li2O (wt\%) & 2.9925 & 3.0909 & 3.0510 & -0.0585 & 0.0399 & $-1.9 \%$ & $1.3 \%$ \\
\hline 14 & SB3-14 & $\mathrm{MgO}(\mathrm{wt} \%)$ & 0.0361 & 0.0361 & 0.0630 & -0.0269 & -0.0269 & $-42.8 \%$ & $-42.7 \%$ \\
\hline 14 & SB3-14 & $\mathrm{MnO}(\mathrm{wt} \%)$ & 2.2725 & 2.1889 & 2.3840 & -0.1115 & -0.1951 & $-4.7 \%$ & $-8.2 \%$ \\
\hline 14 & SB3-14 & $\mathrm{Na} 2 \mathrm{O}(\mathrm{wt} \%)$ & 17.8711 & 16.7095 & 17.1240 & 0.7471 & -0.4145 & $4.4 \%$ & $-2.4 \%$ \\
\hline 14 & SB3-14 & $\mathrm{NiO}(\mathrm{wt} \%)$ & 0.4794 & 0.5232 & 0.5350 & -0.0556 & -0.0118 & $-10.4 \%$ & $-2.2 \%$ \\
\hline 14 & SB3-14 & $\mathrm{PbO}(\mathrm{wt} \%)$ & 0.0854 & 0.0854 & 0.1000 & -0.0146 & -0.0146 & $-14.6 \%$ & $-14.6 \%$ \\
\hline 14 & SB3-14 & $\mathrm{SiO} 2(\mathrm{wt} \%)$ & 46.3693 & 49.8904 & 46.2510 & 0.1183 & 3.6394 & $0.3 \%$ & $7.9 \%$ \\
\hline 14 & SB3-14 & ThO2 (wt $\%)$ & 0.1559 & 0.1559 & 0.0480 & 0.1079 & 0.1079 & $224.8 \%$ & $224.8 \%$ \\
\hline 14 & SB3-14 & $\mathrm{TiO} 2(\mathrm{wt} \%)$ & 0.0083 & 0.0088 & 0.0000 & 0.0083 & 0.0088 & & \\
\hline
\end{tabular}


Table H.4: Average Measured and Bias-Corrected Chemical Compositions Versus Targeted Compositions by Oxide by Glass Number (continued)

(Batch 1: 100; 101-U std)

\begin{tabular}{|c|c|c|c|c|c|c|c|c|c|}
\hline Glass \# & Glass ID & Oxide & $\begin{array}{c}\text { Measured } \\
(\mathrm{wt} \%)\end{array}$ & $\begin{array}{c}\text { Measured } \\
\text { Bias-Corrected } \\
(w t \%)\end{array}$ & $\begin{array}{c}\text { Targeted } \\
(\text { wt } \%)\end{array}$ & $\begin{array}{c}\text { Diff of } \\
\text { Measured }\end{array}$ & $\begin{array}{c}\text { Diff of } \\
\text { Meas BC }\end{array}$ & $\begin{array}{l}\% \text { Diff of } \\
\text { Measured }\end{array}$ & $\begin{array}{l}\% \text { Diff of } \\
\text { Meas BC }\end{array}$ \\
\hline 14 & SB3-14 & U3O8 (wt\%) & 3.2516 & 3.4259 & 3.3840 & -0.1324 & 0.0419 & $-3.9 \%$ & $1.2 \%$ \\
\hline 14 & SB3-14 & $\mathrm{ZnO}(\mathrm{wt} \%)$ & 0.1235 & 0.1235 & 0.1370 & -0.0135 & -0.0135 & $-9.8 \%$ & $-9.8 \%$ \\
\hline 14 & SB3-14 & $\mathrm{ZrO} 2(\mathrm{wt} \%)$ & 0.2242 & 0.2242 & 0.2470 & -0.0228 & -0.0228 & $-9.2 \%$ & $-9.2 \%$ \\
\hline 14 & SB3-14 & Sum of Oxides & 99.9693 & 102.7129 & 100.0010 & -0.0317 & 2.7119 & $0.0 \%$ & $2.7 \%$ \\
\hline 15 & SB3-15 & $\mathrm{Al} 2 \mathrm{O} 3(\mathrm{wt} \%)$ & 4.6104 & 4.8210 & 4.5860 & 0.0244 & 0.2350 & $0.5 \%$ & $5.1 \%$ \\
\hline 15 & SB3-15 & B2O3 (wt\%) & 5.3853 & 5.2242 & 5.3600 & 0.0253 & -0.1358 & $0.5 \%$ & $-2.5 \%$ \\
\hline 15 & SB3-15 & $\mathrm{BaO}(\mathrm{wt} \%)$ & 0.0544 & 0.0604 & 0.0630 & -0.0086 & -0.0026 & $-13.6 \%$ & $-4.1 \%$ \\
\hline 15 & SB3-15 & $\mathrm{CaO}(\mathrm{wt} \%)$ & 0.9843 & 0.9207 & 0.9120 & 0.0723 & 0.0087 & $7.9 \%$ & $1.0 \%$ \\
\hline 15 & SB3-15 & $\mathrm{Ce} 2 \mathrm{O} 3(\mathrm{wt} \%)$ & 0.0846 & 0.0846 & 0.0880 & -0.0034 & -0.0034 & $-3.8 \%$ & $-3.8 \%$ \\
\hline 15 & SB3-15 & $\mathrm{Cr} 2 \mathrm{O} 3(\mathrm{wt} \%)$ & 0.0917 & 0.0975 & 0.0940 & -0.0023 & 0.0035 & $-2.4 \%$ & $3.8 \%$ \\
\hline 15 & SB3-15 & $\mathrm{CuO}(\mathrm{wt} \%)$ & 0.0526 & 0.0560 & 0.0500 & 0.0026 & 0.0060 & $5.2 \%$ & $12.1 \%$ \\
\hline 15 & SB3-15 & $\mathrm{Fe} 2 \mathrm{O} 3(\mathrm{wt} \%)$ & 10.0008 & 9.8997 & 10.1970 & -0.1962 & -0.2973 & $-1.9 \%$ & $-2.9 \%$ \\
\hline 15 & SB3-15 & $\mathrm{K} 2 \mathrm{O}(\mathrm{wt} \%)$ & 0.0852 & 0.0764 & 0.1090 & -0.0238 & -0.0326 & $-21.8 \%$ & $-29.9 \%$ \\
\hline 15 & SB3-15 & $\mathrm{La} 2 \mathrm{O} 3(\mathrm{wt} \%)$ & 0.0460 & 0.0460 & 0.0580 & -0.0120 & -0.0120 & $-20.6 \%$ & $-20.6 \%$ \\
\hline 15 & SB3-15 & $\mathrm{Li} 2 \mathrm{O}(\mathrm{wt} \%)$ & 4.4565 & 4.6187 & 4.6900 & -0.2335 & -0.0713 & $-5.0 \%$ & $-1.5 \%$ \\
\hline 15 & SB3-15 & $\mathrm{MgO}(\mathrm{wt} \%)$ & 1.3244 & 1.3816 & 1.3880 & -0.0636 & -0.0064 & $-4.6 \%$ & $-0.5 \%$ \\
\hline 15 & SB3-15 & $\mathrm{MnO}(\mathrm{wt} \%)$ & 1.7108 & 1.8443 & 1.8190 & -0.1082 & 0.0253 & $-5.9 \%$ & $1.4 \%$ \\
\hline 15 & SB3-15 & $\mathrm{Na} 2 \mathrm{O}(\mathrm{wt} \%)$ & 14.7943 & 13.9104 & 14.3570 & 0.4373 & -0.4466 & $3.0 \%$ & $-3.1 \%$ \\
\hline 15 & SB3-15 & $\mathrm{NiO}(\mathrm{wt} \%)$ & 0.3566 & 0.4058 & 0.4080 & -0.0514 & -0.0022 & $-12.6 \%$ & $-0.5 \%$ \\
\hline 15 & SB3-15 & $\mathrm{PbO}(\mathrm{wt} \%)$ & 0.0668 & 0.0668 & 0.0760 & -0.0092 & -0.0092 & $-12.1 \%$ & $-12.1 \%$ \\
\hline 15 & SB3-15 & $\mathrm{SiO} 2(\mathrm{wt} \%)$ & 51.1828 & 53.5170 & 52.1210 & -0.9382 & 1.3960 & $-1.8 \%$ & $2.7 \%$ \\
\hline 15 & SB3-15 & ThO2 (wt $\%)$ & 0.1044 & 0.1044 & 0.0360 & 0.0684 & 0.0684 & $190.0 \%$ & $190.0 \%$ \\
\hline 15 & SB3-15 & $\mathrm{TiO} 2(\mathrm{wt} \%)$ & 0.6555 & 0.7095 & 0.7140 & -0.0585 & -0.0045 & $-8.2 \%$ & $-0.6 \%$ \\
\hline 15 & SB3-15 & U3O8 (wt\%) & 2.5087 & 2.6250 & 2.5810 & -0.0723 & 0.0440 & $-2.8 \%$ & $1.7 \%$ \\
\hline 15 & SB3-15 & $\mathrm{ZnO}(\mathrm{wt} \%)$ & 0.0890 & 0.0890 & 0.1040 & -0.0150 & -0.0150 & $-14.4 \%$ & $-14.4 \%$ \\
\hline 15 & SB3-15 & $\mathrm{ZrO} 2(\mathrm{wt} \%)$ & 0.1786 & 0.1786 & 0.1880 & -0.0094 & -0.0094 & $-5.0 \%$ & $-5.0 \%$ \\
\hline 15 & SB3-15 & Sum of Oxides & 98.8239 & 100.7380 & 99.9990 & -1.1751 & 0.7390 & $-1.2 \%$ & $0.7 \%$ \\
\hline 16 & SB3-16 & $\mathrm{Al} 2 \mathrm{O} 3(\mathrm{wt} \%)$ & 5.2103 & 5.2876 & 5.1420 & 0.0683 & 0.1456 & $1.3 \%$ & $2.8 \%$ \\
\hline 16 & SB3-16 & $\mathrm{B} 2 \mathrm{O} 3(\mathrm{wt} \%)$ & 5.0150 & 4.9271 & 5.0400 & -0.0250 & -0.1129 & $-0.5 \%$ & $-2.2 \%$ \\
\hline 16 & SB3-16 & $\mathrm{BaO}(\mathrm{wt} \%)$ & 0.0597 & 0.0663 & 0.0710 & -0.0113 & -0.0047 & $-15.9 \%$ & $-6.6 \%$ \\
\hline 16 & SB3-16 & $\mathrm{CaO}(\mathrm{wt} \%)$ & 1.0949 & 1.0241 & 1.0230 & 0.0719 & 0.0011 & $7.0 \%$ & $0.1 \%$ \\
\hline 16 & SB3-16 & $\mathrm{Ce} 2 \mathrm{O} 3(\mathrm{wt} \%)$ & 0.1045 & 0.1045 & 0.0990 & 0.0055 & 0.0055 & $5.6 \%$ & $5.6 \%$ \\
\hline 16 & SB3-16 & $\mathrm{Cr} 2 \mathrm{O} 3(\mathrm{wt} \%)$ & 0.1045 & 0.1111 & 0.1050 & -0.0005 & 0.0061 & $-0.5 \%$ & $5.9 \%$ \\
\hline 16 & SB3-16 & $\mathrm{CuO}(\mathrm{wt} \%)$ & 0.0613 & 0.0654 & 0.0560 & 0.0053 & 0.0094 & $9.5 \%$ & $16.8 \%$ \\
\hline 16 & SB3-16 & $\mathrm{Fe} 2 \mathrm{O} 3(\mathrm{wt} \%)$ & 10.7942 & 10.6859 & 11.4330 & -0.6388 & -0.7471 & $-5.6 \%$ & $-6.5 \%$ \\
\hline 16 & SB3-16 & $\mathrm{K} 2 \mathrm{O}(\mathrm{wt} \%)$ & 0.1042 & 0.0934 & 0.1220 & -0.0178 & -0.0286 & $-14.6 \%$ & $-23.4 \%$ \\
\hline 16 & SB3-16 & $\mathrm{La} 2 \mathrm{O} 3(\mathrm{wt} \%)$ & 0.0613 & 0.0613 & 0.0650 & -0.0037 & -0.0037 & $-5.7 \%$ & $-5.7 \%$ \\
\hline 16 & SB3-16 & $\mathrm{Li} 2 \mathrm{O}$ (wt\%) & 4.2035 & 4.3418 & 4.4100 & -0.2065 & -0.0682 & $-4.7 \%$ & $-1.5 \%$ \\
\hline 16 & SB3-16 & $\mathrm{MgO}(\mathrm{wt} \%)$ & 1.2444 & 1.2982 & 1.3140 & -0.0696 & -0.0158 & $-5.3 \%$ & $-1.2 \%$ \\
\hline 16 & SB3-16 & $\mathrm{MnO}(\mathrm{wt} \%)$ & 1.9045 & 2.0531 & 2.0390 & -0.1345 & 0.0141 & $-6.6 \%$ & $0.7 \%$ \\
\hline 16 & SB3-16 & $\mathrm{Na} 2 \mathrm{O}(\mathrm{wt} \%)$ & 15.7716 & 14.8267 & 15.3700 & 0.4016 & -0.5433 & $2.6 \%$ & $-3.5 \%$ \\
\hline 16 & SB3-16 & $\mathrm{NiO}(\mathrm{wt} \%)$ & 0.3932 & 0.4474 & 0.4570 & -0.0638 & -0.0096 & $-14.0 \%$ & $-2.1 \%$ \\
\hline 16 & SB3-16 & $\mathrm{PbO}(\mathrm{wt} \%)$ & 0.0727 & 0.0727 & 0.0850 & -0.0123 & -0.0123 & $-14.5 \%$ & $-14.5 \%$ \\
\hline 16 & SB3-16 & $\mathrm{SiO} 2(\mathrm{wt} \%)$ & 47.8668 & 51.5040 & 49.1050 & -1.2382 & 2.3990 & $-2.5 \%$ & $4.9 \%$ \\
\hline 16 & SB3-16 & ThO2 (wt $\%)$ & 0.1172 & 0.1172 & 0.0410 & 0.0762 & 0.0762 & $185.9 \%$ & $185.9 \%$ \\
\hline 16 & SB3-16 & $\mathrm{TiO} 2(\mathrm{wt} \%)$ & 0.7076 & 0.7659 & 0.8000 & -0.0924 & -0.0341 & $-11.5 \%$ & $-4.3 \%$ \\
\hline 16 & SB3-16 & $\mathrm{U} 3 \mathrm{O} 8(\mathrm{wt} \%)$ & 2.7829 & 2.9119 & 2.8940 & -0.1111 & 0.0179 & $-3.8 \%$ & $0.6 \%$ \\
\hline 16 & SB3-16 & $\mathrm{ZnO}(\mathrm{wt} \%)$ & 0.0980 & 0.0980 & 0.1170 & -0.0190 & -0.0190 & $-16.2 \%$ & $-16.2 \%$ \\
\hline 16 & SB3-16 & $\mathrm{ZrO} 2(\mathrm{wt} \%)$ & 0.1878 & 0.1878 & 0.2110 & -0.0232 & -0.0232 & $-11.0 \%$ & $-11.0 \%$ \\
\hline 16 & SB3-16 & Sum of Oxides & 97.9604 & 101.0514 & 99.9990 & -2.0386 & 1.0524 & $-2.0 \%$ & $1.1 \%$ \\
\hline 17 & SB3-17 & $\mathrm{Al} 2 \mathrm{O} 3(\mathrm{wt} \%)$ & 5.1961 & 5.3469 & 4.8690 & 0.3271 & 0.4779 & $6.7 \%$ & $9.8 \%$ \\
\hline 17 & SB3-17 & $\mathrm{B} 2 \mathrm{O} 3(\mathrm{wt} \%)$ & 5.5302 & 5.4780 & 5.6000 & -0.0698 & -0.1220 & $-1.2 \%$ & $-2.2 \%$ \\
\hline 17 & SB3-17 & $\mathrm{BaO}(\mathrm{wt} \%)$ & 0.0374 & 0.0449 & 0.0580 & -0.0206 & -0.0131 & $-35.5 \%$ & $-22.6 \%$ \\
\hline
\end{tabular}


Table H.4: Average Measured and Bias-Corrected Chemical Compositions Versus Targeted Compositions by Oxide by Glass Number (continued)

(Batch 1: 100; 101-U std)

\begin{tabular}{|c|c|c|c|c|c|c|c|c|c|}
\hline Glass \# & Glass ID & Oxide & $\begin{array}{c}\text { Measured } \\
(\mathrm{wt} \%)\end{array}$ & $\begin{array}{c}\text { Measured } \\
\text { Bias-Corrected } \\
(w t \%)\end{array}$ & $\begin{array}{c}\text { Targeted } \\
(\text { wt } \%)\end{array}$ & $\begin{array}{c}\text { Diff of } \\
\text { Measured }\end{array}$ & $\begin{array}{c}\text { Diff of } \\
\text { Meas BC }\end{array}$ & $\begin{array}{l}\% \text { Diff of } \\
\text { Measured }\end{array}$ & $\begin{array}{l}\% \text { Diff of } \\
\text { Meas BC }\end{array}$ \\
\hline 17 & SB3-17 & $\mathrm{CaO}(\mathrm{wt} \%)$ & 0.8909 & 0.8215 & 0.8290 & 0.0619 & -0.0075 & $7.5 \%$ & $-0.9 \%$ \\
\hline 17 & SB3-17 & $\mathrm{Ce} 2 \mathrm{O} 3(\mathrm{wt} \%)$ & 0.0726 & 0.0726 & 0.0800 & -0.0074 & -0.0074 & $-9.2 \%$ & $-9.2 \%$ \\
\hline 17 & SB3-17 & $\mathrm{Cr} 2 \mathrm{O} 3(\mathrm{wt} \%)$ & 0.0669 & 0.0794 & 0.0850 & -0.0181 & -0.0056 & $-21.3 \%$ & $-6.6 \%$ \\
\hline 17 & SB3-17 & $\mathrm{CuO}(\mathrm{wt} \%)$ & 0.0466 & 0.0498 & 0.0460 & 0.0006 & 0.0038 & $1.4 \%$ & $8.2 \%$ \\
\hline 17 & SB3-17 & $\mathrm{Fe} 2 \mathrm{O} 3(\mathrm{wt} \%)$ & 10.1294 & 9.8088 & 9.2700 & 0.8594 & 0.5388 & $9.3 \%$ & $5.8 \%$ \\
\hline 17 & SB3-17 & $\mathrm{K} 2 \mathrm{O}(\mathrm{wt} \%)$ & 0.0711 & 0.0632 & 0.0990 & -0.0279 & -0.0358 & $-28.2 \%$ & $-36.1 \%$ \\
\hline 17 & SB3-17 & $\mathrm{La} 2 \mathrm{O} 3(\mathrm{wt} \%)$ & 0.0405 & 0.0405 & 0.0530 & -0.0125 & -0.0125 & $-23.7 \%$ & $-23.7 \%$ \\
\hline 17 & SB3-17 & $\mathrm{Li} 2 \mathrm{O}(\mathrm{wt} \%)$ & 3.3962 & 3.5139 & 3.5000 & -0.1038 & 0.0139 & $-3.0 \%$ & $0.4 \%$ \\
\hline 17 & SB3-17 & $\mathrm{MgO}(\mathrm{wt} \%)$ & 0.0008 & 0.0009 & 0.0430 & -0.0422 & -0.0421 & $-98.1 \%$ & $-97.9 \%$ \\
\hline 17 & SB3-17 & $\mathrm{MnO}(\mathrm{wt} \%)$ & 1.5365 & 1.6998 & 1.6530 & -0.1165 & 0.0468 & $-7.0 \%$ & $2.8 \%$ \\
\hline 17 & SB3-17 & $\mathrm{Na} 2 \mathrm{O}(\mathrm{wt} \%)$ & 14.4236 & 13.9764 & 14.2970 & 0.1266 & -0.3206 & $0.9 \%$ & $-2.2 \%$ \\
\hline 17 & SB3-17 & $\mathrm{NiO}(\mathrm{wt} \%)$ & 0.3181 & 0.3710 & 0.3710 & -0.0529 & 0.0000 & $-14.3 \%$ & $0.0 \%$ \\
\hline 17 & SB3-17 & $\mathrm{PbO}(\mathrm{wt} \%)$ & 0.0555 & 0.0555 & 0.0690 & -0.0135 & -0.0135 & $-19.6 \%$ & $-19.6 \%$ \\
\hline 17 & SB3-17 & $\mathrm{SiO} 2(\mathrm{wt} \%)$ & 54.7661 & 57.6533 & 55.7830 & -1.0169 & 1.8703 & $-1.8 \%$ & $3.4 \%$ \\
\hline 17 & SB3-17 & ThO2 (wt\%) & 0.0916 & 0.0916 & 0.0330 & 0.0586 & 0.0586 & $177.6 \%$ & $177.6 \%$ \\
\hline 17 & SB3-17 & $\mathrm{TiO} 2(\mathrm{wt} \%)$ & 0.4220 & 0.4629 & 0.6490 & -0.2270 & -0.1861 & $-35.0 \%$ & $-28.7 \%$ \\
\hline 17 & SB3-17 & $\mathrm{U} 3 \mathrm{O} 8(\mathrm{wt} \%)$ & 2.2876 & 2.3937 & 2.3460 & -0.0584 & 0.0477 & $-2.5 \%$ & $2.0 \%$ \\
\hline 17 & SB3-17 & $\mathrm{ZnO}(\mathrm{wt} \%)$ & 0.0616 & 0.0616 & 0.0950 & -0.0334 & -0.0334 & $-35.1 \%$ & $-35.1 \%$ \\
\hline 17 & SB3-17 & $\mathrm{ZrO} 2(\mathrm{wt} \%)$ & 0.1449 & 0.1449 & 0.1710 & -0.0261 & -0.0261 & $-15.3 \%$ & $-15.3 \%$ \\
\hline 17 & SB3-17 & Sum of Oxides & 99.5863 & 102.2311 & 99.9990 & -0.4127 & 2.2321 & $-0.4 \%$ & $2.2 \%$ \\
\hline 18 & SB3-18 & $\mathrm{Al} 2 \mathrm{O} 3(\mathrm{wt} \%)$ & 6.4574 & 6.4606 & 6.5460 & -0.0886 & -0.0854 & $-1.4 \%$ & $-1.3 \%$ \\
\hline 18 & SB3-18 & $\mathrm{B} 2 \mathrm{O} 3(\mathrm{wt} \%)$ & 4.5723 & 4.4241 & 4.5600 & 0.0123 & -0.1359 & $0.3 \%$ & $-3.0 \%$ \\
\hline 18 & SB3-18 & $\mathrm{BaO}(\mathrm{wt} \%)$ & 0.0614 & 0.0737 & 0.0830 & -0.0216 & -0.0093 & $-26.0 \%$ & $-11.3 \%$ \\
\hline 18 & SB3-18 & $\mathrm{CaO}(\mathrm{wt} \%)$ & 1.2908 & 1.1902 & 1.1890 & 0.1018 & 0.0012 & $8.6 \%$ & $0.1 \%$ \\
\hline 18 & SB3-18 & $\mathrm{Ce} 2 \mathrm{O} 3(\mathrm{wt} \%)$ & 0.1063 & 0.1063 & 0.1150 & -0.0087 & -0.0087 & $-7.6 \%$ & $-7.6 \%$ \\
\hline 18 & SB3-18 & $\mathrm{Cr} 2 \mathrm{O} 3(\mathrm{wt} \%)$ & 0.1111 & 0.1319 & 0.1220 & -0.0109 & 0.0099 & $-8.9 \%$ & $8.1 \%$ \\
\hline 18 & SB3-18 & $\mathrm{CuO}(\mathrm{wt} \%)$ & 0.0648 & 0.0692 & 0.0650 & -0.0002 & 0.0042 & $-0.3 \%$ & $6.4 \%$ \\
\hline 18 & SB3-18 & $\mathrm{Fe} 2 \mathrm{O} 3(\mathrm{wt} \%)$ & 14.6509 & 14.1837 & 13.2870 & 1.3639 & 0.8967 & $10.3 \%$ & $6.7 \%$ \\
\hline 18 & SB3-18 & $\mathrm{K} 2 \mathrm{O}(\mathrm{wt} \%)$ & 0.1292 & 0.1150 & 0.1420 & -0.0128 & -0.0270 & $-9.0 \%$ & $-19.0 \%$ \\
\hline 18 & SB3-18 & $\mathrm{La} 2 \mathrm{O} 3(\mathrm{wt} \%)$ & 0.0566 & 0.0566 & 0.0760 & -0.0194 & -0.0194 & $-25.5 \%$ & $-25.5 \%$ \\
\hline 18 & SB3-18 & $\mathrm{Li} 2 \mathrm{O}$ (wt\%) & 2.7019 & 2.8086 & 2.8500 & -0.1481 & -0.0414 & $-5.2 \%$ & $-1.5 \%$ \\
\hline 18 & SB3-18 & $\mathrm{MgO}(\mathrm{wt} \%)$ & 0.0104 & 0.0113 & 0.0620 & -0.0516 & -0.0507 & $-83.3 \%$ & $-81.9 \%$ \\
\hline 18 & SB3-18 & $\mathrm{MnO}(\mathrm{wt} \%)$ & 2.1079 & 2.3317 & 2.3700 & -0.2621 & -0.0383 & $-11.1 \%$ & $-1.6 \%$ \\
\hline 18 & SB3-18 & $\mathrm{Na} 2 \mathrm{O}(\mathrm{wt} \%)$ & 18.1306 & 17.5660 & 17.4590 & 0.6716 & 0.1070 & $3.8 \%$ & $0.6 \%$ \\
\hline 18 & SB3-18 & $\mathrm{NiO}(\mathrm{wt} \%)$ & 0.4431 & 0.5169 & 0.5310 & -0.0879 & -0.0141 & $-16.5 \%$ & $-2.7 \%$ \\
\hline 18 & SB3-18 & $\mathrm{PbO}(\mathrm{wt} \%)$ & 0.0789 & 0.0789 & 0.0990 & -0.0201 & -0.0201 & $-20.3 \%$ & $-20.3 \%$ \\
\hline 18 & SB3-18 & $\mathrm{SiO} 2(\mathrm{wt} \%)$ & 45.5671 & 48.9447 & 45.7220 & -0.1549 & 3.2227 & $-0.3 \%$ & $7.0 \%$ \\
\hline 18 & SB3-18 & ThO2 (wt\%) & 0.1311 & 0.1311 & 0.0470 & 0.0841 & 0.0841 & $179.0 \%$ & $179.0 \%$ \\
\hline 18 & SB3-18 & $\mathrm{TiO} 2(\mathrm{wt} \%)$ & 0.8486 & 0.9318 & 0.9300 & -0.0814 & 0.0018 & $-8.8 \%$ & $0.2 \%$ \\
\hline 18 & SB3-18 & U3O8 (wt\%) & 3.2546 & 3.4053 & 3.3630 & -0.1084 & 0.0423 & $-3.2 \%$ & $1.3 \%$ \\
\hline 18 & SB3-18 & $\mathrm{ZnO}(\mathrm{wt} \%)$ & 0.1015 & 0.1015 & 0.1360 & -0.0345 & -0.0345 & $-25.4 \%$ & $-25.4 \%$ \\
\hline 18 & SB3-18 & $\mathrm{ZrO} 2(\mathrm{wt} \%)$ & 0.2073 & 0.2073 & 0.2450 & -0.0377 & -0.0377 & $-15.4 \%$ & $-15.4 \%$ \\
\hline 18 & SB3-18 & Sum of Oxides & 101.0836 & 103.8463 & 99.9990 & 1.0846 & 3.8473 & $1.1 \%$ & $3.8 \%$ \\
\hline 19 & SB3-19 & $\mathrm{A} 12 \mathrm{O} 3$ (wt\%) & 5.0402 & 5.1150 & 4.8690 & 0.1712 & 0.2460 & $3.5 \%$ & $5.1 \%$ \\
\hline 19 & SB3-19 & $\mathrm{B} 2 \mathrm{O} 3(\mathrm{wt} \%)$ & 6.9872 & 6.8646 & 7.0000 & -0.0128 & -0.1354 & $-0.2 \%$ & $-1.9 \%$ \\
\hline 19 & SB3-19 & $\mathrm{BaO}(\mathrm{wt} \%)$ & 0.0469 & 0.0507 & 0.0580 & -0.0111 & -0.0073 & $-19.2 \%$ & $-12.6 \%$ \\
\hline 19 & SB3-19 & $\mathrm{CaO}(\mathrm{wt} \%)$ & 0.9315 & 0.8606 & 0.8290 & 0.1025 & 0.0316 & $12.4 \%$ & $3.8 \%$ \\
\hline 19 & SB3-19 & $\mathrm{Ce} 2 \mathrm{O} 3(\mathrm{wt} \%)$ & 0.0796 & 0.0796 & 0.0800 & -0.0004 & -0.0004 & $-0.4 \%$ & $-0.4 \%$ \\
\hline 19 & SB3-19 & $\mathrm{Cr} 2 \mathrm{O} 3(\mathrm{wt} \%)$ & 0.0899 & 0.0945 & 0.0850 & 0.0049 & 0.0095 & $5.8 \%$ & $11.2 \%$ \\
\hline 19 & SB3-19 & $\mathrm{CuO}(\mathrm{wt} \%)$ & 0.0494 & 0.0523 & 0.0460 & 0.0034 & 0.0063 & $7.5 \%$ & $13.7 \%$ \\
\hline 19 & SB3-19 & $\mathrm{Fe} 2 \mathrm{O} 3(\mathrm{wt} \%)$ & 8.8820 & 8.8163 & 9.2700 & -0.3880 & -0.4537 & $-4.2 \%$ & $-4.9 \%$ \\
\hline 19 & SB3-19 & $\mathrm{K} 2 \mathrm{O}(\mathrm{wt} \%)$ & 0.0644 & 0.0576 & 0.0990 & -0.0346 & -0.0414 & $-34.9 \%$ & $-41.8 \%$ \\
\hline 19 & SB3-19 & $\mathrm{La} 2 \mathrm{O} 3(\mathrm{wt} \%)$ & 0.0413 & 0.0413 & 0.0530 & -0.0117 & -0.0117 & $-22.0 \%$ & $-22.0 \%$ \\
\hline
\end{tabular}


Table H.4: Average Measured and Bias-Corrected Chemical Compositions Versus Targeted Compositions by Oxide by Glass Number (continued)

(Batch 1: 100; 101-U std)

\begin{tabular}{|c|c|c|c|c|c|c|c|c|c|}
\hline Glass \# & Glass ID & Oxide & $\begin{array}{c}\text { Measured } \\
(\mathbf{w t} \%)\end{array}$ & $\begin{array}{c}\text { Measured } \\
\text { Bias-Corrected } \\
(\mathrm{wt} \%)\end{array}$ & $\begin{array}{c}\text { Targeted } \\
(\text { wt } \%)\end{array}$ & $\begin{array}{c}\text { Diff of } \\
\text { Measured }\end{array}$ & $\begin{array}{c}\text { Diff of } \\
\text { Meas BC }\end{array}$ & $\begin{array}{l}\% \text { Diff of } \\
\text { Measured }\end{array}$ & $\begin{array}{l}\% \text { Diff of } \\
\text { Meas BC }\end{array}$ \\
\hline 19 & SB3-19 & $\mathrm{Li} 2 \mathrm{O}(\mathrm{wt} \%)$ & 3.3854 & 3.4967 & 3.5000 & -0.1146 & -0.0033 & $-3.3 \%$ & $-0.1 \%$ \\
\hline 19 & SB3-19 & $\mathrm{MgO}(\mathrm{wt} \%)$ & 0.0075 & 0.0075 & 0.0430 & -0.0355 & -0.0355 & $-82.6 \%$ & $-82.5 \%$ \\
\hline 19 & SB3-19 & $\mathrm{MnO}(\mathrm{wt} \%)$ & 1.6043 & 1.6606 & 1.6530 & -0.0487 & 0.0076 & $-2.9 \%$ & $0.5 \%$ \\
\hline 19 & SB3-19 & $\mathrm{Na} 2 \mathrm{O}(\mathrm{wt} \%)$ & 14.3562 & 13.7965 & 14.2970 & 0.0592 & -0.5005 & $0.4 \%$ & $-3.5 \%$ \\
\hline 19 & SB3-19 & $\mathrm{NiO}(\mathrm{wt} \%)$ & 0.3324 & 0.3671 & 0.3710 & -0.0386 & -0.0039 & $-10.4 \%$ & $-1.1 \%$ \\
\hline 19 & SB3-19 & $\mathrm{PbO}(\mathrm{wt} \%)$ & 0.0646 & 0.0646 & 0.0690 & -0.0044 & -0.0044 & $-6.3 \%$ & $-6.3 \%$ \\
\hline 19 & SB3-19 & $\mathrm{SiO} 2(\mathrm{wt} \%)$ & 53.6964 & 57.7744 & 54.3830 & -0.6866 & 3.3914 & $-1.3 \%$ & $6.2 \%$ \\
\hline 19 & SB3-19 & ThO2 (wt\%) & 0.0970 & 0.0970 & 0.0330 & 0.0640 & 0.0640 & $194.0 \%$ & $194.0 \%$ \\
\hline 19 & SB3-19 & $\mathrm{TiO} 2(\mathrm{wt} \%)$ & 0.5813 & 0.6128 & 0.6490 & -0.0677 & -0.0362 & $-10.4 \%$ & $-5.6 \%$ \\
\hline 19 & SB3-19 & U3O8 (wt\%) & 2.2729 & 2.3580 & 2.3460 & -0.0731 & 0.0120 & $-3.1 \%$ & $0.5 \%$ \\
\hline 19 & SB3-19 & $\mathrm{ZnO}(\mathrm{wt} \%)$ & 0.0769 & 0.0769 & 0.0950 & -0.0181 & -0.0181 & $-19.1 \%$ & $-19.1 \%$ \\
\hline 19 & SB3-19 & $\mathrm{ZrO} 2(\mathrm{wt} \%)$ & 0.1634 & 0.1634 & 0.1710 & -0.0076 & -0.0076 & $-4.4 \%$ & $-4.4 \%$ \\
\hline 19 & SB3-19 & Sum of Oxides & 98.8511 & 102.5081 & 99.9990 & -1.1479 & 2.5091 & $-1.1 \%$ & $2.5 \%$ \\
\hline 20 & SB3-20 & $\mathrm{Al} 2 \mathrm{O} 3(\mathrm{wt} \%)$ & 6.3818 & 6.4764 & 6.2880 & 0.0938 & 0.1884 & $1.5 \%$ & $3.0 \%$ \\
\hline 20 & SB3-20 & $\mathrm{B} 2 \mathrm{O} 3(\mathrm{wt} \%)$ & 5.9085 & 5.8049 & 5.9000 & 0.0085 & -0.0951 & $0.1 \%$ & $-1.6 \%$ \\
\hline 20 & SB3-20 & $\mathrm{BaO}(\mathrm{wt} \%)$ & 0.0706 & 0.0784 & 0.0790 & -0.0084 & -0.0006 & $-10.6 \%$ & $-0.8 \%$ \\
\hline 20 & SB3-20 & $\mathrm{CaO}(\mathrm{wt} \%)$ & 1.1967 & 1.1193 & 1.1340 & 0.0627 & -0.0147 & $5.5 \%$ & $-1.3 \%$ \\
\hline 20 & SB3-20 & $\mathrm{Ce} 2 \mathrm{O} 3(\mathrm{wt} \%)$ & 0.1042 & 0.1042 & 0.1100 & -0.0058 & -0.0058 & $-5.2 \%$ & $-5.2 \%$ \\
\hline 20 & SB3-20 & $\mathrm{Cr} 2 \mathrm{O} 3(\mathrm{wt} \%)$ & 0.1166 & 0.1240 & 0.1170 & -0.0004 & 0.0070 & $-0.4 \%$ & $6.0 \%$ \\
\hline 20 & SB3-20 & $\mathrm{CuO}(\mathrm{wt} \%)$ & 0.0660 & 0.0704 & 0.0620 & 0.0040 & 0.0084 & $6.5 \%$ & $13.5 \%$ \\
\hline 20 & SB3-20 & $\mathrm{Fe} 2 \mathrm{O} 3(\mathrm{wt} \%)$ & 12.7100 & 12.5834 & 12.6690 & 0.0410 & -0.0856 & $0.3 \%$ & $-0.7 \%$ \\
\hline 20 & SB3-20 & $\mathrm{K} 2 \mathrm{O}(\mathrm{wt} \%)$ & 0.1111 & 0.0997 & 0.1360 & -0.0249 & -0.0363 & $-18.3 \%$ & $-26.7 \%$ \\
\hline 20 & SB3-20 & $\mathrm{La} 2 \mathrm{O} 3(\mathrm{wt} \%)$ & 0.0616 & 0.0616 & 0.0720 & -0.0104 & -0.0104 & $-14.5 \%$ & $-14.5 \%$ \\
\hline 20 & SB3-20 & $\mathrm{Li} 2 \mathrm{O}(\mathrm{wt} \%)$ & 2.8687 & 2.9630 & 2.9500 & -0.0813 & 0.0130 & $-2.8 \%$ & $0.4 \%$ \\
\hline 20 & SB3-20 & $\mathrm{MgO}(\mathrm{wt} \%)$ & 0.0174 & 0.0182 & 0.0590 & -0.0416 & -0.0408 & $-70.5 \%$ & $-69.2 \%$ \\
\hline 20 & SB3-20 & $\mathrm{MnO}(\mathrm{wt} \%)$ & 2.1402 & 2.3073 & 2.2600 & -0.1198 & 0.0473 & $-5.3 \%$ & $2.1 \%$ \\
\hline 20 & SB3-20 & $\mathrm{Na} 2 \mathrm{O}(\mathrm{wt} \%)$ & 17.1196 & 16.0937 & 16.9730 & 0.1466 & -0.8793 & $0.9 \%$ & $-5.2 \%$ \\
\hline 20 & SB3-20 & $\mathrm{NiO}(\mathrm{wt} \%)$ & 0.4578 & 0.5209 & 0.5070 & -0.0492 & 0.0139 & $-9.7 \%$ & $2.7 \%$ \\
\hline 20 & SB3-20 & $\mathrm{PbO}(\mathrm{wt} \%)$ & 0.0829 & 0.0829 & 0.0940 & -0.0111 & -0.0111 & $-11.8 \%$ & $-11.8 \%$ \\
\hline 20 & SB3-20 & $\mathrm{SiO} 2(\mathrm{wt} \%)$ & 45.3532 & 48.7964 & 46.0900 & -0.7368 & 2.7064 & $-1.6 \%$ & $5.9 \%$ \\
\hline 20 & SB3-20 & ThO2 (wt\%) & 0.1334 & 0.1334 & 0.0450 & 0.0884 & 0.0884 & $196.5 \%$ & $196.5 \%$ \\
\hline 20 & SB3-20 & $\mathrm{TiO} 2(\mathrm{wt} \%)$ & 0.8473 & 0.9171 & 0.8870 & -0.0397 & 0.0301 & $-4.5 \%$ & $3.4 \%$ \\
\hline 20 & SB3-20 & U3O8 (wt\%) & 3.2192 & 3.3684 & 3.2070 & 0.0122 & 0.1614 & $0.4 \%$ & $5.0 \%$ \\
\hline 20 & SB3-20 & $\mathrm{ZnO}(\mathrm{wt} \%)$ & 0.1170 & 0.1170 & 0.1290 & -0.0120 & -0.0120 & $-9.3 \%$ & $-9.3 \%$ \\
\hline 20 & SB3-20 & $\mathrm{ZrO} 2(\mathrm{wt} \%)$ & 0.2084 & 0.2084 & 0.2340 & -0.0256 & -0.0256 & $-11.0 \%$ & $-11.0 \%$ \\
\hline 20 & SB3-20 & Sum of Oxides & 99.2923 & 102.0489 & 100.0020 & -0.7097 & 2.0469 & $-0.7 \%$ & $2.0 \%$ \\
\hline 21 & SB3-21 & $\mathrm{Al} 2 \mathrm{O} 3(\mathrm{wt} \%)$ & 4.7474 & 4.9641 & 4.7250 & 0.0224 & 0.2391 & $0.5 \%$ & $5.1 \%$ \\
\hline 21 & SB3-21 & $\mathrm{B} 2 \mathrm{O} 3(\mathrm{wt} \%)$ & 5.2967 & 5.1384 & 5.2800 & 0.0167 & -0.1416 & $0.3 \%$ & $-2.7 \%$ \\
\hline 21 & SB3-21 & $\mathrm{BaO}(\mathrm{wt} \%)$ & 0.0564 & 0.0610 & 0.0650 & -0.0086 & -0.0040 & $-13.3 \%$ & $-6.2 \%$ \\
\hline 21 & SB3-21 & $\mathrm{CaO}(\mathrm{wt} \%)$ & 1.0620 & 0.9811 & 0.9400 & 0.1220 & 0.0411 & $13.0 \%$ & $4.4 \%$ \\
\hline 21 & SB3-21 & $\mathrm{Ce} 2 \mathrm{O} 3(\mathrm{wt} \%)$ & 0.0893 & 0.0893 & 0.0910 & -0.0017 & -0.0017 & $-1.9 \%$ & $-1.9 \%$ \\
\hline 21 & SB3-21 & $\mathrm{Cr} 2 \mathrm{O} 3(\mathrm{wt} \%)$ & 0.0764 & 0.0803 & 0.0970 & -0.0206 & -0.0167 & $-21.3 \%$ & $-17.2 \%$ \\
\hline 21 & SB3-21 & $\mathrm{CuO}(\mathrm{wt} \%)$ & 0.0541 & 0.0573 & 0.0520 & 0.0021 & 0.0053 & $4.1 \%$ & $10.2 \%$ \\
\hline 21 & SB3-21 & $\mathrm{Fe} 2 \mathrm{O} 3(\mathrm{wt} \%)$ & 9.7613 & 9.6901 & 10.5060 & -0.7447 & -0.8159 & $-7.1 \%$ & $-7.8 \%$ \\
\hline 21 & SB3-21 & $\mathrm{K} 2 \mathrm{O}(\mathrm{wt} \%)$ & 0.0861 & 0.0770 & 0.1120 & -0.0259 & -0.0350 & $-23.1 \%$ & $-31.2 \%$ \\
\hline 21 & SB3-21 & $\mathrm{La} 2 \mathrm{O} 3$ (wt $\%)$ & 0.0469 & 0.0469 & 0.0600 & -0.0131 & -0.0131 & $-21.8 \%$ & $-21.8 \%$ \\
\hline 21 & SB3-21 & $\mathrm{Li} 2 \mathrm{O}(\mathrm{wt} \%)$ & 2.5620 & 2.6551 & 2.6400 & -0.0780 & 0.0151 & $-3.0 \%$ & $0.6 \%$ \\
\hline 21 & SB3-21 & $\mathrm{MgO}(\mathrm{wt} \%)$ & 0.0108 & 0.0109 & 0.0490 & -0.0382 & -0.0381 & $-78.0 \%$ & $-77.8 \%$ \\
\hline 21 & SB3-21 & $\mathrm{MnO}(\mathrm{wt} \%)$ & 1.7883 & 1.8511 & 1.8740 & -0.0857 & -0.0229 & $-4.6 \%$ & $-1.2 \%$ \\
\hline 21 & SB3-21 & $\mathrm{Na} 2 \mathrm{O}(\mathrm{wt} \%)$ & 14.5247 & 13.9561 & 14.6100 & -0.0853 & -0.6539 & $-0.6 \%$ & $-4.5 \%$ \\
\hline 21 & SB3-21 & $\mathrm{NiO}(\mathrm{wt} \%)$ & 0.3722 & 0.4110 & 0.4200 & -0.0478 & -0.0090 & $-11.4 \%$ & $-2.1 \%$ \\
\hline 21 & SB3-21 & $\mathrm{PbO}(\mathrm{wt} \%)$ & 0.0630 & 0.0630 & 0.0780 & -0.0150 & -0.0150 & $-19.2 \%$ & $-19.2 \%$ \\
\hline 21 & SB3-21 & $\mathrm{SiO} 2(\mathrm{wt} \%)$ & 52.8942 & 55.3067 & 54.6670 & -1.7728 & 0.6397 & $-3.2 \%$ & $1.2 \%$ \\
\hline
\end{tabular}


Table H.4: Average Measured and Bias-Corrected Chemical Compositions Versus Targeted Compositions by Oxide by Glass Number (continued)

(Batch 1: 100; 101-U std)

\begin{tabular}{|c|c|c|c|c|c|c|c|c|c|}
\hline Glass \# & Glass ID & Oxide & $\begin{array}{c}\text { Measured } \\
(\mathrm{wt} \%)\end{array}$ & $\begin{array}{c}\text { Measured } \\
\text { Bias-Corrected } \\
(\mathrm{wt} \%)\end{array}$ & $\begin{array}{c}\text { Targeted } \\
(\mathrm{wt} \%)\end{array}$ & $\begin{array}{c}\text { Diff of } \\
\text { Measured }\end{array}$ & $\begin{array}{c}\text { Diff of } \\
\text { Meas BC }\end{array}$ & $\begin{array}{c}\% \text { Diff of } \\
\text { Measured }\end{array}$ & $\begin{array}{l}\% \text { Diff of } \\
\text { Meas BC }\end{array}$ \\
\hline 21 & SB3-21 & ThO2 (wt \%) & 0.1050 & 0.1050 & 0.0370 & 0.0680 & 0.0680 & $183.7 \%$ & $183.7 \%$ \\
\hline 21 & SB3-21 & $\mathrm{TiO} 2(\mathrm{wt} \%)$ & 0.6476 & 0.6827 & 0.7350 & -0.0874 & -0.0523 & $-11.9 \%$ & $-7.1 \%$ \\
\hline 21 & SB3-21 & $\mathrm{U} 3 \mathrm{O} 8(\mathrm{wt} \%)$ & 2.5087 & 2.6027 & 2.6590 & -0.1503 & -0.0563 & $-5.7 \%$ & $-2.1 \%$ \\
\hline 21 & SB3-21 & $\mathrm{ZnO}(\mathrm{wt} \%)$ & 0.0946 & 0.0946 & 0.1070 & -0.0124 & -0.0124 & $-11.6 \%$ & $-11.6 \%$ \\
\hline 21 & SB3-21 & $\mathrm{ZrO} 2(\mathrm{wt} \%)$ & 0.1689 & 0.1689 & 0.1940 & -0.0252 & -0.0252 & $-13.0 \%$ & $-13.0 \%$ \\
\hline 21 & SB3-21 & Sum of Oxides & 97.0165 & 99.0931 & 99.9980 & -2.9815 & -0.9049 & $-3.0 \%$ & $-0.9 \%$ \\
\hline 22 & SB3-22 & $\mathrm{Al} 2 \mathrm{O} 3(\mathrm{wt} \%)$ & 6.6794 & 6.6791 & 6.6700 & 0.0094 & 0.0091 & $0.1 \%$ & $0.1 \%$ \\
\hline 22 & SB3-22 & B2O3 (wt\%) & 4.1215 & 3.9880 & 4.1600 & -0.0385 & -0.1720 & $-0.9 \%$ & $-4.1 \%$ \\
\hline 22 & SB3-22 & $\mathrm{BaO}(\mathrm{wt} \%)$ & 0.0790 & 0.0863 & 0.0920 & -0.0130 & -0.0057 & $-14.1 \%$ & $-6.2 \%$ \\
\hline 22 & SB3-22 & $\mathrm{CaO}(\mathrm{wt} \%)$ & 1.3754 & 1.3107 & 1.3270 & 0.0484 & -0.0163 & $3.6 \%$ & $-1.2 \%$ \\
\hline 22 & SB3-22 & $\mathrm{Ce} 2 \mathrm{O} 3(\mathrm{wt} \%)$ & 0.1233 & 0.1233 & 0.1290 & -0.0057 & -0.0057 & $-4.4 \%$ & $-4.4 \%$ \\
\hline 22 & SB3-22 & $\mathrm{Cr} 2 \mathrm{O} 3(\mathrm{wt} \%)$ & 0.1396 & 0.1485 & 0.1360 & 0.0036 & 0.0125 & $2.6 \%$ & $9.2 \%$ \\
\hline 22 & SB3-22 & $\mathrm{CuO}(\mathrm{wt} \%)$ & 0.0663 & 0.0696 & 0.0730 & -0.0067 & -0.0034 & $-9.1 \%$ & $-4.7 \%$ \\
\hline 22 & SB3-22 & $\mathrm{Fe} 2 \mathrm{O} 3(\mathrm{wt} \%)$ & 14.0003 & 14.2129 & 14.8320 & -0.8317 & -0.6191 & $-5.6 \%$ & $-4.2 \%$ \\
\hline 22 & SB3-22 & $\mathrm{K} 2 \mathrm{O}(\mathrm{wt} \%)$ & 0.1488 & 0.1331 & 0.1590 & -0.0102 & -0.0259 & $-6.4 \%$ & $-16.3 \%$ \\
\hline 22 & SB3-22 & $\mathrm{La} 2 \mathrm{O} 3(\mathrm{wt} \%)$ & 0.0677 & 0.0677 & 0.0850 & -0.0173 & -0.0173 & $-20.3 \%$ & $-20.3 \%$ \\
\hline 22 & SB3-22 & $\mathrm{Li} 2 \mathrm{O}(\mathrm{wt} \%)$ & 2.0329 & 2.1131 & 2.0800 & -0.0471 & 0.0331 & $-2.3 \%$ & $1.6 \%$ \\
\hline 22 & SB3-22 & $\mathrm{MgO}(\mathrm{wt} \%)$ & 0.0435 & 0.0436 & 0.0700 & -0.0265 & -0.0264 & $-37.8 \%$ & $-37.7 \%$ \\
\hline 22 & SB3-22 & $\mathrm{MnO}(\mathrm{wt} \%)$ & 2.4597 & 2.3703 & 2.6450 & -0.1853 & -0.2747 & $-7.0 \%$ & $-10.4 \%$ \\
\hline 22 & SB3-22 & $\mathrm{Na} 2 \mathrm{O}(\mathrm{wt} \%)$ & 18.2317 & 17.0475 & 18.1550 & 0.0767 & -1.1075 & $0.4 \%$ & $-6.1 \%$ \\
\hline 22 & SB3-22 & $\mathrm{NiO}(\mathrm{wt} \%)$ & 0.5077 & 0.5540 & 0.5930 & -0.0853 & -0.0390 & $-14.4 \%$ & $-6.6 \%$ \\
\hline 22 & SB3-22 & $\mathrm{PbO}(\mathrm{wt} \%)$ & 0.0948 & 0.0948 & 0.1110 & -0.0162 & -0.0162 & $-14.6 \%$ & $-14.6 \%$ \\
\hline 22 & SB3-22 & $\mathrm{SiO} 2(\mathrm{wt} \%)$ & 43.1604 & 46.3599 & 43.4120 & -0.2516 & 2.9479 & $-0.6 \%$ & $6.8 \%$ \\
\hline 22 & SB3-22 & ThO2 (wt $\%)$ & 0.1715 & 0.1715 & 0.0530 & 0.1185 & 0.1185 & $223.7 \%$ & $223.7 \%$ \\
\hline 22 & SB3-22 & $\mathrm{TiO} 2(\mathrm{wt} \%)$ & 0.9733 & 1.0226 & 1.0380 & -0.0647 & -0.0154 & $-6.2 \%$ & $-1.5 \%$ \\
\hline 22 & SB3-22 & $\mathrm{U} 3 \mathrm{O} 8$ (wt\%) & 3.4963 & 3.6835 & 3.7540 & -0.2577 & -0.0705 & $-6.9 \%$ & $-1.9 \%$ \\
\hline 22 & SB3-22 & $\mathrm{ZnO}(\mathrm{wt} \%)$ & 0.1304 & 0.1304 & 0.1520 & -0.0216 & -0.0216 & $-14.2 \%$ & $-14.2 \%$ \\
\hline 22 & SB3-22 & $\mathrm{ZrO} 2(\mathrm{wt} \%)$ & 0.2401 & 0.2401 & 0.2740 & -0.0339 & -0.0339 & $-12.4 \%$ & $-12.4 \%$ \\
\hline 22 & SB3-22 & Sum of Oxides & 98.3437 & 100.6506 & 100.0000 & -1.6563 & 0.6506 & $-1.7 \%$ & $0.7 \%$ \\
\hline 23 & SB3-23 & $\mathrm{Al} 2 \mathrm{O} 3(\mathrm{wt} \%)$ & 4.7190 & 4.9347 & 4.5860 & 0.1330 & 0.3487 & $2.9 \%$ & $7.6 \%$ \\
\hline 23 & SB3-23 & $\mathrm{B} 2 \mathrm{O} 3(\mathrm{wt} \%)$ & 5.3853 & 5.2242 & 5.3600 & 0.0253 & -0.1358 & $0.5 \%$ & $-2.5 \%$ \\
\hline 23 & SB3-23 & $\mathrm{BaO}(\mathrm{wt} \%)$ & 0.0553 & 0.0597 & 0.0630 & -0.0077 & -0.0033 & $-12.3 \%$ & $-5.2 \%$ \\
\hline 23 & SB3-23 & $\mathrm{CaO}(\mathrm{wt} \%)$ & 1.0095 & 0.9326 & 0.9120 & 0.0975 & 0.0206 & $10.7 \%$ & $2.3 \%$ \\
\hline 23 & SB3-23 & $\mathrm{Ce} 2 \mathrm{O} 3(\mathrm{wt} \%)$ & 0.0864 & 0.0864 & 0.0880 & -0.0016 & -0.0016 & $-1.8 \%$ & $-1.8 \%$ \\
\hline 23 & SB3-23 & $\mathrm{Cr} 2 \mathrm{O} 3(\mathrm{wt} \%)$ & 0.0639 & 0.0672 & 0.0940 & -0.0301 & -0.0268 & $-32.0 \%$ & $-28.5 \%$ \\
\hline 23 & SB3-23 & $\mathrm{CuO}(\mathrm{wt} \%)$ & 0.0535 & 0.0566 & 0.0500 & 0.0035 & 0.0066 & $7.0 \%$ & $13.3 \%$ \\
\hline 23 & SB3-23 & $\mathrm{Fe} 2 \mathrm{O} 3(\mathrm{wt} \%)$ & 10.0079 & 9.9350 & 10.1970 & -0.1891 & -0.2620 & $-1.9 \%$ & $-2.6 \%$ \\
\hline 23 & SB3-23 & $\mathrm{K} 2 \mathrm{O}(\mathrm{wt} \%)$ & 0.0843 & 0.0754 & 0.1090 & -0.0247 & -0.0336 & $-22.6 \%$ & $-30.8 \%$ \\
\hline 23 & SB3-23 & $\mathrm{La} 2 \mathrm{O} 3(\mathrm{wt} \%)$ & 0.0463 & 0.0463 & 0.0580 & -0.0117 & -0.0117 & $-20.1 \%$ & $-20.1 \%$ \\
\hline 23 & SB3-23 & $\mathrm{Li} 2 \mathrm{O}(\mathrm{wt} \%)$ & 3.1648 & 3.2799 & 3.3500 & -0.1852 & -0.0701 & $-5.5 \%$ & $-2.1 \%$ \\
\hline 23 & SB3-23 & $\mathrm{MgO}(\mathrm{wt} \%)$ & 0.0087 & 0.0088 & 0.0480 & -0.0393 & -0.0392 & $-81.9 \%$ & $-81.7 \%$ \\
\hline 23 & SB3-23 & $\mathrm{MnO}(\mathrm{wt} \%)$ & 1.8303 & 1.8942 & 1.8190 & 0.0113 & 0.0752 & $0.6 \%$ & $4.1 \%$ \\
\hline 23 & SB3-23 & $\mathrm{Na} 2 \mathrm{O}(\mathrm{wt} \%)$ & 13.8507 & 13.3080 & 13.6870 & 0.1637 & -0.3790 & $1.2 \%$ & $-2.8 \%$ \\
\hline 23 & SB3-23 & $\mathrm{NiO}(\mathrm{wt} \%)$ & 0.3846 & 0.4246 & 0.4080 & -0.0234 & 0.0166 & $-5.7 \%$ & $4.1 \%$ \\
\hline 23 & SB3-23 & $\mathrm{PbO}(\mathrm{wt} \%)$ & 0.0619 & 0.0619 & 0.0760 & -0.0141 & -0.0141 & $-18.5 \%$ & $-18.5 \%$ \\
\hline 23 & SB3-23 & $\mathrm{SiO} 2(\mathrm{wt} \%)$ & 54.5522 & 57.0416 & 55.4710 & -0.9188 & 1.5706 & $-1.7 \%$ & $2.8 \%$ \\
\hline 23 & SB3-23 & ThO2 (wt $\%)$ & 0.1095 & 0.1095 & 0.0360 & 0.0735 & 0.0735 & $204.2 \%$ & $204.2 \%$ \\
\hline 23 & SB3-23 & $\mathrm{TiO} 2(\mathrm{wt} \%)$ & 0.6864 & 0.7236 & 0.7140 & -0.0276 & 0.0096 & $-3.9 \%$ & $1.3 \%$ \\
\hline 23 & SB3-23 & $\mathrm{U} 3 \mathrm{O} 8(\mathrm{wt} \%)$ & 2.5854 & 2.6823 & 2.5810 & 0.0044 & 0.1013 & $0.2 \%$ & $3.9 \%$ \\
\hline 23 & SB3-23 & $\mathrm{ZnO}(\mathrm{wt} \%)$ & 0.1024 & 0.1024 & 0.1040 & -0.0016 & -0.0016 & $-1.6 \%$ & $-1.6 \%$ \\
\hline 23 & SB3-23 & $\mathrm{ZrO} 2(\mathrm{wt} \%)$ & 0.1766 & 0.1766 & 0.1880 & -0.0114 & -0.0114 & $-6.1 \%$ & $-6.1 \%$ \\
\hline 23 & SB3-23 & Sum of Oxides & 99.0249 & 101.2314 & 99.9990 & -0.9741 & 1.2324 & $-1.0 \%$ & $1.2 \%$ \\
\hline 24 & SB3-24 & $\mathrm{Al} 2 \mathrm{O} 3(\mathrm{wt} \%)$ & 6.7361 & 7.0438 & 6.6700 & 0.0661 & 0.3738 & $1.0 \%$ & $5.6 \%$ \\
\hline
\end{tabular}


Table H.4: Average Measured and Bias-Corrected Chemical Compositions Versus Targeted Compositions by Oxide by Glass Number (continued)

(Batch 1: 100; 101-U std)

\begin{tabular}{|c|c|c|c|c|c|c|c|c|c|}
\hline Glass \# & Glass ID & Oxide & $\begin{array}{c}\text { Measured } \\
(\mathbf{w t} \%)\end{array}$ & $\begin{array}{c}\text { Measured } \\
\text { Bias-Corrected } \\
(w \mathrm{t} \%)\end{array}$ & $\begin{array}{c}\text { Targeted } \\
(\text { wt } \%)\end{array}$ & $\begin{array}{c}\text { Diff of } \\
\text { Measured }\end{array}$ & $\begin{array}{c}\text { Diff of } \\
\text { Meas BC }\end{array}$ & $\begin{array}{l}\% \text { Diff of } \\
\text { Measured }\end{array}$ & $\begin{array}{l}\% \text { Diff of } \\
\text { Meas BC }\end{array}$ \\
\hline 24 & SB3-24 & B2O3 (wt\%) & 4.2986 & 4.1698 & 4.1600 & 0.1386 & 0.0098 & $3.3 \%$ & $0.2 \%$ \\
\hline 24 & SB3-24 & $\mathrm{BaO}(\mathrm{wt} \%)$ & 0.0809 & 0.0884 & 0.0920 & -0.0111 & -0.0036 & $-12.0 \%$ & $-3.9 \%$ \\
\hline 24 & SB3-24 & $\mathrm{CaO}(\mathrm{wt} \%)$ & 1.3982 & 1.3324 & 1.3270 & 0.0712 & 0.0054 & $5.4 \%$ & $0.4 \%$ \\
\hline 24 & SB3-24 & $\mathrm{Ce} 2 \mathrm{O} 3(\mathrm{wt} \%)$ & 0.1265 & 0.1265 & 0.1290 & -0.0025 & -0.0025 & $-1.9 \%$ & $-1.9 \%$ \\
\hline 24 & SB3-24 & $\mathrm{Cr} 2 \mathrm{O} 3(\mathrm{wt} \%)$ & 0.1224 & 0.1302 & 0.1360 & -0.0136 & -0.0058 & $-10.0 \%$ & $-4.3 \%$ \\
\hline 24 & SB3-24 & $\mathrm{CuO}(\mathrm{wt} \%)$ & 0.0704 & 0.0739 & 0.0730 & -0.0026 & 0.0009 & $-3.5 \%$ & $1.2 \%$ \\
\hline 24 & SB3-24 & $\mathrm{Fe} 2 \mathrm{O} 3(\mathrm{wt} \%)$ & 14.1612 & 14.3748 & 14.8320 & -0.6708 & -0.4572 & $-4.5 \%$ & $-3.1 \%$ \\
\hline 24 & SB3-24 & $\mathrm{K} 2 \mathrm{O}(\mathrm{wt} \%)$ & 0.1605 & 0.1437 & 0.1590 & 0.0015 & -0.0153 & $1.0 \%$ & $-9.7 \%$ \\
\hline 24 & SB3-24 & $\mathrm{La} 2 \mathrm{O} 3(\mathrm{wt} \%)$ & 0.0692 & 0.0692 & 0.0850 & -0.0158 & -0.0158 & $-18.6 \%$ & $-18.6 \%$ \\
\hline 24 & SB3-24 & Li2O (wt\%) & 2.5297 & 2.6217 & 2.6000 & -0.0703 & 0.0217 & $-2.7 \%$ & $0.8 \%$ \\
\hline 24 & SB3-24 & $\mathrm{MgO}(\mathrm{wt} \%)$ & 0.0431 & 0.0432 & 0.0700 & -0.0269 & -0.0268 & $-38.4 \%$ & $-38.3 \%$ \\
\hline 24 & SB3-24 & $\mathrm{MnO}(\mathrm{wt} \%)$ & 2.4856 & 2.3952 & 2.6450 & -0.1594 & -0.2498 & $-6.0 \%$ & $-9.4 \%$ \\
\hline 24 & SB3-24 & $\mathrm{Na} 2 \mathrm{O}(\mathrm{wt} \%)$ & 18.7709 & 17.5504 & 17.6350 & 1.1359 & -0.0846 & $6.4 \%$ & $-0.5 \%$ \\
\hline 24 & SB3-24 & $\mathrm{NiO}(\mathrm{wt} \%)$ & 0.5173 & 0.5643 & 0.5930 & -0.0757 & -0.0287 & $-12.8 \%$ & $-4.8 \%$ \\
\hline 24 & SB3-24 & $\mathrm{PbO}(\mathrm{wt} \%)$ & 0.0967 & 0.0967 & 0.1110 & -0.0143 & -0.0143 & $-12.9 \%$ & $-12.9 \%$ \\
\hline 24 & SB3-24 & $\mathrm{SiO} 2(\mathrm{wt} \%)$ & 43.3743 & 45.3525 & 43.4120 & -0.0377 & 1.9405 & $-0.1 \%$ & $4.5 \%$ \\
\hline 24 & SB3-24 & ThO2 (wt $\%)$ & 0.1713 & 0.1713 & 0.0530 & 0.1183 & 0.1183 & $223.1 \%$ & $223.1 \%$ \\
\hline 24 & SB3-24 & $\mathrm{TiO} 2(\mathrm{wt} \%)$ & 0.9908 & 1.0409 & 1.0380 & -0.0472 & 0.0029 & $-4.5 \%$ & $0.3 \%$ \\
\hline 24 & SB3-24 & $\mathrm{U} 3 \mathrm{O} 8(\mathrm{wt} \%)$ & 3.5376 & 3.7265 & 3.7540 & -0.2164 & -0.0275 & $-5.8 \%$ & $-0.7 \%$ \\
\hline 24 & SB3-24 & $\mathrm{ZnO}(\mathrm{wt} \%)$ & 0.1341 & 0.1341 & 0.1520 & -0.0179 & -0.0179 & $-11.8 \%$ & $-11.8 \%$ \\
\hline 24 & SB3-24 & $\mathrm{ZrO} 2(\mathrm{wt} \%)$ & 0.2398 & 0.2398 & 0.2740 & -0.0342 & -0.0342 & $-12.5 \%$ & $-12.5 \%$ \\
\hline 24 & SB3-24 & Sum of Oxides & 100.1150 & 101.4892 & 100.0000 & 0.1150 & 1.4892 & $0.1 \%$ & $1.5 \%$ \\
\hline 25 & SB3-25 & $\mathrm{Al} 2 \mathrm{O} 3(\mathrm{wt} \%)$ & 4.4781 & 4.5445 & 4.3080 & 0.1701 & 0.2365 & $3.9 \%$ & $5.5 \%$ \\
\hline 25 & SB3-25 & $\mathrm{B} 2 \mathrm{O} 3(\mathrm{wt} \%)$ & 5.5221 & 5.4252 & 5.5200 & 0.0021 & -0.0948 & $0.0 \%$ & $-1.7 \%$ \\
\hline 25 & SB3-25 & $\mathrm{BaO}(\mathrm{wt} \%)$ & 0.0438 & 0.0526 & 0.0600 & -0.0162 & -0.0074 & $-27.0 \%$ & $-12.4 \%$ \\
\hline 25 & SB3-25 & $\mathrm{CaO}(\mathrm{wt} \%)$ & 0.9242 & 0.8521 & 0.8570 & 0.0672 & -0.0049 & $7.8 \%$ & $-0.6 \%$ \\
\hline 25 & SB3-25 & $\mathrm{Ce} 2 \mathrm{O} 3(\mathrm{wt} \%)$ & 0.0767 & 0.0767 & 0.0830 & -0.0063 & -0.0063 & $-7.6 \%$ & $-7.6 \%$ \\
\hline 25 & SB3-25 & $\mathrm{Cr} 2 \mathrm{O} 3(\mathrm{wt} \%)$ & 0.0760 & 0.0902 & 0.0880 & -0.0120 & 0.0022 & $-13.6 \%$ & $2.5 \%$ \\
\hline 25 & SB3-25 & $\mathrm{CuO}(\mathrm{wt} \%)$ & 0.0488 & 0.0521 & 0.0470 & 0.0018 & 0.0051 & $3.9 \%$ & $10.9 \%$ \\
\hline 25 & SB3-25 & $\mathrm{Fe} 2 \mathrm{O} 3(\mathrm{wt} \%)$ & 10.3081 & 9.9821 & 9.5790 & 0.7291 & 0.4031 & $7.6 \%$ & $4.2 \%$ \\
\hline 25 & SB3-25 & $\mathrm{K} 2 \mathrm{O}(\mathrm{wt} \%)$ & 0.0771 & 0.0686 & 0.1030 & -0.0259 & -0.0344 & $-25.2 \%$ & $-33.4 \%$ \\
\hline 25 & SB3-25 & $\mathrm{La} 2 \mathrm{O} 3(\mathrm{wt} \%)$ & 0.0419 & 0.0419 & 0.0550 & -0.0131 & -0.0131 & $-23.8 \%$ & $-23.8 \%$ \\
\hline 25 & SB3-25 & $\mathrm{Li} 2 \mathrm{O}(\mathrm{wt} \%)$ & 5.3769 & 5.5535 & 5.5200 & -0.1431 & 0.0335 & $-2.6 \%$ & $0.6 \%$ \\
\hline 25 & SB3-25 & $\mathrm{MgO}(\mathrm{wt} \%)$ & 0.0008 & 0.0009 & 0.0450 & -0.0442 & -0.0441 & $-98.2 \%$ & $-98.0 \%$ \\
\hline 25 & SB3-25 & $\mathrm{MnO}(\mathrm{wt} \%)$ & 1.5462 & 1.7105 & 1.7090 & -0.1628 & 0.0015 & $-9.5 \%$ & $0.1 \%$ \\
\hline 25 & SB3-25 & $\mathrm{Na} 2 \mathrm{O}(\mathrm{wt} \%)$ & 11.9197 & 11.5447 & 11.7800 & 0.1397 & -0.2353 & $1.2 \%$ & $-2.0 \%$ \\
\hline 25 & SB3-25 & $\mathrm{NiO}(\mathrm{wt} \%)$ & 0.3328 & 0.3881 & 0.3830 & -0.0502 & 0.0051 & $-13.1 \%$ & $1.3 \%$ \\
\hline 25 & SB3-25 & $\mathrm{PbO}(\mathrm{wt} \%)$ & 0.0557 & 0.0557 & 0.0710 & -0.0153 & -0.0153 & $-21.5 \%$ & $-21.5 \%$ \\
\hline 25 & SB3-25 & $\mathrm{SiO} 2(\mathrm{wt} \%)$ & 55.6218 & 59.8457 & 56.3890 & -0.7672 & 3.4567 & $-1.4 \%$ & $6.1 \%$ \\
\hline 25 & SB3-25 & ThO2 (wt\%) & 0.0916 & 0.0916 & 0.0340 & 0.0576 & 0.0576 & $169.4 \%$ & $169.4 \%$ \\
\hline 25 & SB3-25 & $\mathrm{TiO} 2(\mathrm{wt} \%)$ & 0.6147 & 0.6749 & 0.6700 & -0.0553 & 0.0049 & $-8.3 \%$ & $0.7 \%$ \\
\hline 25 & SB3-25 & $\mathrm{U} 3 \mathrm{O} 8(\mathrm{wt} \%)$ & 2.3731 & 2.4831 & 2.4250 & -0.0519 & 0.0581 & $-2.1 \%$ & $2.4 \%$ \\
\hline 25 & SB3-25 & $\mathrm{ZnO}(\mathrm{wt} \%)$ & 0.0666 & 0.0666 & 0.0980 & -0.0314 & -0.0314 & $-32.0 \%$ & $-32.0 \%$ \\
\hline 25 & SB3-25 & $\mathrm{ZrO} 2(\mathrm{wt} \%)$ & 0.1469 & 0.1469 & 0.1770 & -0.0301 & -0.0301 & $-17.0 \%$ & $-17.0 \%$ \\
\hline 25 & SB3-25 & Sum of Oxides & 99.7437 & 103.7483 & 100.0010 & -0.2573 & 3.7473 & $-0.3 \%$ & $3.7 \%$ \\
\hline 26 & SB3-26 & $\mathrm{Al} 2 \mathrm{O} 3$ (wt $\%)$ & 6.4479 & 6.6350 & 6.2530 & 0.1949 & 0.3820 & $3.1 \%$ & $6.1 \%$ \\
\hline 26 & SB3-26 & $\mathrm{B} 2 \mathrm{O} 3(\mathrm{wt} \%)$ & 4.3871 & 4.3458 & 4.4000 & -0.0129 & -0.0542 & $-0.3 \%$ & $-1.2 \%$ \\
\hline 26 & SB3-26 & $\mathrm{BaO}(\mathrm{wt} \%)$ & 0.0729 & 0.0809 & 0.0870 & -0.0141 & -0.0061 & $-16.3 \%$ & $-7.0 \%$ \\
\hline 26 & SB3-26 & $\mathrm{CaO}(\mathrm{wt} \%)$ & 1.3621 & 1.2741 & 1.2440 & 0.1181 & 0.0301 & $9.5 \%$ & $2.4 \%$ \\
\hline 26 & SB3-26 & $\mathrm{Ce} 2 \mathrm{O} 3(\mathrm{wt} \%)$ & 0.1174 & 0.1174 & 0.1210 & -0.0036 & -0.0036 & $-3.0 \%$ & $-3.0 \%$ \\
\hline 26 & SB3-26 & $\mathrm{Cr} 2 \mathrm{O} 3(\mathrm{wt} \%)$ & 0.1202 & 0.1279 & 0.1280 & -0.0078 & -0.0001 & $-6.1 \%$ & $-0.1 \%$ \\
\hline 26 & SB3-26 & $\mathrm{CuO}(\mathrm{wt} \%)$ & 0.0735 & 0.0784 & 0.0690 & 0.0045 & 0.0094 & $6.6 \%$ & $13.6 \%$ \\
\hline 26 & SB3-26 & $\mathrm{Fe} 2 \mathrm{O} 3(\mathrm{wt} \%)$ & 13.6179 & 13.4754 & 13.9050 & -0.2871 & -0.4296 & $-2.1 \%$ & $-3.1 \%$ \\
\hline
\end{tabular}


Table H.4: Average Measured and Bias-Corrected Chemical Compositions Versus Targeted Compositions by Oxide by Glass Number (continued)

(Batch 1: 100; 101-U std)

\begin{tabular}{|c|c|c|c|c|c|c|c|c|c|}
\hline Glass \# & Glass ID & Oxide & $\begin{array}{c}\text { Measured } \\
(\mathrm{wt} \%)\end{array}$ & $\begin{array}{c}\text { Measured } \\
\text { Bias-Corrected } \\
(w t \%)\end{array}$ & $\begin{array}{c}\text { Targeted } \\
\text { (wt\%) }\end{array}$ & $\begin{array}{c}\text { Diff of } \\
\text { Measured }\end{array}$ & $\begin{array}{c}\text { Diff of } \\
\text { Meas BC }\end{array}$ & $\begin{array}{l}\% \text { Diff of } \\
\text { Measured }\end{array}$ & $\begin{array}{l}\% \text { Diff of } \\
\text { Meas BC }\end{array}$ \\
\hline 26 & SB3-26 & $\mathrm{K} 2 \mathrm{O}(\mathrm{wt} \%)$ & 0.1464 & 0.1312 & 0.1490 & -0.0026 & -0.0178 & $-1.8 \%$ & $-11.9 \%$ \\
\hline 26 & SB3-26 & $\mathrm{La} 2 \mathrm{O} 3(\mathrm{wt} \%)$ & 0.0639 & 0.0639 & 0.0790 & -0.0151 & -0.0151 & $-19.1 \%$ & $-19.1 \%$ \\
\hline 26 & SB3-26 & $\mathrm{Li} 2 \mathrm{O}(\mathrm{wt} \%)$ & 4.2520 & 4.3993 & 4.4000 & -0.1480 & -0.0007 & $-3.4 \%$ & $0.0 \%$ \\
\hline 26 & SB3-26 & $\mathrm{MgO}(\mathrm{wt} \%)$ & 0.0166 & 0.0173 & 0.0650 & -0.0484 & -0.0477 & $-74.5 \%$ & $-73.4 \%$ \\
\hline 26 & SB3-26 & $\mathrm{MnO}(\mathrm{wt} \%)$ & 2.2176 & 2.3908 & 2.4800 & -0.2624 & -0.0892 & $-10.6 \%$ & $-3.6 \%$ \\
\hline 26 & SB3-26 & $\mathrm{Na} 2 \mathrm{O}(\mathrm{wt} \%)$ & 16.1423 & 15.1762 & 15.7450 & 0.3973 & -0.5688 & $2.5 \%$ & $-3.6 \%$ \\
\hline 26 & SB3-26 & $\mathrm{NiO}(\mathrm{wt} \%)$ & 0.4606 & 0.5242 & 0.5560 & -0.0954 & -0.0318 & $-17.2 \%$ & $-5.7 \%$ \\
\hline 26 & SB3-26 & $\mathrm{PbO}(\mathrm{wt} \%)$ & 0.0770 & 0.0770 & 0.1040 & -0.0270 & -0.0270 & $-25.9 \%$ & $-25.9 \%$ \\
\hline 26 & SB3-26 & $\mathrm{SiO} 2(\mathrm{wt} \%)$ & 45.1392 & 47.5184 & 45.2740 & -0.1348 & 2.2444 & $-0.3 \%$ & $5.0 \%$ \\
\hline 26 & SB3-26 & ThO2 (wt\%) & 0.1334 & 0.1334 & 0.0490 & 0.0844 & 0.0844 & $172.3 \%$ & $172.3 \%$ \\
\hline 26 & SB3-26 & $\mathrm{TiO} 2(\mathrm{wt} \%)$ & 0.8836 & 0.9564 & 0.9730 & -0.0894 & -0.0166 & $-9.2 \%$ & $-1.7 \%$ \\
\hline 26 & SB3-26 & $\mathrm{U} 3 \mathrm{O} 8(\mathrm{wt} \%)$ & 3.3548 & 3.5104 & 3.5190 & -0.1642 & -0.0086 & $-4.7 \%$ & $-0.2 \%$ \\
\hline 26 & SB3-26 & $\mathrm{ZnO}(\mathrm{wt} \%)$ & 0.1158 & 0.1158 & 0.1420 & -0.0262 & -0.0262 & $-18.5 \%$ & $-18.5 \%$ \\
\hline 26 & SB3-26 & $\mathrm{ZrO} 2(\mathrm{wt} \%)$ & 0.2205 & 0.2205 & 0.2570 & -0.0365 & -0.0365 & $-14.2 \%$ & $-14.2 \%$ \\
\hline 26 & SB3-26 & Sum of Oxides & 99.4229 & 101.3697 & 99.9990 & -0.5761 & 1.3707 & $-0.6 \%$ & $1.4 \%$ \\
\hline 27 & SB3-27 & $\mathrm{Al} 2 \mathrm{O} 3(\mathrm{wt} \%)$ & 4.4262 & 4.6284 & 4.3080 & 0.1182 & 0.3204 & $2.7 \%$ & $7.4 \%$ \\
\hline 27 & SB3-27 & $\mathrm{B} 2 \mathrm{O} 3(\mathrm{wt} \%)$ & 6.9147 & 6.7075 & 6.9000 & 0.0147 & -0.1925 & $0.2 \%$ & $-2.8 \%$ \\
\hline 27 & SB3-27 & $\mathrm{BaO}(\mathrm{wt} \%)$ & 0.0480 & 0.0524 & 0.0600 & -0.0120 & -0.0076 & $-20.0 \%$ & $-12.6 \%$ \\
\hline 27 & SB3-27 & $\mathrm{CaO}(\mathrm{wt} \%)$ & 0.9095 & 0.8667 & 0.8570 & 0.0525 & 0.0097 & $6.1 \%$ & $1.1 \%$ \\
\hline 27 & SB3-27 & $\mathrm{Ce} 2 \mathrm{O} 3(\mathrm{wt} \%)$ & 0.0835 & 0.0835 & 0.0830 & 0.0005 & 0.0005 & $0.5 \%$ & $0.5 \%$ \\
\hline 27 & SB3-27 & $\mathrm{Cr} 2 \mathrm{O} 3(\mathrm{wt} \%)$ & 0.0943 & 0.1003 & 0.0880 & 0.0063 & 0.0123 & $7.1 \%$ & $14.0 \%$ \\
\hline 27 & SB3-27 & $\mathrm{CuO}(\mathrm{wt} \%)$ & 0.0507 & 0.0532 & 0.0470 & 0.0037 & 0.0062 & $7.9 \%$ & $13.1 \%$ \\
\hline 27 & SB3-27 & $\mathrm{Fe} 2 \mathrm{O} 3(\mathrm{wt} \%)$ & 8.9785 & 9.1135 & 9.5790 & -0.6005 & -0.4655 & $-6.3 \%$ & $-4.9 \%$ \\
\hline 27 & SB3-27 & $\mathrm{K} 2 \mathrm{O}(\mathrm{wt} \%)$ & 0.0891 & 0.0798 & 0.1030 & -0.0139 & -0.0232 & $-13.5 \%$ & $-22.5 \%$ \\
\hline 27 & SB3-27 & La2O3 (wt $\%)$ & 0.0425 & 0.0425 & 0.0550 & -0.0125 & -0.0125 & $-22.7 \%$ & $-22.7 \%$ \\
\hline 27 & SB3-27 & $\mathrm{Li} 2 \mathrm{O}(\mathrm{wt} \%)$ & 5.2800 & 5.4721 & 5.5200 & -0.2400 & -0.0479 & $-4.3 \%$ & $-0.9 \%$ \\
\hline 27 & SB3-27 & $\mathrm{MgO}(\mathrm{wt} \%)$ & 0.0133 & 0.0133 & 0.0450 & -0.0317 & -0.0317 & $-70.5 \%$ & $-70.6 \%$ \\
\hline 27 & SB3-27 & $\mathrm{MnO}(\mathrm{wt} \%)$ & 1.5882 & 1.5300 & 1.7090 & -0.1208 & -0.1790 & $-7.1 \%$ & $-10.5 \%$ \\
\hline 27 & SB3-27 & $\mathrm{Na} 2 \mathrm{O}(\mathrm{wt} \%)$ & 12.6577 & 11.8339 & 11.7800 & 0.8777 & 0.0539 & $7.5 \%$ & $0.5 \%$ \\
\hline 27 & SB3-27 & $\mathrm{NiO}(\mathrm{wt} \%)$ & 0.3331 & 0.3634 & 0.3830 & -0.0499 & -0.0196 & $-13.0 \%$ & $-5.1 \%$ \\
\hline 27 & SB3-27 & $\mathrm{PbO}(\mathrm{wt} \%)$ & 0.0547 & 0.0547 & 0.0710 & -0.0163 & -0.0163 & $-23.0 \%$ & $-23.0 \%$ \\
\hline 27 & SB3-27 & $\mathrm{SiO} 2(\mathrm{wt} \%)$ & 54.1243 & 56.5949 & 55.0090 & -0.8847 & 1.5859 & $-1.6 \%$ & $2.9 \%$ \\
\hline 27 & SB3-27 & ThO2 (wt\%) & 0.1070 & 0.1070 & 0.0340 & 0.0730 & 0.0730 & $214.6 \%$ & $214.6 \%$ \\
\hline 27 & SB3-27 & $\mathrm{TiO} 2(\mathrm{wt} \%)$ & 0.6101 & 0.6410 & 0.6700 & -0.0599 & -0.0290 & $-8.9 \%$ & $-4.3 \%$ \\
\hline 27 & SB3-27 & U3O8 (wt\%) & 2.2523 & 2.3728 & 2.4250 & -0.1727 & -0.0522 & $-7.1 \%$ & $-2.2 \%$ \\
\hline 27 & SB3-27 & $\mathrm{ZnO}(\mathrm{wt} \%)$ & 0.0784 & 0.0784 & 0.0980 & -0.0196 & -0.0196 & $-20.0 \%$ & $-20.0 \%$ \\
\hline 27 & SB3-27 & $\mathrm{ZrO} 2(\mathrm{wt} \%)$ & 0.1510 & 0.1510 & 0.1770 & -0.0260 & -0.0260 & $-14.7 \%$ & $-14.7 \%$ \\
\hline 27 & SB3-27 & Sum of Oxides & 98.8868 & 100.9402 & 100.0010 & -1.1142 & 0.9392 & $-1.1 \%$ & $0.9 \%$ \\
\hline 28 & SB3-28 & $\mathrm{Al} 2 \mathrm{O} 3(\mathrm{wt} \%)$ & 6.0417 & 6.0444 & 5.9760 & 0.0657 & 0.0684 & $1.1 \%$ & $1.1 \%$ \\
\hline 28 & SB3-28 & $\mathrm{B} 2 \mathrm{O} 3(\mathrm{wt} \%)$ & 5.7556 & 5.5692 & 5.7000 & 0.0556 & -0.1308 & $1.0 \%$ & $-2.3 \%$ \\
\hline 28 & SB3-28 & $\mathrm{BaO}(\mathrm{wt} \%)$ & 0.0634 & 0.0761 & 0.0830 & -0.0196 & -0.0069 & $-23.7 \%$ & $-8.3 \%$ \\
\hline 28 & SB3-28 & $\mathrm{CaO}(\mathrm{wt} \%)$ & 1.2845 & 1.1844 & 1.1890 & 0.0955 & -0.0046 & $8.0 \%$ & $-0.4 \%$ \\
\hline 28 & SB3-28 & $\mathrm{Ce} 2 \mathrm{O} 3(\mathrm{wt} \%)$ & 0.1075 & 0.1075 & 0.1150 & -0.0075 & -0.0075 & $-6.6 \%$ & $-6.6 \%$ \\
\hline 28 & SB3-28 & $\mathrm{Cr} 2 \mathrm{O} 3(\mathrm{wt} \%)$ & 0.1100 & 0.1306 & 0.1220 & -0.0120 & 0.0086 & $-9.8 \%$ & $7.0 \%$ \\
\hline 28 & SB3-28 & $\mathrm{CuO}(\mathrm{wt} \%)$ & 0.0645 & 0.0688 & 0.0650 & -0.0005 & 0.0038 & $-0.8 \%$ & $5.9 \%$ \\
\hline 28 & SB3-28 & $\mathrm{Fe} 2 \mathrm{O} 3(\mathrm{wt} \%)$ & 13.8681 & 13.4288 & 13.2870 & 0.5811 & 0.1418 & $4.4 \%$ & $1.1 \%$ \\
\hline 28 & SB3-28 & $\mathrm{K} 2 \mathrm{O}(\mathrm{wt} \%)$ & 0.1355 & 0.1206 & 0.1420 & -0.0065 & -0.0214 & $-4.6 \%$ & $-15.1 \%$ \\
\hline 28 & SB3-28 & $\mathrm{La} 2 \mathrm{O} 3(\mathrm{wt} \%)$ & 0.0598 & 0.0598 & 0.0760 & -0.0162 & -0.0162 & $-21.3 \%$ & $-21.3 \%$ \\
\hline 28 & SB3-28 & $\mathrm{Li} 2 \mathrm{O}(\mathrm{wt} \%)$ & 4.3973 & 4.5711 & 4.5600 & -0.1627 & 0.0111 & $-3.6 \%$ & $0.2 \%$ \\
\hline 28 & SB3-28 & $\mathrm{MgO}(\mathrm{wt} \%)$ & 0.0099 & 0.0108 & 0.0620 & -0.0521 & -0.0512 & $-84.0 \%$ & $-82.6 \%$ \\
\hline 28 & SB3-28 & $\mathrm{MnO}(\mathrm{wt} \%)$ & 2.1531 & 2.3822 & 2.3700 & -0.2169 & 0.0122 & $-9.2 \%$ & $0.5 \%$ \\
\hline 28 & SB3-28 & $\mathrm{Na} 2 \mathrm{O}(\mathrm{wt} \%)$ & 15.1987 & 14.7225 & 15.1790 & 0.0197 & -0.4565 & $0.1 \%$ & $-3.0 \%$ \\
\hline 28 & SB3-28 & $\mathrm{NiO}(\mathrm{wt} \%)$ & 0.4479 & 0.5224 & 0.5310 & -0.0831 & -0.0086 & $-15.6 \%$ & $-1.6 \%$ \\
\hline
\end{tabular}


Table H.4: Average Measured and Bias-Corrected Chemical Compositions Versus Targeted Compositions by Oxide by Glass Number (continued)

(Batch 1: 100; 101-U std)

\begin{tabular}{|c|c|c|c|c|c|c|c|c|c|}
\hline Glass \# & Glass ID & Oxide & $\begin{array}{c}\text { Measured } \\
(\mathrm{wt} \%)\end{array}$ & $\begin{array}{c}\text { Measured } \\
\text { Bias-Corrected } \\
(\mathrm{wt} \%)\end{array}$ & $\begin{array}{c}\text { Targeted } \\
(\text { wt } \%)\end{array}$ & $\begin{array}{c}\text { Diff of } \\
\text { Measured }\end{array}$ & $\begin{array}{c}\text { Diff of } \\
\text { Meas BC }\end{array}$ & $\begin{array}{l}\% \text { Diff of } \\
\text { Measured }\end{array}$ & $\begin{array}{l}\% \text { Diff of } \\
\text { Meas BC }\end{array}$ \\
\hline 28 & SB3-28 & $\mathrm{PbO}(\mathrm{wt} \%)$ & 0.0792 & 0.0792 & 0.0990 & -0.0198 & -0.0198 & $-20.0 \%$ & $-20.0 \%$ \\
\hline 28 & SB3-28 & $\mathrm{SiO} 2(\mathrm{wt} \%)$ & 45.7810 & 49.1722 & 45.7220 & 0.0590 & 3.4502 & $0.1 \%$ & $7.5 \%$ \\
\hline 28 & SB3-28 & ThO2 (wt\%) & 0.1306 & 0.1306 & 0.0470 & 0.0836 & 0.0836 & $177.8 \%$ & $177.8 \%$ \\
\hline 28 & SB3-28 & $\mathrm{TiO} 2(\mathrm{wt} \%)$ & 0.8599 & 0.9440 & 0.9300 & -0.0701 & 0.0140 & $-7.5 \%$ & $1.5 \%$ \\
\hline 28 & SB3-28 & $\mathrm{U} 3 \mathrm{O} 8(\mathrm{wt} \%)$ & 3.2693 & 3.4209 & 3.3630 & -0.0937 & 0.0579 & $-2.8 \%$ & $1.7 \%$ \\
\hline 28 & SB3-28 & $\mathrm{ZnO}(\mathrm{wt} \%)$ & 0.0999 & 0.0999 & 0.1360 & -0.0361 & -0.0361 & $-26.5 \%$ & $-26.5 \%$ \\
\hline 28 & SB3-28 & $\mathrm{ZrO} 2(\mathrm{wt} \%)$ & 0.2057 & 0.2057 & 0.2450 & -0.0393 & -0.0393 & $-16.1 \%$ & $-16.1 \%$ \\
\hline 28 & SB3-28 & Sum of Oxides & 100.1229 & 103.0517 & 99.9990 & 0.1239 & 3.0527 & $0.1 \%$ & $3.1 \%$ \\
\hline 29 & SB3-29 & $\mathrm{Al} 2 \mathrm{O} 3(\mathrm{wt} \%)$ & 4.5395 & 4.7469 & 4.5040 & 0.0355 & 0.2429 & $0.8 \%$ & $5.4 \%$ \\
\hline 29 & SB3-29 & B2O3 (wt\%) & 5.6831 & 5.5131 & 5.6800 & 0.0031 & -0.1669 & $0.1 \%$ & $-2.9 \%$ \\
\hline 29 & SB3-29 & $\mathrm{BaO}(\mathrm{wt} \%)$ & 0.0441 & 0.0529 & 0.0620 & -0.0179 & -0.0091 & $-28.9 \%$ & $-14.6 \%$ \\
\hline 29 & SB3-29 & $\mathrm{CaO}(\mathrm{wt} \%)$ & 0.9682 & 0.8928 & 0.8960 & 0.0722 & -0.0032 & $8.1 \%$ & $-0.4 \%$ \\
\hline 29 & SB3-29 & $\mathrm{Ce} 2 \mathrm{O} 3(\mathrm{wt} \%)$ & 0.3927 & 0.3927 & 0.0870 & 0.3057 & 0.3057 & $351.4 \%$ & $351.4 \%$ \\
\hline 29 & SB3-29 & $\mathrm{Cr} 2 \mathrm{O} 3(\mathrm{wt} \%)$ & 0.0446 & 0.0529 & 0.0920 & -0.0474 & -0.0391 & $-51.5 \%$ & $-42.5 \%$ \\
\hline 29 & SB3-29 & $\mathrm{CuO}(\mathrm{wt} \%)$ & 0.0510 & 0.0545 & 0.0490 & 0.0020 & 0.0055 & $4.1 \%$ & $11.2 \%$ \\
\hline 29 & SB3-29 & $\mathrm{Fe} 2 \mathrm{O} 3(\mathrm{wt} \%)$ & 11.2231 & 10.8645 & 10.0160 & 1.2071 & 0.8485 & $12.1 \%$ & $8.5 \%$ \\
\hline 29 & SB3-29 & $\mathrm{K} 2 \mathrm{O}(\mathrm{wt} \%)$ & 0.0753 & 0.0670 & 0.1070 & -0.0317 & -0.0400 & $-29.6 \%$ & $-37.4 \%$ \\
\hline 29 & SB3-29 & $\mathrm{La} 2 \mathrm{O} 3(\mathrm{wt} \%)$ & 0.3759 & 0.3759 & 0.0570 & 0.3189 & 0.3189 & $559.4 \%$ & $559.4 \%$ \\
\hline 29 & SB3-29 & $\mathrm{Li} 2 \mathrm{O}(\mathrm{wt} \%)$ & 4.7471 & 4.9198 & 4.9700 & -0.2229 & -0.0502 & $-4.5 \%$ & $-1.0 \%$ \\
\hline 29 & SB3-29 & $\mathrm{MgO}(\mathrm{wt} \%)$ & 1.3468 & 1.4621 & 1.4670 & -0.1202 & -0.0049 & $-8.2 \%$ & $-0.3 \%$ \\
\hline 29 & SB3-29 & $\mathrm{MnO}(\mathrm{wt} \%)$ & 1.6527 & 1.8284 & 1.7860 & -0.1333 & 0.0424 & $-7.5 \%$ & $2.4 \%$ \\
\hline 29 & SB3-29 & $\mathrm{Na} 2 \mathrm{O}(\mathrm{wt} \%)$ & 11.0974 & 10.7499 & 11.0000 & 0.0974 & -0.2501 & $0.9 \%$ & $-2.3 \%$ \\
\hline 29 & SB3-29 & $\mathrm{NiO}(\mathrm{wt} \%)$ & 0.3493 & 0.4074 & 0.4010 & -0.0517 & 0.0064 & $-12.9 \%$ & $1.6 \%$ \\
\hline 29 & SB3-29 & $\mathrm{PbO}(\mathrm{wt} \%)$ & 0.0714 & 0.0714 & 0.0750 & -0.0036 & -0.0036 & $-4.8 \%$ & $-4.8 \%$ \\
\hline 29 & SB3-29 & $\mathrm{SiO} 2(\mathrm{wt} \%)$ & 54.0708 & 56.5378 & 55.1920 & -1.1212 & 1.3458 & $-2.0 \%$ & $2.4 \%$ \\
\hline 29 & SB3-29 & ThO2 (wt\%) & 0.0993 & 0.0993 & 0.0360 & 0.0633 & 0.0633 & $175.8 \%$ & $175.8 \%$ \\
\hline 29 & SB3-29 & $\mathrm{TiO} 2(\mathrm{wt} \%)$ & 0.6639 & 0.7289 & 0.7010 & -0.0371 & 0.0279 & $-5.3 \%$ & $4.0 \%$ \\
\hline 29 & SB3-29 & U3O8 (wt\%) & 2.4350 & 2.5479 & 2.5350 & -0.1000 & 0.0129 & $-3.9 \%$ & $0.5 \%$ \\
\hline 29 & SB3-29 & $\mathrm{ZnO}(\mathrm{wt} \%)$ & 0.0728 & 0.0728 & 0.1020 & -0.0292 & -0.0292 & $-28.6 \%$ & $-28.6 \%$ \\
\hline 29 & SB3-29 & $\mathrm{ZrO} 2(\mathrm{wt} \%)$ & 0.2384 & 0.2384 & 0.1850 & 0.0534 & 0.0534 & $28.9 \%$ & $28.9 \%$ \\
\hline 29 & SB3-29 & Sum of Oxides & 100.2426 & 102.6771 & 100.0000 & 0.2426 & 2.6771 & $0.2 \%$ & $2.7 \%$ \\
\hline 30 & SB3-30 & $\mathrm{Al} 2 \mathrm{O} 3(\mathrm{wt} \%)$ & 7.6100 & 7.8307 & 6.9900 & 0.6200 & 0.8407 & $8.9 \%$ & $12.0 \%$ \\
\hline 30 & SB3-30 & $\mathrm{B} 2 \mathrm{O} 3(\mathrm{wt} \%)$ & 4.3791 & 4.3377 & 4.4000 & -0.0209 & -0.0623 & $-0.5 \%$ & $-1.4 \%$ \\
\hline 30 & SB3-30 & $\mathrm{BaO}(\mathrm{wt} \%)$ & 0.0871 & 0.0951 & 0.0970 & -0.0099 & -0.0019 & $-10.2 \%$ & $-1.9 \%$ \\
\hline 30 & SB3-30 & $\mathrm{CaO}(\mathrm{wt} \%)$ & 1.4167 & 1.3500 & 1.3910 & 0.0257 & -0.0410 & $1.8 \%$ & $-2.9 \%$ \\
\hline 30 & SB3-30 & $\mathrm{Ce} 2 \mathrm{O} 3(\mathrm{wt} \%)$ & 0.1294 & 0.1294 & 0.1350 & -0.0056 & -0.0056 & $-4.1 \%$ & $-4.1 \%$ \\
\hline 30 & SB3-30 & $\mathrm{Cr} 2 \mathrm{O} 3(\mathrm{wt} \%)$ & 0.1235 & 0.1314 & 0.1430 & -0.0195 & -0.0116 & $-13.6 \%$ & $-8.1 \%$ \\
\hline 30 & SB3-30 & $\mathrm{CuO}(\mathrm{wt} \%)$ & 0.0726 & 0.0762 & 0.0770 & -0.0044 & -0.0008 & $-5.7 \%$ & $-1.1 \%$ \\
\hline 30 & SB3-30 & $\mathrm{Fe} 2 \mathrm{O} 3(\mathrm{wt} \%)$ & 15.2978 & 15.5315 & 15.5420 & -0.2442 & -0.0105 & $-1.6 \%$ & $-0.1 \%$ \\
\hline 30 & SB3-30 & $\mathrm{K} 2 \mathrm{O}(\mathrm{wt} \%)$ & 0.1473 & 0.1318 & 0.1660 & -0.0187 & -0.0342 & $-11.3 \%$ & $-20.6 \%$ \\
\hline 30 & SB3-30 & $\mathrm{La} 2 \mathrm{O} 3(\mathrm{wt} \%)$ & 0.0765 & 0.0765 & 0.0890 & -0.0125 & -0.0125 & $-14.0 \%$ & $-14.0 \%$ \\
\hline 30 & SB3-30 & $\mathrm{Li} 2 \mathrm{O}(\mathrm{wt} \%)$ & 3.7353 & 3.8648 & 3.8500 & -0.1147 & 0.0148 & $-3.0 \%$ & $0.4 \%$ \\
\hline 30 & SB3-30 & $\mathrm{MgO}(\mathrm{wt} \%)$ & 1.1806 & 1.1835 & 1.1730 & 0.0076 & 0.0105 & $0.6 \%$ & $0.9 \%$ \\
\hline 30 & SB3-30 & $\mathrm{MnO}(\mathrm{wt} \%)$ & 2.7244 & 2.6263 & 2.7720 & -0.0476 & -0.1457 & $-1.7 \%$ & $-5.3 \%$ \\
\hline 30 & SB3-30 & $\mathrm{Na} 2 \mathrm{O}(\mathrm{wt} \%)$ & 14.5247 & 13.5806 & 13.7580 & 0.7667 & -0.1774 & $5.6 \%$ & $-1.3 \%$ \\
\hline 30 & SB3-30 & $\mathrm{NiO}(\mathrm{wt} \%)$ & 0.5354 & 0.5842 & 0.6210 & -0.0856 & -0.0368 & $-13.8 \%$ & $-5.9 \%$ \\
\hline 30 & SB3-30 & $\mathrm{PbO}(\mathrm{wt} \%)$ & 0.1058 & 0.1058 & 0.1160 & -0.0102 & -0.0102 & $-8.8 \%$ & $-8.8 \%$ \\
\hline 30 & SB3-30 & $\mathrm{SiO} 2(\mathrm{wt} \%)$ & 43.1069 & 45.3818 & 43.1590 & -0.0521 & 2.2228 & $-0.1 \%$ & $5.2 \%$ \\
\hline 30 & SB3-30 & ThO2 (wt $\%)$ & 0.1849 & 0.1849 & 0.0550 & 0.1299 & 0.1299 & $236.2 \%$ & $236.2 \%$ \\
\hline 30 & SB3-30 & $\mathrm{TiO} 2(\mathrm{wt} \%)$ & 1.0713 & 1.1255 & 1.0880 & -0.0167 & 0.0375 & $-1.5 \%$ & $3.5 \%$ \\
\hline 30 & SB3-30 & $\mathrm{U} 3 \mathrm{O} 8(\mathrm{wt} \%)$ & 3.8265 & 4.0313 & 3.9340 & -0.1075 & 0.0973 & $-2.7 \%$ & $2.5 \%$ \\
\hline 30 & SB3-30 & $\mathrm{ZnO}(\mathrm{wt} \%)$ & 0.1425 & 0.1425 & 0.1590 & -0.0165 & -0.0165 & $-10.4 \%$ & $-10.4 \%$ \\
\hline 30 & SB3-30 & $\mathrm{ZrO} 2(\mathrm{wt} \%)$ & 0.2644 & 0.2644 & 0.2870 & -0.0226 & -0.0226 & $-7.9 \%$ & $-7.9 \%$ \\
\hline
\end{tabular}


Table H.4: Average Measured and Bias-Corrected Chemical Compositions Versus Targeted Compositions by Oxide by Glass Number (continued)

(Batch 1: 100; 101-U std)

\begin{tabular}{|c|c|c|c|c|c|c|c|c|c|}
\hline Glass \# & Glass ID & Oxide & $\begin{array}{c}\text { Measured } \\
(\mathrm{wt} \%)\end{array}$ & $\begin{array}{c}\text { Measured } \\
\text { Bias-Corrected } \\
(w \mathrm{w} \%)\end{array}$ & $\begin{array}{c}\text { Targeted } \\
(\mathrm{wt} \%)\end{array}$ & $\begin{array}{c}\text { Diff of } \\
\text { Measured }\end{array}$ & $\begin{array}{c}\text { Diff of } \\
\text { Meas BC }\end{array}$ & $\begin{array}{l}\% \text { Diff of } \\
\text { Measured }\end{array}$ & $\begin{array}{l}\% \text { Diff of } \\
\text { Meas BC }\end{array}$ \\
\hline 30 & SB3-30 & Sum of Oxides & 100.7427 & 102.7662 & 100.0020 & 0.7407 & 2.7642 & $0.7 \%$ & $2.8 \%$ \\
\hline 31 & SB3-31 & $\mathrm{Al} 2 \mathrm{O} 3(\mathrm{wt} \%)$ & 6.1976 & 6.4807 & 6.0860 & 0.1116 & 0.3947 & $1.8 \%$ & $6.5 \%$ \\
\hline 31 & SB3-31 & $\mathrm{B} 2 \mathrm{O} 3(\mathrm{wt} \%)$ & 5.2726 & 5.1148 & 5.2000 & 0.0726 & -0.0852 & $1.4 \%$ & $-1.6 \%$ \\
\hline 31 & SB3-31 & $\mathrm{BaO}(\mathrm{wt} \%)$ & 0.0662 & 0.0715 & 0.0750 & -0.0088 & -0.0035 & $-11.8 \%$ & $-4.7 \%$ \\
\hline 31 & SB3-31 & $\mathrm{CaO}(\mathrm{wt} \%)$ & 1.1718 & 1.0825 & 1.0820 & 0.0898 & 0.0005 & $8.3 \%$ & $0.0 \%$ \\
\hline 31 & SB3-31 & $\mathrm{Ce} 2 \mathrm{O} 3(\mathrm{wt} \%)$ & 0.1060 & 0.1060 & 0.1050 & 0.0010 & 0.0010 & $1.0 \%$ & $1.0 \%$ \\
\hline 31 & SB3-31 & $\mathrm{Cr} 2 \mathrm{O} 3(\mathrm{wt} \%)$ & 0.0826 & 0.0868 & 0.1110 & -0.0284 & -0.0242 & $-25.6 \%$ & $-21.8 \%$ \\
\hline 31 & SB3-31 & $\mathrm{CuO}(\mathrm{wt} \%)$ & 0.0588 & 0.0623 & 0.0600 & -0.0012 & 0.0023 & $-1.9 \%$ & $3.8 \%$ \\
\hline 31 & SB3-31 & $\mathrm{Fe} 2 \mathrm{O} 3(\mathrm{wt} \%)$ & 11.5448 & 11.4599 & 12.0880 & -0.5432 & -0.6281 & $-4.5 \%$ & $-5.2 \%$ \\
\hline 31 & SB3-31 & $\mathrm{K} 2 \mathrm{O}(\mathrm{wt} \%)$ & 0.1153 & 0.1031 & 0.1290 & -0.0137 & -0.0259 & $-10.6 \%$ & $-20.1 \%$ \\
\hline 31 & SB3-31 & $\mathrm{La} 2 \mathrm{O} 3(\mathrm{wt} \%)$ & 0.0534 & 0.0534 & 0.0690 & -0.0156 & -0.0156 & $-22.7 \%$ & $-22.7 \%$ \\
\hline 31 & SB3-31 & $\mathrm{Li} 2 \mathrm{O}(\mathrm{wt} \%)$ & 3.1325 & 3.2464 & 3.2500 & -0.1175 & -0.0036 & $-3.6 \%$ & $-0.1 \%$ \\
\hline 31 & SB3-31 & $\mathrm{MgO}(\mathrm{wt} \%)$ & 0.0174 & 0.0175 & 0.0570 & -0.0396 & -0.0395 & $-69.5 \%$ & $-69.3 \%$ \\
\hline 31 & SB3-31 & $\mathrm{MnO}(\mathrm{wt} \%)$ & 2.0691 & 2.1413 & 2.1560 & -0.0869 & -0.0147 & $-4.0 \%$ & $-0.7 \%$ \\
\hline 31 & SB3-31 & $\mathrm{Na} 2 \mathrm{O}(\mathrm{wt} \%)$ & 13.1868 & 12.6708 & 12.6840 & 0.5028 & -0.0132 & $4.0 \%$ & $-0.1 \%$ \\
\hline 31 & SB3-31 & $\mathrm{NiO}(\mathrm{wt} \%)$ & 0.4301 & 0.4747 & 0.4830 & -0.0529 & -0.0083 & $-11.0 \%$ & $-1.7 \%$ \\
\hline 31 & SB3-31 & $\mathrm{PbO}(\mathrm{wt} \%)$ & 0.0827 & 0.0827 & 0.0900 & -0.0073 & -0.0073 & $-8.1 \%$ & $-8.1 \%$ \\
\hline 31 & SB3-31 & $\mathrm{SiO} 2(\mathrm{wt} \%)$ & 51.5571 & 53.9097 & 51.9790 & -0.4219 & 1.9307 & $-0.8 \%$ & $3.7 \%$ \\
\hline 31 & SB3-31 & ThO2 (wt\%) & 0.1311 & 0.1311 & 0.0430 & 0.0881 & 0.0881 & $205.0 \%$ & $205.0 \%$ \\
\hline 31 & SB3-31 & $\mathrm{TiO} 2(\mathrm{wt} \%)$ & 0.7890 & 0.8317 & 0.8460 & -0.0570 & -0.0143 & $-6.7 \%$ & $-1.7 \%$ \\
\hline 31 & SB3-31 & $\mathrm{U} 3 \mathrm{O} 8(\mathrm{wt} \%)$ & 3.2133 & 3.3337 & 3.0600 & 0.1533 & 0.2737 & $5.0 \%$ & $8.9 \%$ \\
\hline 31 & SB3-31 & $\mathrm{ZnO}(\mathrm{wt} \%)$ & 0.1058 & 0.1058 & 0.1240 & -0.0182 & -0.0182 & $-14.7 \%$ & $-14.7 \%$ \\
\hline 31 & SB3-31 & $\mathrm{ZrO} 2(\mathrm{wt} \%)$ & 0.2087 & 0.2087 & 0.2230 & -0.0143 & -0.0143 & $-6.4 \%$ & $-6.4 \%$ \\
\hline 31 & SB3-31 & Sum of Oxides & 99.5928 & 101.7750 & 100.0000 & -0.4072 & 1.7750 & $-0.4 \%$ & $1.8 \%$ \\
\hline 32 & SB3-32 & $\mathrm{Al} 2 \mathrm{O} 3(\mathrm{wt} \%)$ & 8.3232 & 8.4468 & 7.8300 & 0.4932 & 0.6168 & $6.3 \%$ & $7.9 \%$ \\
\hline 32 & SB3-32 & $\mathrm{B} 2 \mathrm{O} 3(\mathrm{wt} \%)$ & 4.3147 & 4.2390 & 4.2400 & 0.0747 & -0.0010 & $1.8 \%$ & $0.0 \%$ \\
\hline 32 & SB3-32 & $\mathrm{BaO}(\mathrm{wt} \%)$ & 0.0837 & 0.1005 & 0.1010 & -0.0173 & -0.0005 & $-17.1 \%$ & $-0.5 \%$ \\
\hline 32 & SB3-32 & $\mathrm{CaO}(\mathrm{wt} \%)$ & 1.6703 & 1.5401 & 1.4520 & 0.2183 & 0.0881 & $15.0 \%$ & $6.1 \%$ \\
\hline 32 & SB3-32 & $\mathrm{Ce} 2 \mathrm{O} 3(\mathrm{wt} \%)$ & 0.1362 & 0.1362 & 0.1410 & -0.0048 & -0.0048 & $-3.4 \%$ & $-3.4 \%$ \\
\hline 32 & SB3-32 & $\mathrm{Cr} 2 \mathrm{O} 3(\mathrm{wt} \%)$ & 0.1114 & 0.1323 & 0.1490 & -0.0376 & -0.0167 & $-25.2 \%$ & $-11.2 \%$ \\
\hline 32 & SB3-32 & $\mathrm{CuO}(\mathrm{wt} \%)$ & 0.0832 & 0.0889 & 0.0800 & 0.0032 & 0.0089 & $4.1 \%$ & $11.1 \%$ \\
\hline 32 & SB3-32 & $\mathrm{Fe} 2 \mathrm{O} 3(\mathrm{wt} \%)$ & 16.0484 & 15.5436 & 16.2330 & -0.1846 & -0.6894 & $-1.1 \%$ & $-4.2 \%$ \\
\hline 32 & SB3-32 & $\mathrm{K} 2 \mathrm{O}(\mathrm{wt} \%)$ & 0.1970 & 0.1753 & 0.1740 & 0.0230 & 0.0013 & $13.2 \%$ & $0.7 \%$ \\
\hline 32 & SB3-32 & $\mathrm{La} 2 \mathrm{O} 3(\mathrm{wt} \%)$ & 0.0748 & 0.0748 & 0.0930 & -0.0182 & -0.0182 & $-19.6 \%$ & $-19.6 \%$ \\
\hline 32 & SB3-32 & $\mathrm{Li} 2 \mathrm{O}(\mathrm{wt} \%)$ & 2.5835 & 2.6685 & 2.6500 & -0.0665 & 0.0185 & $-2.5 \%$ & $0.7 \%$ \\
\hline 32 & SB3-32 & $\mathrm{MgO}(\mathrm{wt} \%)$ & 0.0265 & 0.0288 & 0.0760 & -0.0495 & -0.0472 & $-65.1 \%$ & $-62.1 \%$ \\
\hline 32 & SB3-32 & $\mathrm{MnO}(\mathrm{wt} \%)$ & 2.6050 & 2.8817 & 2.8950 & -0.2900 & -0.0133 & $-10.0 \%$ & $-0.5 \%$ \\
\hline 32 & SB3-32 & $\mathrm{Na} 2 \mathrm{O}(\mathrm{wt} \%)$ & 16.8163 & 16.2905 & 14.6330 & 2.1833 & 1.6575 & $14.9 \%$ & $11.3 \%$ \\
\hline 32 & SB3-32 & $\mathrm{NiO}(\mathrm{wt} \%)$ & 0.5147 & 0.6004 & 0.6490 & -0.1343 & -0.0486 & $-20.7 \%$ & $-7.5 \%$ \\
\hline 32 & SB3-32 & $\mathrm{PbO}(\mathrm{wt} \%)$ & 0.0953 & 0.0953 & 0.1210 & -0.0257 & -0.0257 & $-21.2 \%$ & $-21.2 \%$ \\
\hline 32 & SB3-32 & $\mathrm{SiO} 2(\mathrm{wt} \%)$ & 41.9838 & 45.1737 & 42.7150 & -0.7312 & 2.4587 & $-1.7 \%$ & $5.8 \%$ \\
\hline 32 & SB3-32 & ThO2 (wt $\%)$ & 0.1644 & 0.1644 & 0.0580 & 0.1064 & 0.1064 & $183.5 \%$ & $183.5 \%$ \\
\hline 32 & SB3-32 & $\mathrm{TiO} 2(\mathrm{wt} \%)$ & 1.0671 & 1.1717 & 1.1360 & -0.0689 & 0.0357 & $-6.1 \%$ & $3.1 \%$ \\
\hline 32 & SB3-32 & $\mathrm{U} 3 \mathrm{O} 8(\mathrm{wt} \%)$ & 4.0417 & 4.2289 & 4.1090 & -0.0673 & 0.1199 & $-1.6 \%$ & $2.9 \%$ \\
\hline 32 & SB3-32 & $\mathrm{ZnO}(\mathrm{wt} \%)$ & 0.1319 & 0.1319 & 0.1660 & -0.0341 & -0.0341 & $-20.5 \%$ & $-20.5 \%$ \\
\hline 32 & SB3-32 & $\mathrm{ZrO} 2(\mathrm{wt} \%)$ & 0.2553 & 0.2553 & 0.3000 & -0.0447 & -0.0447 & $-14.9 \%$ & $-14.9 \%$ \\
\hline 32 & SB3-32 & Sum of Oxides & 101.3285 & 104.1684 & 100.0010 & 1.3275 & 4.1674 & $1.3 \%$ & $4.2 \%$ \\
\hline 33 & SB3-33 & $\mathrm{Al} 2 \mathrm{O} 3(\mathrm{wt} \%)$ & 5.9755 & 5.9743 & 5.6500 & 0.3255 & 0.3243 & $5.8 \%$ & $5.7 \%$ \\
\hline 33 & SB3-33 & $\mathrm{B} 2 \mathrm{O} 3(\mathrm{wt} \%)$ & 6.8020 & 6.5831 & 6.8000 & 0.0020 & -0.2169 & $0.0 \%$ & $-3.2 \%$ \\
\hline 33 & SB3-33 & $\mathrm{BaO}(\mathrm{wt} \%)$ & 0.0597 & 0.0646 & 0.0690 & -0.0093 & -0.0044 & $-13.4 \%$ & $-6.4 \%$ \\
\hline 33 & SB3-33 & $\mathrm{CaO}(\mathrm{wt} \%)$ & 1.0372 & 0.9581 & 0.9890 & 0.0482 & -0.0309 & $4.9 \%$ & $-3.1 \%$ \\
\hline 33 & SB3-33 & $\mathrm{Ce} 2 \mathrm{O} 3(\mathrm{wt} \%)$ & 0.0943 & 0.0943 & 0.0960 & -0.0017 & -0.0017 & $-1.8 \%$ & $-1.8 \%$ \\
\hline 33 & SB3-33 & $\mathrm{Cr} 2 \mathrm{O} 3(\mathrm{wt} \%)$ & 0.0815 & 0.0856 & 0.1020 & -0.0205 & -0.0164 & $-20.1 \%$ & $-16.0 \%$ \\
\hline
\end{tabular}


Table H.4: Average Measured and Bias-Corrected Chemical Compositions Versus Targeted Compositions by Oxide by Glass Number (continued)

(Batch 1: 100; 101-U std)

\begin{tabular}{|c|c|c|c|c|c|c|c|c|c|}
\hline Glass \# & Glass ID & Oxide & $\begin{array}{c}\text { Measured } \\
(\mathbf{w t} \%)\end{array}$ & $\begin{array}{c}\text { Measured } \\
\text { Bias-Corrected } \\
(w t \%)\end{array}$ & $\begin{array}{c}\text { Targeted } \\
(\text { wt } \%)\end{array}$ & $\begin{array}{c}\text { Diff of } \\
\text { Measured }\end{array}$ & $\begin{array}{c}\text { Diff of } \\
\text { Meas BC }\end{array}$ & $\begin{array}{l}\% \text { Diff of } \\
\text { Measured }\end{array}$ & $\begin{array}{l}\% \text { Diff of } \\
\text { Meas BC }\end{array}$ \\
\hline 33 & SB3-33 & $\mathrm{CuO}(\mathrm{wt} \%)$ & 0.0598 & 0.0633 & 0.0540 & 0.0058 & 0.0093 & $10.7 \%$ & $17.1 \%$ \\
\hline 33 & SB3-33 & $\mathrm{Fe} 2 \mathrm{O} 3(\mathrm{wt} \%)$ & 10.8264 & 10.7468 & 11.0520 & -0.2256 & -0.3052 & $-2.0 \%$ & $-2.8 \%$ \\
\hline 33 & SB3-33 & $\mathrm{K} 2 \mathrm{O}(\mathrm{wt} \%)$ & 0.0750 & 0.0671 & 0.1180 & -0.0430 & -0.0509 & $-36.5 \%$ & $-43.2 \%$ \\
\hline 33 & SB3-33 & La2O3 (wt\%) & 0.0516 & 0.0516 & 0.0630 & -0.0114 & -0.0114 & $-18.1 \%$ & $-18.1 \%$ \\
\hline 33 & SB3-33 & $\mathrm{Li} 2 \mathrm{O}(\mathrm{wt} \%)$ & 3.2509 & 3.3795 & 3.4000 & -0.1491 & -0.0205 & $-4.4 \%$ & $-0.6 \%$ \\
\hline 33 & SB3-33 & $\mathrm{MgO}(\mathrm{wt} \%)$ & 0.0137 & 0.0138 & 0.0520 & -0.0383 & -0.0382 & $-73.7 \%$ & $-73.5 \%$ \\
\hline 33 & SB3-33 & $\mathrm{MnO}(\mathrm{wt} \%)$ & 2.0110 & 2.0815 & 1.9710 & 0.0400 & 0.1105 & $2.0 \%$ & $5.6 \%$ \\
\hline 33 & SB3-33 & $\mathrm{Na} 2 \mathrm{O}(\mathrm{wt} \%)$ & 12.5162 & 12.0275 & 12.1970 & 0.3192 & -0.1695 & $2.6 \%$ & $-1.4 \%$ \\
\hline 33 & SB3-33 & $\mathrm{NiO}(\mathrm{wt} \%)$ & 0.4250 & 0.4693 & 0.4420 & -0.0170 & 0.0273 & $-3.8 \%$ & $6.2 \%$ \\
\hline 33 & SB3-33 & $\mathrm{PbO}(\mathrm{wt} \%)$ & 0.0792 & 0.0792 & 0.0820 & -0.0028 & -0.0028 & $-3.4 \%$ & $-3.4 \%$ \\
\hline 33 & SB3-33 & $\mathrm{SiO} 2(\mathrm{wt} \%)$ & 52.5733 & 56.4750 & 52.9360 & -0.3627 & 3.5390 & $-0.7 \%$ & $6.7 \%$ \\
\hline 33 & SB3-33 & ThO2 (wt $\%)$ & 0.1181 & 0.1181 & 0.0390 & 0.0791 & 0.0791 & $202.7 \%$ & $202.7 \%$ \\
\hline 33 & SB3-33 & $\mathrm{TiO} 2(\mathrm{wt} \%)$ & 0.7489 & 0.7896 & 0.7730 & -0.0241 & 0.0166 & $-3.1 \%$ & $2.1 \%$ \\
\hline 33 & SB3-33 & $\mathrm{U} 3 \mathrm{O} 8(\mathrm{wt} \%)$ & 2.7741 & 2.8780 & 2.7970 & -0.0229 & 0.0810 & $-0.8 \%$ & $2.9 \%$ \\
\hline 33 & SB3-33 & $\mathrm{ZnO}(\mathrm{wt} \%)$ & 0.1039 & 0.1039 & 0.1130 & -0.0091 & -0.0091 & $-8.0 \%$ & $-8.0 \%$ \\
\hline 33 & SB3-33 & $\mathrm{ZrO} 2(\mathrm{wt} \%)$ & 0.1976 & 0.1976 & 0.2040 & -0.0064 & -0.0064 & $-3.2 \%$ & $-3.2 \%$ \\
\hline 33 & SB3-33 & Sum of Oxides & 99.8748 & 103.3017 & 99.9990 & -0.1242 & 3.3027 & $-0.1 \%$ & $3.3 \%$ \\
\hline 34 & SB3-34 & $\mathrm{A} 12 \mathrm{O} 3(\mathrm{wt} \%)$ & 7.8320 & 8.1899 & 7.8300 & 0.0020 & 0.3599 & $0.0 \%$ & $4.6 \%$ \\
\hline 34 & SB3-34 & $\mathrm{B} 2 \mathrm{O} 3(\mathrm{wt} \%)$ & 5.4497 & 5.2864 & 5.3000 & 0.1497 & -0.0136 & $2.8 \%$ & $-0.3 \%$ \\
\hline 34 & SB3-34 & $\mathrm{BaO}(\mathrm{wt} \%)$ & 0.0949 & 0.1026 & 0.1010 & -0.0061 & 0.0016 & $-6.0 \%$ & $1.6 \%$ \\
\hline 34 & SB3-34 & $\mathrm{CaO}(\mathrm{wt} \%)$ & 1.5440 & 1.4264 & 1.4520 & 0.0920 & -0.0256 & $6.3 \%$ & $-1.8 \%$ \\
\hline 34 & SB3-34 & $\mathrm{Ce} 2 \mathrm{O} 3(\mathrm{wt} \%)$ & 0.1411 & 0.1411 & 0.1410 & 0.0001 & 0.0001 & $0.1 \%$ & $0.1 \%$ \\
\hline 34 & SB3-34 & $\mathrm{Cr} 2 \mathrm{O} 3(\mathrm{wt} \%)$ & 0.1319 & 0.1386 & 0.1490 & -0.0171 & -0.0104 & $-11.5 \%$ & $-6.9 \%$ \\
\hline 34 & SB3-34 & $\mathrm{CuO}(\mathrm{wt} \%)$ & 0.0811 & 0.0858 & 0.0800 & 0.0011 & 0.0058 & $1.3 \%$ & $7.2 \%$ \\
\hline 34 & SB3-34 & $\mathrm{Fe} 2 \mathrm{O} 3(\mathrm{wt} \%)$ & 15.7624 & 15.6471 & 16.2330 & -0.4706 & -0.5859 & $-2.9 \%$ & $-3.6 \%$ \\
\hline 34 & SB3-34 & $\mathrm{K} 2 \mathrm{O}(\mathrm{wt} \%)$ & 0.1705 & 0.1524 & 0.1740 & -0.0035 & -0.0216 & $-2.0 \%$ & $-12.4 \%$ \\
\hline 34 & SB3-34 & La2O3 (wt\%) & 0.0786 & 0.0786 & 0.0930 & -0.0144 & -0.0144 & $-15.5 \%$ & $-15.5 \%$ \\
\hline 34 & SB3-34 & $\mathrm{Li} 2 \mathrm{O}(\mathrm{wt} \%)$ & 2.5889 & 2.6831 & 2.6500 & -0.0611 & 0.0331 & $-2.3 \%$ & $1.2 \%$ \\
\hline 34 & SB3-34 & $\mathrm{MgO}(\mathrm{wt} \%)$ & 0.0398 & 0.0401 & 0.0760 & -0.0362 & -0.0359 & $-47.6 \%$ & $-47.2 \%$ \\
\hline 34 & SB3-34 & $\mathrm{MnO}(\mathrm{wt} \%)$ & 2.7922 & 2.8901 & 2.8950 & -0.1028 & -0.0049 & $-3.6 \%$ & $-0.2 \%$ \\
\hline 34 & SB3-34 & $\mathrm{Na} 2 \mathrm{O}(\mathrm{wt} \%)$ & 15.0302 & 14.4446 & 14.6330 & 0.3972 & -0.1884 & $2.7 \%$ & $-1.3 \%$ \\
\hline 34 & SB3-34 & $\mathrm{NiO}(\mathrm{wt} \%)$ & 0.5427 & 0.5992 & 0.6490 & -0.1063 & -0.0498 & $-16.4 \%$ & $-7.7 \%$ \\
\hline 34 & SB3-34 & $\mathrm{PbO}(\mathrm{wt} \%)$ & 0.1034 & 0.1034 & 0.1210 & -0.0176 & -0.0176 & $-14.5 \%$ & $-14.5 \%$ \\
\hline 34 & SB3-34 & $\mathrm{SiO} 2(\mathrm{wt} \%)$ & 41.5024 & 43.3992 & 41.6550 & -0.1526 & 1.7442 & $-0.4 \%$ & $4.2 \%$ \\
\hline 34 & SB3-34 & ThO2 (wt $\%)$ & 0.1769 & 0.1769 & 0.0580 & 0.1189 & 0.1189 & $205.1 \%$ & $205.1 \%$ \\
\hline 34 & SB3-34 & $\mathrm{TiO} 2(\mathrm{wt} \%)$ & 1.1076 & 1.1676 & 1.1360 & -0.0284 & 0.0316 & $-2.5 \%$ & $2.8 \%$ \\
\hline 34 & SB3-34 & U3O8 (wt\%) & 4.0270 & 4.1778 & 4.1090 & -0.0820 & 0.0688 & $-2.0 \%$ & $1.7 \%$ \\
\hline 34 & SB3-34 & $\mathrm{ZnO}(\mathrm{wt} \%)$ & 0.1590 & 0.1590 & 0.1660 & -0.0070 & -0.0070 & $-4.2 \%$ & $-4.2 \%$ \\
\hline 34 & SB3-34 & $\mathrm{ZrO} 2(\mathrm{wt} \%)$ & 0.2823 & 0.2823 & 0.3000 & -0.0177 & -0.0177 & $-5.9 \%$ & $-5.9 \%$ \\
\hline 34 & SB3-34 & Sum of Oxides & 99.6386 & 101.3724 & 100.0010 & -0.3624 & 1.3714 & $-0.4 \%$ & $1.4 \%$ \\
\hline 35 & SB3-35 & $\mathrm{A} 12 \mathrm{O} 3(\mathrm{wt} \%)$ & 4.7285 & 4.7304 & 4.6330 & 0.0955 & 0.0974 & $2.1 \%$ & $2.1 \%$ \\
\hline 35 & SB3-35 & $\mathrm{B} 2 \mathrm{O} 3(\mathrm{wt} \%)$ & 6.0373 & 5.8438 & 6.0000 & 0.0373 & -0.1562 & $0.6 \%$ & $-2.6 \%$ \\
\hline 35 & SB3-35 & $\mathrm{BaO}(\mathrm{wt} \%)$ & 0.0452 & 0.0502 & 0.0540 & -0.0088 & -0.0038 & $-16.3 \%$ & $-7.1 \%$ \\
\hline 35 & SB3-35 & $\mathrm{CaO}(\mathrm{wt} \%)$ & 0.8213 & 0.7682 & 0.7730 & 0.0483 & -0.0048 & $6.3 \%$ & $-0.6 \%$ \\
\hline 35 & SB3-35 & $\mathrm{Ce} 2 \mathrm{O} 3(\mathrm{wt} \%)$ & 0.0741 & 0.0741 & 0.0750 & -0.0009 & -0.0009 & $-1.2 \%$ & $-1.2 \%$ \\
\hline 35 & SB3-35 & $\mathrm{Cr} 2 \mathrm{O} 3(\mathrm{wt} \%)$ & 0.0683 & 0.0727 & 0.0790 & -0.0107 & -0.0063 & $-13.5 \%$ & $-8.0 \%$ \\
\hline 35 & SB3-35 & $\mathrm{CuO}(\mathrm{wt} \%)$ & 0.0432 & 0.0460 & 0.0430 & 0.0002 & 0.0030 & $0.4 \%$ & $7.1 \%$ \\
\hline 35 & SB3-35 & $\mathrm{Fe} 2 \mathrm{O} 3(\mathrm{wt} \%)$ & 8.5067 & 8.4195 & 8.6340 & -0.1273 & -0.2145 & $-1.5 \%$ & $-2.5 \%$ \\
\hline 35 & SB3-35 & $\mathrm{K} 2 \mathrm{O}(\mathrm{wt} \%)$ & 0.0623 & 0.0559 & 0.0920 & -0.0297 & -0.0361 & $-32.2 \%$ & $-39.2 \%$ \\
\hline 35 & SB3-35 & La2O3 (wt\%) & 0.0396 & 0.0396 & 0.0490 & -0.0094 & -0.0094 & $-19.2 \%$ & $-19.2 \%$ \\
\hline 35 & SB3-35 & $\mathrm{Li} 2 \mathrm{O}(\mathrm{wt} \%)$ & 3.5738 & 3.7153 & 3.7500 & -0.1762 & -0.0347 & $-4.7 \%$ & $-0.9 \%$ \\
\hline 35 & SB3-35 & $\mathrm{MgO}(\mathrm{wt} \%)$ & 0.0044 & 0.0045 & 0.0400 & -0.0356 & -0.0355 & $-89.1 \%$ & $-88.7 \%$ \\
\hline 35 & SB3-35 & $\mathrm{MnO}(\mathrm{wt} \%)$ & 1.4687 & 1.5831 & 1.5400 & -0.0713 & 0.0431 & $-4.6 \%$ & $2.8 \%$ \\
\hline
\end{tabular}


Table H.4: Average Measured and Bias-Corrected Chemical Compositions Versus Targeted Compositions by Oxide by Glass Number (continued)

(Batch 1: 100; 101-U std)

\begin{tabular}{|c|c|c|c|c|c|c|c|c|c|}
\hline Glass \# & Glass ID & Oxide & $\begin{array}{c}\text { Measured } \\
(\mathrm{wt} \%)\end{array}$ & $\begin{array}{c}\text { Measured } \\
\text { Bias-Corrected } \\
(\mathrm{wt} \%)\end{array}$ & $\begin{array}{c}\text { Targeted } \\
(\text { wt } \%)\end{array}$ & $\begin{array}{c}\text { Diff of } \\
\text { Measured }\end{array}$ & $\begin{array}{c}\text { Diff of } \\
\text { Meas BC }\end{array}$ & $\begin{array}{l}\% \text { Diff of } \\
\text { Measured }\end{array}$ & $\begin{array}{l}\% \text { Diff of } \\
\text { Meas BC }\end{array}$ \\
\hline 35 & SB3-35 & $\mathrm{Na} 2 \mathrm{O}(\mathrm{wt} \%)$ & 15.7042 & 14.7645 & 14.8100 & 0.8942 & -0.0455 & $6.0 \%$ & $-0.3 \%$ \\
\hline 35 & SB3-35 & $\mathrm{NiO}(\mathrm{wt} \%)$ & 0.3035 & 0.3453 & 0.3450 & -0.0415 & 0.0003 & $-12.0 \%$ & $0.1 \%$ \\
\hline 35 & SB3-35 & $\mathrm{PbO}(\mathrm{wt} \%)$ & 0.0557 & 0.0557 & 0.0640 & -0.0083 & -0.0083 & $-12.9 \%$ & $-12.9 \%$ \\
\hline 35 & SB3-35 & $\mathrm{SiO} 2(\mathrm{wt} \%)$ & 55.4079 & 59.5229 & 55.9500 & -0.5421 & 3.5729 & $-1.0 \%$ & $6.4 \%$ \\
\hline 35 & SB3-35 & ThO2 (wt\%) & 0.0913 & 0.0913 & 0.0310 & 0.0603 & 0.0603 & $194.6 \%$ & $194.6 \%$ \\
\hline 35 & SB3-35 & $\mathrm{TiO} 2(\mathrm{wt} \%)$ & 0.5238 & 0.5669 & 0.6040 & -0.0802 & -0.0371 & $-13.3 \%$ & $-6.1 \%$ \\
\hline 35 & SB3-35 & $\mathrm{U} 3 \mathrm{O} 8(\mathrm{wt} \%)$ & 2.1432 & 2.2425 & 2.1850 & -0.0418 & 0.0575 & $-1.9 \%$ & $2.6 \%$ \\
\hline 35 & SB3-35 & $\mathrm{ZnO}(\mathrm{wt} \%)$ & 0.0753 & 0.0753 & 0.0880 & -0.0127 & -0.0127 & $-14.4 \%$ & $-14.4 \%$ \\
\hline 35 & SB3-35 & $\mathrm{ZrO} 2(\mathrm{wt} \%)$ & 0.1364 & 0.1364 & 0.1590 & -0.0226 & -0.0226 & $-14.2 \%$ & $-14.2 \%$ \\
\hline 35 & SB3-35 & Sum of Oxides & 99.9148 & 103.2043 & 99.9980 & -0.0832 & 3.2063 & $-0.1 \%$ & $3.2 \%$ \\
\hline 36 & SB3-36 & $\mathrm{Al} 2 \mathrm{O} 3(\mathrm{wt} \%)$ & 7.2746 & 7.2779 & 7.5400 & -0.2654 & -0.2621 & $-3.5 \%$ & $-3.5 \%$ \\
\hline 36 & SB3-36 & $\mathrm{B} 2 \mathrm{O} 3(\mathrm{wt} \%)$ & 4.5320 & 4.3856 & 4.4000 & 0.1320 & -0.0144 & $3.0 \%$ & $-0.3 \%$ \\
\hline 36 & SB3-36 & $\mathrm{BaO}(\mathrm{wt} \%)$ & 0.0829 & 0.0920 & 0.0970 & -0.0141 & -0.0050 & $-14.5 \%$ & $-5.1 \%$ \\
\hline 36 & SB3-36 & $\mathrm{CaO}(\mathrm{wt} \%)$ & 1.4478 & 1.3543 & 1.3910 & 0.0568 & -0.0367 & $4.1 \%$ & $-2.6 \%$ \\
\hline 36 & SB3-36 & $\mathrm{Ce} 2 \mathrm{O} 3(\mathrm{wt} \%)$ & 0.1250 & 0.1250 & 0.1350 & -0.0100 & -0.0100 & $-7.4 \%$ & $-7.4 \%$ \\
\hline 36 & SB3-36 & $\mathrm{Cr} 2 \mathrm{O} 3(\mathrm{wt} \%)$ & 0.1326 & 0.1411 & 0.1430 & -0.0104 & -0.0019 & $-7.2 \%$ & $-1.4 \%$ \\
\hline 36 & SB3-36 & $\mathrm{CuO}(\mathrm{wt} \%)$ & 0.0760 & 0.0811 & 0.0770 & -0.0010 & 0.0041 & $-1.2 \%$ & $5.3 \%$ \\
\hline 36 & SB3-36 & $\mathrm{Fe} 2 \mathrm{O} 3(\mathrm{wt} \%)$ & 14.8045 & 14.6503 & 15.5420 & -0.7375 & -0.8917 & $-4.7 \%$ & $-5.7 \%$ \\
\hline 36 & SB3-36 & $\mathrm{K} 2 \mathrm{O}(\mathrm{wt} \%)$ & 0.1539 & 0.1380 & 0.1660 & -0.0121 & -0.0280 & $-7.3 \%$ & $-16.9 \%$ \\
\hline 36 & SB3-36 & $\mathrm{La} 2 \mathrm{O} 3(\mathrm{wt} \%)$ & 0.0710 & 0.0710 & 0.0890 & -0.0180 & -0.0180 & $-20.3 \%$ & $-20.3 \%$ \\
\hline 36 & SB3-36 & $\mathrm{Li} 2 \mathrm{O}(\mathrm{wt} \%)$ & 2.7019 & 2.8087 & 2.7500 & -0.0481 & 0.0587 & $-1.7 \%$ & $2.1 \%$ \\
\hline 36 & SB3-36 & $\mathrm{MgO}(\mathrm{wt} \%)$ & 0.0253 & 0.0264 & 0.0730 & -0.0477 & -0.0466 & $-65.4 \%$ & $-63.8 \%$ \\
\hline 36 & SB3-36 & $\mathrm{MnO}(\mathrm{wt} \%)$ & 2.4597 & 2.6517 & 2.7720 & -0.3123 & -0.1203 & $-11.3 \%$ & $-4.3 \%$ \\
\hline 36 & SB3-36 & $\mathrm{Na} 2 \mathrm{O}(\mathrm{wt} \%)$ & 17.7262 & 16.6658 & 17.0580 & 0.6682 & -0.3922 & $3.9 \%$ & $-2.3 \%$ \\
\hline 36 & SB3-36 & $\mathrm{NiO}(\mathrm{wt} \%)$ & 0.5128 & 0.5835 & 0.6210 & -0.1082 & -0.0375 & $-17.4 \%$ & $-6.0 \%$ \\
\hline 36 & SB3-36 & $\mathrm{PbO}(\mathrm{wt} \%)$ & 0.0978 & 0.0978 & 0.1160 & -0.0182 & -0.0182 & $-15.7 \%$ & $-15.7 \%$ \\
\hline 36 & SB3-36 & $\mathrm{SiO} 2(\mathrm{wt} \%)$ & 41.9838 & 45.0960 & 41.5090 & 0.4748 & 3.5870 & $1.1 \%$ & $8.6 \%$ \\
\hline 36 & SB3-36 & ThO2 (wt\%) & 0.1567 & 0.1567 & 0.0550 & 0.1017 & 0.1017 & $185.0 \%$ & $185.0 \%$ \\
\hline 36 & SB3-36 & $\mathrm{TiO} 2(\mathrm{wt} \%)$ & 0.9883 & 1.0697 & 1.0880 & -0.0997 & -0.0183 & $-9.2 \%$ & $-1.7 \%$ \\
\hline 36 & SB3-36 & U3O8 (wt\%) & 3.7145 & 3.8866 & 3.9340 & -0.2195 & -0.0474 & $-5.6 \%$ & $-1.2 \%$ \\
\hline 36 & SB3-36 & $\mathrm{ZnO}(\mathrm{wt} \%)$ & 0.1254 & 0.1254 & 0.1590 & -0.0336 & -0.0336 & $-21.1 \%$ & $-21.1 \%$ \\
\hline 36 & SB3-36 & $\mathrm{ZrO} 2(\mathrm{wt} \%)$ & 0.2452 & 0.2452 & 0.2870 & -0.0418 & -0.0418 & $-14.6 \%$ & $-14.6 \%$ \\
\hline 36 & SB3-36 & Sum of Oxides & 99.4380 & 101.7297 & 100.0020 & -0.5640 & 1.7277 & $-0.6 \%$ & $1.7 \%$ \\
\hline 37 & SB3-37 & $\mathrm{Al} 2 \mathrm{O} 3(\mathrm{wt} \%)$ & 4.9410 & 5.0844 & 4.8150 & 0.1260 & 0.2694 & $2.6 \%$ & $5.6 \%$ \\
\hline 37 & SB3-37 & $\mathrm{B} 2 \mathrm{O} 3(\mathrm{wt} \%)$ & 5.3531 & 5.3026 & 5.5200 & -0.1669 & -0.2174 & $-3.0 \%$ & $-3.9 \%$ \\
\hline 37 & SB3-37 & $\mathrm{BaO}(\mathrm{wt} \%)$ & 0.0550 & 0.0601 & 0.0670 & -0.0120 & -0.0069 & $-17.9 \%$ & $-10.4 \%$ \\
\hline 37 & SB3-37 & $\mathrm{CaO}(\mathrm{wt} \%)$ & 1.0099 & 0.9624 & 0.9580 & 0.0519 & 0.0044 & $5.4 \%$ & $0.5 \%$ \\
\hline 37 & SB3-37 & $\mathrm{Ce} 2 \mathrm{O} 3(\mathrm{wt} \%)$ & 0.0940 & 0.0940 & 0.0930 & 0.0010 & 0.0010 & $1.1 \%$ & $1.1 \%$ \\
\hline 37 & SB3-37 & $\mathrm{Cr} 2 \mathrm{O} 3(\mathrm{wt} \%)$ & 0.0855 & 0.0910 & 0.0980 & -0.0125 & -0.0070 & $-12.8 \%$ & $-7.2 \%$ \\
\hline 37 & SB3-37 & $\mathrm{CuO}(\mathrm{wt} \%)$ & 0.0504 & 0.0529 & 0.0530 & -0.0026 & -0.0001 & $-4.9 \%$ & $-0.3 \%$ \\
\hline 37 & SB3-37 & $\mathrm{Fe} 2 \mathrm{O} 3(\mathrm{wt} \%)$ & 10.2724 & 10.4288 & 10.7070 & -0.4346 & -0.2782 & $-4.1 \%$ & $-2.6 \%$ \\
\hline 37 & SB3-37 & $\mathrm{K} 2 \mathrm{O}(\mathrm{wt} \%)$ & 0.0979 & 0.0876 & 0.1150 & -0.0171 & -0.0274 & $-14.9 \%$ & $-23.8 \%$ \\
\hline 37 & SB3-37 & $\mathrm{La} 2 \mathrm{O} 3$ (wt $\%)$ & 0.0493 & 0.0493 & 0.0610 & -0.0117 & -0.0117 & $-19.2 \%$ & $-19.2 \%$ \\
\hline 37 & SB3-37 & $\mathrm{Li} 2 \mathrm{O}$ (wt\%) & 5.2800 & 5.4630 & 5.5200 & -0.2400 & -0.0570 & $-4.3 \%$ & $-1.0 \%$ \\
\hline 37 & SB3-37 & $\mathrm{MgO}(\mathrm{wt} \%)$ & 0.0224 & 0.0224 & 0.0500 & -0.0276 & -0.0276 & $-55.2 \%$ & $-55.2 \%$ \\
\hline 37 & SB3-37 & $\mathrm{MnO}(\mathrm{wt} \%)$ & 1.8755 & 1.8074 & 1.9100 & -0.0345 & -0.1026 & $-1.8 \%$ & $-5.4 \%$ \\
\hline 37 & SB3-37 & $\mathrm{Na} 2 \mathrm{O}(\mathrm{wt} \%)$ & 9.8943 & 9.2516 & 9.2740 & 0.6203 & -0.0224 & $6.7 \%$ & $-0.2 \%$ \\
\hline 37 & SB3-37 & $\mathrm{NiO}(\mathrm{wt} \%)$ & 0.3891 & 0.4246 & 0.4280 & -0.0389 & -0.0034 & $-9.1 \%$ & $-0.8 \%$ \\
\hline 37 & SB3-37 & $\mathrm{PbO}(\mathrm{wt} \%)$ & 0.0681 & 0.0681 & 0.0800 & -0.0119 & -0.0119 & $-14.8 \%$ & $-14.8 \%$ \\
\hline 37 & SB3-37 & $\mathrm{SiO} 2(\mathrm{wt} \%)$ & 54.9800 & 57.8805 & 56.4480 & -1.4680 & 1.4325 & $-2.6 \%$ & $2.5 \%$ \\
\hline 37 & SB3-37 & ThO2 (wt $\%)$ & 0.1220 & 0.1220 & 0.0380 & 0.0840 & 0.0840 & $221.2 \%$ & $221.2 \%$ \\
\hline 37 & SB3-37 & $\mathrm{TiO} 2(\mathrm{wt} \%)$ & 0.7214 & 0.7580 & 0.7490 & -0.0276 & 0.0090 & $-3.7 \%$ & $1.2 \%$ \\
\hline 37 & SB3-37 & $\mathrm{U} 3 \mathrm{O} 8(\mathrm{wt} \%)$ & 2.6149 & 2.7548 & 2.7100 & -0.0951 & 0.0448 & $-3.5 \%$ & $1.7 \%$ \\
\hline
\end{tabular}


Table H.4: Average Measured and Bias-Corrected Chemical Compositions Versus Targeted Compositions by Oxide by Glass Number (continued)

(Batch 1: 100; 101-U std)

\begin{tabular}{|c|c|c|c|c|c|c|c|c|c|}
\hline Glass \# & Glass ID & Oxide & $\begin{array}{c}\text { Measured } \\
(\mathrm{wt} \%)\end{array}$ & $\begin{array}{c}\text { Measured } \\
\text { Bias-Corrected } \\
(w t \%)\end{array}$ & $\begin{array}{c}\text { Targeted } \\
(\text { wt } \%)\end{array}$ & $\begin{array}{c}\text { Diff of } \\
\text { Measured }\end{array}$ & $\begin{array}{c}\text { Diff of } \\
\text { Meas BC }\end{array}$ & $\begin{array}{l}\% \text { Diff of } \\
\text { Measured }\end{array}$ & $\begin{array}{l}\% \text { Diff of } \\
\text { Meas BC }\end{array}$ \\
\hline 37 & SB3-37 & $\mathrm{ZnO}(\mathrm{wt} \%)$ & 0.0962 & 0.0962 & 0.1090 & -0.0128 & -0.0128 & $-11.8 \%$ & $-11.8 \%$ \\
\hline 37 & SB3-37 & $\mathrm{ZrO} 2(\mathrm{wt} \%)$ & 0.1753 & 0.1753 & 0.1980 & -0.0227 & -0.0227 & $-11.5 \%$ & $-11.5 \%$ \\
\hline 37 & SB3-37 & Sum of Oxides & 98.2475 & 101.0368 & 100.0010 & -1.7535 & 1.0358 & $-1.8 \%$ & $1.0 \%$ \\
\hline 38 & SB3-38 & $\mathrm{A} 12 \mathrm{O} 3(\mathrm{wt} \%)$ & 7.2368 & 7.4467 & 7.1450 & 0.0918 & 0.3017 & $1.3 \%$ & $4.2 \%$ \\
\hline 38 & SB3-38 & $\mathrm{B} 2 \mathrm{O} 3(\mathrm{wt} \%)$ & 4.2825 & 4.2422 & 4.3200 & -0.0375 & -0.0778 & $-0.9 \%$ & $-1.8 \%$ \\
\hline 38 & SB3-38 & $\mathrm{BaO}(\mathrm{wt} \%)$ & 0.0776 & 0.0931 & 0.0990 & -0.0214 & -0.0059 & $-21.6 \%$ & $-5.9 \%$ \\
\hline 38 & SB3-38 & $\mathrm{CaO}(\mathrm{wt} \%)$ & 1.5269 & 1.4079 & 1.4220 & 0.1049 & -0.0141 & $7.4 \%$ & $-1.0 \%$ \\
\hline 38 & SB3-38 & $\mathrm{Ce} 2 \mathrm{O} 3(\mathrm{wt} \%)$ & 0.1335 & 0.1335 & 0.1380 & -0.0045 & -0.0045 & $-3.2 \%$ & $-3.2 \%$ \\
\hline 38 & SB3-38 & $\mathrm{Cr} 2 \mathrm{O} 3$ (wt\%) & 0.0775 & 0.0920 & 0.1460 & -0.0685 & -0.0540 & $-46.9 \%$ & $-37.0 \%$ \\
\hline 38 & SB3-38 & $\mathrm{CuO}(\mathrm{wt} \%)$ & 0.0764 & 0.0815 & 0.0780 & -0.0016 & 0.0035 & $-2.1 \%$ & $4.5 \%$ \\
\hline 38 & SB3-38 & $\mathrm{Fe} 2 \mathrm{O} 3(\mathrm{wt} \%)$ & 16.3701 & 15.8518 & 15.8870 & 0.4831 & -0.0352 & $3.0 \%$ & $-0.2 \%$ \\
\hline 38 & SB3-38 & $\mathrm{K} 2 \mathrm{O}(\mathrm{wt} \%)$ & 0.1735 & 0.1544 & 0.1700 & 0.0035 & -0.0156 & $2.0 \%$ & $-9.2 \%$ \\
\hline 38 & SB3-38 & $\mathrm{La} 2 \mathrm{O} 3(\mathrm{wt} \%)$ & 0.0695 & 0.0695 & 0.0910 & -0.0215 & -0.0215 & $-23.6 \%$ & $-23.6 \%$ \\
\hline 38 & SB3-38 & $\mathrm{Li} 2 \mathrm{O}(\mathrm{wt} \%)$ & 4.1497 & 4.2935 & 4.3200 & -0.1703 & -0.0265 & $-3.9 \%$ & $-0.6 \%$ \\
\hline 38 & SB3-38 & $\mathrm{MgO}(\mathrm{wt} \%)$ & 0.0195 & 0.0212 & 0.0740 & -0.0545 & -0.0528 & $-73.7 \%$ & $-71.4 \%$ \\
\hline 38 & SB3-38 & $\mathrm{MnO}(\mathrm{wt} \%)$ & 2.4985 & 2.7639 & 2.8340 & -0.3355 & -0.0701 & $-11.8 \%$ & $-2.5 \%$ \\
\hline 38 & SB3-38 & $\mathrm{Na} 2 \mathrm{O}(\mathrm{wt} \%)$ & 12.6207 & 12.2235 & 12.3100 & 0.3106 & -0.0865 & $2.5 \%$ & $-0.7 \%$ \\
\hline 38 & SB3-38 & $\mathrm{NiO}(\mathrm{wt} \%)$ & 0.5023 & 0.5859 & 0.6350 & -0.1327 & -0.0491 & $-20.9 \%$ & $-7.7 \%$ \\
\hline 38 & SB3-38 & $\mathrm{PbO}(\mathrm{wt} \%)$ & 0.0926 & 0.0926 & 0.1180 & -0.0254 & -0.0254 & $-21.5 \%$ & $-21.5 \%$ \\
\hline 38 & SB3-38 & $\mathrm{SiO} 2(\mathrm{wt} \%)$ & 44.0161 & 46.3385 & 44.5670 & -0.5509 & 1.7715 & $-1.2 \%$ & $4.0 \%$ \\
\hline 38 & SB3-38 & ThO2 (wt $\%)$ & 0.1599 & 0.1599 & 0.0570 & 0.1029 & 0.1029 & $180.5 \%$ & $180.5 \%$ \\
\hline 38 & SB3-38 & $\mathrm{TiO} 2(\mathrm{wt} \%)$ & 1.0179 & 1.1176 & 1.1120 & -0.0941 & 0.0056 & $-8.5 \%$ & $0.5 \%$ \\
\hline 38 & SB3-38 & U3O8 (wt\%) & 3.8707 & 4.0501 & 4.0210 & -0.1503 & 0.0291 & $-3.7 \%$ & $0.7 \%$ \\
\hline 38 & SB3-38 & $\mathrm{ZnO}(\mathrm{wt} \%)$ & 0.1189 & 0.1189 & 0.1620 & -0.0431 & -0.0431 & $-26.6 \%$ & $-26.6 \%$ \\
\hline 38 & SB3-38 & $\mathrm{ZrO} 2(\mathrm{wt} \%)$ & 0.3880 & 0.3880 & 0.2930 & 0.0950 & 0.0950 & $32.4 \%$ & $32.4 \%$ \\
\hline 38 & SB3-38 & Sum of Oxides & 99.4789 & 101.7261 & 99.9990 & -0.5201 & 1.7271 & $-0.5 \%$ & $1.7 \%$ \\
\hline 39 & SB3-39 & $\mathrm{Al} 2 \mathrm{O} 3$ (wt\%) & 4.4734 & 4.4715 & 4.3490 & 0.1244 & 0.1225 & $2.9 \%$ & $2.8 \%$ \\
\hline 39 & SB3-39 & B2O3 (wt\%) & 7.2126 & 6.9809 & 7.2000 & 0.0126 & -0.2191 & $0.2 \%$ & $-3.0 \%$ \\
\hline 39 & SB3-39 & $\mathrm{BaO}(\mathrm{wt} \%)$ & 0.0511 & 0.0552 & 0.0600 & -0.0089 & -0.0048 & $-14.9 \%$ & $-8.0 \%$ \\
\hline 39 & SB3-39 & $\mathrm{CaO}(\mathrm{wt} \%)$ & 0.9490 & 0.8767 & 0.8650 & 0.0840 & 0.0117 & $9.7 \%$ & $1.4 \%$ \\
\hline 39 & SB3-39 & $\mathrm{Ce} 2 \mathrm{O} 3$ (wt\%) & 0.0805 & 0.0805 & 0.0840 & -0.0035 & -0.0035 & $-4.1 \%$ & $-4.1 \%$ \\
\hline 39 & SB3-39 & $\mathrm{Cr} 2 \mathrm{O} 3(\mathrm{wt} \%)$ & 0.1842 & 0.1935 & 0.0890 & 0.0952 & 0.1045 & $106.9 \%$ & $117.4 \%$ \\
\hline 39 & SB3-39 & $\mathrm{CuO}(\mathrm{wt} \%)$ & 0.0557 & 0.0590 & 0.0480 & 0.0077 & 0.0110 & $16.1 \%$ & $22.8 \%$ \\
\hline 39 & SB3-39 & $\mathrm{Fe} 2 \mathrm{O} 3(\mathrm{wt} \%)$ & 10.1044 & 10.0309 & 9.6710 & 0.4334 & 0.3599 & $4.5 \%$ & $3.7 \%$ \\
\hline 39 & SB3-39 & $\mathrm{K} 2 \mathrm{O}(\mathrm{wt} \%)$ & 0.0711 & 0.0635 & 0.1030 & -0.0319 & -0.0395 & $-31.0 \%$ & $-38.3 \%$ \\
\hline 39 & SB3-39 & La2O3 (wt\%) & 0.0449 & 0.0449 & 0.0550 & -0.0101 & -0.0101 & $-18.4 \%$ & $-18.4 \%$ \\
\hline 39 & SB3-39 & $\mathrm{Li} 2 \mathrm{O}(\mathrm{wt} \%)$ & 5.5330 & 5.7524 & 5.7600 & -0.2270 & -0.0076 & $-3.9 \%$ & $-0.1 \%$ \\
\hline 39 & SB3-39 & $\mathrm{MgO}(\mathrm{wt} \%)$ & 0.0041 & 0.0042 & 0.0450 & -0.0409 & -0.0408 & $-90.8 \%$ & $-90.7 \%$ \\
\hline 39 & SB3-39 & $\mathrm{MnO}(\mathrm{wt} \%)$ & 1.6818 & 1.7404 & 1.7250 & -0.0432 & 0.0154 & $-2.5 \%$ & $0.9 \%$ \\
\hline 39 & SB3-39 & $\mathrm{Na} 2 \mathrm{O}(\mathrm{wt} \%)$ & 8.8597 & 8.5126 & 8.6670 & 0.1927 & -0.1544 & $2.2 \%$ & $-1.8 \%$ \\
\hline 39 & SB3-39 & $\mathrm{NiO}(\mathrm{wt} \%)$ & 0.3770 & 0.4160 & 0.3870 & -0.0100 & 0.0290 & $-2.6 \%$ & $7.5 \%$ \\
\hline 39 & SB3-39 & $\mathrm{PbO}(\mathrm{wt} \%)$ & 0.0633 & 0.0633 & 0.0720 & -0.0087 & -0.0087 & $-12.1 \%$ & $-12.1 \%$ \\
\hline 39 & SB3-39 & $\mathrm{SiO} 2(\mathrm{wt} \%)$ & 56.6380 & 60.8426 & 57.3840 & -0.7460 & 3.4586 & $-1.3 \%$ & $6.0 \%$ \\
\hline 39 & SB3-39 & ThO2 (wt $\%)$ & 0.1013 & 0.1013 & 0.0340 & 0.0673 & 0.0673 & $197.9 \%$ & $197.9 \%$ \\
\hline 39 & SB3-39 & $\mathrm{TiO} 2(\mathrm{wt} \%)$ & 0.6351 & 0.6695 & 0.6770 & -0.0419 & -0.0075 & $-6.2 \%$ & $-1.1 \%$ \\
\hline 39 & SB3-39 & $\mathrm{U} 3 \mathrm{O} 8(\mathrm{wt} \%)$ & 2.4115 & 2.5018 & 2.4480 & -0.0365 & 0.0538 & $-1.5 \%$ & $2.2 \%$ \\
\hline 39 & SB3-39 & $\mathrm{ZnO}(\mathrm{wt} \%)$ & 0.0918 & 0.0918 & 0.0990 & -0.0072 & -0.0072 & $-7.3 \%$ & $-7.3 \%$ \\
\hline 39 & SB3-39 & $\mathrm{ZrO} 2(\mathrm{wt} \%)$ & 0.2198 & 0.2198 & 0.1790 & 0.0408 & 0.0408 & $22.8 \%$ & $22.8 \%$ \\
\hline 39 & SB3-39 & Sum of Oxides & 99.8431 & 103.7724 & 100.0010 & -0.1579 & 3.7714 & $-0.2 \%$ & $3.8 \%$ \\
\hline 40 & SB3-40 & $\mathrm{A} 12 \mathrm{O} 3(\mathrm{wt} \%)$ & 7.3029 & 7.4111 & 7.1450 & 0.1579 & 0.2661 & $2.2 \%$ & $3.7 \%$ \\
\hline 40 & SB3-40 & $\mathrm{B} 2 \mathrm{O} 3(\mathrm{wt} \%)$ & 5.4980 & 5.4015 & 5.4000 & 0.0980 & 0.0015 & $1.8 \%$ & $0.0 \%$ \\
\hline 40 & SB3-40 & $\mathrm{BaO}(\mathrm{wt} \%)$ & 0.0851 & 0.0945 & 0.0990 & -0.0139 & -0.0045 & $-14.0 \%$ & $-4.5 \%$ \\
\hline 40 & SB3-40 & $\mathrm{CaO}(\mathrm{wt} \%)$ & 1.5118 & 1.4142 & 1.4220 & 0.0898 & -0.0078 & $6.3 \%$ & $-0.6 \%$ \\
\hline
\end{tabular}


Table H.4: Average Measured and Bias-Corrected Chemical Compositions Versus Targeted Compositions by Oxide by Glass Number (continued)

(Batch 1: 100; 101-U std)

\begin{tabular}{|c|c|c|c|c|c|c|c|c|c|}
\hline Glass \# & Glass ID & Oxide & $\begin{array}{c}\text { Measured } \\
(\mathrm{wt} \%)\end{array}$ & $\begin{array}{c}\text { Measured } \\
\text { Bias-Corrected } \\
(w t \%)\end{array}$ & $\begin{array}{c}\text { Targeted } \\
\text { (wt\%) }\end{array}$ & $\begin{array}{c}\text { Diff of } \\
\text { Measured }\end{array}$ & $\begin{array}{c}\text { Diff of } \\
\text { Meas BC }\end{array}$ & $\begin{array}{l}\% \text { Diff of } \\
\text { Measured }\end{array}$ & $\begin{array}{l}\% \text { Diff of } \\
\text { Meas BC }\end{array}$ \\
\hline 40 & SB3-40 & $\mathrm{Ce} 2 \mathrm{O} 3(\mathrm{wt} \%)$ & 0.1324 & 0.1324 & 0.1380 & -0.0056 & -0.0056 & $-4.1 \%$ & $-4.1 \%$ \\
\hline 40 & SB3-40 & $\mathrm{Cr} 2 \mathrm{O} 3(\mathrm{wt} \%)$ & 0.1345 & 0.1430 & 0.1460 & -0.0115 & -0.0030 & $-7.9 \%$ & $-2.0 \%$ \\
\hline 40 & SB3-40 & $\mathrm{CuO}(\mathrm{wt} \%)$ & 0.0792 & 0.0844 & 0.0780 & 0.0012 & 0.0064 & $1.5 \%$ & $8.2 \%$ \\
\hline 40 & SB3-40 & $\mathrm{Fe} 2 \mathrm{O} 3(\mathrm{wt} \%)$ & 15.2620 & 15.1037 & 15.8870 & -0.6250 & -0.7833 & $-3.9 \%$ & $-4.9 \%$ \\
\hline 40 & SB3-40 & $\mathrm{K} 2 \mathrm{O}(\mathrm{wt} \%)$ & 0.1738 & 0.1558 & 0.1700 & 0.0038 & -0.0142 & $2.2 \%$ & $-8.3 \%$ \\
\hline 40 & SB3-40 & $\mathrm{La} 2 \mathrm{O} 3(\mathrm{wt} \%)$ & 0.0756 & 0.0756 & 0.0910 & -0.0154 & -0.0154 & $-16.9 \%$ & $-16.9 \%$ \\
\hline 40 & SB3-40 & $\mathrm{Li} 2 \mathrm{O}(\mathrm{wt} \%)$ & 4.2251 & 4.3639 & 4.3200 & -0.0949 & 0.0439 & $-2.2 \%$ & $1.0 \%$ \\
\hline 40 & SB3-40 & $\mathrm{MgO}(\mathrm{wt} \%)$ & 0.0298 & 0.0312 & 0.0740 & -0.0442 & -0.0428 & $-59.7 \%$ & $-57.9 \%$ \\
\hline 40 & SB3-40 & $\mathrm{MnO}(\mathrm{wt} \%)$ & 2.5469 & 2.7460 & 2.8340 & -0.2871 & -0.0880 & $-10.1 \%$ & $-3.1 \%$ \\
\hline 40 & SB3-40 & $\mathrm{Na} 2 \mathrm{O}(\mathrm{wt} \%)$ & 12.9846 & 12.2080 & 12.3100 & 0.6746 & -0.1020 & $5.5 \%$ & $-0.8 \%$ \\
\hline 40 & SB3-40 & $\mathrm{NiO}(\mathrm{wt} \%)$ & 0.5030 & 0.5723 & 0.6350 & -0.1320 & -0.0627 & $-20.8 \%$ & $-9.9 \%$ \\
\hline 40 & SB3-40 & $\mathrm{PbO}(\mathrm{wt} \%)$ & 0.0999 & 0.0999 & 0.1180 & -0.0181 & -0.0181 & $-15.3 \%$ & $-15.3 \%$ \\
\hline 40 & SB3-40 & $\mathrm{SiO} 2(\mathrm{wt} \%)$ & 43.4813 & 46.7828 & 43.4870 & -0.0057 & 3.2958 & $0.0 \%$ & $7.6 \%$ \\
\hline 40 & SB3-40 & ThO2 $(\mathrm{wt} \%)$ & 0.1656 & 0.1656 & 0.0570 & 0.1086 & 0.1086 & $190.5 \%$ & $190.5 \%$ \\
\hline 40 & SB3-40 & $\mathrm{TiO} 2(\mathrm{wt} \%)$ & 1.0421 & 1.1279 & 1.1120 & -0.0699 & 0.0159 & $-6.3 \%$ & $1.4 \%$ \\
\hline 40 & SB3-40 & $\mathrm{U} 3 \mathrm{O} 8(\mathrm{wt} \%)$ & 3.8471 & 4.0254 & 4.0210 & -0.1739 & 0.0044 & $-4.3 \%$ & $0.1 \%$ \\
\hline 40 & SB3-40 & $\mathrm{ZnO}(\mathrm{wt} \%)$ & 0.1422 & 0.1422 & 0.1620 & -0.0198 & -0.0198 & $-12.2 \%$ & $-12.2 \%$ \\
\hline 40 & SB3-40 & $\mathrm{ZrO} 2(\mathrm{wt} \%)$ & 0.2556 & 0.2556 & 0.2930 & -0.0374 & -0.0374 & $-12.8 \%$ & $-12.8 \%$ \\
\hline 40 & SB3-40 & Sum of Oxides & 99.5785 & 102.5372 & 99.9990 & -0.4205 & 2.5382 & $-0.4 \%$ & $2.5 \%$ \\
\hline 41 & SB3-41 & $\mathrm{Al} 2 \mathrm{O} 3(\mathrm{wt} \%)$ & 4.6434 & 4.7782 & 4.3590 & 0.2844 & 0.4192 & $6.5 \%$ & $9.6 \%$ \\
\hline 41 & SB3-41 & $\mathrm{B} 2 \mathrm{O} 3(\mathrm{wt} \%)$ & 5.8522 & 5.7970 & 5.9200 & -0.0678 & -0.1230 & $-1.1 \%$ & $-2.1 \%$ \\
\hline 41 & SB3-41 & $\mathrm{BaO}(\mathrm{wt} \%)$ & 0.0508 & 0.0564 & 0.0600 & -0.0092 & -0.0036 & $-15.3 \%$ & $-6.0 \%$ \\
\hline 41 & SB3-41 & $\mathrm{CaO}(\mathrm{wt} \%)$ & 0.9266 & 0.8667 & 0.8670 & 0.0596 & -0.0003 & $6.9 \%$ & $0.0 \%$ \\
\hline 41 & SB3-41 & $\mathrm{Ce} 2 \mathrm{O} 3(\mathrm{wt} \%)$ & 0.0820 & 0.0820 & 0.0860 & -0.0040 & -0.0040 & $-4.7 \%$ & $-4.7 \%$ \\
\hline 41 & SB3-41 & $\mathrm{Cr} 2 \mathrm{O} 3(\mathrm{wt} \%)$ & 0.0859 & 0.0913 & 0.0900 & -0.0041 & 0.0013 & $-4.6 \%$ & $1.5 \%$ \\
\hline 41 & SB3-41 & $\mathrm{CuO}(\mathrm{wt} \%)$ & 0.0494 & 0.0527 & 0.0480 & 0.0014 & 0.0047 & $3.0 \%$ & $9.8 \%$ \\
\hline 41 & SB3-41 & $\mathrm{Fe} 2 \mathrm{O} 3(\mathrm{wt} \%)$ & 9.7613 & 9.6609 & 9.6970 & 0.0643 & -0.0361 & $0.7 \%$ & $-0.4 \%$ \\
\hline 41 & SB3-41 & $\mathrm{K} 2 \mathrm{O}(\mathrm{wt} \%)$ & 0.0705 & 0.0632 & 0.1040 & -0.0335 & -0.0408 & $-32.2 \%$ & $-39.2 \%$ \\
\hline 41 & SB3-41 & $\mathrm{La} 2 \mathrm{O} 3(\mathrm{wt} \%)$ & 0.0393 & 0.0393 & 0.0490 & -0.0097 & -0.0097 & $-19.8 \%$ & $-19.8 \%$ \\
\hline 41 & SB3-41 & Li2O (wt\%) & 5.7752 & 5.9753 & 5.9210 & -0.1458 & 0.0543 & $-2.5 \%$ & $0.9 \%$ \\
\hline 41 & SB3-41 & $\mathrm{MgO}(\mathrm{wt} \%)$ & 0.0021 & 0.0022 & 0.0450 & -0.0429 & -0.0428 & $-95.4 \%$ & $-95.2 \%$ \\
\hline 41 & SB3-41 & $\mathrm{MnO}(\mathrm{wt} \%)$ & 1.6075 & 1.7330 & 1.7280 & -0.1205 & 0.0050 & $-7.0 \%$ & $0.3 \%$ \\
\hline 41 & SB3-41 & $\mathrm{Na} 2 \mathrm{O}(\mathrm{wt} \%)$ & 14.2214 & 13.3702 & 13.7140 & 0.5074 & -0.3438 & $3.7 \%$ & $-2.5 \%$ \\
\hline 41 & SB3-41 & $\mathrm{NiO}(\mathrm{wt} \%)$ & 0.3372 & 0.3837 & 0.3880 & -0.0508 & -0.0043 & $-13.1 \%$ & $-1.1 \%$ \\
\hline 41 & SB3-41 & $\mathrm{PbO}(\mathrm{wt} \%)$ & 0.0617 & 0.0617 & 0.0730 & -0.0113 & -0.0113 & $-15.5 \%$ & $-15.5 \%$ \\
\hline 41 & SB3-41 & $\mathrm{SiO} 2(\mathrm{wt} \%)$ & 53.6964 & 56.5261 & 54.0850 & -0.3886 & 2.4411 & $-0.7 \%$ & $4.5 \%$ \\
\hline 41 & SB3-41 & ThO2 (wt $\%)$ & 0.0979 & 0.0979 & 0.0340 & 0.0639 & 0.0639 & $187.8 \%$ & $187.8 \%$ \\
\hline 41 & SB3-41 & $\mathrm{TiO} 2(\mathrm{wt} \%)$ & 0.0046 & 0.0050 & 0.0000 & 0.0046 & 0.0050 & & \\
\hline 41 & SB3-41 & $\mathrm{U} 3 \mathrm{O} 8(\mathrm{wt} \%)$ & 2.3761 & 2.4862 & 2.4530 & -0.0769 & 0.0332 & $-3.1 \%$ & $1.4 \%$ \\
\hline 41 & SB3-41 & $\mathrm{ZnO}(\mathrm{wt} \%)$ & 0.0794 & 0.0794 & 0.0990 & -0.0196 & -0.0196 & $-19.8 \%$ & $-19.8 \%$ \\
\hline 41 & SB3-41 & $\mathrm{ZrO} 2(\mathrm{wt} \%)$ & 0.1614 & 0.1614 & 0.1790 & -0.0176 & -0.0176 & $-9.8 \%$ & $-9.8 \%$ \\
\hline 41 & SB3-41 & Sum of Oxides & 99.9822 & 102.3696 & 99.9990 & -0.0168 & 2.3706 & $0.0 \%$ & $2.4 \%$ \\
\hline 42 & SB3-42 & $\mathrm{Al} 2 \mathrm{O} 3(\mathrm{wt} \%)$ & 7.0573 & 7.2620 & 6.8740 & 0.1833 & 0.3880 & $2.7 \%$ & $5.6 \%$ \\
\hline 42 & SB3-42 & $\mathrm{B} 2 \mathrm{O} 3(\mathrm{wt} \%)$ & 4.7574 & 4.7125 & 4.7200 & 0.0374 & -0.0075 & $0.8 \%$ & $-0.2 \%$ \\
\hline 42 & SB3-42 & $\mathrm{BaO}(\mathrm{wt} \%)$ & 0.0740 & 0.0887 & 0.0950 & -0.0210 & -0.0063 & $-22.1 \%$ & $-6.6 \%$ \\
\hline 42 & SB3-42 & $\mathrm{CaO}(\mathrm{wt} \%)$ & 1.4992 & 1.3824 & 1.3670 & 0.1322 & 0.0154 & $9.7 \%$ & $1.1 \%$ \\
\hline 42 & SB3-42 & $\mathrm{Ce} 2 \mathrm{O} 3(\mathrm{wt} \%)$ & 0.1227 & 0.1227 & 0.1350 & -0.0123 & -0.0123 & $-9.1 \%$ & $-9.1 \%$ \\
\hline 42 & SB3-42 & $\mathrm{Cr} 2 \mathrm{O} 3(\mathrm{wt} \%)$ & 0.1323 & 0.1570 & 0.1420 & -0.0097 & 0.0150 & $-6.8 \%$ & $10.6 \%$ \\
\hline 42 & SB3-42 & $\mathrm{CuO}(\mathrm{wt} \%)$ & 0.0779 & 0.0832 & 0.0750 & 0.0029 & 0.0082 & $3.9 \%$ & $10.9 \%$ \\
\hline 42 & SB3-42 & $\mathrm{Fe} 2 \mathrm{O} 3(\mathrm{wt} \%)$ & 12.7279 & 12.3277 & 15.2920 & -2.5641 & -2.9643 & $-16.8 \%$ & $-19.4 \%$ \\
\hline 42 & SB3-42 & $\mathrm{K} 2 \mathrm{O}(\mathrm{wt} \%)$ & 0.1596 & 0.1420 & 0.1630 & -0.0034 & -0.0210 & $-2.1 \%$ & $-12.9 \%$ \\
\hline 42 & SB3-42 & $\mathrm{La} 2 \mathrm{O} 3(\mathrm{wt} \%)$ & 0.0598 & 0.0598 & 0.0780 & -0.0182 & -0.0182 & $-23.3 \%$ & $-23.3 \%$ \\
\hline 42 & SB3-42 & $\mathrm{Li} 2 \mathrm{O}(\mathrm{wt} \%)$ & 6.0873 & 6.2984 & 4.7210 & 1.3663 & 1.5774 & $28.9 \%$ & $33.4 \%$ \\
\hline
\end{tabular}


Table H.4: Average Measured and Bias-Corrected Chemical Compositions Versus Targeted Compositions by Oxide by Glass Number (continued)

(Batch 1: 100; 101-U std)

\begin{tabular}{|c|c|c|c|c|c|c|c|c|c|}
\hline Glass \# & Glass ID & Oxide & $\begin{array}{c}\text { Measured } \\
(\mathbf{w t} \%)\end{array}$ & $\begin{array}{c}\text { Measured } \\
\text { Bias-Corrected } \\
(w t \%)\end{array}$ & $\begin{array}{c}\text { Targeted } \\
(\text { wt } \%)\end{array}$ & $\begin{array}{c}\text { Diff of } \\
\text { Measured }\end{array}$ & $\begin{array}{c}\text { Diff of } \\
\text { Meas BC }\end{array}$ & $\begin{array}{l}\% \text { Diff of } \\
\text { Measured }\end{array}$ & $\begin{array}{l}\% \text { Diff of } \\
\text { Meas BC }\end{array}$ \\
\hline 42 & SB3-42 & $\mathrm{MgO}(\mathrm{wt} \%)$ & 0.0257 & 0.0279 & 0.0720 & -0.0463 & -0.0441 & $-64.3 \%$ & $-61.2 \%$ \\
\hline 42 & SB3-42 & $\mathrm{MnO}(\mathrm{wt} \%)$ & 2.4533 & 2.7139 & 2.7250 & -0.2717 & -0.0111 & $-10.0 \%$ & $-0.4 \%$ \\
\hline 42 & SB3-42 & $\mathrm{Na} 2 \mathrm{O}(\mathrm{wt} \%)$ & 15.3335 & 14.8531 & 14.7020 & 0.6315 & 0.1511 & $4.3 \%$ & $1.0 \%$ \\
\hline 42 & SB3-42 & $\mathrm{NiO}(\mathrm{wt} \%)$ & 0.5227 & 0.6096 & 0.6120 & -0.0893 & -0.0024 & $-14.6 \%$ & $-0.4 \%$ \\
\hline 42 & SB3-42 & $\mathrm{PbO}(\mathrm{wt} \%)$ & 0.0961 & 0.0961 & 0.1150 & -0.0189 & -0.0189 & $-16.4 \%$ & $-16.4 \%$ \\
\hline 42 & SB3-42 & $\mathrm{SiO} 2(\mathrm{wt} \%)$ & 44.1765 & 46.5069 & 43.7500 & 0.4265 & 2.7569 & $1.0 \%$ & $6.3 \%$ \\
\hline 42 & SB3-42 & ThO2 (wt $\%)$ & 0.1530 & 0.1530 & 0.0540 & 0.0990 & 0.0990 & $183.4 \%$ & $183.4 \%$ \\
\hline 42 & SB3-42 & $\mathrm{TiO} 2(\mathrm{wt} \%)$ & 0.0083 & 0.0092 & 0.0000 & 0.0083 & 0.0092 & & \\
\hline 42 & SB3-42 & $\mathrm{U} 3 \mathrm{O} 8(\mathrm{wt} \%)$ & 3.8029 & 3.9791 & 3.8690 & -0.0661 & 0.1101 & $-1.7 \%$ & $2.8 \%$ \\
\hline 42 & SB3-42 & $\mathrm{ZnO}(\mathrm{wt} \%)$ & 0.1207 & 0.1207 & 0.1560 & -0.0353 & -0.0353 & $-22.6 \%$ & $-22.6 \%$ \\
\hline 42 & SB3-42 & $\mathrm{ZrO} 2(\mathrm{wt} \%)$ & 0.2371 & 0.2371 & 0.2820 & -0.0449 & -0.0449 & $-15.9 \%$ & $-15.9 \%$ \\
\hline 42 & SB3-42 & Sum of Oxides & 99.6854 & 101.9434 & 99.9990 & -0.3136 & 1.9444 & $-0.3 \%$ & $1.9 \%$ \\
\hline 100 & Batch 1 & $\mathrm{Al} 2 \mathrm{O} 3(\mathrm{wt} \%)$ & 4.7720 & 4.8770 & 4.8770 & -0.1050 & 0.0000 & $-2.2 \%$ & $0.0 \%$ \\
\hline 100 & Batch 1 & $\mathrm{~B} 2 \mathrm{O} 3(\mathrm{wt} \%)$ & 7.9552 & 7.7770 & 7.7770 & 0.1782 & 0.0000 & $2.3 \%$ & $0.0 \%$ \\
\hline 100 & Batch 1 & $\mathrm{BaO}(\mathrm{wt} \%)$ & 0.1350 & 0.1510 & 0.1510 & -0.0160 & 0.0000 & $-10.6 \%$ & $0.0 \%$ \\
\hline 100 & Batch 1 & $\mathrm{CaO}(\mathrm{wt} \%)$ & 1.3071 & 1.2200 & 1.2200 & 0.0871 & 0.0000 & $7.1 \%$ & $0.0 \%$ \\
\hline 100 & Batch 1 & $\mathrm{Ce} 2 \mathrm{O} 3(\mathrm{wt} \%)$ & 0.0059 & 0.0059 & 0.0000 & 0.0059 & 0.0059 & & \\
\hline 100 & Batch 1 & $\mathrm{Cr} 2 \mathrm{O} 3(\mathrm{wt} \%)$ & 0.0983 & 0.1070 & 0.1070 & -0.0087 & 0.0000 & $-8.1 \%$ & $0.0 \%$ \\
\hline 100 & Batch 1 & $\mathrm{CuO}(\mathrm{wt} \%)$ & 0.3763 & 0.3990 & 0.3990 & -0.0227 & 0.0000 & $-5.7 \%$ & $0.0 \%$ \\
\hline 100 & Batch 1 & $\mathrm{Fe} 2 \mathrm{O} 3(\mathrm{wt} \%)$ & 12.9513 & 12.8390 & 12.8390 & 0.1123 & 0.0000 & $0.9 \%$ & $0.0 \%$ \\
\hline 100 & Batch 1 & $\mathrm{~K} 2 \mathrm{O}(\mathrm{wt} \%)$ & 3.7217 & 3.3270 & 3.3270 & 0.3947 & 0.0000 & $11.9 \%$ & $0.0 \%$ \\
\hline 100 & Batch 1 & $\mathrm{La} 2 \mathrm{O} 3(\mathrm{wt} \%)$ & 0.0059 & 0.0059 & 0.0000 & 0.0059 & 0.0059 & & \\
\hline 100 & Batch 1 & $\mathrm{Li} 2 \mathrm{O}(\mathrm{wt} \%)$ & 4.2757 & 4.4290 & 4.4290 & -0.1533 & 0.0000 & $-3.5 \%$ & $0.0 \%$ \\
\hline 100 & Batch 1 & $\mathrm{MgO}(\mathrm{wt} \%)$ & 1.3735 & 1.4190 & 1.4190 & -0.0455 & 0.0000 & $-3.2 \%$ & $0.0 \%$ \\
\hline 100 & Batch 1 & $\mathrm{MnO}(\mathrm{wt} \%)$ & 1.6560 & 1.7260 & 1.7260 & -0.0700 & 0.0000 & $-4.1 \%$ & $0.0 \%$ \\
\hline 100 & Batch 1 & $\mathrm{Na} 2 \mathrm{O}(\mathrm{wt} \%)$ & 9.4669 & 9.0030 & 9.0030 & 0.4639 & 0.0000 & $5.2 \%$ & $0.0 \%$ \\
\hline 100 & Batch 1 & $\mathrm{NiO}(\mathrm{wt} \%)$ & 0.6682 & 0.7510 & 0.7510 & -0.0828 & 0.0000 & $-11.0 \%$ & $0.0 \%$ \\
\hline 100 & Batch 1 & $\mathrm{PbO}(\mathrm{wt} \%)$ & 0.0054 & 0.0054 & 0.0000 & 0.0054 & 0.0054 & & \\
\hline 100 & Batch 1 & $\mathrm{SiO} 2(\mathrm{wt} \%)$ & 47.2902 & 50.2200 & 50.2200 & -2.9298 & 0.0000 & $-5.8 \%$ & $0.0 \%$ \\
\hline 100 & Batch 1 & ThO2 $(\mathrm{wt} \%)$ & 0.0057 & 0.0057 & 0.0000 & 0.0057 & 0.0057 & & \\
\hline 100 & Batch 1 & $\mathrm{TiO} 2$ (wt\%) & 0.6322 & 0.6770 & 0.6770 & -0.0448 & 0.0000 & $-6.6 \%$ & $0.0 \%$ \\
\hline 100 & Batch 1 & $\mathrm{U} 3 \mathrm{O} 8(\mathrm{wt} \%)$ & 0.0590 & 0.0617 & 0.0000 & 0.0590 & 0.0617 & & \\
\hline 100 & Batch 1 & $\mathrm{ZnO}(\mathrm{wt} \%)$ & 0.0062 & 0.0062 & 0.0000 & 0.0062 & 0.0062 & & \\
\hline 100 & Batch 1 & $\mathrm{ZrO} 2(\mathrm{wt} \%)$ & 0.0810 & 0.0810 & 0.0980 & -0.0170 & -0.0170 & $-17.3 \%$ & $-17.3 \%$ \\
\hline 100 & Batch 1 & Sum of Oxides & 96.8484 & 99.0937 & 99.0200 & -2.1716 & 0.0737 & $-2.2 \%$ & $0.1 \%$ \\
\hline 101 & $\mathrm{U}$ std & $\mathrm{A} 12 \mathrm{O} 3$ (wt\%) & 3.7349 & 3.8195 & 4.1000 & -0.3651 & -0.2805 & $-8.9 \%$ & $-6.8 \%$ \\
\hline 101 & $\mathrm{U}$ std & $\mathrm{B} 2 \mathrm{O} 3(\mathrm{wt} \%)$ & 8.8990 & 8.6996 & 9.2090 & -0.3100 & -0.5094 & $-3.4 \%$ & $-5.5 \%$ \\
\hline 101 & U std & $\mathrm{BaO}(\mathrm{wt} \%)$ & 0.0056 & 0.0063 & 0.0000 & 0.0056 & 0.0063 & & \\
\hline 101 & $\mathrm{U}$ std & $\mathrm{CaO}(\mathrm{wt} \%)$ & 1.3252 & 1.2370 & 1.3010 & 0.0242 & -0.0640 & $1.9 \%$ & $-4.9 \%$ \\
\hline 101 & U std & $\mathrm{Ce} 2 \mathrm{O} 3(\mathrm{wt} \%)$ & 0.0059 & 0.0059 & 0.0000 & 0.0059 & 0.0059 & & \\
\hline 101 & $\mathrm{U}$ std & $\mathrm{Cr} 2 \mathrm{O} 3(\mathrm{wt} \%)$ & 0.2302 & 0.2508 & 0.0000 & 0.2302 & 0.2508 & & \\
\hline 101 & U std & $\mathrm{CuO}(\mathrm{wt} \%)$ & 0.0068 & 0.0072 & 0.0000 & 0.0068 & 0.0072 & & \\
\hline 101 & $\mathrm{U}$ std & $\mathrm{Fe} 2 \mathrm{O} 3(\mathrm{wt} \%)$ & 13.1747 & 13.0616 & 13.1960 & -0.0213 & -0.1344 & $-0.2 \%$ & $-1.0 \%$ \\
\hline 101 & $\mathrm{U}$ std & $\mathrm{K} 2 \mathrm{O}(\mathrm{wt} \%)$ & 3.0486 & 2.7253 & 2.9990 & 0.0496 & -0.2737 & $1.7 \%$ & $-9.1 \%$ \\
\hline 101 & U std & $\mathrm{La} 2 \mathrm{O} 3(\mathrm{wt} \%)$ & 0.0059 & 0.0059 & 0.0000 & 0.0059 & 0.0059 & & \\
\hline 101 & $\mathrm{U}$ std & $\mathrm{Li} 2 \mathrm{O}(\mathrm{wt} \%)$ & 2.7548 & 2.8536 & 3.0570 & -0.3022 & -0.2034 & $-9.9 \%$ & $-6.7 \%$ \\
\hline 101 & $\mathrm{U}$ std & $\mathrm{MgO}(\mathrm{wt} \%)$ & 1.1488 & 1.1869 & 1.2100 & -0.0612 & -0.0231 & $-5.1 \%$ & $-1.9 \%$ \\
\hline 101 & U std & $\mathrm{MnO}(\mathrm{wt} \%)$ & 2.5840 & 2.6992 & 2.8920 & -0.3080 & -0.1928 & $-10.6 \%$ & $-6.7 \%$ \\
\hline 101 & $\mathrm{U}$ std & $\mathrm{Na} 2 \mathrm{O}(\mathrm{wt} \%)$ & 11.7169 & 11.1410 & 11.7950 & -0.0781 & -0.6540 & $-0.7 \%$ & $-5.5 \%$ \\
\hline 101 & U std & $\mathrm{NiO}(\mathrm{wt} \%)$ & 0.9689 & 1.0892 & 1.1200 & -0.1511 & -0.0308 & $-13.5 \%$ & $-2.8 \%$ \\
\hline 101 & $\mathrm{U}$ std & $\mathrm{PbO}(\mathrm{wt} \%)$ & 0.0054 & 0.0054 & 0.0000 & 0.0054 & 0.0054 & & \\
\hline 101 & U std & $\mathrm{SiO} 2(\mathrm{wt} \%)$ & 47.5727 & 50.5260 & 45.3530 & 2.2197 & 5.1730 & $4.9 \%$ & $11.4 \%$ \\
\hline 101 & $\mathrm{U}$ std & ThO2 $(\mathrm{wt} \%)$ & 0.0683 & 0.0683 & 0.0000 & 0.0683 & 0.0683 & & \\
\hline
\end{tabular}


Immobilization Technology Section

WSRC-TR-2002-00549

Savannah River Technology Center

Rev. 0

Westinghouse Savannah River Company

Table H.4: Average Measured and Bias-Corrected Chemical Compositions Versus Targeted Compositions by Oxide by Glass Number (continued)

(Batch 1: 100; 101-U std)

\begin{tabular}{|c|c|c|c|c|c|c|c|c|c|}
\hline Glass \# & Glass ID & Oxide & $\begin{array}{c}\text { Measured } \\
(\mathrm{wt} \%)\end{array}$ & $\begin{array}{c}\text { Measured } \\
\text { Bias-Corrected } \\
(\mathrm{wt} \%)\end{array}$ & $\begin{array}{c}\text { Targeted } \\
\text { (wt\%) }\end{array}$ & $\begin{array}{c}\text { Diff of } \\
\text { Measured }\end{array}$ & $\begin{array}{c}\text { Diff of } \\
\text { Meas BC }\end{array}$ & $\begin{array}{l}\% \text { Diff of } \\
\text { Measured }\end{array}$ & $\begin{array}{l}\% \text { Diff of } \\
\text { Meas BC }\end{array}$ \\
\hline 101 & $\bar{U}$ std & $\mathrm{TiO} 2(\mathrm{wt} \%)$ & 0.9059 & 0.9702 & 1.0490 & -0.1431 & -0.0788 & $-13.6 \%$ & $-7.5 \%$ \\
\hline 101 & U std & U3O8 (wt\%) & 2.3004 & 2.4060 & 2.4060 & -0.1056 & 0.0000 & $-4.4 \%$ & $0.0 \%$ \\
\hline 101 & $\mathrm{U}$ std & $\mathrm{ZnO}(\mathrm{wt} \%)$ & 0.0062 & 0.0062 & 0.0000 & 0.0062 & 0.0062 & & \\
\hline 101 & U std & $\mathrm{ZrO} 2(\mathrm{wt} \%)$ & 0.0066 & 0.0066 & 0.0000 & 0.0066 & 0.0066 & & \\
\hline 101 & $\mathrm{U}$ std & Sum of Oxides & 100.4758 & 102.7777 & 99.6870 & 0.7888 & 3.0907 & $0.8 \%$ & $3.1 \%$ \\
\hline
\end{tabular}


Immobilization Technology Section

WSRC-TR-2002-00549

Savannah River Technology Center

Rev. 0

Westinghouse Savannah River Company

\section{Exhibit H.1: SRTC-ML Measurements in Analytical Sequence for Samples Prepared Using the LM Method}

$\mathrm{BaO}(\mathrm{wt} \%)$ By Analytical Sequence

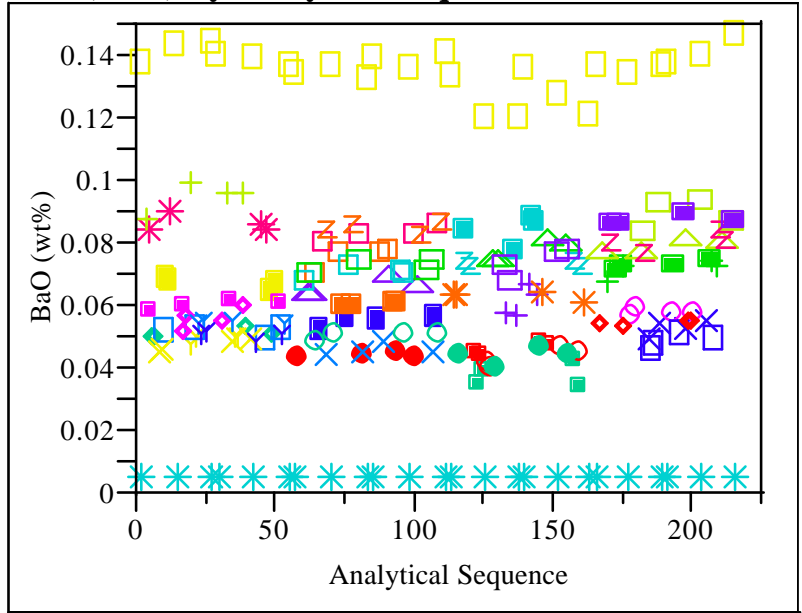

$\mathrm{CaO}(w \mathrm{t} \%)$ By Analytical Sequence

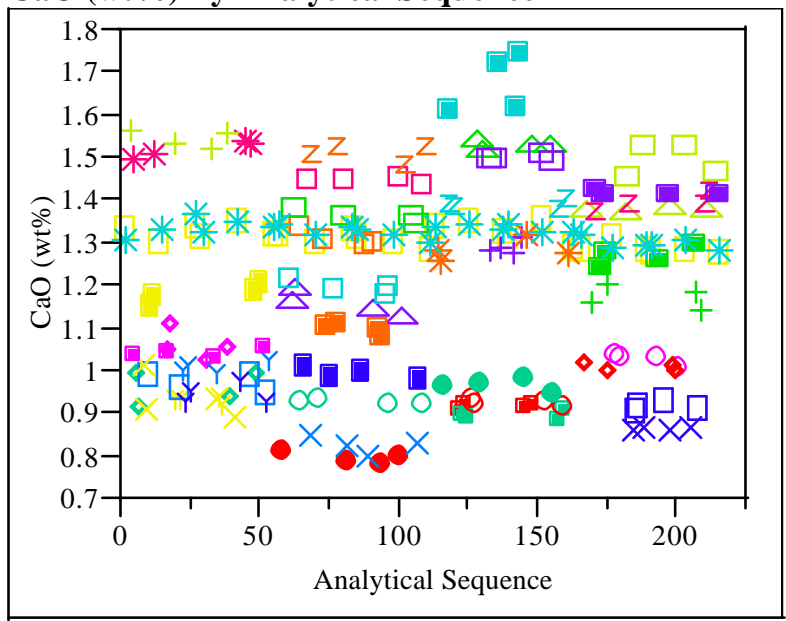

Ce2O3 (wt\%) By Analytical Sequence

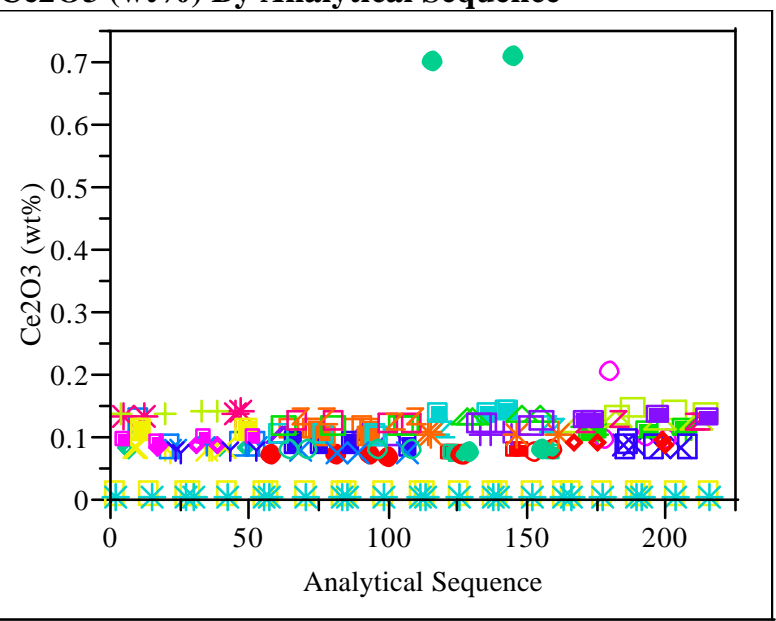

Cr2O3 (wt\%) By Analytical Sequence

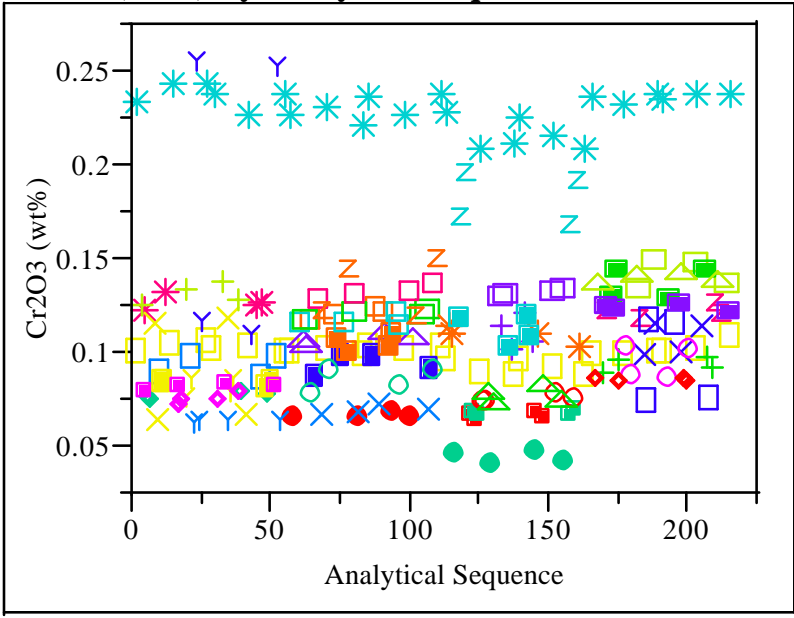

$\mathrm{CuO}(\mathrm{wt} \%)$ By Analytical Sequence

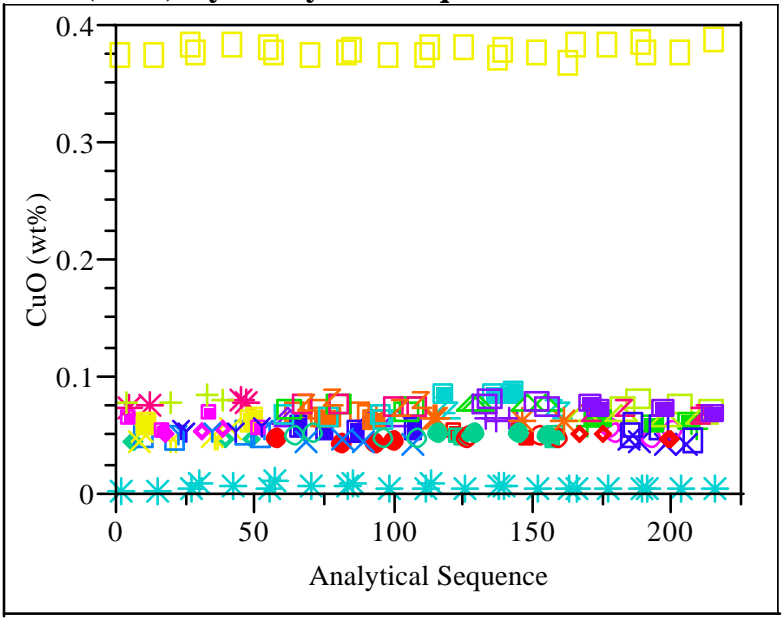

Fe2O3 (wt\%) By Analytical Sequence

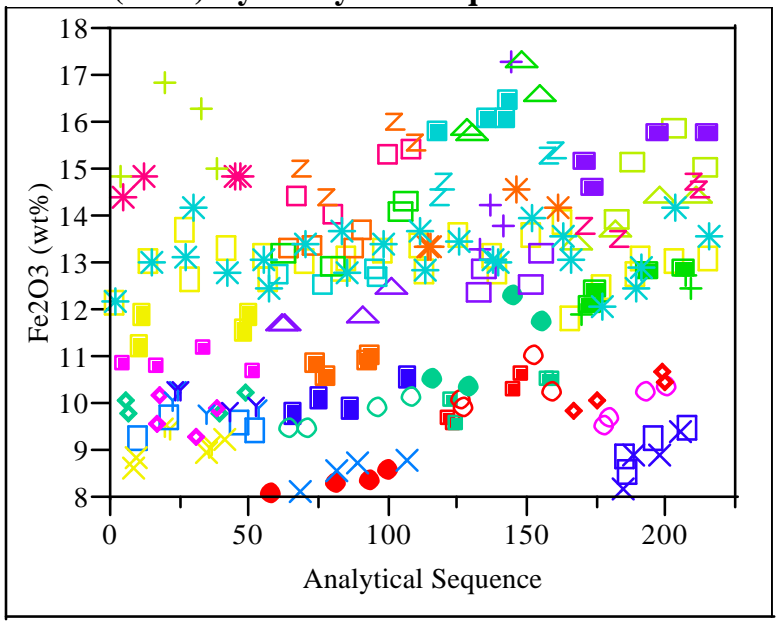


Immobilization Technology Section

WSRC-TR-2002-00549

Savannah River Technology Center

Rev. 0

Westinghouse Savannah River Company

\section{Exhibit H.1: SRTC-ML Measurements in Analytical Sequence for Samples Prepared Using the LM Method (continued)}

K2O (wt\%) By Analytical Sequence

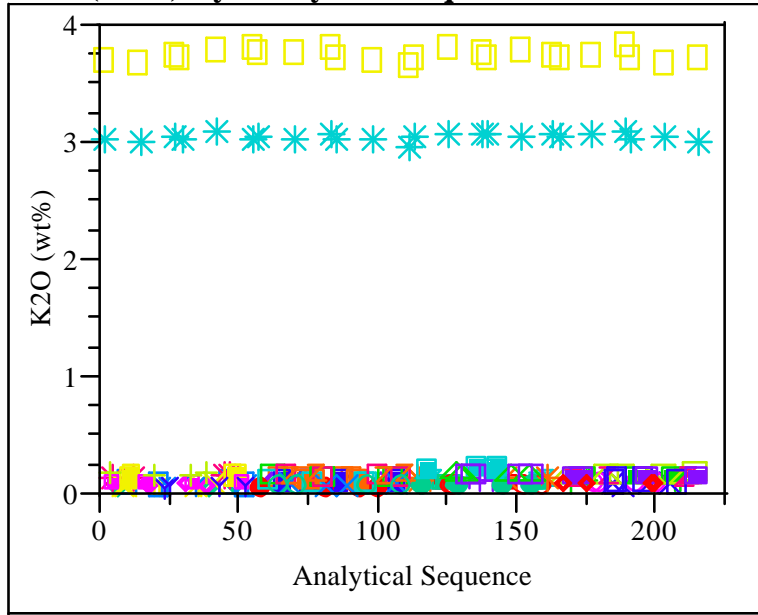

La2O3 (wt\%) By Analytical Sequence

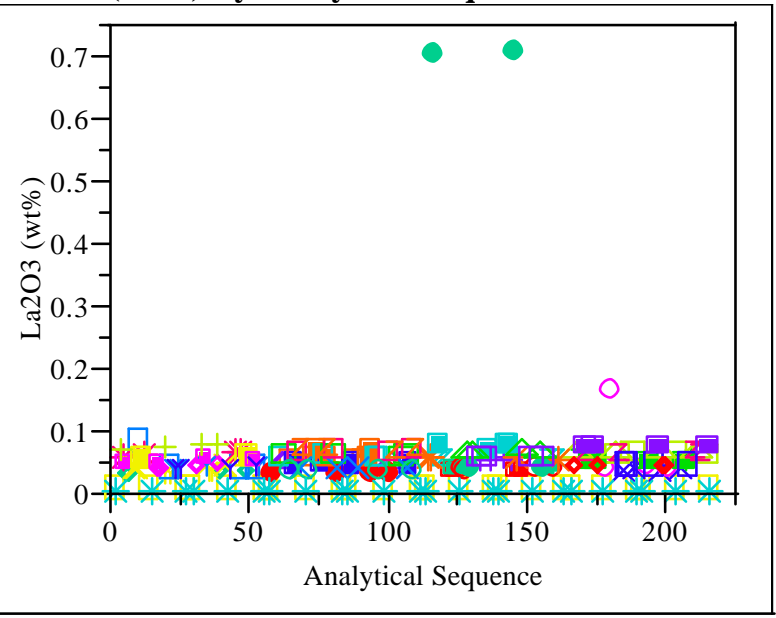

$\mathrm{MgO}(\mathrm{wt} \%)$ By Analytical Sequence

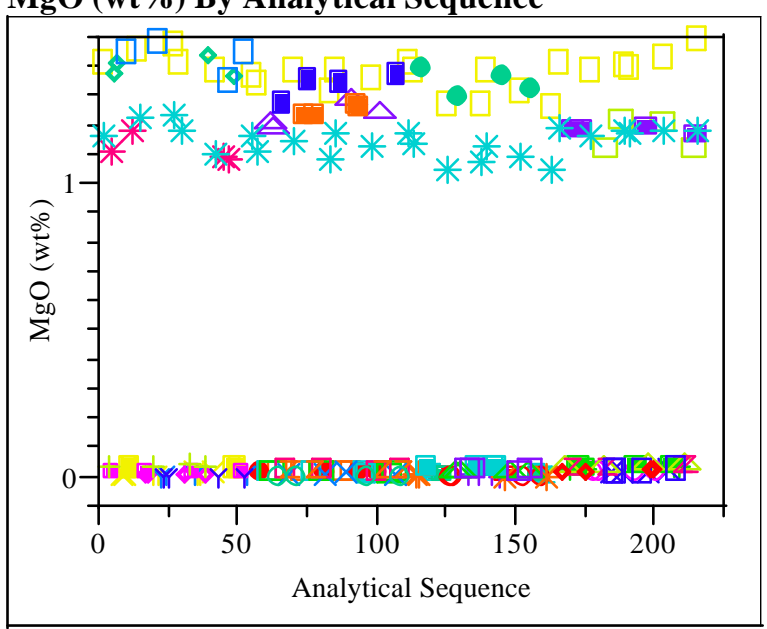

MnO (wt \%) By Analytical Sequence
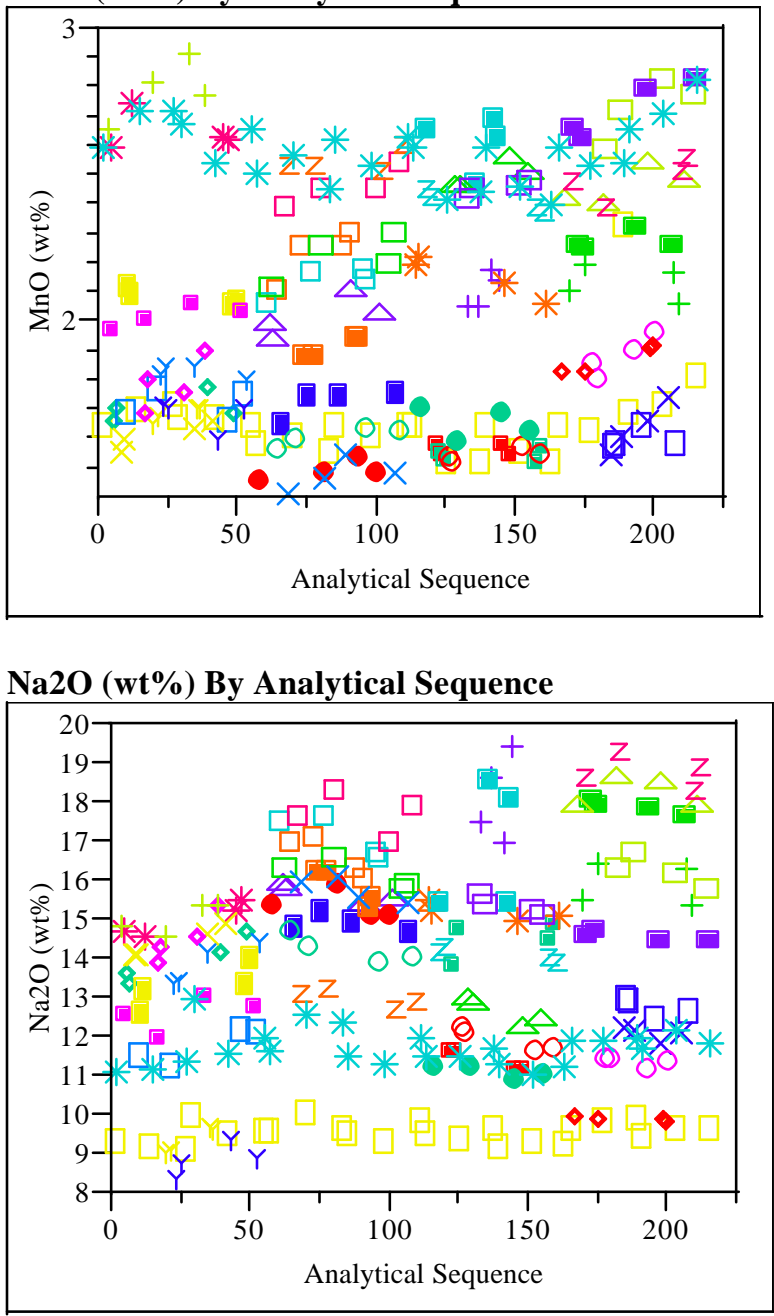

$\mathrm{NiO}(w \mathrm{t} \%)$ By Analytical Sequence

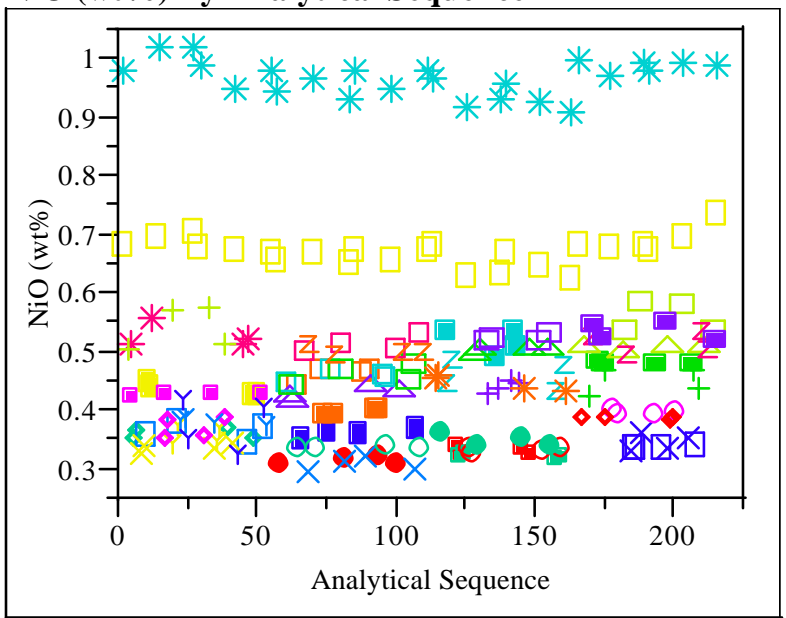




\section{Exhibit H.1: SRTC-ML Measurements in Analytical Sequence for Samples Prepared Using the LM Method (continued)}

PbO (wt \%) By Analytical Sequence

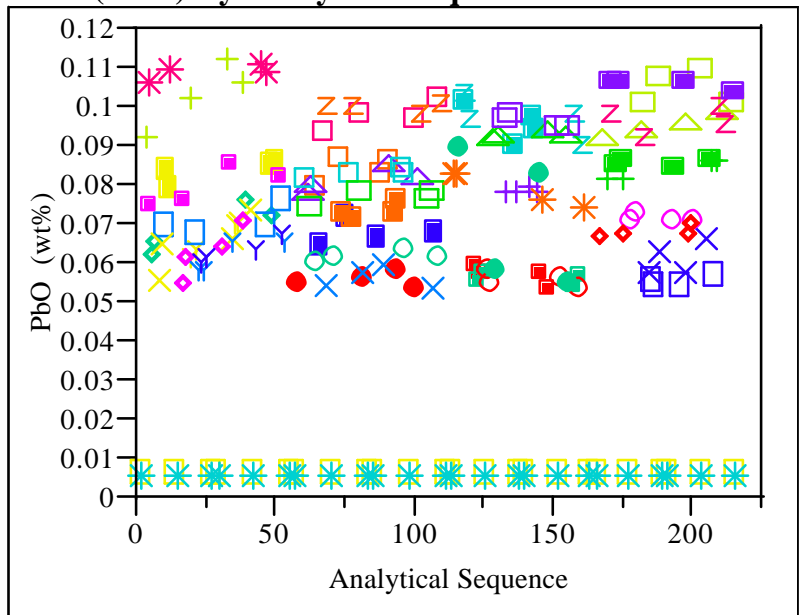

ThO2 (wt\%) By Analytical Sequence

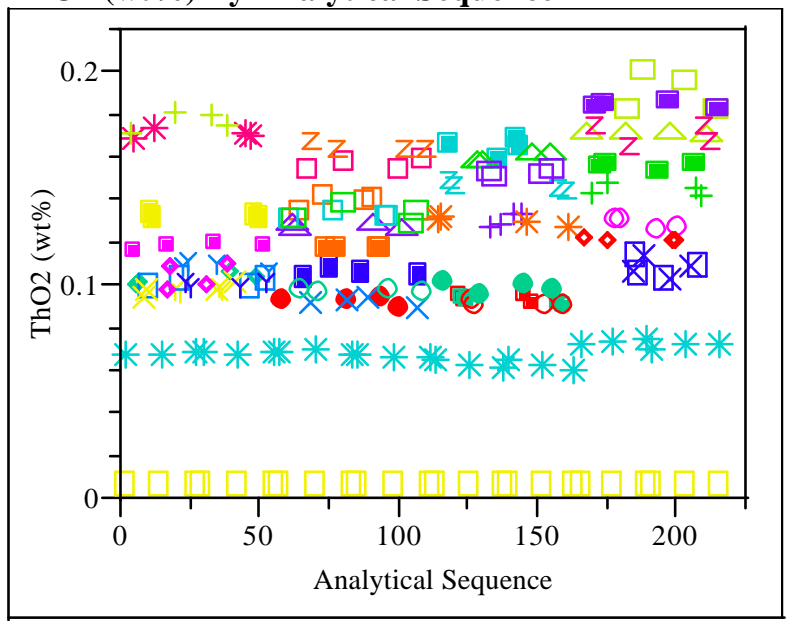

TiO2 (wt \%) By Analytical Sequence

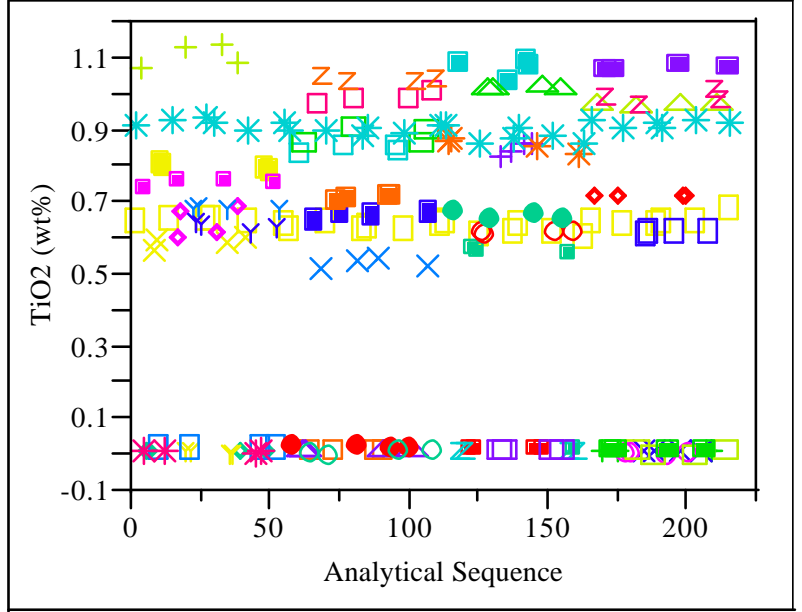

U3O8 (wt \%) By Analytical Sequence

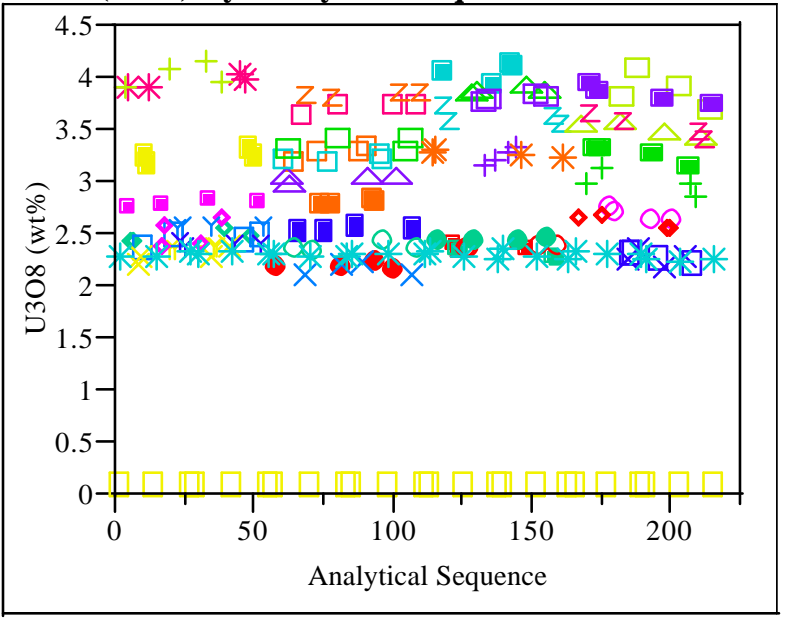

\section{ZnO (wt\%) By Analytical Sequence}

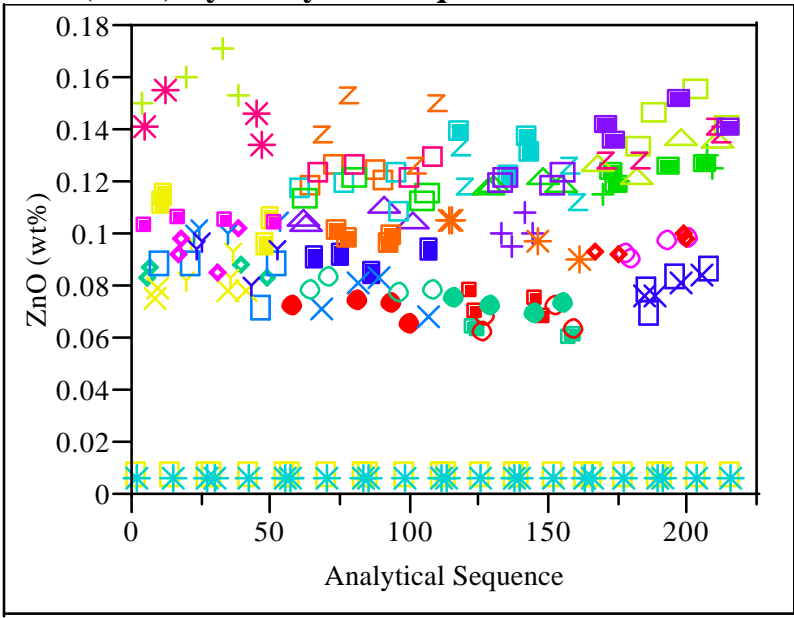

ZrO2 (wt \%) By Analytical Sequence

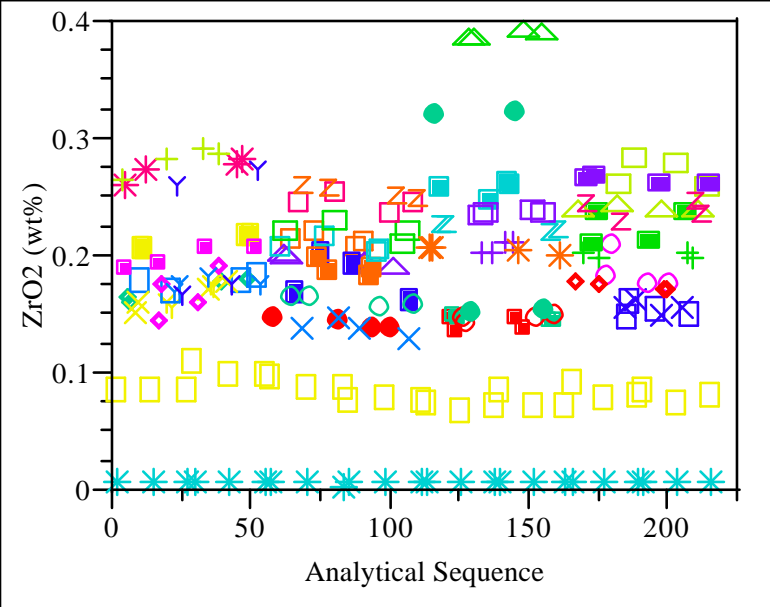


Immobilization Technology Section

WSRC-TR-2002-00549

Savannah River Technology Center

Rev. 0

Westinghouse Savannah River Company

\section{Exhibit H.2: SRTC-ML Measurements in Analytical Sequence for Samples Prepared Using the PF Method}

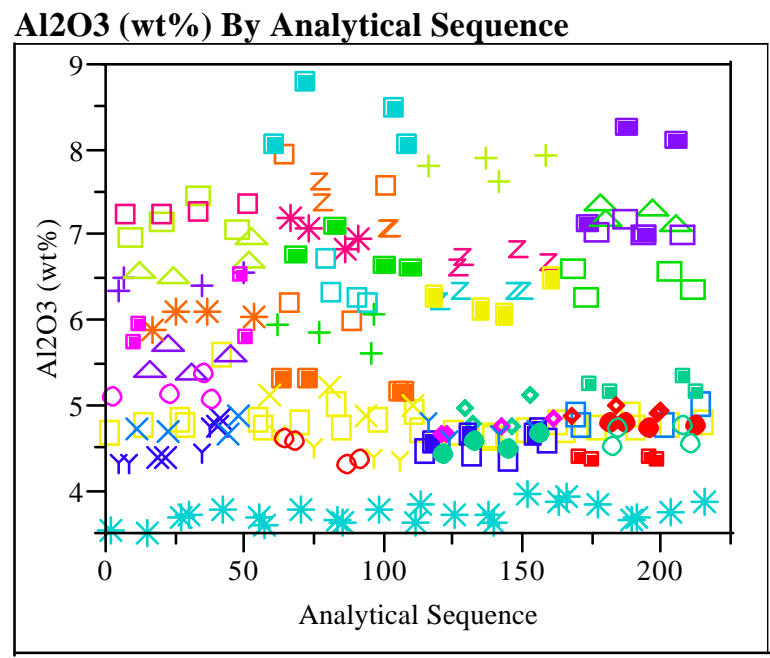

Li2O (wt\%) By Analytical Sequence

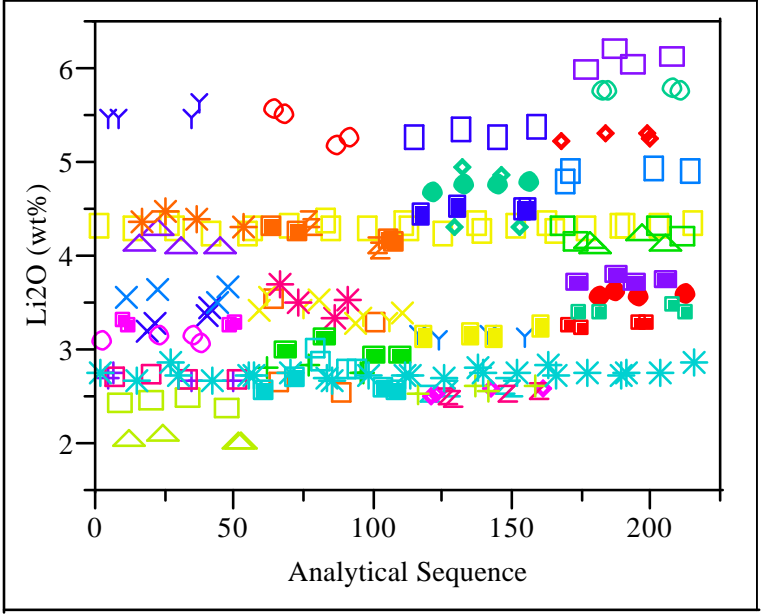

B2O3 (wt \%) By Analytical Sequence

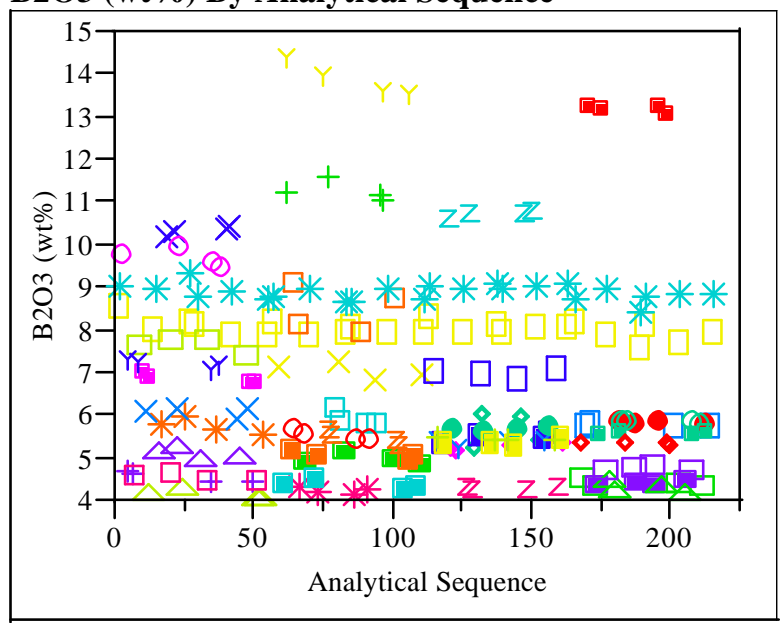

$\mathrm{SiO2}(w \mathrm{t} \%)$ By Analytical Sequence

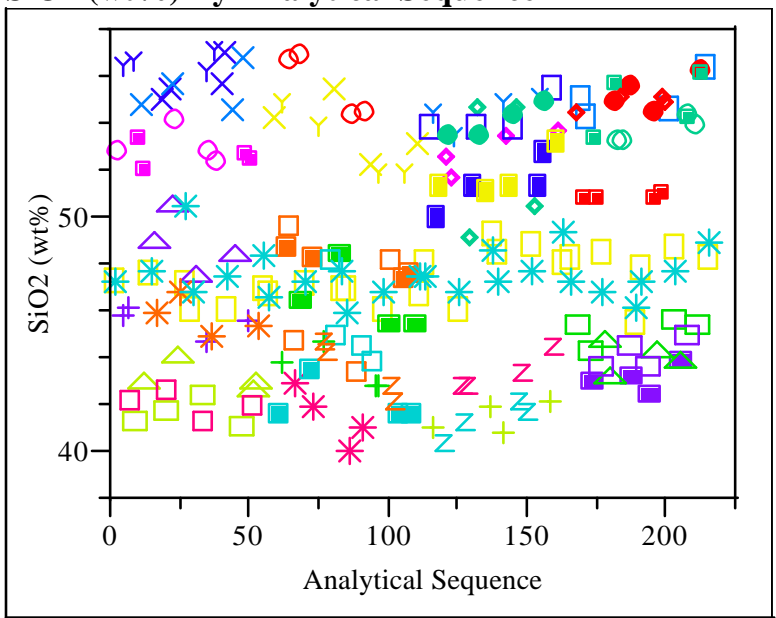


Westinghouse Savannah River Company

Exhibit H.4: Statistical Analysis by Analytical Block of Measurements of Standard Glasses Prepared Using the PF Method

SB3 Glass \#=100; Reference Value for $\mathrm{BaO}$ is $0.151 \mathrm{wt} \%$

Oneway Analysis of BaO (wt\%) By Analytical Blks (LM)

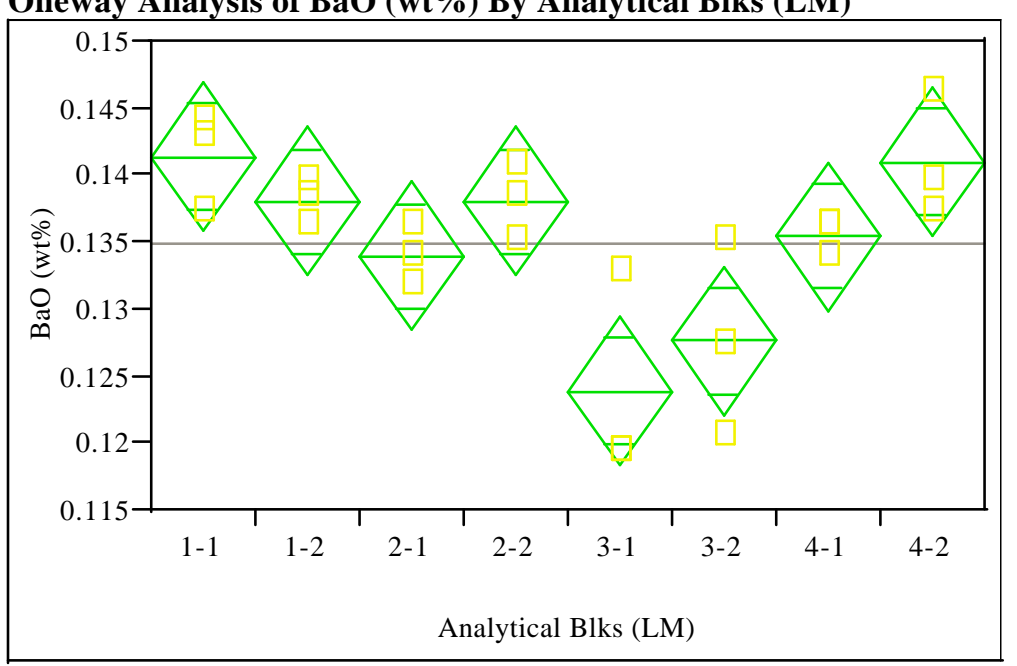

\section{Oneway Anova}

Root Mean Square Error

\subsection{9}

0.004535

Mean of Response

$$
\begin{array}{r}
0.134957 \\
24
\end{array}
$$

Analysis of Variance

Analysis of Variance

$\begin{array}{lrrrrr}\text { Source } & \text { DF } & \text { Sum of Squares } & \text { Mean Square } & \text { F Ratio } & \text { Prob > F } \\ \text { Analytical Blks (LM) } & 7 & 0.00082352 & 0.000118 & 5.7197 & 0.0019 \\ \text { Error } & 16 & 0.00032910 & 0.000021 & & \\ \text { C. Total } & 23 & 0.00115261 & & & \end{array}$

\section{Means for Oneway Anova}

$\begin{array}{lrrrrr}\text { Level } & \text { Number } & \text { Mean } & \text { Std Error } & \text { Lower 95\% } & \text { Upper 95\% } \\ 1-1 & 3 & 0.141423 & 0.00262 & 0.13587 & 0.14697 \\ 1-2 & 3 & 0.138074 & 0.00262 & 0.13252 & 0.14362 \\ 2-1 & 3 & 0.133980 & 0.00262 & 0.12843 & 0.13953 \\ 2-2 & 3 & 0.138074 & 0.00262 & 0.13252 & 0.14362 \\ 3-1 & 3 & 0.123932 & 0.00262 & 0.11838 & 0.12948 \\ 3-2 & 3 & 0.127653 & 0.00262 & 0.12210 & 0.13320 \\ 4-1 & 3 & 0.135469 & 0.00262 & 0.12992 & 0.14102 \\ 4-2 & 3 & 0.141051 & 0.00262 & 0.13550 & 0.14660\end{array}$

Std Error uses a pooled estimate of error variance
SB3 Glass \#=100; Reference Value for $\mathrm{CaO}$ is $1.22 \mathrm{wt} \%$

Oneway Analysis of CaO (wt\%) By Analytical Blks (LM)

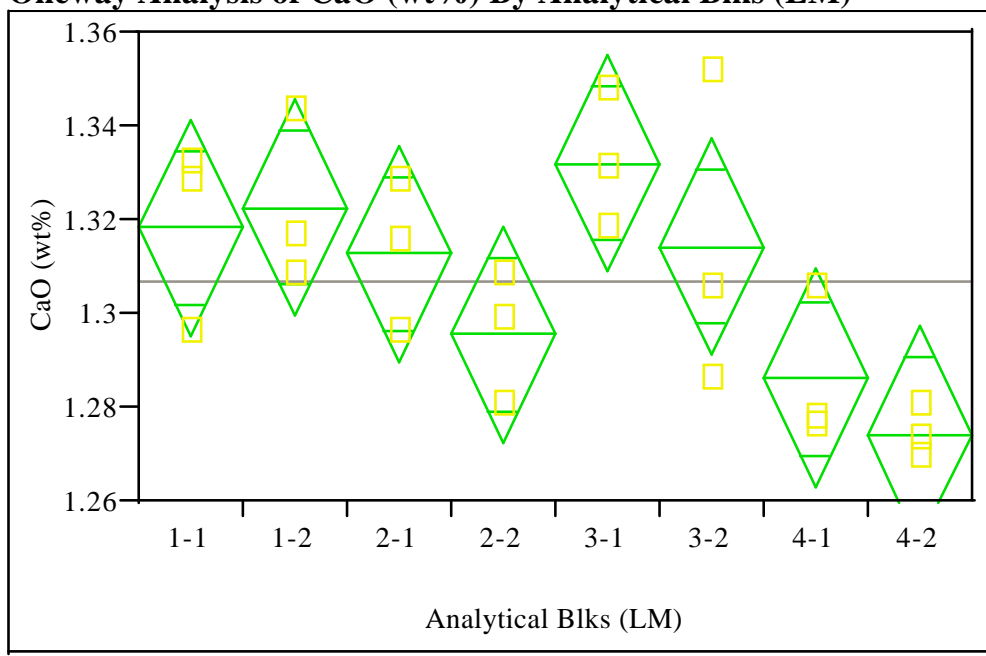

\section{Oneway Anova}

Rsquare

Root Mean Square Error

(1)

Mean of Response 0.018902
1.307086

(or Sum Wgts)

Analysis of Variance

Source DF Sum of Squares Mean Square F Ratio Prob $>$ F

$\begin{array}{lrrrrr}\text { Analytical Blks (LM) } & 7 & 0.00817822 & 0.001168 & 3.2699 & 0.0235\end{array}$

Error $\begin{array}{lll}0.001168 & 3.2699 & 0.0235 \\ 0.000357 & & \end{array}$

C. Total

$23 \quad 0.01389488$

\section{Means for Oneway Anova}

Level Number Mean Std Error Lower 95\% Upper 95\%

$\begin{array}{rrrrrr}1-1 & 3 & 1.31851 & 0.01091 & 1.2954 & 1.3416\end{array}$

$\begin{array}{llllll}1-2 & 3 & 1.32271 & 0.01091 & 1.2996 & 1.3458\end{array}$

$\begin{array}{llllll}2-1 & 3 & 1.31292 & 0.01091 & 1.2898 & 1.3361\end{array}$

$\begin{array}{llllll}2-2 & 3 & 1.29566 & 0.01091 & 1.2725 & 1.3188 \\ 3-1 & 3 & 1.33204 & 0.01091 & 1.3089 & 1.3552\end{array}$

$\begin{array}{llllll}3-1 & 3 & 1.33204 & 0.01091 & 1.3089 & 1.3552 \\ 3-2 & 3 & 1.31432 & 0.01091 & 1.2912 & 1.3375\end{array}$

$\begin{array}{llllll}3-2 & 3 & 1.31432 & 0.01091 & 1.2912 & 1.3375 \\ 4-1 & 3 & 1.28633 & 0.01091 & 1.2632 & 1.3095\end{array}$

$\begin{array}{llllll}4-2 & 3 & 1.27420 & 0.01091 & 1.2511 & 1.2973\end{array}$

Std Error uses a pooled estimate of error variance 
Westinghouse Savannah River Company

Exhibit H.4: Statistical Analysis by Analytical Block of Measurements of Standard Glasses Prepared Using the PF Method (continued)

SB3 Glass \#=100; Reference Value for Ce2O3 is 0 wt\%

Oneway Analysis of Ce2O3 (wt\%) By Analytical Blks (LM)

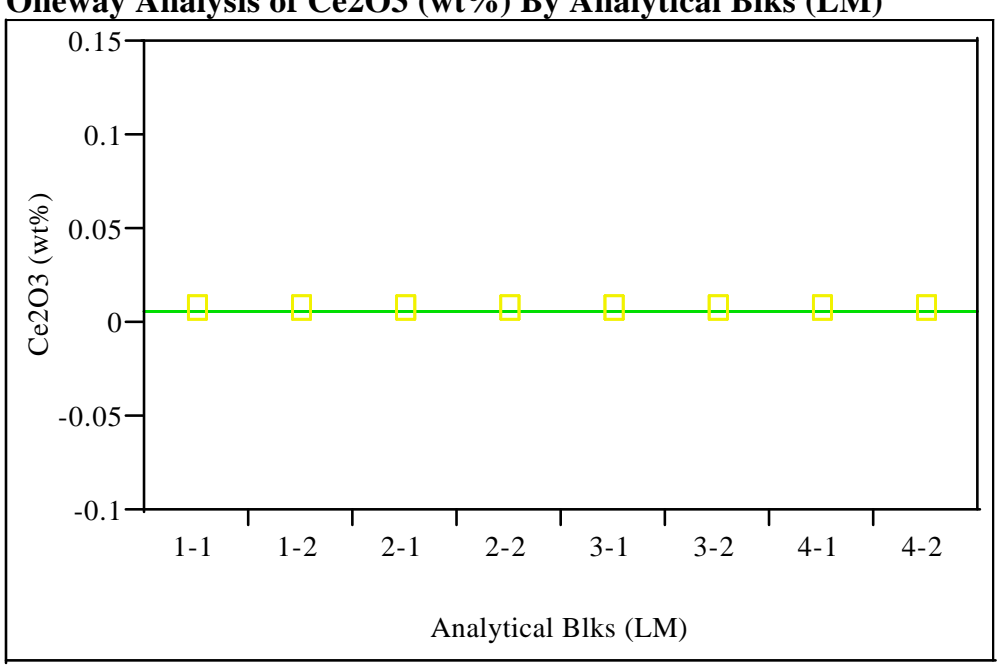

\section{Oneway Anova}

Rsquare

Root Mean Square Error

Mean of Response

Observations (or Sum Wgts)

24

Analysis of Variance

Source DF Sum of Squares Mean Square F Ratio Prob $>$ F

$\begin{array}{rrrrr}7 & \text { Sum of Squares } & \text { Mean Square } & \text { F Ratio } & \text { Prob }>\text { F } \\ 0 & 0 & . & \\ 0 & 0 & & \end{array}$

Error

C. Total

23

Means for Oneway Anova

$\begin{array}{lrrrrr}\text { Level } & \text { Number } & \text { Mean } & \text { Std Error } & \text { Lower 95\% } & \text { Upper 95\% } \\ 1-1 & 3 & 0.005857 & 0 & 0.00586 & 0.00586 \\ 1-2 & 3 & 0.005857 & 0 & 0.00586 & 0.00586 \\ 2-1 & 3 & 0.005857 & 0 & 0.00586 & 0.00586 \\ 2-2 & 3 & 0.005857 & 0 & 0.00586 & 0.00586 \\ 3-1 & 3 & 0.005857 & 0 & 0.00586 & 0.00586 \\ 3-2 & 3 & 0.005857 & 0 & 0.00586 & 0.00586 \\ 4-1 & 3 & 0.005857 & 0 & 0.00586 & 0.00586 \\ 4-2 & 3 & 0.005857 & 0 & 0.00586 & 0.00586\end{array}$

Std Error uses a pooled estimate of error variance
SB3 Glass \#=100; Reference Value for $\mathrm{Cr} 2 \mathrm{O3}$ is $0.107 \mathrm{wt} \%$

Oneway Analysis of Cr2O3 (wt\%) By Analytical Blks (LM)

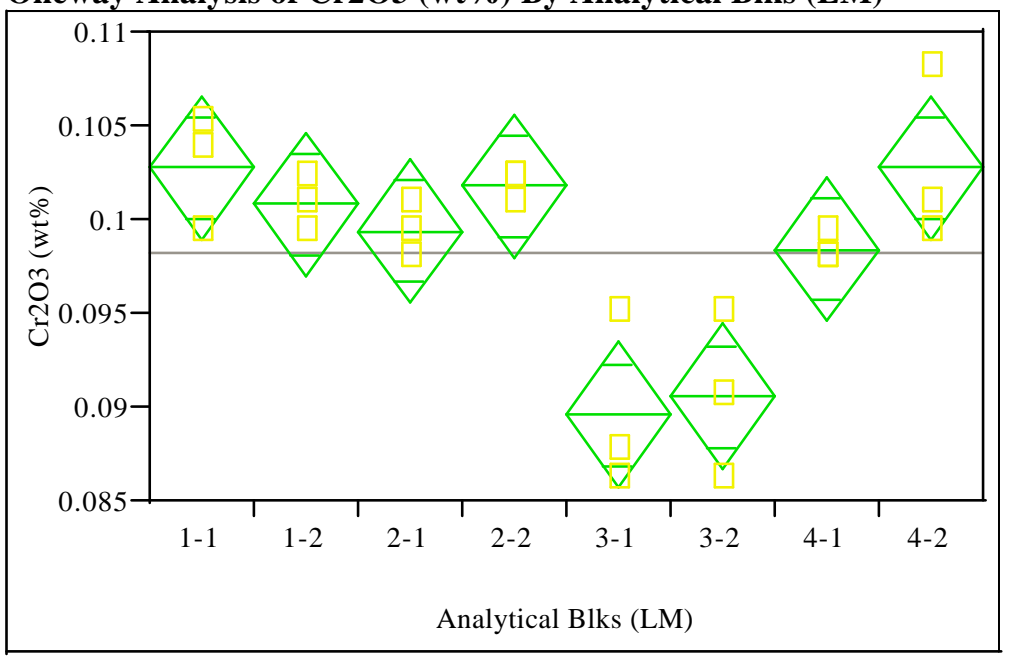

\section{Oneway Anova}

Rsquare

Root Mean Square Error

Mean of Response

0.78836

0.003129

0.098293

Observations (or Sum Wgts)

Analysis of Variance

Source DF Sum of Squares Mean Square F Ratio Prob $>$ F

$\begin{array}{lrrrrr}\text { Analytical Blks (LM) } & 7 & 0.00058356 & 0.000083 & 8.5143 & 0.0002\end{array}$

0.000010

C. Total

$23 \quad 0.00074022$

Means for Oneway Anova

Level Number Mean Std Error Lower 95\% Upper 95\%

$\begin{array}{rrrrrr}1-1 & 3 & 0.102799 & 0.00181 & 0.09897 & 0.10663\end{array}$

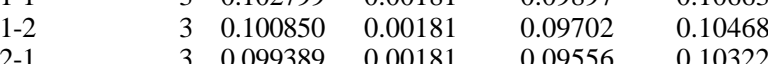

$\begin{array}{llllll}2-1 & 3 & 0.099389 & 0.00181 & 0.09556 & 0.10322\end{array}$

$\begin{array}{llllll}2-2 & 3 & 0.101825 & 0.00181 & 0.09800 & 0.10565\end{array}$

$\begin{array}{llllll}3-1 & 3 & 0.089645 & 0.00181 & 0.08582 & 0.09347\end{array}$

$\begin{array}{llllll}3-2 & 3 & 0.090619 & 0.00181 & 0.08679 & 0.09445 \\ 4-1 & 3 & 0.098414 & 0.00181 & 0.09458 & 0.10224\end{array}$

$\begin{array}{llllll}4-1 & 3 & 0.098414 & 0.00181 & 0.09458 & 0.10224 \\ 4-2 & 3 & 0.102799 & 0.00181 & 0.09897 & 0.10663\end{array}$

Std Error uses a pooled estimate of error variance 
Westinghouse Savannah River Company

\section{Exhibit H.4: Statistical Analysis by Analytical Block of Measurements of Standard Glasses Prepared Using the PF Method (continued)}

SB3 Glass \#=100; Reference Value for $\mathrm{CuO}$ is 0.399 wt \%

Oneway Analysis of $\mathrm{CuO}$ (wt\%) By Analytical Blks (LM)

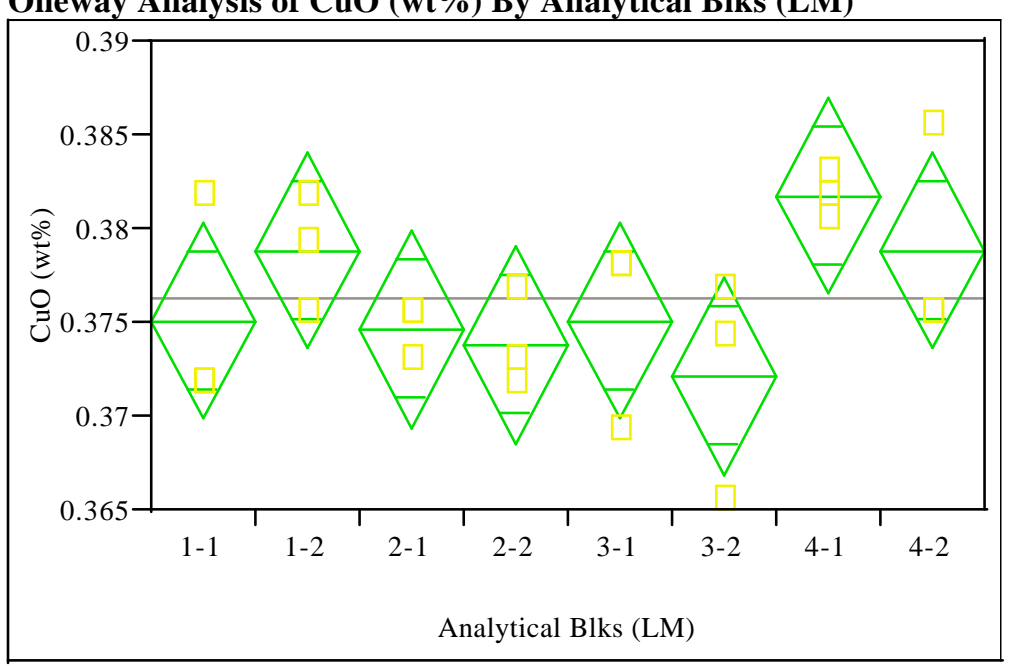

\section{Oneway Anova}

Rsquare

Root Mean Square Erro

0.420601

0.004299

Mean of Response

$$
\begin{array}{r}
0.376322 \\
24
\end{array}
$$

Analysis of Variance

Analysis of Variance

$\begin{array}{lrrrrr}\text { Source } & \text { DF } & \text { Sum of Squares } & \text { Mean Square } & \text { F Ratio } & \text { Prob > F } \\ \text { Analytical Blks (LM) } & 7 & 0.00021461 & 0.000031 & 1.6593 & 0.1898 \\ \text { Error } & 16 & 0.00029564 & 0.000018 & & \\ \text { C. Total } & 23 & 0.00051026 & & & \end{array}$

\section{Means for Oneway Anova}

Level Number Mean Std Error Lower 95\% Upper 95\%

$\begin{array}{llllrr}1-1 & 3 & 0.375123 & 0.00248 & 0.36986 & 0.38038\end{array}$

$\begin{array}{llllll}1-2 & 3 & 0.378878 & 0.00248 & 0.37362 & 0.38414\end{array}$

$\begin{array}{llllll}2-1 & 3 & 0.374705 & 0.00248 & 0.36944 & 0.37997\end{array}$

$\begin{array}{llllll}2-2 & 3 & 0.373871 & 0.00248 & 0.36861 & 0.37913 \\ 3-1 & 3 & 0.375123 & 0.00248 & 0.36986 & 0.38038\end{array}$

$\begin{array}{llllll}3-1 & 3 & 0.375123 & 0.00248 & 0.36986 & 0.38038 \\ 3-2 & 3 & 0.372202 & 0.00248 & 0.36694 & 0.37746\end{array}$

$\begin{array}{llllll}3-2 & 3 & 0.372202 & 0.00248 & 0.36694 & 0.37746 \\ 4-1 & 3 & 0.381799 & 0.00248 & 0.37654 & 0.38706\end{array}$

$\begin{array}{llllll}4-2 & 3 & 0.378878 & 0.00248 & 0.37362 & 0.38414\end{array}$

Std Error uses a pooled estimate of error variance
SB3 Glass \#=100; Reference Value for Fe2O3 is $12.839 \mathrm{wt} \%$

Oneway Analysis of Fe2O3 (wt\%) By Analytical Blks (LM)

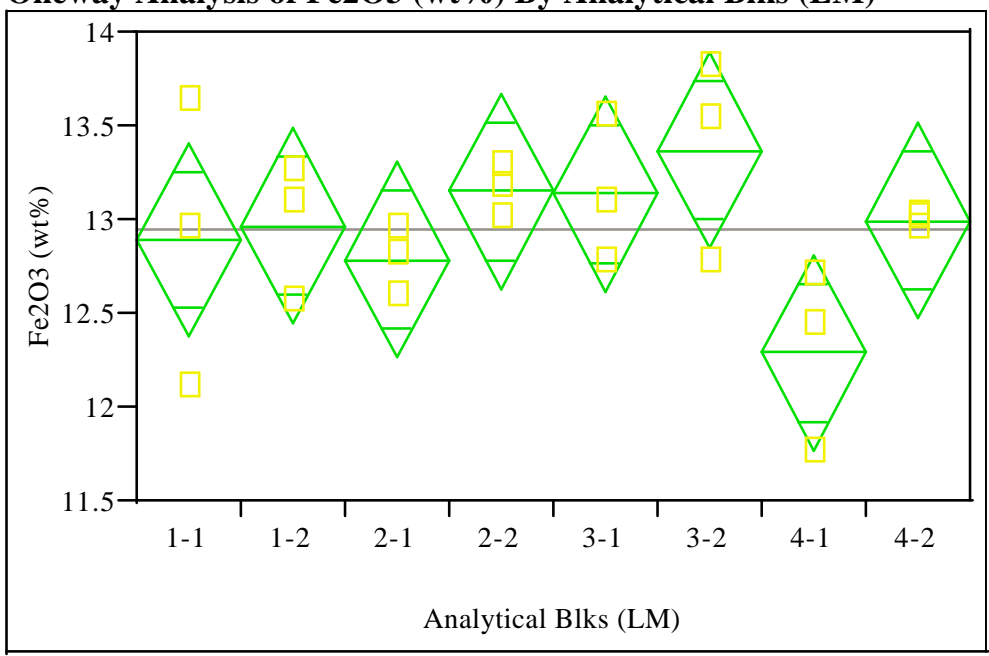

\section{Oneway Anova}

\section{Rsquare}

Root Mean Square Error

Mean of Response

0.42505
12.95129

(or Sum Wgts)

Analysis of Variance

Source DF Sum of Squares Mean Square F Ratio Prob > F

$\begin{array}{lrrrrr}\text { Analytical Blks (LM) } & 7 & 2.1488248 & 0.306975 & 1.6991 & 0.1796\end{array}$

Error

2.1488248
2.8906843

0.180668

$23 \quad 5.0395091$

\section{Means for Oneway Anova}

Level Number Mean Std Error Lower 95\% Upper 95\%

$\begin{array}{lllrrr}1-1 & 3 & 12.8959 & 0.24540 & 12.376 & 13.416\end{array}$

$\begin{array}{llllll}1-2 & 3 & 12.9721 & 0.24540 & 12.452 & 13.492 \\ 2-1 & 3 & 12.7863 & 0.24540 & 12.266 & 13.307\end{array}$

$\begin{array}{llllll}2-1 & 3 & 12.7863 & 0.24540 & 12.266 & 13.307\end{array}$

$\begin{array}{llllll}2-2 & 3 & 13.1532 & 0.24540 & 12.633 & 13.673 \\ 3-1 & 3 & 13.1389 & 0.24540 & 12.619 & 13.659\end{array}$

$\begin{array}{llllll}3-1 & 3 & 13.1389 & 0.24540 & 12.619 & 13.659 \\ 3-2 & 3 & 13.3725 & 0.24540 & 12.852 & 13.893\end{array}$

$\begin{array}{llllll}3-2 & 3 & 13.3725 & 0.24540 & 12.852 & 13.893 \\ 4-1 & 3 & 12.2954 & 0.24540 & 11.775 & 12.816\end{array}$

$\begin{array}{llllll}4-1 & 3 & 12.2954 & 0.24540 & 11.775 & 12.816 \\ 4-2 & 3 & 12.9960 & 0.24540 & 12.476 & 13.516\end{array}$

Std Error uses a pooled estimate of error variance 
Westinghouse Savannah River Company

Exhibit H.4: Statistical Analysis by Analytical Block of Measurements of Standard Glasses Prepared Using the PF Method (continued)

SB3 Glass \#=100; Reference Value for K2) is $3.327 \mathrm{wt} \%$

Oneway Analysis of K2O (wt\%) By Analytical Blks (LM)

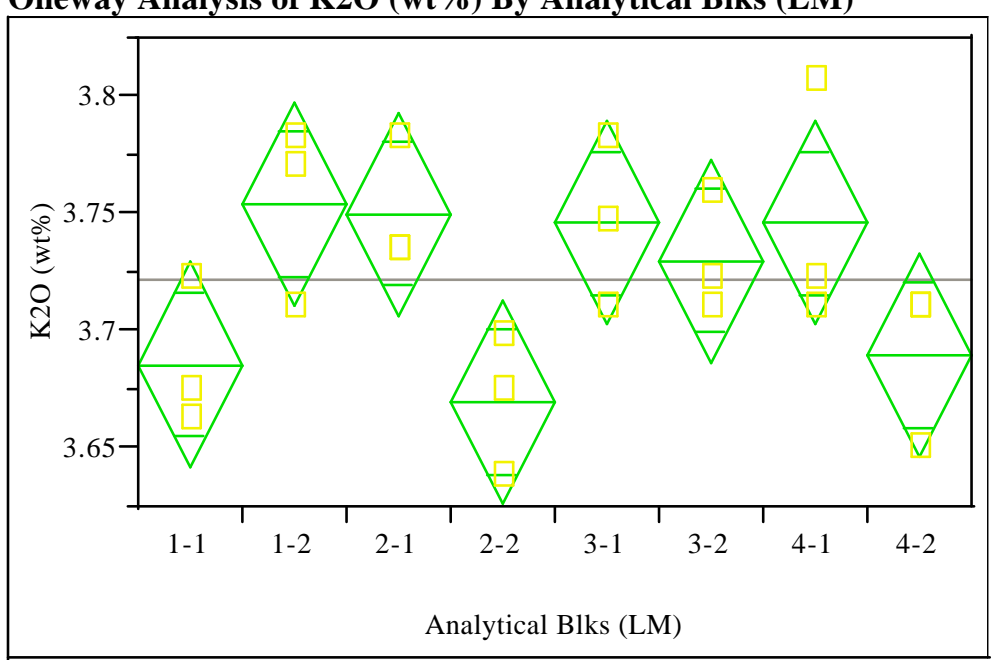

\section{Oneway Anova}

Root Mean Square Error $\quad 0.035548$

Mean of Response

$$
\begin{array}{r}
3.721712 \\
24
\end{array}
$$

Analysis of Variance

Analysis of Variance

$\begin{array}{lrrrrr}\text { Source } & \text { DF } & \text { Sum of Squares } & \text { Mean Square } & \text { F Ratio } & \text { Prob > F } \\ \text { Analytical Blks (LM) } & 7 & 0.02432341 & 0.003475 & 2.7498 & 0.0445 \\ \text { Error } & 16 & 0.02021812 & 0.001264 & & \\ \text { C. Total } & 23 & 0.04454153 & & & \end{array}$

Means for Oneway Anova

Level Number Mean Std Error Lower 95\% Upper 95\%

$\begin{array}{lrrrrr}\text { Level } & \text { Number } & \text { Mean } & \text { Std Error } & \text { Lower 95\% } & \text { Upper 95\% } \\ 1-1 & 3 & 3.68608 & 0.02052 & 3.6426 & 3.7296\end{array}$

$\begin{array}{llllll}1-1 & 3 & 3.68608 & 0.02052 & 3.6426 & 3.7296 \\ 1-2 & 3 & 3.75434 & 0.02052 & 3.7108 & 3.7978\end{array}$

$\begin{array}{llllll}2-1 & 3 & 3.75032 & 0.02052 & 3.7068 & 3.7938\end{array}$

$\begin{array}{llllll}2-2 & 3 & 3.67001 & 0.02052 & 3.6265 & 3.7135\end{array}$

$\begin{array}{llllll}3-1 & 3 & 3.74631 & 0.02052 & 3.7028 & 3.7898\end{array}$

$\begin{array}{llllll}3-2 & 3 & 3.73024 & 0.02052 & 3.6867 & 3.7738 \\ 4-1 & 3 & 3.74631 & 0.02052 & 3.7028 & 3.7898\end{array}$

$\begin{array}{llllll}4-1 & 3 & 3.74631 & 0.02052 & 3.7028 & 3.7898 \\ 4-2 & 3 & 3.69009 & 0.02052 & 3.6466 & 3.7336\end{array}$

Std Error uses a pooled estimate of error variance
SB3 Glass \#=100; Reference Value for La2O3 is 0 wt\%

Oneway Analysis of La2O3 (wt\%) By Analytical Blks (LM)

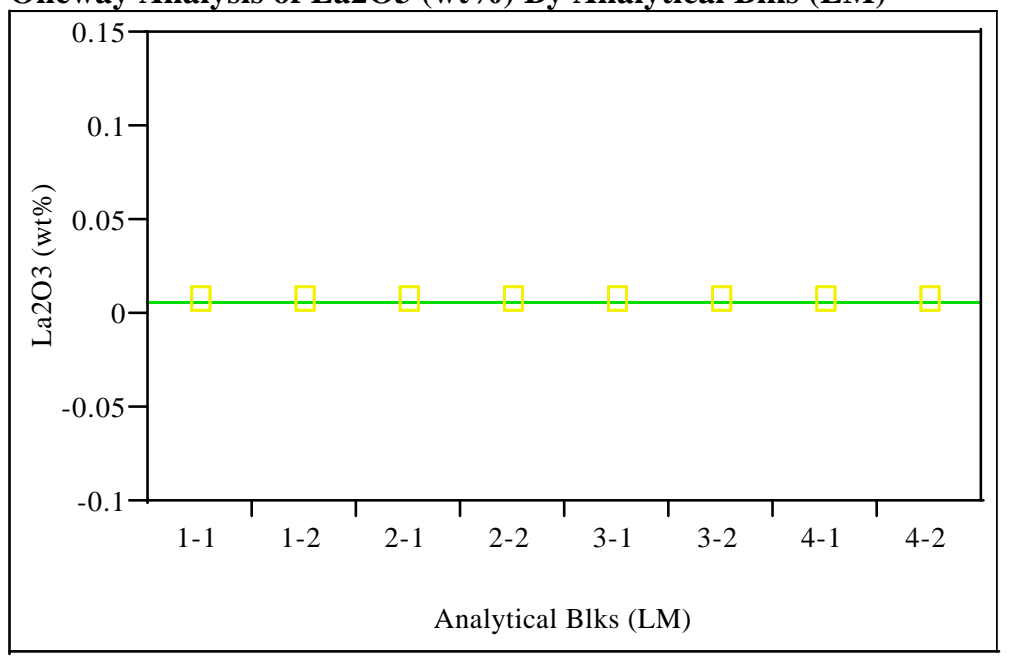

\section{Oneway Anova}

\section{Rsquare}

Root Mean Square Error

Mean of Response 0.005864

Observations (or Sum Wgts)

Analysis of Variance

Source DF Sum of Squares Mean Square F Ratio Prob $>$ F

Analytical Blks (LM)

Error

76

C. Total

23

0 0

Means for Oneway Anova

Level Number Mean Std Error Lower 95\% Upper 95\%

$\begin{array}{rrrrrr}1-1 & 3 & 0.005864 & 0 & 0.00586 & 0.00586\end{array}$

$\begin{array}{llllll}1-2 & 3 & 0.005864 & 0 & 0.00586 & 0.00586 \\ 2-1 & 3 & 0.005864 & 0 & 0.00586 & 0.00586\end{array}$

$\begin{array}{llllll}2-1 & 3 & 0.005864 & 0 & 0.00586 & 0.00586\end{array}$

$\begin{array}{llllll}2-2 & 3 & 0.005864 & 0 & 0.00586 & 0.00586\end{array}$

$\begin{array}{llllll}3-1 & 3 & 0.005864 & 0 & 0.00586 & 0.00586\end{array}$

$\begin{array}{llllll}3-2 & 3 & 0.005864 & 0 & 0.00586 & 0.00586 \\ 4-1 & 3 & 0.005864 & 0 & 0.00586 & 0.00586\end{array}$

$\begin{array}{llllll}4-1 & 3 & 0.005864 & 0 & 0.00586 & 0.00586\end{array}$

Std Error uses a pooled estimate of error variance 
Westinghouse Savannah River Company

\section{Exhibit H.4: Statistical Analysis by Analytical Block of Measurements of Standard Glasses Prepared Using the PF Method (continued)}

SB3 Glass \#=100; Reference Value for $\mathrm{MgO}$ is $1.419 \mathrm{wt} \%$

Oneway Analysis of $\mathrm{MgO}$ (wt\%) By Analytical Blks (LM)

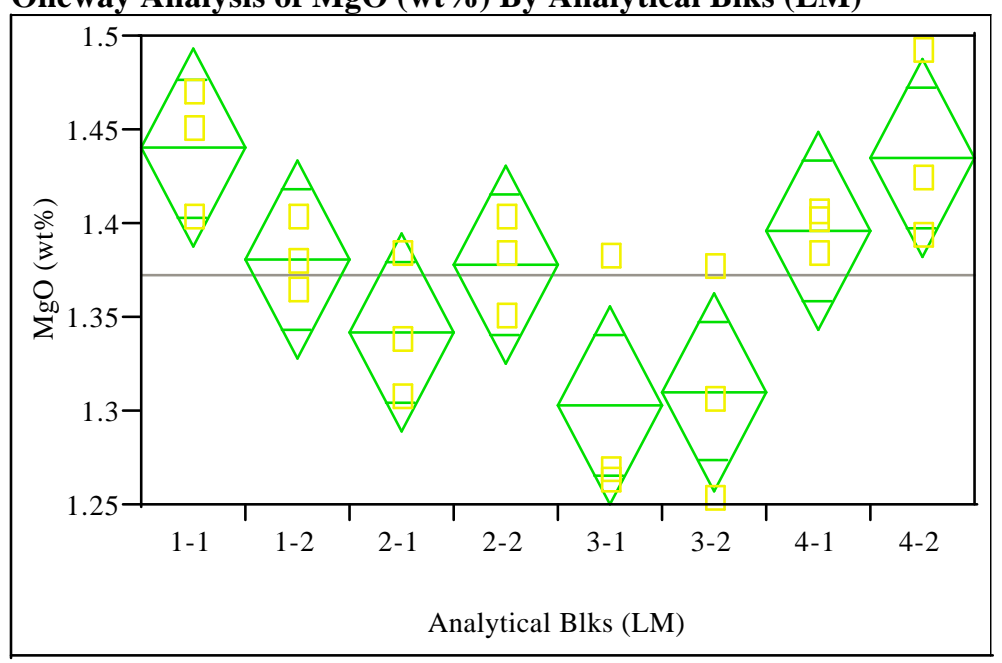

\section{Oneway Anova}

Rsquare

Root Mean Square Error $\quad 0.043101$

Mean of Response $\quad 1.373459$

Observations (or Sum Wgts)

$$
24
$$

Analysis of Variance

$\begin{array}{lrrrrr}\text { Source } & \text { DF } & \text { Sum of Squares } & \text { Mean Square } & \text { F Ratio } & \text { Prob > F } \\ \text { Analytical Blks (LM) } & 7 & 0.05616811 & 0.008024 & 4.3193 & 0.0073 \\ \text { Error } & 16 & 0.02972355 & 0.001858 & & \\ \text { C. Total } & 23 & 0.08589166 & & & \end{array}$

\section{Means for Oneway Anova}

$\begin{array}{lrrrrr}\text { Level } & \text { Number } & \text { Mean } & \text { Std Error } & \text { Lower 95\% } & \text { Upper 95\% } \\ 1-1 & 3 & 1.44034 & 0.02488 & 1.3876 & 1.4931 \\ 1-2 & 3 & 1.38120 & 0.02488 & 1.3284 & 1.4340 \\ 2-1 & 3 & 1.34196 & 0.02488 & 1.2892 & 1.3947 \\ 2-2 & 3 & 1.37843 & 0.02488 & 1.3257 & 1.4312 \\ 3-1 & 3 & 1.30327 & 0.02488 & 1.2505 & 1.3560 \\ 3-2 & 3 & 1.3100 & 0.02488 & 1.2583 & 1.3638 \\ 4-1 & 3 & 1.39612 & 0.02488 & 1.3434 & 1.4489 \\ 4-2 & 3 & 1.43536 & 0.02488 & 1.3826 & 1.4881\end{array}$

Std Error uses a pooled estimate of error variance
SB3 Glass \#=100; Reference Value for $\mathrm{MnO}$ is $1.726 \mathrm{wt} \%$

Oneway Analysis of MnO (wt\%) By Analytical Blks (LM)

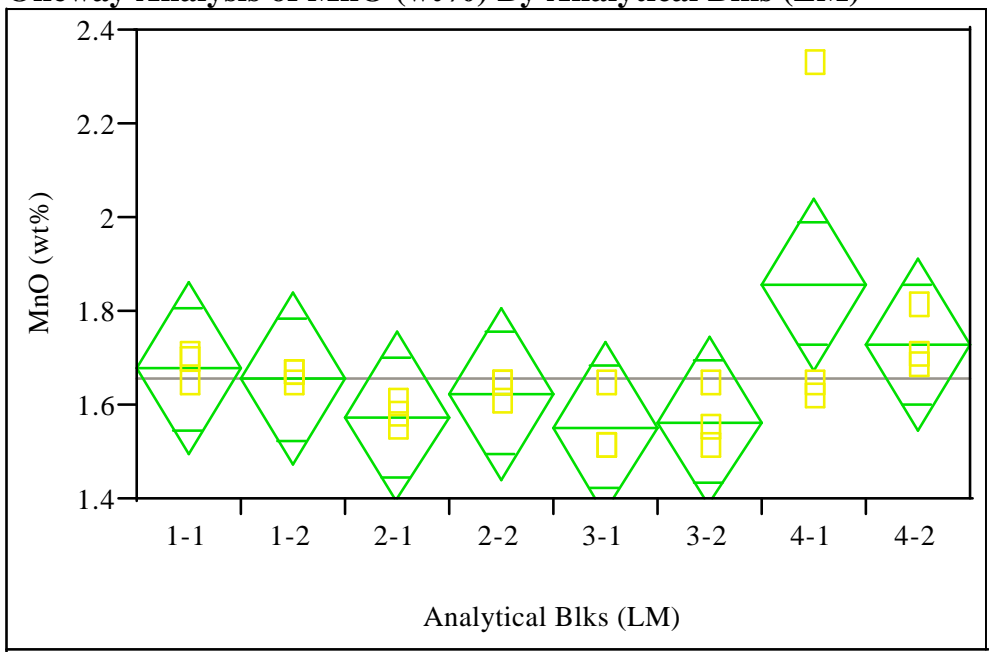

\section{Oneway Anova}

\section{Rsquare}

0.379559

Root Mean Square Error $\quad 0.149746$

Mean of Response

0.149746
1.655964

Observations (or Sum Wgts)

Analysis of Variance

Source DF Sum of Squares Mean Square F Ratio Prob $>$ F

$\begin{array}{lrrrrr}\text { Analytical Blks (LM) } & 7 & 0.21948654 & 0.031355 & 1.3983 & 0.2723 \\ \text { Error } & 16 & 0.35878089 & 0.022424 & & \end{array}$

Error

0.022424

\section{Means for Oneway Anova}

Level Number Mean Std Error Lower 95\% Upper 95\%

$\begin{array}{lrrrrr}\text { Level } & \text { Number } & \text { Mean } & \text { Std Error } & \text { Lower 95\% } & \text { Upper 95\% } \\ 1-1 & 3 & 1.67856 & 0.08646 & 1.4953 & 1.8618\end{array}$

$\begin{array}{llllll}1-1 & 3 & 1.67856 & 0.08646 & 1.4953 & 1.8618 \\ 1-2 & 3 & 1.65704 & 0.08646 & 1.4738 & 1.8403\end{array}$

$\begin{array}{llllll}2-1 & 3 & 1.57526 & 0.08646 & 1.3920 & 1.7585\end{array}$

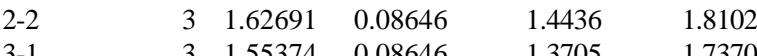

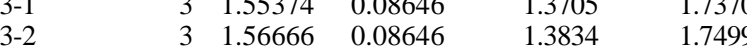

$\begin{array}{llllll}3-2 & 3 & 1.56666 & 0.08646 & 1.3834 & 1.7499 \\ 4-1 & 3 & 1.85933 & 0.08646 & 1.6760 & 2.0426\end{array}$

$\begin{array}{llllll}4-1 & 3 & 1.85933 & 0.08646 & 1.6760 & 2.0426 \\ 4-2 & 3 & 1.73021 & 0.08646 & 1.5469 & 1.9135\end{array}$

Std Error uses a pooled estimate of error variance 
Westinghouse Savannah River Company

\section{Exhibit H.4: Statistical Analysis by Analytical Block of Measurements of Standard Glasses Prepared Using the PF Method (continued)}

SB3 Glass \#=100; Reference Value for $\mathrm{Na2O}$ is $9.003 \mathrm{wt} \%$ Oneway Analysis of Na2O (wt\%) By Analytical Blks (LM)

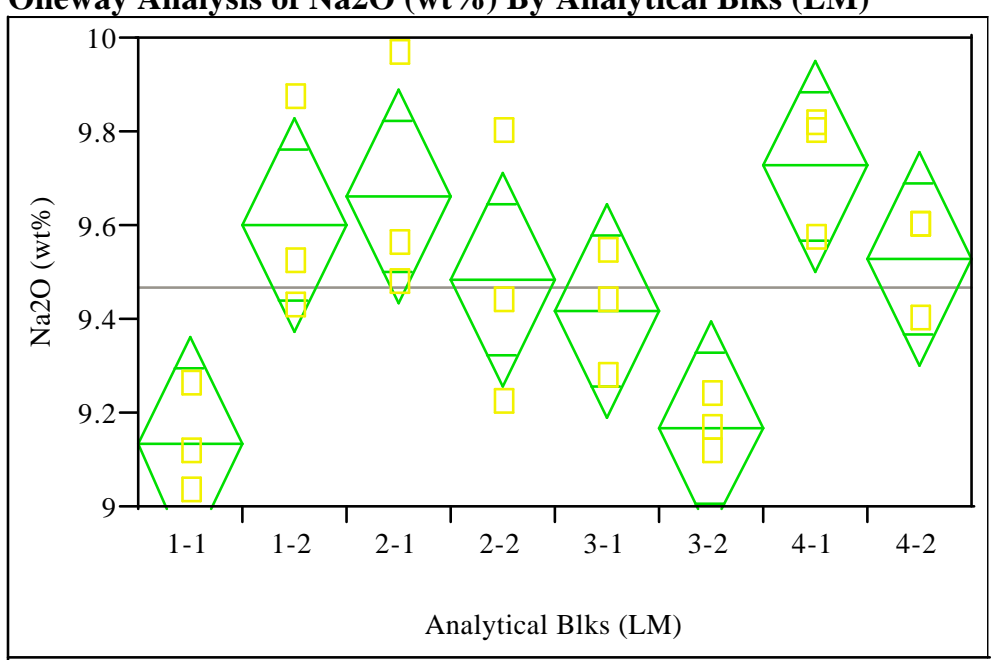

\section{Oneway Anova}

Rsquare

Root Mean Square Error $\quad 0.642141$
$\quad \begin{aligned} & 9.468892 \\ & \text { Rean }\end{aligned}$

Mean of Response

$$
\begin{array}{r}
9.466892 \\
24
\end{array}
$$

Analysis of Variance

Analysis of Variance

$\begin{array}{lrrrrr}\text { Source } & \text { DF } & \text { Sum of Squares } & \text { Mean Square } & \text { F Ratio } & \text { Prob > F } \\ \text { Analytical Blks (LM) } & 7 & 0.9912227 & 0.141603 & 4.1015 & 0.0092 \\ \text { Error } & 16 & 0.5523996 & 0.034525 & & \\ \text { C. Total } & 23 & 1.5436223 & & & \end{array}$

Means for Oneway Anova

$\begin{array}{lrrrrr}\text { Level } & \text { Number } & \text { Mean } & \text { Std Error } & \text { Lower 95\% } & \text { Upper 95\% } \\ 1-1 & 3 & 9.13495 & 0.10728 & 8.9075 & 9.3624\end{array}$

$\begin{array}{llllll}1-1 & 3 & 9.13495 & 0.10728 & 8.9075 & 9.3624 \\ 1-2 & 3 & 9.60225 & 0.10728 & 9.3748 & 9.8297\end{array}$

$\begin{array}{llllll}2-1 & 3 & 9.66516 & 0.10728 & 9.4377 & 9.8926\end{array}$

$\begin{array}{llllll}2-2 & 3 & 9.48543 & 0.10728 & 9.2580 & 9.7128\end{array}$

$\begin{array}{llllll}3-1 & 3 & 9.41803 & 0.10728 & 9.1906 & 9.6454 \\ 3-2 & 3 & 9.17089 & 0.10728 & 8.9435 & 9.3983\end{array}$

$\begin{array}{llllll}3-2 & 3 & 9.17089 & 0.10728 & 8.9435 & 9.3983 \\ 4-1 & 3 & 9.72807 & 0.10728 & 9.5006 & 9.9555\end{array}$

$\begin{array}{llllll}4-1 & 3 & 9.72807 & 0.10728 & 9.5006 & 9.9555 \\ 4-2 & 3 & 9.53036 & 0.10728 & 9.3029 & 9.7578\end{array}$

Std Error uses a pooled estimate of error variance
SB3 Glass \#=100; Reference Value for $\mathrm{NiO}$ is $0.751 \mathrm{wt} \%$

Oneway Analysis of $\mathrm{NiO}$ (wt\%) By Analytical Blks (LM)

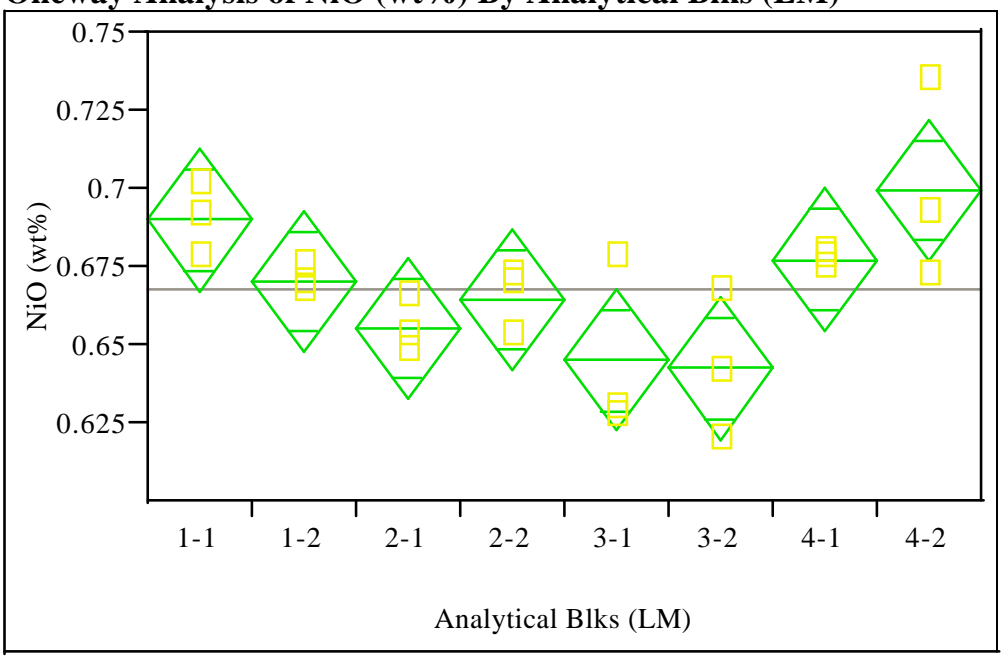

\section{Oneway Anova}

Rsquare

Root Mean Square Error

0.018508

0.668169

Observations (or Sum Wgts)

Analysis of Variance

Source DF Sum of Squares Mean Square F Ratio Prob $>$ F

$\begin{array}{lrrrrr}\text { Analytical Blks (LM) } & 7 & 0.00873292 & 0.001248 & 3.6421 & 0.0153\end{array}$

$\begin{array}{llll}\text { Error } & 16 & 0.00548064 & 0.000343 \\ \text { C. Total } & 23 & 0.01421356 & \end{array}$

$23 \quad 0.01421356$

\section{Means for Oneway Anova}

Level Number Mean Std Error Lower 95\% Upper 95\%

$\begin{array}{rrrrrr}1-1 & 3 & 0.690119 & 0.01069 & 0.66747 & 0.71277\end{array}$

$\begin{array}{llllll}1-2 & 3 & 0.670608 & 0.01069 & 0.64796 & 0.69326\end{array}$

$\begin{array}{llllll}2-1 & 3 & 0.655337 & 0.01069 & 0.63269 & 0.67799\end{array}$

$\begin{array}{llllll}2-2 & 3 & 0.664669 & 0.01069 & 0.64202 & 0.68732 \\ 3-1 & 3 & 0.645157 & 0.01069 & 0.62251 & 0.66781\end{array}$

$\begin{array}{llllll}3-1 & 3 & 0.645157 & 0.01069 & 0.62251 & 0.66781 \\ 3-2 & 3 & 0.642612 & 0.01069 & 0.61996 & 0.66526\end{array}$

$\begin{array}{llllll}3-2 & 3 & 0.642612 & 0.01069 & 0.61996 & 0.66526 \\ 4-1 & 3 & 0.677394 & 0.01069 & 0.65474 & 0.70005\end{array}$

$\begin{array}{llllll}4-1 & 3 & 0.677394 & 0.01069 & 0.65474 & 0.70005 \\ 4-2 & 3 & 0.699451 & 0.01069 & 0.67680 & 0.72210\end{array}$

Std Error uses a pooled estimate of error variance 
Westinghouse Savannah River Company

Exhibit H.4: Statistical Analysis by Analytical Block of Measurements of Standard Glasses Prepared Using the PF Method (continued)

SB3 Glass \#=100; Reference Value for $\mathrm{PbO}$ is $0 \mathrm{wt} \%$

Oneway Analysis of PbO (wt\%) By Analytical Blks (LM)

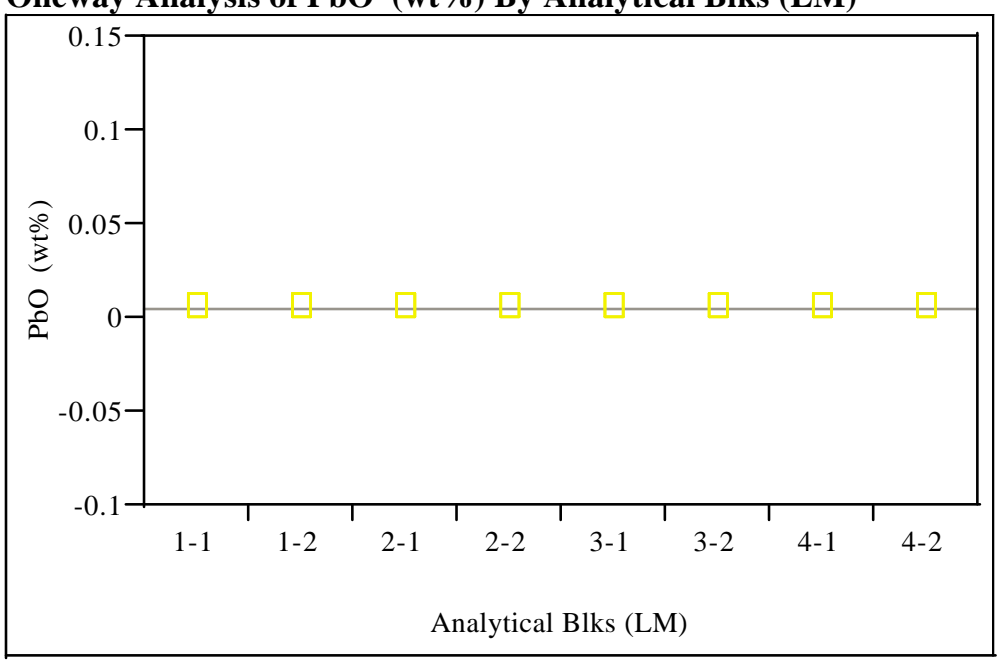

\section{Oneway Anova}

Rsquare

Root Mean Square Error

Mean of Response

4

Observations (or Sum Wgts)

0.005386

Analysis of Variance

$\begin{array}{lrrrrr}\text { Source } & \text { DF } & \text { Sum of Squares } & \text { Mean Square } & \text { F Ratio } & \text { Prob > F } \\ \text { Analytical Blks (LM) } & 7 & 7.2222 \mathrm{e}-35 & 1.032 \mathrm{e}-35 & -3.0476 & -1.0000 \\ \text { Error } & 16 & -5.417 \mathrm{e}-35 & -3.39 \mathrm{e}-36 & & \\ \text { C. Total } & 23 & 1.8056 \mathrm{e}-35 & & & \end{array}$

Means for Oneway Anova

Level Number Mean Std Error Lower 95\% Upper 95\%

$1-1 \quad 3 \quad 0.005386$

$\begin{array}{lll}1-2 & 3 & 0.005386\end{array}$

$\begin{array}{lll}2-1 & 3 & 0.005386 \\ 2-2 & 3 & 0.005386 \\ 3-1 & 3 & 0.005386\end{array}$

$3-1 \quad 3 \quad 0.005386$

$\begin{array}{lll}3-2 & 3 & 0.005386\end{array}$

$\begin{array}{lll}4-1 & 3 & 0.005386\end{array}$

Std Error uses a pooled estimate of error variance
SB3 Glass \#=100; Reference Value for ThO2 is $0 \mathrm{wt} \%$

Oneway Analysis of ThO2 (wt\%) By Analytical Blks (LM)

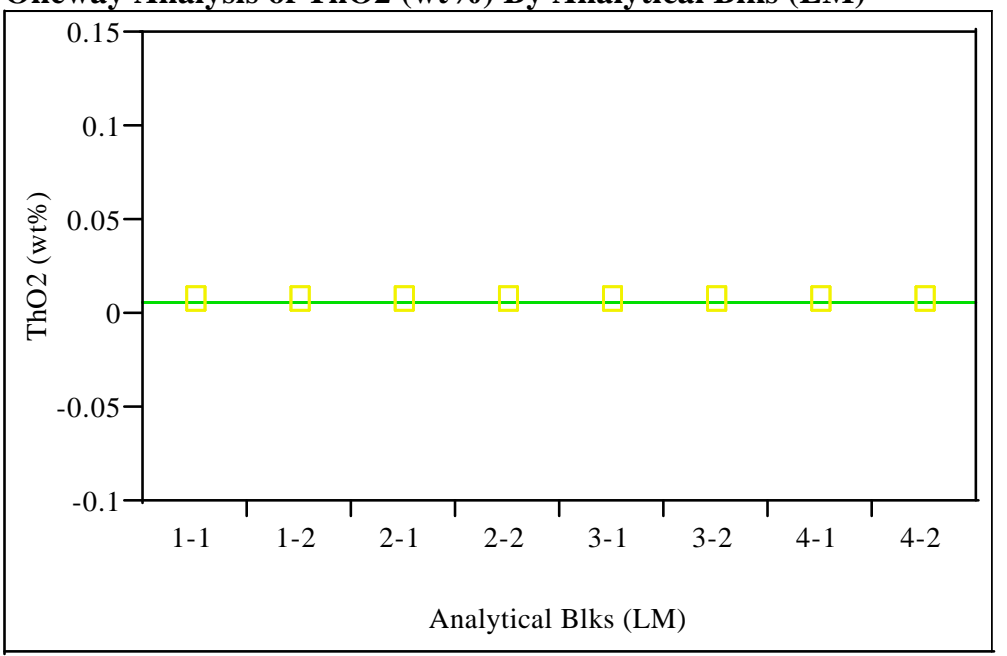

\section{Oneway Anova}

\section{Rsquare}

Root Mean Square Error

Mean of Response

0
0.005689
24

Analysis of Variance

Source DF Sum of Squares Mean Square F Ratio Prob > F

$\begin{array}{rrrrr}7 & \text { Sum of Squares } & \text { Mean Square } & \text { F Ratio } & \text { Prob }>\text { F } \\ 7 & 0 & 0 & \text {. }\end{array}$

Analytical Blks (LM)

Error

16
23

Means for Oneway Anova

Level Number Mean Std Error Lower 95\% Upper 95\%

$\begin{array}{lrrrrr}\text { Level } & \text { Number } & \text { Mean } & \text { Std Error } & \text { Lower 95\% } & \text { Upper 95\% } \\ 1-1 & 3 & 0.005689 & 0 & 0.00569 & 0.00569\end{array}$

$\begin{array}{llllll}1-1 & 3 & 0.005689 & 0 & 0.00569 & 0.00569 \\ 1-2 & 3 & 0.005689 & 0 & 0.00569 & 0.00569\end{array}$

$\begin{array}{llllll}2-1 & 3 & 0.005689 & 0 & 0.00569 & 0.00569 \\ 2-2 & 3 & 0.005689 & 0 & 0.00569 & 0.00569\end{array}$

$\begin{array}{llllll}2-2 & 3 & 0.005689 & 0 & 0.00569 & 0.00569\end{array}$

$\begin{array}{llllll}3-1 & 3 & 0.005689 & 0 & 0.00569 & 0.00569\end{array}$

$\begin{array}{llllll}3-2 & 3 & 0.005689 & 0 & 0.00569 & 0.00569\end{array}$

$\begin{array}{lll}4-1 & 3 & 0.005689 \\ 4-2 & 3 & 0.005689\end{array}$

Std Error uses a pooled estimate of error variance 
Westinghouse Savannah River Company

\section{Exhibit H.4: Statistical Analysis by Analytical Block of Measurements of Standard Glasses Prepared Using the PF Method (continued)}

SB3 Glass \#=100; Reference Value for TiO2 is $0.677 \mathrm{wt} \%$

Oneway Analysis of TiO2 (wt\%) By Analytical Blks (LM)

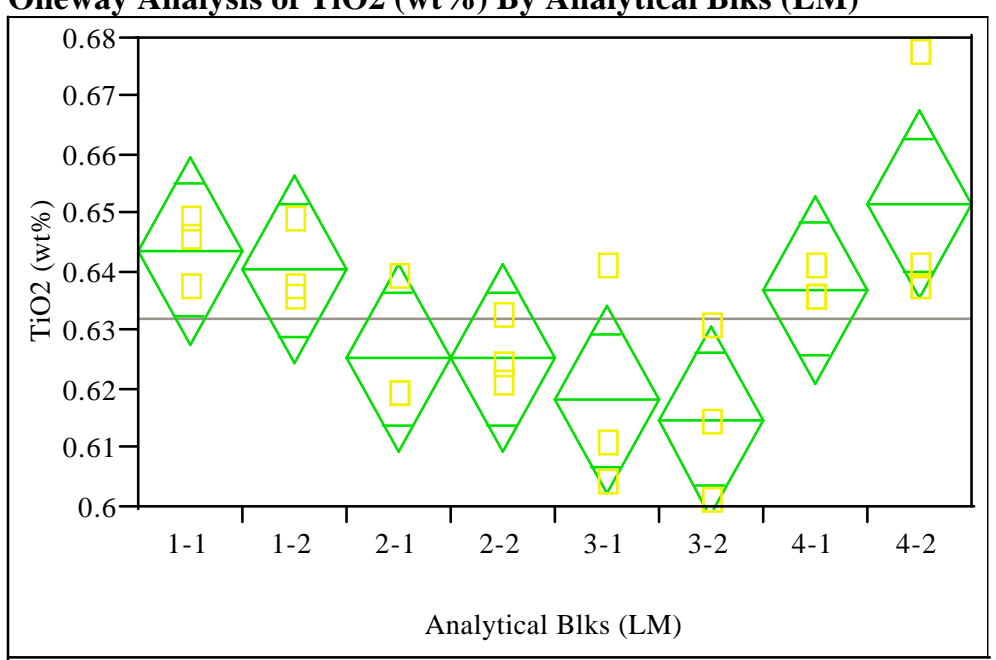

\section{Oneway Anova}

Root Mean Square Erro

\subsection{5}

0.013081

0.632172

Observations (or Sum Wgts)

$$
24
$$

Analysis of Variance

$\begin{array}{lrrrrr}\text { Source } & \text { DF } & \text { Sum of Squares } & \text { Mean Square } & \text { F Ratio } & \text { Prob > F } \\ \text { Analytical Blks (LM) } & 7 & 0.00356681 & 0.000510 & 2.9779 & 0.0335 \\ \text { Error } & 16 & 0.00273771 & 0.000171 & & \\ \text { C. Total } & 23 & 0.00630452 & & & \end{array}$

$23 \quad 0.00630452$

\section{Means for Oneway Anova}

Level Number Mean Std Error Lower 95\% Upper 95\%

$\begin{array}{rrrrrr}1-1 & 3 & 0.643848 & 0.00755 & 0.62784 & 0.65986\end{array}$

$\begin{array}{llllll}1-2 & 3 & 0.640512 & 0.00755 & 0.62450 & 0.65652\end{array}$

$\begin{array}{llllll}2-1 & 3 & 0.625500 & 0.00755 & 0.60949 & 0.64151\end{array}$

$\begin{array}{llllll}2-2 & 3 & 0.625500 & 0.00755 & 0.60949 & 0.6415 \\ 3-1 & 3 & 0.618272 & 0.00755 & 0.60226 & 0.63428\end{array}$

$\begin{array}{llllll}3-1 & 3 & 0.618272 & 0.00755 & 0.60226 & 0.63428 \\ 3-2 & 3 & 0.614936 & 0.00755 & 0.59893 & 0.63095\end{array}$

$\begin{array}{llllll}3-2 & 3 & 0.614936 & 0.00755 & 0.59893 & 0.63095 \\ 4-1 & 3 & 0.637176 & 0.00755 & 0.62117 & 0.65319\end{array}$

$\begin{array}{llllll}4-1 & 3 & 0.637176 & 0.00755 & 0.62117 & 0.65319 \\ 4-2 & 3 & 0.651632 & 0.00755 & 0.63562 & 0.66764\end{array}$

Std Error uses a pooled estimate of error variance
SB3 Glass \#=100; Reference Value for U3O8 is $0 \mathrm{wt} \%$

Oneway Analysis of U3O8 (wt\%) By Analytical Blks (LM)

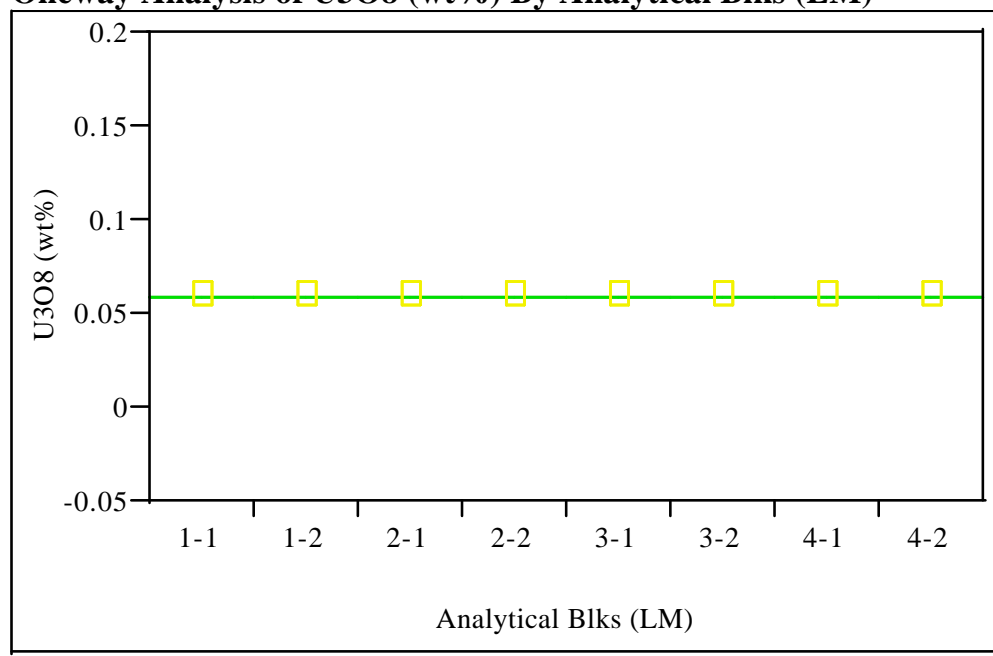

\section{Oneway Anova}

\section{Rsquare}

Root Mean Square Error

Mean of Response

1
0
0.05896
24

Analysis of Variance

sis of Variance

Source DF Sum of Squares Mean Square F Ratio Prob > F

Analytical Blks (LM) $\quad 7 \quad 4.6222 \mathrm{e}-33 \quad 6.603 \mathrm{e}-34$

Error

$\begin{array}{rrr}7 & 4.6222 \mathrm{e}-33 & 6.603 \mathrm{e}-34 \\ 6 & 0 & 0\end{array}$

C. Total

$23 \quad 4.6222 \mathrm{e}-33$

\section{Means for Oneway Anova}

Level Number Mean Std Error Lower 95\% Upper 95\%

$\begin{array}{lrrrrr}\text { Level } & \text { Number } & \text { Mean } & \text { Std Error } & \text { Lower 95\% } & \text { Upper 95\% } \\ 1-1 & 3 & 0.058960 & 0 & 0.05896 & 0.05896 \\ 1-2 & 3 & 0.058960 & 0 & 0.05896 & 0.05896\end{array}$

$\begin{array}{llllll}1-1 & 3 & 0.058960 & 0 & 0.05896 & 0.05896 \\ 1-2 & 3 & 0.058960 & 0 & 0.05896 & 0.05896\end{array}$

$\begin{array}{llllll}2-1 & 3 & 0.058960 & 0 & 0.05896 & 0.05896 \\ 2-2 & 3 & 0.058960 & 0 & 0.05896 & 0.05896\end{array}$

$\begin{array}{llllll}2-2 & 3 & 0.058960 & 0 & 0.05896 & 0.05896\end{array}$

$\begin{array}{llllll}3-1 & 3 & 0.058960 & 0 & 0.05896 & 0.05896\end{array}$

$\begin{array}{llllll}3-2 & 3 & 0.058960 & 0 & 0.05896 & 0.05896 \\ 4-1 & 3 & 0.058960 & 0 & 0.05896 & 0.05896\end{array}$

$\begin{array}{llllll}4-1 & 3 & 0.058960 & 0 & 0.05896 & 0.05896\end{array}$

Std Error uses a pooled estimate of error variance 
Westinghouse Savannah River Company

Exhibit H.4: Statistical Analysis by Analytical Block of Measurements of Standard Glasses Prepared Using the PF Method (continued)

SB3 Glass \#=100; Reference Value for $\mathrm{ZnO}$ is $0 \mathrm{wt} \%$

Oneway Analysis of ZnO (wt \%) By Analytical Blks (LM)

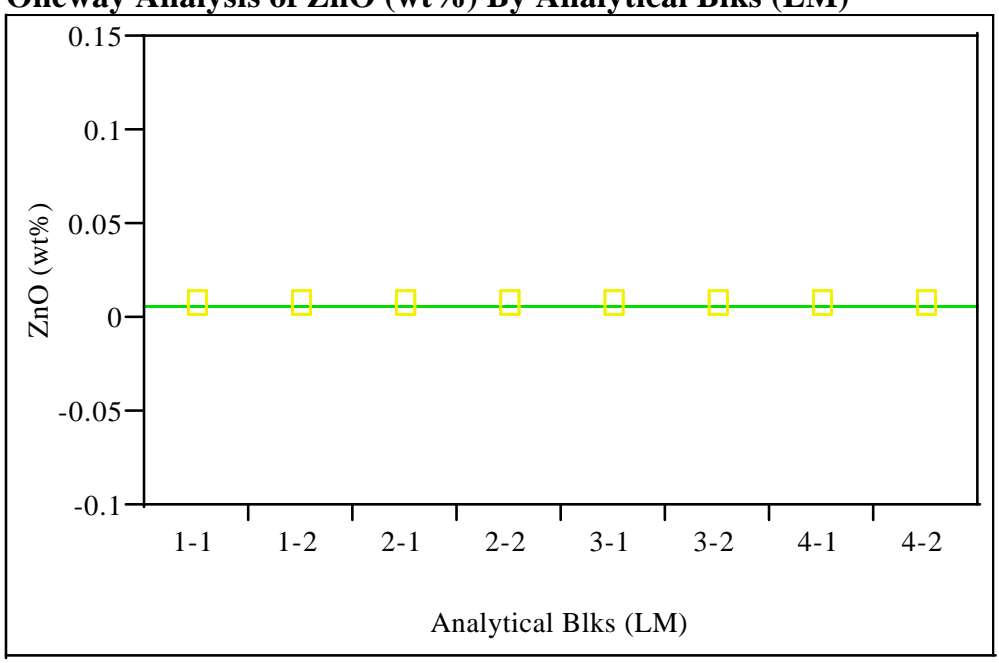

\section{Oneway Anova}

Rsquare

Root Mean Square Error

Mean of Response

Observations (or Sum Wgts)

$$
24
$$

Analysis of Variance

Source DF Sum of Squares Mean Square F Ratio Prob $>$ F

$\begin{array}{rrrrr}7 & \text { Sum of Squares } & \text { Mean Square } & \text { F Ratio } & \text { Prob }>\text { F } \\ 0 & 0 & . & \\ 0 & 0 & & \end{array}$

23

0

Means for Oneway Anova

Level Number Mean Std Error Lower 95\% Upper 95\%

$\begin{array}{llllll}1-1 & 3 & 0.006224 & 0 & 0.00622 & 0.00622 \\ 1-2 & 3 & 0.006224 & 0 & 0.00622 & 0.00622\end{array}$

$\begin{array}{llllll}2-1 & 3 & 0.006224 & 0 & 0.00622 & 0.00622\end{array}$

$\begin{array}{llllll}2-2 & 3 & 0.006224 & 0 & 0.00622 & 0.00622\end{array}$

$\begin{array}{llllll}3-1 & 3 & 0.006224 & 0 & 0.00622 & 0.00622\end{array}$

$\begin{array}{llllll}3-2 & 3 & 0.006224 & 0 & 0.00622 & 0.00622 \\ 4-1 & 3 & 0.006224 & 0 & 0.00622 & 0.00622\end{array}$

$\begin{array}{llllll}4-1 & 3 & 0.006224 & 0 & 0.00622 & 0.00622 \\ 4-2 & 3 & 0.006224 & 0 & 0.00622 & 0.00622\end{array}$

Std Error uses a pooled estimate of error variance
SB3 Glass \#=100; Reference Value for $\mathrm{ZrO2}$ is $0.098 \mathrm{wt} \%$

Oneway Analysis of $\mathrm{ZrO2}$ (wt\%) By Analytical Blks (LM)

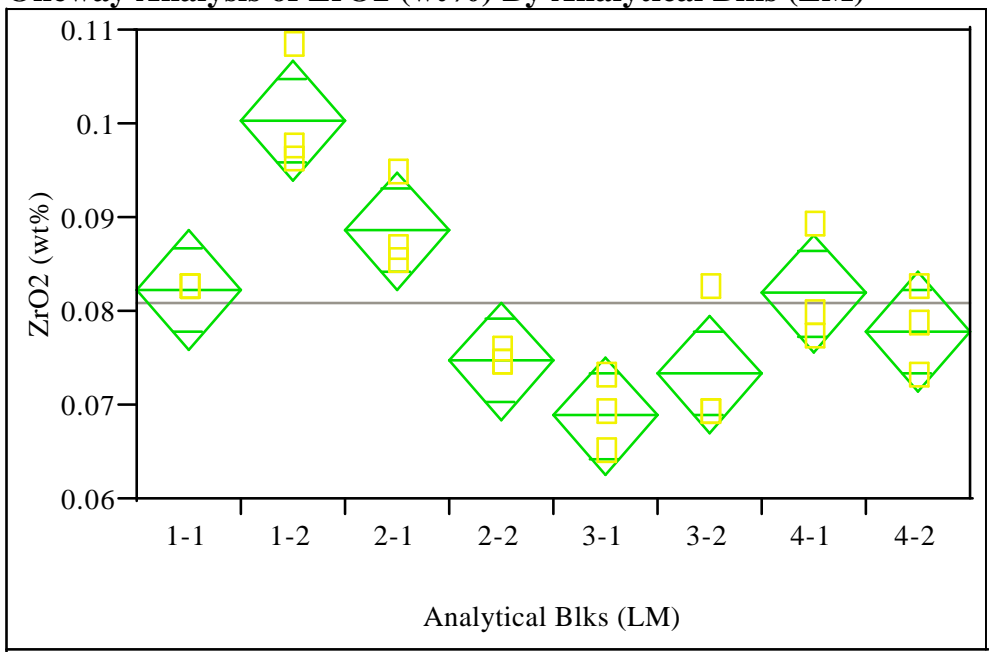

\section{Oneway Anova}

\section{Rsquare}

Root Mean Square Erro

\subsection{7}

Mean of Response

0.005144

0.081048

Observations (or Sum Wgts)

Analysis of Variance

Source DF Sum of Squares Mean Square F Ratio Prob $>$ F

$\begin{array}{lrrrrr}\text { Analytical Blks (LM) } & 7 & 0.00207646 & 0.000297 & 11.2118 & <.0001\end{array}$

$\begin{array}{llll}\text { Error } & 16 & 0.00042332 & 0.000026\end{array}$

C. Total

$23 \quad 0.00249979$

Means for Oneway Anova

Level Number Mean Std Error Lower 95\% Upper 95\%

$\begin{array}{rrrrrr}1-1 & 3 & 0.082399 & 0.00297 & 0.07610 & 0.08869\end{array}$

$\begin{array}{llllll}1-2 & 3 & 0.100409 & 0.00297 & 0.09411 & 0.10670 \\ 2-1 & 3 & 0.088703 & 0.00297 & 0.08241 & 0.09500\end{array}$

$\begin{array}{llllll}2-1 & 3 & 0.088703 & 0.00297 & 0.08241 & 0.09500\end{array}$

$\begin{array}{llllll}2-2 & 3 & 0.074744 & 0.00297 & 0.06845 & 0.08104\end{array}$

$\begin{array}{llllll}3-1 & 3 & 0.068891 & 0.00297 & 0.06260 & 0.07519\end{array}$

$\begin{array}{llllll}3-2 & 3 & 0.073393 & 0.00297 & 0.06710 & 0.07969 \\ 4-1 & 3 & 0.081949 & 0.00297 & 0.07565 & 0.08824\end{array}$

$\begin{array}{llllll}4-1 & 3 & 0.081949 & 0.00297 & 0.07565 & 0.08824 \\ 4-2 & 3 & 0.077896 & 0.00297 & 0.07160 & 0.08419\end{array}$

Std Error uses a pooled estimate of error variance 
Westinghouse Savannah River Company

Exhibit H.4: Statistical Analysis by Analytical Block of Measurements of Standard Glasses Prepared Using the PF Method (continued)

SB3 Glass \#=101; Reference Value for $\mathrm{BaO}$ is $0 \mathrm{wt} \%$

Oneway Analysis of $\mathrm{BaO}$ (wt\%) By Analytical Blks (LM)

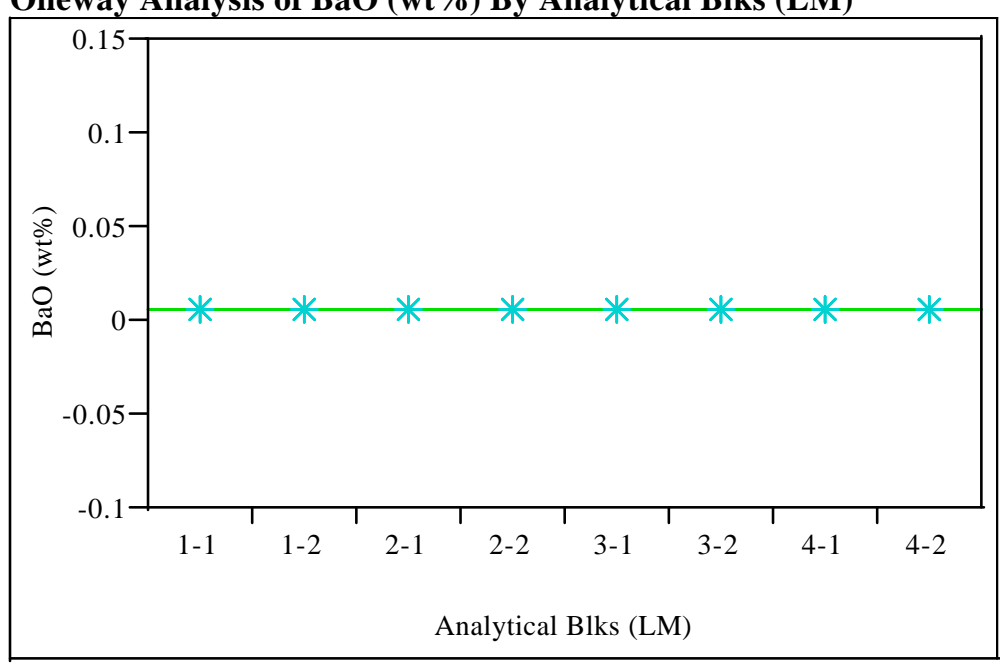

\section{Oneway Anova}

Rsquare

Root Mean Square Error

Mean of Response

Observations (or Sum Wgts)

24

Analysis of Variance

Source DF Sum of Squares Mean Square F Ratio Prob $>$ F

$\begin{array}{rrrrr}7 & \text { Sum of Squares } & \text { Mean Square } & \text { F Ratio } & \text { Prob }>\text { F } \\ 0 & 0 & \text {. } & \\ 0 & 0 & & \end{array}$

Error

C. Total

23

Means for Oneway Anova

Level Number Mean Std Error Lower 95\% Upper 95\%

$\begin{array}{rrrrrr}1-1 & 3 & 0.005583 & 0 & 0.00558 & 0.00558\end{array}$

$\begin{array}{llllll}1-2 & 3 & 0.005583 & 0 & 0.00558 & 0.00558\end{array}$

$\begin{array}{llllll}2-1 & 3 & 0.005583 & 0 & 0.00558 & 0.00558\end{array}$

$\begin{array}{llllll}2-2 & 3 & 0.005583 & 0 & 0.00558 & 0.00558\end{array}$

$\begin{array}{llllll}3-1 & 3 & 0.005583 & 0 & 0.00558 & 0.00558 \\ 3-2 & 3 & 0.005583 & 0 & 0.00558 & 0.00558\end{array}$

$\begin{array}{llllll}3-2 & 3 & 0.005583 & 0 & 0.00558 & 0.00558 \\ 4-1 & 3 & 0.005583 & 0 & 0.00558 & 0.00558\end{array}$

$\begin{array}{llllll}3-1 & 3 & 0.005583 & 0 & 0.00558 & 0.00558 \\ 4-2 & 3 & 0.005583 & 0 & 0.00558 & 0.00558\end{array}$

Std Error uses a pooled estimate of error variance
SB3 Glass \#=101; Reference Value for $\mathrm{CaO}$ is $1.301 \mathrm{wt} \%$

Oneway Analysis of $\mathrm{CaO}$ (wt\%) By Analytical Blks (LM)

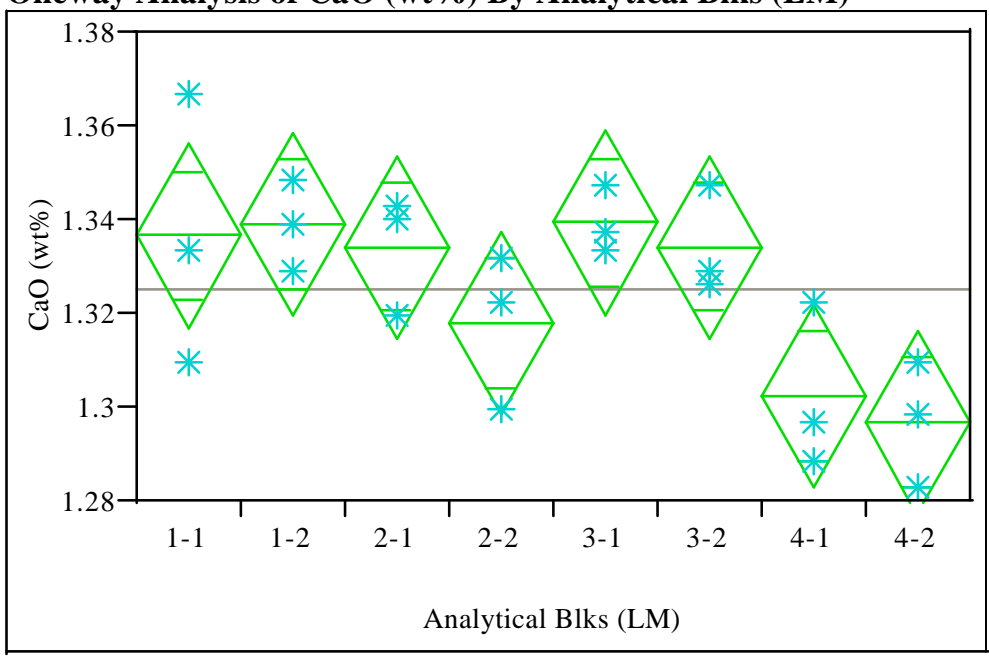

\section{Oneway Anova}

\section{Rsquare}

Root Mean Square Error

0.601761

Mean of Response

0.015941

1.325217

Observations (or Sum Wgts)

Analysis of Variance

Source DF Sum of Squares Mean Square F Ratio Prob > F

$\begin{array}{lrrrrr}\text { Analytical Blks (LM) } & 7 & 0.00614337 & 0.000878 & 3.4538 & 0.0190\end{array}$

Error $\begin{array}{lll}0.000878 & 3.4538 & 0.0190 \\ 0.000254 & & \end{array}$

C. Total

$23 \quad 0.01020899$

\section{Means for Oneway Anova}

Level Number Mean Std Error Lower 95\% Upper 95\%

$\begin{array}{rrrrrr}1-1 & 3 & 1.33670 & 0.00920 & 1.3172 & 1.3562\end{array}$

$\begin{array}{llllll}1-2 & 3 & 1.33903 & 0.00920 & 1.3195 & 1.3585\end{array}$

$\begin{array}{llllll}2-1 & 3 & 1.33437 & 0.00920 & 1.3149 & 1.3539 \\ 2-2 & 3 & 1.31805 & 0.00920 & 1.2985 & 1.3376\end{array}$

$\begin{array}{llllll}3-1 & 3 & 1.33950 & 0.00920 & 1.3200 & 1.3590\end{array}$

$\begin{array}{llllll}3-2 & 3 & 1.33437 & 0.00920 & 1.3149 & 1.3539 \\ 4-1 & 3 & 1.30266 & 0.00920 & 1.2831 & 1.3222\end{array}$

$\begin{array}{llllll}4-1 & 3 & 1.30266 & 0.00920 & 1.2831 & 1.3222\end{array}$

Std Error uses a pooled estimate of error variance 
Westinghouse Savannah River Company

Exhibit H.4: Statistical Analysis by Analytical Block of Measurements of Standard Glasses Prepared Using the PF Method (continued)

SB3 Glass \#=101; Reference Value for Ce2O3 is 0 wt\%

Oneway Analysis of Ce2O3 (wt\%) By Analytical Blks (LM)

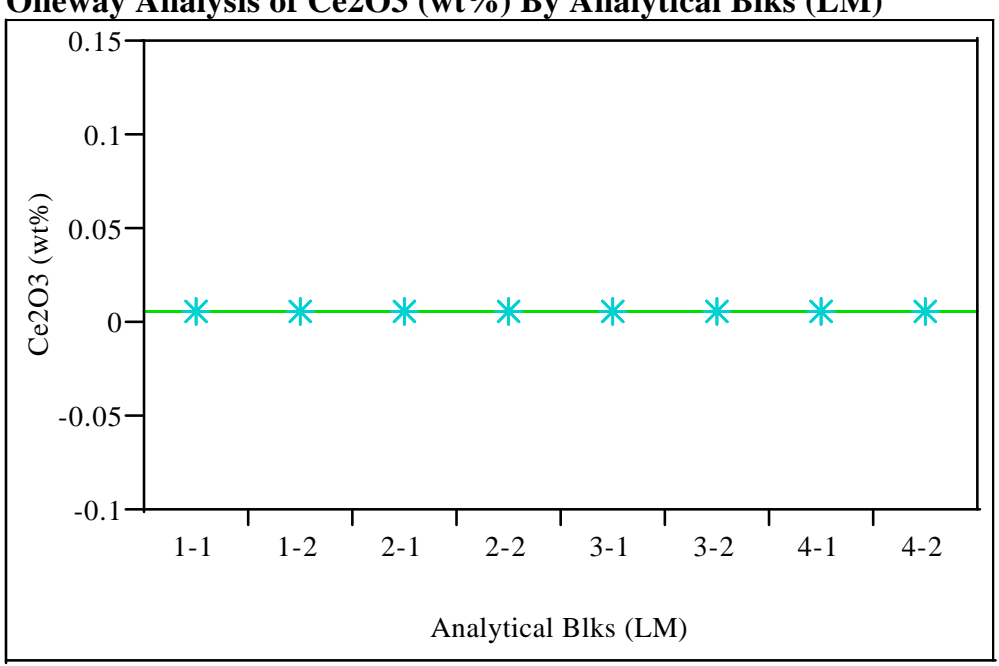

\section{Oneway Anova}

Rsquare

Root Mean Square Error

Mean of Response

Observations (or Sum Wgts)

24

Analysis of Variance

Source DF Sum of Squares Mean Square F Ratio Prob $>$ F

$\begin{array}{rrrrr}7 & \text { Sum of Squares } & \text { Mean Square } & \text { F Ratio } & \text { Prob }>\text { F } \\ 0 & 0 & . & \\ 0 & 0 & & \end{array}$

Error

C. Total

23

Means for Oneway Anova

$\begin{array}{lrrrrr}\text { Level } & \text { Number } & \text { Mean } & \text { Std Error } & \text { Lower 95\% } & \text { Upper 95\% } \\ 1-1 & 3 & 0.005857 & 0 & 0.00586 & 0.00586 \\ 1-2 & 3 & 0.005857 & 0 & 0.00586 & 0.00586 \\ 2-1 & 3 & 0.005857 & 0 & 0.00586 & 0.00586 \\ 2-2 & 3 & 0.005857 & 0 & 0.00586 & 0.00586 \\ 3-1 & 3 & 0.005857 & 0 & 0.00586 & 0.00586 \\ 3-2 & 3 & 0.005857 & 0 & 0.00586 & 0.00586 \\ 4-1 & 3 & 0.005857 & 0 & 0.00586 & 0.00586 \\ 4-2 & 3 & 0.005857 & 0 & 0.00586 & 0.00586\end{array}$

Std Error uses a pooled estimate of error variance
SB3 Glass \#=101; Reference Value for $\mathrm{Cr} 2 \mathrm{O3}$ is $0 \mathrm{wt} \%$

Oneway Analysis of Cr2O3 (wt\%) By Analytical Blks (LM)

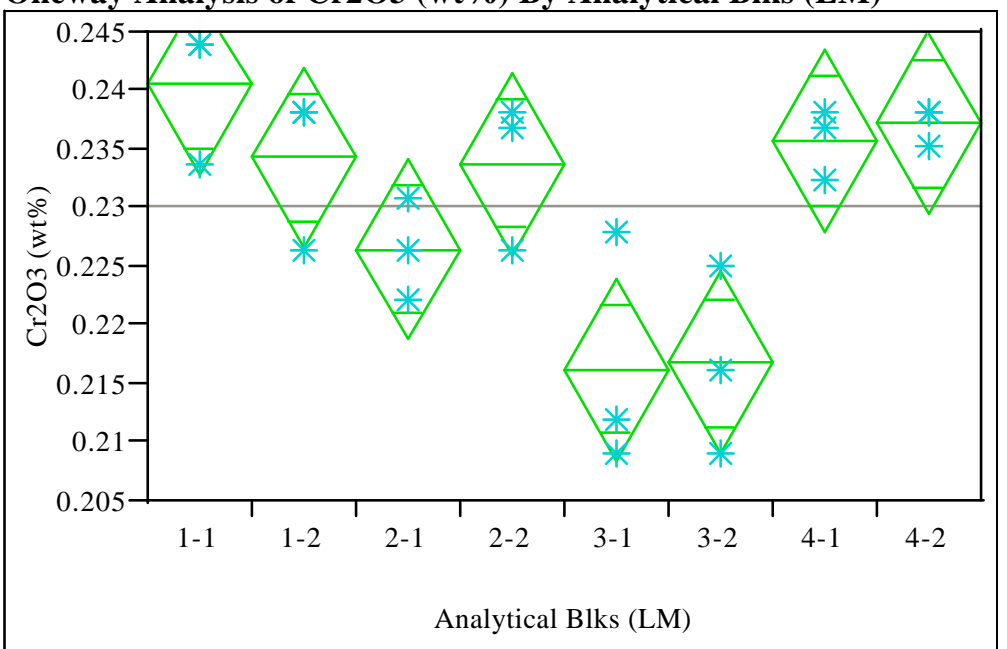

\section{Oneway Anova}

Rsquare

Root Mean Square Error

Mean of Response

\subsection{9}

0.006343

Observations (or Sum Wgts)

Analysis of Variance

$\begin{array}{lrrrrr}\text { Source } & \text { DF } & \text { Sum of Squares } & \text { Mean Square } & \text { F Ratio } & \text { Prob > F } \\ \text { Analytical Blks (LM) } & 7 & 0.00182153 & 0.000260 & 6.4678 & 0.0010 \\ \text { Error } & 16 & 0.00064373 & 0.000040 & & \\ \text { C. Total } & 23 & 0.00246526 & & & \end{array}$

Means for Oneway Anova
Level Number Mean Std Error Lower 95\% Upper 95\%

$\begin{array}{lrrrrr}\text { Level } & \text { Number } & \text { Mean } & \text { Std Error } & \text { Lower 95\% } & \text { Upper 95\% } \\ 1-1 & 3 & 0.240677 & 0.00366 & 0.23291 & 0.24844\end{array}$

$\begin{array}{llllll}1-1 & 3 & 0.240677 & 0.00366 & 0.23291 & 0.24844 \\ 1-2 & 3 & 0.234343 & 0.00366 & 0.22658 & 0.24211\end{array}$

$\begin{array}{llllll}2-1 & 3 & 0.226548 & 0.00366 & 0.22658 & 0.24211 \\ 2-2 & 3 & 0.233856 & 0.00366 & 0.21878 & 0.23431\end{array}$

$\begin{array}{llllll}2-2 & 3 & 0.233856 & 0.00366 & 0.22609 & 0.24162\end{array}$

$\begin{array}{llllll}3-1 & 3 & 0.216317 & 0.00366 & 0.20855 & 0.22408\end{array}$

$\begin{array}{llllll}3-2 & 3 & 0.216804 & 0.00366 & 0.20904 & 0.22457 \\ 4-1 & 3 & 0.235805 & 0.00366 & 0.22804 & 0.24357\end{array}$

$\begin{array}{llllll}4-1 & 3 & 0.235805 & 0.00366 & 0.22804 & 0.24357 \\ 4-2 & 3 & 0.237266 & 0.00366 & 0.22950 & 0.24503\end{array}$

Std Error uses a pooled estimate of error variance 
Westinghouse Savannah River Company

\section{Exhibit H.4: Statistical Analysis by Analytical Block of Measurements of Standard Glasses Prepared Using the PF Method (continued)}

SB3 Glass \#=101; Reference Value for $\mathrm{CuO}$ is $\mathrm{O} \mathrm{wt} \%$

Oneway Analysis of $\mathrm{CuO}$ (wt\%) By Analytical Blks (LM)

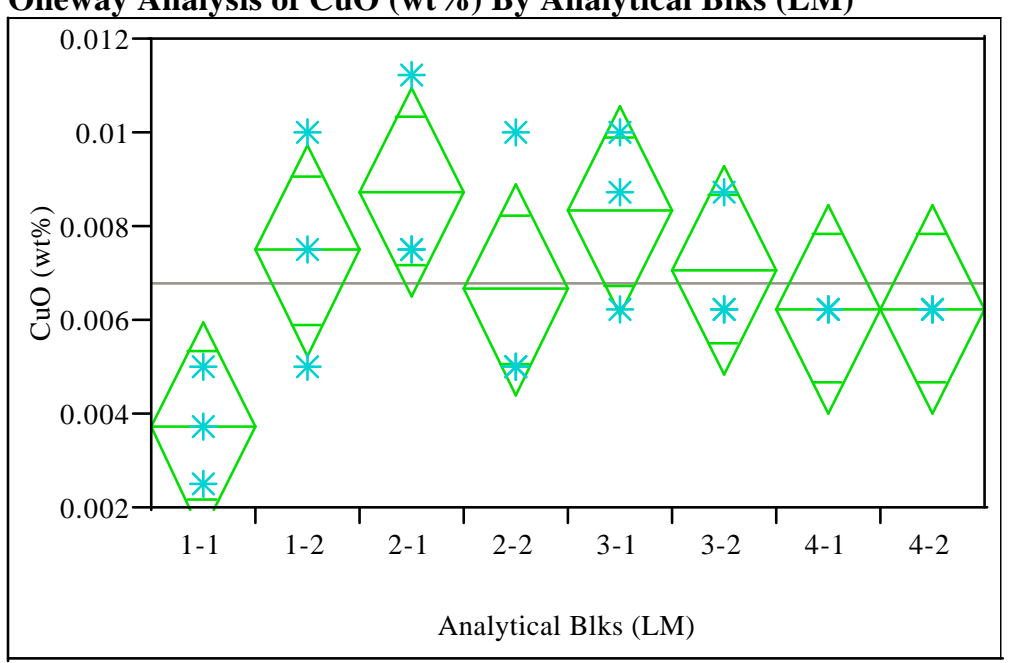

\section{Oneway Anova}

Rsquare

Root Mean Square Erro

0.484523

0.001825

Mean of Response

$$
\begin{array}{r}
0.006833 \\
24
\end{array}
$$

Analysis of Variance

Analysis of Variance

$\begin{array}{lrrrrr}\text { Source } & \text { DF } & \text { Sum of Squares } & \text { Mean Square } & \text { F Ratio } & \text { Prob > F } \\ \text { Analytical Blks (LM) } & 7 & 0.00005008 & 0.0000072 & 2.1485 & 0.0973 \\ \text { Error } & 16 & 0.00005328 & 0.0000033 & & \\ \text { C. Total } & 23 & 0.00010336 & & & \end{array}$

\section{Means for Oneway Anova}

Level Number Mean Std Error Lower 95\% Upper 95\%

$\begin{array}{lrrrrr}1-1 & 3 & 0.003755 & 0.00105 & 0.00152 & 0.00599\end{array}$

$\begin{array}{llllll}1-2 & 3 & 0.007511 & 0.00105 & 0.00528 & 0.00974\end{array}$

$\begin{array}{llllll}2-1 & 3 & 0.008763 & 0.00105 & 0.00653 & 0.01100\end{array}$

$\begin{array}{llllll}2-2 & 3 & 0.006676 & 0.00105 & 0.00444 & 0.00891 \\ 3-1 & 3 & 0.008345 & 0.00105 & 0.00611 & 0.01058\end{array}$

$\begin{array}{llllll}3-1 & 3 & 0.008345 & 0.00105 & 0.00611 & 0.01058 \\ 3-2 & 3 & 0.007094 & 0.00105 & 0.00486 & 0.00933\end{array}$

$\begin{array}{llllll}3-2 & 3 & 0.006259 & 0.00105 & 0.00486 & 0.00933 \\ 4-1 & 3 & 0.006259 & 0.00105 & 0.00403 & 0.00849\end{array}$

$\begin{array}{llllll}4-2 & 3 & 0.006259 & 0.00105 & 0.00403 & 0.00849\end{array}$

Std Error uses a pooled estimate of error variance
SB3 Glass \#=101; Reference Value for Fe2O3 is $13.196 \mathrm{wt} \%$

Oneway Analysis of Fe2O3 (wt\%) By Analytical Blks (LM)

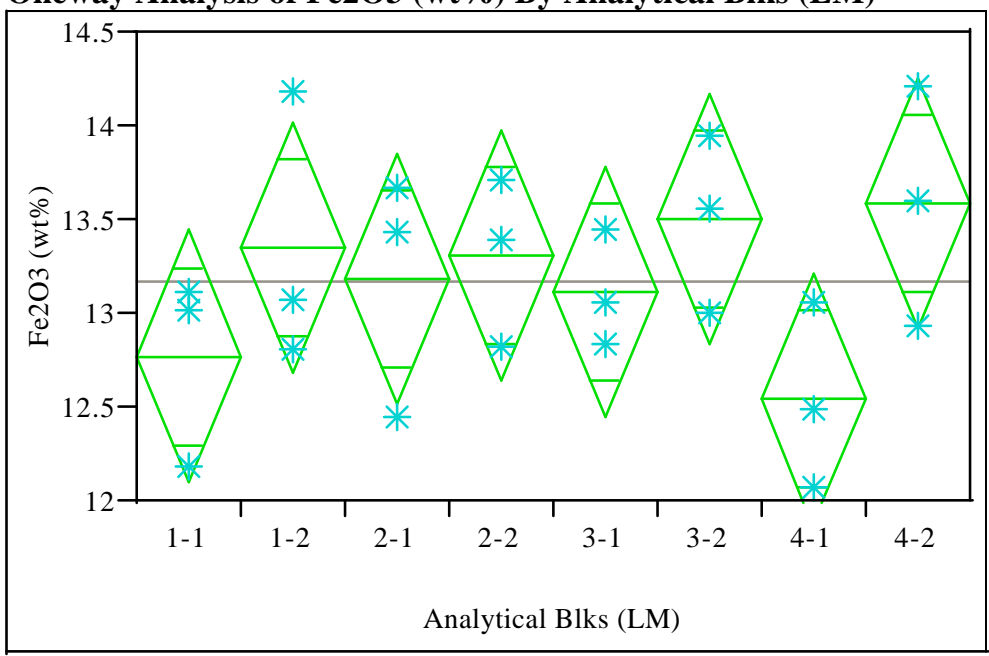

\section{Oneway Anova}

Rsquare

0.358359

Root Mean Square Error $\quad 0.546358$

Mean of Response 13.17469

Observations (or Sum Wgts)

Analysis of Variance

Source DF Sum of Squares Mean Square F Ratio Prob $>$ F

$\begin{array}{lrrrrr}\text { Analytical Blks (LM) } & 7 & 2.6674749 & 0.381068 & 1.2766 & 0.3219\end{array}$

$\begin{array}{llll}\text { Error } & 16 & 4.7761087 & 0.298507 \\ \text { C. Total } & 23 & 7.4435837 & \end{array}$ 7.4435837

\section{Means for Oneway Anova}

Level Number Mean Std Error Lower 95\% Upper 95\%

$\begin{array}{rrrrrr}1-1 & 3 & 12.7768 & 0.31544 & 12.108 & 13.445\end{array}$

$\begin{array}{llllll}1-2 & 3 & 13.3582 & 0.31544 & 12.689 & 14.027\end{array}$

$\begin{array}{llllll}2-1 & 3 & 13.1866 & 0.31544 & 12.518 & 13.855\end{array}$

$\begin{array}{llllll}2-2 & 3 & 13.3105 & 0.31544 & 12.642 & 13.979 \\ 3-1 & 3 & 13.1199 & 0.31544 & 12.451 & 13.789\end{array}$

$\begin{array}{llllll}3-1 & 3 & 13.1199 & 0.31544 & 12.451 & 13.789 \\ 3-2 & 3 & 13.5107 & 0.31544 & 12.842 & 14.179\end{array}$

$\begin{array}{llllll}3-2 & 3 & 13.5107 & 0.31544 & 12.842 & 14.179 \\ 4-1 & 3 & 12.5480 & 0.31544 & 11.879 & 13.217\end{array}$

$\begin{array}{llllll}4-1 & 3 & 12.5480 & 0.31544 & 11.879 & 13.217 \\ 4-2 & 3 & 13.5869 & 0.31544 & 12.918 & 14.256\end{array}$

Std Error uses a pooled estimate of error variance 
Westinghouse Savannah River Company

Exhibit H.4: Statistical Analysis by Analytical Block of Measurements of Standard Glasses Prepared Using the PF Method (continued)

SB3 Glass \#=101; Reference Value for K2O is $2.999 \mathrm{wt} \%$

Oneway Analysis of K2O (wt\%) By Analytical Blks (LM)

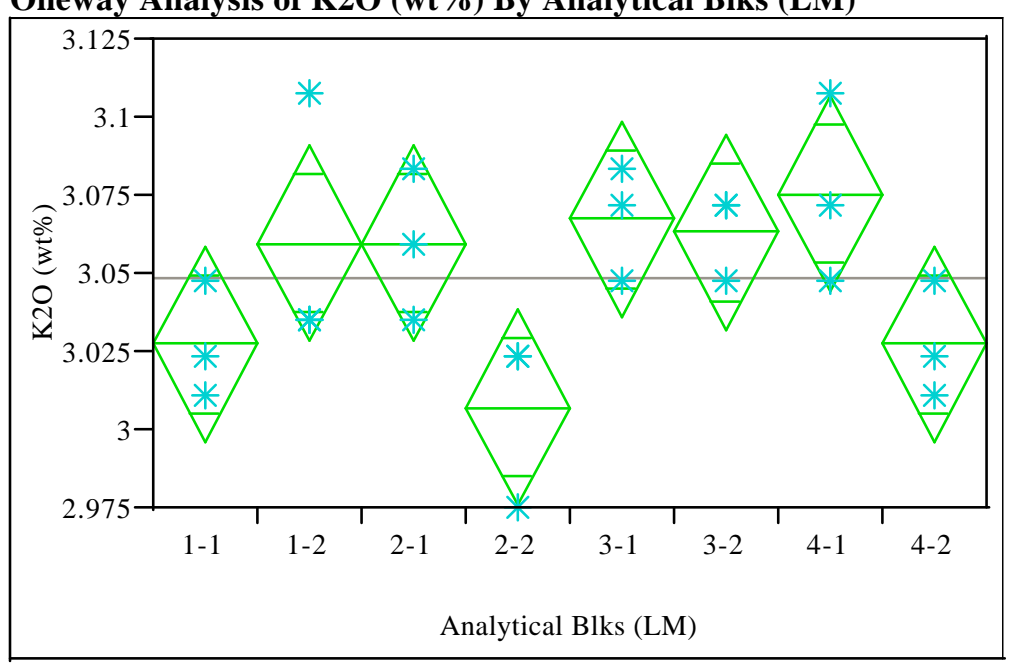

\section{Oneway Anova}

Rsquare

Root Mean Square Error $\quad 0.025553$

Mean of Response $\quad 3.048642$

Observations (or Sum Wgts)$$
24
$$

Analysis of Variance

$\begin{array}{lrrrrr}\text { Source } & \text { DF } & \text { Sum of Squares } & \text { Mean Square } & \text { F Ratio } & \text { Prob > F } \\ \text { Analytical Blks (LM) } & 7 & 0.01245494 & 0.001779 & 2.7249 & 0.0459 \\ \text { Error } & 16 & 0.01044764 & 0.000653 & & \\ \text { C. Total } & 23 & 0.02290258 & & & \end{array}$

C. Tor

Means for Oneway Anova

Level Number Mean Std Error Lower 95\% Upper 95\%

$\begin{array}{lrrrrr}\text { Level } & \text { Number } & \text { Mean } & \text { Std Error } & \text { Lower 95\% } & \text { Upper 95\% } \\ 1-1 & 3 & 3.02756 & 0.01475 & 2.9963 & 3.0588\end{array}$

$\begin{array}{llllll}1-1 & 3 & 3.02756 & 0.01475 & 2.9963 & 3.0588 \\ 1-2 & 3 & 3.05968 & 0.01475 & 3.0284 & 3.0910\end{array}$

$\begin{array}{llllll}2-1 & 3 & 3.05968 & 0.01475 & 3.0284 & 3.0910\end{array}$

$\begin{array}{llllll}2-2 & 3 & 3.00748 & 0.01475 & 2.9762 & 3.0388\end{array}$

$\begin{array}{llllll}3-1 & 3 & 3.06771 & 0.01475 & 3.0364 & 3.0990\end{array}$

$\begin{array}{llllll}3-2 & 3 & 3.06370 & 0.01475 & 3.0324 & 3.0950 \\ 4-1 & 3 & 3.07575 & 0.01475 & 3.0445 & 3.1070\end{array}$

$\begin{array}{llllll}4-1 & 3 & 3.07575 & 0.01475 & 3.0445 & 3.1070 \\ 4-2 & 3 & 3.02756 & 0.01475 & 2.9963 & 3.0588\end{array}$

Std Error uses a pooled estimate of error variance
SB3 Glass \#=101; Reference Value for La2O3 is 0 wt\%

Oneway Analysis of La2O3 (wt\%) By Analytical Blks (LM)

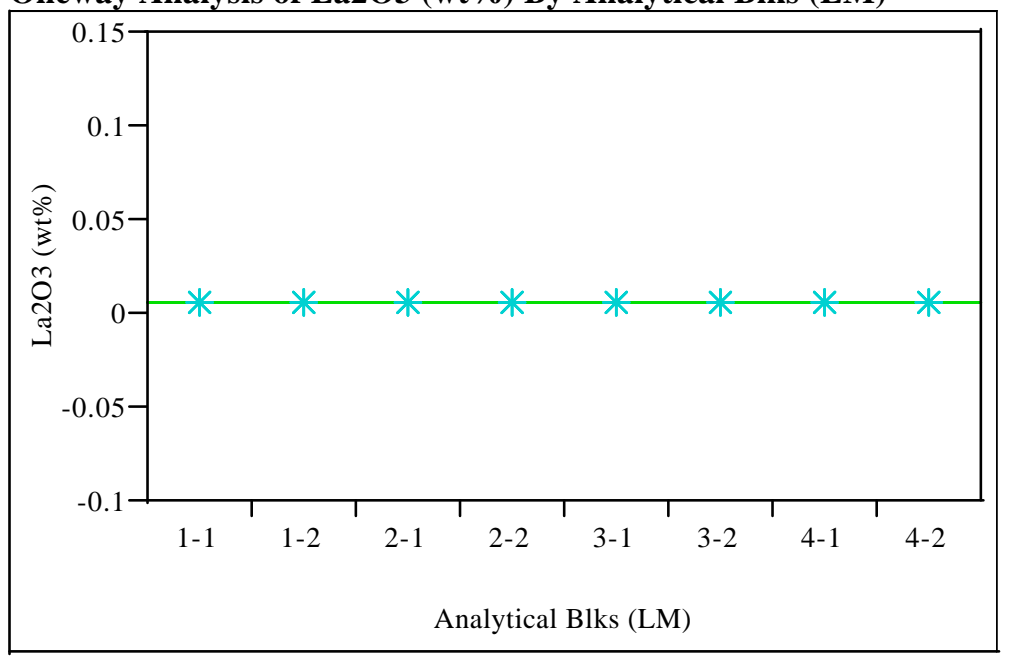

\section{Oneway Anova}

\section{Rsquare}

Root Mean Square Error

Mean of Response 0.005864

Observations (or Sum Wgts)

Analysis of Variance

Source DF Sum of Squares Mean Square F Ratio Prob $>$ F

Analytical Blks (LM)

Error

7
16
23

0

C. Total

23

0

Means for Oneway Anova

Level Number Mean Std Error Lower 95\% Upper 95\%

$\begin{array}{lllllr}1-1 & 3 & 0.005864 & 0 & 0.00586 & 0.00586\end{array}$

$\begin{array}{llllll}1-2 & 3 & 0.005864 & 0 & 0.00586 & 0.00586\end{array}$

$\begin{array}{llllll}2-1 & 3 & 0.005864 & 0 & 0.00586 & 0.00586\end{array}$

$\begin{array}{llllll}2-2 & 3 & 0.005864 & 0 & 0.00586 & 0.00586\end{array}$

$\begin{array}{llllll}3-1 & 3 & 0.005864 & 0 & 0.00586 & 0.00586\end{array}$

$\begin{array}{llllll}3-2 & 3 & 0.005864 & 0 & 0.00586 & 0.00586 \\ -1 & 3 & 0.005864 & 0 & 0.00586 & 0.00586\end{array}$

$\begin{array}{llllll}4-1 & 3 & 0.005864 & 0 & 0.00586 & 0.00586 \\ 4-2 & 3 & 0.005864 & 0 & 0.00586 & 0.00586\end{array}$

Std Error uses a pooled estimate of error variance 
Westinghouse Savannah River Company

Exhibit H.4: Statistical Analysis by Analytical Block of Measurements of Standard Glasses Prepared Using the PF Method (continued)

SB3 Glass \#=101; Reference Value for $\mathrm{MgO}$ is $1.21 \mathrm{wt} \%$

Oneway Analysis of $\mathrm{MgO}$ (wt\%) By Analytical Blks (LM)

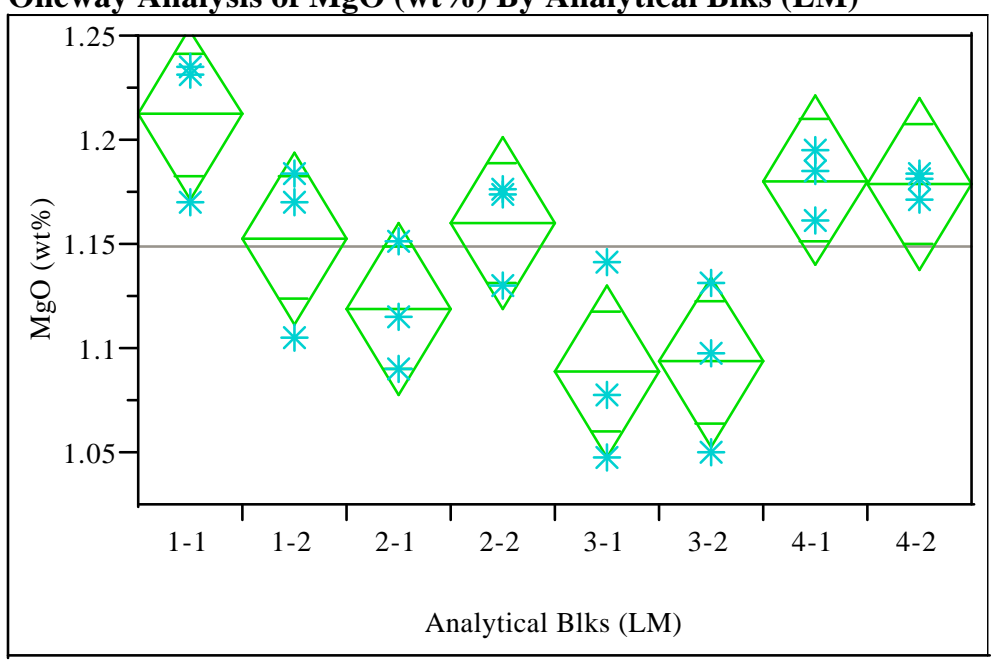

\section{Oneway Anova}

Rsquare

Root Mean Square Error $\quad 0.033564$

Mean of Response $\quad 1.148787$

Observations (or Sum Wgts)$$
24
$$

Analysis of Variance

$\begin{array}{lrrrrr}\text { Source } & \text { DF } & \text { Sum of Squares } & \text { Mean Square } & \text { F Ratio } & \text { Prob > F } \\ \text { Analytical Blks (LM) } & 7 & 0.04086370 & 0.005838 & 5.1820 & 0.0031 \\ \text { Error } & 16 & 0.01802438 & 0.001127 & & \\ \text { C. Total } & 23 & 0.05888808 & & & \end{array}$

C. Total

\section{Means for Oneway Anova}

$\begin{array}{lrrrrr}\text { Level } & \text { Number } & \text { Mean } & \text { Std Error } & \text { Lower 95\% } & \text { Upper 95\% } \\ 1-1 & 3 & 1.21262 & 0.01938 & 1.1715 & 1.2537 \\ 1-2 & 3 & 1.15348 & 0.01938 & 1.1124 & 1.1946 \\ 2-1 & 3 & 1.11977 & 0.01938 & 1.0787 & 1.1608 \\ 2-2 & 3 & 1.16067 & 0.01938 & 1.1196 & 1.2017 \\ 3-1 & 3 & 1.08937 & 0.01938 & 1.0483 & 1.1305 \\ 3-2 & 3 & 1.09379 & 0.01938 & 1.0527 & 1.1349 \\ 4-1 & 3 & 1.18112 & 0.01938 & 1.1400 & 1.2222 \\ 4-2 & 3 & 1.17946 & 0.01938 & 1.1384 & 1.2205\end{array}$

Std Error uses a pooled estimate of error variance
SB3 Glass \#=101; Reference Value for $\mathrm{MnO}$ is $\mathbf{2 . 8 9 2} \mathrm{wt} \%$

Oneway Analysis of MnO (wt\%) By Analytical Blks (LM)

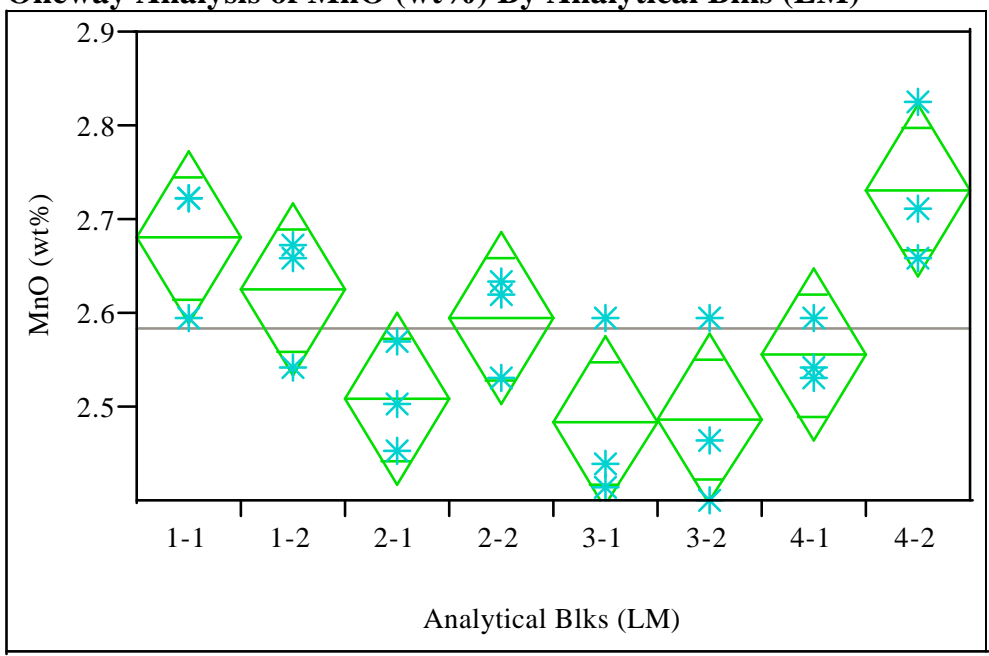

\section{Oneway Anova}

Rsquare

Root Mean Square Error

- 0.075012

Mean of Response 2.584014
24

(

Analysis of Variance

Source DF Sum of Squares Mean Square F Ratio Prob > F

$\begin{array}{lrrrrr}\text { Analytical Blks (LM) } & 7 & 0.17782745 & 0.025404 & 4.5148 & 0.0060\end{array}$

Error

0.17782745
0.09002866 0.025404
0.005627

C. Total

$23 \quad 0.26785611$

\section{Means for Oneway Anova}

Level Number Mean Std Error Lower 95\% Upper 95\%

$\begin{array}{rrrrrr}1-1 & 3 & 2.68139 & 0.04331 & 2.5896 & 2.7732\end{array}$

$\begin{array}{llllll}1-2 & 3 & 2.62544 & 0.04331 & 2.5336 & 2.7172 \\ 2-1 & 3 & 2.50923 & 0.04331 & 2.4174 & 2.6010\end{array}$

$\begin{array}{llllll}2-1 & 3 & 2.50923 & 0.04331 & 2.4174 & 2.6010 \\ 2-2 & 3 & 2.59531 & 0.04331 & 2.5035 & 2.6871\end{array}$

$\begin{array}{llllll}3-1 & 3 & 2.48341 & 0.04331 & 2.3916 & 2.5752\end{array}$

\begin{tabular}{llllll}
$3-1$ & 3 & 2.48771 & 0.04331 & 2.3959 & 2.5795 \\
\hline & 3 & 2.55658 & 0.04331 & 2.4648 & 2.6484
\end{tabular}

$\begin{array}{llllll}4-1 & 3 & 2.55658 & 0.04331 & 2.4648 & 2.6484\end{array}$

$\begin{array}{llllll}4-2 & 3 & 2.73304 & 0.04331 & 2.6412 & 2.8248\end{array}$

Std Error uses a pooled estimate of error variance 
Westinghouse Savannah River Company

Exhibit H.4: Statistical Analysis by Analytical Block of Measurements of Standard Glasses Prepared Using the PF Method (continued)

SB3 Glass \#=101; Reference Value for Na2O is $11.795 \mathrm{wt} \%$ Oneway Analysis of Na2O (wt\%) By Analytical Blks (LM)

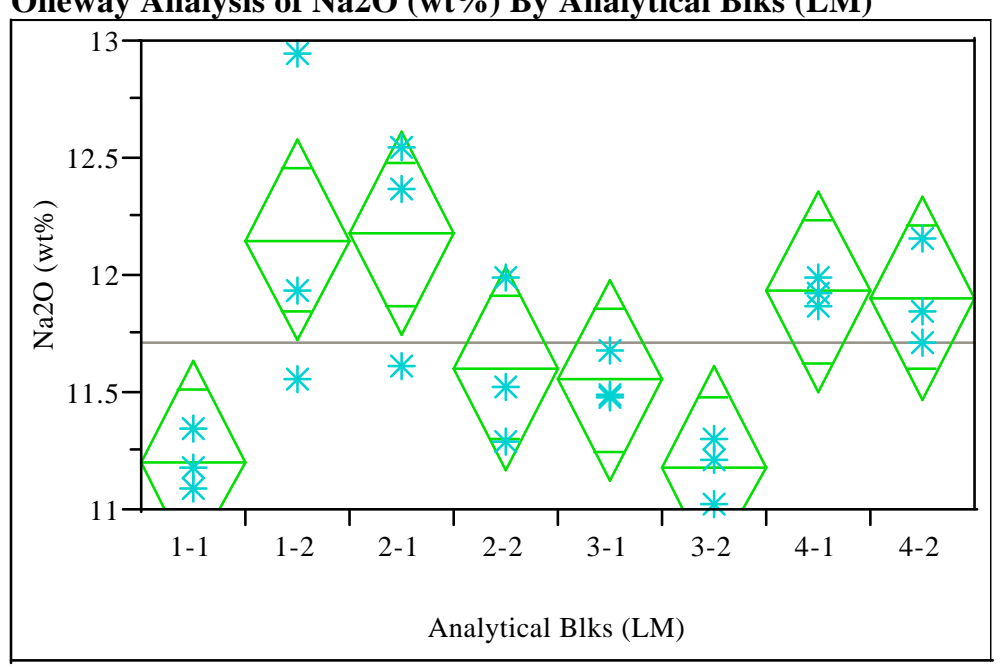

\section{Oneway Anova}

Rsquare

$\begin{array}{ll}\text { Root Mean Square Error } & 0.352397\end{array}$

Mean of Response

$$
\begin{array}{r}
11.71693 \\
24
\end{array}
$$

Analysis of Variance

Analysis of Variance

Source

Analytical Blks (LM)

Sum of Squares

$$
\text { Error }
$$$$
7 \quad 3.2063330
$$

Mean Square F Ratio Prob $>$ F

C. Total

$23 \quad 5.1932757$

$\begin{array}{lll}0.458048 & 3.6885 & 0.0145\end{array}$

0.124184

Means for Oneway Anova

$\begin{array}{lrrrrr}\text { Level } & \text { Number } & \text { Mean } & \text { Std Error } & \text { Lower 95\% } & \text { Upper 95\% } \\ 1-1 & 3 & 11.2109 & 0.20346 & 10.780 & 11.642 \\ 1-2 & 3 & 12.1545 & 0.20346 & 11.723 & 12.586 \\ 2-1 & 3 & 12.1814 & 0.20346 & 11.750 & 12.613 \\ 2-2 & 3 & 11.6063 & 0.20346 & 11.175 & 12.038 \\ 3-1 & 3 & 11.5569 & 0.20346 & 11.126 & 11.988 \\ 3-2 & 3 & 11.1839 & 0.20346 & 10.753 & 11.615 \\ 4-1 & 3 & 11.9343 & 0.20346 & 11.503 & 12.366 \\ 4-2 & 3 & 11.9073 & 0.20346 & 11.476 & 12.339\end{array}$

Std Error uses a pooled estimate of error variance
SB3 Glass \#=101; Reference Value for $\mathrm{NiO}$ is $1.12 \mathrm{wt} \%$

Oneway Analysis of $\mathrm{NiO}$ (wt\%) By Analytical Blks (LM)

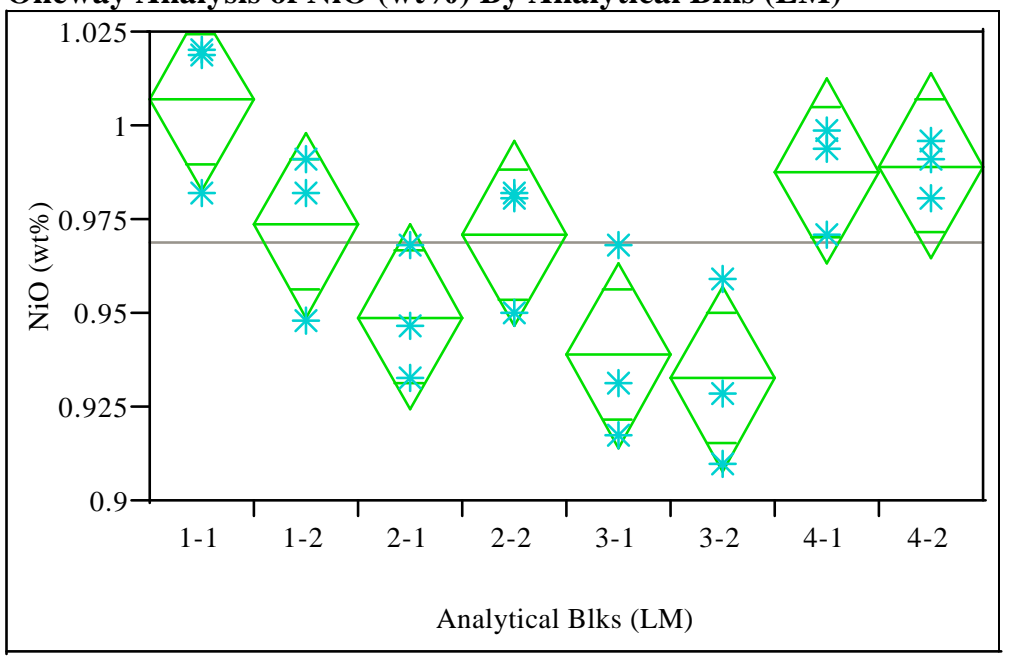

\section{Oneway Anova}

Rsquare

0.693239

Root Mean Square Error

Mean of Response

0.968903

Observations (or Sum Wgts)

Analysis of Variance

Source DF Sum of Squares Mean Square F Ratio Prob $>$ F

$\begin{array}{lrrrrr}\text { Analytical Blks (LM) } & 7 & 0.01464212 & 0.002092 & 5.1654 & 0.0032\end{array}$

Error

0.01464212 0.000405

\section{Means for Oneway Anova}

Level Number Mean Std Error Lower 95\% Upper 95\%

$\begin{array}{lrrrrr}\text { Level } & \text { Number } & \text { Mean } & \text { Std Error } & \text { Lower 95\% } & \text { Upper 95\% } \\ 1-1 & 3 & 1.00740 & 0.01162 & 0.98277 & 1.0320\end{array}$

$\begin{array}{llllll}1-1 & 3 & 1.00740 & 0.01162 & 0.98277 & 1.0320 \\ 1-2 & 3 & 0.97389 & 0.01162 & 0.94926 & 0.9985\end{array}$

$\begin{array}{llllll}2-1 & 3 & 0.94928 & 0.01162 & 0.92466 & 0.9739\end{array}$

$\begin{array}{llllll}2-2 & 3 & 0.97134 & 0.01162 & 0.94671 & 0.9960 \\ 3-1 & 3 & 0.93910 & 0.01162 & 0.91448 & 0.9637\end{array}$

$\begin{array}{llllll}3-1 & 3 & 0.93910 & 0.01162 & 0.91448 & 0.9637 \\ 3-2 & 3 & 0.93274 & 0.01162 & 0.90811 & 0.9574\end{array}$

$\begin{array}{llllll}3-2 & 3 & 0.93274 & 0.01162 & 0.90811 & 0.9574 \\ 4-1 & 3 & 0.98788 & 0.01162 & 0.96325 & 1.0125\end{array}$

$\begin{array}{llllll}4-1 & 3 & 0.98788 & 0.01162 & 0.96325 & 1.0125 \\ 4-2 & 3 & 0.98958 & 0.01162 & 0.96495 & 1.0142\end{array}$

Std Error uses a pooled estimate of error variance 
Westinghouse Savannah River Company

Exhibit H.4: Statistical Analysis by Analytical Block of Measurements of Standard Glasses Prepared Using the PF Method (continued)

SB3 Glass \#=101; Reference Value for $\mathrm{PbO}$ is $0 \mathrm{wt} \%$

Oneway Analysis of PbO (wt\%) By Analytical Blks (LM)

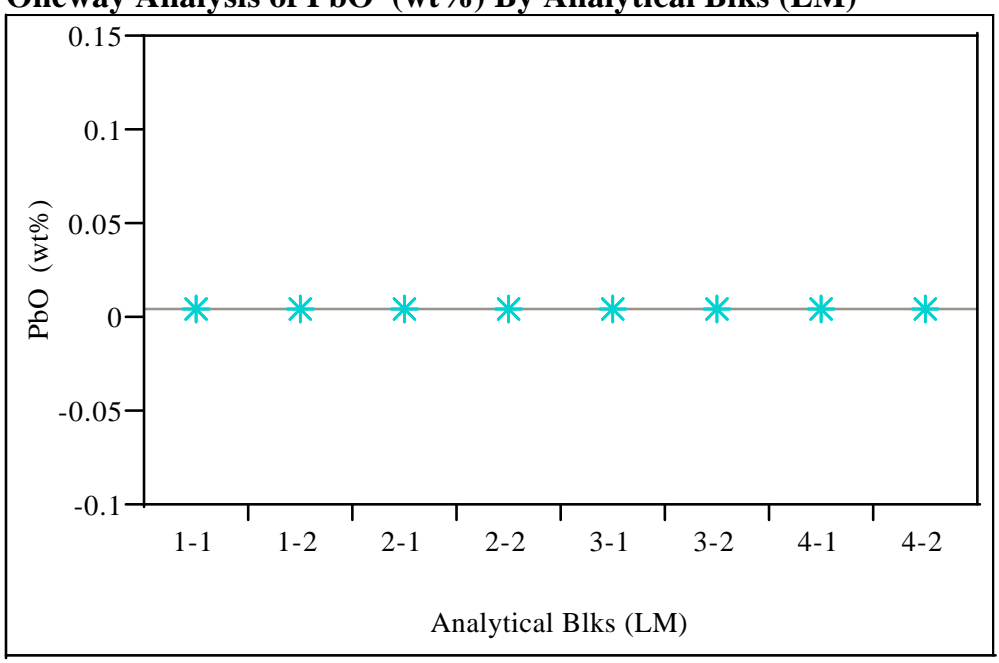

\section{Oneway Anova}

Rsquare

Root Mean Square Error

Mean of Response

4

Observations (or Sum Wgts)

0.005386

Analysis of Variance

$\begin{array}{lrrrrr}\text { Source } & \text { DF } & \text { Sum of Squares } & \text { Mean Square } & \text { F Ratio } & \text { Prob > F } \\ \text { Analytical Blks (LM) } & 7 & 7.2222 \mathrm{e}-35 & 1.032 \mathrm{e}-35 & -3.0476 & -1.0000 \\ \text { Error } & 16 & -5.417 \mathrm{e}-35 & -3.39 \mathrm{e}-36 & & \\ \text { C. Total } & 23 & 1.8056 \mathrm{e}-35 & & & \end{array}$

Means for Oneway Anova

Level Number Mean Std Error Lower 95\% Upper 95\%

$1-1 \quad 3 \quad 0.005386$

$\begin{array}{lll}1-2 & 3 & 0.005386\end{array}$

$\begin{array}{lll}2-1 & 3 & 0.005386 \\ 2-2 & 3 & 0.005386 \\ 3-1 & 3 & 0.005386\end{array}$

$\begin{array}{lll}3-1 & 3 & 0.005386\end{array}$

$\begin{array}{lll}3-2 & 3 & 0.005386\end{array}$

$\begin{array}{cccc}4-1 & 3 & 0.005386 & 0.005386\end{array}$

Std Error uses a pooled estimate of error variance
SB3 Glass \#=101; Reference Value for ThO2 is $0 \mathrm{wt} \%$

Oneway Analysis of ThO2 (wt\%) By Analytical Blks (LM)

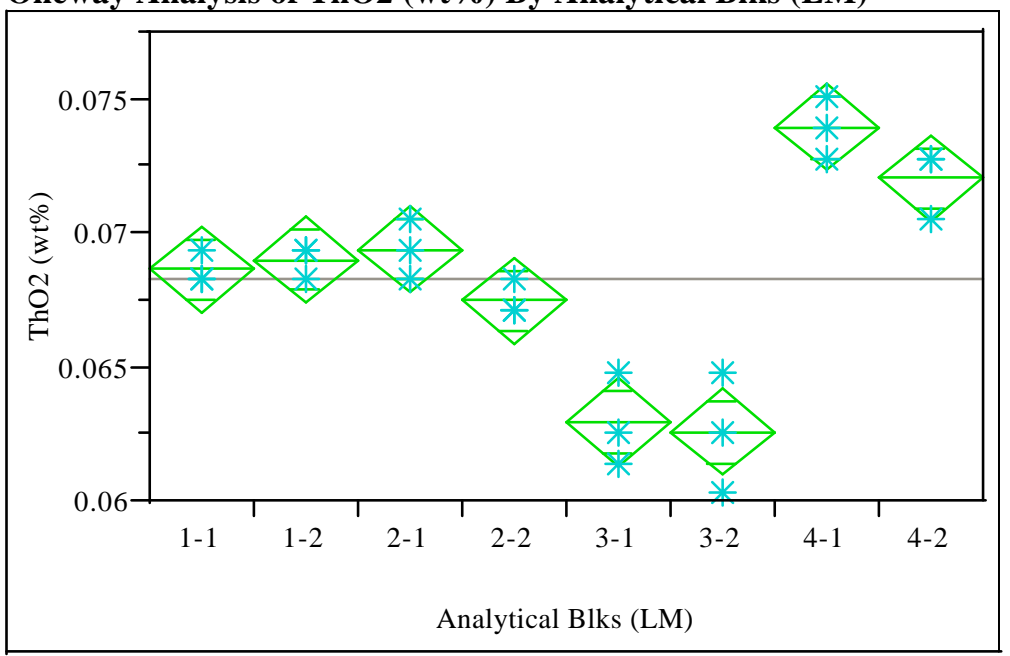

\section{Oneway Anova}

Rsquare

Root Mean Square Error

0.922705

Mean of Response

0.001314

0.068274

Observations (or Sum Wgts)

Analysis of Variance

Source $\quad$ DF Sum of Squares Mean Square $\quad$ F Ratio Prob $>$ F

$\begin{array}{lrrrrr}\text { Analytical Blks (LM) } & 7 & 0.00032975 & 0.000047 & 27.2857 & <.0001\end{array}$

Error

$\begin{array}{lll}0.000047 & 27.2857<.0001 \\ 0.000002 & & \end{array}$

C. Total

$23 \quad 0.00035737$

Means for Oneway Anova

Level Number Mean Std Error Lower 95\% Upper 95\%

$\begin{array}{lrrrrr}\text { Level } & \text { Number } & \text { Mean } & \text { Std Error } & \text { Lower 95\% } & \text { Upper 95\% } \\ 1-1 & 3 & 0.068653 & 0.00076 & 0.06705 & 0.07026\end{array}$

$\begin{array}{llllll}1-1 & 3 & 0.068653 & 0.00076 & 0.06705 & 0.07026 \\ 1-2 & 3 & 0.069033 & 0.00076 & 0.06742 & 0.07064\end{array}$

$\begin{array}{llllll}2-1 & 3 & 0.06933 & 0.00076 & 0.06742 & 0.07064 \\ 2-2 & 3 & 0.069412 & 0.00076 & 0.06780 & 0.07102\end{array}$

$\begin{array}{llllll}2-2 & 3 & 0.067515 & 0.00076 & 0.06591 & 0.06912\end{array}$

$\begin{array}{llllll}3-1 & 3 & 0.062964 & 0.00076 & 0.06136 & 0.06457\end{array}$

$\begin{array}{llllll}3-2 & 3 & 0.062585 & 0.00076 & 0.06098 & 0.06419 \\ 4-1 & 3 & 0.073964 & 0.00076 & 0.07236 & 0.07557\end{array}$

$\begin{array}{llllll}4-1 & 3 & 0.073964 & 0.00076 & 0.07236 & 0.07557 \\ 4-2 & 3 & 0.072067 & 0.00076 & 0.07046 & 0.07368\end{array}$

Std Error uses a pooled estimate of error variance 
Westinghouse Savannah River Company

\section{Exhibit H.4: Statistical Analysis by Analytical Block of Measurements of Standard Glasses Prepared Using the PF Method (continued)}

SB3 Glass \#=101; Reference Value for TiO2 is $1.049 \mathrm{wt} \%$

Oneway Analysis of TiO2 (wt\%) By Analytical Blks (LM)

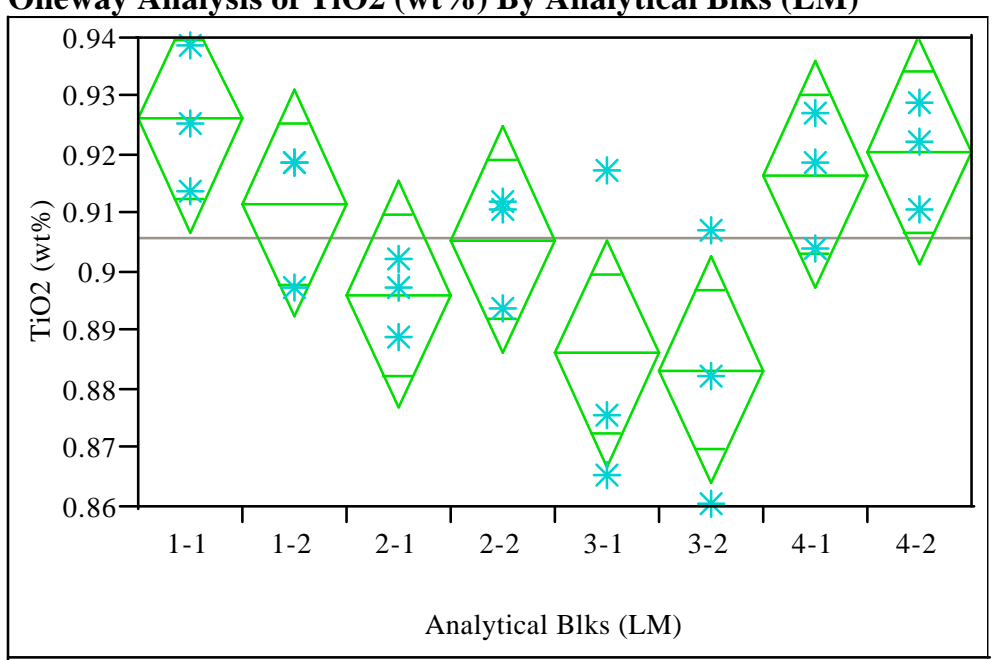

\section{Oneway Anova}

Root Mean Square Eror

0.572279

0.01575

Mean of Response

$$
\begin{array}{r}
0.905933 \\
24
\end{array}
$$

rvations (or Sum

Analysis of Variance

$\begin{array}{lrrrrr}\text { Source } & \text { DF } & \text { Sum of Squares } & \text { Mean Square } & \text { F Ratio } & \text { Prob > F } \\ \text { Analytical Blks (LM) } & 7 & 0.00531579 & 0.000759 & 3.0582 & 0.0304 \\ \text { Error } & 16 & 0.00397302 & 0.000248 & & \\ \text { C. Total } & 23 & 0.00928880 & & & \end{array}$

Means for Oneway Anova

Level Number Mean Std Error Lower 95\% Upper 95\%

$\begin{array}{rrrrrr}1-1 & 3 & 0.926296 & 0.00910 & 0.90701 & 0.94558\end{array}$

$\begin{array}{llllll}1-2 & 3 & 0.911840 & 0.00910 & 0.89255 & 0.93113\end{array}$

$\begin{array}{llllll}2-1 & 3 & 0.896272 & 0.00910 & 0.87699 & 0.91556\end{array}$

$\begin{array}{llllll}2-2 & 3 & 0.905724 & 0.00910 & 0.88644 & 0.92501\end{array}$

$\begin{array}{llllll}3-1 & 3 & 0.886264 & 0.00910 & 0.86698 & 0.90555 \\ 3-2 & 3 & 0.883484 & 0.00910 & 0.86420 & 0.90277\end{array}$

$\begin{array}{llllll}3-2 & 3 & 0.883484 & 0.00910 & 0.86420 & 0.90277 \\ 4-1 & 3 & 0.916844 & 0.00910 & 0.89756 & 0.93613\end{array}$

$\begin{array}{llllll}4-2 & 3 & 0.920736 & 0.00910 & 0.90145 & 0.94002\end{array}$

Std Error uses a pooled estimate of error variance
SB3 Glass \#=101; Reference Value for U308 is $2.406 \mathrm{wt} \%$

Oneway Analysis of U3O8 (wt\%) By Analytical Blks (LM)

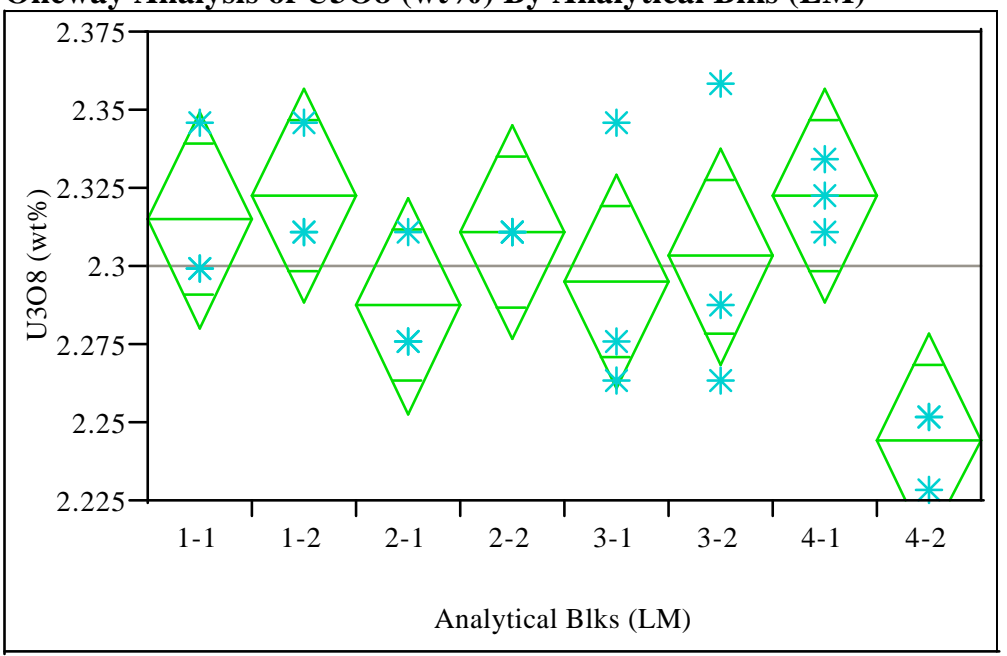

\section{Oneway Anova}

Rsquare

Root Mean Square Error

Mean of Response

0.028071
2.300423

Observations (or Sum Wgts)

Analysis of Variance

Source DF Sum of Squares Mean Square F Ratio Prob $>$ F

$\begin{array}{lrrrrr}\text { Analytical Blks (LM) } & 7 & 0.01406735 & 0.002010 & 2.5504 & 0.0573\end{array}$

Error 0.000788

C. Total

$23 \quad 0.02667467$

\section{Means for Oneway Anova}

Level Number Mean Std Error Lower 95\% Upper 95\%

$\begin{array}{rrrrrr}1-1 & 3 & 2.31516 & 0.01621 & 2.2808 & 2.3495\end{array}$

$\begin{array}{llllll}1-2 & 3 & 2.32302 & 0.01621 & 2.2887 & 2.3574 \\ 2-1 & 3 & 2.28765 & 0.01621 & 2.2533 & 2.3220\end{array}$

$\begin{array}{llllll}2-1 & 3 & 2.28765 & 0.01621 & 2.2533 & 2.3220\end{array}$

$\begin{array}{llllll}2-2 & 3 & 2.31123 & 0.01621 & 2.2769 & 2.3456 \\ 3-1 & 3 & 2.29551 & 0.01621 & 2.2612 & 2.3299\end{array}$

$\begin{array}{llllll}3-1 & 3 & 2.29551 & 0.01621 & 2.2612 & 2.3299 \\ 3-2 & 3 & 2.30337 & 0.01621 & 2.2690 & 2.3377\end{array}$

$\begin{array}{llllll}3-2 & 3 & 2.30337 & 0.01621 & 2.2690 & 2.3377 \\ 4-1 & 3 & 2.32302 & 0.01621 & 2.2887 & 2.3574\end{array}$

$\begin{array}{llllll}4-2 & 3 & 2.24441 & 0.01621 & 2.2101 & 2.2788\end{array}$

Std Error uses a pooled estimate of error variance 
Westinghouse Savannah River Company

Exhibit H.4: Statistical Analysis by Analytical Block of Measurements of Standard Glasses Prepared Using the PF Method (continued)

SB3 Glass \#=101; Reference Value for $\mathrm{ZnO}$ is $0 \mathrm{wt} \%$

Oneway Analysis of ZnO (wt \%) By Analytical Blks (LM)

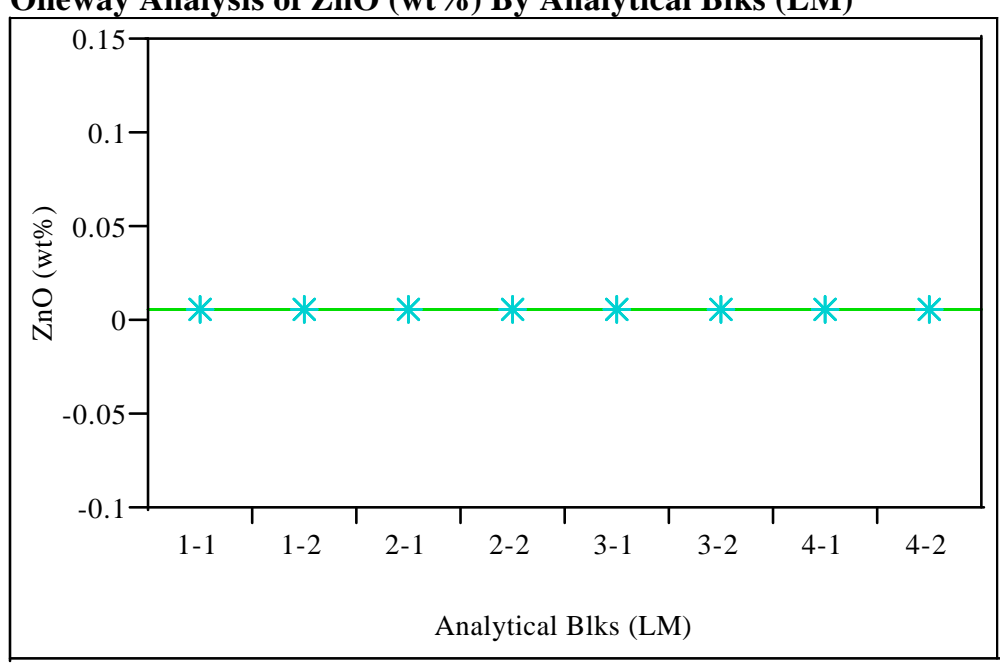

\section{Oneway Anova}

Rsquare

Root Mean Square Error

Mean of Response

Observations (or Sum Wgts)

$$
24
$$

Analysis of Variance

Source DF Sum of Squares Mean Square F Ratio Prob $>$ F

$\begin{array}{rrrrr}7 & \text { Sum of Squares } & \text { Mean Square } & \text { F Ratio } & \text { Prob }>\text { F } \\ 0 & 0 & . & \\ 0 & 0 & & \end{array}$

23

Means for Oneway Anova

Level Number Mean Std Error Lower 95\% Upper 95\%

$\begin{array}{llllll}1-1 & 3 & 0.006224 & 0 & 0.00622 & 0.00622 \\ 1-2 & 3 & 0.006224 & 0 & 0.00622 & 0.00622\end{array}$

$\begin{array}{llllll}2-1 & 3 & 0.006224 & 0 & 0.00622 & 0.00622\end{array}$

$\begin{array}{llllll}2-2 & 3 & 0.006224 & 0 & 0.00622 & 0.00622\end{array}$

$\begin{array}{llllll}3-1 & 3 & 0.006224 & 0 & 0.00622 & 0.00622\end{array}$

$\begin{array}{llllll}3-2 & 3 & 0.006224 & 0 & 0.00622 & 0.00622 \\ 4-1 & 3 & 0.006224 & 0 & 0.00622 & 0.00622\end{array}$

$\begin{array}{llllll}4-1 & 3 & 0.006224 & 0 & 0.00622 & 0.00622 \\ 4-2 & 3 & 0.006224 & 0 & 0.00622 & 0.00622\end{array}$

Std Error uses a pooled estimate of error variance
SB3 Glass \#=101; Reference Value for $\mathrm{ZrO2}$ is $0 \mathrm{wt} \%$

Oneway Analysis of ZrO2 (wt\%) By Analytical Blks (LM)

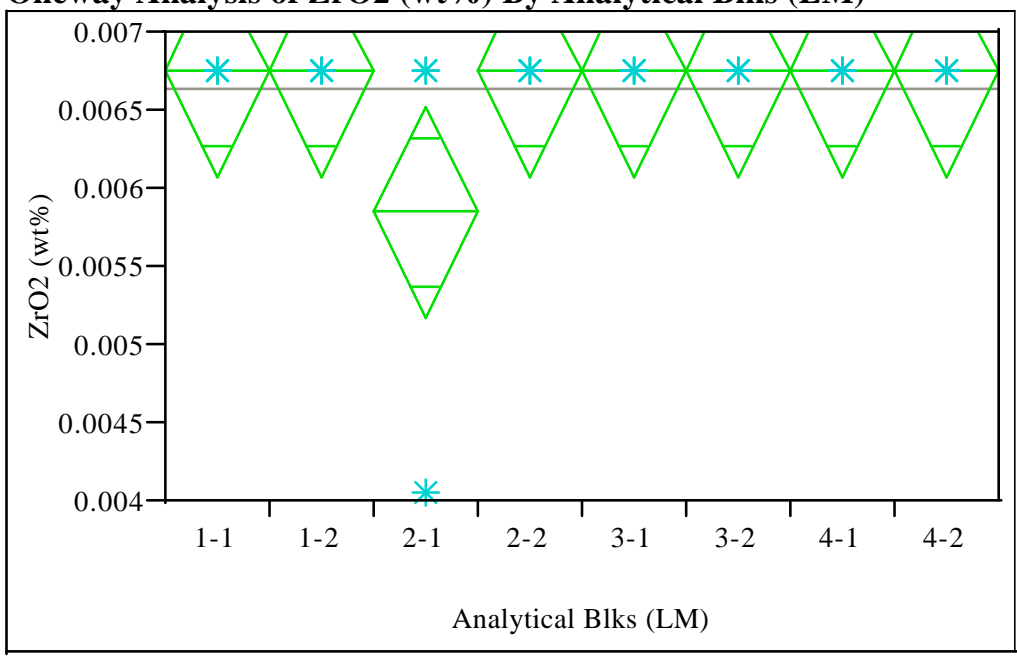

\section{Oneway Anova}

\section{Rsquare}

Root Mean Square Erro

Mean of Response

0.304348

0.000551

0.006641

Observations (or Sum Wgts)

Analysis of Variance

Source DF Sum of Squares Mean Square F Ratio Prob $>$ F

$\begin{array}{llrrrr}\text { Analytical Blks (LM) } & 7 & 0.00000213 & 3.0411 \mathrm{e}-7 & 1.0000 & 0.4663\end{array}$

Error

0.00000213 $3.0411 \mathrm{e}-7$

C. Total

$23 \quad 0.00000699$

Means for Oneway Anova

Level Number Mean Std Error Lower 95\% Upper 95\%

$\begin{array}{rrrrrr}1-1 & 3 & 0.006754 & 0.00032 & 0.00608 & 0.00743\end{array}$

$\begin{array}{llllll}1-2 & 3 & 0.006754 & 0.00032 & 0.00608 & 0.00743 \\ 2-1 & 3 & 0.005853 & 0.00032 & 0.00518 & 0.00653\end{array}$

$\begin{array}{llllll}2-1 & 3 & 0.005853 & 0.00032 & 0.00518 & 0.00653\end{array}$

$\begin{array}{llllll}2-2 & 3 & 0.006754 & 0.00032 & 0.00608 & 0.00743 \\ 3-1 & 3 & 0.006754 & 0.00032 & 0.00608 & 0.00743\end{array}$

$\begin{array}{llllll}3-1 & 3 & 0.006754 & 0.00032 & 0.00608 & 0.00743 \\ 3-2 & 3 & 0.006754 & 0.00032 & 0.00608 & 0.00743\end{array}$

$\begin{array}{llllll}3-2 & 3 & 0.006754 & 0.00032 & 0.00608 & 0.00743 \\ 4-1 & 3 & 0.006754 & 0.00032 & 0.00608 & 0.00743\end{array}$

$\begin{array}{llllll}4-1 & 3 & 0.006754 & 0.00032 & 0.00608 & 0.00743 \\ 4-2 & 3 & 0.006754 & 0.00032 & 0.00608 & 0.00743\end{array}$

Std Error uses a pooled estimate of error variance 
Westinghouse Savannah River Company

\section{Exhibit H.4: Statistical Analysis by Analytical Block of Measurements of Standard Glasses Prepared Using the PF Method (continued)}

SB3 Glass \#=100; Reference Value for Al2O3 is $4.877 \mathrm{wt} \%$ Oneway Analysis of Al2O3 (wt\%) By Analytical Block

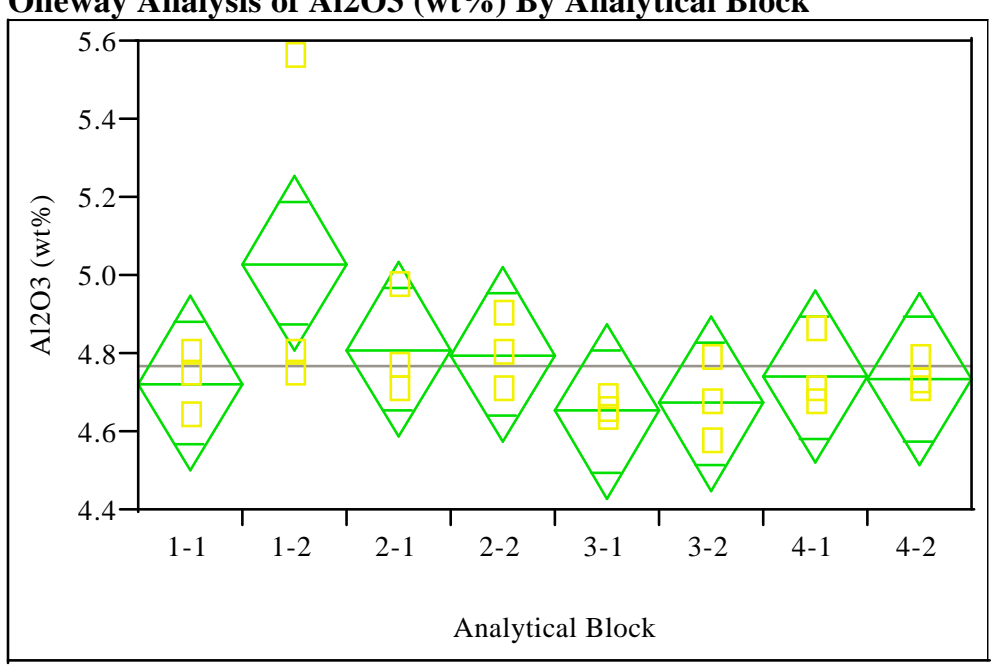

\section{Oneway Anova}

Root Mean Square Error $\quad 0.181786$

Mean of Response

Observations (or Sum Wgts)

24

Analysis of Variance

$\begin{array}{lrrrrr}\text { Source } & \text { DF } & \text { Sum of Squares } & \text { Mean Square } & \text { F Ratio } & \text { Prob > F } \\ \text { Analytical Block } & 7 & 0.29397890 & 0.041997 & 1.2709 & 0.3244 \\ \text { Error } & 16 & 0.52873805 & 0.033046 & & \\ \text { C. Total } & 23 & 0.82271694 & & & \end{array}$

\section{Means for Oneway Anova}

Level Number Mean Std Error Lower 95\% Upper 95\%

$\begin{array}{rrrrrr}1-1 & 3 & 4.72526 & 0.10495 & 4.5028 & 4.9478\end{array}$

$\begin{array}{llllll}1-2 & 3 & 5.03237 & 0.10495 & 4.8099 & 5.2549\end{array}$

$\begin{array}{llllll}2-1 & 3 & 4.81193 & 0.10495 & 4.5894 & 5.0344\end{array}$

$\begin{array}{llllll}2-2 & 3 & 4.79933 & 0.10495 & 4.5768 & 5.0218\end{array}$

$\begin{array}{llllll}3-1 & 3 & 4.65447 & 0.10495 & 4.4320 & 4.8770 \\ 3-2 & 3 & 4.67336 & 0.10495 & 4.4509 & 4.8959\end{array}$

$\begin{array}{llllll}3-2 & 3 & 4.67336 & 0.10495 & 4.4509 & 4.8959 \\ 4-1 & 3 & 4.74264 & 0.10495 & 4.5202 & 4.9651\end{array}$

$\begin{array}{llllll}4-1 & 3 & 4.74264 & 0.10495 & 4.5202 & 4.9651 \\ 4-2 & 3 & 4.73635 & 0.10495 & 4.5139 & 4.9588\end{array}$

Std Error uses a pooled estimate of error variance
SB3 Glass \#=100; Reference Value for B2O3 is $7.777 \mathrm{wt} \%$

Oneway Analysis of B2O3 (wt\%) By Analytical Block

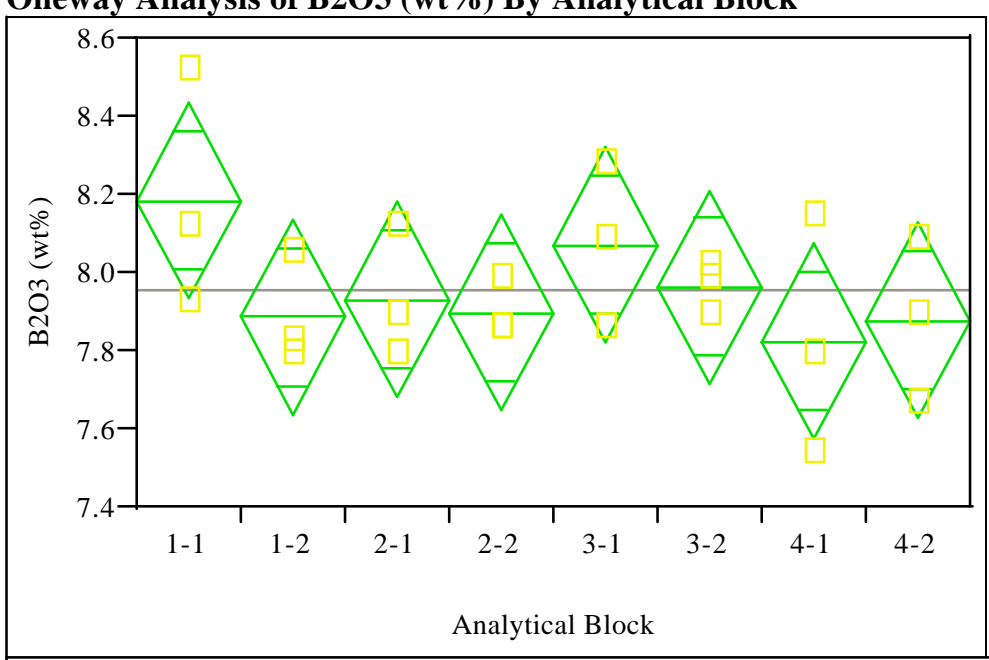

\section{Oneway Anova}

Rsquare

Root Mean Square Error

0.305087

Mean of Response

0.203631

7.955192

Observations (or Sum Wgts)

Analysis of Variance

Source DF Sum of Squares Mean Square F Ratio Prob $>$ F

$\begin{array}{lrrrrr}\text { Analytical Block } & 7 & 0.29127229 & 0.041610 & 1.0035 & 0.4642\end{array}$

0.29127229

0.041465

C. Total

$23 \quad 0.95471910$

\section{Means for Oneway Anova}

Level Number Mean Std Error Lower 95\% Upper 95\%

$\begin{array}{llllrr}1-1 & 3 & 8.18413 & 0.11757 & 7.9349 & 8.4334\end{array}$

$\begin{array}{llllll}1-2 & 3 & 7.88875 & 0.11757 & 7.6395 & 8.1380 \\ 2-1 & 3 & 7.93169 & 0.11757 & 7.6825 & 8.1809\end{array}$

$\begin{array}{llllll}2-1 & 3 & 7.93169 & 0.11757 & 7.6825 & 8.1809\end{array}$

$\begin{array}{llllll}2-2 & 3 & 7.89949 & 0.11757 & 7.6503 & 8.1487\end{array}$

$\begin{array}{llllll}3-1 & 3 & 8.07122 & 0.11757 & 7.8220 & 8.3204 \\ 3-2 & 3 & 7.96389 & 0.11757 & 7.7147 & 8.2131\end{array}$

$\begin{array}{llllll}3-2 & 3 & 7.96389 & 0.11757 & 7.7147 & 8.2131 \\ 4-1 & 3 & 7.82436 & 0.11757 & 7.5751 & 8.0736\end{array}$

$\begin{array}{llllll}4-1 & 3 & 7.82436 & 0.11757 & 7.5751 & 8.0736 \\ 4-2 & 3 & 7.87802 & 0.11757 & 7.6288 & 8.1273\end{array}$

Std Error uses a pooled estimate of error variance 
Westinghouse Savannah River Company

\section{Exhibit H.4: Statistical Analysis by Analytical Block of Measurements of Standard Glasses Prepared Using the PF Method (continued)}

SB3 Glass \#=100; Reference Value for Li2O is $4.429 \mathrm{wt} \%$ Oneway Analysis of Li2O (wt\%) By Analytical Block

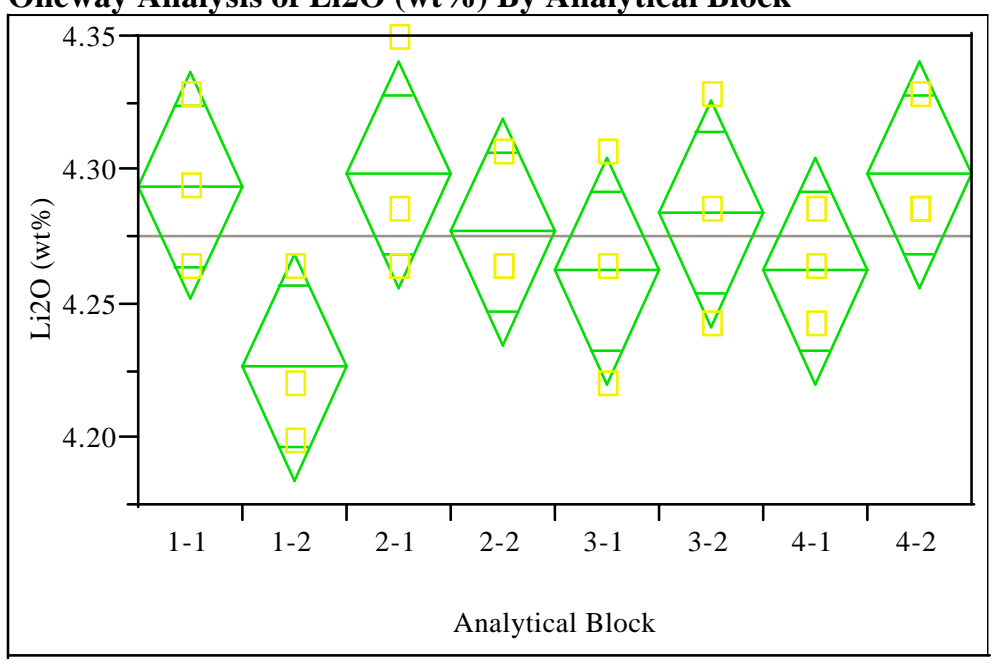

\section{Oneway Anova}

$\begin{array}{ll}\text { Rsquare } & 0.397185 \\ \text { Root Mean Square Error } & 0.034537\end{array}$

Mean of Response

$$
\begin{array}{r}
4.27565 \\
24
\end{array}
$$

Observations (or Sum Wgts)

Analysis of Variance

$\begin{array}{lrrrrr}\text { Source } & \text { DF } & \text { Sum of Squares } & \text { Mean Square } & \text { F Ratio } & \text { Prob > F } \\ \text { Analytical Block } & 7 & 0.01257440 & 0.001796 & 1.5060 & 0.2346\end{array}$

$\begin{array}{lrr}\text { Analytical Block } & 7 & 0.01257440 \\ \text { Error } & 16 & 0.01908440\end{array}$

$23 \quad 0.03165880$

0.001193

\section{Means for Oneway Anova}

Level Number Mean Std Error Lower 95\% Upper 95\%

$\begin{array}{rrrrrr}1-1 & 3 & 4.29425 & 0.01994 & 4.2520 & 4.3365\end{array}$

$\begin{array}{llllll}1-2 & 3 & 4.22686 & 0.01994 & 4.1846 & 4.2691\end{array}$

$\begin{array}{llllll}2-1 & 3 & 4.29862 & 0.01994 & 4.2564 & 4.3409\end{array}$

$\begin{array}{llllll}2-2 & 3 & 4.27709 & 0.01994 & 4.2348 & 4.3194\end{array}$

$\begin{array}{llllll}3-1 & 3 & 4.26274 & 0.01994 & 4.2205 & 4.3050\end{array}$

$\begin{array}{llllll}3-2 & 3 & 4.28427 & 0.01994 & 4.2420 & 4.3265 \\ 4-1 & 3 & 4.26274 & 0.01994 & 4.2205 & 4.3050\end{array}$

$\begin{array}{llllll}4-1 & 3 & 4.26274 & 0.01994 & 4.2205 & 4.3050 \\ 4-2 & 3 & 4.29862 & 0.01994 & 4.2564 & 4.3409\end{array}$

Std Error uses a pooled estimate of error variance
SB3 Glass \#=100; Reference Value for SiO2 is $50.22 \mathrm{wt} \%$

Oneway Analysis of $\mathrm{SiO} 2$ (wt\%) By Analytical Block

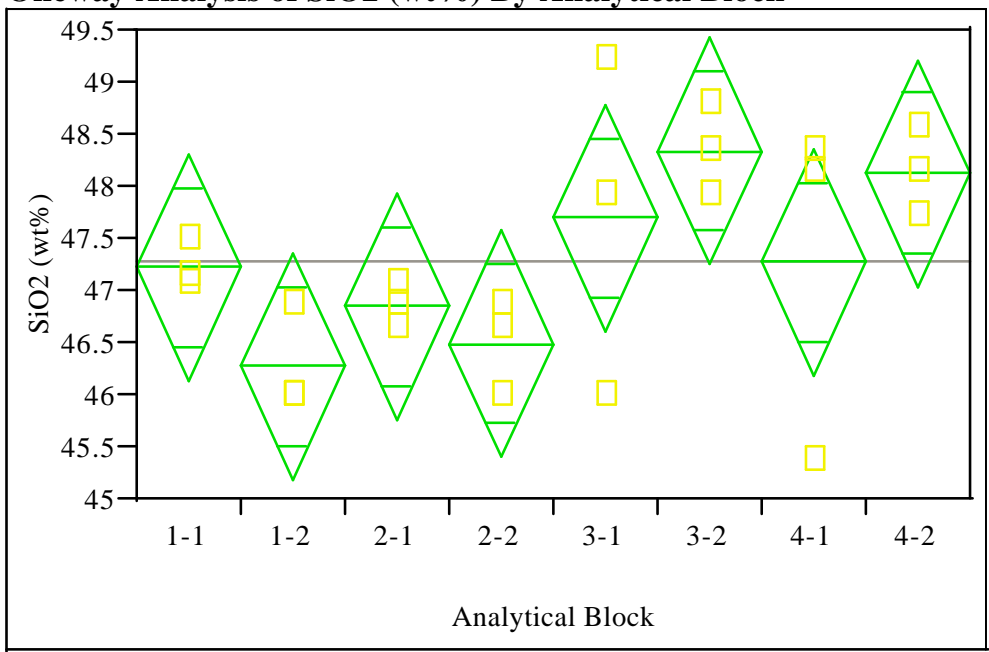

\section{Oneway Anova}

Rsquare

Root Mean Square Error

Square Error

Mean of Response

$$
\begin{array}{r}
47.29021 \\
24
\end{array}
$$

Analysis of Varianc

Source DF Sum of Squares Mean Square F Ratio Prob > F

$\begin{array}{lrrrrr}\text { Source } & \text { DF } & \text { Sum of Squares } & \text { Mean Square } & \text { F Ratio } & \text { Prob > F } \\ \text { Analytical Block } & 7 & 11.567545 & 1.65251 & 2.0959 & 0.1044\end{array}$

Anty

11.567545
12.615444

0.78847

C. Total

$23 \quad 24.182988$

\section{Means for Oneway Anova}

Level Number Mean Std Error Lower 95\% Upper 95\%

$\begin{array}{rrrrrr}1-1 & 3 & 47.2293 & 0.51266 & 46.143 & 48.316\end{array}$

$\begin{array}{llllll}1-2 & 3 & 46.2802 & 0.51266 & 45.193 & 47.367\end{array}$

$\begin{array}{llllll}2-1 & 3 & 46.8507 & 0.51266 & 45.764 & 47.937\end{array}$

$\begin{array}{llllll}2-2 & 3 & 46.4941 & 0.51266 & 45.407 & 47.581\end{array}$

$\begin{array}{llllll}3-1 & 3 & 47.7064 & 0.51266 & 46.620 & 48.793 \\ 3-2 & 3 & 48.3482 & 0.51266 & 47.261 & 49.435\end{array}$

$\begin{array}{llllll}3-2 & 3 & 48.3482 & 0.51266 & 47.261 & 49.435 \\ 4-1 & 3 & 47.2785 & 0.51266 & 46.192 & 48.365\end{array}$

$\begin{array}{llllll}4-1 & 3 & 47.2785 & 0.51266 & 46.192 & 48.365 \\ 4-2 & 3 & 48.1343 & 0.51266 & 47.047 & 49.221\end{array}$

Std Error uses a pooled estimate of error variance 
Westinghouse Savannah River Company

\section{Exhibit H.4: Statistical Analysis by Analytical Block of Measurements of Standard Glasses Prepared Using the PF Method (continued)}

SB3 Glass \#=101; Reference Value for Al203 is $4.1 \mathrm{wt} \%$ Oneway Analysis of Al2O3 (wt\%) By Analytical Block

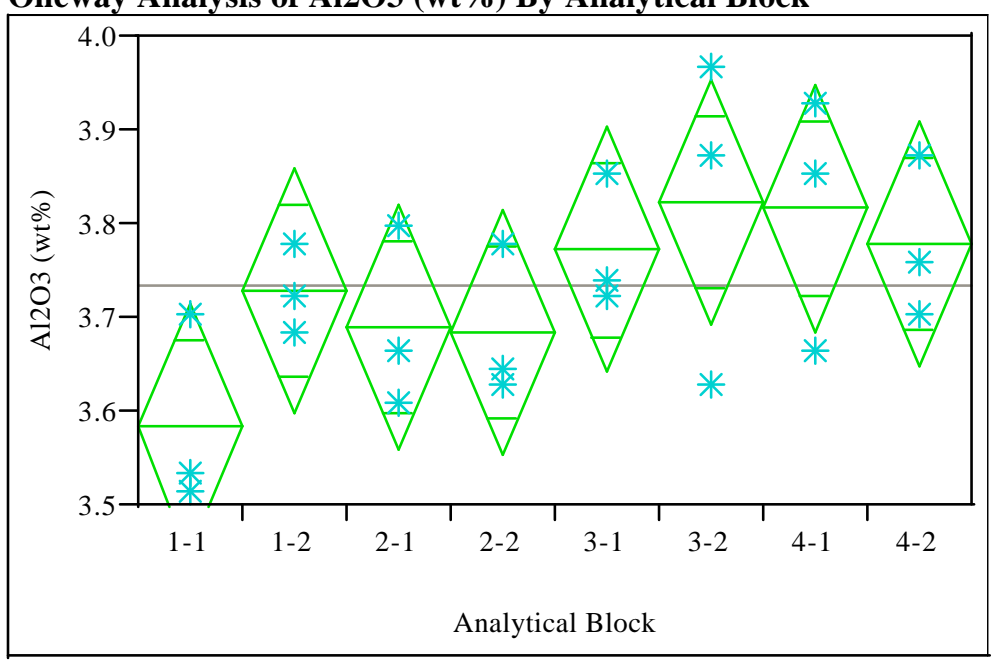

\section{Oneway Anova}

Root Mean Square Erro

0.426328

Mean of Response

0.106817

3.734912

Observations (or Sum Wgts)

24

Analysis of Variance

$\begin{array}{lrrrrr}\text { Source } & \text { DF } & \text { Sum of Squares } & \text { Mean Square } & \text { F Ratio } & \text { Prob > F } \\ \text { Analytical Block } & 7 & 0.13566799 & 0.019381 & 1.6986 & 0.1797\end{array}$

Error

$\begin{array}{ll}16 & 0.18255675 \\ 23 & 0.31822474\end{array}$

0.011410

\section{Means for Oneway Anova}

Level Number Mean Std Error Lower 95\% Upper 95\%

$\begin{array}{lrrrrr}1-1 & 3 & 3.58375 & 0.06167 & 3.4530 & 3.7145\end{array}$

$\begin{array}{llllll}1-2 & 3 & 3.72861 & 0.06167 & 3.5979 & 3.8593\end{array}$

$\begin{array}{llllll}2-1 & 3 & 3.69082 & 0.06167 & 3.5601 & 3.8216\end{array}$

$\begin{array}{llllll}2-2 & 3 & 3.68452 & 0.06167 & 3.5538 & 3.8153 \\ 3-1 & 3 & 3.77270 & 0.06167 & 3.6420 & 3.9034\end{array}$

$\begin{array}{llllll}3-1 & 3 & 3.77270 & 0.06167 & 3.6420 & 3.9034 \\ 3-2 & 3 & 3.82309 & 0.06167 & 3.6924 & 3.9538\end{array}$

$\begin{array}{llllll}3-2 & 3 & 3.82309 & 0.06167 & 3.6924 & 3.9538 \\ 4-1 & 3 & 3.81679 & 0.06167 & 3.6861 & 3.9475\end{array}$

$\begin{array}{llllll}4-1 & 3 & 3.81679 & 0.06167 & 3.6861 & 3.9475 \\ 4-2 & 3 & 3.77900 & 0.06167 & 3.6483 & 3.9097\end{array}$

Std Error uses a pooled estimate of error variance
SB3 Glass \#=101; Reference Value for B2O3 is $9.209 \mathrm{wt} \%$

Oneway Analysis of B2O3 (wt\%) By Analytical Block

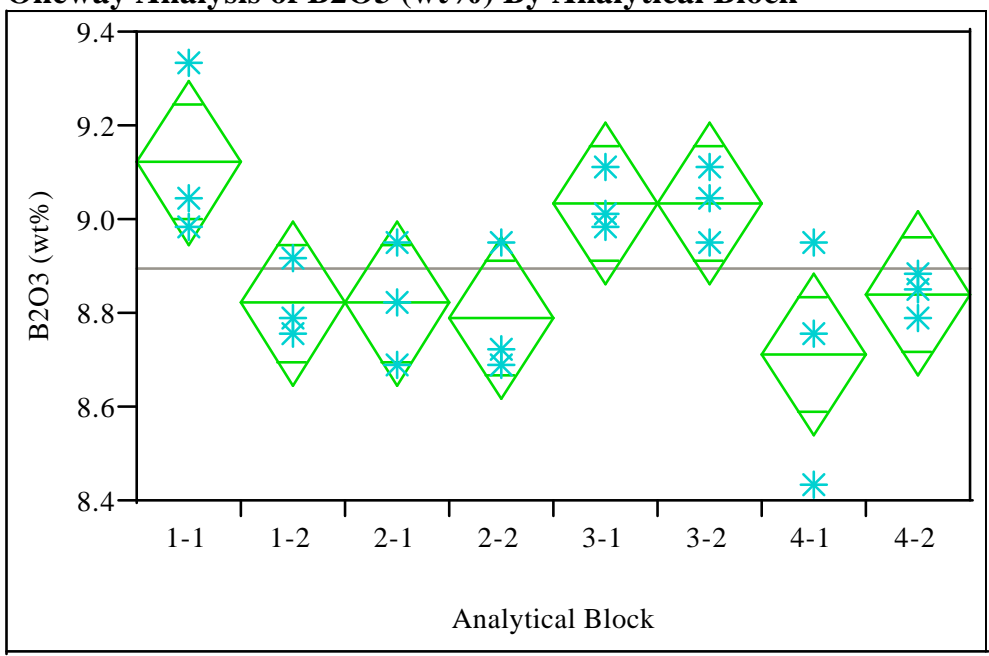

\section{Oneway Anova}

\section{Rsquare}

Root Mean Square Error

Mean of Response

0.58177

0.141578 8.898999

Observations (or Sum Wgts)

Analysis of Variance

Source DF Sum of Squares Mean Square F Ratio Prob $>$ F

$\begin{array}{lrrrrr}\text { Analytical Block } & 7 & 0.44611590 & 0.063731 & 3.1795 & 0.0262\end{array}$

0.44611590 0.020044

C. Total

$23 \quad 0.76682515$

\section{Means for Oneway Anova}

Level Number Mean Std Error Lower 95\% Upper 95\%

$\begin{array}{llllll}1-1 & 3 & 9.12305 & 0.08174 & 8.9498 & 9.2963\end{array}$

$\begin{array}{llllll}1-2 & 3 & 8.82253 & 0.08174 & 8.6492 & 8.9958 \\ 2-1 & 3 & 8.82253 & 0.08174 & 8.6492 & 8.9958\end{array}$

$\begin{array}{llllll}2-1 & 3 & 8.82253 & 0.08174 & 8.6492 & 8.9958\end{array}$

$\begin{array}{llllll}2-2 & 3 & 8.79033 & 0.08174 & 8.6170 & 8.9636 \\ 3-1 & 3 & 9.03719 & 0.08174 & 8.8639 & 9.2105\end{array}$

$\begin{array}{llllll}3-1 & 3 & 9.03719 & 0.08174 & 8.8639 & 9.2105 \\ 3-2 & 3 & 9.03719 & 0.08174 & 8.8639 & 9.2105\end{array}$

$\begin{array}{llllll}3-2 & 3 & 9.03719 & 0.08174 & 8.8639 & 9.2105 \\ 4-1 & 3 & 8.71520 & 0.08174 & 8.5419 & 8.8885\end{array}$

$\begin{array}{llllll}4-1 & 3 & 8.71520 & 0.08174 & 8.5419 & 8.8885 \\ 4-2 & 3 & 8.84399 & 0.08174 & 8.6707 & 9.0173\end{array}$

Std Error uses a pooled estimate of error variance 
Westinghouse Savannah River Company

\section{Exhibit H.4: Statistical Analysis by Analytical Block of Measurements of Standard Glasses Prepared Using the PF Method (continued)}

SB3 Glass \#=101; Reference Value for Li2O is $3.057 \mathrm{wt} \%$ Oneway Analysis of Li2O (wt\%) By Analytical Block

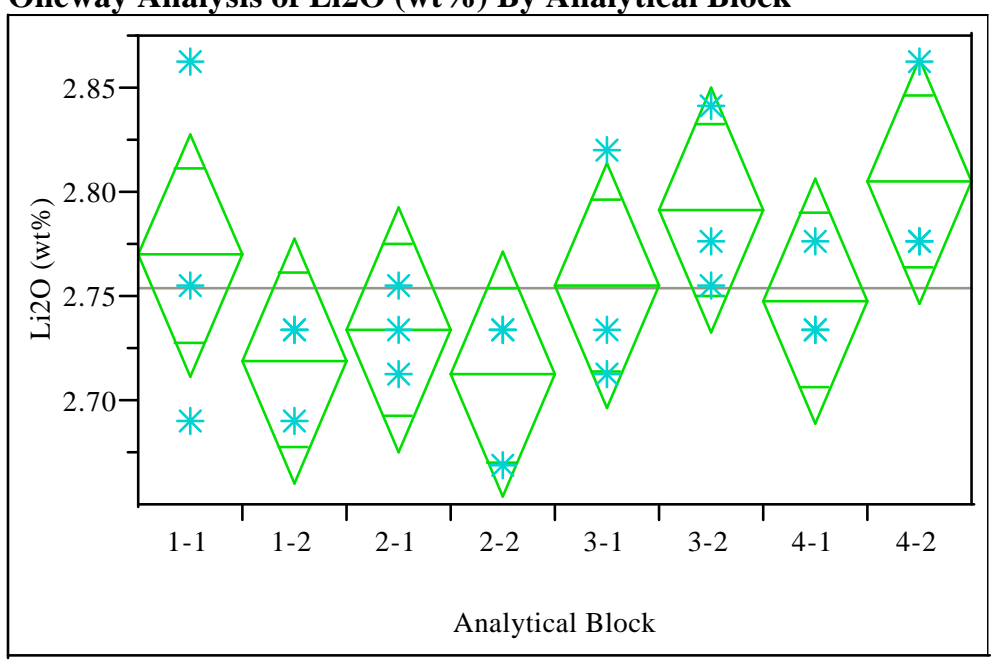

\section{Oneway Anova}

Root Mean Square Error

\subsection{4}

0.047939

Mean of Response

$$
\begin{array}{r}
2.754815 \\
24
\end{array}
$$

Analysis of Varia

$\begin{array}{lrrrrr}\text { Analysis of Variance } & & & \\ \text { Source } & \text { DF } & \text { Sum of Squares } & \text { Mean Square } & \text { F Ratio } & \text { Prob > F } \\ \text { Analytical Block } & 7 & 0.02300108 & 0.003286 & 1.4298 & 0.2607 \\ \text { Error } & 16 & 0.03677083 & 0.002298 & & \\ \text { C. Total } & 23 & 0.05977191 & & & \end{array}$

C. $23-0.05977191$

\section{Means for Oneway Anova}

Level Number Mean Std Error Lower 95\% Upper 95\%

$\begin{array}{rrrrrr}1-1 & 3 & 2.77006 & 0.02768 & 2.7114 & 2.8287\end{array}$

$\begin{array}{llllll}1-2 & 3 & 2.71983 & 0.02768 & 2.6612 & 2.7785\end{array}$

$\begin{array}{llllll}2-1 & 3 & 2.73418 & 0.02768 & 2.6755 & 2.7929\end{array}$

$\begin{array}{llllll}2-2 & 3 & 2.71265 & 0.02768 & 2.6540 & 2.7713\end{array}$

$\begin{array}{llllll}3-1 & 3 & 2.75571 & 0.02768 & 2.6970 & 2.8144 \\ 3-2 & 3 & 2.79159 & 0.02768 & 2.7329 & 2.8503\end{array}$

$\begin{array}{llllll}3-2 & 3 & 2.79159 & 0.02768 & 2.7329 & 2.8503 \\ 4-1 & 3 & 2.74854 & 0.02768 & 2.6899 & 2.8072\end{array}$

$\begin{array}{llllll}4-2 & 3 & 2.80595 & 0.02768 & 2.7473 & 2.8646\end{array}$

Std Error uses a pooled estimate of error variance
SB3 Glass \#=101; Reference Value for $\mathrm{SiO} 2$ is $45.353 \mathrm{wt} \%$

Oneway Analysis of $\mathrm{SiO} 2(w t \%)$ By Analytical Block

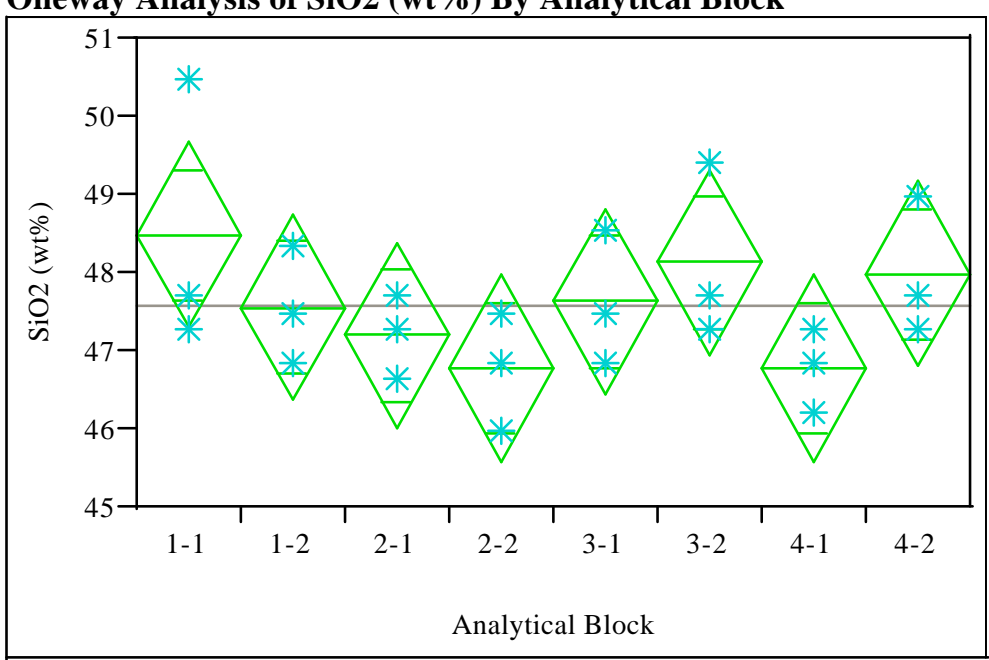

\section{Oneway Anova}

Rsquare

0.351157

Root Mean Square Error $\quad 0.972539$

Mean of Response 47.57268

Observations (or Sum Wgts)

Analysis of Variance

Source DF Sum of Squares Mean Square F Ratio Prob $>$ F

$\begin{array}{lrrrrr}\text { Analytical Block } & 7 & 8.190215 & 1.17003 & 1.2370 & 0.3397 \\ \text { Error } & 16 & 15.133306 & 0.94583 & & \end{array}$

$\begin{array}{rr}7 & 8.190215 \\ 16 & 15.133306\end{array}$

0.94583

C. Total

23

23.32352

\section{Means for Oneway Anova}

Level Number Mean Std Error Lower 95\% Upper 95\%

$\begin{array}{lllrrr}1-1 & 3 & 48.4908 & 0.56150 & 47.300 & 49.681\end{array}$

$\begin{array}{llllll}1-2 & 3 & 47.5638 & 0.56150 & 46.373 & 48.754\end{array}$

$\begin{array}{llllll}2-1 & 3 & 47.2072 & 0.56150 & 46.017 & 48.398\end{array}$

$\begin{array}{llllll}2-2 & 3 & 46.7794 & 0.56150 & 45.589 & 47.970 \\ 3-1 & 3 & 47.6351 & 0.56150 & 46.445 & 48.825\end{array}$

$\begin{array}{llllll}3-1 & 3 & 47.6351 & 0.56150 & 46.445 & 48.825 \\ 3-2 & 3 & 48.1343 & 0.56150 & 46.944 & 49.325\end{array}$

$\begin{array}{llllll}3-2 & 3 & 48.1343 & 0.56150 & 46.944 & 49.325 \\ 4-1 & 3 & 46.7794 & 0.56150 & 45.589 & 47.970\end{array}$

$\begin{array}{llllll}4-1 & 3 & 46.7794 & 0.56150 & 45.589 & 47.970 \\ 4-2 & 3 & 47.9916 & 0.56150 & 46.801 & 49.182\end{array}$

Std Error uses a pooled estimate of error variance 


\section{Exhibit H.5: LM Measurements by SB3 Glass ID Number by Oxide}

(Batch 1 - 100 and Ustd - 101)

BaO (wt\%) By SB3 Glass \#

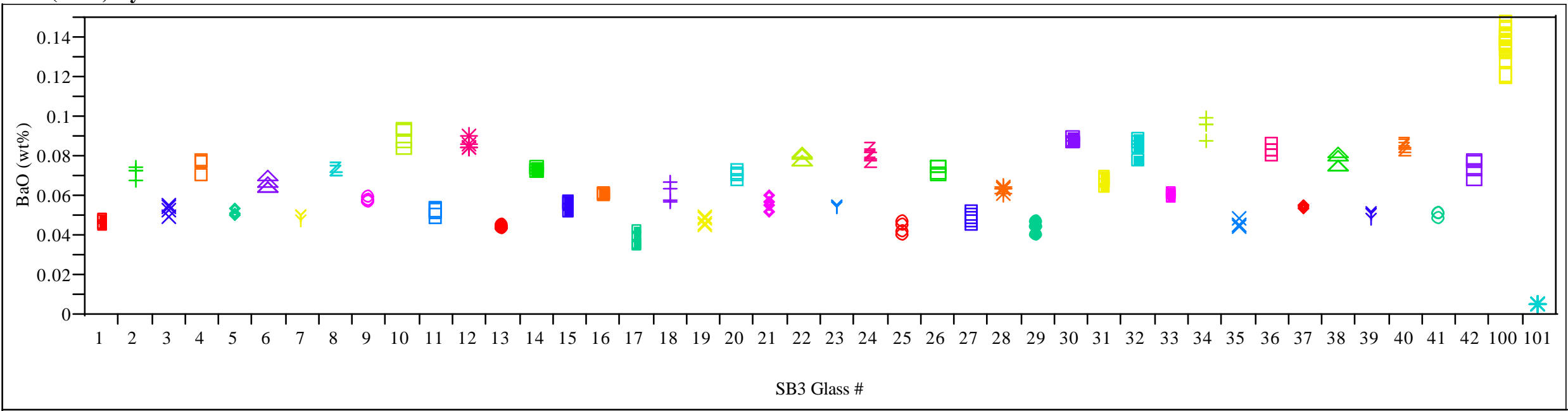

BaO bc (wt\%) By SB3 Glass \#

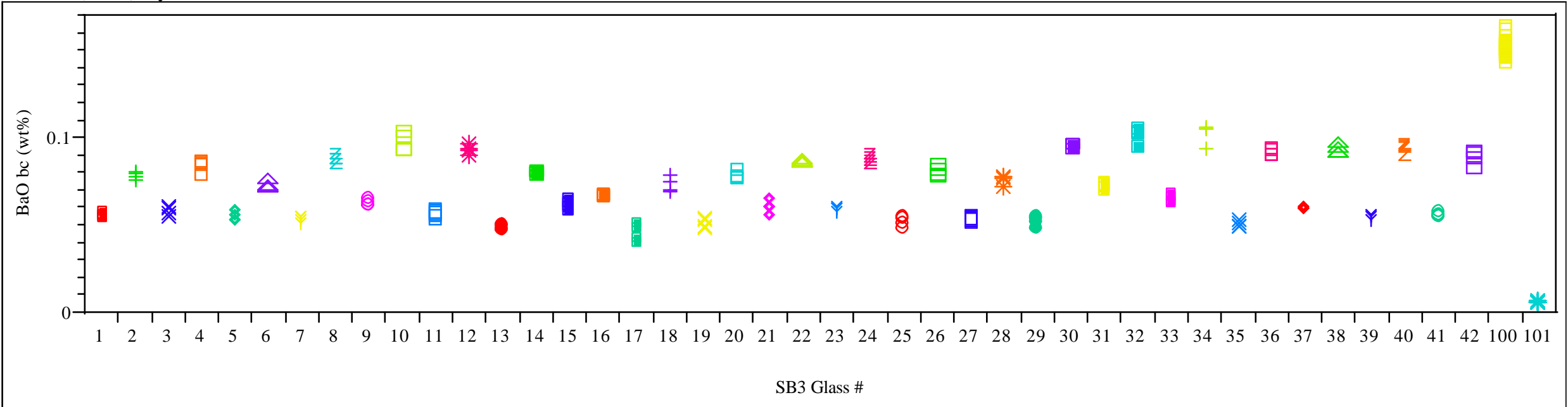


Exhibit H.5: LM Measurements by SB3 Glass ID Number by Oxide (continued) (Batch 1 - 100 and Ustd - 101)

CaO (wt\%) By SB3 Glass \#

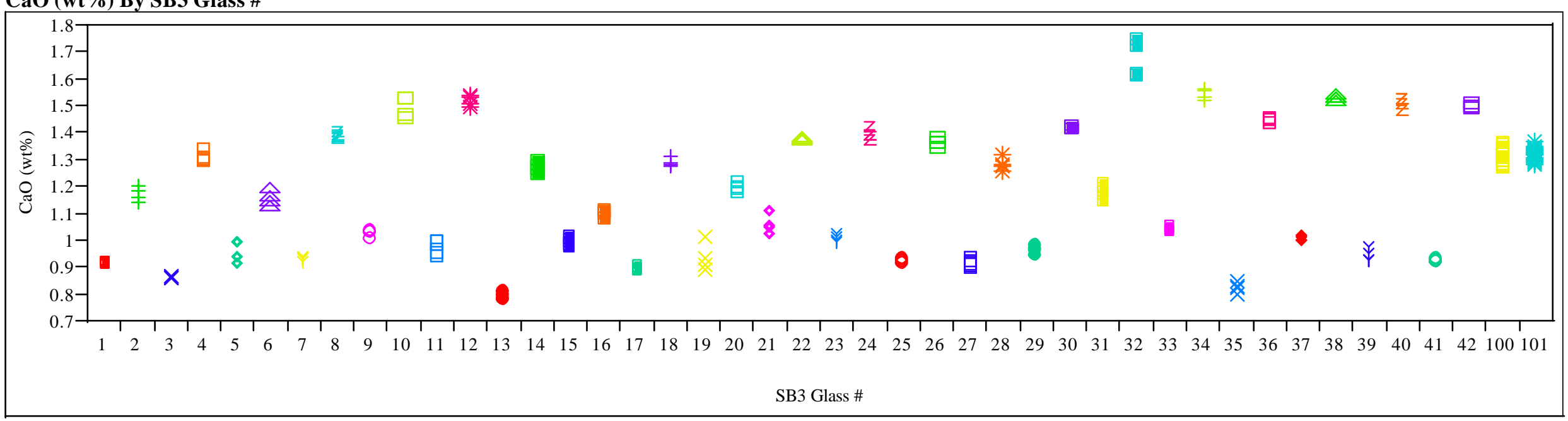

\section{CaO bc (wt\%) By SB3 Glass \#}

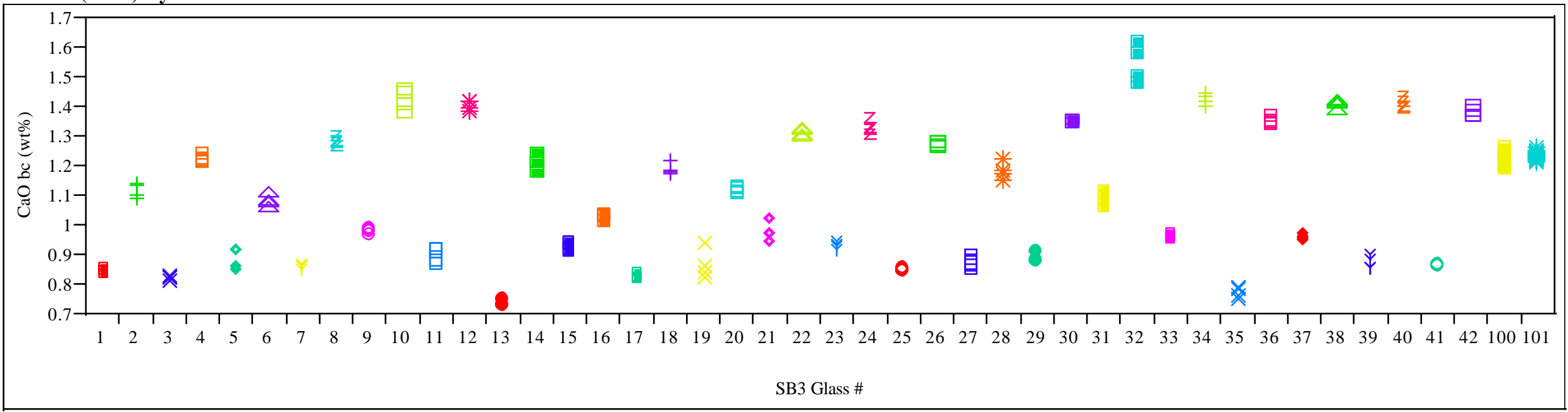


Exhibit H.5: LM Measurements by SB3 Glass ID Number by Oxide (continued) (Batch 1 - 100 and Ustd - 101)

\section{Ce2O3 (wt \%) By SB3 Glass \#}

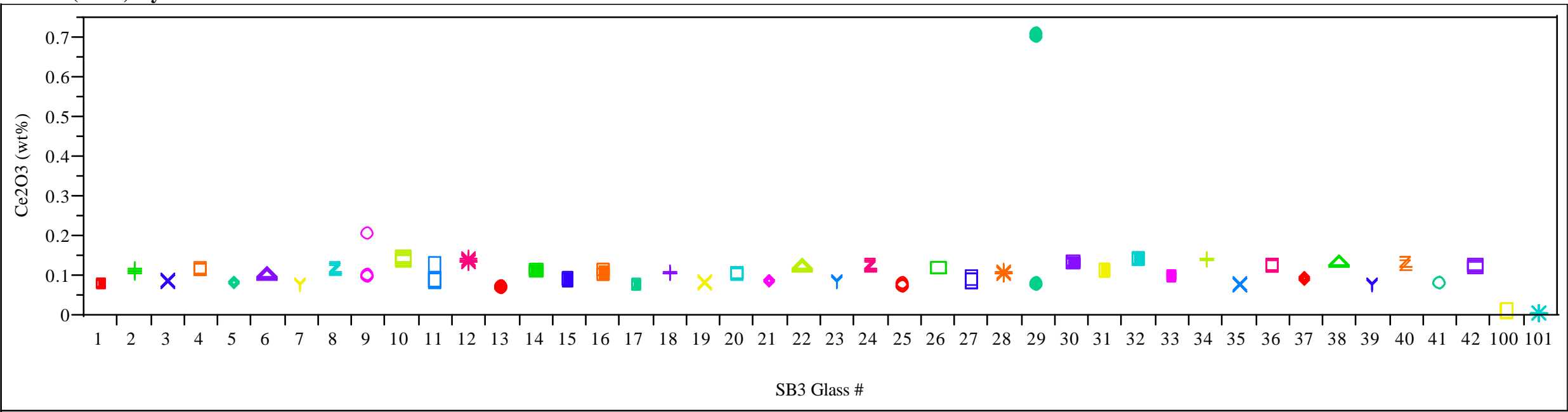

\section{Ce2O3 bc (wt\%) By SB3 Glass \#}

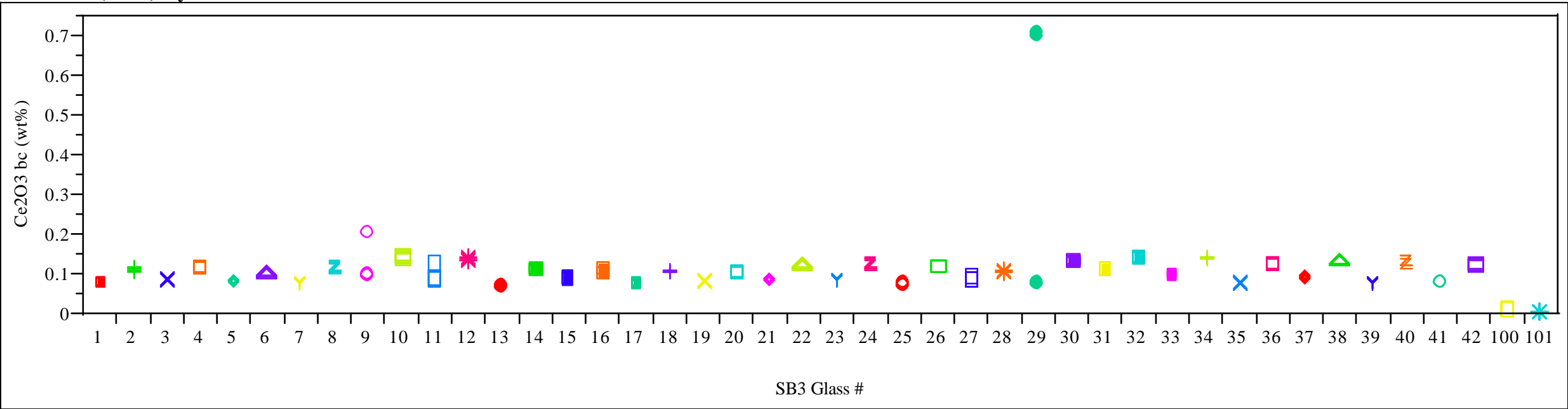


Exhibit H.5: LM Measurements by SB3 Glass ID Number by Oxide (continued) (Batch 1 - 100 and Ustd - 101)

Cr2O3 (wt\%) By SB3 Glass \#

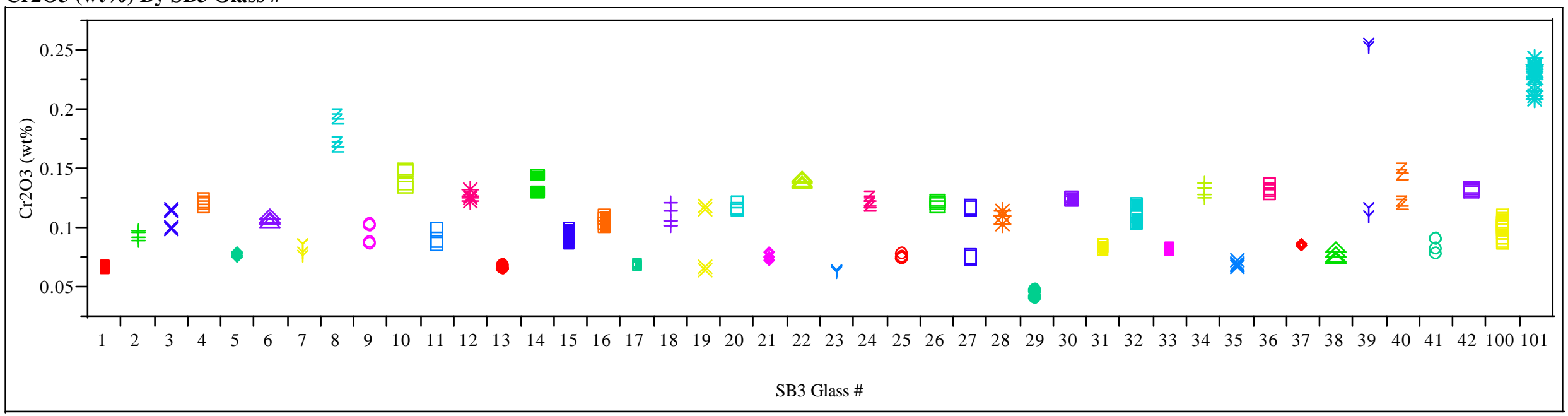

\section{Cr2O3 bc (wt\%) By SB3 Glass \#}

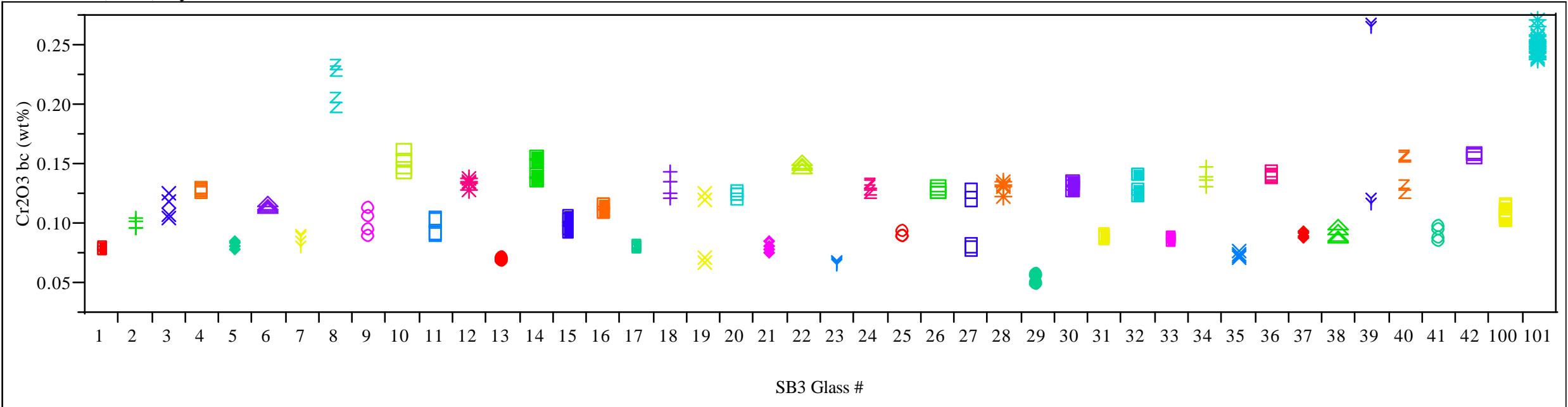


Exhibit H.5: LM Measurements by SB3 Glass ID Number by Oxide (continued) (Batch 1 - 100 and Ustd - 101)

$\mathrm{CuO}(\mathrm{wt} \%)$ By SB3 Glass \#

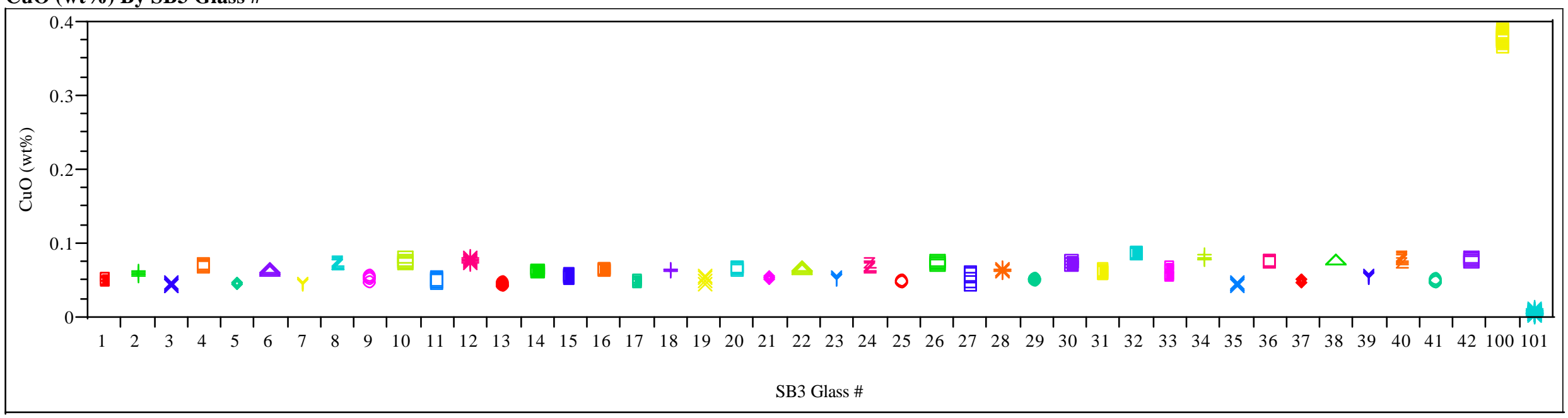

\section{CuO bc (wt \%) By SB3 Glass \#}

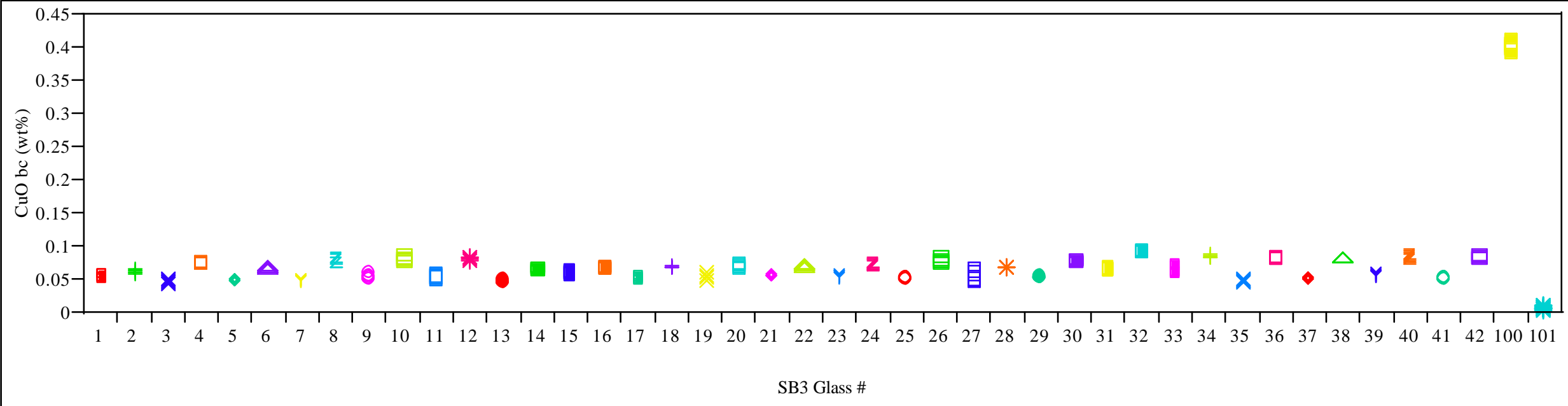


Exhibit H.5: LM Measurements by SB3 Glass ID Number by Oxide (continued) (Batch 1-100 and Ustd - 101)

Fe2O3 (wt \%) By SB3 Glass \#

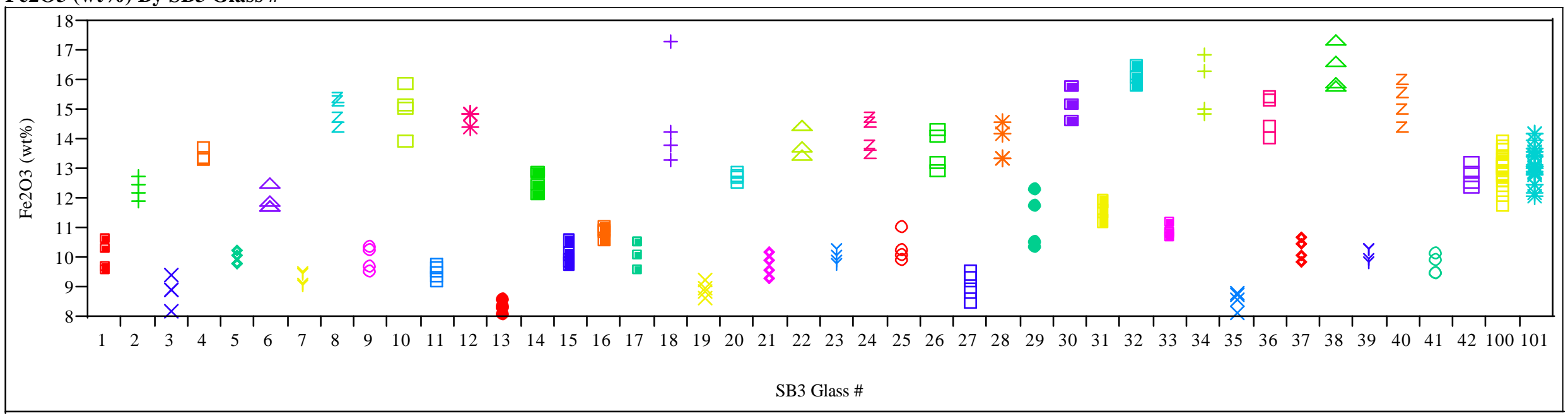

Fe2O3 bc (wt \%) By SB3 Glass \#

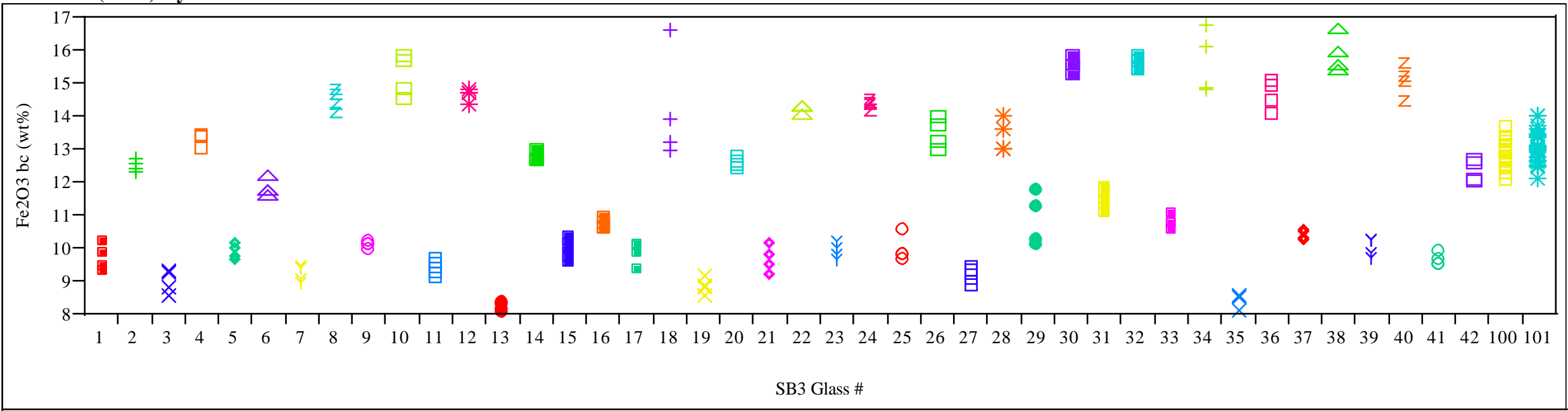


Exhibit H.5: LM Measurements by SB3 Glass ID Number by Oxide (continued) (Batch 1 - 100 and Ustd - 101)

\section{K2O (wt\%) By SB3 Glass \#}

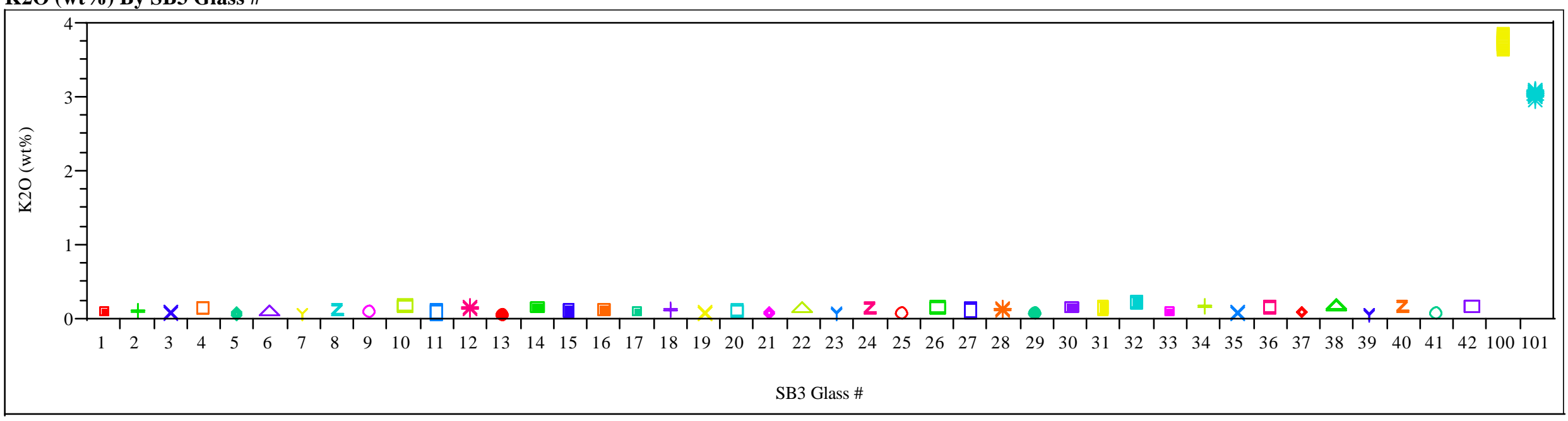

\section{K2O bc (wt\%) By SB3 Glass \#}

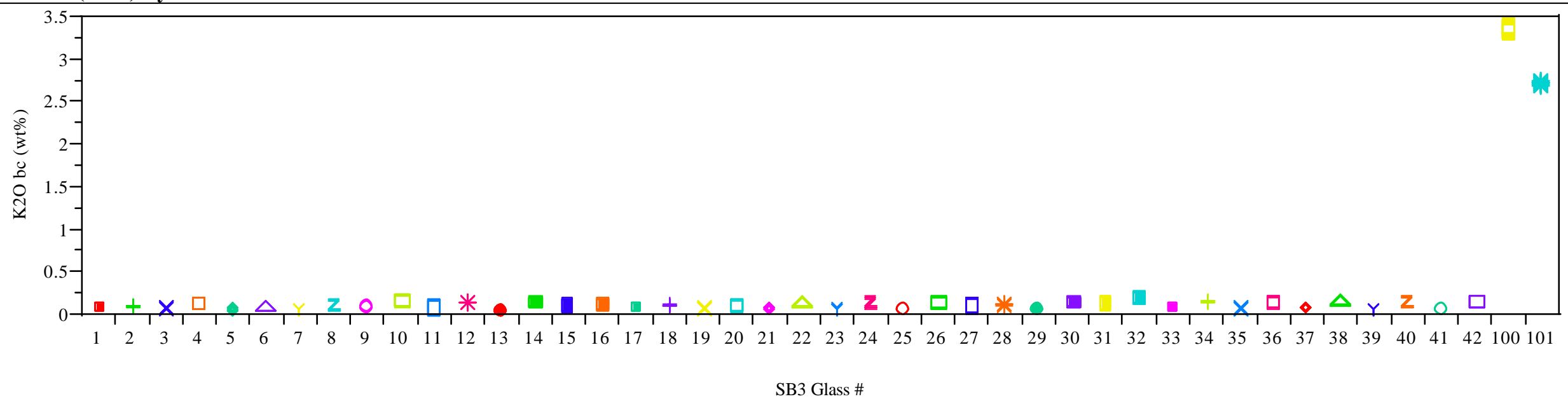


Exhibit H.5: LM Measurements by SB3 Glass ID Number by Oxide (continued) (Batch 1 - 100 and Ustd - 101)

\section{La2O3 (wt \%) By SB3 Glass \#}

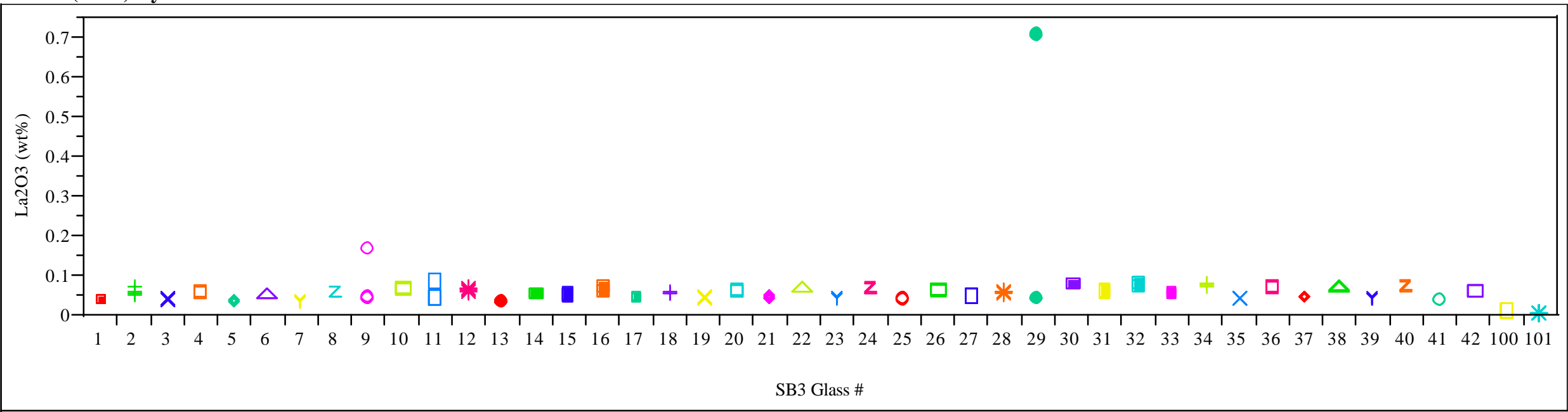

\section{La2O3 bc (wt \%) By SB3 Glass \#}

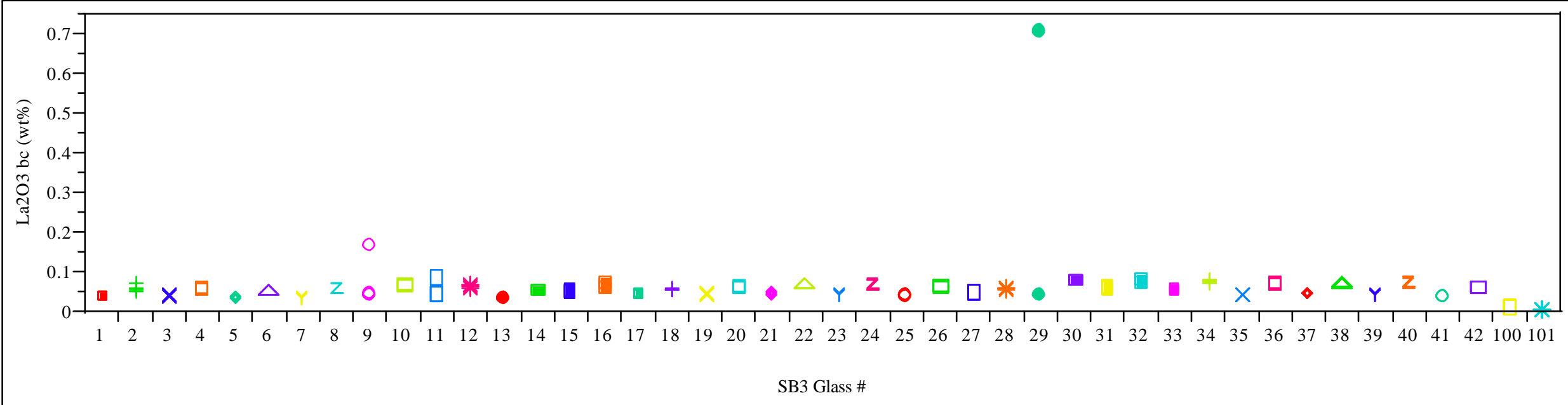


Exhibit H.5: LM Measurements by SB3 Glass ID Number by Oxide (continued) (Batch 1 - 100 and Ustd - 101)

MgO (wt \%) By SB3 Glass \#

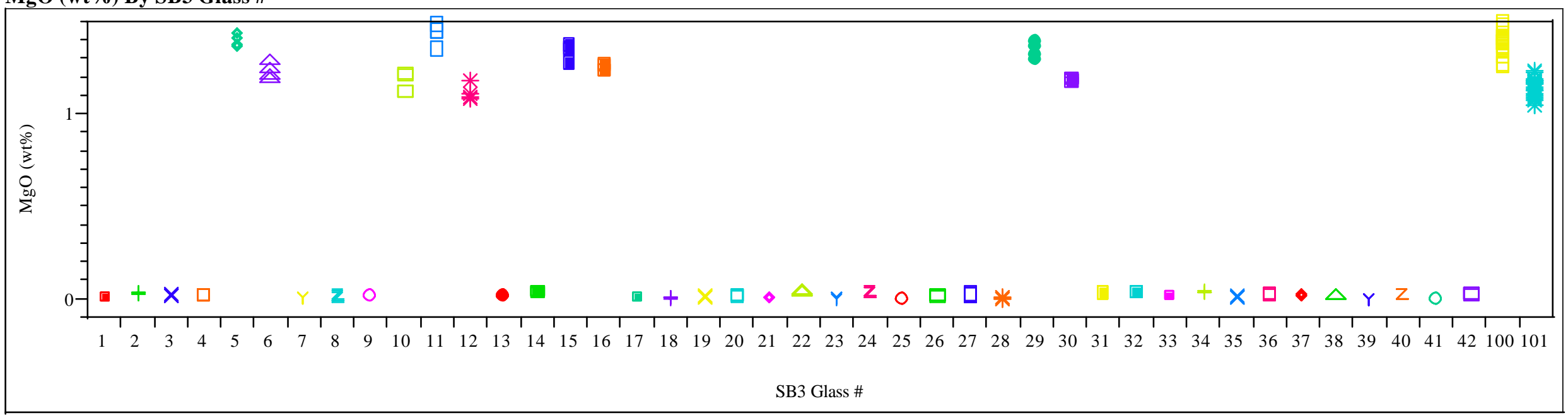

MgO bc (wt \%) By SB3 Glass \#

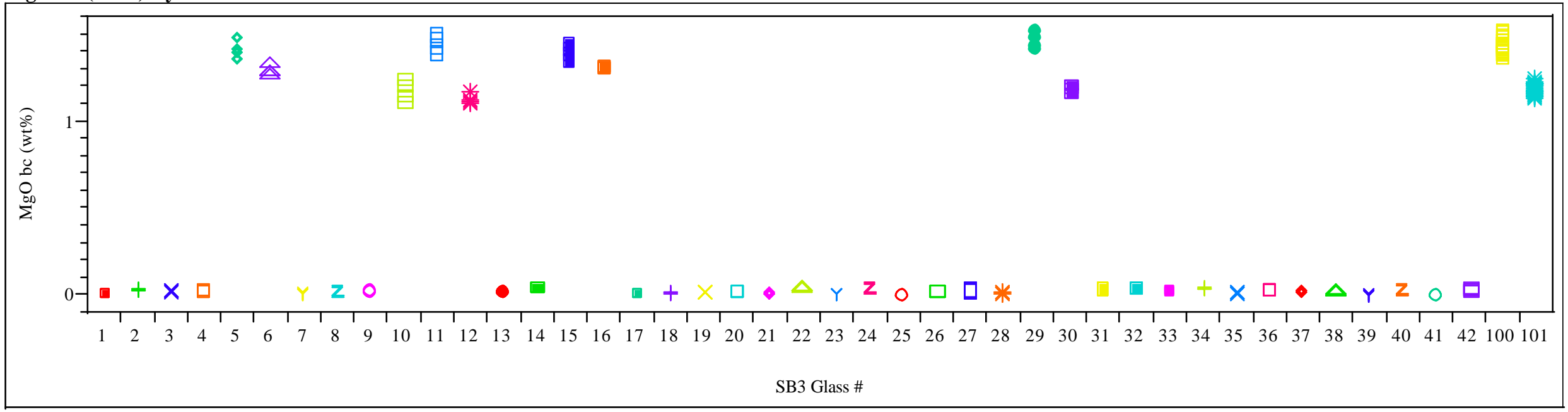


Exhibit H.5: LM Measurements by SB3 Glass ID Number by Oxide (continued) (Batch 1-100 and Ustd - 101)

MnO (wt \%) By SB3 Glass \#

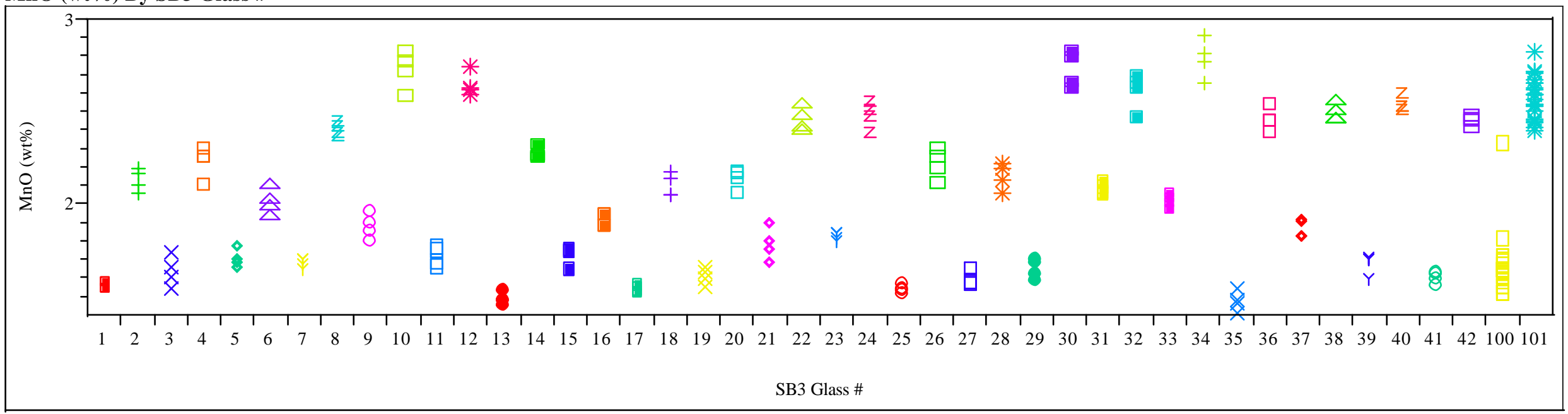

\section{MnO bc (wt\%) By SB3 Glass \#}

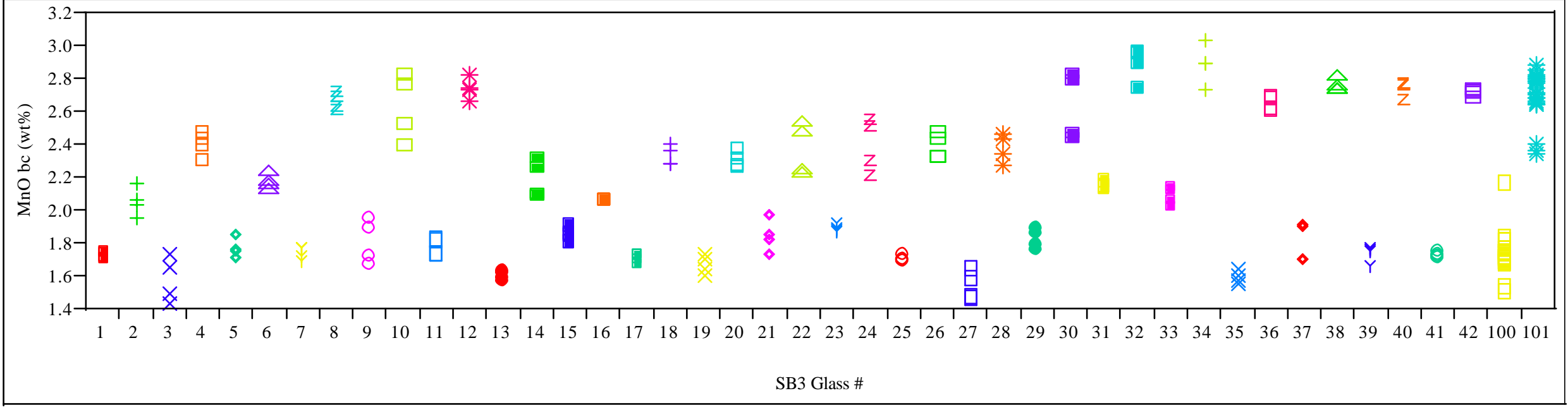


Exhibit H.5: LM Measurements by SB3 Glass ID Number by Oxide (continued) (Batch 1-100 and Ustd - 101)

\section{Na2O (wt\%) By SB3 Glass \#}

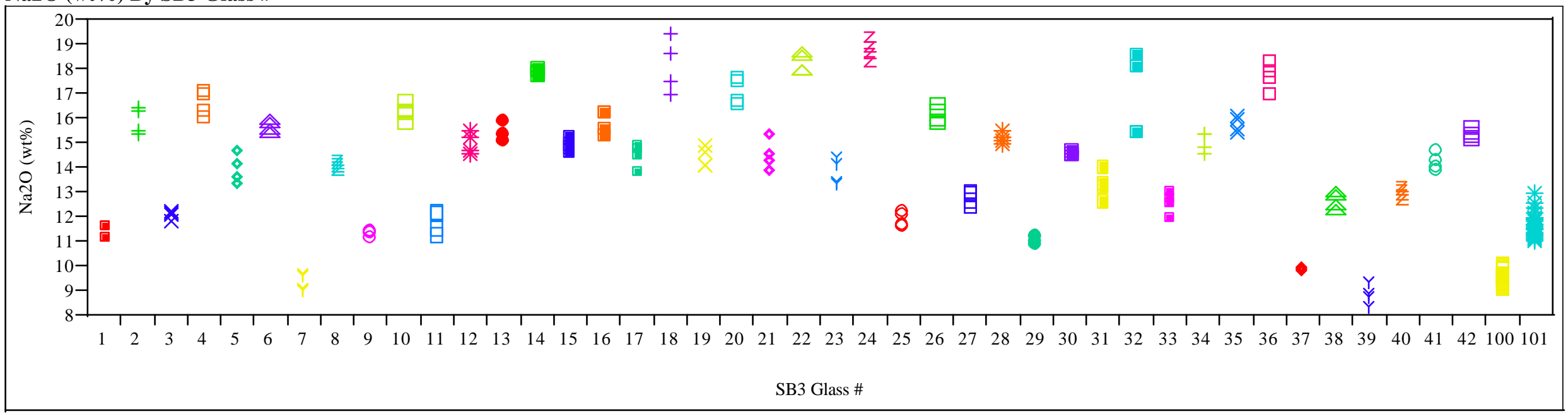

Na2O bc (wt\%) By SB3 Glass \#

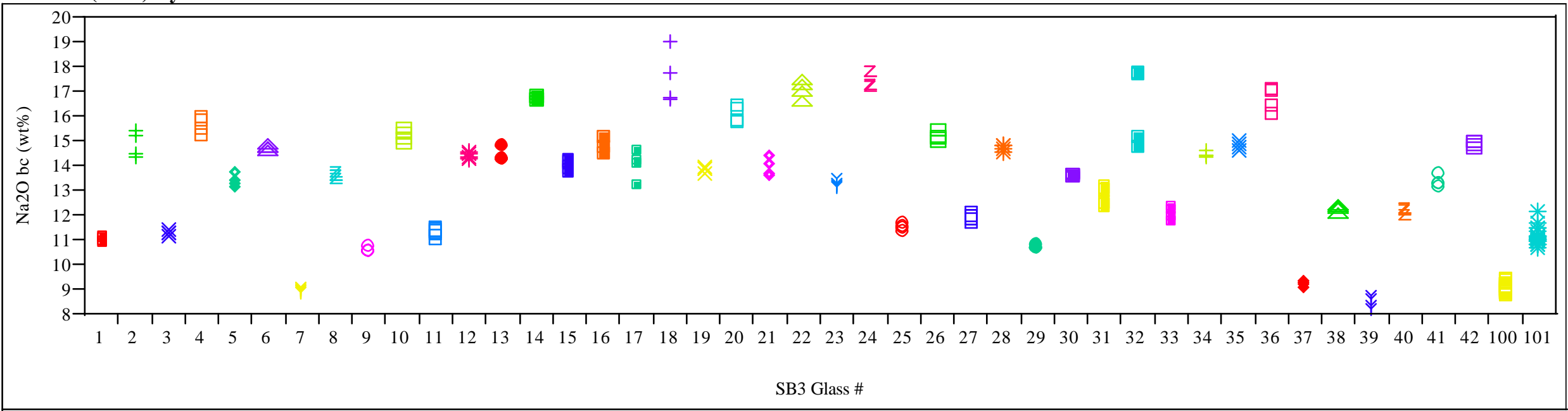


Exhibit H.5: LM Measurements by SB3 Glass ID Number by Oxide (continued) (Batch 1 - 100 and Ustd - 101)

\section{NiO (wt\%) By SB3 Glass \#}

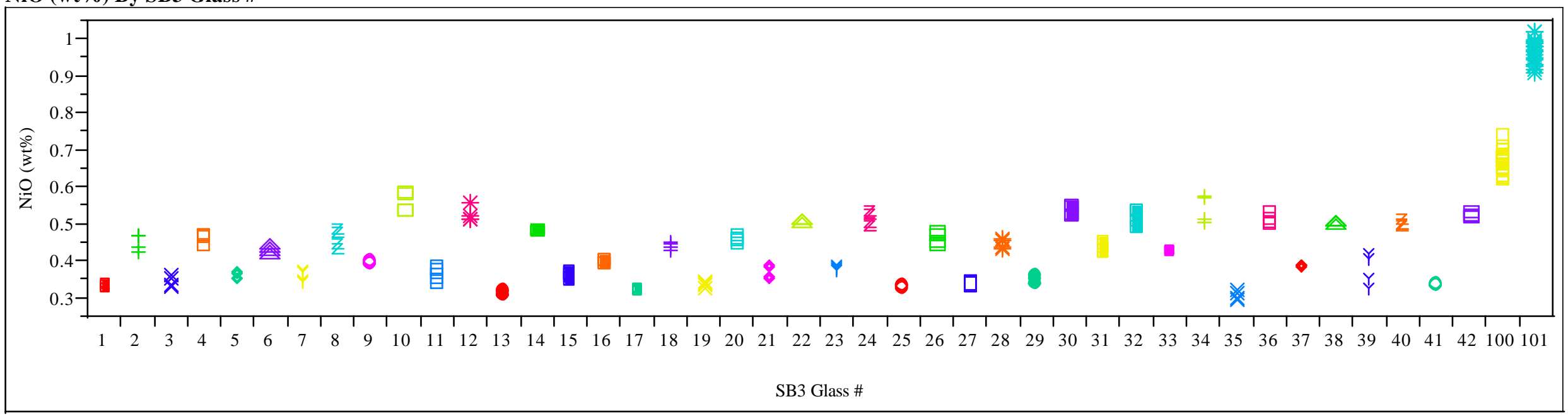

\section{NiO bc (wt \%) By SB3 Glass \#}

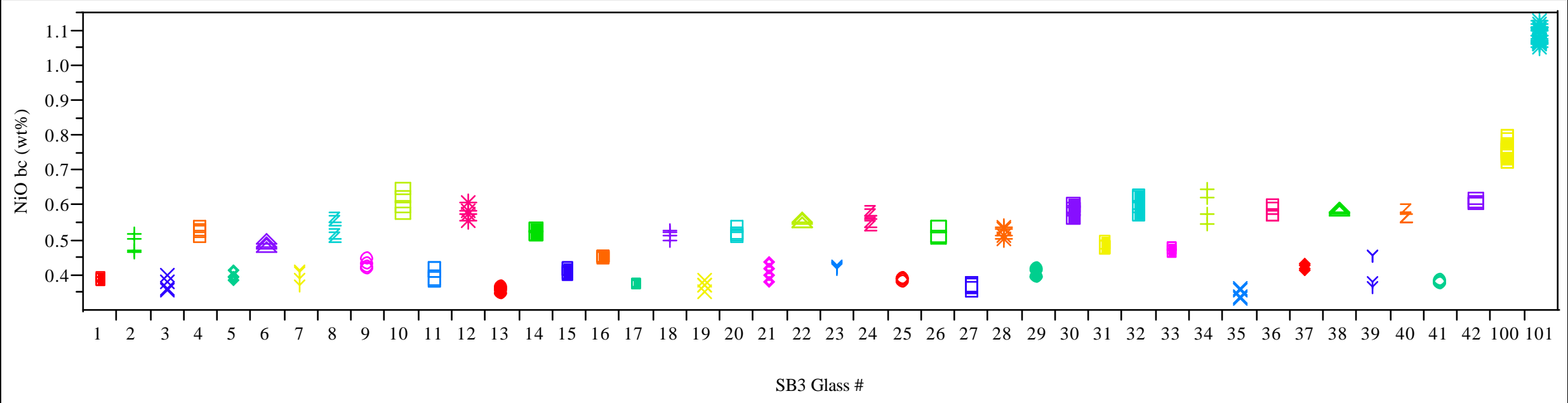


Exhibit H.5: LM Measurements by SB3 Glass ID Number by Oxide (continued) (Batch 1 - 100 and Ustd - 101)

PbO (wt\%) By SB3 Glass \#

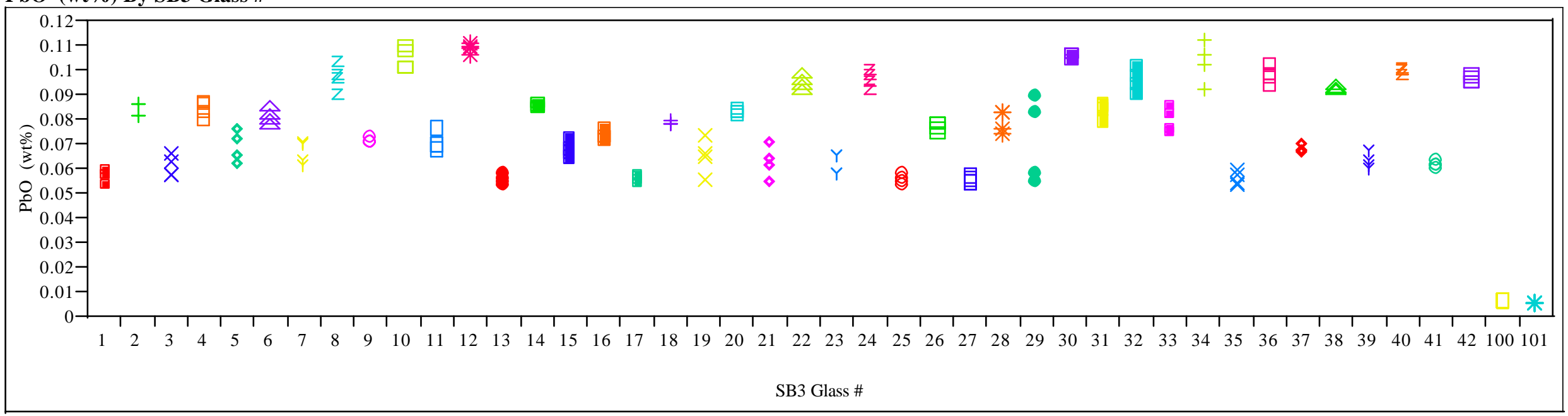

PbO bc (wt\%) By SB3 Glass \#

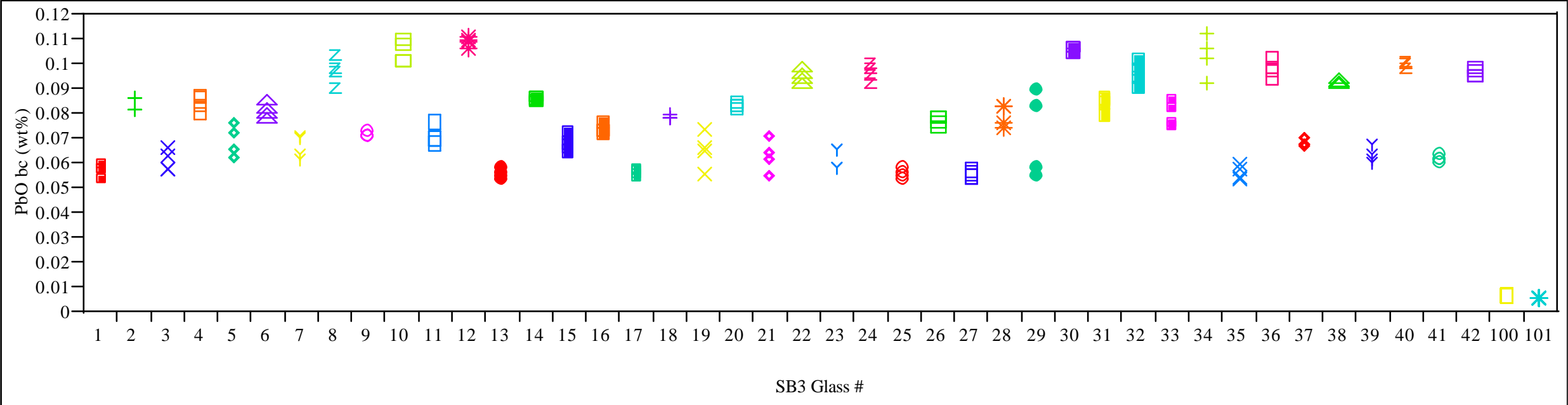


Exhibit H.5: LM Measurements by SB3 Glass ID Number by Oxide (continued) (Batch 1-100 and Ustd - 101)

ThO2 (wt\%) By SB3 Glass \#

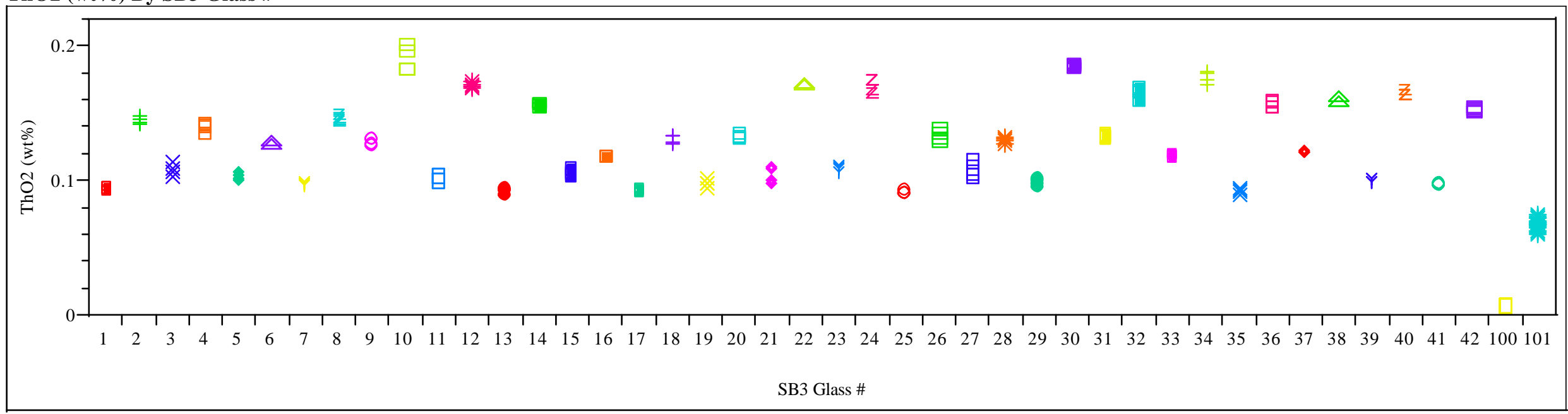

ThO2 bc (wt\%) By SB3 Glass \#

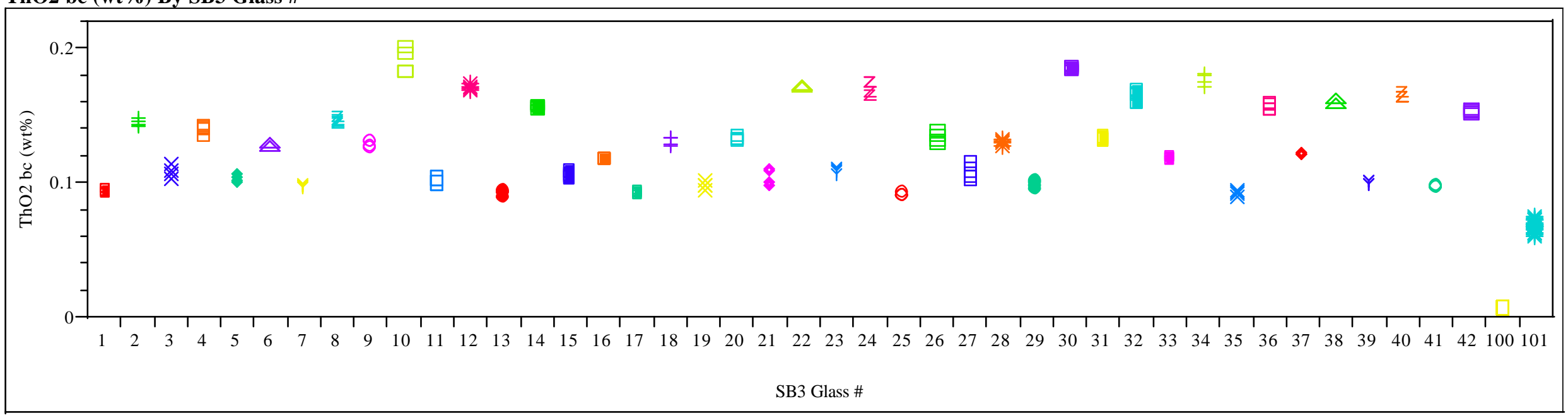


Exhibit H.5: LM Measurements by SB3 Glass ID Number by Oxide (continued) (Batch 1 - 100 and Ustd - 101)

TiO2 (wt\%) By SB3 Glass \#

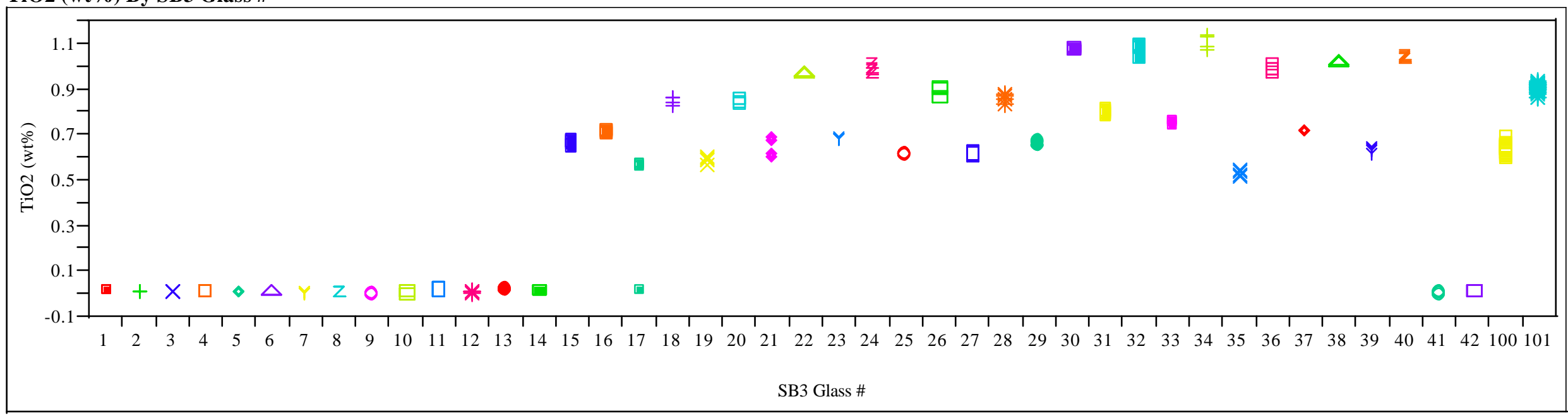

\section{TiO2 bc (wt\%) By SB3 Glass \#}

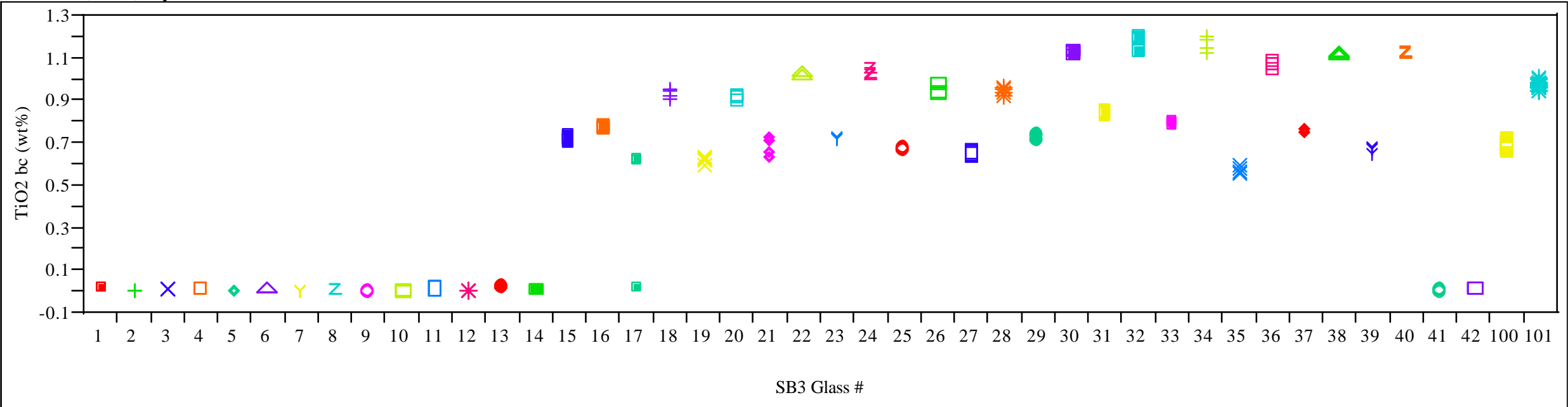


Exhibit H.5: LM Measurements by SB3 Glass ID Number by Oxide (continued) (Batch 1 - 100 and Ustd - 101)

\section{U3O8 (wt\%) By SB3 Glass \#}

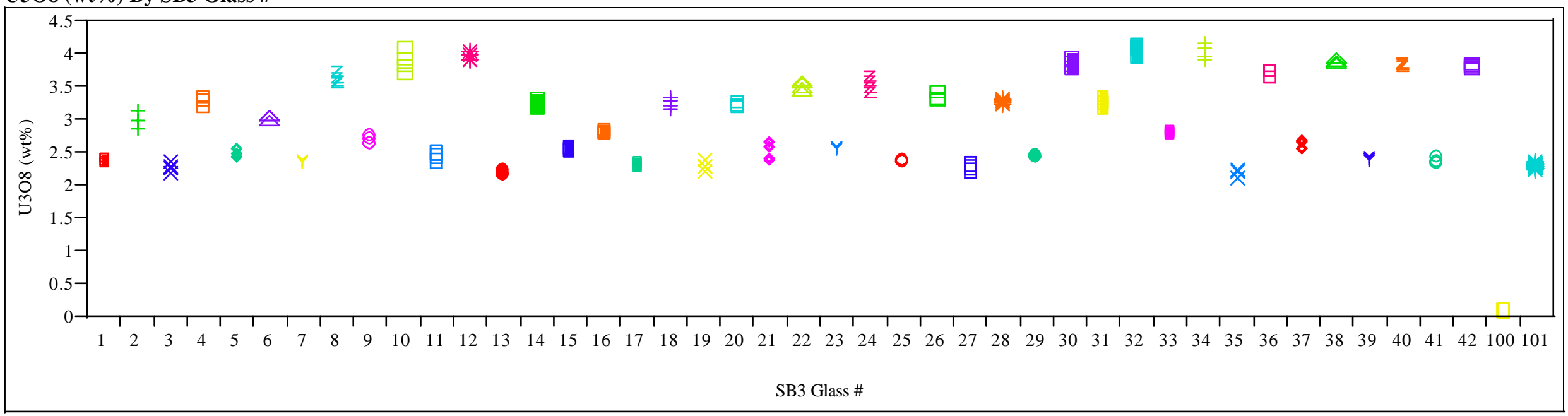

\section{U308 bc (wt\%) By SB3 Glass \#}

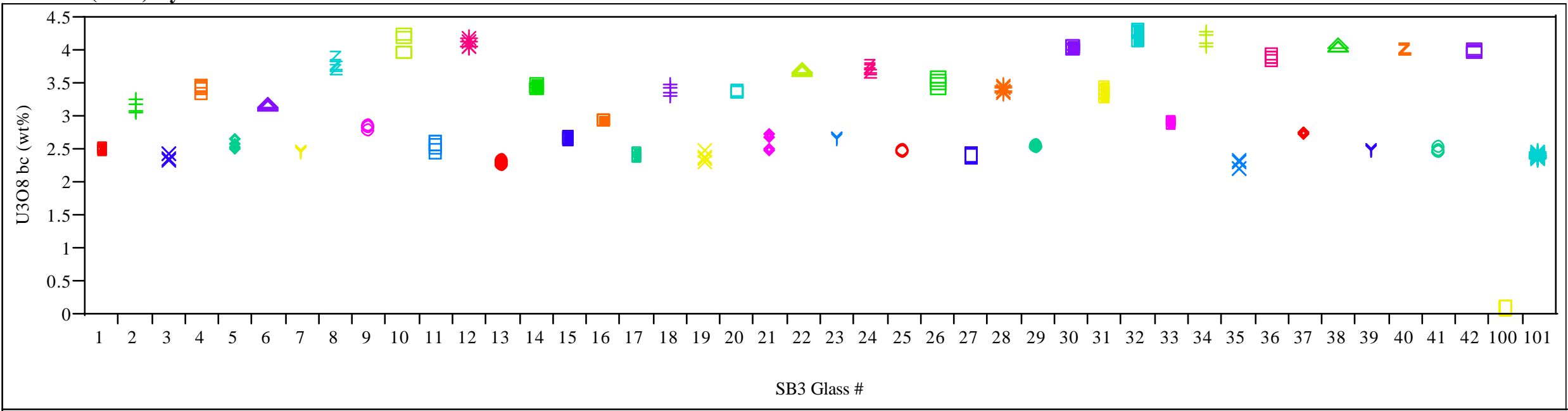


Exhibit H.5: LM Measurements by SB3 Glass ID Number by Oxide (continued) (Batch 1 - 100 and Ustd - 101)

ZnO (wt \%) By SB3 Glass \#

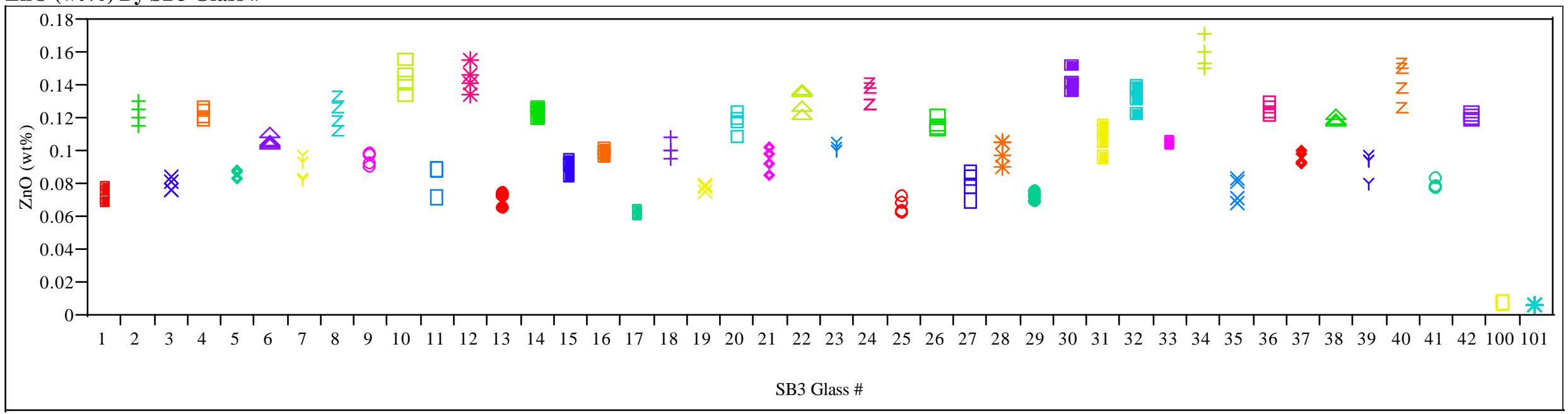

\section{ZnO bc (wt\%) By SB3 Glass \#}

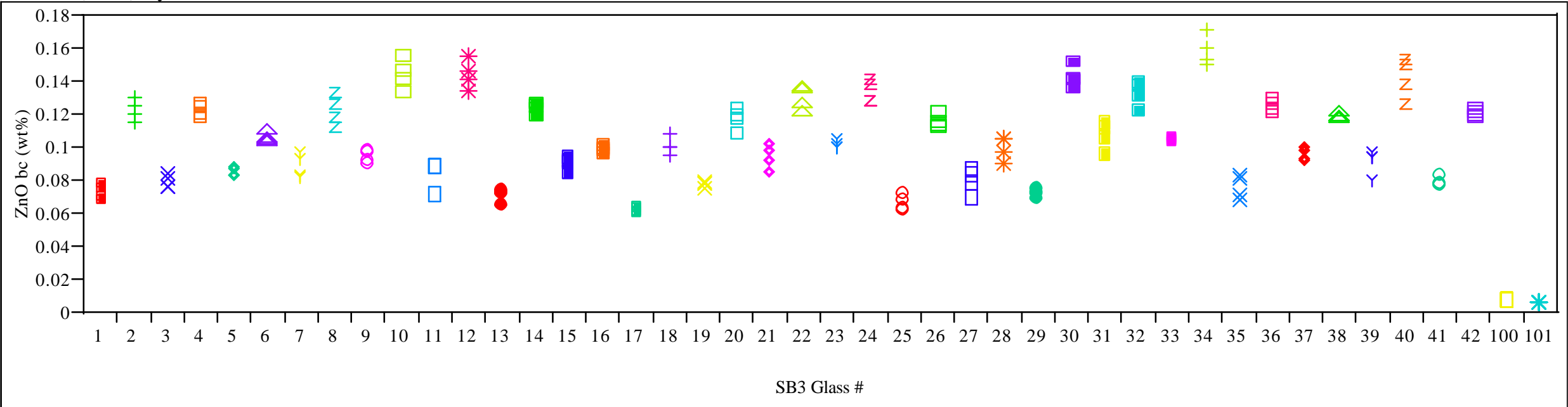


Exhibit H.5: LM Measurements by SB3 Glass ID Number by Oxide (continued) (Batch 1 - 100 and Ustd - 101)

ZrO2 (wt\%) By SB3 Glass \#

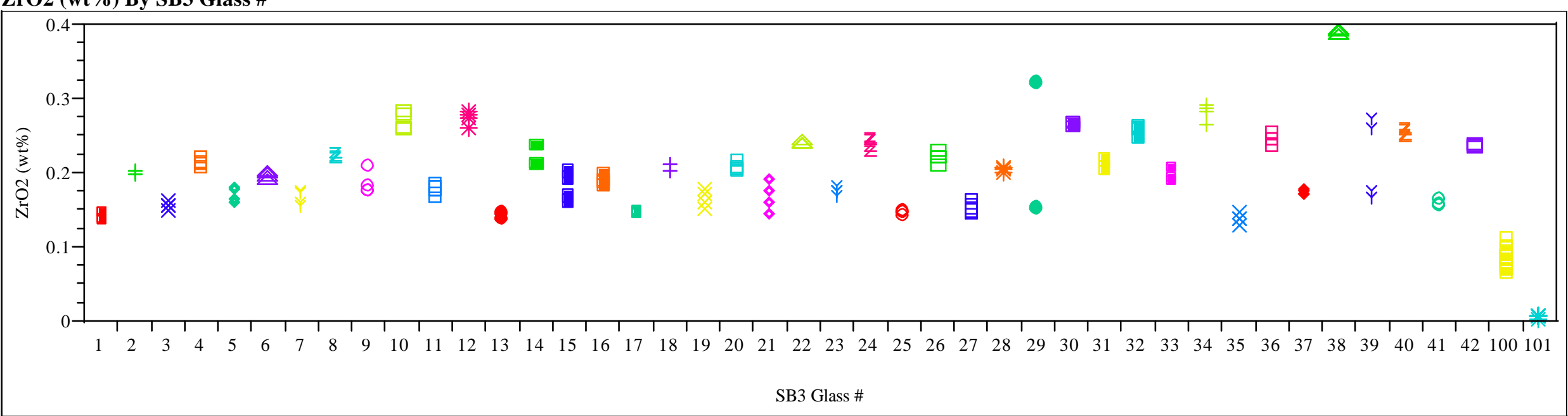

\section{ZrO2 bc (wt\%) By SB3 Glass \#}

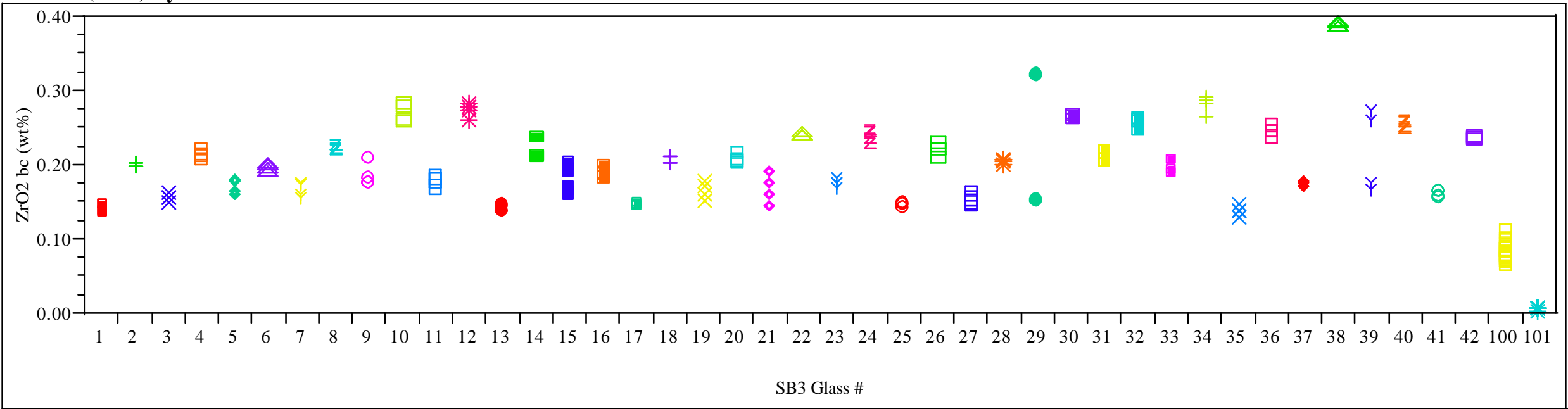


Exhibit H.6: PF Measurements by SB3 Glass ID Number by Oxide (continued) (Batch 1 - 100 and Ustd - 101)

\section{$\operatorname{Al2O3}(w t \%)$ By SB3 Glass \#}

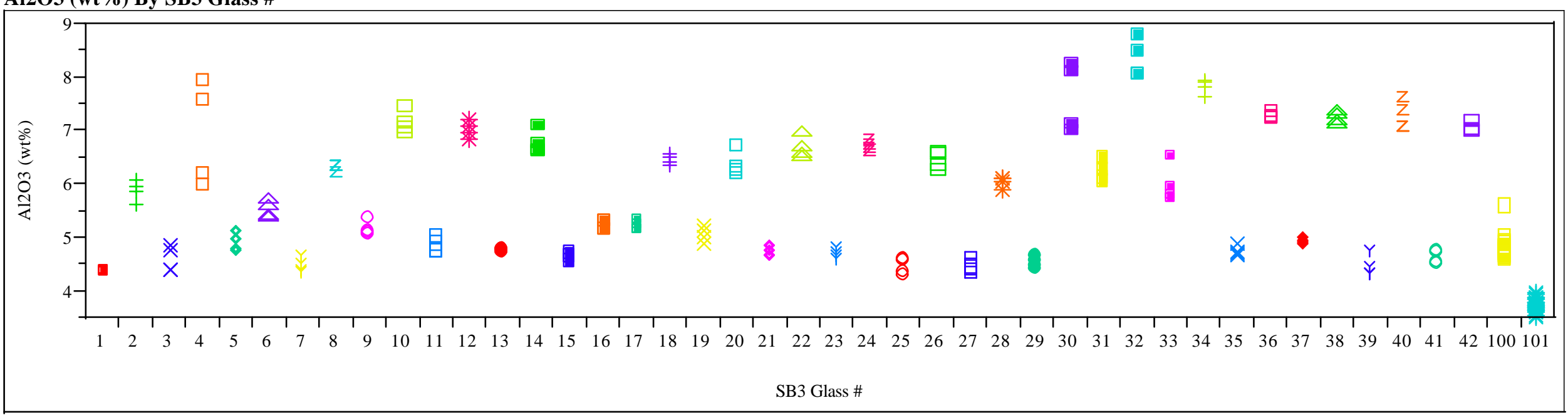

\section{Al2O3 bc (wt\%) By SB3 Glass \#}

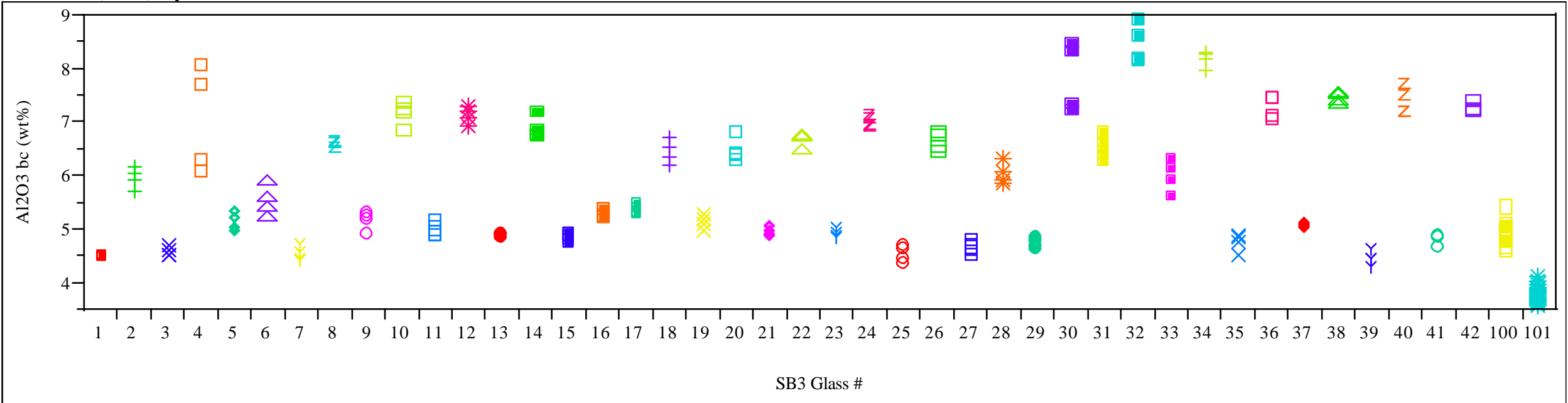


Exhibit H.6: PF Measurements by SB3 Glass ID Number by Oxide (continued) (Batch 1 - 100 and Ustd - 101)

\section{B2O3 (wt \%) By SB3 Glass \#}

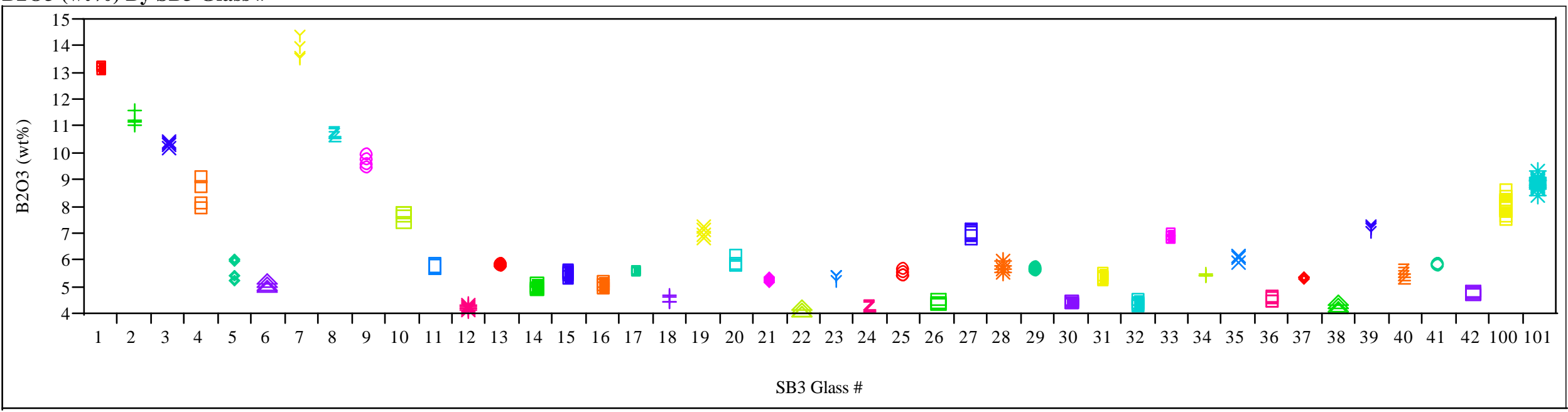

\section{B2O3 bc (wt \%) By SB3 Glass \#}

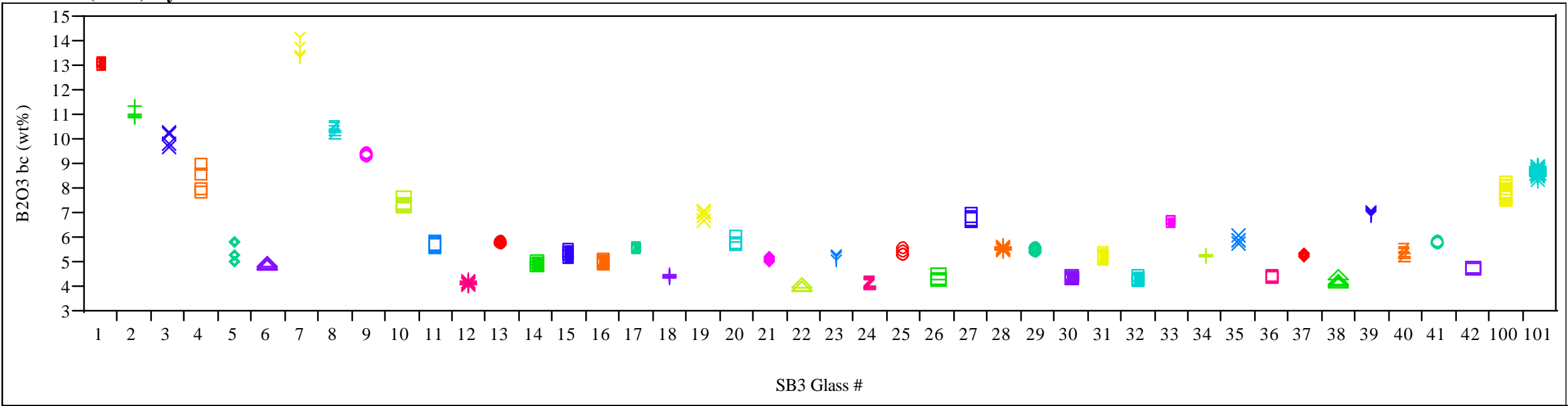


Exhibit H.6: PF Measurements by SB3 Glass ID Number by Oxide (continued) (Batch 1 - 100 and Ustd - 101)

Li2O (wt\%) By SB3 Glass \#

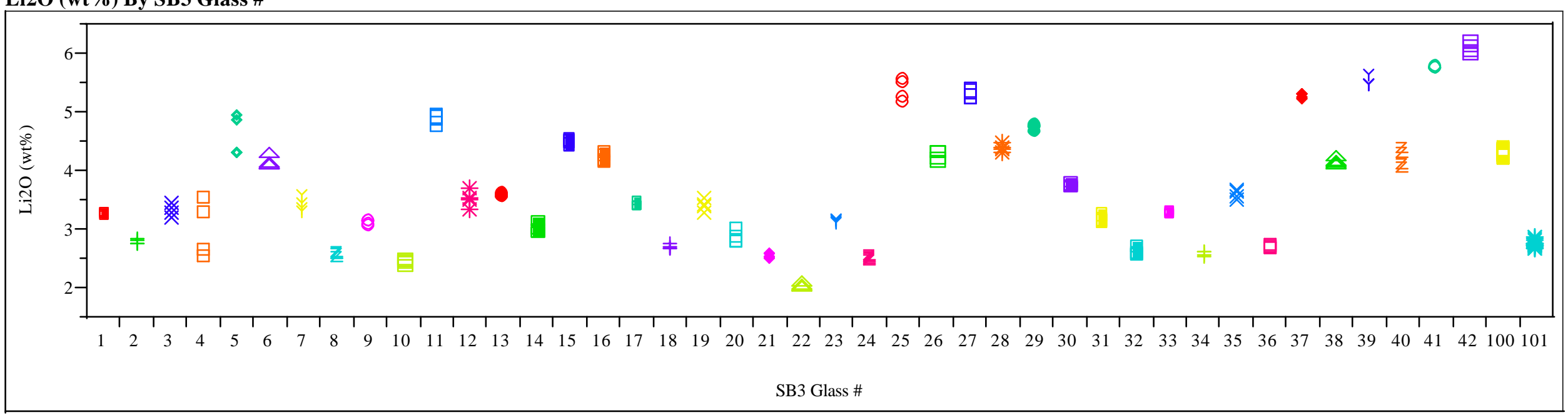

\section{Li2O bc (wt\%) By SB3 Glass \#}

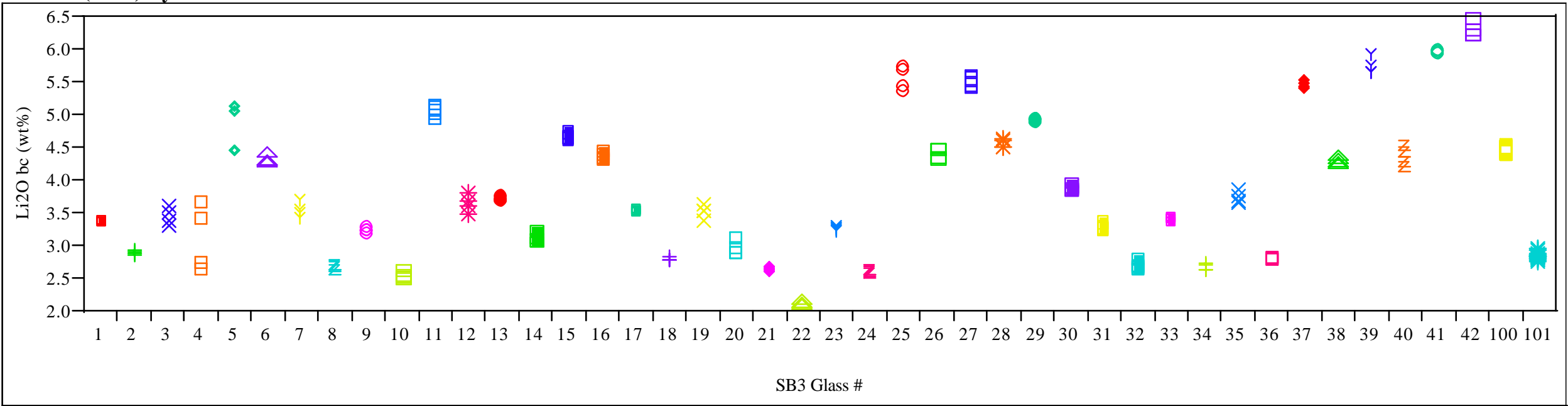


Exhibit H.6: PF Measurements by SB3 Glass ID Number by Oxide (continued) (Batch 1 - 100 and Ustd - 101)

SiO2 (wt\%) By SB3 Glass \#

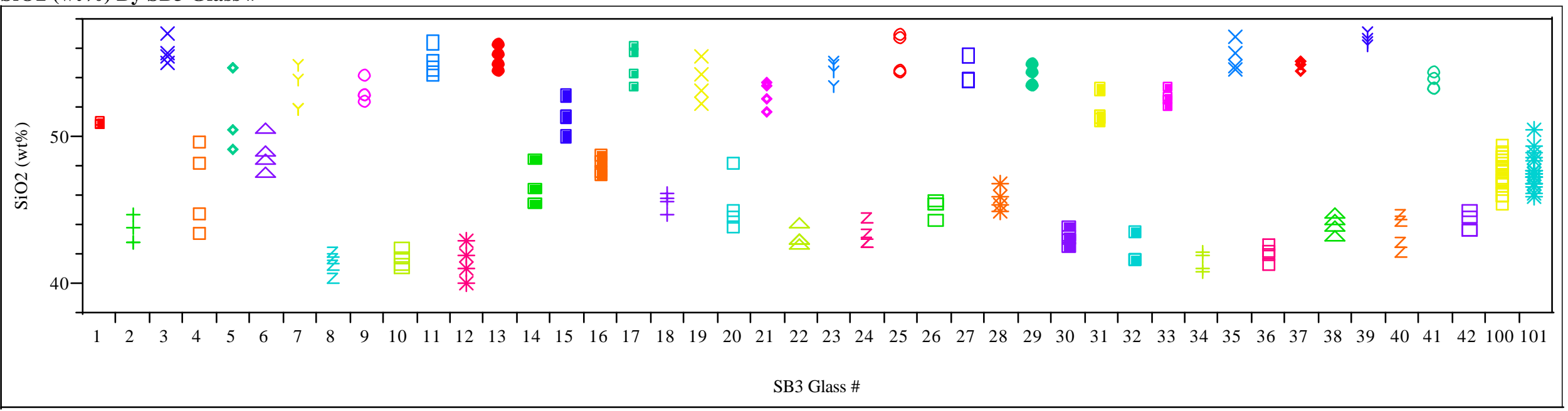

\section{SiO2 bc (wt \%) By SB3 Glass \#}

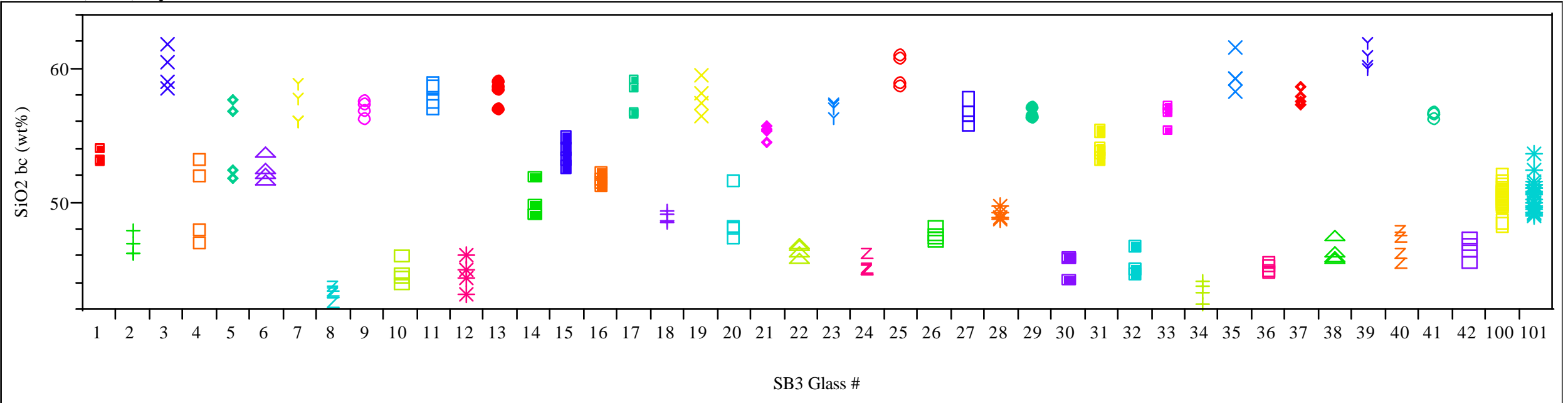


Exhibit H.7: Measured, Measured Bias-Corrected, and Targeted Compositions for the SB3 Glasses by Oxide (continued) (Batch 1 - 100 and Ustd - 101)

Oxide $=\mathbf{A} 1203(\mathrm{wt} \%)$

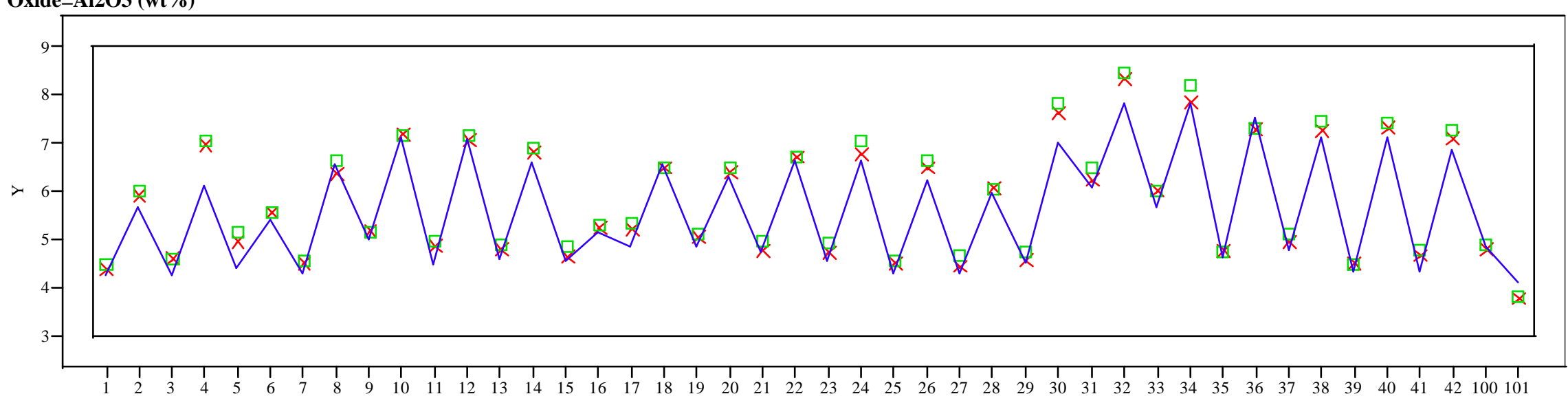
Glass \#

\section{Oxide $=\mathbf{B 2 O 3}(\mathrm{wt} \%)$}

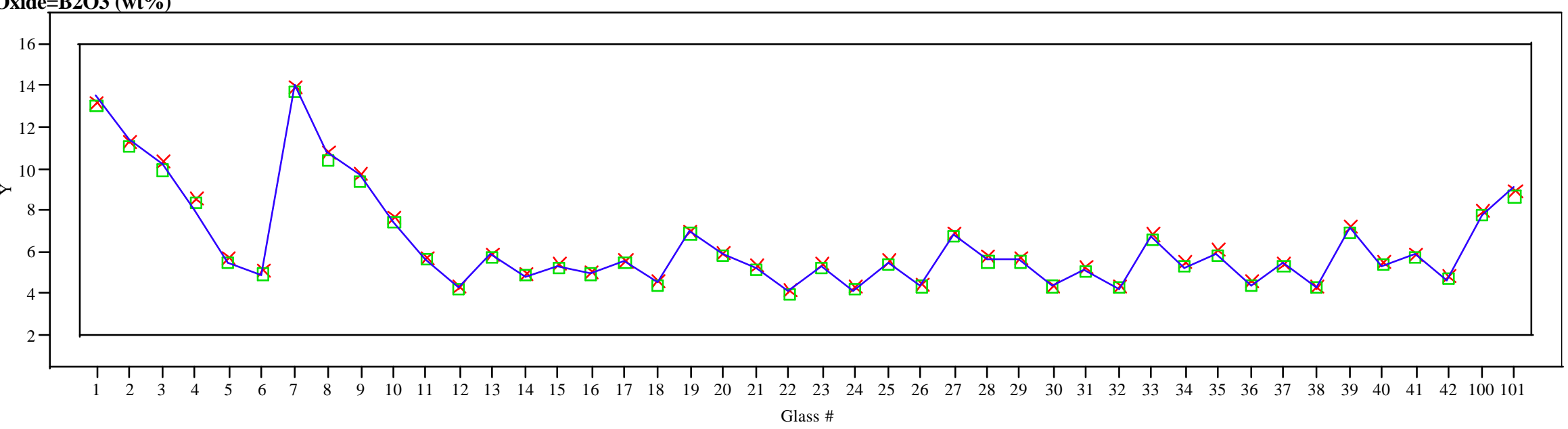

Y $\times$ Measured $\square$ Measured bc - Targeted 
Westinghouse Savannah River Company

Exhibit H.7: Measured, Measured Bias-Corrected, and Targeted Compositions for the SB3 Glasses by Oxide (continued) (Batch 1 - 100 and Ustd - 101)

\section{Oxide $=\mathrm{BaO}(\mathrm{wt} \%)$}

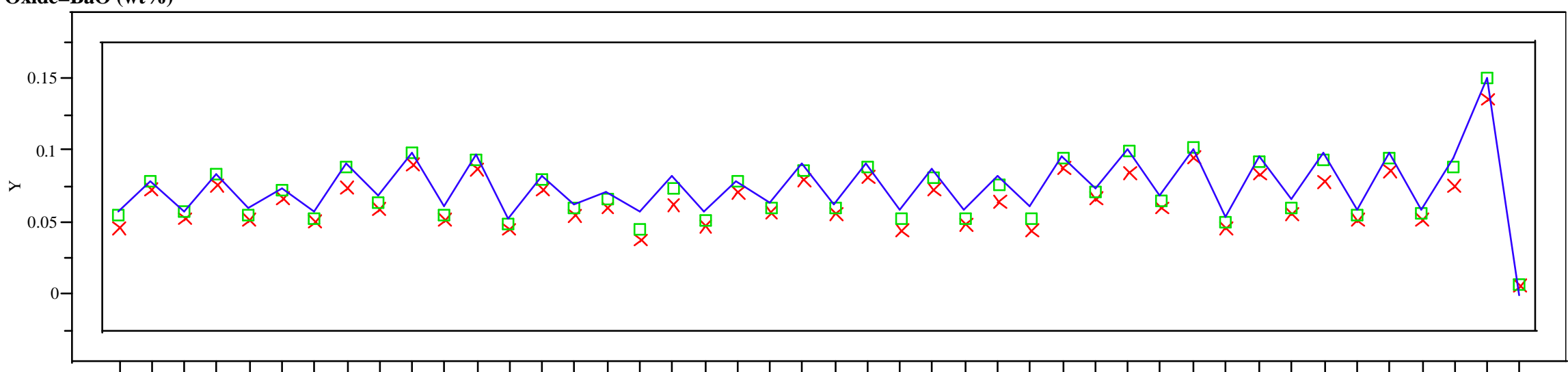

$\begin{array}{lllllllllllllllllllllllllllllllllllllllllllllllll}1 & 2 & 1 & 4 & 1 & 1 & 1 & 1 & 1 & 1 & 1 & 1 & 1 & 1 & 1 & 1 & 1 & 1 & 1 & 1 & 1 & 1 & 1 & 1 & 1 & 1 & 1 & 1 & 1 & 1 & 1 & 1 & 1 & 1 & 1 & 1 & 1 & 1 & 1 & 1 & 1 & 1 & 1 & 1 \\ 1 & 3 & 4 & 5 & 6 & 7 & 8 & 9 & 10 & 11 & 12 & 13 & 14 & 15 & 16 & 17 & 18 & 19 & 20 & 21 & 22 & 23 & 24 & 25 & 26 & 27 & 28 & 29 & 30 & 31 & 32 & 33 & 34 & 35 & 36 & 37 & 38 & 39 & 40 & 41 & 42 & 100 & 101\end{array}$ Glass \#

\section{Oxide $=\mathrm{CaO}(\mathrm{wt} \%)$}

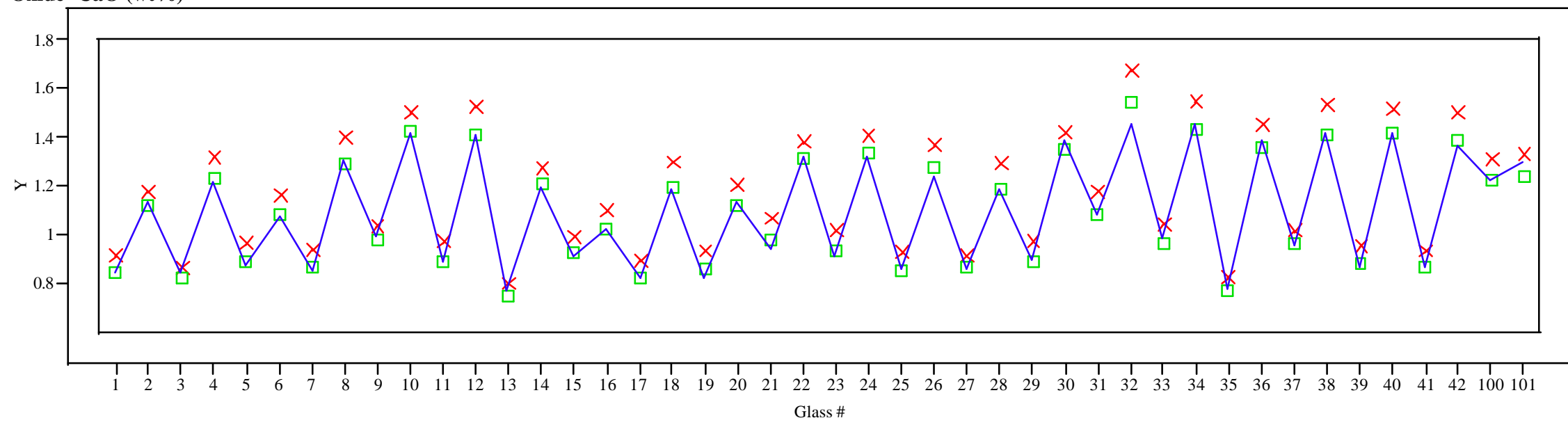

Y $\times$ Measured $\square$ Measured bc - Targeted 
Exhibit H.7: Measured, Measured Bias-Corrected, and Targeted Compositions for the SB3 Glasses by Oxide (continued) (Batch 1 - 100 and Ustd - 101)

\section{Oxide $=\mathrm{Ce} 2 \mathrm{O} 3(\mathrm{wt} \%)$}

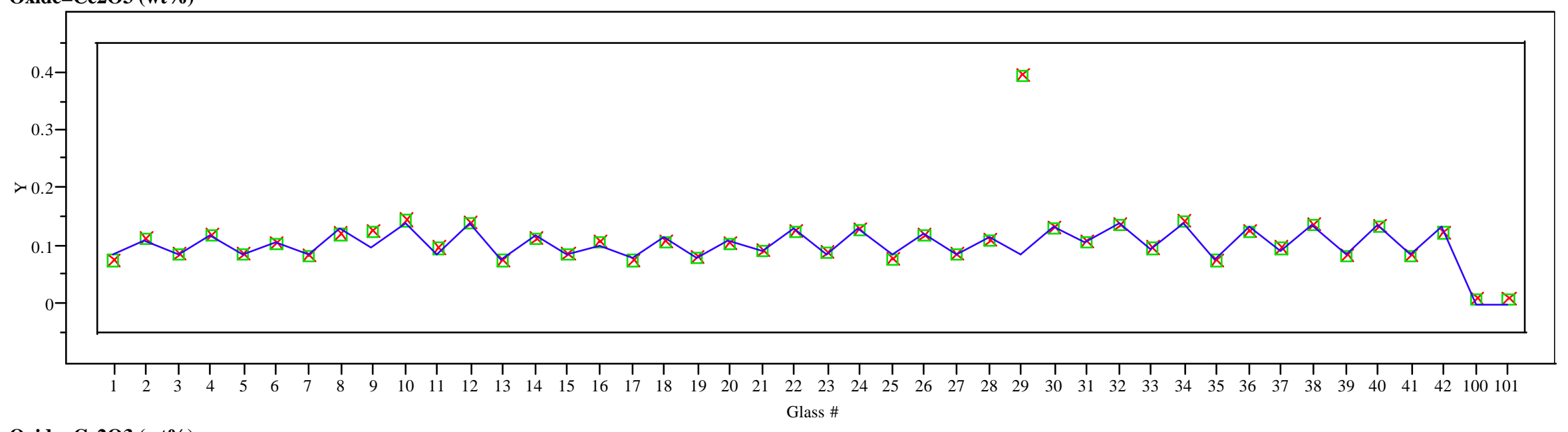

\section{Oxide $=\mathrm{Cr} 203(\mathrm{wt} \%)$}

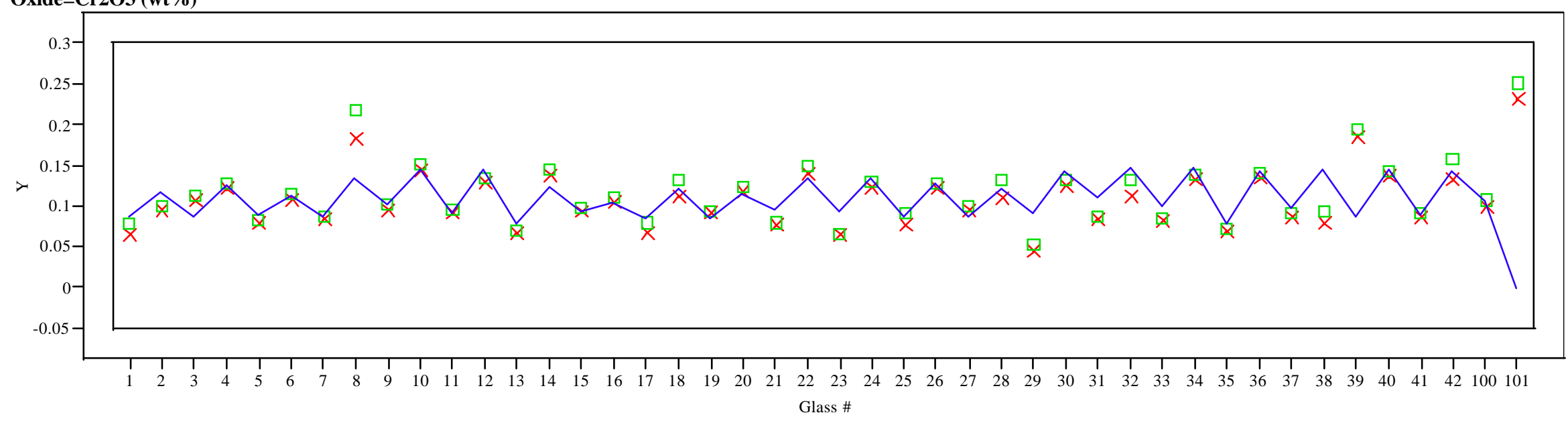

Y $\times$ Measured Measured b-Targeted 
Exhibit H.7: Measured, Measured Bias-Corrected, and Targeted Compositions for the SB3 Glasses by Oxide (continued) (Batch 1 - 100 and Ustd - 101)

Oxide $=\mathrm{CuO}(\mathrm{wt} \%)$

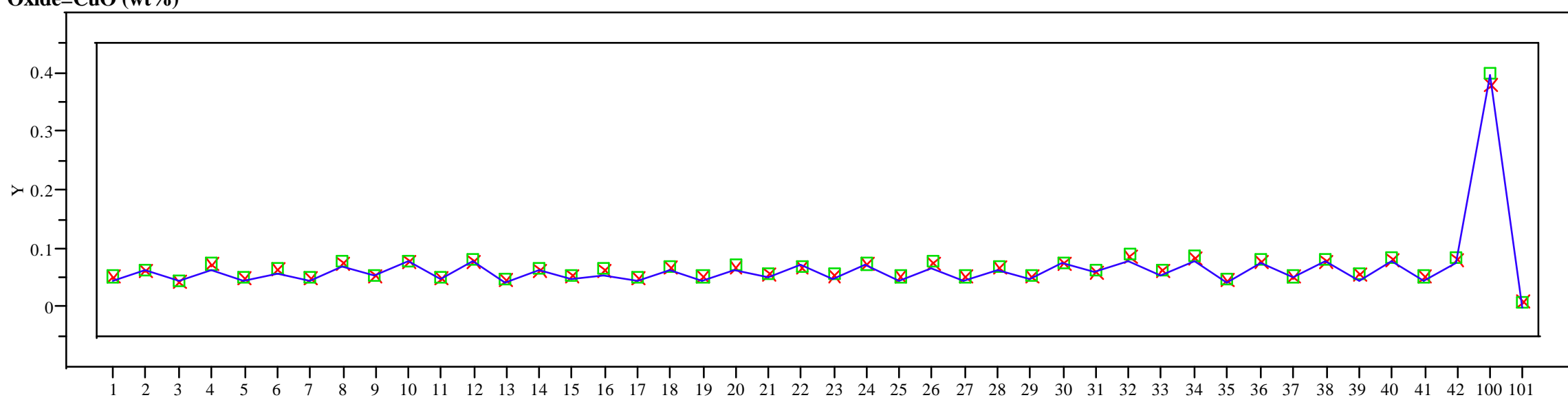

Oxide $=\mathrm{Fe} 2 \mathrm{O} 3(\mathrm{wt} \%)$

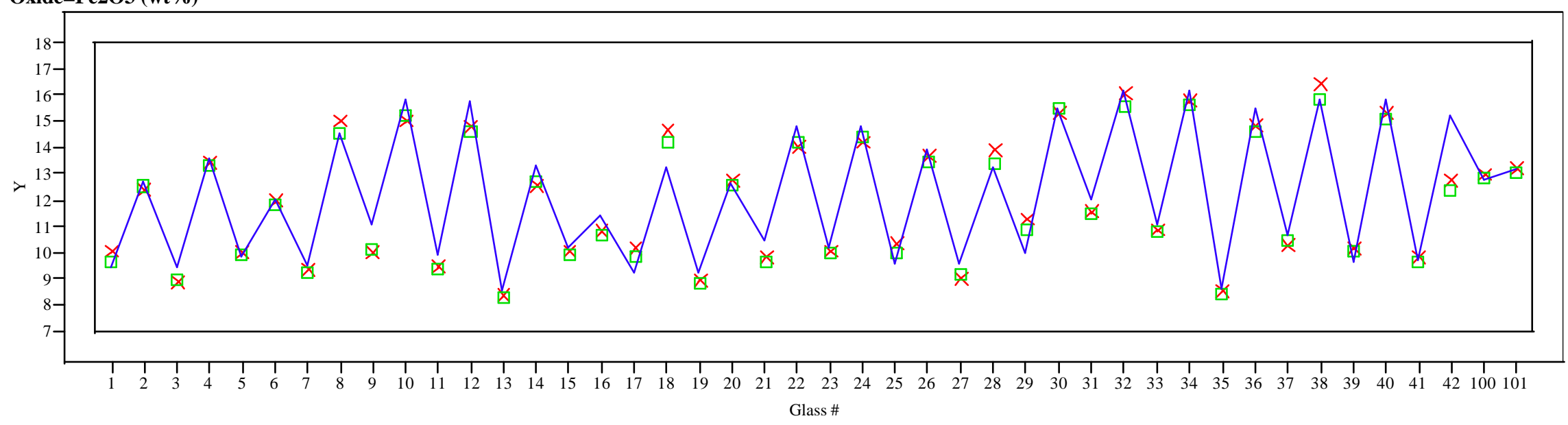

Y $\times$ Measured $\square$ Measured bc - Targeted 
Exhibit H.7: Measured, Measured Bias-Corrected, and Targeted Compositions for the SB3 Glasses by Oxide (continued)

(Batch 1 - 100 and Ustd - 101)

\section{Oxide $=\mathrm{K} 20(\mathrm{wt} \%)$}

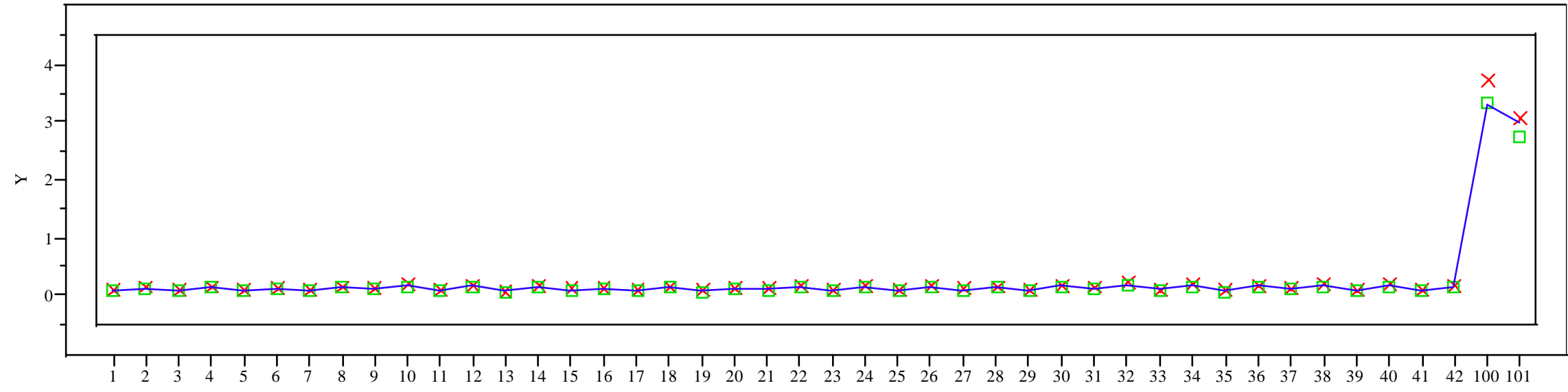
Glass \#

\section{Oxide $=\mathrm{La2O3}(\mathrm{wt} \%)$}

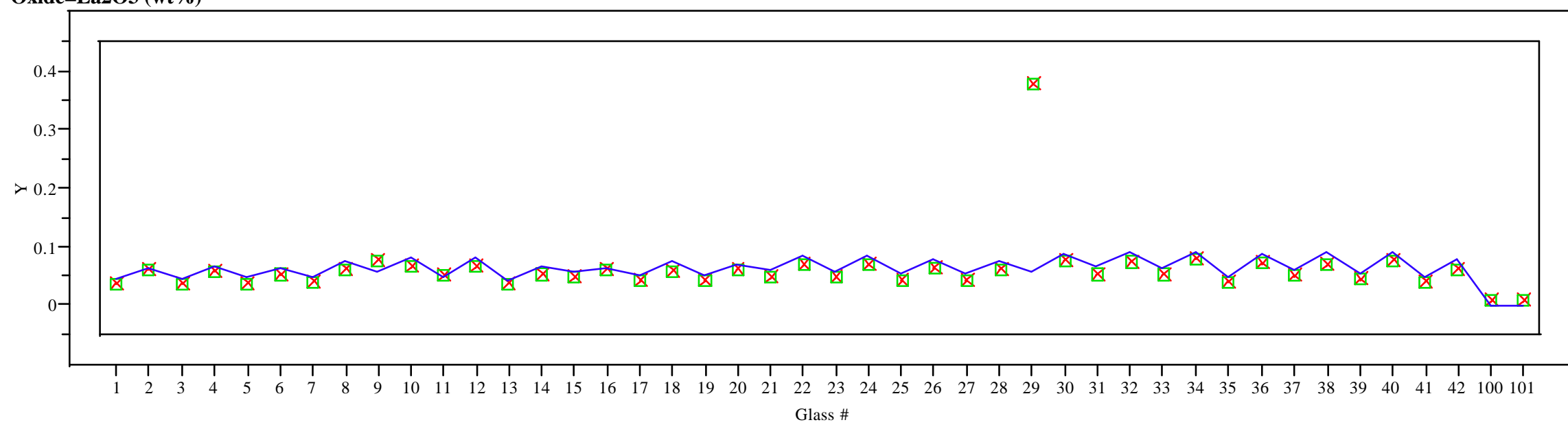

Y $\times$ Measured $\square$ Measured bc- Targeted 
Exhibit H.7: Measured, Measured Bias-Corrected, and Targeted Compositions for the SB3 Glasses by Oxide (continued) (Batch 1 - 100 and Ustd - 101)

Oxide $=\mathrm{Li} 20($ wt \%)

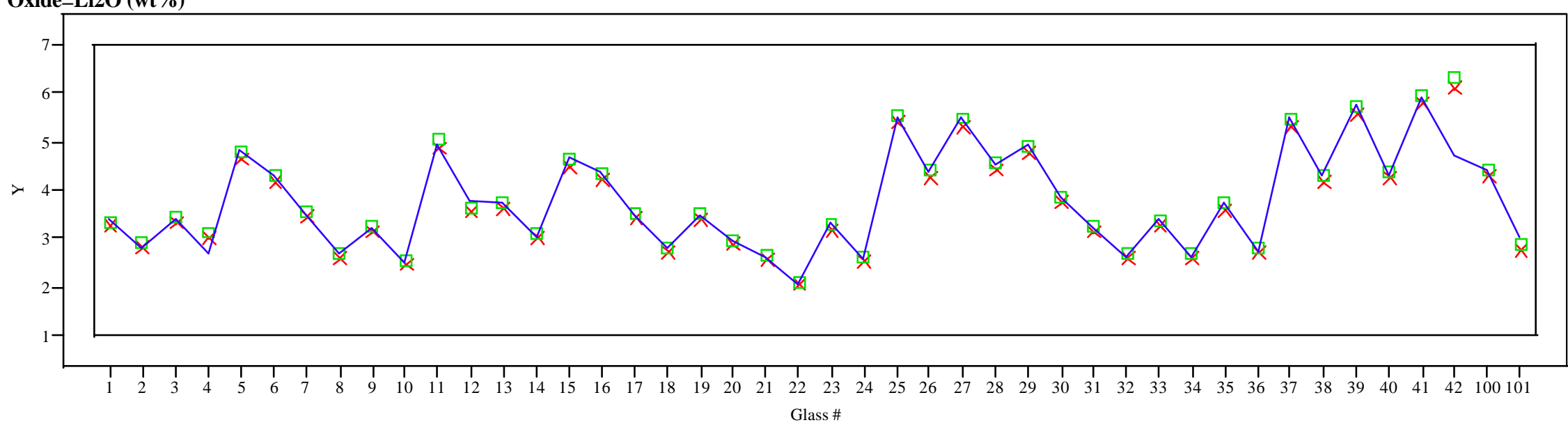

Oxide $=\operatorname{MgO}(w t \%)$

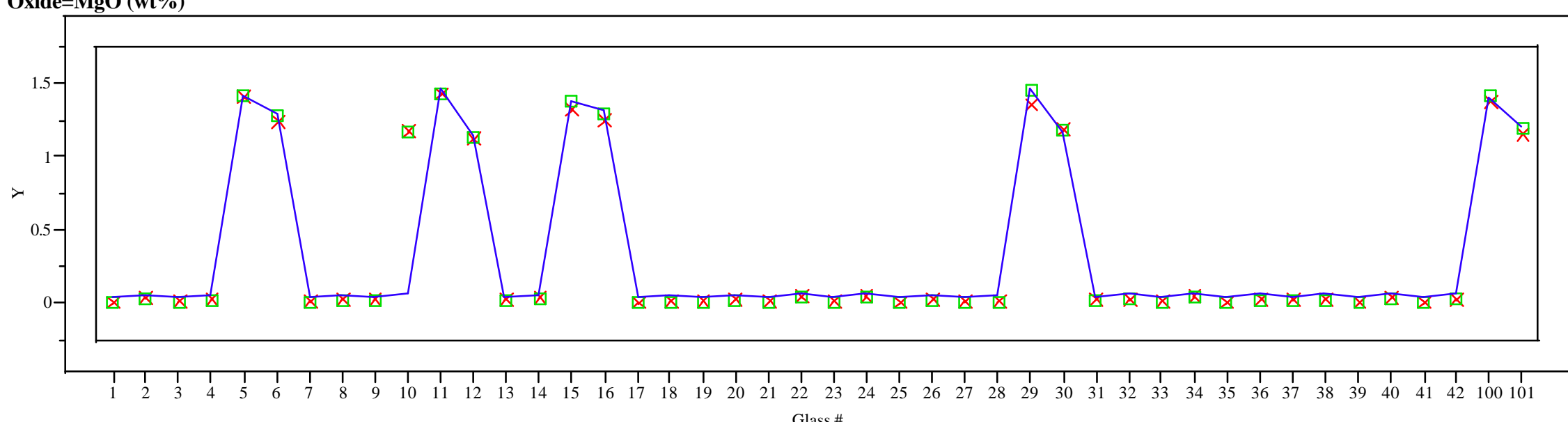

Y $\times$ Measured $\square$ Measured bc - Targeted 
Westinghouse Savannah River Company

Exhibit H.7: Measured, Measured Bias-Corrected, and Targeted Compositions for the SB3 Glasses by Oxide (continued) (Batch 1 - 100 and Ustd - 101)

\section{Oxide $=\mathrm{MnO}(\mathrm{wt} \%)$}

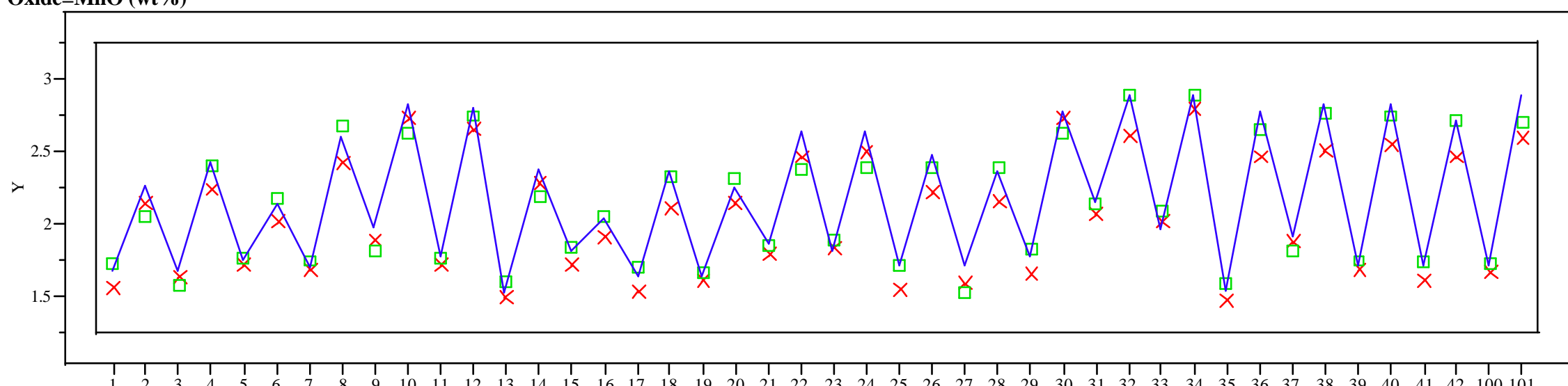

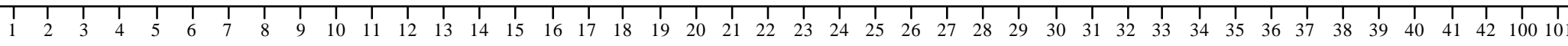
Glass \#

\section{Oxide $=\mathrm{Na} 2 \mathrm{O}(\mathrm{wt} \%)$}

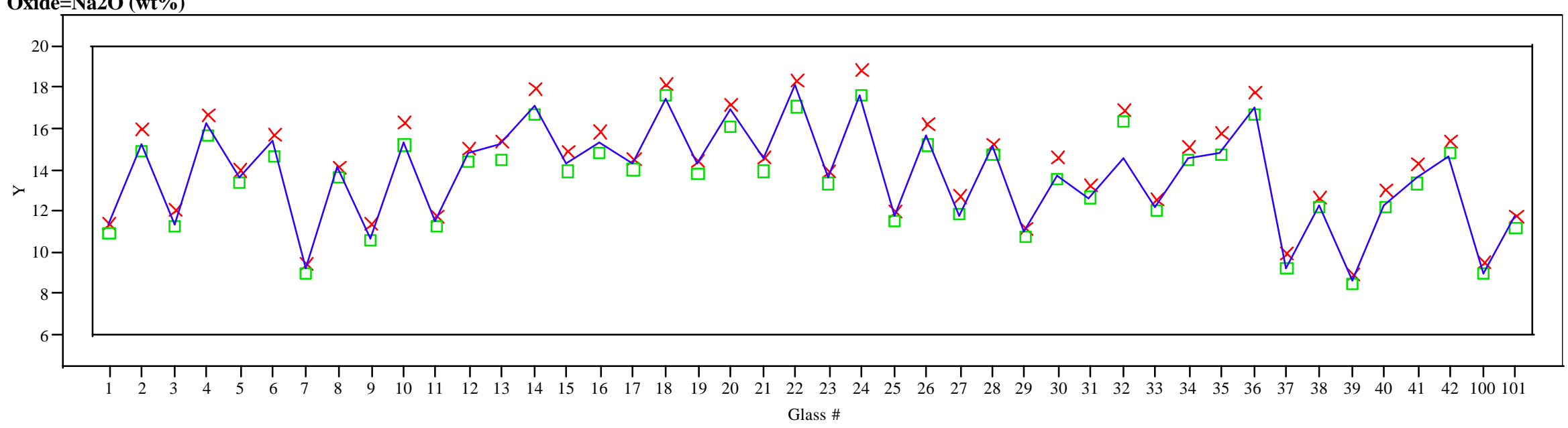

Y $\times$ Measured $\square$ Measured bc - Targeted 
Exhibit H.7: Measured, Measured Bias-Corrected, and Targeted Compositions for the SB3 Glasses by Oxide (continued) (Batch 1 - 100 and Ustd - 101)

\section{Oxide $=\mathrm{NiO}(\mathrm{wt} \%)$}

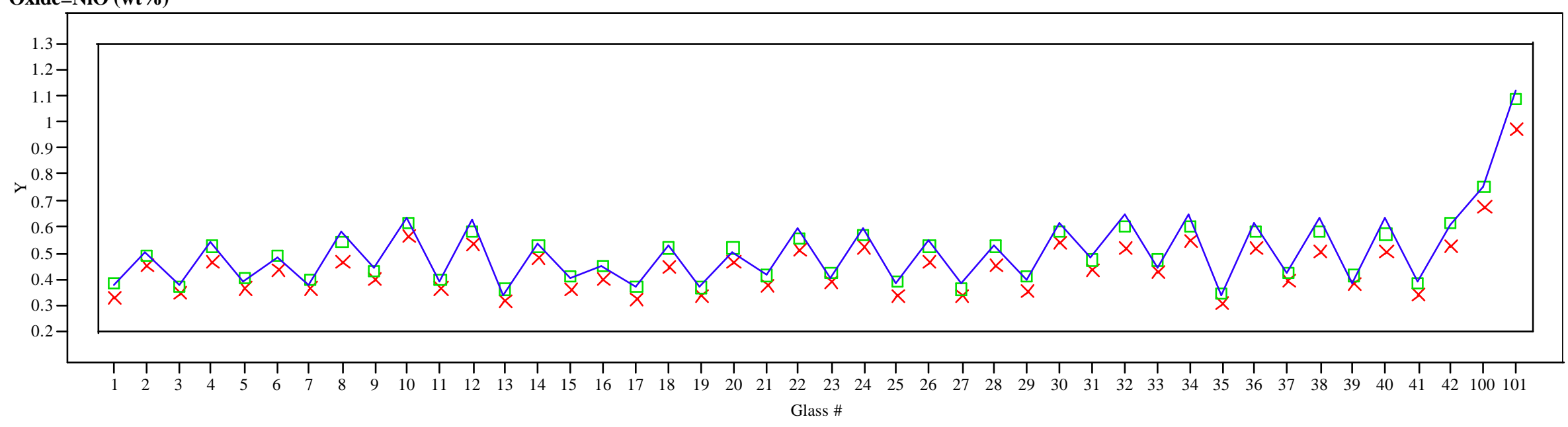

\section{Oxide $=\mathrm{PbO}(\mathrm{wt} \%)$}

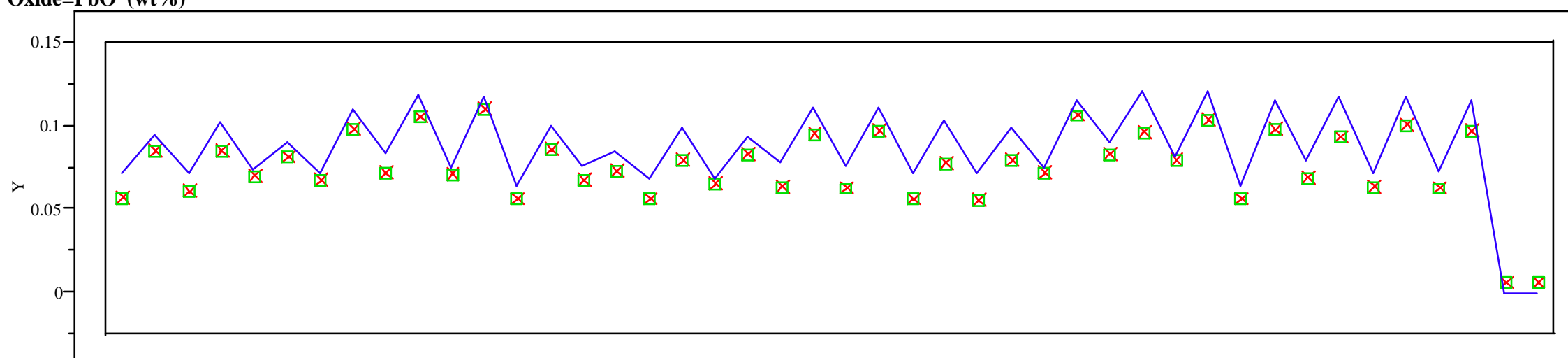

$\begin{array}{llllllllllllllllllllllllllllllllllllllllllllllllll}1 & 1 & 1 & 1 & 1 & 1 & 1 & 1 & 1 & 1 & 1 & 1 & 1 & 1 & 1 & 1 & 1 & 1 & 1 & 1 & 1 & 1 & 1 & 1 & 1 & 1 & 1 & 1 & 1 & 1 & 1 & 1 & 1 & 1 & 1 & 1 & 1 & 1 & 1 & 1 & 1 & 1 & 1 \\ 1 & 2 & 3 & 4 & 5 & 6 & 7 & 8 & 9 & 10 & 11 & 12 & 13 & 14 & 15 & 16 & 17 & 18 & 19 & 20 & 21 & 22 & 23 & 24 & 25 & 26 & 27 & 28 & 29 & 30 & 31 & 32 & 33 & 34 & 35 & 36 & 37 & 38 & 39 & 40 & 41 & 42 & 100 & 101\end{array}$ Glass \#

Y $\times$ Measured $\square$ Measured bc - Targeted 
Exhibit H.7: Measured, Measured Bias-Corrected, and Targeted Compositions for the SB3 Glasses by Oxide (continued) (Batch 1 - 100 and Ustd - 101)

Oxide=SiO2 $(w t \%)$

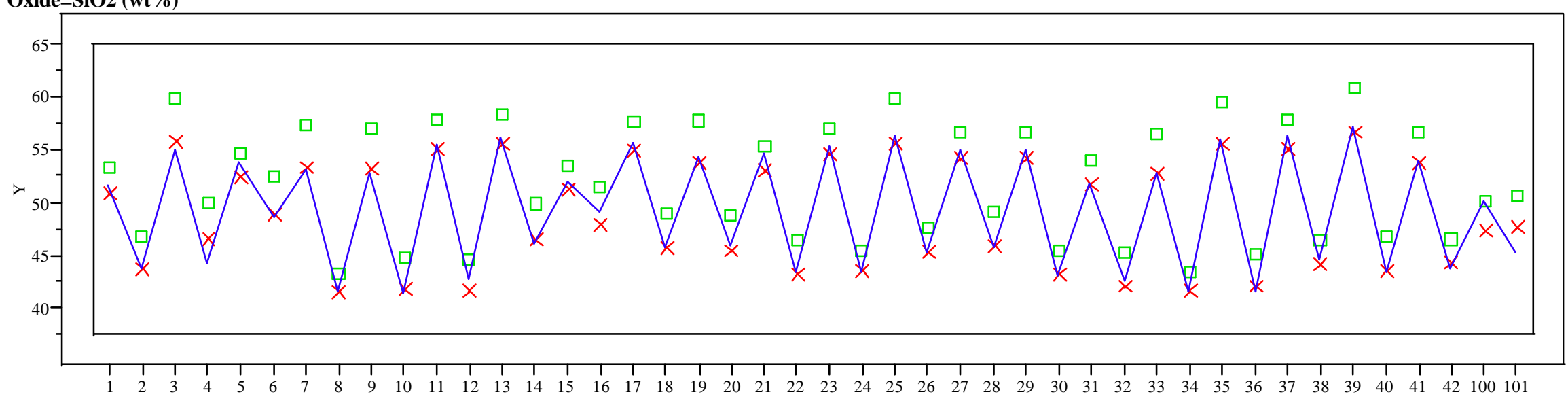

Oxide $=\mathrm{ThO} 2(\mathrm{wt} \%)$

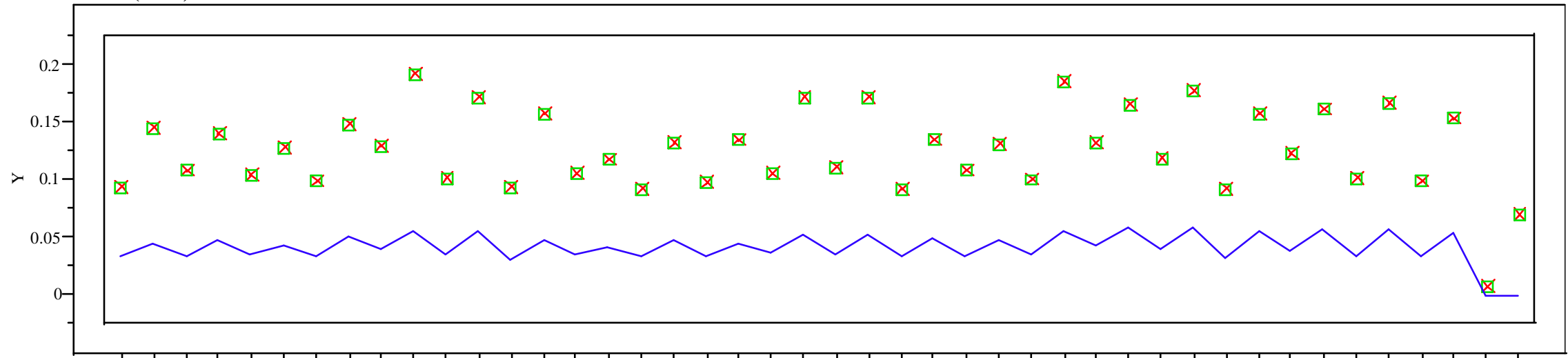

$\begin{array}{lllllllllllllllllllllllllllllllllllllllllllllllllll}1 & 1 & 1 & 4 & 1 & 1 & 1 & 1 & 1 & 1 & 1 & 1 & 1 & 1 & 1 & 1 & 1 & 1 & 1 & 1 & 1 & 1 & 1 & 1 & 1 & 1 & 1 & 1 & 1 & 1 & 1 & 1 & 1 & 1 & 1 & 1 & 1 & 1 & 1 & 1 & 1 & 1 & 1 & 1 \\ 1 & 2 & 3 & 4 & 5 & 6 & 7 & 8 & 9 & 10 & 11 & 12 & 13 & 14 & 15 & 16 & 17 & 18 & 19 & 20 & 21 & 22 & 23 & 24 & 25 & 26 & 27 & 28 & 29 & 30 & 31 & 32 & 33 & 34 & 35 & 36 & 37 & 38 & 39 & 40 & 41 & 42 & 100 & 101\end{array}$ Glass \#

Y X Measured $\square$ Measured bc - Targeted 
Exhibit H.7: Measured, Measured Bias-Corrected, and Targeted Compositions for the SB3 Glasses by Oxide (continued) (Batch 1 - 100 and Ustd - 101)

\section{Oxide $=\mathrm{TiO} 2(\mathrm{wt} \%)$}

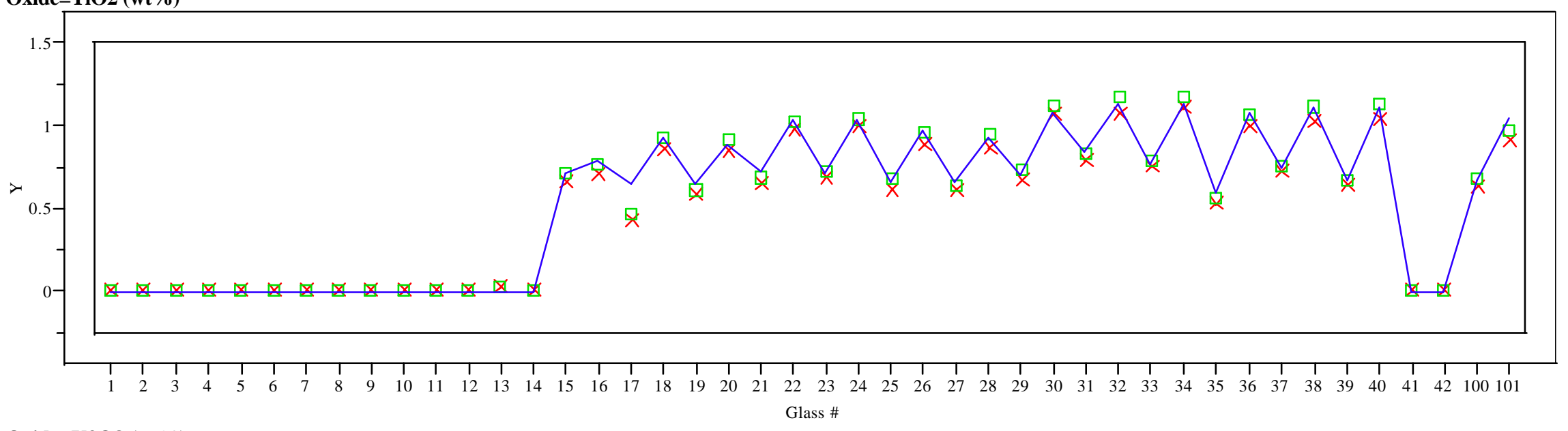

Oxide $=\mathrm{U} 308(\mathrm{wt} \%)$

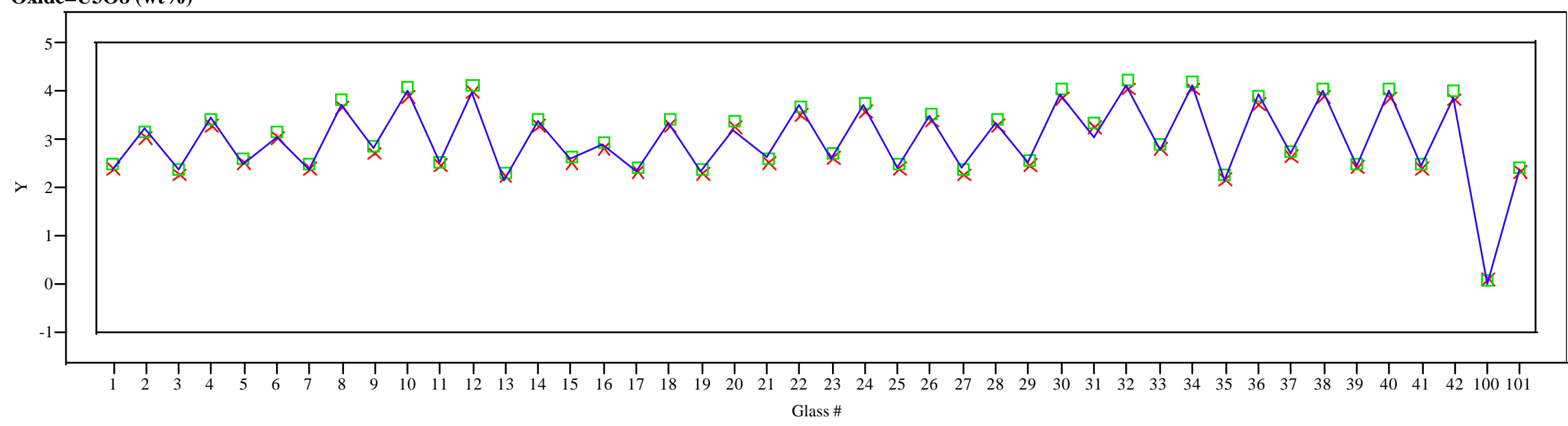

Y $\times$ Measured $\square$ Measured bc- Targeted 
Exhibit H.7: Measured, Measured Bias-Corrected, and Targeted Compositions for the SB3 Glasses by Oxide (continued) (Batch 1 - 100 and Ustd - 101)

\section{Oxide $=\mathrm{ZnO}(\mathrm{wt} \%)$}

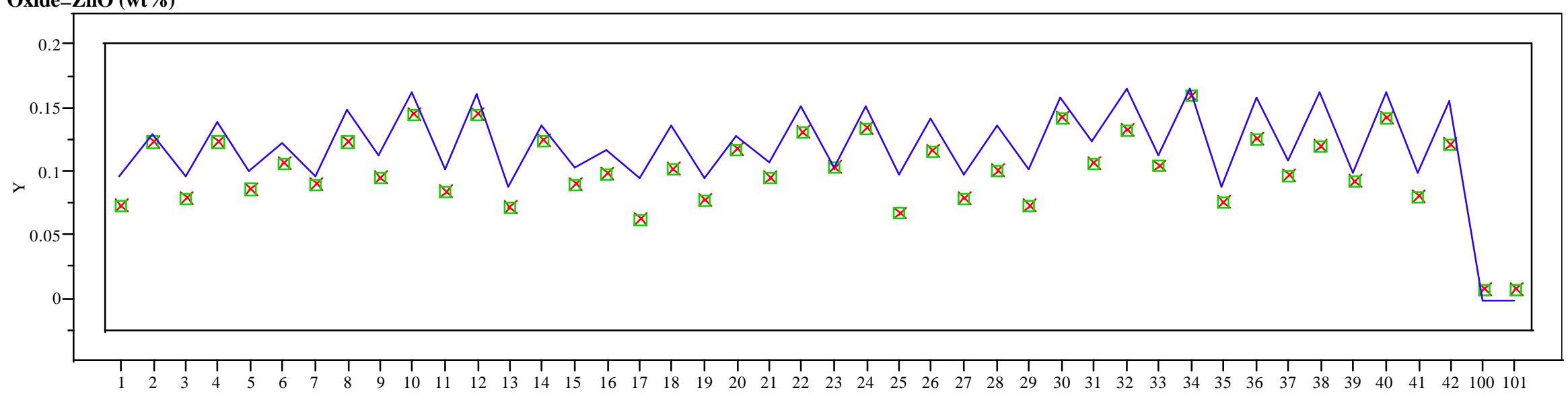

Oxide $=\mathrm{ZrO} 2(\mathrm{wt} \%)$

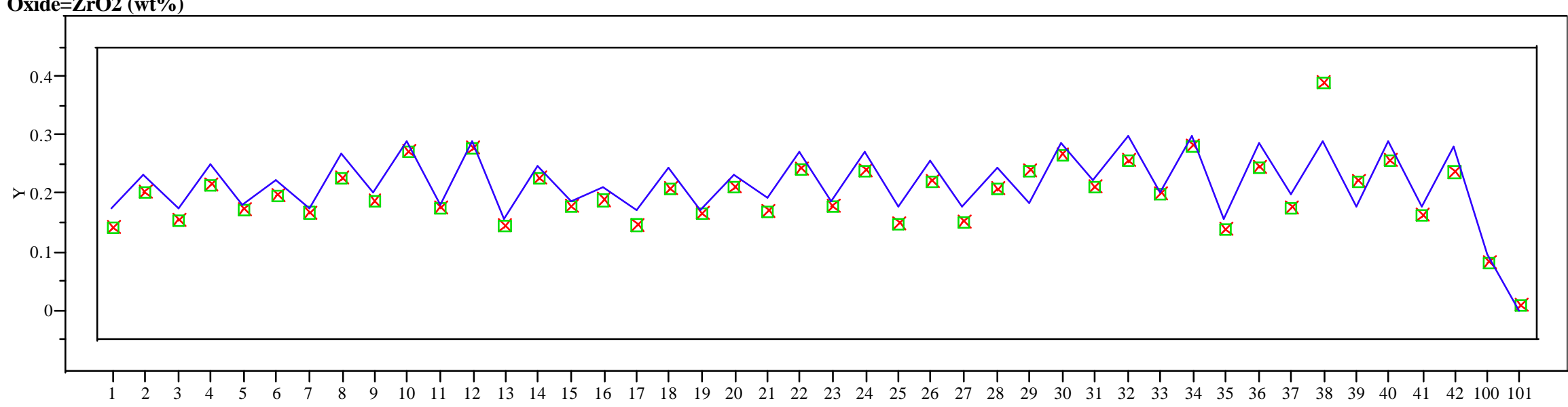
Glass \#

Y $\times$ Measured $\square$ Measured bc- Targeted 
Exhibit H.7: Measured, Measured Bias-Corrected, and Targeted Compositions for the SB3 Glasses by Oxide (continued) (Batch 1 - 100 and Ustd - 101)

Oxide $=$ Sum of Oxides

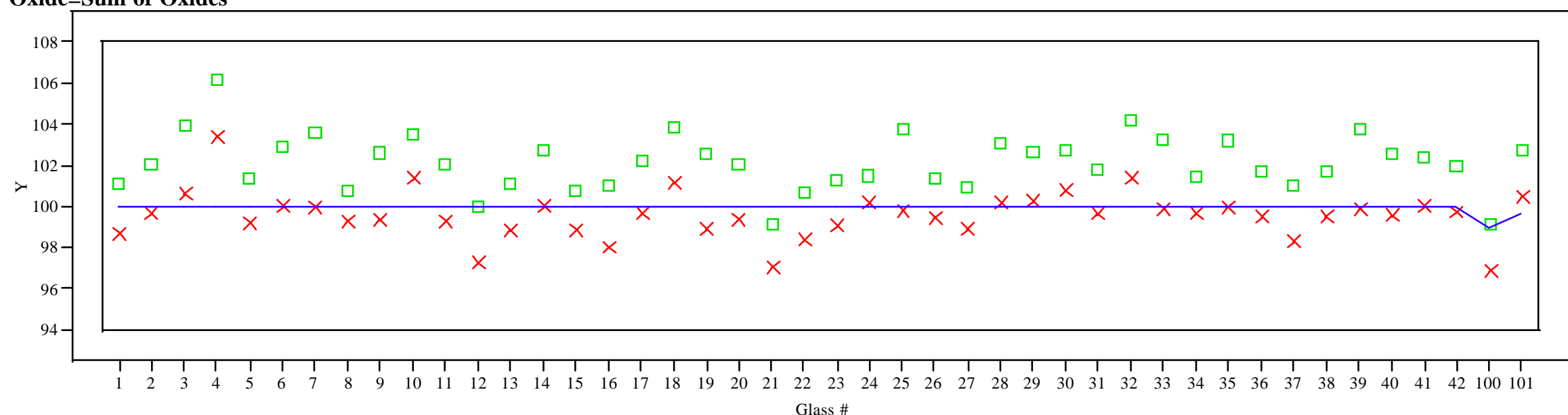

$\mathrm{Y} \times$ Measured $\square$ Measured bc - Targeted 
Immobilization Technology Section

WSRC-TR-2002-00549

Savannah River Technology Center

Rev. 0

Westinghouse Savannah River Company

\section{Appendix I}

Tables and Exhibits Supporting the Statistical Analysis of the Measured PCTs of the Study Glasses 
Table I.1. SRTC-ML Mesaurements of PCT Leachate Solutions for SB3 Phase 1 glasses

\begin{tabular}{|c|c|c|c|c|c|c|c|c|c|c|c|c|c|c|c|c|c|c|c|}
\hline \multirow{2}{*}{$\begin{array}{c}\text { Analytical } \\
\text { Plan }\end{array}$} & \multirow{2}{*}{$\begin{array}{c}\text { Glass } \\
\text { ID }\end{array}$} & \multirow{2}{*}{$\begin{array}{c}\text { SRTC-ML } \\
\text { ID }\end{array}$} & \multirow{2}{*}{$\begin{array}{c}\text { Heat } \\
\text { Treatment }\end{array}$} & \multirow{2}{*}{\multicolumn{2}{|c|}{$\begin{array}{lc} & \text { Seq } \\
\text { Block } & \#\end{array}$}} & \multicolumn{7}{|c|}{ As Reported (in ppm) by SRTC-ML } & \multicolumn{7}{|c|}{ Values (in ppm) after Adjusting for Dilution Effects } \\
\hline & & & & & & Al & B & $\mathrm{Fe}$ & $\mathrm{Li}$ & $\mathrm{Na}$ & $\mathrm{Si}$ & $\mathbf{U}$ & Al & B & $\mathbf{F e}$ & $\mathbf{L i}$ & $\mathbf{N a}$ & $\mathbf{S i}$ & $\mathbf{U}$ \\
\hline SRT-SCS-2002-00062 & soln std & stdb1-1 & & 1 & 1 & 3.65 & 20.3 & 3.75 & 9.08 & 81.8 & 47.5 & $<1.00$ & 3.65 & 20.30 & 3.75 & 9.08 & 81.80 & 47.50 & 0.50 \\
\hline SRT-SCS-2002-00062 & SB3-08ccc & $\mathrm{J} 48$ & $\mathrm{ccc}$ & 1 & 2 & 7.05 & 15.5 & 0.976 & 5.47 & 44.8 & 45.9 & 2.13 & 11.75 & 25.83 & 1.63 & 9.12 & 74.67 & 76.50 & 3.55 \\
\hline SRT-SCS-2002-00062 & SB3-05 & $\mathrm{J} 41$ & quenched & 1 & 3 & 5.72 & 9.81 & 3.45 & 12.5 & 61.9 & 90.4 & 2.33 & 9.53 & 16.35 & 5.75 & 20.83 & 103.17 & 150.67 & 3.88 \\
\hline SRT-SCS-2002-00062 & SB3-14ccc & $\mathrm{J} 51$ & $\mathrm{ccc}$ & 1 & 4 & 12.1 & 7.98 & 5.81 & 7.26 & 78.9 & 77.4 & 2.36 & 20.17 & 13.30 & 9.68 & 12.10 & 131.50 & 129.00 & 3.93 \\
\hline SRT-SCS-2002-00062 & SB3-03ccc & $\mathrm{J} 23$ & $\mathrm{ccc}$ & 1 & 5 & 5.18 & 10.3 & 3.88 & 5.51 & 24.4 & 58.7 & 2.67 & 8.63 & 17.17 & 6.47 & 9.18 & 40.67 & 97.84 & 4.45 \\
\hline SRT-SCS-2002-00062 & SB3-13 & $\mathrm{J} 32$ & quenched & 1 & 6 & 7.77 & 8.86 & 13.4 & 8.48 & 66 & 93.3 & 5.05 & 12.95 & 14.77 & 22.33 & 14.13 & 110.00 & 155.50 & 8.42 \\
\hline SRT-SCS-2002-00062 & SB3-10 & J37 & quenched & 1 & 7 & 12.6 & 12.1 & 2.47 & 5.3 & 58.1 & 54.8 & 1.78 & 21.00 & 20.17 & 4.12 & 8.83 & 96.84 & 91.34 & 2.97 \\
\hline SRT-SCS-2002-00062 & SB3-11ccc & J17 & $\mathrm{ccc}$ & 1 & 8 & 7.09 & 8.38 & 2.65 & 11 & 38.2 & 81.8 & 3.62 & 11.82 & 13.97 & 4.42 & 18.33 & 63.67 & 136.34 & 6.03 \\
\hline SRT-SCS-2002-00062 & SB3-14 & J53 & uenched & 1 & 9 & 12.8 & 8.3 & 7.48 & 7.07 & 85.7 & 82.8 & 2.3 & 21.33 & 13.83 & 12.47 & 11.78 & 142.84 & 138.00 & 3.83 \\
\hline SRT-SCS-2002-00062 & soln std & std-b-1-2 & & 1 & 10 & 3.9 & 21.1 & 3.8 & 9.32 & 79.5 & 50.9 & $<1.00$ & 3.90 & 21.10 & 3.80 & 9.32 & 79.50 & 50.90 & 0.50 \\
\hline SRT-SCS-2002-00062 & SB3-05ccc & $\mathrm{J} 31$ & $\mathrm{ccc}$ & 1 & 11 & 5.43 & 9.32 & 3.45 & 11.6 & 51.4 & 89.5 & 2.47 & 9.05 & 15.53 & 5.75 & 19.33 & 85.67 & 149.17 & 4.12 \\
\hline RT-SCS-2002-00062 & SB3-04ccc & J13 & $\mathrm{ccc}$ & 1 & 12 & 8.55 & 11.7 & 3.23 & 5.37 & 54.9 & 60.2 & 2.26 & 14.25 & 19.50 & 5.38 & & & 100.34 & 3.77 \\
\hline T-SCS-2002-00062 & $\mathrm{SR} 303$ & I88 & uenched & 1 & 13 & 4.32 & 11.7 & $45-15$ & 6. & & 63.7 & 2.78 & 7.20 & 19.50 & 7.58 & & & & 4.63 \\
\hline SRT-SCS-2002-00062 & SB3-08 & $\mathrm{J} 25$ & quenched & 1 & 14 & 8.03 & 17.1 & 1.25 & 5.59 & 44.1 & 49.5 & 2.2 & 13.38 & 28.50 & 2.08 & 9.32 & & 2.50 & 3.67 \\
\hline SRT-SCS-2002-00062 & SB3-04 & $\mathrm{J} 75$ & quenched & 1 & 15 & 9.16 & 12.3 & 2.78 & 5.19 & 59.2 & 62.7 & 2.41 & 15.27 & 20.50 & 4.63 & 8.65 & 98.67 & 104.50 & 4.02 \\
\hline RT-SCS-2002-00062 & SB3-13ccc & J57 & $\mathrm{ccc}$ & 1 & 16 & 7.87 & 7.98 & 14.3 & & & 88 & 4.72 & 13.12 & 13.30 & 23.83 & & & 67 & 7.87 \\
\hline RT-SCS-2002-00062 & SB3-10ccc & $\mathrm{J} 16$ & $\mathrm{ccc}$ & 1 & 17 & 12.6 & 12.4 & 2.6 & 5. & 55.7 & 58.3 & 2.08 & 21.00 & 20.67 & 4.42 & & & 17 & 3.47 \\
\hline SRT-SCS-2002-00062 & SB3-11 & $\mathrm{J} 62$ & uenched & 1 & 18 & 7.14 & 8. & 3.14 & 11.7 & 39.6 & 87.7 & 3. & 11.90 & 14.83 & 5.23 & 19.50 & & 146.17 & 5.33 \\
\hline SRT-SCS-2002-00062 & soln std & std-b1-3 & & 1 & 19 & 4.16 & 20.7 & 4.07 & 9.05 & 76.8 & 50.7 & $<1.00$ & 4.16 & 20.70 & 4.07 & 9.05 & & 50.70 & 0.50 \\
\hline T-SCS-2002-00062 & soln std & std-b2-1 & & 2 & 1 & 4.07 & 20.3 & 3.88 & 9. & & 49.9 & $<1.00$ & 4.07 & 20.30 & 3.88 & 9.39 & & 90 & 0.50 \\
\hline RT-SCS-2002-00062 & SB3-10 & $\mathrm{J} 65$ & iched & 2 & 2 & 13.7 & 12.1 & 4.1 & 5.67 & 62.3 & 55.6 & 2.13 & 22.83 & 20.17 & 6.83 & 9. & 10 & 92.67 & 3.55 \\
\hline TT-SCS-2002-00062 & SB3-14 & $\mathrm{J} 78$ & quenched & 2 & 3 & 12.8 & 8.15 & 6.97 & 7.24 & 91.9 & 79.9 & 2.62 & 21.33 & 13.58 & 11.62 & 12.07 & 153.17 & 133.17 & 4.37 \\
\hline RT-SCS-2002-00062 & SB3-08 & J85 & quenched & 2 & 4 & 8.31 & 16.3 & 2.24 & 5.77 & 48.3 & 47.4 & 2.43 & 13.85 & 27.17 & 3.73 & & & 79.00 & 4.05 \\
\hline 0062 & SB3 & $\mathrm{J} 34$ & quenched & 2 & 5 & 5. & 11 & 3 & 6. & 2 & 60.5 & 2.91 & 9.25 & 18 & 6.62 & 10 & & 100.84 & 4.85 \\
\hline T-SCS-2002-00062 & SB3-04cce & J87 & $\operatorname{ccc}$ & 2 & 6 & 8.06 & 11.1 & 2.48 & 5.36 & 57.4 & 57.2 & 2.24 & 13.43 & 18.50 & 4.13 & 8.93 & 95.67 & 95.34 & 3.73 \\
\hline RT-SCS-2002-00062 & SB3-08ccc & J14 & $\mathrm{ccc}$ & 2 & 7 & 7.81 & 15 & 1.35 & 5.58 & 44.6 & 46.6 & 2.39 & 13.02 & 25.00 & 2.25 & 9.30 & 74.33 & 77.67 & 3.98 \\
\hline$-2002-00062$ & SB3-13ccc & $\mathrm{J} 70$ & $\mathrm{ccc}$ & 2 & 8 & & & 1000 & & & 87.9 & 5.19 & & & & & & & 8.65 \\
\hline$-2002-00062$ & SB3-05ccc & J10 & $\mathrm{ccc}$ & 2 & 9 & 6.35 & 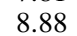 & 4.16 & 11.8 & 5 & 87.6 & 2.68 & 10.58 & 14.80 & 6.93 & 19.67 & 93.17 & 146.00 & 4.47 \\
\hline SRT-SCS-2002-00062 & soln std & std-b-2-2 & & 2 & 10 & 4.38 & 21.2 & 4.13 & 9.51 & 85.7 & 51.1 & $<1.00$ & 4.38 & 21.20 & 4.13 & 9.51 & 85.70 & 51.10 & 0.50 \\
\hline SRT-SCS-2002-00062 & SB3-13 & J39 & quenched & 2 & 11 & 8.53 & 8.89 & 14.7 & 8.6 & 68.9 & 94.3 & 5.34 & 14.22 & 14.82 & 24.50 & 14.33 & 114.84 & 157.17 & 8.90 \\
\hline SRT-SCS-2002-00062 & SB3-03ccc & & $\mathrm{ccc}$ & 2 & 12 & 5. & & & & & 61.8 & 0 & & & 8.90 & & & .00 & 4.83 \\
\hline 0062 & SB3-10ccc & $\mathrm{J} 54$ & $\mathrm{ccc}$ & 2 & 13 & 12 & 11 & 2.36 & & & 55.9 & 2.36 & 20.83 & 19.50 & 3.93 & 9. & 97 & 93.17 & 3.93 \\
\hline SRT-SCS-2002-00062 & SB3-11 & $\mathrm{J} 40$ & quenched & 2 & 14 & 7.36 & 8.25 & 3.2 & 11.3 & 41.3 & 83.2 & 3.68 & 12.27 & 13.75 & 5.33 & 18.83 & 68.83 & 138.67 & 6.13 \\
\hline SRT-SCS-2002-00062 & SB3-14ccc & J09 & $\mathrm{ccc}$ & 2 & 15 & 12.7 & 7.12 & 6.67 & 6.9 & 75.6 & 74.1 & 2.97 & 21.17 & 11.87 & 11.12 & 11.50 & 126.00 & 123.50 & 4.95 \\
\hline RT-SCS-2002-00062 & SB3-11ccc & $\mathrm{J} 12$ & $\mathrm{ccc}$ & 2 & 16 & 10.1 & 8.03 & 2.91 & 10 & & 80.2 & 3.88 & 16.83 & 13.38 & 4.85 & 18.00 & & 133.67 & 6.47 \\
\hline T-SCS-2002-00062 & CD 304 & $\mathrm{~J} 72$ & uenched & 2 & 17 & 13.6 & 11.7 & 2.74 & 5. & 63.3 & 60.8 & 2.78 & 22.67 & 19.50 & 4.57 & 8.63 & 105.50 & 101.34 & 4.63 \\
\hline T-SCS-2002-00062 & SB3-05 & J19 & quenched & 2 & 18 & 5.55 & 9. & 4.3 & 12 & 61.2 & 92.5 & 2.68 & 9.25 & 15.50 & 7.17 & 20 & & 154.17 & 4.47 \\
\hline SRT-SCS-2002-00062 & soln std & std-bl-2-3 & & 2 & 19 & 4.12 & 20.7 & 4.15 & 9.24 & 83.3 & 51 & $<1.00$ & 4.12 & 20.70 & 4.15 & 9.2 r -2.0 & 83.30 & 51.00 & 0.50 \\
\hline-00062 & & std-b3-1 & & 3 & 1 & 4.06 & 21.2 & 3.88 & & 8 & 50 & $<1.00$ & 4.06 & 21.20 & 3.88 & 9.6 & & 50.00 & 0.50 \\
\hline SRT-SCS-2002-00062 & SB3-10 & J29 & quenched & 3 & 2 & 12.5 & 12 & 2.2 & 5.49 & 62.9 & 54 & 2.16 & 20.83 & 20.00 & 3.67 & 9.15 & 104.84 & 90.00 & 3.60 \\
\hline SRT-SCS-2002-00062 & SB3-14 & J58 & quenched & 3 & 3 & 12.5 & 8.16 & 7.47 & 7.24 & 91.3 & 79.2 & 2.56 & 20.83 & 13.60 & 12.45 & 12.07 & 152.17 & 132.00 & 4.27 \\
\hline SRT-SCS-2002-00062 & SB3-05ccc & J86 & $\mathrm{ccc}$ & 3 & 4 & 5.6 & 9.14 & 3.93 & 12 & 56.2 & 87.9 & 2.87 & 9.33 & 15.23 & 6.55 & 20.00 & 93.67 & 146.50 & 4.78 \\
\hline
\end{tabular}


Table I.1. SRTC-ML Mesaurements of PCT Leachate Solutions for SB3 Phase 1 glasses

\begin{tabular}{|c|c|c|c|c|c|c|c|c|c|c|c|c|c|c|c|c|c|c|c|}
\hline \multirow{2}{*}{$\begin{array}{c}\text { Analytical } \\
\text { Plan }\end{array}$} & \multirow{2}{*}{$\begin{array}{c}\text { Glass } \\
\text { ID }\end{array}$} & \multirow{2}{*}{$\begin{array}{c}\text { SRTC-ML } \\
\text { ID }\end{array}$} & \multirow{2}{*}{$\begin{array}{c}\text { Heat } \\
\text { Treatment }\end{array}$} & \multirow{2}{*}{\multicolumn{2}{|c|}{$\begin{array}{lc} & \text { Seq } \\
\text { Block } & \#\end{array}$}} & \multicolumn{7}{|c|}{ As Reported (in ppm) by SRTC-ML } & \multicolumn{7}{|c|}{ Values (in ppm) after Adjusting for Dilution Effects } \\
\hline & & & & & & Al & B & $\mathbf{F e}$ & $\mathbf{L i}$ & $\mathbf{N a}$ & $\mathbf{S i}$ & $\mathbf{U}$ & Al & B & $\mathrm{Fe}$ & $\mathbf{L i}$ & $\mathbf{N a}$ & $\mathbf{S i}$ & $\mathbf{U}$ \\
\hline SRT-SCS-2002-00062 & SB3-03ccc & $\mathrm{J} 49$ & $\mathrm{ccc}$ & 3 & 5 & 17.6 & 10.3 & 4.08 & 5.81 & 25.8 & $\overline{59.1}$ & 3.03 & 29.33 & 17.17 & 6.80 & 9.68 & 43.00 & 98.50 & 5.05 \\
\hline SRT-SCS-2002-00062 & SB3-13ccc & $\mathrm{J} 22$ & $\mathrm{ccc}$ & 3 & 6 & 11.5 & 8.84 & 16.3 & 8.77 & 63.6 & 90.3 & 4.79 & 19.17 & 14.73 & 27.17 & 14.62 & 106.00 & 150.50 & 7.98 \\
\hline SRT-SCS-2002-00062 & SB3-08ccc & J84 & $\mathrm{ccc}$ & 3 & 7 & 7.57 & 16 & 1.2 & 5.84 & 46.5 & 47.6 & 2.6 & 2.62 & 26.67 & 2.00 & 9.73 & 77.50 & 79.33 & 4.33 \\
\hline SRT-SCS-2002-00062 & SB3-08 & $\mathrm{J} 15$ & quenched & 3 & 8 & 8.12 & 16.8 & 1.21 & 5.93 & 50.3 & 48 & 2.63 & 13.53 & 28.00 & 2.02 & 9.88 & 83.84 & 80.00 & 4.38 \\
\hline RT-SCS-2002-00062 & SB3-11 ccc & J59 & $\mathrm{ccc}$ & 3 & 9 & 7.16 & 8.33 & 2.53 & 11.3 & 41 & 80.8 & 4.19 & 11.93 & 13.88 & 4.22 & 18.83 & 68.33 & 134.67 & 6.98 \\
\hline RT-SCS-2002-00062 & soln std & std-b3-2 & & 3 & 10 & 3.97 & 21.2 & 4.02 & 9.66 & 85.8 & 50.4 & $<1.00$ & 3.97 & 21.20 & 4.02 & 9.66 & 85.80 & 50.40 & 0.50 \\
\hline SRT-SCS-2002-00062 & SB3-03 & J04 & quenched & 3 & 11 & 4.55 & 11.9 & 4.28 & 6.54 & 28 & 62.7 & 3.22 & 7.58 & 19.83 & 7.13 & 10.90 & 46.67 & 104.50 & 5.37 \\
\hline SRT-SCS-2002-00062 & SB3-14ccc & J63 & $\mathrm{ccc}$ & 3 & 12 & 13.7 & 7.79 & 7.01 & 7.65 & 81.3 & 77.7 & 3.24 & 22.83 & 12.98 & 11.68 & 12.75 & 135.50 & 129.50 & 5.40 \\
\hline RT-SCS-2002-00062 & SB3-05 & $\mathrm{J} 33$ & uenche & 3 & 13 & 6.47 & 9.75 & 4.78 & 12.6 & 63.9 & 92.6 & 2.87 & 10.78 & 16.25 & 7.97 & 21.00 & & 54.34 & 4.78 \\
\hline T-SCS-2002-00062 & SB3-10ccc & J79 & ccc & 3 & 14 & 13.4 & 12 & 3.86 & 5.86 & 60.1 & 56.3 & 2.69 & 22.33 & 20.00 & 6.43 & 9.77 & 100.17 & 93.84 & 4.48 \\
\hline RT-SCS-2002-00062 & SB3-04 & J69 & quenched & 3 & 15 & 9.94 & 12 & 4.78 & 5.39 & 66.3 & 60.5 & 2.81 & 16.57 & 20.00 & 7.97 & 8.98 & & 100.84 & 4.68 \\
\hline T-SCS-2002-00062 & SB3-13 & $\mathrm{J} 47$ & quenched & 3 & 16 & 8.72 & 9.02 & 14.5 & & $72=$ & 94.3 & 5.7 & & 15.03 & 24.17 & & & .17 & 9.50 \\
\hline T-SCS-2002-00062 & SB3-04ccc & $\mathrm{J} 27$ & $\mathrm{ccc}$ & 2 & 17 & 9.16 & 11.9 & 226 & 5.8 & 6 & 61 & 2.49 & & 19.83 & & & & & 4.15 \\
\hline SRT-SCS-2002-00062 & SB3-11 & $\mathrm{J} 38$ & quenched & 3 & 18 & 7.96 & 8.72 & 3.23 & 12 & 43.3 & 84.8 & 3.63 & 13.27 & 14.53 & 5.38 & 20.00 & & 41.34 & 6.05 \\
\hline SRT-SCS-2002-00062 & soln std & std-b3-3 & & 3 & 19 & 4.21 & 21.4 & 4.03 & 9.87 & 88.7 & 51.6 & $<1.00$ & 4.21 & 21.40 & 4.03 & 9.87 & 88.70 & 51.60 & 0.50 \\
\hline RT-SCS-2002-00062 & soln std & std-b4-1 & & 4 & 1 & 3.84 & 21.2 & & & 8 & 49.7 & $<1.00$ & & & & & & & 0.50 \\
\hline RT-SCS-2002-00062 & SB3-06ccc & J01 & $\mathrm{ccc}$ & 4 & 2 & 7.5 & 9.5 & 6.6 & 11. & 74 & 86.9 & 2.66 & 12.50 & 15.83 & 11.13 & 18.67 & & 44.84 & 4.43 \\
\hline RT-SCS-2002-00062 & ARM & $\mathrm{J} 60$ & & 4 & 3 & 2.49 & 12.4 & $<0.040$ & 8.6 & 24 & 38 & $<1.00$ & 4.15 & 20.67 & 0.03 & 14 & & & 0.83 \\
\hline RT-SCS-2002-00062 & SB3-02 & J05 & quenched & 4 & 4 & 6.52 & 18.9 & 1. & 6 & 56.3 & 50.5 & 2.55 & 10.87 & 31.50 & 2.50 & 10.00 & & 17 & 4.25 \\
\hline $02-00062$ & SB3-06 & $\mathrm{J} 50$ & quenched & 4 & 5 & 8.33 & 10.4 & 6.08 & 12.2 & 87.5 & 94.6 & 2.12 & 13.88 & 17.33 & 10.13 & 20 & & 157.67 & 3.53 \\
\hline 0062 & EA & J52 & & 4 & 6 & $<0.090$ & 38.7 & $<0.040$ & 10.9 & 10 & 52.6 & $<1.00$ & 0.75 & 645.00 & 0.33 & 181.67 & & 876.67 & 8.33 \\
\hline T-SCS-2002-00062 & SB3-07ccc & J36 & $\mathrm{ccc}$ & 4 & 7 & 2.9 & 18 & 1.6 & 7.0 & 20.9 & 53.6 & 2.39 & 4.83 & 30.00 & 2.67 & 11.80 & 34.83 & 89.34 & 3.98 \\
\hline RT-SCS-2002-00062 & SR $3-07$ & $\mathrm{~J} 42$ & quenched & 4 & 8 & 4.43 & 19.1 & 2.78 & 708 & 21 & 55.8 & 2.77 & 7.38 & 31.83 & & 13.30 & & 3.00 & 4.62 \\
\hline 062 & SB3-01ccc & $\mathrm{J} 28$ & $\mathrm{ccc}$ & 4 & 9 & 3. & 29.2 & 1 & 10 & 44.4 & 67.3 & 2. & 6.48 & 7 & 2. & 17 & & 112.17 & 4.67 \\
\hline RT-SCS-2002-00062 & soln std & std-b4-2 & & 4 & 10 & 3.9 & 20.8 & 3.5 & 9. & 85 & 49.7 & $<1.00$ & 3.99 & 20.80 & 3.54 & 9.40 & & 49.70 & 0.50 \\
\hline SRT-SCS-2002-00062 & SB3-12 & J74 & quenched & 4 & 11 & 13.8 & 9.74 & 6.12 & 10.2 & 80.3 & 75.9 & 2.04 & 23.00 & 16.23 & 10.20 & 17.00 & 133.84 & 126.50 & 3.40 \\
\hline-00062 & SB3-09 & J90 & quenched & 4 & 12 & & & & & & 52.8 & 2.69 & & & & & & & 4.48 \\
\hline 062 & blank & J80 & & 4 & 13 & $<0.090$ & $<0.150$ & $<0.040$ & $<0.500$ & $<0.100$ & $<0.790$ & $<1.00$ & 0.08 & 0.13 & 0. & 0. & 0 & 0.66 & 0.83 \\
\hline SRT-SCS-2002-00062 & SB3-12ccc & J26 & $\mathrm{ccc}$ & 4 & 14 & 13.9 & 9.42 & 4.96 & 11 & 78.2 & 76.9 & 2.18 & 23.17 & 15.70 & 8.27 & 18.33 & 130.34 & 128.17 & 3.63 \\
\hline SRT-SCS-2002-00062 & SB3-01 & J61 & quenched & 4 & 15 & 2.28 & 30 & 3.05 & & 45. & 65.4 & 2.98 & 3.80 & 50.00 & 5.08 & 18.00 & & 109.00 & 4.97 \\
\hline T-SCS-2002-00062 & SB3-09cec & J71 & $\mathrm{ccc}$ & 4 & 16 & & & & & & 53.3 & ? & & & & & & & 4.50 \\
\hline 0062 & SB3- & J11 & $\mathrm{ccc}$ & 4 & 17 & 6. & & 3. & 6 & 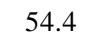 & 51.9 & 2.82 & 11.62 & 29.33 & 5 . & 10 & & 86.50 & 4.70 \\
\hline T-SCS-2002-00062 & soln std & std4-3 & & 4 & 18 & 3.7 & 20.7 & 3.7 & 9.48 & 86.3 & 49.7 & $<1.00$ & 3.70 & 20.70 & 3.70 & 9.48 & 86.30 & 49.70 & 0.50 \\
\hline SRT-SCS-2002-00062 & soln std & std-b5-1 & & 5 & 1 & 4.06 & 21.2 & 3.81 & 9.6 & 86.2 & 49.9 & $<1.00$ & 4.06 & 21.20 & 3.81 & 9.60 & 86.20 & 49.90 & 0.50 \\
\hline RT-SCS-2002-00062 & blank & J82 & & 5 & 2 & $<0.090$ & 0.374 & $<0.040$ & $<0.500$ & 0.829 & $<0.790$ & 0.04 & 0.08 & & 0.03 & & & 0.66 & 0.07 \\
\hline$-2002-00062$ & EA & J55 & & 5 & 3 & 0.559 & 38.3 & 0.065 & 11 & 99.3 & 53 & $<1.00$ & 9.32 & 638.33 & 1.08 & 185.00 & 1655.00 & 883.34 & 8.33 \\
\hline -2002-00062 & SB3-02ccc & J68 & $\mathrm{ccc}$ & 5 & 4 & & & 2.2 & & & 50.6 & 2.5 & 11.62 & 33 & 3.70 & & & & 4.17 \\
\hline T-SCS-2002-00062 & SB3-01ccc & $\mathrm{J} 73$ & $\mathrm{ccc}$ & 5 & 5 & 4.8 & 30.4 & 2.93 & 10.7 & 45 & 68.4 & 2.83 & 8.07 & 50.67 & 4. & 17.83 & & 114.00 & 4.72 \\
\hline & & J92 & lenched & 5 & 6 & & 9.34 & 2.8 & & 22.2 & 54.2 & 2.78 & 9.25 & & & & & 90.34 & 4.63 \\
\hline T-SCS-2002-00062 & SB3-12ccc & J91 & $\mathrm{ccc}$ & 5 & 7 & 14.2 & 9.76 & 5.45 & 11.1 & 77.5 & 77.6 & 2.14 & 23.67 & 16.27 & 9.08 & 18.50 & 129.17 & 129.34 & 3.57 \\
\hline$-2002-00062$ & & J18 & nch & 5 & 8 & 2.44 & 30.1 & 2 & 10 & 44.4 & 66.2 & 2.86 & 4.07 & 50.17 & 3.33 & 18.00 & & 110.34 & 4.77 \\
\hline SRT-SCS-2002-00062 & SB3-07 & J06 & quenched & 5 & 9 & 9.83 & 19.1 & 2.85 & 8.12 & 21.4 & 57.4 & 2.52 & 16.38 & 31.83 & 4.75 & 13.53 & 35.67 & 95.67 & \\
\hline
\end{tabular}


Table I.1. SRTC-ML Mesaurements of PCT Leachate Solutions for SB3 Phase 1 glasses

\begin{tabular}{|c|c|c|c|c|c|c|c|c|c|c|c|c|c|c|c|c|c|c|c|}
\hline \multirow{2}{*}{$\begin{array}{c}\text { Analytical } \\
\text { Plan }\end{array}$} & \multirow{2}{*}{$\begin{array}{c}\text { Glass } \\
\text { ID }\end{array}$} & \multirow{2}{*}{$\begin{array}{c}\text { SRTC-ML } \\
\text { ID }\end{array}$} & \multirow{2}{*}{$\begin{array}{c}\text { Heat } \\
\text { Treatment }\end{array}$} & \multirow{2}{*}{\multicolumn{2}{|c|}{$\begin{array}{c}\text { Seq } \\
\#\end{array}$}} & \multicolumn{7}{|c|}{ As Reported (in ppm) by SRTC-ML } & \multicolumn{7}{|c|}{ Values (in ppm) after Adjusting for Dilution Effects } \\
\hline & & & & & & Al & B & $\mathrm{Fe}$ & $\mathrm{Li}$ & $\mathbf{N a}$ & $\mathbf{S i}$ & $\mathbf{U}$ & Al & B & $\mathbf{F e}$ & $\mathbf{L i}$ & $\mathbf{N a}$ & $\mathbf{S i}$ & $\mathbf{U}$ \\
\hline SRT-SCS-2002-00062 & soln std & std-b4-2 & & 5 & 10 & 3.96 & 20.9 & 3.7 & 9.54 & 84.7 & 49.8 & $<1.00$ & 3.96 & 20.90 & 3.70 & 9.54 & 84.70 & 49.80 & 0.50 \\
\hline SRT-SCS-2002-00062 & SB3-02 & J03 & quenched & 5 & 11 & 6.83 & 20 & 1.65 & 6.3 & 58.5 & 52.2 & 2.62 & 11.38 & 33.33 & 2.75 & 10.50 & 97.50 & 87.00 & 4.37 \\
\hline SRT-SCS-2002-00062 & SB3-06ccc & J77 & $\mathrm{ccc}$ & 5 & 12 & 7.78 & 9.4 & 6.49 & 11.4 & 74.7 & 86.6 & 2.65 & 12.97 & 15.67 & 10.82 & 19.00 & 124.50 & 144.34 & 4.42 \\
\hline SRT-SCS-2002-00062 & SB3-07ccc & J83 & $\mathrm{ccc}$ & 5 & 13 & 3.67 & 18 & 2.72 & 7.28 & 21.1 & 55.1 & 2.46 & 6.12 & 30.00 & 4.53 & 12.13 & 35.17 & 91.84 & 4.10 \\
\hline SRT-SCS-2002-00062 & SB3-12 & J81 & uenched & 5 & 14 & 14.5 & 10.1 & 4.95 & 10.7 & 83.3 & 78.9 & 1.95 & 24.17 & 16.83 & 8.25 & 17.83 & 138.84 & 131.50 & 3.25 \\
\hline RT-SCS-2002-00062 & SB3-06 & $\mathrm{J} 24$ & quenched & 5 & 15 & 7.97 & 10.1 & 5.4 & 11.9 & 83.9 & 92.8 & 2 & 13.28 & 16.83 & 9.00 & 19.83 & 139.84 & 154.67 & 3.33 \\
\hline SRT-SCS-2002-00062 & SB3-09ccc & J56 & $\mathrm{ccc}$ & 5 & 16 & 11 & 9.08 & 2.9 & 5.43 & 21.9 & 55.4 & 2.82 & 18.33 & 15.13 & 4.83 & 9.05 & 36.50 & 92.34 & 4.70 \\
\hline SRT-SCS-2002-00062 & ARM & $\mathrm{J} 67$ & & 5 & 17 & 3.02 & 11.7 & $<0.040$ & 8.42 & 23 & 37.6 & $<1.00$ & 5.03 & 19.50 & 0.03 & 14.03 & 38.33 & 62.67 & 0.83 \\
\hline SRT-SCS-2002-00062 & soln std & std-b5-3 & & 5 & 18 & 4.15 & 20.7 & 4.03 & 9.47 & 84.8 & 49.9 & $<1.00$ & 4.15 & 20.70 & 4.03 & 9.47 & 84.80 & 49.90 & 0.50 \\
\hline SRT-SCS-2002-00062 & soln std & std-b6-1 & & 6 & 1 & 3.94 & 21.5 & 3.8 & 9.46 & 85.3 & 50.4 & $<1.00$ & 3.94 & 21.50 & 3.80 & 9.46 & 85.30 & 0.40 & 0.50 \\
\hline SRT-SCS-2002-00062 & SB3-12ccc & $\mathrm{J} 20$ & $\mathrm{ccc}$ & 6 & 2 & 14.2 & 10.1 & 5.67 & 11.1 & 75.6 & 79.4 & 1.86 & 23.67 & 16.83 & 9.45 & 18.50 & 126.00 & 132.34 & 3.10 \\
\hline SRT-SCS-2002-00062 & ARM & J66 & & 6 & 3 & 2.83 & 11.6 & $<0.040$ & 8.12 & 22.2 & 37.7 & $<1.00$ & 4.72 & 19.33 & 0.03 & 13.53 & 37.00 & 62.83 & 0.83 \\
\hline SRT-SCS-2002-00062 & EA & J44 & & 6 & 4 & 0.565 & 39.4 & 1.75 & 11 & 96.8 & 54.7 & $<1.00$ & 9.42 & 656.67 & 29.17 & 183.33 & 3.34 & 911.67 & 8.33 \\
\hline T-SCS-2002-00062 & SB3-07 & $\mathrm{J} 21$ & quenched & 6 & 5 & 4.19 & $18+2$ & 3.6 & 7.97 & 21.1 & 57.5 & 2.31 & 6.98 & 32.33 & 6.03 & & & & 3.85 \\
\hline SRT-SCS-2002-00062 & SB3-07ccc & $\mathrm{J} 35$ & $\mathrm{ccc}$ & 6 & 6 & 3.77 & 19.5 & 3.1 & 7.75 & 23.3 & 57.9 & 2.32 & 6.28 & 32.50 & 5.27 & 12.92 & & 50 & 3.87 \\
\hline SRT-SCS-2002-00062 & SB3-02 & J64 & quenched & 6 & 7 & 6.41 & 18.9 & 1.48 & 5.83 & 53.8 & 50.3 & 2.18 & 10.68 & 31.50 & 2.47 & 9.72 & 67 & 83.84 & 3.63 \\
\hline RT-SCS-2002-00062 & SB3-01ccc & J89 & $\mathrm{ccc}$ & 6 & 8 & 2.46 & 31.4 & 2.85 & 10.9 & 45 & 70.5 & 2.57 & 4.10 & 52.33 & 4.75 & 18.17 & .00 & 117.50 & 4.28 \\
\hline 0062 & SB3- & $\mathrm{J} 0$ & $\mathrm{ccc}$ & 6 & 9 & 7.1 & 10 & & & $\tilde{s}$ & 53.6 & 2.32 & 11.83 & 31.67 & 5.02 & & & & 3.87 \\
\hline 2002-00062 & soln std & std-b6-2 & & 6 & 10 & 4.14 & 21.7 & 4. & 9 & 84.1 & 51.7 & $<1.00$ & 4.14 & 21.70 & 4.17 & & & 1.70 & 0.50 \\
\hline SRT-SCS-2002-00062 & SB3-12 & $\mathrm{J} 07$ & quenched & 6 & 11 & 14 & 10.5 & 5.25 & 10.7 & 79.3 & 80.9 & 1.65 & 23.33 & 17.50 & 8.75 & 17.83 & 132.17 & 134.84 & 2.75 \\
\hline TT-SCS-2002-00062 & SB3-06ccc & J76 & $\mathrm{ccc}$ & 6 & 12 & 8.86 & 10.4 & 7 & 12 & 9 & 93.2 & 1.86 & 14.77 & 17.33 & 12.27 & 50 & 50 & .34 & 3.10 \\
\hline $02-00062$ & SB3-06 & J46 & Iench & 6 & 13 & 8.39 & 10 & -5 & 12.5 & 84.5 & 97.8 & 1.84 & 13.98 & 18.00 & 8.90 & 20.83 & & 63.00 & 3.07 \\
\hline T-SCS-2002-00062 & SB3-01 & $\mathrm{J} 30$ & quenched & 6 & 14 & 7.77 & 3 & 2.8 & 11.8 & 43 & 70.4 & 2.76 & 12.95 & 55.17 & 4.72 & 19.67 & 73. & 117.34 & 4.60 \\
\hline T-SCS-2002-00062 & SB3-09 & J02 & quenched & 6 & 15 & 5.7 & & & & & 57.8 & 2.52 & & & & & & & 4.20 \\
\hline 02-00062 & SB3-09ccc & $\mathrm{J} 45$ & $\operatorname{ccc}$ & 6 & 16 & 7.74 & 9. & 2 & 5. & 21.6 & 57.5 & 2.41 & 12.90 & 15.72 & 4.30 & 9 & 20 & 84 & 4.02 \\
\hline TT-SCS-2 & soln std & std-b6-3 & & 6 & 17 & 4.3 & & 1 & & 8 & 52.2 & $<1.00$ & 4.39 & 21.60 & 4.97 & & & 52.20 & 0.50 \\
\hline SRT-SCS-2002-00063 & soln std & std-b1-1 & & 1 & 1 & 4.04 & 21.3 & 3.9 & 9.61 & 85.7 & 50.1 & $<1.00$ & 4.04 & 21.30 & 3.99 & 9.61 & 85.70 & 50.10 & 0.50 \\
\hline T-SCS-2002-00063 & SB3-23 & $\mathrm{m} 03$ & quenched & 1 & 2 & 6.1 & 561 & 7 & 6 & 433 & 67.4 & 2.98 & & 9.35 & 12.42 & & & 34 & 4.97 \\
\hline RT-SCS-2002-00063 & SB3-26ccc & $\mathrm{m} 90$ & $\mathrm{ccc}$ & 1 & 3 & 11 & 8 & 63 & 1 & 82 & 87.4 & 1.97 & 19.50 & 14.67 & 10.42 & 7 & 50 & 145.67 & 3.28 \\
\hline-00063 & SB3 & $\mathrm{m}$ & $\mathrm{co}$ & 1 & 4 & 18 & & & & 7 & 72.8 & 1.71 & & 11.70 & 8.43 & & & 21.34 & 2.85 \\
\hline T-SCS-2002-00063 & SB3-24 & $\mathrm{m} 44$ & quenched & 1 & 5 & 12.2 & 7.59 & 5.24 & 6.16 & 93.6 & 76.6 & 1.51 & 20.33 & 12.65 & 8.73 & 10.27 & & 127.67 & 2.52 \\
\hline T-SCS-2002-00063 & SB3-18 & 24 & Tuenched & 1 & 6 & 11.6 & 75 & $5 f->$ & & 88.9 & 78.5 & 1.67 & 19.33 & 12.50 & 9.35 & & & 130.84 & 2.78 \\
\hline$-2002-00063$ & SB3-27 & $\mathrm{m} 21$ & quenched & 1 & 7 & 6.7 & 9.87 & 0 & 13.1 & 41 & 88.3 & 3.62 & 11.22 & 16.45 & 15.20 & 21.83 & 69.83 & 147.17 & 6.03 \\
\hline Г-SCS-2002-00063 & SB3-27ccc & $\mathrm{m} 1$ & $\mathrm{ccc}$ & 1 & 8 & 6. & & $7 .{ }^{\circ}$ & & 39.1 & 84.3 & 3.42 & & 15.57 & 12.87 & & & 40.50 & 5.70 \\
\hline SRT-SCS-2002-00063 & SB3-16ccc & $\mathrm{m} 28$ & $\mathrm{ccc}$ & 1 & 9 & 7.28 & 10.1 & 4.29 & 12.2 & 75.3 & 92.2 & 2.57 & 12.13 & 16.83 & 7.15 & 20.33 & 125.50 & 153.67 & 4.28 \\
\hline SRT-SCS-2002-00063 & soln std & std-b1-2 & & 1 & 10 & 4.02 & 21.4 & 4.24 & 9.47 & 82.1 & 51 & $<1.00$ & 4.02 & 21.40 & 4.24 & 9.47 & 82.10 & 51.00 & 0.50 \\
\hline $02-00063$ & SB3-26 & $\mathrm{m} 36$ & ong & 1 & 11 & & $9.2-2 \cdot 0.0 .0$ & $6=$ & & 88 & 92.1 & 1.95 & 20.33 & 15.38 & 11.27 & 20.83 & & 53.50 & 3.25 \\
\hline T-SCS-2002-00063 & SB3-16 & m77 & quenched & 1 & 12 & 7.49 & 10.4 & 5.1 & 11.9 & 78.9 & 96.1 & 2.66 & 12 & 17.33 & 8.62 & 19.83 & 50 & 160.17 & 4.43 \\
\hline T-SCS-2002-00063 & SB3-15 & m52 & quenched & 1 & 13 & 6.12 & 9.95 & 4.45 & 12.1 & 67.7 & 94.7 & 3.05 & 10.20 & 16.58 & 7.42 & 20.17 & 112.84 & 157.84 & 5.08 \\
\hline$-2002-00063$ & SB3-24ccc & m08 & $\mathrm{ccc}$ & 1 & 14 & 11.9 & 7.73 & 4.4 & 6.67 & 86. & 76.5 & 1.48 & 19.83 & 12.88 & 7.40 & 11.12 & 144.84 & 127.50 & 2.47 \\
\hline 00063 & SB3 & $\mathrm{m}$ & $\mathrm{ccc}$ & 1 & 15 & 6.51 & 7. & & & & & 19 & & & 13.72 & & & .50 & 4.98 \\
\hline SRT-SCS-2002-00063 & SB3-19 & m79 & quenched & 1 & 16 & 6.5 & 8.06 & 8.84 & 6.33 & 44.1 & 71.2 & 3.13 & 10.83 & 13.43 & 14.73 & 10.55 & 73.50 & 118.67 & 5.22 \\
\hline
\end{tabular}


Table I.1. SRTC-ML Mesaurements of PCT Leachate Solutions for SB3 Phase 1 glasses

\begin{tabular}{|c|c|c|c|c|c|c|c|c|c|c|c|c|c|c|c|c|c|c|c|}
\hline \multirow{2}{*}{$\begin{array}{c}\text { Analytical } \\
\text { Plan }\end{array}$} & \multirow{2}{*}{$\begin{array}{c}\text { Glass } \\
\text { ID }\end{array}$} & \multirow{2}{*}{$\begin{array}{c}\text { SRTC-ML } \\
\text { ID }\end{array}$} & \multirow{2}{*}{$\begin{array}{c}\text { Heat } \\
\text { Treatment }\end{array}$} & \multicolumn{3}{|c|}{ Seq } & \multicolumn{5}{|c|}{ As Reported (in ppm) by SRTC-ML } & \multirow[b]{2}{*}{$\mathbf{U}$} & \multicolumn{7}{|c|}{ Values (in ppm) after Adjusting for Dilution Effects } \\
\hline & & & & Block & \# & Al & B & $\mathbf{F e}$ & $\mathbf{L i}$ & $\mathrm{Na}$ & $\mathbf{S i}$ & & Al & B & $\mathbf{F e}$ & $\mathbf{L i}$ & $\mathbf{N a}$ & $\mathbf{S i}$ & $\mathbf{U}$ \\
\hline SRT-SCS-2002-00063 & SB3-15ccc & m59 & $\mathrm{ccc}$ & 1 & 17 & 5.91 & 9.94 & 4.28 & 12.3 & 62.9 & 93.7 & 2.78 & 9.85 & 16.57 & 7.13 & 20.50 & 104.84 & 156.17 & 4.63 \\
\hline SRT-SCS-2002-00063 & SB3-23ccc & m86 & $\mathrm{ccc}$ & 1 & 18 & 6.43 & 5.78 & 7.54 & 6.14 & 39.5 & 72 & 2.88 & 10.72 & 9.63 & 12.57 & 10.23 & 65.83 & 120.00 & 4.80 \\
\hline SRT-SCS-2002-00063 & soln std & std-b1-3 & & 1 & 19 & 4.12 & 21.5 & 4.4 & 9.56 & 82.8 & 52.1 & $<1.00$ & 4.12 & 21.50 & 4.40 & 9.56 & 82.80 & 52.10 & 0.50 \\
\hline RT-SCS-2002-00063 & soln std & std-b2-1 & & 2 & 1 & 4.1 & 21.3 & 4.2 & 9.59 & 85.9 & 50.5 & $<1.00$ & 4.10 & 21.30 & 4.20 & 9.59 & 85.90 & 50.50 & 0.50 \\
\hline SRT-SCS-2002-00063 & SB3-24 & $\mathrm{m} 01$ & quenched & 2 & 2 & 13.1 & 7.95 & 5.35 & 6.38 & 99.2 & 77.8 & 1.6 & 21.83 & 13.25 & 8.92 & 10.63 & 165.34 & 129.67 & 2.67 \\
\hline SRT-SCS-2002-00063 & SB3-15ccc & $\mathrm{m} 87$ & $\mathrm{ccc}$ & 2 & 3 & 6.11 & 9.61 & 4.2 & 12.27 & 65.3 & 89.8 & 2.85 & 10.18 & 16.02 & 7.00 & 20.45 & 108.84 & 149.67 & 4.75 \\
\hline SRT-SCS-2002-00063 & SB3-16 & m58 & uenched & 2 & 4 & 8.45 & 9.93 & 7.88 & 11.77 & 83.9 & 92.5 & 2.71 & 14.08 & 16.55 & 13.13 & 19.62 & 139.84 & 154.17 & 4.52 \\
\hline SRT-SCS-2002-00063 & SB3-19 & $\mathrm{m} 12$ & quenched & 2 & 5 & 7.08 & 7.55 & 9.15 & 6.29 & 46.4 & 68.5 & 3.22 & 11.80 & 12.58 & 15.25 & 10.48 & 77.33 & 114.17 & 5.37 \\
\hline SRT-SCS-2002-00063 & SB3-26 & $\mathrm{m} 10$ & quenched & 2 & 6 & 12.1 & 8.74 & 6.68 & 12.3 & 91.5 & 89.3 & 1.82 & 20.17 & 14.57 & 11.13 & 20.50 & 152.50 & 148.84 & 3.03 \\
\hline SRT-SCS-2002-00063 & SB3-26ccc & $\mathrm{m} 15$ & $\mathrm{ccc}$ & 2 & 7 & 12.1 & 8.68 & 6.85 & 13.4 & 84.9 & 88.7 & 1.99 & 20.17 & 14.47 & 11.42 & 22.33 & 141.50 & 147.84 & 3.32 \\
\hline SRT-SCS-2002-00063 & SB3-16ccc & m06 & $\mathrm{ccc}$ & 2 & 8 & 7.66 & 10 & 4.65 & 12.3 & 76.7 & 93.1 & 2.61 & 2.77 & 16.67 & 7.75 & 20.50 & 127.84 & 55.17 & 4.35 \\
\hline TT-SCS-2002-00063 & SB3-18 & m74 & quenched & 2 & 9 & 11.7 & 7.15 & 6.48 & 6.33 & 88.4 & 76.9 & 1.64 & 19.50 & 11.92 & 10.80 & 10.55 & 147.34 & 128.17 & 2.73 \\
\hline SRT-SCS-2002-00063 & soln std & std-b2-2 & & 2 & 10 & 4.33 & 21.2 & 4.6 & 9.55 & 86.1 & 50.7 & $<1.00$ & 4.33 & 21.20 & 4.60 & 9.55 & 86.10 & 50.70 & 0.50 \\
\hline SRT-SCS-2002-00063 & SB3-23ccc & m55 & $\mathrm{ccc}$ & 2 & 11 & 6.6 & 5.71 & 7.83 & 6.23 & 40.7 & 70.1 & 2.9 & 11.00 & 9.52 & 13.05 & 10.38 & 67.83 & 116.84 & 4.83 \\
\hline RT-SCS-2002-00063 & SB3-27ccc & m53 & $\mathrm{ccc}$ & 2 & 12 & 6.66 & 9.01 & 7.95 & 12.3 & 39.4 & 83.1 & 3.49 & 11.10 & 15.02 & 13.25 & 20.50 & 65.67 & 138.50 & 5.82 \\
\hline T-SCS-2002-00063 & SB3-23 & $\mathrm{m} 31$ & quenched & 2 & 13 & 6.8 & 5.62 & 8.98 & 6.28 & 44.5 & 71.8 & 3.07 & 11.33 & 9.37 & 14.97 & 10.47 & 74.17 & 119.67 & 5.12 \\
\hline TT-SCS-2002-00063 & SB3-15 & $\mathrm{m} 13$ & quenched & 2 & 14 & 7.12 & 9.67 & 5.52 & 12.2 & 70.6 & 94.7 & 3.03 & 11.87 & 16.12 & 9.20 & 20.33 & 117.67 & 157.84 & 5.05 \\
\hline 0063 & SB3-19ccc & 73 & $\mathrm{ccc}$ & 2 & 15 & 6.5 & 7.33 & 9.02 & 6.09 & 41 & 68.8 & 3 & 98 & 12.22 & 15.03 & 10.15 & & 67 & 5.00 \\
\hline 0063 & SB3-24ccc & $\mathrm{m} 47$ & $\mathrm{ccc}$ & 2 & 16 & 12.1 & 7.33 & 4.93 & 6.67 & 89. & 75.6 & 1.55 & 20.17 & 12.22 & 8.22 & 11.12 & & 126.00 & 2.58 \\
\hline SRT-SCS-2002-00063 & SB3-18ccc & $\mathrm{m} 41$ & $\mathrm{ccc}$ & 2 & 17 & 11.35 & 6.89 & 5.95 & 6.56 & 79.5 & 73.8 & 1.75 & 18.92 & 11.48 & 9.92 & 10.93 & 132.50 & 123.00 & 2.92 \\
\hline T-SCS-2002-00063 & SB3-27 & $\mathrm{m} 46$ & quenched & 2 & 18 & 6.59 & 9.17 & 9.2 & 12.4 & 41 & 84.5 & 3.53 & 10.98 & 15.28 & 15.33 & 20.67 & 33 & 140.84 & 5.88 \\
\hline 0063 & soln std & std-b2-3 & & 2 & 19 & 4 & 20.8 & 4.34 & 9.41 & 84.8 & 50.6 & $<1.00$ & 4.10 & 20.80 & 4.34 & 9.41 & 30 & 60 & 0.50 \\
\hline T-SCS-2002-00063 & soln std & std-b3-1 & & 3 & 1 & 4.12 & 21 & 3.96 & 9.47 & 87.2 & 49.7 & $<1.00$ & 4.12 & 21.00 & 3.96 & 9.47 & 87.20 & 49.70 & 0.50 \\
\hline T-SCS-2002-00063 & SB3-19 & $\mathrm{m} 81$ & quenched & 3 & 2 & 6.93 & 7.77 & 8.95 & 6.27 & 46.3 & 69.4 & 3.14 & 11.55 & 12.95 & 14.92 & 10.45 & 77.17 & 115.67 & 5.23 \\
\hline T-SCS-2002-00063 & SB3-27ccc & 129 & $\mathrm{ccc}$ & 3 & 3 & 7.29 & 9.36 & 9.01 & 12.9 & 42.4 & 86 & 3.5 & 12.15 & 15.60 & 15.02 & 21.50 & 67 & 143.34 & 5.83 \\
\hline 0063 & SB3-18ccc & $\mathrm{m} 83$ & $\mathrm{ccc}$ & 3 & 4 & 11 & 6.95 & 5.11 & 6.6 & 83.6 & 74.6 & 1.66 & 19.00 & 11.58 & 8.52 & 11.05 & 34 & 124.34 & 2.77 \\
\hline T-SCS-2002-00063 & SB3-18 & m54 & quenched & 3 & 5 & 11.2 & 6.79 & 5.52 & 5.99 & 87.3 & 73.8 & 1.68 & 18.83 & 11.32 & 9.20 & 9.98 & 145.50 & 123.00 & 2.80 \\
\hline T-SCS-2002-00063 & SB3-23ccc & $\mathrm{m} 50$ & $\mathrm{ccc}$ & 3 & 6 & 6.49 & 5.31 & 7.35 & 6.03 & 41.8 & 69.7 & 2.96 & 10.82 & 8.85 & 12.25 & 10.05 & 69.67 & 116.17 & 4.93 \\
\hline $02-00063$ & SB3-16 & $\mathrm{m} 64$ & quenched & 3 & 7 & 8.1 & 9.61 & 6.45 & 11.5 & 83 & 90.7 & 2.56 & 13.53 & 16.02 & 10.75 & 19.17 & 67 & 151.17 & 4.27 \\
\hline 0063 & SB3-15ccc & $\mathrm{m} 69$ & $\mathrm{ccc}$ & 3 & 8 & & 9.38 & 5.28 & 12 & 65 & 91 & 2.89 & 10.67 & 15.63 & 8.80 & 20.33 & 84 & 152.00 & 4.82 \\
\hline T-SCS-2002-00063 & SB3-24ccc & $\mathrm{m} 26$ & $\mathrm{ccc}$ & 3 & 9 & 12.6 & 7.14 & 5.07 & 6.59 & 91.8 & 74.6 & 1.42 & 21.00 & 11.90 & 8.45 & 10.98 & 153.00 & 124.34 & 2.37 \\
\hline SRT-SCS-2002-00063 & soln std & std-b3-2 & & 3 & 10 & 4.04 & 20.9 & 3.95 & 9.41 & 86.6 & 49.9 & $<1.00$ & 4.04 & 20.90 & 3.95 & 9.41 & 86.60 & 49.90 & 0.50 \\
\hline SRT-SCS-2002-00063 & SB3-15 & $\mathrm{m} 51$ & uenched & 3 & 11 & 6.3 & 9.12 & 5.7 & 11.4 & 67.6 & 89.6 & 2.64 & 10.53 & 15.20 & 9.50 & 19.00 & 112.67 & 149.34 & 4.40 \\
\hline T-SCS-2002-00063 & SB3-26ccc & $\mathrm{m} 09$ & $\mathrm{ccc}$ & 3 & 12 & 12 & 8.76 & 7.02 & 13. & 85 & 90 & 2 & 20.67 & 14.60 & 11.70 & 22.50 & 17 & .00 & 3.33 \\
\hline T-SCS-2002-00063 & SB3-24 & m57 & quenched & 3 & 13 & 12.5 & 7.39 & 4.82 & 6.1 & 98 & 77.2 & 1.42 & 20.83 & 12.32 & 8.03 & 10.17 & 163.34 & 128.67 & 2.37 \\
\hline SRT-SCS-2002-00063 & SB3-27 & $\mathrm{m} 70$ & quenched & 3 & 14 & 6.67 & 9.49 & 8.52 & 13 & 42.7 & 87 & 3.54 & 11.12 & 15.82 & 14.20 & 21.67 & 71.17 & 145.00 & 5.90 \\
\hline SRT-SCS-2002-00063 & SB3-23 & $\mathrm{m} 92$ & quenched & 3 & 15 & 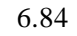 & 5.55 & 8.85 & 6.3 & 45 & 7 & 3.04 & & 9.25 & 14.75 & 10.58 & & 67 & 5.07 \\
\hline 0063 & SB3-26 & $\mathrm{m} 82$ & quenched & 3 & 16 & & 8.79 & 6.73 & 12.5 & 93.7 & 9 & 1.77 & 20.33 & 14.65 & 11.22 & 20.83 & 17 & 153.34 & 2.95 \\
\hline SRT-SCS-2002-00063 & SB3-16ccc & $\mathrm{m} 30$ & $\mathrm{ccc}$ & 3 & 17 & 7.67 & 9.75 & 5.32 & 12 & 76.6 & 92.2 & 2.47 & 12.78 & 16.25 & 8.87 & 20.00 & 127.67 & 153.67 & 4.12 \\
\hline SRT-SCS-2002-00063 & SB3-19ccc & $\mathrm{m} 84$ & $\mathrm{ccc}$ & 3 & 18 & 6.81 & 7.09 & 8.13 & 5.99 & 42.6 & 67.5 & 2.84 & 11.35 & 11.82 & 13.55 & 9.98 & 71.00 & 112.50 & 4.73 \\
\hline-00063 & & std-b. & & 3 & 19 & 4.0 & 20.6 & 3.97 & 9.3 & 85 & 50.4 & $<1.00$ & 4.02 & 20.60 & 3.97 & 9.35 & 85.60 & 50.40 & 0.50 \\
\hline SRT-SCS-2002-00063 & soln std & std-b4-1 & & 4 & 1 & 4.2 & 21 & 4 & 9.44 & 85.3 & 48.9 & $<1.00$ & 4.20 & 21.00 & 4.00 & 9.44 & 85.30 & 48.90 & 0.50 \\
\hline
\end{tabular}


Table I.1. SRTC-ML Mesaurements of PCT Leachate Solutions for SB3 Phase 1 glasses

\begin{tabular}{|c|c|c|c|c|c|c|c|c|c|c|c|c|c|c|c|c|c|c|c|}
\hline \multirow{2}{*}{$\begin{array}{c}\text { Analytical } \\
\text { Plan }\end{array}$} & \multirow{2}{*}{$\begin{array}{c}\text { Glass } \\
\text { ID }\end{array}$} & \multirow{2}{*}{$\begin{array}{c}\text { SRTC-ML } \\
\text { ID }\end{array}$} & \multirow{2}{*}{$\begin{array}{c}\text { Heat } \\
\text { Treatment } \\
\end{array}$} & \multicolumn{3}{|c|}{ Seq } & \multicolumn{5}{|c|}{ As Reported (in ppm) by SRTC-ML } & \multirow[b]{2}{*}{$\mathbf{U}$} & \multicolumn{7}{|c|}{ Values (in ppm) after Adjusting for Dilution Effects } \\
\hline & & & & Block & \# & Al & B & $\mathbf{F e}$ & $\mathbf{L i}$ & $\mathbf{N a}$ & $\mathrm{Si}$ & & Al & B & $\mathbf{F e}$ & $\mathbf{L i}$ & $\mathbf{N a}$ & $\mathbf{S i}$ & $\mathbf{U}$ \\
\hline SRT-SCS-2002-00063 & EA & $\mathrm{m} 43$ & & 4 & 2 & 0.841 & 38.4 & 0.319 & 10.8 & 98.1 & 52.1 & $<1.00$ & 14.02 & 640.00 & 5.32 & 180.00 & 1635.00 & 868.34 & 8.33 \\
\hline SRT-SCS-2002-00063 & SB3-21ccc & m76 & $\operatorname{ccc}$ & 4 & 3 & 6.82 & 5.53 & 6.55 & 4.56 & 43.1 & 63.9 & 2.57 & 11.37 & 9.22 & 10.92 & 7.60 & 71.83 & 106.50 & 4.28 \\
\hline RT-SCS-2002-00063 & SB3-20 & $\mathrm{m} 18$ & quenched & 4 & 4 & 10.5 & 8.76 & 4.76 & 6.15 & 78.2 & 69.5 & 1.77 & 17.50 & 14.60 & 7.93 & 10.25 & 130.34 & 115.84 & 2.95 \\
\hline TT-SCS-2002-00063 & B3-28 & $\mathrm{m} 60$ & quenched & 4 & 5 & 11 & 11 & 6.04 & 12.5 & 82.7 & 85.5 & 1.97 & 18.33 & 18.33 & 10.07 & 20.83 & 137.84 & 142.50 & 3.28 \\
\hline RT-SCS-2002-00063 & SB3-22ccc & $\mathrm{m} 22$ & $\operatorname{ccc}$ & 4 & 6 & 12.1 & 6.88 & 6.37 & 4.94 & 88.9 & 69.3 & 1.6 & 20.17 & 11.47 & 10.62 & 8.23 & 148.17 & 115.50 & 2.67 \\
\hline SRT-SCS-2002-00063 & SB3-17 & $\mathrm{m} 75$ & quenched & 4 & 7 & 7.59 & 5.99 & 11.5 & 6.67 & 48.8 & 74.3 & 3.85 & 12.65 & 9.98 & 19.17 & 11.12 & 81.33 & 123.84 & 6.42 \\
\hline SRT-SCS-2002-00063 & SB3-17ccc & $\mathrm{m} 89$ & $\mathrm{ccc}$ & 4 & 8 & 7.36 & 5.76 & 11.1 & 6.4 & 44.2 & 70.6 & 3.49 & 12.27 & 9.60 & 18.50 & 10.67 & 73.67 & 17.67 & 5.82 \\
\hline RT-SCS-2002-00063 & SB3-25ccc & $\mathrm{m} 27$ & $\mathrm{ccc}$ & 4 & 9 & 7.63 & 7.01 & 11.6 & 12.7 & 40.9 & 87.2 & 3.86 & 12.72 & 11.68 & 19.33 & 21.17 & 68.17 & 45.34 & 6.43 \\
\hline SRT-SCS-2002-00063 & soln std & std-b4-2 & & 4 & 10 & 4.13 & 20.4 & 4.11 & 9.3 & 83.4 & 49.1 & $<1.00$ & 4.13 & 20.40 & 4.11 & 9.30 & 83.40 & 49.10 & 0.50 \\
\hline SRT-SCS-2002-00063 & SB3-22 & $\mathrm{m} 16$ & quenched & 4 & 11 & 13 & 7.35 & 4.99 & 4.67 & 97.5 & 72.1 & 1.6 & 21.67 & 12.25 & 8.32 & 7.78 & 162.50 & 120.17 & 2.67 \\
\hline T-SCS-2002-00063 & SB3-25 & m05 & quenched & 4 & 12 & 7.76 & 7.24 & 12.9 & 13.1 & 42.5 & 89.5 & 4.13 & 12.93 & 12.07 & 21.50 & 21.83 & & 49.17 & 6.88 \\
\hline T-SCS-2002-00063 & SB3-28ccc & m33 & $\mathrm{ccc}$ & 4 & 13 & 10.7 & 10.4 & 5.85 & 12.4 & 72.9 & 82.5 & 2.02 & 17.83 & 17.33 & 9.75 & 20.67 & & 37.50 & 3.37 \\
\hline RT-SCS-2002-00063 & SB3-21 & $\mathrm{m} 62$ & quenched & 4 & 14 & 6.96 & 5.55 & 9.57 & 4.72 & 47.4 & 68.8 & 2.73 & 11.60 & 9.25 & 15.95 & 7.87 & & 14.67 & 4.55 \\
\hline T-SCS-2002-00063 & SB3-20ccc & $\mathrm{m} 11$ & $\mathrm{ccc}$ & 4 & 15 & 9.42 & 7.88 & 4.3 & 5.83 & 65.5 & 63.5 & 1.72 & 15.70 & 13.13 & 7.17 & 9.72 & 109.17 & 105.84 & 2.87 \\
\hline $02-00063$ & blank & m35 & & 4 & 16 & 0.731 & $<0.150$ & 0.786 & $<0.500$ & $<0.100$ & $<0.790$ & $<1.00$ & 1.22 & 0.13 & 1.31 & 0.42 & 8 & 0.66 & 0.83 \\
\hline T-SCS-2002-00063 & ARM & $\mathrm{m} 78$ & & 4 & 17 & 3.67 & 10.7 & 0.761 & 7.84 & 22.1 & 36.1 & $<1.00$ & 6.12 & 17.83 & 1.27 & 13.07 & 3 & 60.17 & 0.83 \\
\hline RT-SCS-2002-00063 & soln std & std-b4-3 & & 4 & 18 & 4.15 & 20.4 & 4.06 & 9.36 & 84.9 & 49.4 & $<1.00$ & 4.15 & 20.40 & 4.06 & 9.36 & & 49.40 & 0.50 \\
\hline 0063 & soln & std-b5-1 & & 5 & 1 & 4. & 20.8 & 3. & 9.35 & 86 & 49.2 & $<1.00$ & 4.21 & 80 & 3.91 & & & 20 & 0.50 \\
\hline 063 & SB3-25 & m32 & uenched & 5 & 2 & 7.39 & 7.27 & 11.2 & 12.8 & 42. & 87.6 & 4.22 & 12.32 & 12.12 & 18.67 & 21.33 & & 146.00 & 7.03 \\
\hline SRT-SCS-2002-00063 & SB3-17 & $\mathrm{m} 02$ & quenched & 5 & 3 & 7.15 & 5.84 & 10.6 & 6.39 & 48.7 & 72.5 & 3.85 & 11.92 & 9.73 & 17.67 & 10.65 & 81.17 & 120.84 & 6.42 \\
\hline T-SCS-2002-00063 & blank & $\mathrm{m} 42$ & & 5 & 4 & 2.05 & 0.432 & 0.621 & $<0.500$ & 2.1 & 1.11 & $<1.00$ & 3.42 & 0.72 & 1.04 & 0.42 & & 1.85 & 0.83 \\
\hline 0063 & SB3-21 & 23 & uenched & 5 & 5 & 6. & 5.32 & 6.47 & 4.59 & 48.2 & 68 & 2.65 & 10.82 & 8.87 & 10.78 & 7. & 0 & 34 & 4.42 \\
\hline [-SCS-2002-00063 & SB3-20 & $\mathrm{m} 25$ & quenched & 5 & 6 & 10.2 & 8.35 & 4.8 & 5.95 & 78 & 69.3 & 1.84 & 17.00 & 13.92 & 8.00 & 9.92 & 13 & 15.50 & 3.07 \\
\hline T-SCS-2002-00063 & SB3-17ccc & $\mathrm{m} 88$ & $\mathrm{ccc}$ & 5 & 7 & 7.55 & 5.56 & 10.9 & 6.26 & 44 & 69.9 & 3.46 & 12.58 & 9.27 & 18.17 & 10.43 & 73 & 16.50 & 5.77 \\
\hline T-SCS-2002-00063 & SB3-25ccc & 37 & $\mathrm{ccc}$ & 5 & 8 & 7.47 & 6.88 & 11.6 & 12.6 & 41.6 & 87.5 & 3.96 & 12.45 & 11.47 & 19.33 & 21.00 & 69 & 84 & 6.60 \\
\hline 0063 & SB3-20ccc & m34 & $\mathrm{ccc}$ & 5 & 9 & 9.5 & 7.71 & 4.1 & 5.87 & 6 & 63.5 & 1.7 & 15.98 & 12.85 & 6.85 & 9. & & 84 & 2.93 \\
\hline T-SCS-2002-00063 & soln std & std-b5-2 & & 5 & 10 & 4.1 & 20.4 & 3.95 & 9.33 & 86.6 & 49.4 & $<1.00$ & 4.14 & 20.40 & 3.95 & 9.3 & 86 & 49.40 & 0.50 \\
\hline T-SCS-2002-00063 & ARM & $\mathrm{m} 63$ & & 5 & 11 & 3.37 & 10.7 & $<0.040$ & 7.81 & 22.2 & 35.9 & $<1.00$ & 5.62 & 17.83 & 0.03 & 13.02 & 3 & .83 & 0.83 \\
\hline T-SCS-2002-00063 & SB3-21ccc & n65 & $\mathrm{cc}$ & 5 & 12 & 6.7 & 5.17 & 698 & 4.51 & 42.6 & 64.7 & 2.64 & 11.17 & 8.62 & 11.63 & 7.52 & & 107.84 & 4.40 \\
\hline 0063 & & $\mathrm{~m} 71$ & quenche & 5 & 13 & 11.9 & 6.74 & 3 & 4.37 & 95 & 69.9 & 1.69 & 19.83 & 11.23 & 6.58 & 7. & & 50 & 2.82 \\
\hline T-SCS-2002-00063 & SB3-28 & m56 & quenched & 5 & 14 & 11 & 10.73 & 6.06 & 12.4 & 90 & 86.2 & 2.06 & 18.33 & 17.88 & 10.10 & 20.67 & & 143.67 & 3.43 \\
\hline $02-00063$ & SB3-22ccc & m07 & $\operatorname{ccc}$ & 5 & 15 & 11.7 & 6.71 & 4.07 & 4.85 & 92 & 69.9 & 1.75 & 19.50 & 11.18 & 6.78 & & & .50 & 2.92 \\
\hline T-SCS-2002-00063 & EA & m67 & & 5 & 16 & 0.804 & 36.1 & 0.864 & 10.3 & 95 & 50.6 & $<1.00$ & 13.40 & 601.67 & 14.40 & 171.67 & 34 & 3.34 & 8.33 \\
\hline $2-00063$ & SB3-28ccc & m39 & $\mathrm{ccc}$ & 5 & 17 & 10.7 & 9.56 & 5.51 & 11.6 & 69.7 & 76.3 & 2.24 & 17.83 & 15.93 & 9.18 & 19.33 & 17 & 127.17 & 3.73 \\
\hline T-SCS-2002-00063 & soln std & std-b5-3 & & 5 & 18 & 4.07 & 20.1 & 3.93 & 9.25 & 84.9 & 49.3 & $<1.00$ & 4.07 & 20.10 & 3.93 & 9.25 & 84.90 & 49.30 & 0.50 \\
\hline SRT-SCS-2002-00063 & soln std & std-b6-1 & & 6 & 1 & 4.07 & 20.7 & 3.88 & 9.31 & 83.7 & 49.1 & $<1.00$ & 4.07 & 20.70 & 3.88 & 9.31 & 83.70 & 49.10 & 0.50 \\
\hline SRT-SCS-2002-00063 & SB3-17ccc & $\mathrm{m} 40$ & & 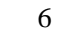 & 2 & 7.36 & 5.73 & 10 & 6.26 & 43 & 69.4 & 3. & 12.27 & 9.55 & 18.00 & 10.43 & & & 5.50 \\
\hline 063 & & & ench & 6 & 3 & & 7.21 & 11 & 13.3 & 4 & 89.7 & 4 & 12.42 & 12.02 & 19.33 & 22.17 & 7 & 50 & 6.83 \\
\hline T-SCS-2002-00063 & SB3-21 & m66 & quenched & 6 & 4 & 6.33 & 5.11 & 6.61 & 4.48 & 46.3 & 65.7 & 2.6 & 10.55 & 8.52 & 11.02 & 7.47 & 77.17 & 109.50 & 4.33 \\
\hline T-SCS-2002-00063 & SB3-20 & $\mathrm{m} 80$ & quenched & 6 & 5 & 10.2 & 8.43 & 4.44 & 6.1 & 77.5 & 69.8 & 1.77 & 17.00 & 14.05 & 7.40 & 10.17 & 129.17 & 116.34 & 2.95 \\
\hline 0063 & SB3-22 & $\mathrm{m} 19$ & quenched & 6 & 6 & 14. & 6.9 & 4. & 4.61 & 96.2 & 72.4 & 1.53 & 24.33 & 11.50 & 7.50 & & & 120.67 & 2.55 \\
\hline SRT-SCS-2002-00063 & SB3-28ccc & $\mathrm{m} 17$ & $\mathrm{ccc}$ & 6 & 7 & 10.7 & 9.64 & 7.11 & 12 & 69.2 & 78.4 & 2.23 & 17.83 & 16.07 & 11.85 & 20.00 & 115.34 & 130.67 & 3.72 \\
\hline
\end{tabular}


Table I.1. SRTC-ML Mesaurements of PCT Leachate Solutions for SB3 Phase 1 glasses

\begin{tabular}{|c|c|c|c|c|c|c|c|c|c|c|c|c|c|c|c|c|c|c|c|}
\hline \multirow{2}{*}{$\begin{array}{l}\text { Analytical } \\
\text { Plan }\end{array}$} & \multirow{2}{*}{$\begin{array}{c}\text { Glass } \\
\text { ID }\end{array}$} & \multirow{2}{*}{$\begin{array}{c}\text { SRTC-ML } \\
\text { ID }\end{array}$} & \multirow{2}{*}{$\begin{array}{c}\text { Heat } \\
\text { Treatment }\end{array}$} & \multirow{2}{*}{\multicolumn{2}{|c|}{$\begin{array}{c}\text { Seq } \\
\#\end{array}$}} & \multicolumn{7}{|c|}{ As Reported (in ppm) by SRTC-ML } & \multicolumn{7}{|c|}{ Values (in ppm) after Adjusting for Dilution Effects } \\
\hline & & & & & & Al & B & $\mathrm{Fe}$ & $\mathrm{Li}$ & $\mathbf{N a}$ & $\mathbf{S i}$ & $\mathbf{U}$ & Al & B & $\mathrm{Fe}$ & $\mathbf{L i}$ & $\mathbf{N a}$ & $\mathbf{S i}$ & $\mathbf{U}$ \\
\hline SRT-SCS-2002-00063 & $\overline{E A}$ & $\mathrm{~m} 91$ & & $\overline{6}$ & 8 & 0.741 & 36.8 & 1.36 & 10.4 & 93.8 & 51.5 & $<1.00$ & 12.35 & 613.33 & 22.67 & 173.33 & 1563.34 & 858.34 & 8.33 \\
\hline SRT-SCS-2002-00063 & SB3-17 & $\mathrm{m} 45$ & quenched & 6 & 9 & 8.08 & 5.92 & 13.8 & 6.58 & 47.8 & 74.2 & 3.77 & 13.47 & 9.87 & 23.00 & 10.97 & 79.67 & 123.67 & 6.28 \\
\hline SRT-SCS-2002-00063 & soln std & std-b6-2 & & 6 & 10 & 4.24 & 20.8 & 4.23 & 9.43 & 84.9 & 49.6 & $<1.00$ & 4.24 & 20.80 & 4.23 & 9.43 & 84.90 & 49.60 & 0.50 \\
\hline SRT-SCS-2002-00063 & SB3-22ccc & $\mathrm{m} 04$ & $\mathrm{ccc}$ & 6 & 11 & 13 & 7.07 & 4.81 & 5 & 90.2 & 72.9 & 1.55 & 21.67 & 11.78 & 8.02 & 8.33 & 150.34 & 121.50 & 2.58 \\
\hline SRT-SCS-2002-00063 & SB3-20ccc & m61 & $\mathrm{ccc}$ & 6 & 12 & 10.4 & 8.32 & 4.18 & 6.33 & 70.9 & 68.7 & 1.69 & 17.33 & 13.87 & 6.97 & 10.55 & 118.17 & 114.50 & 2.82 \\
\hline RT-SCS-2002-00063 & SB3-28 & $\mathrm{m} 38$ & quenched & 6 & 13 & 11.1 & 10.6 & 6.01 & 12.2 & 81.5 & 85.6 & 1.93 & 18.50 & 17.67 & 10.02 & 20.33 & 135.84 & 142.67 & 3.22 \\
\hline SRT-SCS-2002-00063 & SB3-21ccc & $\mathrm{m} 85$ & $\mathrm{ccc}$ & 6 & 14 & 6.22 & 5.01 & 6.23 & 4.46 & 42.1 & 64.9 & 2.57 & 10.37 & 8.35 & 10.38 & 7.43 & 70.17 & 08.17 & 4.28 \\
\hline SRT-SCS-2002-00063 & SB3-25ccc & $\mathrm{m} 72$ & $\mathrm{ccc}$ & 6 & 15 & 8.35 & 6.9 & 10.6 & 12.6 & 40.7 & 88.9 & 3.97 & 13.92 & 11.50 & 17.67 & 21.00 & 67.83 & 148.17 & 6.62 \\
\hline SRT-SCS-2002-00063 & ARM & $\mathrm{m} 49$ & & 6 & 16 & 3.31 & 11.3 & $<0.040$ & 8.05 & 22.8 & 37 & $<1.00$ & 5.52 & 18.83 & 0.03 & 13.42 & 38.00 & 61.67 & 0.83 \\
\hline SRT-SCS-2002-00063 & soln std & std-b6-3 & & 6 & 17 & 4.16 & 20.7 & 4 & 9.42 & 86.1 & 50.2 & $<1.00$ & 4.16 & 20.70 & 4.00 & 9.42 & 10 & 50.20 & 0.50 \\
\hline SRT-SCS-2002-00066 & soln std & std-b1-1 & & 1 & 1 & 3.85 & 21 & 3.8 & 9.54 & 86.1 & 49.1 & $<1.00$ & 3.85 & 21.00 & 3.80 & 9.54 & 10 & 49.10 & 0.50 \\
\hline SRT-SCS-2002-00066 & SB3-37ccc & n87 & $\mathrm{ccc}$ & 1 & 2 & 6.6 & 6.12 & 6.75 & 10.9 & 22.1 & 73.4 & 3.02 & 11.00 & 10.20 & 11.25 & 18.17 & 33 & 122.34 & 5.03 \\
\hline T-SCS-2002-00066 & SB3-31ccc & n61 & $\mathrm{ccc}$ & 1 & 3 & 7.33 & 5.14 & 3.88 & 5.62 & 33.1 & 57.3 & 1.63 & 12.22 & 8.57 & 6.47 & & & 95.50 & 2.72 \\
\hline T-SCS-2002-00066 & SB3-34ccc & $\mathrm{n} 45$ & $\mathrm{ccc}$ & 1 & 4 & 10.8 & .54 & 2.98 & 5.27 & 48.9 & 50.6 & 1.29 & 18.00 & 10.90 & 4.97 & & & 34 & 2.15 \\
\hline SRT-SCS-2002-00066 & SB3-29 & n47 & quenched & 1 & 5 & 4.88 & 7.78 & 2.81 & 10.8 & 35.8 & 76.4 & 2.19 & 8.13 & 12.97 & 4.68 & 18 & 59 & 127.34 & 3.65 \\
\hline SRT-SCS-2002-00066 & SB3-34 & n59 & quenched & 1 & 6 & 10.9 & 7.46 & 1.37 & 5.32 & 53.5 & 51.5 & 1.04 & 18.17 & 12.43 & 2.28 & 8. & 89.17 & 85.84 & 1.73 \\
\hline T-SCS-2002-00066 & SB3-40ccc & n26 & $\mathrm{ccc}$ & 1 & 7 & 11.5 & 7.55 & 3.69 & 10.2 & 44.9 & 61.7 & 1.9 & 19.17 & 12.58 & 6.15 & 17.00 & 7 & 102.84 & 3.17 \\
\hline 0066 & & & quenches & 1 & 8 & 7.4 & & 1 & 5.76 & 347 & 57.9 & 1.72 & & & 6.90 & & & & 2.87 \\
\hline -2002-00066 & SB3-36ccc & n60 & $\mathrm{ccc}$ & 1 & 9 & 13. & 7.08 & 4.21 & 6 & 79.1 & 64.8 & 1.49 & 22.33 & 11.80 & 7.02 & 11.00 & & 108.00 & 2.48 \\
\hline SRT-SCS-2002-00066 & soln std & std-b1-2 & & 1 & 10 & 3.73 & 20.9 & 3.96 & 9.5 & 83.5 & 49.9 & $<1.00$ & 3.73 & 20.90 & 3.96 & 9.50 & & 49.90 & 0.50 \\
\hline RT-SCS-2002-00066 & SB3-32 & n53 & quenched & 1 & 11 & 11. & 6.24 & 1.92 & 5.46 & 56.1 & 55.2 & $<1.00$ & 19.17 & 10.40 & 3.20 & 9. & & 2.00 & 0.83 \\
\hline $02-00066$ & SB3-32ccc & n77 & (set & 1 & 12 & 11. & 42 & 2.91 & 5.49 & 5 & 54.5 & 1.17 & 19.33 & 9.03 & 4.85 & & & 90.84 & 1.95 \\
\hline T-SCS-2002-00066 & EA & $\mathrm{n} 13$ & & 1 & 13 & 0.103 & 38.9 & $<0.040$ & 10.8 & 9 & 52.8 & $<1.00$ & 1.72 & 648.33 & 0.33 & 180.00 & 16 & 880.00 & 8.33 \\
\hline T-SCS-2002-00066 & SB3-29ccc & & $\mathrm{ccc}$ & 1 & 14 & 4.9 & 7.89 & 272 & & & & & & & & & & & 3.57 \\
\hline T-SCS-2002-00066 & ARM & n37 & & 1 & 15 & 2.87 & 11.33 & 0.188 & 8.04 & 22 & 36.2 & $<1.00$ & 4. & 18.88 & 0.31 & $132+3$ & 37.17 & 33 & 0.83 \\
\hline 066 & SB3-37 & n35 & enched & 1 & 16 & 6.4 & & 7. & 10 & 20 & 71.8 & 3.12 & & & 12.22 & & & & 5.20 \\
\hline T-SCS-2002-00066 & SB3-36 & $\mathrm{n} 71$ & quenched & 1 & 17 & 12. & 7.75 & 3.48 & 6.18 & 84.2 & 66.9 & 1.32 & 21.33 & 12.92 & 5.80 & 10.30 & 140.34 & 111.50 & 2.20 \\
\hline T-SCS-2002-00066 & SB3-40 & $\mathrm{n} 23$ & quenched & 1 & 18 & 10 & 851 & 200 & 10 & 464 & 62.1 & 1.29 & 18.00 & 14.18 & 4.98 & 17.00 & & 103.50 & 2.15 \\
\hline RT-SCS-2002-00066 & soln std & std-b1-3 & & 1 & 19 & $3.8>-2$ & 20 & $30+2>$ & 9.46 & 84 & 49.8 & $<1.00$ & 3.88 & 20.70 & 3.97 & 9. & Q4 & 49.80 & 0.50 \\
\hline Г-SCS-2002-00066 & sols & std-b2-1 & & 2 & 1 & 4 & & & 9 & & 5 & & & & 4.24 & & & 50.00 & 0.50 \\
\hline T-SCS-2002-00066 & SB3-32 & n57 & quenched & 2 & 2 & 12.1 & 6.31 & 2.11 & 5.6 & 58.3 & 55.5 & 1.21 & 20.17 & 10.52 & 3.52 & 9.33 & 97.17 & 92.50 & 2.02 \\
\hline T-SCS-2002-00066 & SB3-36 & & 1 & 2 & 3 & 13 & 751 & 332 & 6.1 & 840 & 64.3 & 1.57 & 21.67 & 12.52 & 5.53 & 10.28 & & 107.17 & 2.62 \\
\hline 0066 & SB3-37 & $\mathrm{n}$ & quenched & 2 & 4 & 7.1 & 5.91 & 7.82 & 11.2 & 21 & 73.1 & 3.39 & 11.92 & 9.85 & 13.03 & 18.67 & 36.50 & 121.84 & 5.65 \\
\hline Г-SCS-2002-00066 & SB3-34 & $\mathrm{n} 43$ & quenched & 2 & 5 & 11 & 7. & 1. & & 5 & 51.8 & 1 & 18.67 & 12.33 & 2.83 & & & 86.34 & 2.00 \\
\hline T-SCS-2002-00066 & EA & n10 & & 2 & 6 & 0.632 & 37.9 & 0.411 & 10.9 & 97.2 & 52.4 & $<1.00$ & 10.53 & 631.67 & 6.85 & 181.67 & 1620.00 & 873.34 & 8.33 \\
\hline SRT-SCS-2002-00066 & ARM & n05 & & 2 & 7 & 3.31 & 10.9 & 0.183 & 7.91 & 22.1 & 35.8 & $<1.00$ & 5.52 & 18.17 & 0.31 & 13.18 & 36.83 & 59.67 & 0.83 \\
\hline$-2002-00066$ & SB3-40ccc & $\mathrm{n} 21$ & $\mathrm{ccc}$ & 2 & 8 & & 7.4 & $4=5$ & & 44 & 6 & 2.09 & 19.33 & 12.47 & 7.62 & & & 03.34 & 3.48 \\
\hline T-SCS-2002-00066 & SB3-40 & n18 & quenched & 2 & 9 & 11.1 & 8.47 & 2.93 & 10.2 & 47.1 & 62.5 & 1.46 & 18.50 & 14.12 & 4.88 & 17.00 & 78.50 & 104.17 & 2.43 \\
\hline TT-SCS-2002-00066 & soln std & std-b2-2 & & 2 & 10 & 4.2 & 20.8 & 4.15 & 9.45 & 85.8 & 49.5 & $<1.00$ & 4.20 & 20.80 & 4.15 & 9.45 & 85.80 & 49.50 & 0.50 \\
\hline S-2002-00066 & SB3-31 ccc & $\mathrm{n} 89$ & $\mathrm{ccc}$ & 2 & 11 & 7.98 & 5.11 & 4.18 & 5.58 & 32.6 & 57.3 & 1.82 & 13.30 & 8.52 & 6.97 & 9.30 & 54.33 & 95.50 & 3.03 \\
\hline $2-00066$ & SB3 & $\mathrm{n} 8$ & $\mathrm{ccc}$ & 2 & 12 & & & 2 & & & & 2.33 & & & 4.93 & 0 & & 127.84 & 3.88 \\
\hline SRT-SCS-2002-00066 & SB3-31 & n34 & quenched & 2 & 13 & 7.76 & 5.09 & 4.4 & 5.81 & 34.8 & 58.8 & 1.9 & 12.93 & 8.48 & 7.33 & 9.68 & 58.00 & 98.00 & 3.17 \\
\hline
\end{tabular}


Table I.1. SRTC-ML Mesaurements of PCT Leachate Solutions for SB3 Phase 1 glasses

\begin{tabular}{|c|c|c|c|c|c|c|c|c|c|c|c|c|c|c|c|c|c|c|c|}
\hline \multirow{2}{*}{$\begin{array}{c}\text { Analytical } \\
\text { Plan }\end{array}$} & \multirow{2}{*}{$\begin{array}{c}\text { Glass } \\
\text { ID }\end{array}$} & \multirow{2}{*}{$\begin{array}{c}\text { SRTC-ML } \\
\text { ID }\end{array}$} & \multirow{2}{*}{$\begin{array}{c}\text { Heat } \\
\text { Treatment }\end{array}$} & \multicolumn{3}{|c|}{ Seq } & \multicolumn{5}{|c|}{ As Reported (in ppm) by SRTC-ML } & \multirow[b]{2}{*}{$\mathbf{U}$} & \multicolumn{7}{|c|}{ Values (in ppm) after Adjusting for Dilution Effects } \\
\hline & & & & Block & \# & Al & B & $\mathbf{F e}$ & $\mathbf{L i}$ & $\mathbf{N a}$ & $\mathrm{Si}$ & & Al & B & $\mathbf{F e}$ & $\mathbf{L i}$ & $\mathbf{N a}$ & $\mathbf{S i}$ & $\mathbf{U}$ \\
\hline SRT-SCS-2002-00066 & SB3-36ccc & $\mathrm{n} 28$ & $\mathrm{ccc}$ & 2 & 14 & 13.7 & 7.14 & 4.52 & 6.72 & 81.5 & 66.3 & 1.71 & 22.83 & 11.90 & 7.53 & 11.20 & 135.84 & 110.50 & 2.85 \\
\hline SRT-SCS-2002-00066 & SB3-37ccc & $\mathrm{n} 02$ & $\mathrm{ccc}$ & 2 & 15 & 7.16 & 5.93 & 6.95 & 11 & 22.7 & 73.4 & 3.27 & 11.93 & 9.88 & 11.58 & 18.33 & 37.83 & 122.34 & 5.45 \\
\hline SRT-SCS-2002-00066 & SB3-29 & n62 & quenched & 2 & 16 & 5.25 & 7.59 & 3.1 & 10.5 & 35.9 & 75.4 & 2.42 & 8.75 & 12.65 & 5.17 & 17.50 & 59.83 & 125.67 & 4.03 \\
\hline RT-SCS-2002-00066 & SB3-34ccc & $\mathrm{n} 78$ & $\mathrm{ccc}$ & 2 & 17 & 10.9 & 6.34 & 2.9 & 5.24 & 48.8 & 50 & 1.43 & 18.17 & 10.57 & 4.83 & 8.73 & 81.33 & 83.34 & 2.38 \\
\hline SRT-SCS-2002-00066 & SB3-32ccc & n69 & $\operatorname{ccc}$ & 2 & 18 & 12.1 & 5.38 & 2.86 & 5.62 & 53.9 & 55.6 & 1.41 & 20.17 & 8.97 & 4.77 & 9.37 & 89.84 & 92.67 & 2.35 \\
\hline SRT-SCS-2002-00066 & soln std & std-b2-3 & & 2 & 19 & 4.19 & 20.6 & 4.25 & 9.52 & 86.5 & 50.3 & $<1.00$ & 4.19 & 20.60 & 4.25 & 9.52 & 86.50 & 50.30 & 0.50 \\
\hline SRT-SCS-2002-00066 & soln std & std-b3-1 & & 3 & 1 & 3.85 & 20.5 & 3.48 & 9.37 & 85.1 & 48.9 & $<1.00$ & 3.85 & 20.50 & 3.48 & 9.37 & & 48.90 & 0.50 \\
\hline ISRT-SCS-2002-00066 & SB3-29ccc & $\mathrm{n} 80$ & $\mathrm{ccc}$ & 3 & 2 & 4.98 & 7.65 & 2.73 & 10.7 & 35.6 & 75.9 & 2.25 & 8.30 & 12.75 & 4.55 & 17.83 & 59.33 & 126.50 & 3.75 \\
\hline SRT-SCS-2002-00066 & SB3-34 & n16 & quenched & 3 & 3 & 11.6 & 7.55 & 1.24 & 5.57 & 56.8 & 53.8 & 1.13 & 19.33 & 12.58 & 2.07 & 9.28 & 94.67 & 89.67 & 1.88 \\
\hline SRT-SCS-2002-00066 & SB3-40 & n64 & quenched & 3 & 4 & 11.3 & 8.36 & 2.5 & 10.4 & 48 & 63.2 & 1.35 & 18.83 & 13.93 & 4.17 & 17.33 & 80.00 & 105.34 & 2.25 \\
\hline TT-SCS-2002-00066 & SB3-32ccc & n92 & $\mathrm{ccc}$ & 3 & 5 & 12.2 & 5.22 & 2.5 & 5.69 & 55 & 56 & 1.26 & 20.33 & 8.70 & 4.17 & 9.48 & 7 & 93.34 & 2.10 \\
\hline T-SCS-2002-00066 & SB3-36ccc & n06 & $\mathrm{ccc}$ & 3 & 6 & 13.8 & 7.08 & 3.81 & 6.86 & 83 & 67.7 & 1.54 & 23.00 & 11.80 & 6.35 & 11.43 & 138.34 & 112.84 & 2.57 \\
\hline RT-SCS-2002-00066 & SB3-34ccc & $\mathrm{n} 86$ & $\operatorname{ccc}$ & 3 & 7 & 11.8 & 6.42 & 2.35 & 5.46 & 51.5 & 53.1 & 1.29 & 19.67 & 10.70 & 3.92 & 9.10 & 85.84 & 88.50 & 2.15 \\
\hline $02-00066$ & SB3-32 & $\mathrm{n} 46$ & quenched & 3 & 8 & 11.9 & 5.84 & 1.71 & 5.52 & 58.2 & 56.2 & 1.03 & 19.83 & 9.73 & 2.85 & 9.20 & 97.00 & 93.67 & 1.72 \\
\hline-00066 & SB3-31 & $\mathrm{n} 40$ & quenched & 3 & 9 & 7.65 & 4.84 & 3.96 & 5.82 & 35.3 & 59.8 & 1.75 & 12.75 & 8.07 & 6.60 & 9.70 & 58.83 & 99.67 & 2.92 \\
\hline RT-SCS-2002-00066 & soln std & std-b3-2 & & 3 & 10 & 4 & 20.7 & 3.75 & 9.42 & 85.3 & 50.2 & $<1.00$ & 4.00 & 20.70 & 3.75 & 9.42 & 85.30 & 50.20 & 0.50 \\
\hline SRT-SCS-2002-00066 & EA & n39 & & 3 & 11 & 2.42 & 38.3 & 0.496 & 10.7 & 97.8 & 53.1 & $<1.00$ & 40.33 & 638.33 & 8.27 & 178.33 & 1630.00 & 885.00 & 8.33 \\
\hline 0066 & SB3-36 & n68 & quenche & 3 & 12 & 13 & 7.83 & 3.41 & 6 & 8 & 68.6 & 8 & 00 & 13.05 & 5.68 & 63 & & 1.34 & 2.30 \\
\hline 0066 & SB3-31ccc & $\mathrm{n} 85$ & $\mathrm{ccc}$ & 3 & 13 & 7. & 4.81 & 3.6 & 5.5 & 32.7 & 58.2 & 1.63 & 12.17 & 8.02 & 6.05 & 9.23 & & 97.00 & 2.72 \\
\hline SRT-SCS-2002-00066 & SB3-29 & n72 & quenched & 3 & 14 & 4.95 & 7.49 & 2.85 & 10.6 & 36 & 77.3 & 2.18 & 8.25 & 12.48 & 4.75 & 17.67 & 60.00 & 128.84 & 3.63 \\
\hline T-SCS-2002-00066 & SB3-40ccc & $\mathrm{n} 81$ & $\operatorname{ccc}$ & 3 & 15 & 11.5 & 7.3 & 3.74 & 10.2 & 45.2 & 63.4 & 1.99 & 19.17 & 12.17 & 6.23 & 17.00 & 75.33 & 105.67 & 3.32 \\
\hline 0066 & SB & $\mathrm{n} 22$ & uenched & 3 & 16 & 6 & 5.65 & 7 & 1 & 21.8 & 74.7 & 3.24 & 11.33 & 9.42 & 12.55 & 33 & & .50 & 5.40 \\
\hline r-SCS-2002-00066 & SB3-37ccc & n14 & $\mathrm{ccc}$ & 3 & 17 & 6.61 & 5.64 & 6.42 & 10.8 & 21.9 & 74 & 2.93 & 11.02 & 9.40 & 10.70 & 18.00 & 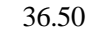 & 123.34 & 4.88 \\
\hline T-SCS-2002-00066 & ARM & n51 & & 3 & 18 & 2.84 & 10.4 & $<0.040$ & 7.79 & 21.4 & 36 & $<1.00$ & 4.73 & 17.33 & 0.03 & 12.98 & 7 & 60.00 & 0.83 \\
\hline TT-SCS-2002-00066 & soln std & std-b3-3 & & 3 & 19 & 3.76 & 20.6 & 37 & 9.45 & 85.6 & 50.2 & $<1.00$ & 3.76 & 20.60 & 3.70 & 9.45 & 0 & 20 & 0.50 \\
\hline 0066 & soln std & std-b4-1 & & 4 & 1 & 4.2 & 21.2 & 4 & 9. & 8 & 49.4 & $<1.00$ & 4.23 & 21.20 & 4.06 & 0 & 0 & 40 & 0.50 \\
\hline T-SCS-2002-00066 & SB3-35ccc & n76 & $\mathrm{ccc}$ & 4 & 2 & 6.9 & 6.95 & 11.2 & 7.31 & 52.3 & 74 & 4.03 & 11.65 & 11.58 & 18.67 & 12.18 & 87.17 & 123.34 & 6.72 \\
\hline T-SCS-2002-00066 & SB3-33 & n70 & quenched & 4 & 3 & 6.85 & 6.43 & 4.56 & 5.8 & 30.9 & 57.7 & 2.03 & 11.42 & 10.72 & 7.60 & 9.67 & & 6.17 & 3.38 \\
\hline T-SCS-2002-00066 & SB3-35 & $\mathrm{n} 42$ & quenched & 4 & 4 & 7.3 & 7.34 & 125 & 7.64 & 58.8 & 80.7 & 4 & 12.23 & 12.23 & 20.83 & 12.73 & & 134.50 & 7.00 \\
\hline 0066 & SB3- & $\mathrm{n} 27$ & $\mathrm{ccc}$ & 4 & 5 & 6 & 6.36 & 4 & 5. & 30 & 58.1 & 1.93 & 11.42 & 10.60 & 7.35 & 947 & & 84 & 3.22 \\
\hline T-SCS-2002-00066 & SB3-42ccc & $\mathrm{n} 01$ & $\mathrm{ccc}$ & 4 & 6 & 17.1 & 14.5 & 14.3 & 38.9 & 114 & 140 & 3.66 & 28.50 & 24.17 & 23.83 & 64.83 & .00 & 233.34 & 6.10 \\
\hline $02-00066$ & SB3-42 & $\mathrm{n} 48$ & quenched & 4 & 7 & 15.6 & 12.2 & 8.58 & & 11 & 106 & 2.87 & 26.00 & 20.33 & 14.30 & & & 5.67 & 4.78 \\
\hline T-SCS-2002-00066 & SB3-30ccc & $\mathrm{n} 20$ & $\mathrm{ccc}$ & 4 & 8 & 11 & 7.5 & 40 & 9.25 & 59. & 67.8 & 1.33 & 18.33 & 12.50 & 6.82 & 15.42 & 17 & 113.00 & 2.22 \\
\hline T-SCS-2002-00066 & SB3-39ccc & $\mathrm{n} 90$ & $\mathrm{ccc}$ & 4 & 9 & 6 & 7.73 & 7.3 & 11 & 19 & 74.5 & 3.2 & 10.17 & 12.88 & 12.30 & 18.67 & 32.50 & 124.17 & 5.43 \\
\hline T-SCS-2002-00066 & soln std & std-b4-2 & & 4 & 10 & 4.12 & 20.9 & 4.14 & 9.49 & 86.5 & 49.9 & $<1.00$ & 4.12 & 20.90 & 4.14 & 9.49 & 86.50 & 49.90 & 0.50 \\
\hline SRT-SCS-2002-00066 & SB3-41ccc & $\mathrm{n} 29$ & $\operatorname{ccc}$ & 4 & 11 & 8.3 & 10.9 & 12.5 & 19.4 & 70.4 & 111 & 4.07 & 13.83 & 18.17 & 20.83 & 32.33 & 117.34 & 185.00 & 6.78 \\
\hline SRT-SCS-2002-00066 & & n07 & quenchec & 4 & 12 & 11.1 & 6.29 & 2.77 & 9.6 & 45 & 61.2 & 1. & 18.50 & 10.48 & 4.62 & 16.08 & & 2.00 & 2.90 \\
\hline 22-00066 & SB3-38ccc & n09 & $\mathrm{ccc}$ & 4 & 13 & 11 & 5.72 & 4.04 & 07 & 43.6 & 62 & 28 & 19.00 & 9.53 & 6.73 & 16.20 & 72.67 & 103.34 & 3.48 \\
\hline T-SCS-2002-00066 & blank & n50 & & 4 & 14 & 0.38 & $<0.150$ & $<0.040$ & $<0.500$ & $<0.100$ & $<0.790$ & $<1.00$ & 0.63 & 0.13 & 0.03 & 0.42 & 0.08 & 0.66 & 0.83 \\
\hline SRT-SCS-2002-00066 & SB3-39 & n03 & quenched & 4 & 15 & 6.26 & 7.53 & 7.79 & 11.4 & 18.6 & 73.2 & 3.5 & 10.43 & 12.55 & 12.98 & 19.00 & 31.00 & 122.00 & 5.83 \\
\hline 066 & & n91 & & 4 & 16 & 8. & 10.8 & 13.8 & 18 & 75. & 108 & 4.13 & 14.32 & 18.00 & 23.00 & 30.00 & 126.17 & 180.00 & 6.88 \\
\hline SRT-SCS-2002-00066 & SB3-30 & n58 & quenched & 4 & 17 & 10.8 & 8.51 & 3.55 & 9.64 & 65.1 & 68.3 & 1.67 & 18.00 & 14.18 & 5.92 & 16.07 & 108.50 & 113.84 & 2.78 \\
\hline
\end{tabular}


Table I.1. SRTC-ML Mesaurements of PCT Leachate Solutions for SB3 Phase 1 glasses

\begin{tabular}{|c|c|c|c|c|c|c|c|c|c|c|c|c|c|c|c|c|c|c|c|}
\hline \multirow{2}{*}{$\begin{array}{l}\text { Analytical } \\
\text { Plan }\end{array}$} & \multirow{2}{*}{$\begin{array}{c}\text { Glass } \\
\text { ID }\end{array}$} & \multirow{2}{*}{$\begin{array}{c}\text { SRTC-ML } \\
\text { ID }\end{array}$} & \multirow{2}{*}{$\begin{array}{c}\text { Heat } \\
\text { Treatment }\end{array}$} & \multirow{2}{*}{\multicolumn{2}{|c|}{$\begin{array}{c}\text { Seq } \\
\#\end{array}$}} & \multicolumn{7}{|c|}{ As Reported (in ppm) by SRTC-ML } & \multicolumn{7}{|c|}{ Values (in ppm) after Adjusting for Dilution Effects } \\
\hline & & & & & & Al & B & $\mathrm{Fe}$ & $\mathbf{L i}$ & $\mathbf{N a}$ & $\mathbf{S i}$ & $\mathbf{U}$ & Al & B & $\mathrm{Fe}$ & $\mathbf{L i}$ & $\mathbf{N a}$ & $\mathbf{S i}$ & $\mathbf{U}$ \\
\hline SRT-SCS-2002-00066 & soln std & std-b4-3 & & $\overline{4}$ & 18 & 4.08 & 20.2 & 4.03 & 9.34 & 85.4 & 48.9 & $<1.00$ & 4.08 & 20.20 & 4.03 & 9.34 & 85.40 & 48.90 & 0.50 \\
\hline SRT-SCS-2002-00066 & soln std & std-b5-1 & & 5 & 1 & 3.97 & 21 & 3.71 & 9.44 & 86 & 49.8 & $<1.00$ & 3.97 & 21.00 & 3.71 & 9.44 & 86.00 & 49.80 & 0.50 \\
\hline SRT-SCS-2002-00066 & SB3-42 & n55 & quenched & 5 & 2 & 16.6 & 12.7 & 9.08 & 24.5 & 113 & 111 & 3.23 & 27.67 & 1.17 & 15.13 & 40.83 & 188.34 & 185.00 & 5.38 \\
\hline SRT-SCS-2002-00066 & SB3-38 & $\mathrm{n} 15$ & quenched & 5 & 3 & 10.9 & 6.22 & 2.4 & 9.64 & 45.4 & 62.2 & 1.61 & 8.17 & 10.37 & 4.00 & 16.07 & 75.67 & 103.67 & 2.68 \\
\hline SRT-SCS-2002-00066 & SB3-38ccc & n67 & $\mathrm{ccc}$ & 5 & 4 & 12.9 & 6.02 & 6.49 & 10.3 & 46.4 & 66.5 & 2 & 21.50 & 10.03 & 10.82 & 17.17 & 77.33 & 110.84 & 3.33 \\
\hline SRT-SCS-2002-00066 & SB3-39ccc & n17 & $\operatorname{ccc}$ & 5 & 5 & 6.82 & 7.89 & 7.98 & 11.5 & 21.5 & 77.5 & 3.21 & 11.37 & 13.15 & 13.30 & 19.17 & 35.83 & 29.17 & 5.35 \\
\hline SRT-SCS-2002-00066 & SB3-30 & n54 & quenched & 5 & 6 & 10.8 & 8.64 & 3.42 & 9.82 & 66.1 & 70 & 1.64 & 18.00 & 14.40 & 5.70 & 16.37 & 110.17 & 16.67 & 2.73 \\
\hline SRT-SCS-2002-00066 & SB3-30ccc & n36 & $\mathrm{ccc}$ & 5 & 7 & 11.2 & 7.26 & 4.19 & 9.21 & 59.2 & 67.9 & 1.32 & 8.67 & 12.10 & 6.98 & 15.35 & 98.67 & 113.17 & 2.20 \\
\hline SRT-SCS-2002-00066 & SB3-33ccc & $\mathrm{n} 25$ & $\mathrm{ccc}$ & 5 & 8 & 7.15 & 6.12 & 3.93 & 5.62 & 29.6 & 58.1 & 1.81 & 11.92 & 10.20 & 6.55 & 9.37 & & 96.84 & 3.02 \\
\hline SRT-SCS-2002-00066 & blank & $\mathrm{n} 88$ & & 5 & 9 & 0.786 & $<0.150$ & $<0.040$ & $<0.500$ & $<0.100$ & $<0.790$ & $<1.00$ & 1.31 & 0.13 & 0.03 & 0 & & .66 & 0.83 \\
\hline SRT-SCS-2002-00066 & soln std & std-b5-2 & & 5 & 10 & 3.97 & 20.7 & 3.76 & 036 & 85.5 & 49.9 & $<1.00$ & 3.97 & 20.70 & 3.76 & & & 90 & 0.50 \\
\hline SRT-SCS-2002-00066 & SB3-42ccc & n04 & $\mathrm{ccc}$ & 5 & 11 & 17.3 & 14.1 & 14.9 & 39.4 & 112 & 141 & 3.81 & 28.83 & 23.50 & 24.83 & 65.67 & 67 & 235.00 & 6.35 \\
\hline T-SCS-2002-00066 & SB3-33 & n24 & quenched & 5 & 12 & 7.19 & 6.31 & 4.81 & 5.82 & 31.2 & 59.4 & 1.92 & 11.98 & 10.52 & 8.02 & & & 99.00 & 3.20 \\
\hline T-SCS-2002-00066 & SB3-35ccc & $\mathrm{n} 11$ & $\mathrm{ccc}$ & 5 & 13 & 8.26 & 671 & 18 & 7.42 & 52.4 & 79 & 4.02 & 13.77 & 11.18 & 22.33 & & & 1.67 & 6.70 \\
\hline T-SCS-2002-00066 & SB3-41 & n33 & quenched & 5 & 14 & 9.53 & & 16 & 18 & 75.6 & 111 & 4.58 & 15.88 & 18.17 & 26.83 & 30 & & 85.00 & 7.63 \\
\hline SRT-SCS-2002-00066 & SB3-41ccc & n82 & $\mathrm{ccc}$ & 5 & 15 & 10.7 & 10.9 & 12.7 & 19.7 & 71.8 & 115 & 3.81 & 17.83 & 18.17 & 21.17 & 32 & 119.67 & 191.67 & 6.35 \\
\hline T-SCS-2002-00066 & SB3-35 & n19 & quenched & 5 & 16 & 7.37 & 7.18 & 11.6 & 7.66 & 59.2 & 82 & 4.02 & 12.28 & 11.97 & 19.33 & 12 & 98.67 & 136.67 & 6.70 \\
\hline 0066 & SB3-39 & & quenched & 5 & 17 & 6.42 & 740 & 83 & 11 & 10 & 77 & & 10.70 & 12.48 & 13.97 & & & 128.34 & 5.67 \\
\hline T-SCS-2002-00066 & soln std & std-b5-3 & & 5 & 18 & 3.96 & 20.6 & 3.8 & 9.3 & 86.8 & 50.1 & $<1.00$ & 3.96 & 20.60 & 3.81 & 9.36 & & 50.10 & 0.50 \\
\hline SRT-SCS-2002-00066 & soln std & std-b6-1 & & 6 & 1 & 4.05 & 20.8 & 4.13 & 9.4 & 84.2 & 49.1 & $<1.00$ & 4.05 & 20.80 & 4.13 & 9.40 & 84.20 & 49.10 & 0.50 \\
\hline T-SCS-2002-00066 & SB3-41 & n65 & quenched & 6 & 2 & 9.1 & & 1 & 18.5 & 76 & 113 & 4.64 & 15 & 19.00 & 24.50 & 30 & & 188.34 & 7.73 \\
\hline $02-00066$ & SB3-39 & n49 & quenched & 6 & 3 & 651 & 80 & -2 & 118 & 18.6 & 75.9 & 3.47 & 10.85 & 13.37 & 14.08 & & & 126.50 & 5.78 \\
\hline T-SCS-2002-00066 & SB3-30ccc & $\mathrm{n} 12$ & $\mathrm{ccc}$ & 6 & 4 & 11.2 & 7. & 4.2 & 9.17 & 58.4 & 66.4 & 1.37 & 18.67 & 12.50 & 7.15 & 15 & 97 & 110.67 & 2.28 \\
\hline TT-SCS-2002-00066 & SB3-38ccc & n08 & $\mathrm{ccc}$ & 6 & 5 & 11.6 & 5.81 & 3.98 & & 42.9 & 62 & 2.04 & 19.33 & 9.68 & 6. & & & 34 & 3.40 \\
\hline T-SCS-2002-00066 & SB3-42ccc & $\mathrm{n}$ & $\mathrm{ccc}$ & 6 & 6 & 20 & 134 & 23 & 37 & 1 & 139 & 4.83 & 34.17 & 22.33 & 38.33 & 62 & 00 & .67 & 8.05 \\
\hline $2002-00066$ & SB3-38 & n56 & uenched & 6 & 7 & 12.38 & & 5 & 10 & 47.5 & 65.9 & 1. & 20 & 11.42 & 9.08 & & & 109.84 & 2.83 \\
\hline T-SCS-2002-00066 & SB3-41ccc & $\mathrm{n} 31$ & $\mathrm{ccc}$ & 6 & 8 & 8.7 & 11.2 & 13.5 & 20.1 & 72.1 & 115 & 3.9 & 14.50 & 18.67 & 22.50 & 33.50 & 12 & 191.67 & 6.50 \\
\hline SRT-SCS-2002-00066 & SB3-30 & n52 & quenched & 6 & 9 & 11 & 8.53 & 4.76 & 9.4 & 62.9 & 67.1 & 1.62 & 18.33 & 14.22 & 7.93 & 15.67 & 10 & 111.84 & 2.70 \\
\hline SRT-SCS-2002-00066 & soln std & std-b6-2 & & 6 & 10 & 4.08 & 20 & 414 & 9.32 & 842 & 48.9 & $<1.00$ & 4.08 & 20.60 & 4.14 & 93 & 84.30 & 48.90 & 0.50 \\
\hline 02-00066 & SB3-33ccc & n79 & $\mathrm{cc}$ & 6 & 11 & 71 & & & & & 58.9 & 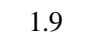 & & & & & & & 3.17 \\
\hline T-SCS-2002-00066 & SB3-39ccc & n38 & $\mathrm{ccc}$ & 6 & 12 & 6.85 & 7.9 & 8.29 & 11.4 & 19.7 & 75.9 & 3.39 & 11.42 & 13.17 & 13.82 & 19.00 & 32.83 & 126.50 & 5.65 \\
\hline T-SCS-2002-00066 & SB3-35 & n66 & quenched & 6 & 13 & 7.73 & 6.88 & 12 & 7.09 & 54.7 & 77.3 & 3.59 & 12.88 & 11.47 & 20.00 & 11.82 & & 128.84 & 5.98 \\
\hline T-SCS-2002-00066 & SB3-42 & n73 & quenched & 6 & 14 & 15.7 & 12.1 & 8.24 & 23.5 & 108 & 106 & 3.17 & 26.17 & 20.17 & 13.73 & 39.17 & 180.00 & 176.67 & 5.28 \\
\hline r-SCS-2002-00066 & SB3-35ccc & $\mathrm{n} 44$ & $\mathrm{ccc}$ & 6 & 15 & 7. & & 12.7 & & 50 & 77.7 & 3.99 & & 11.43 & 21.17 & 12 & 84 & 129.50 & 6.65 \\
\hline SRT-SCS-2002-00066 & SB3-33 & $\mathrm{n} 41$ & quenched & 6 & 16 & 6.72 & 6.23 & 5 & 5.59 & 29.1 & 57.1 & 1.9 & 11.20 & 10.38 & 8.33 & 9.3 & 48.50 & 95.17 & 3.17 \\
\hline SRT-SCS-2002-00066 & soln std & std-b6-3 & & 6 & 17 & 3.96 & 20.1 & 4.26 & 9.18 & 82 & 49.1 & $<1.00$ & 3.96 & 20.10 & 4.26 & 9.18 & 82.00 & 49.10 & 0.50 \\
\hline
\end{tabular}




\section{Exhibit I.1. SRTC-ML Mesaurements of PCT Leachate Solutions for SB3 Phase 1 glasses In Analytical Sequence Over All Three Groups}
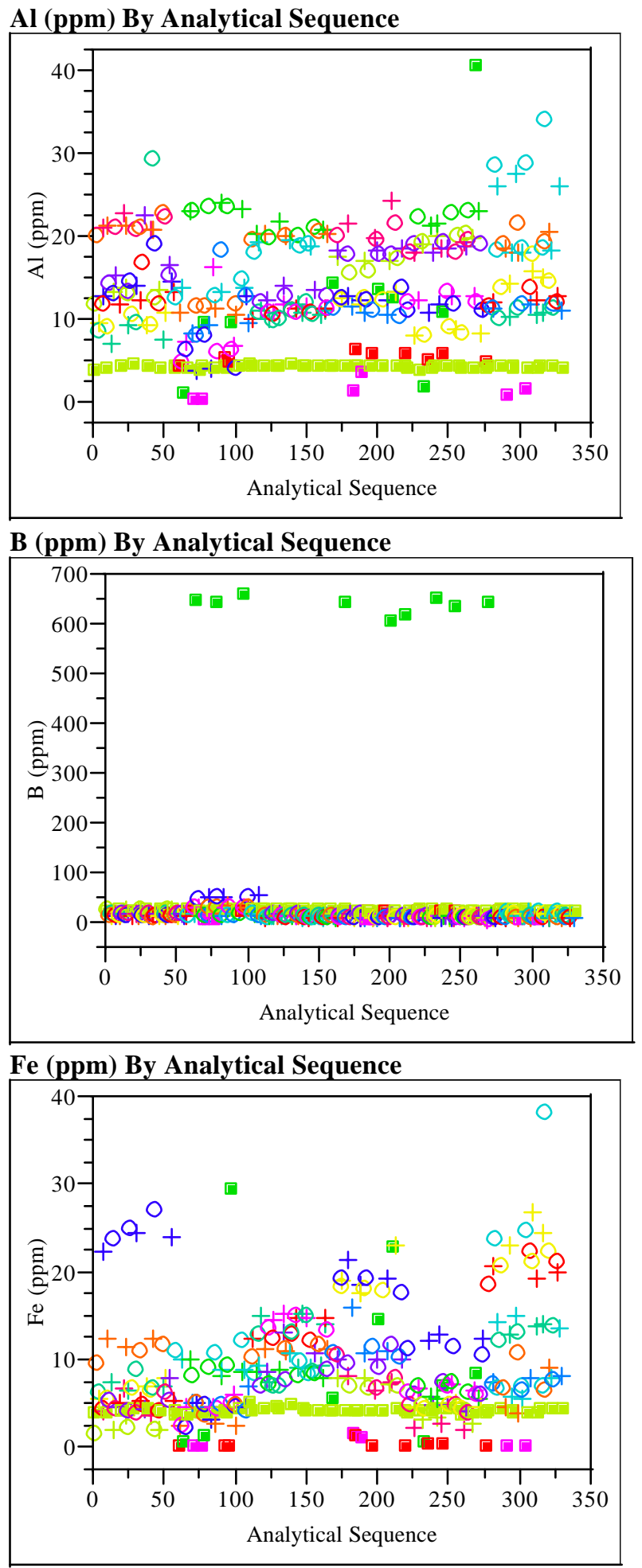

Li (ppm) By Analytical Sequence

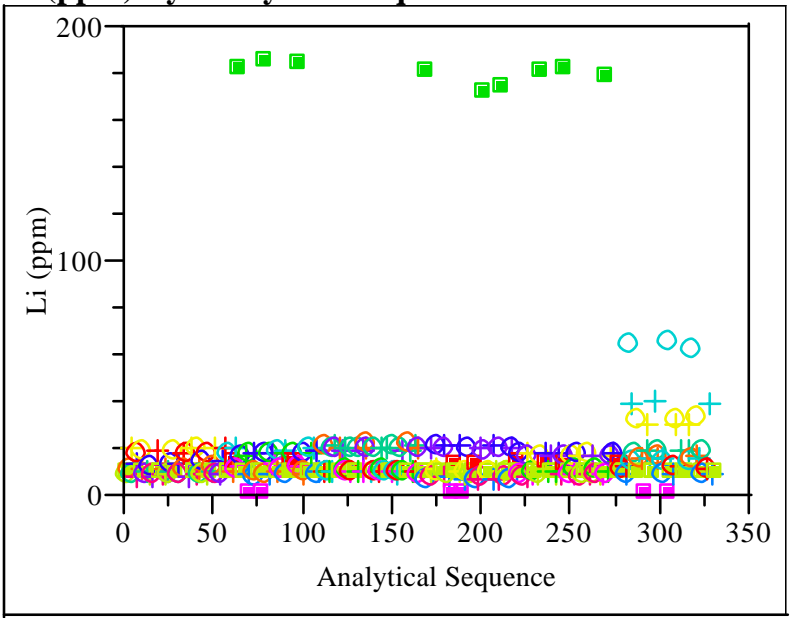

\section{Na (ppm) By Analytical Sequence}

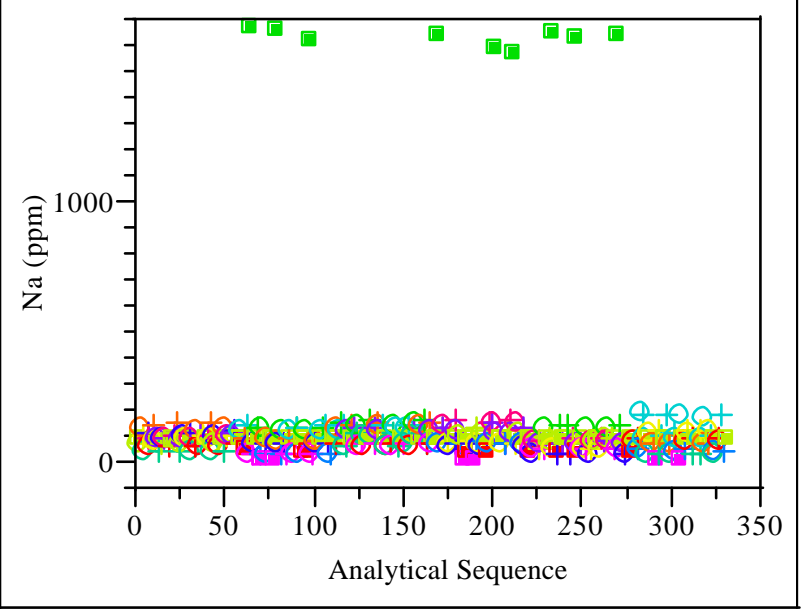

\section{Si (ppm) By Analytical Sequence}

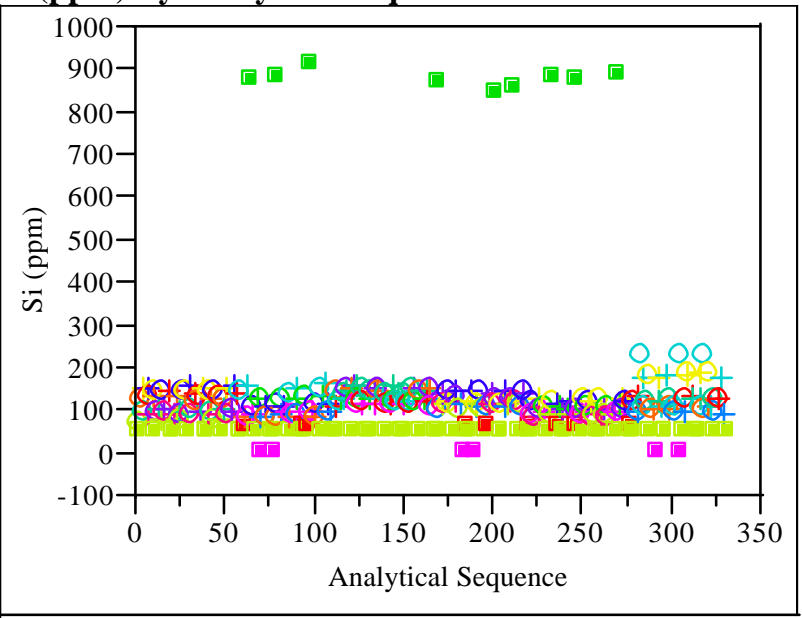


Exhibit I.1. SRTC-ML Mesaurements of PCT Leachate Solutions for SB3 Phase 1 glasses In Analytical Sequence Over All Three Groups

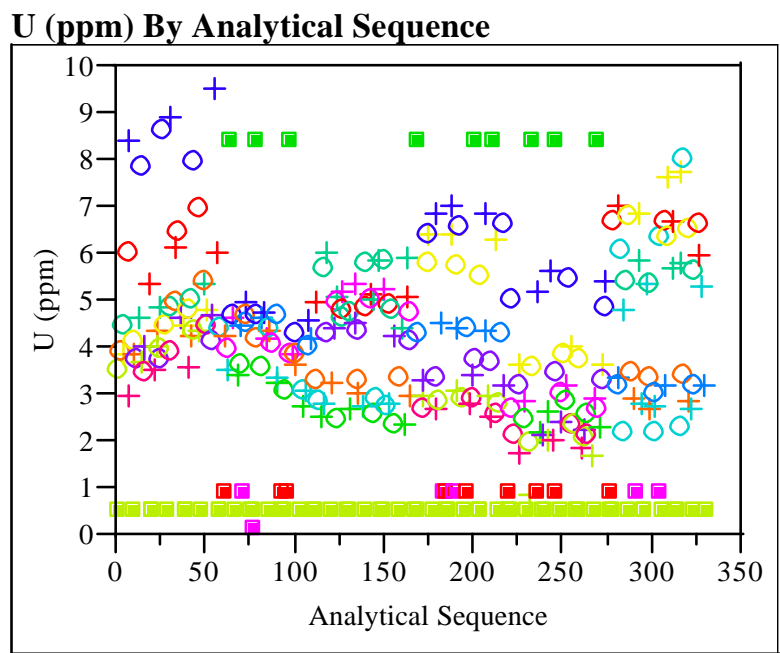




\section{Exhibit I.2. SRTC-ML Mesaurements of PCT Leachate Solutions for SB3 Phase 1 glasses In Analytical Sequence Over All Three Groups Excluding the EA Glass}
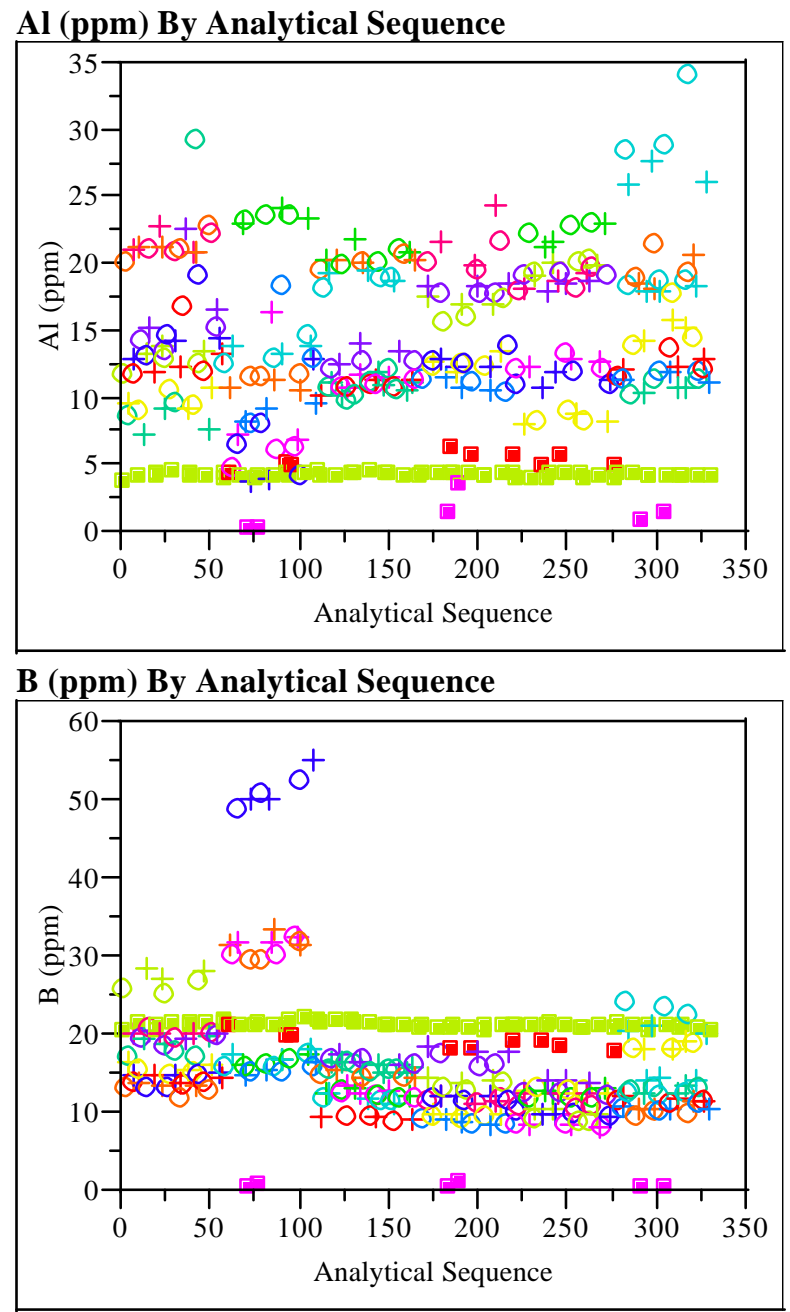

Fe (ppm) By Analytical Sequence

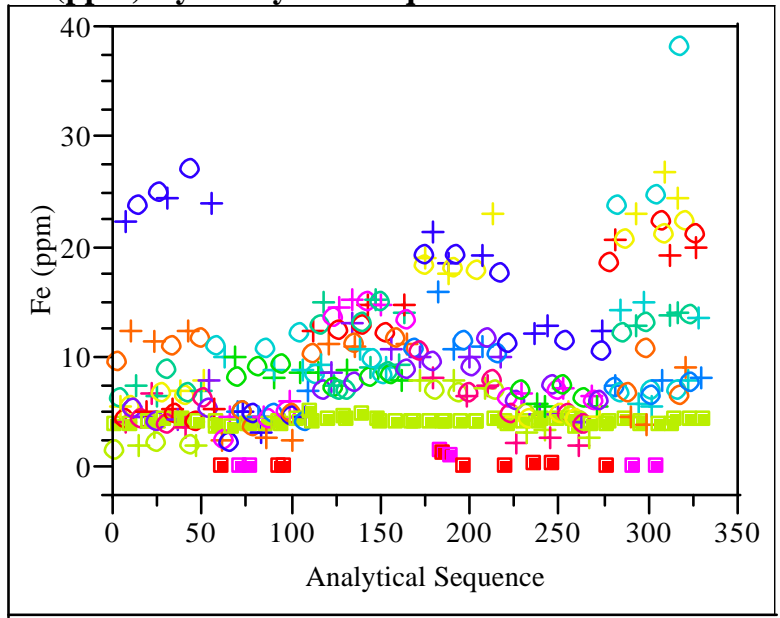

Li (ppm) By Analytical Sequence
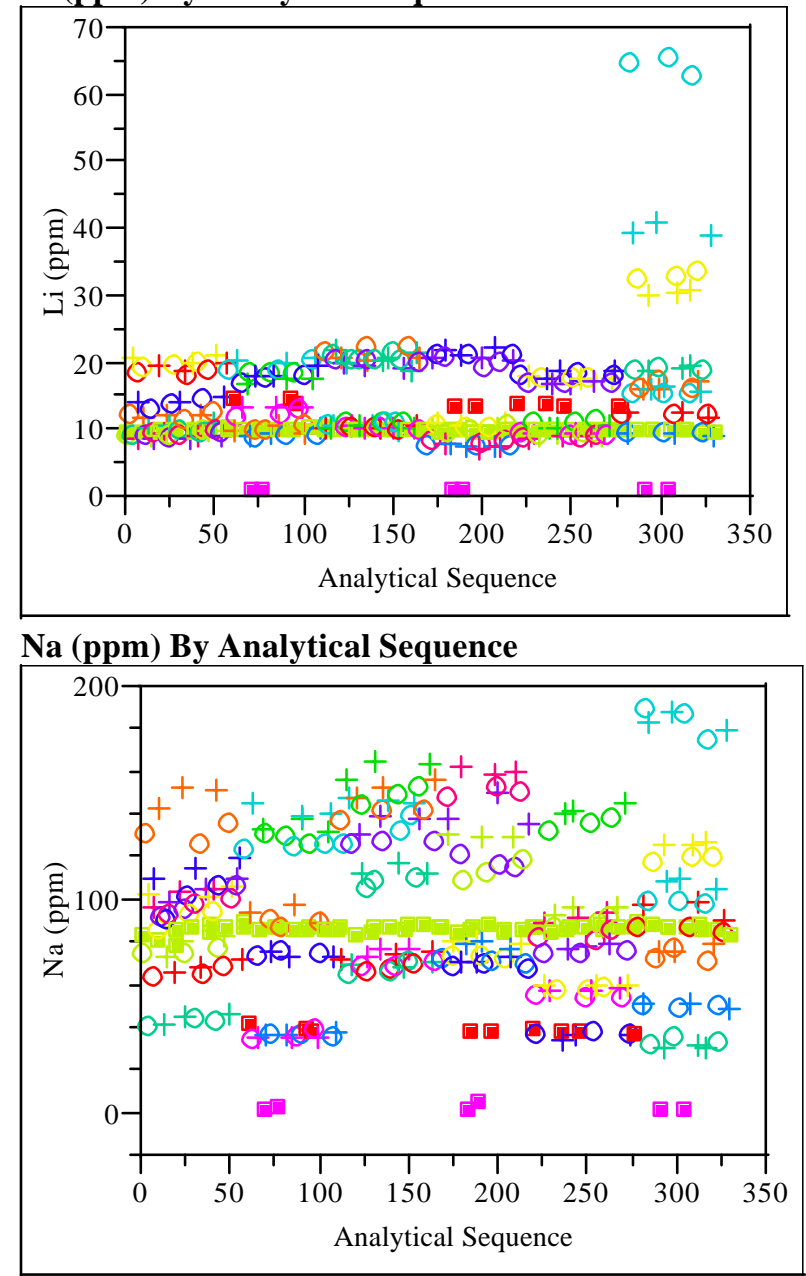

Si (ppm) By Analytical Sequence

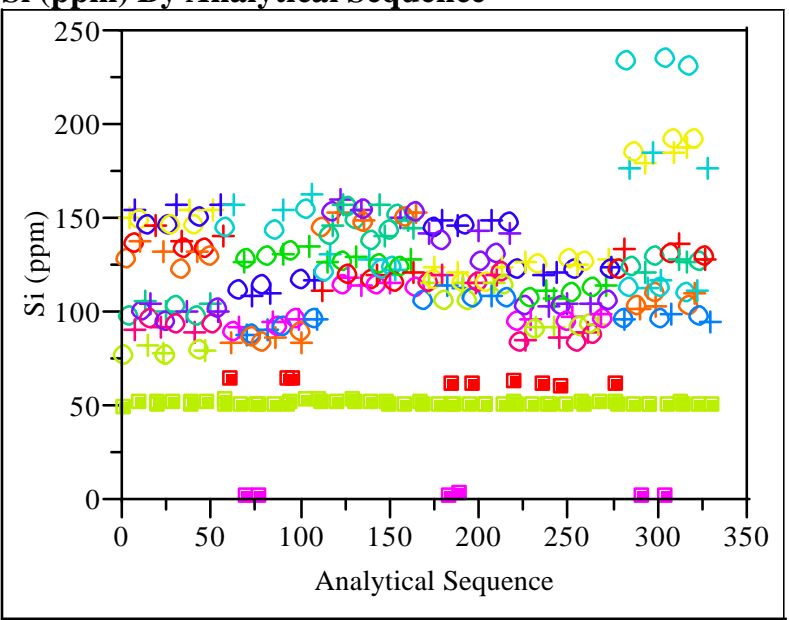


Exhibit I.2. SRTC-ML Mesaurements of PCT Leachate Solutions for SB3 Phase 1 glasses In Analytical Sequence Over All Three Groups Excluding the EA Glass

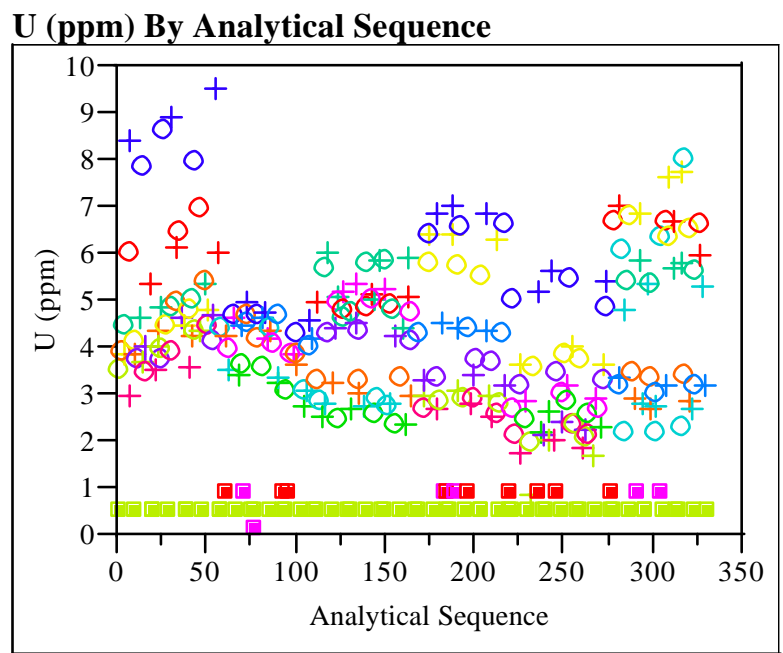


Exhibit I.3. SRTC-ML Mesaurements of PCT Leachate Solutions by SB3 Phase 1 Glass ID (Plus - Quenched; Circle - Centerline Canister Cooled (ccc))

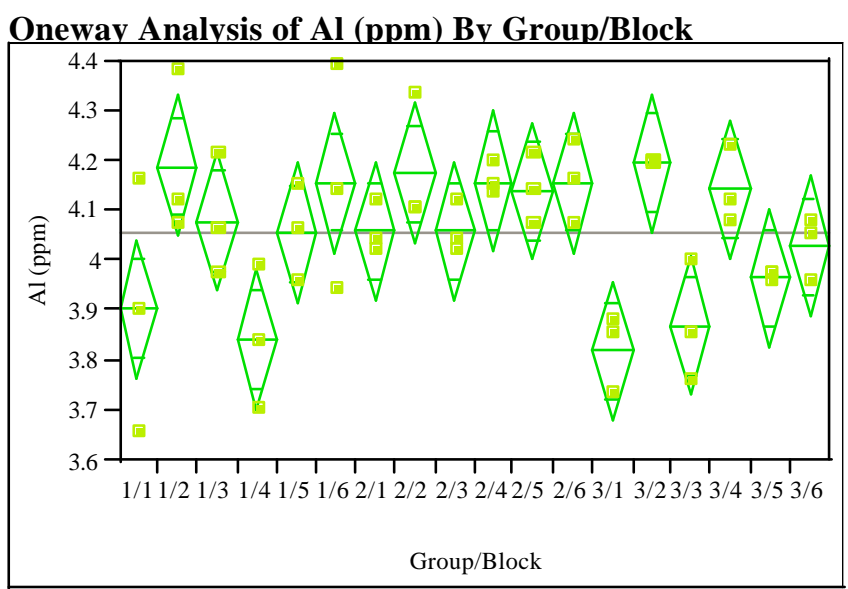

\section{Oneway Anova}

\section{Summary of Fit}

$\begin{array}{lr}\text { Rsquare } & 0.609304 \\ \text { Adj Rsquare } & 0.424809 \\ \text { Root Mean Square Error } & 0.119257 \\ \text { Mean of Response } & 4.056111 \\ \text { Observations (or Sum Wgts) } & 54\end{array}$

\section{Analysis of Variance}

$\begin{array}{lrrrrr}\text { Source } & \text { DF } & \text { Sum of Squares } & \text { Mean Square } & \text { F Ratio } & \text { Prob > } \\ \text { Group/Block } & 17 & 0.7984833 & 0.046970 & 3.3026 & 0.0013 \\ \text { Error } & 36 & 0.5120000 & 0.014222 & & \\ \text { C. } & 53 & 1.3104833 & & & \end{array}$

\section{Means for Oneway Anova}

$\begin{array}{lrrrr}\text { Level } & \text { Number } & \text { Mean } & \text { Std Error } & \text { Lower 95\% } \\ 1 / 1 & 3 & 3.90333 & 0.06885 & 3.7637 \\ 1 / 2 & 3 & 4.19000 & 0.06885 & 4.0504 \\ 1 / 3 & 3 & 4.08000 & 0.06885 & 3.9404 \\ 1 / 4 & 3 & 3.84333 & 0.06885 & 3.7037 \\ 1 / 5 & 3 & 4.05667 & 0.06885 & 3.9170 \\ 1 / 6 & 3 & 4.15667 & 0.06885 & 4.0170 \\ 2 / 1 & 3 & 4.06000 & 0.06885 & 3.9204 \\ 2 / 2 & 3 & 4.17667 & 0.06885 & 4.0370 \\ 2 / 3 & 3 & 4.06000 & 0.06885 & 3.9204 \\ 2 / 4 & 3 & 4.16000 & 0.06885 & 4.0204 \\ 2 / 5 & 3 & 4.14000 & 0.06885 & 4.0004 \\ 2 / 6 & 3 & 4.15667 & 0.06885 & 4.0170 \\ 3 / 1 & 3 & 3.82000 & 0.06885 & 3.6804 \\ 3 / 2 & 3 & 4.19667 & 0.06885 & 4.0570 \\ 3 / 3 & 3 & 3.87000 & 0.06885 & 3.7304 \\ 3 / 4 & 3 & 4.14333 & 0.06885 & 4.0037 \\ 3 / 5 & 3 & 3.96667 & 0.06885 & 3.8270 \\ 3 / 6 & 3 & 4.03000 & 0.06885 & 3.8904 \\ \text { Std Error uses a pooled estimate of error variance }\end{array}$

Oneway Analysis of B (ppm) By Group/Block

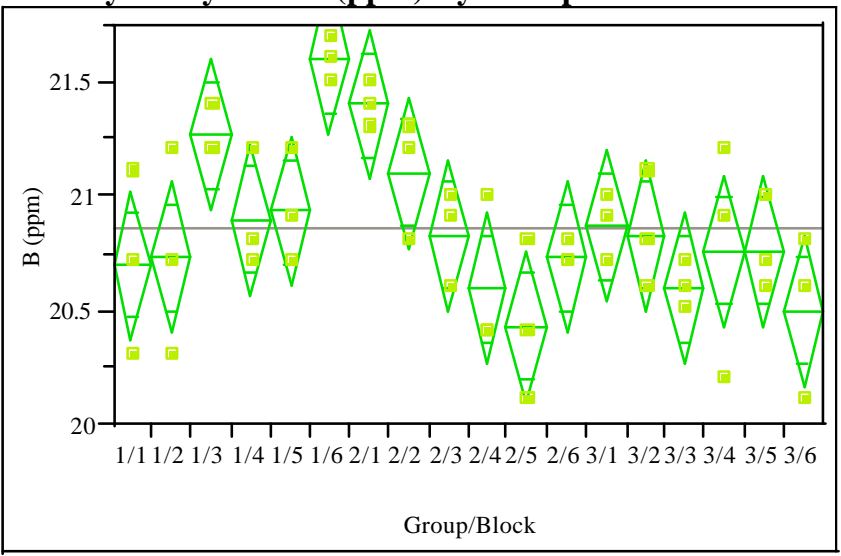

Oneway Anova Summary of Fit

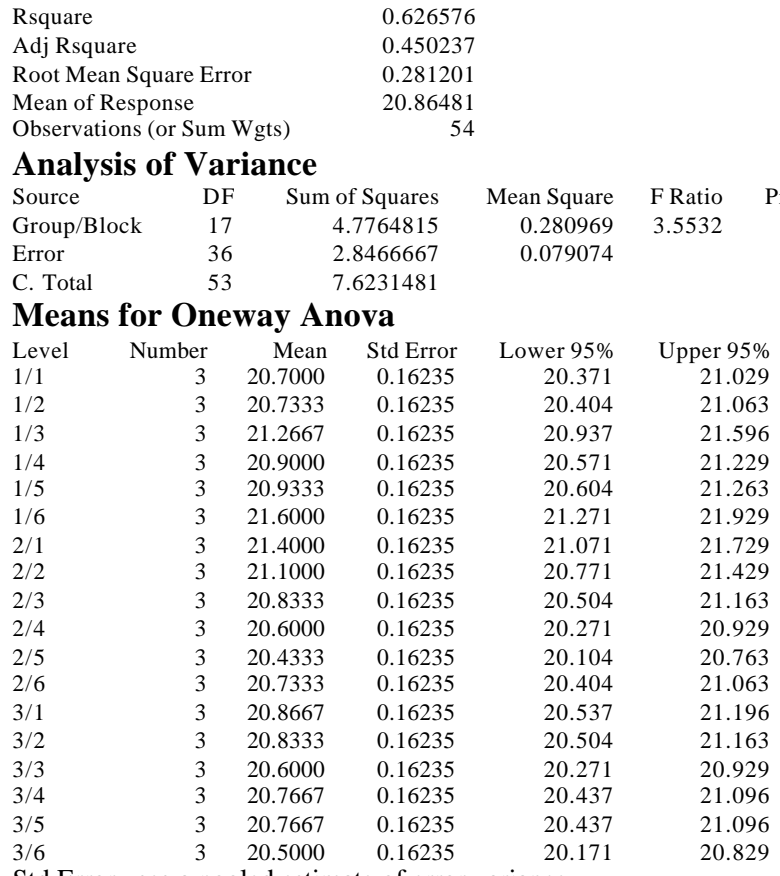

Std Error uses a pooled estimate of error variance 
Exhibit I.3. SRTC-ML Mesaurements of PCT Leachate Solutions by SB3 Phase 1 Glass ID (Plus - Quenched; Circle - Centerline Canister Cooled (ccc))

Oneway Analysis of Fe (ppm) By Group/Block

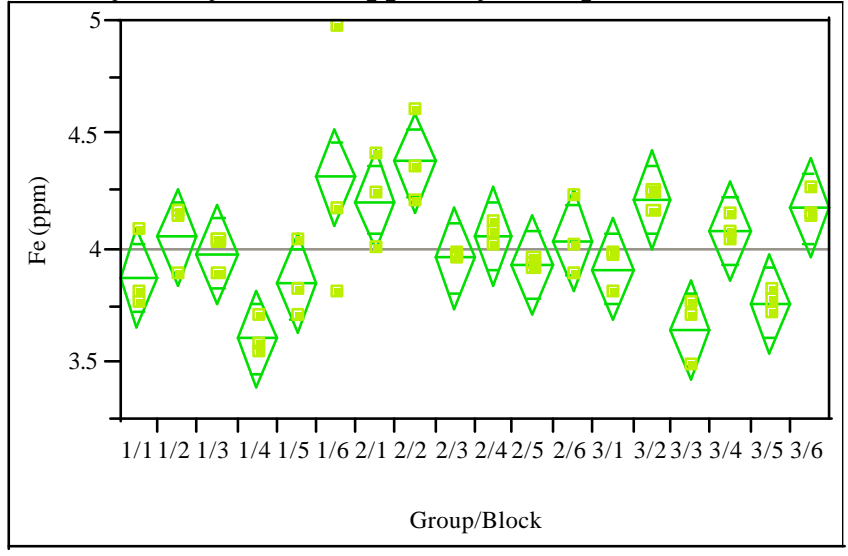

Oneway Anova

Summary of Fit

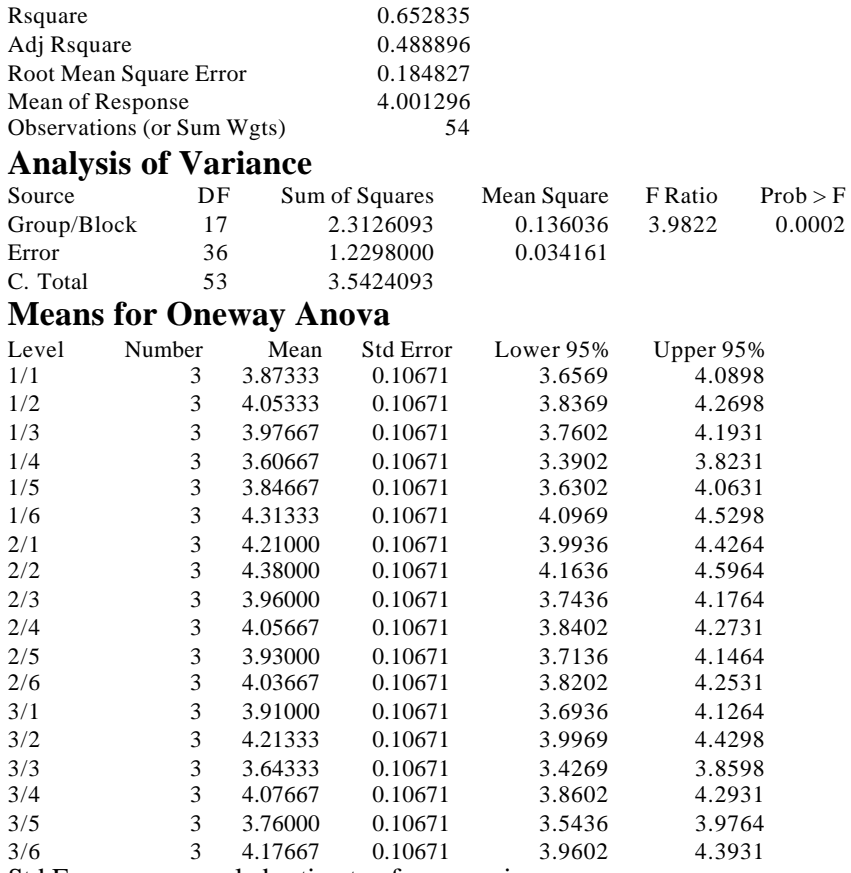

Oneway Analysis of Li (ppm) By Group/Block

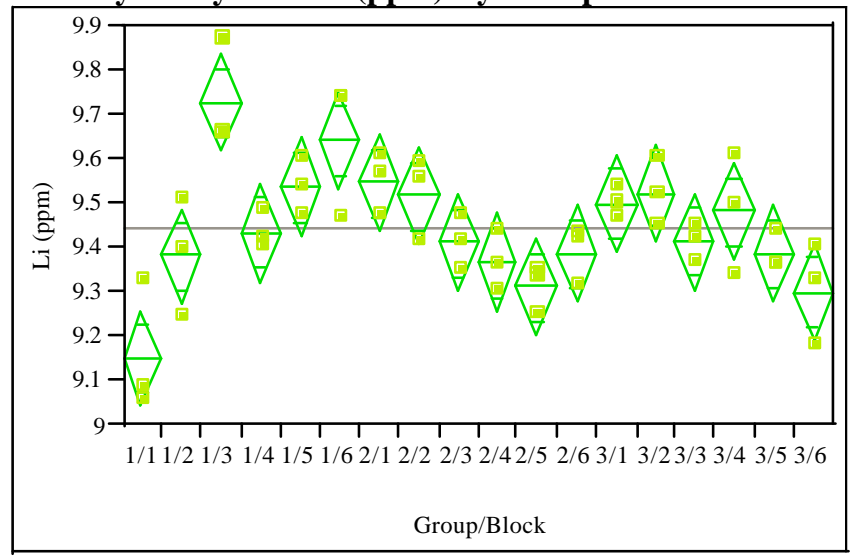

Oneway Anova

Summary of Fit

$\begin{array}{lr}\text { Rsquare } & 0.739287 \\ \text { Adj Rsquare } & 0.616173 \\ \text { Root Mean Square Error } & 0.093492 \\ \text { Mean of Response } & 9.444815 \\ \text { Observations (or Sum Wgts) } & 54\end{array}$

Analysis of Variance

Source DF Sum of Squares Mean Square F Ratio Prob > F

$\begin{array}{lrrrrr}\text { Group/Block } & 17 & 0.8922815 & 0.052487 & 6.0049 & <.0001\end{array}$

$\begin{array}{llll}\text { Error } & 36 & 0.3146667 & 0.008741\end{array}$

$\begin{array}{lll}\text { C. Total } & 53 & 1.2069481\end{array}$

Means for Oneway Anova

Level Number Mean Std Error Lower 95\% Upper 95\%

$\begin{array}{lrrrrr}1 / 1 & 3 & 9.15000 & 0.05398 & 9.0405 & 9.2595\end{array}$

$\begin{array}{llllll}1 / 2 & 3 & 9.38000 & 0.05398 & 9.2705 & 9.4895\end{array}$

$\begin{array}{llllll}1 / 3 & 3 & 9.72667 & 0.05398 & 9.6172 & 9.8361\end{array}$

$\begin{array}{llllll}1 / 4 & 3 & 9.43333 & 0.05398 & 9.3239 & 9.5428\end{array}$

$\begin{array}{llllll}1 / 5 & 3 & 9.53667 & 0.05398 & 9.4272 & 9.6461\end{array}$

$\begin{array}{llllll}1 / 6 & 3 & 9.64000 & 0.05398 & 9.5305 & 9.7495\end{array}$

$\begin{array}{llllll}2 / 1 & 3 & 9.54667 & 0.05398 & 9.4372 & 9.6561\end{array}$

$\begin{array}{llllll}2 / 2 & 3 & 9.51667 & 0.05398 & 9.4072 & 9.6261\end{array}$

$\begin{array}{llllll}2 / 3 & 3 & 9.41000 & 0.05398 & 9.3005 & 9.5195\end{array}$

$\begin{array}{llllll}2 / 4 & 3 & 9.36667 & 0.05398 & 9.2572 & 9.4761\end{array}$

$\begin{array}{llllll}2 / 5 & 3 & 9.31000 & 0.05398 & 9.2005 & 9.4195\end{array}$

$\begin{array}{llllll}2 / 6 & 3 & 9.38667 & 0.05398 & 9.2772 & 9.4961\end{array}$

$\begin{array}{llllll}3 / 1 & 3 & 9.50000 & 0.05398 & 9.3905 & 9.6095\end{array}$

$\begin{array}{llllll}3 / 2 & 3 & 9.52333 & 0.05398 & 9.4139 & 9.6328 \\ 3 / 3 & 3 & 9.41333 & 0.05398 & 9.3039 & 9.5228\end{array}$

$\begin{array}{llllll}3 / 3 & 3 & 9.41333 & 0.05398 & 9.3039 & 9.5228 \\ 3 / 4 & 3 & 9.48000 & 0.05398 & 9.3705 & 9.5895\end{array}$

$\begin{array}{llllll}3 / 4 & 3 & 9.48000 & 0.05398 & 9.3705 & 9.5895\end{array}$

$\begin{array}{llllll}3 / 5 & 3 & 9.38667 & 0.05398 & 9.2772 & 9.4961\end{array}$

$\begin{array}{llllll}3 / 6 & 3 & 9.30000 & 0.05398 & 9.1905 & 9.4095\end{array}$

Std Error uses a pooled estimate of error variance 
Exhibit I.3. SRTC-ML Mesaurements of PCT Leachate Solutions by SB3 Phase 1 Glass ID (Plus - Quenched; Circle - Centerline Canister Cooled (ccc))

Oneway Analysis of Na (ppm) By Group/Block

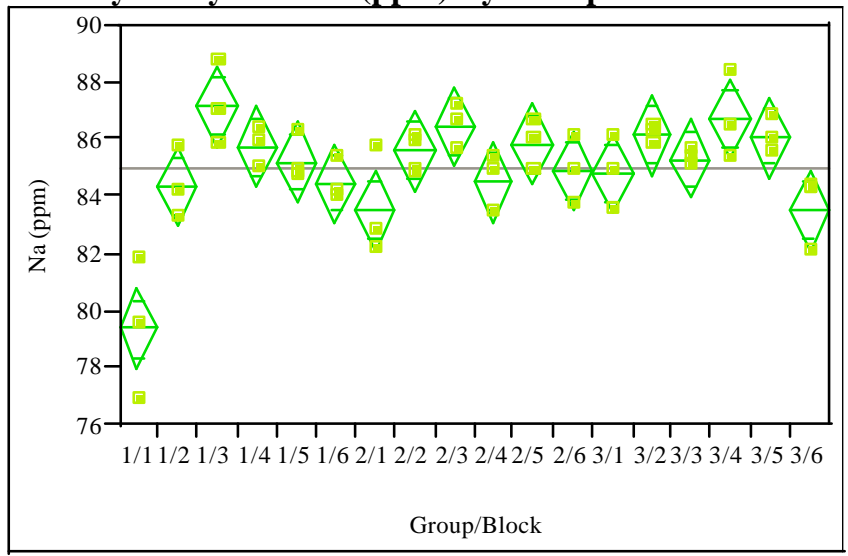

Oneway Anova

Summary of Fit

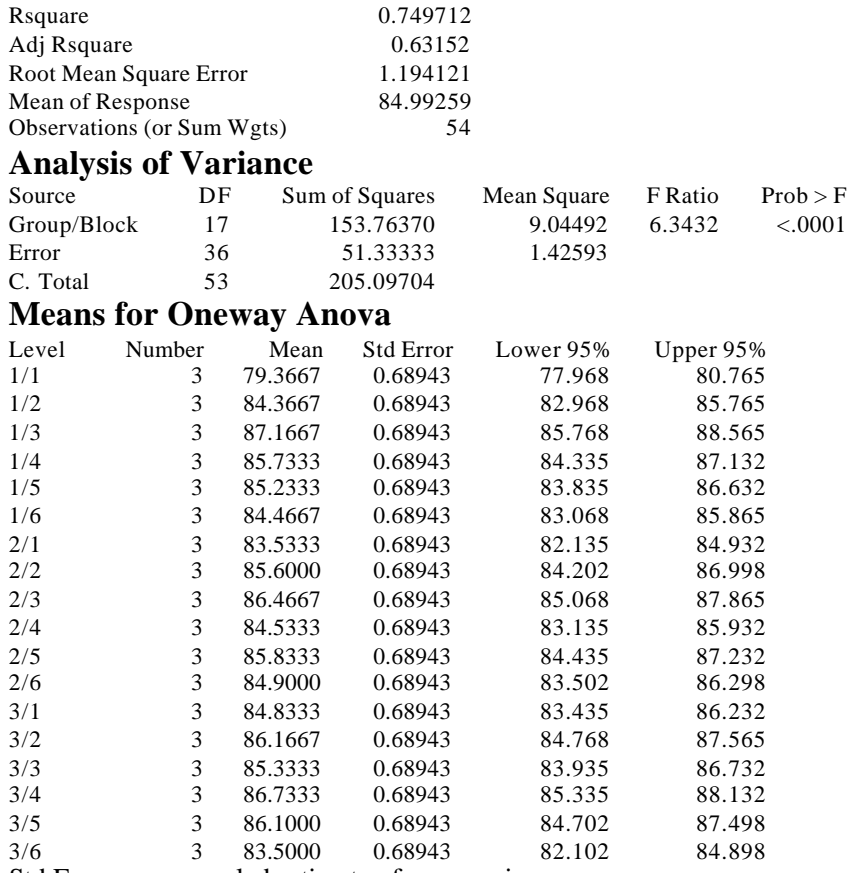

Oneway Analysis of Si (ppm) By Group/Block

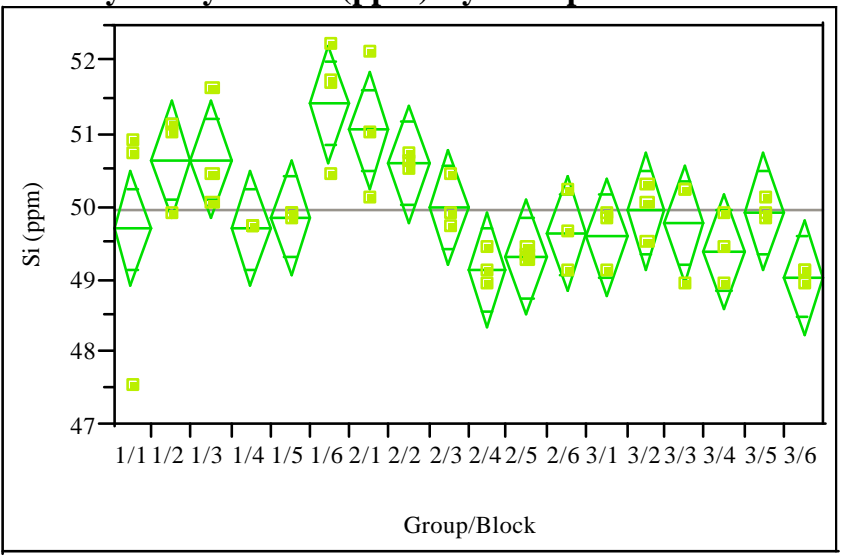

Oneway Anova

Summary of Fit

\begin{tabular}{lrrrrr} 
Rsquare & 0.57396 & & & \\
Adj Rsquare & 0.372775 & & & \\
Root Mean Square Error & 0.681773 & & & \\
Mean of Response & 49.96852 & & & \\
Observations (or Sum Wgts) & \multicolumn{2}{l}{54} & & & \\
\multicolumn{2}{l}{ Analysis of Variance } & & & & \\
Source & DF & Sum of Squares & Mean Square & F Ratio & Prob > F \\
Group/Block & 17 & 22.543148 & 1.32607 & 2.8529 & 0.0040 \\
Error & 36 & 16.733333 & 0.46481 & &
\end{tabular}

\section{Means for Oneway Anova}

$\begin{array}{lrrrrr}\text { Level } & \text { Number } & \text { Mean } & \text { Std Error } & \text { Lower 95\% } & \text { Upper 95\% } \\ 1 / 1 & 3 & 49.7000 & 0.39362 & 48.902 & 50.498\end{array}$

$\begin{array}{llllll}1 / 2 & 3 & 50.6667 & 0.39362 & 49.868 & 51.465\end{array}$

$\begin{array}{llllll}1 / 3 & 3 & 50.6667 & 0.39362 & 49.868 & 51.465\end{array}$

$\begin{array}{llllll}1 / 4 & 3 & 49.7000 & 0.39362 & 48.902 & 50.498\end{array}$

$\begin{array}{llllll}1 / 5 & 3 & 49.8667 & 0.39362 & 49.068 & 50.665\end{array}$

$\begin{array}{llllll}1 / 6 & 3 & 51.4333 & 0.39362 & 50.635 & 52.232\end{array}$

$\begin{array}{llllll}2 / 1 & 3 & 51.0667 & 0.39362 & 50.268 & 51.865\end{array}$

$\begin{array}{llllll}2 / 2 & 3 & 50.6000 & 0.39362 & 49.802 & 51.398\end{array}$

$\begin{array}{llllll}2 / 3 & 3 & 50.0000 & 0.39362 & 49.202 & 50.798\end{array}$

$\begin{array}{llllll}2 / 4 & 3 & 49.1333 & 0.39362 & 48.335 & 49.932\end{array}$

$\begin{array}{llllll}2 / 5 & 3 & 49.3000 & 0.39362 & 48.502 & 50.098\end{array}$

$\begin{array}{llllrl}2 / 6 & 3 & 49.6333 & 0.39362 & 48.835 & 50.432 \\ 3 / 1 & 3 & 49.6000 & 0.39362 & 48.802 & 50.398\end{array}$

$\begin{array}{llllll}3 / 1 & 3 & 49.6000 & 0.39362 & 48.802 & 50.398\end{array}$

$\begin{array}{llllll}3 / 2 & 3 & 49.9333 & 0.39362 & 49.135 & 50.732\end{array}$

$\begin{array}{llllll}3 / 3 & 3 & 49.7667 & 0.39362 & 48.968 & 50.565\end{array}$

$\begin{array}{llllll}3 / 4 & 3 & 49.4000 & 0.39362 & 48.602 & 50.198\end{array}$

$\begin{array}{llllll}3 / 5 & 3 & 49.9333 & 0.39362 & 49.135 & 50.732\end{array}$

$\begin{array}{llllll}3 / 6 & 3 & 49.0333 & 0.39362 & 48.235 & 49.832\end{array}$

Std Error uses a pooled estimate of error variance 
Exhibit I.3. SRTC-ML Mesaurements of PCT Leachate Solutions by SB3 Phase 1 Glass ID (Plus - Quenched; Circle - Centerline Canister Cooled (ccc))

Oneway Analysis of U (ppm) By Group/Block

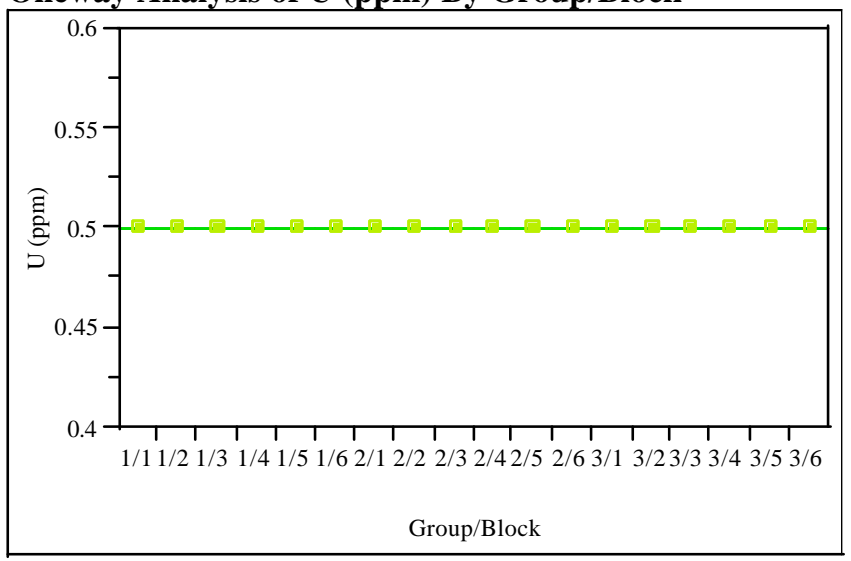

Oneway Anova

Summary of Fit

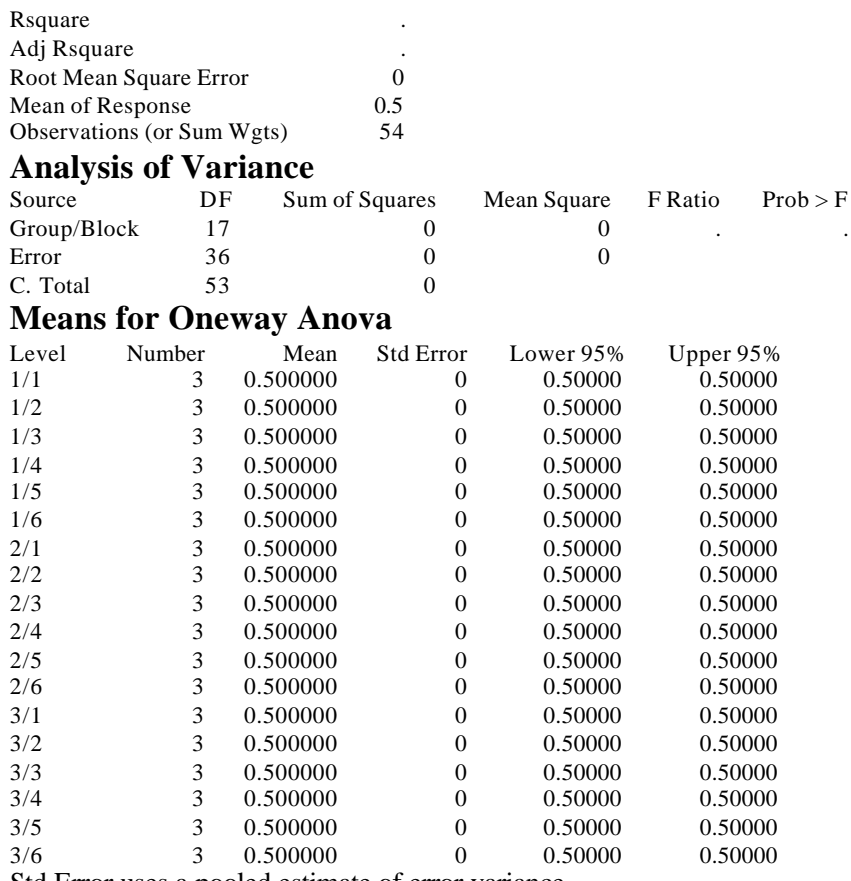


Exhibit I.4. SRTC-ML Mesaurements of PCT Leachate Solutions by SB3 Phase 1 Glass ID (Plus - Quenched; Circle - Centerline Canister Cooled (ccc))

\section{Al (ppm) By Glass ID w/o Heat Treatment (i.e., a Short ID)}

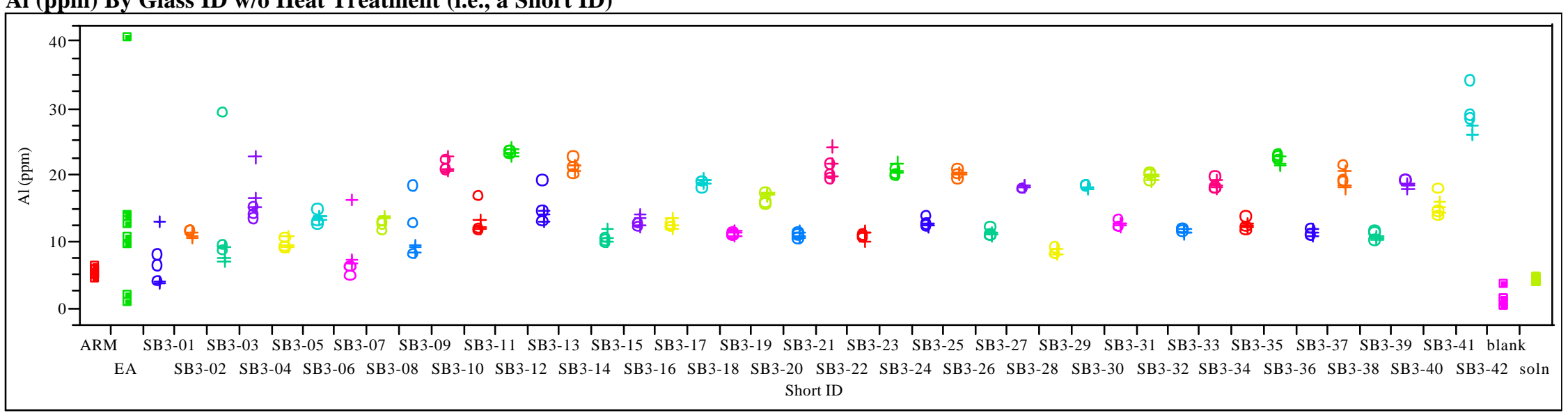

\section{B (ppm) By Glass ID w/o Heat Treatment (i.e., a Short ID)}

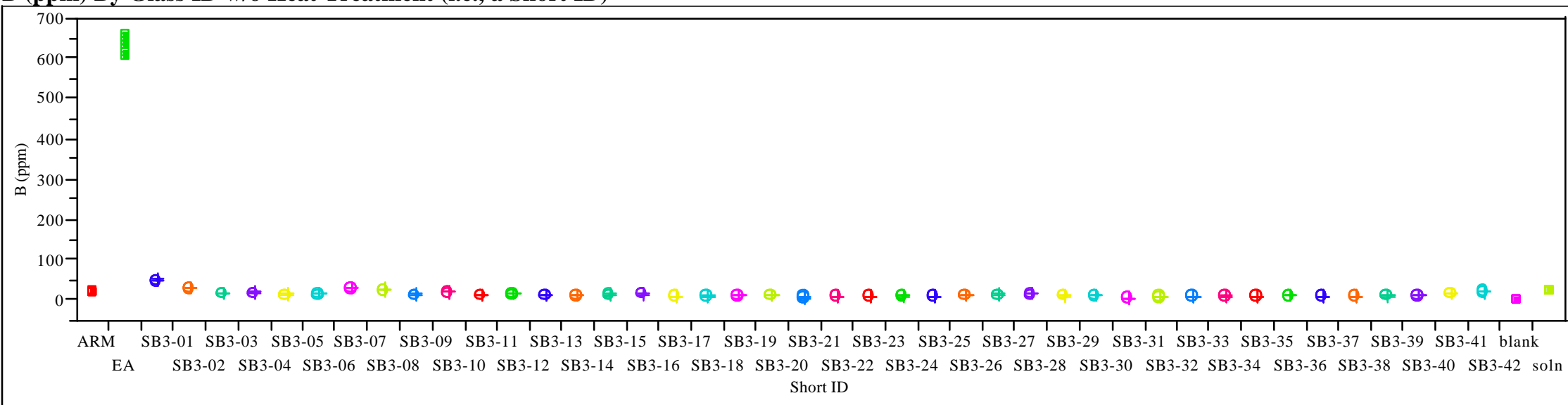


Exhibit I.4. SRTC-ML Mesaurements of PCT Leachate Solutions by SB3 Phase 1 Glass ID (Plus - Quenched; Circle - Centerline Canister Cooled (ccc))

\section{Fe (ppm) By Glass ID w/o Heat Treatment (i.e., a Short ID)}

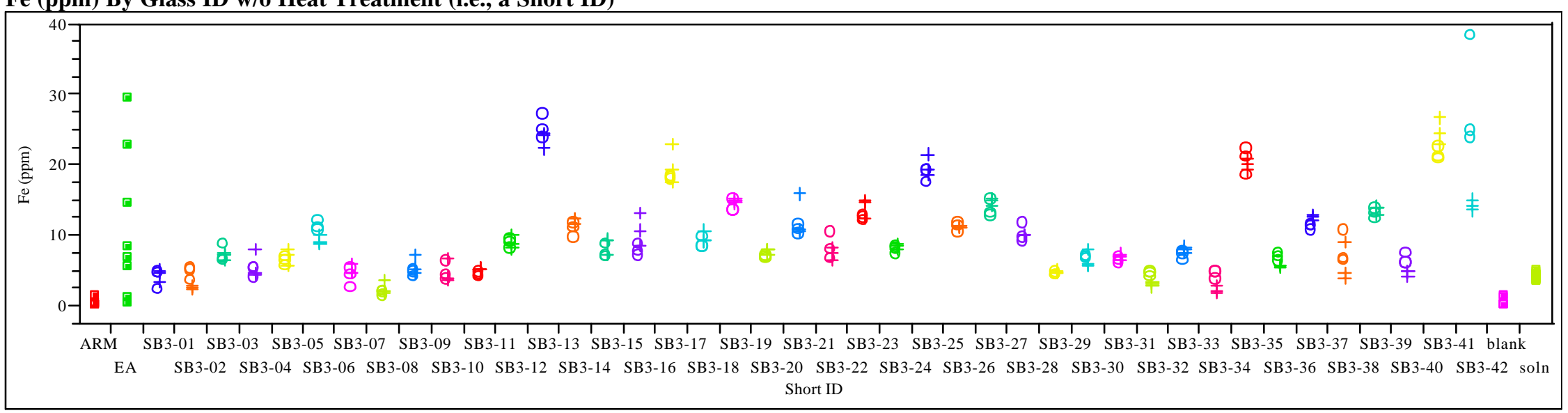

\section{Li (ppm) By Glass ID w/o Heat Treatment (i.e., a Short ID)}

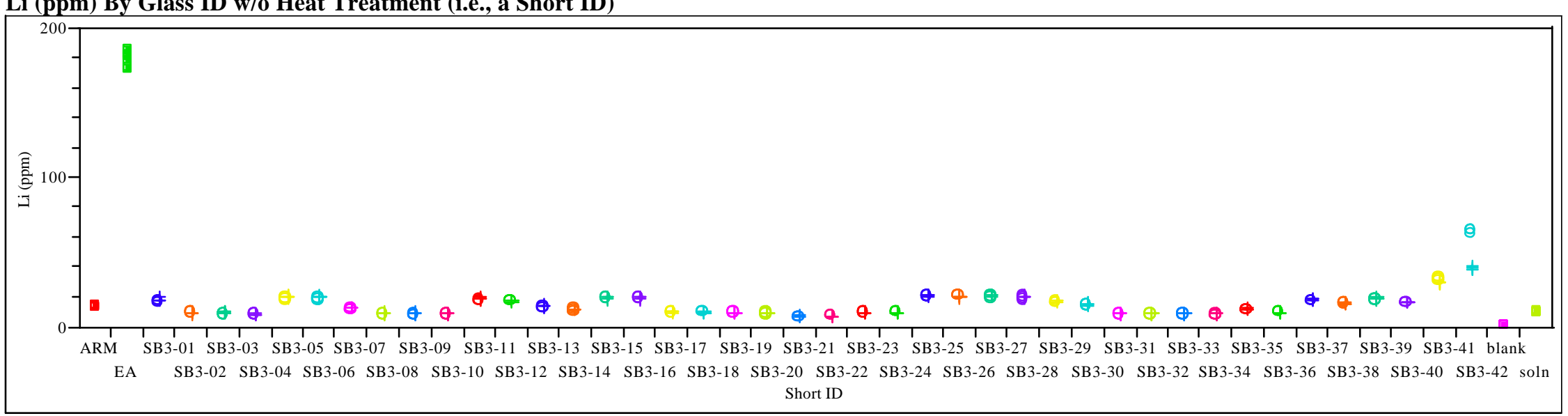


Exhibit I.4. SRTC-ML Mesaurements of PCT Leachate Solutions by SB3 Phase 1 Glass ID

(Plus - Quenched; Circle - Centerline Canister Cooled (ccc))

\section{Na (ppm) By Glass ID w/o Heat Treatment (i.e., a Short ID)}

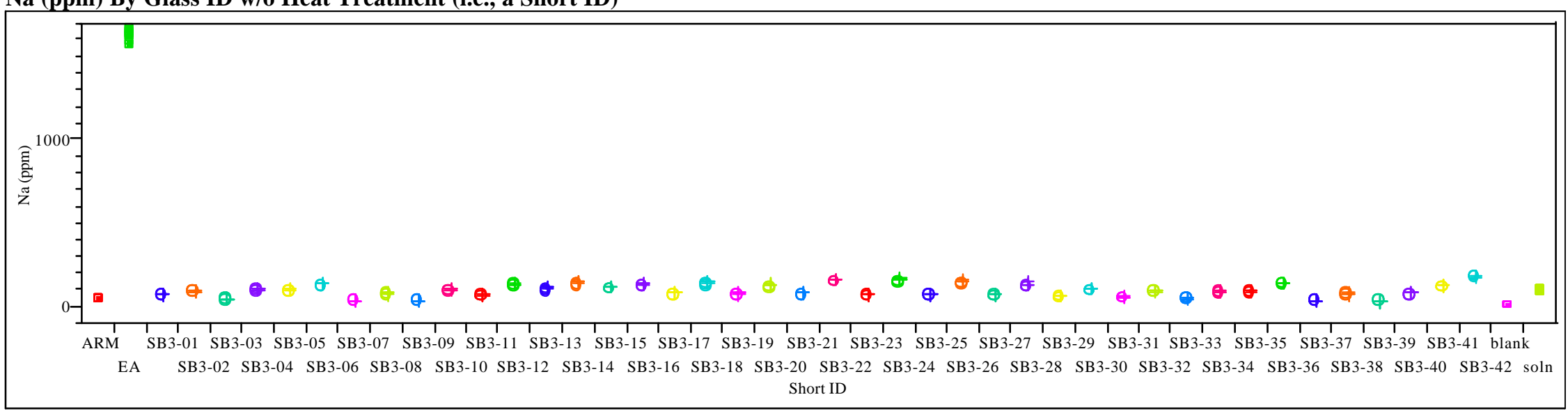

\section{Si (ppm) By Glass ID w/o Heat Treatment (i.e., a Short ID)}

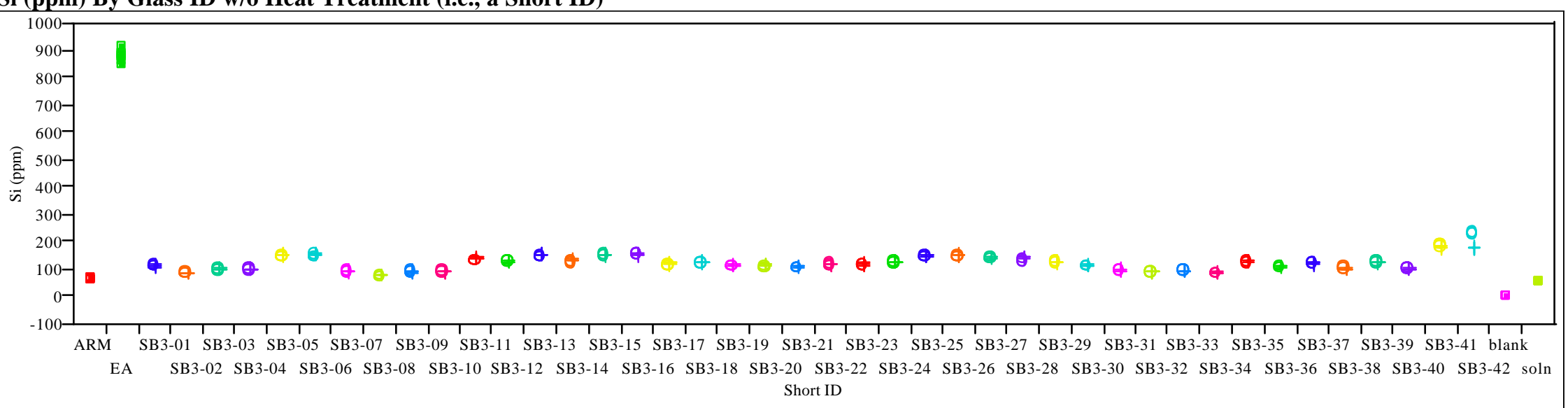


Exhibit I.4. SRTC-ML Mesaurements of PCT Leachate Solutions by SB3 Phase 1 Glass ID (Plus - Quenched; Circle - Centerline Canister Cooled (ccc))

\section{U (ppm) By Glass ID w/o Heat Treatment (i.e., a Short ID)}

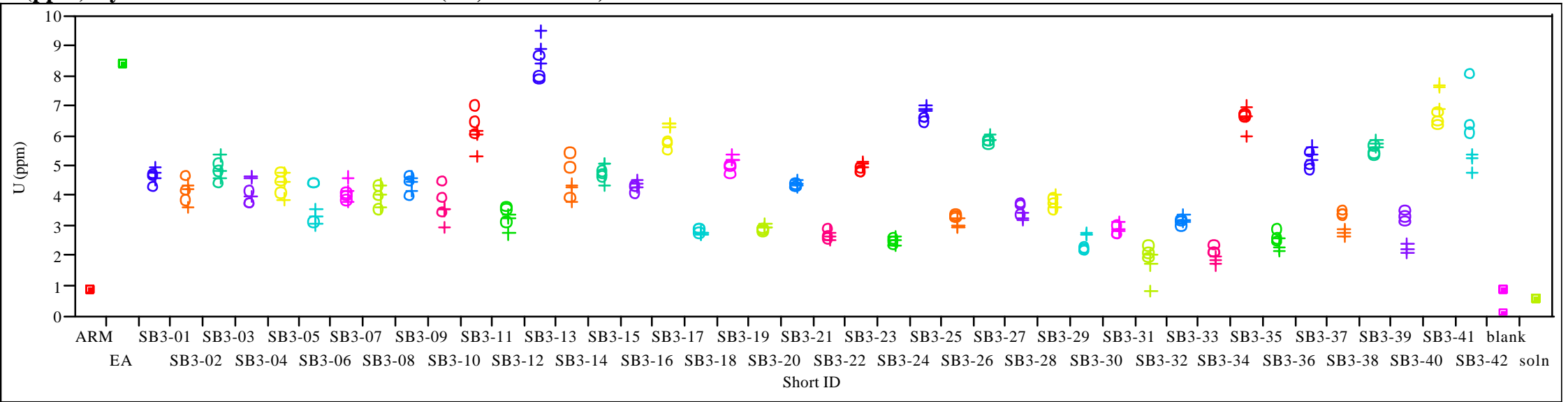


Westinghouse Savannah River Company

Exhibit I.5. SRTC-ML Mesaurements of PCT Leachate Solutions by SB3 Phase 1 Glass ID without EA (Plus - Quenched; Circle - Centerline Canister Cooled (ccc))

Al (ppm) By Glass ID w/o Heat Treatment (i.e., a Short ID)

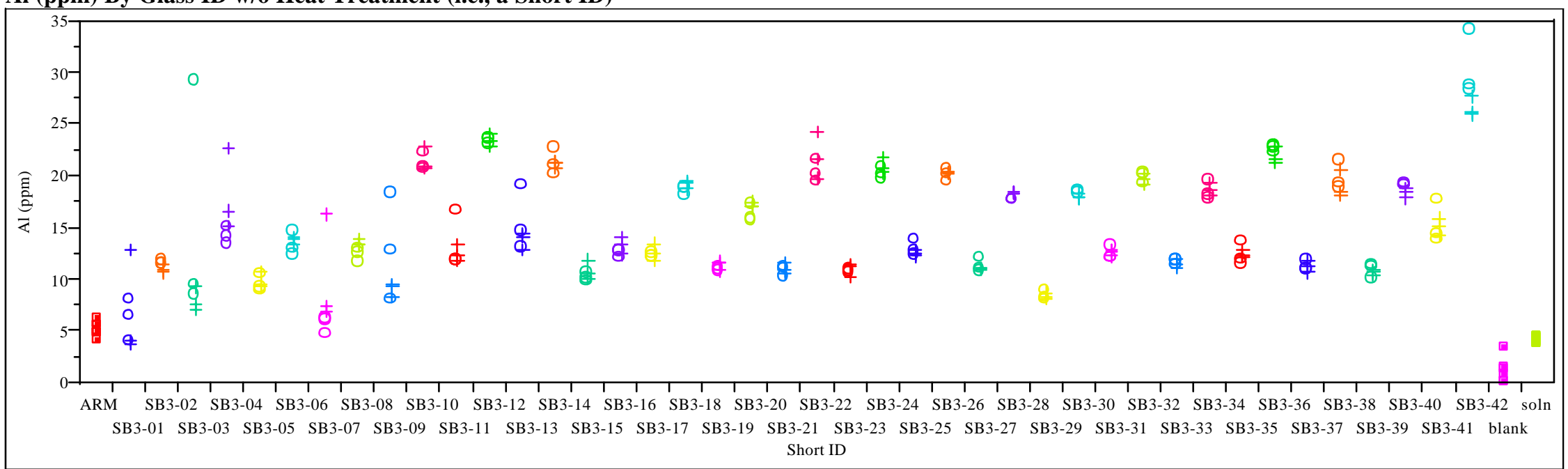

B (ppm) By Glass ID w/o Heat Treatment (i.e., a Short ID)

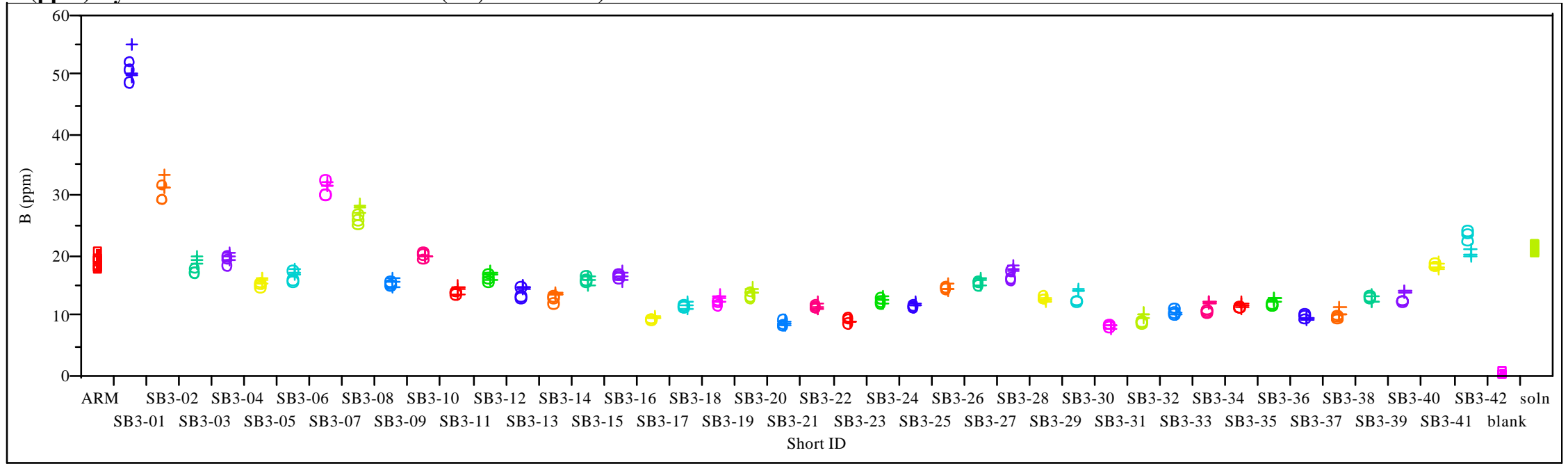


Westinghouse Savannah River Company

Exhibit I.5. SRTC-ML Mesaurements of PCT Leachate Solutions by SB3 Phase 1 Glass ID without EA (Plus - Quenched; Circle - Centerline Canister Cooled (ccc))

\section{Fe (ppm) By Glass ID w/o Heat Treatment (i.e., a Short ID)}

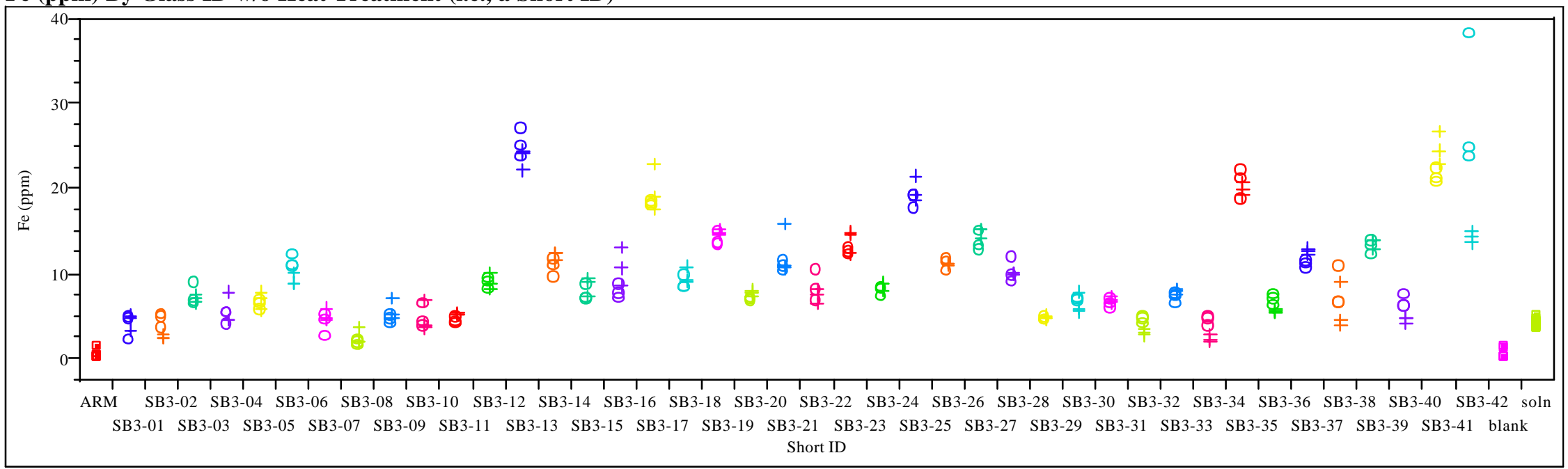

Li (ppm) By Glass ID w/o Heat Treatment (i.e., a Short ID)

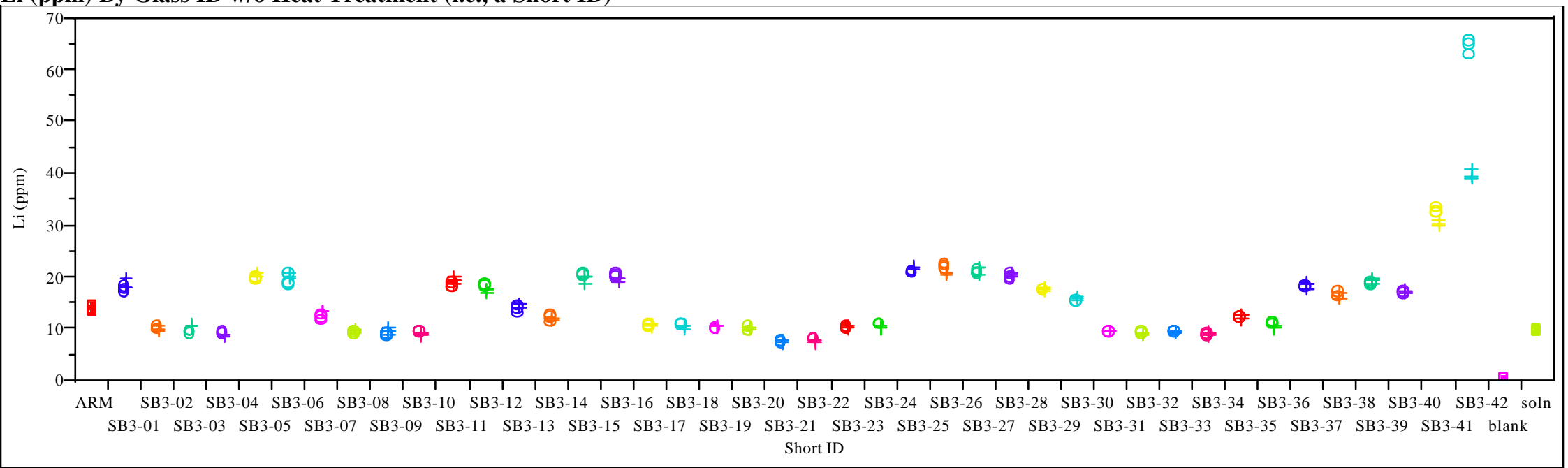


Westinghouse Savannah River Company

Exhibit I.5. SRTC-ML Mesaurements of PCT Leachate Solutions by SB3 Phase 1 Glass ID without EA (Plus - Quenched; Circle - Centerline Canister Cooled (ccc))

Na (ppm) By Glass ID w/o Heat Treatment (i.e., a Short ID)

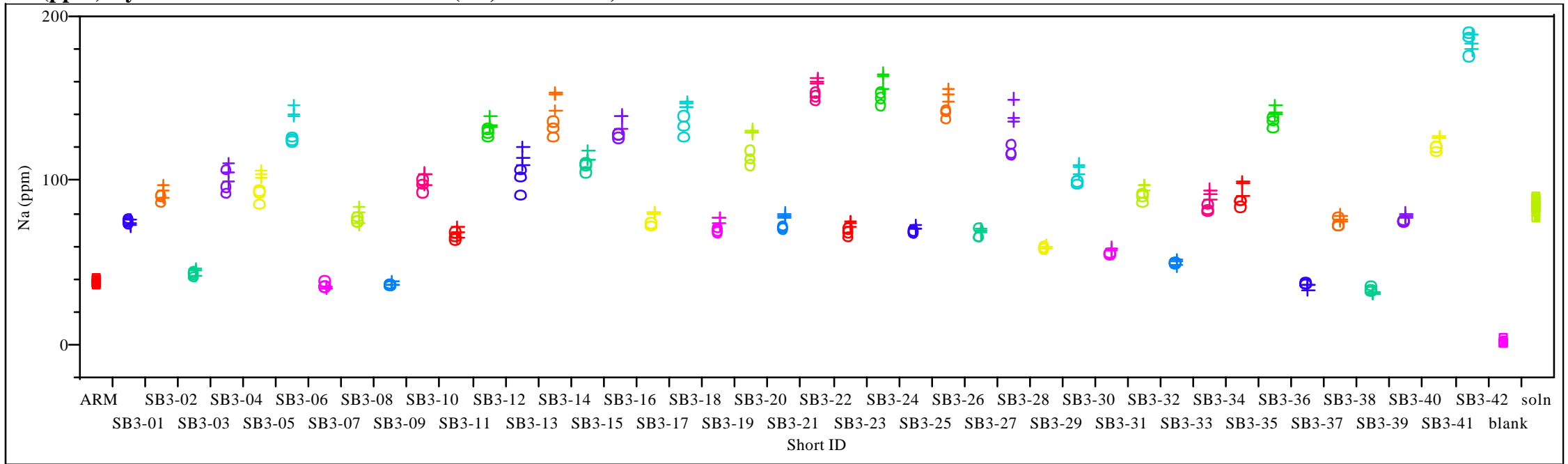

Si (ppm) By Glass ID w/o Heat Treatment (i.e., a Short ID)

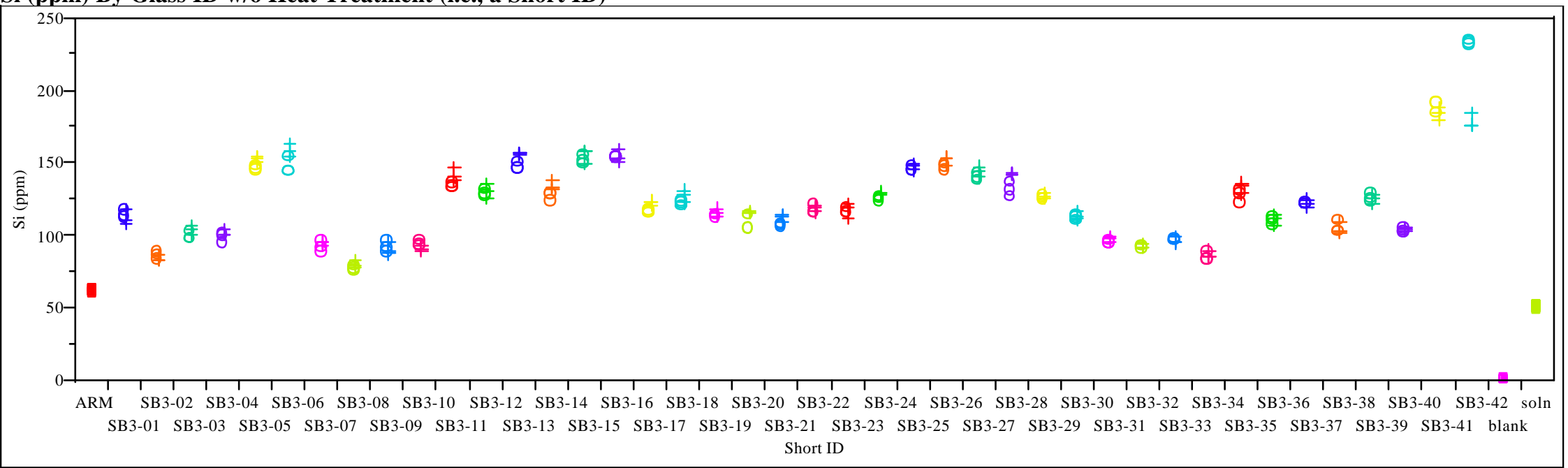


Westinghouse Savannah River Company

Exhibit I.5. SRTC-ML Mesaurements of PCT Leachate Solutions by SB3 Phase 1 Glass ID without EA (Plus - Quenched; Circle - Centerline Canister Cooled (ccc))

U (ppm) By Glass ID w/o Heat Treatment (i.e., a Short ID)

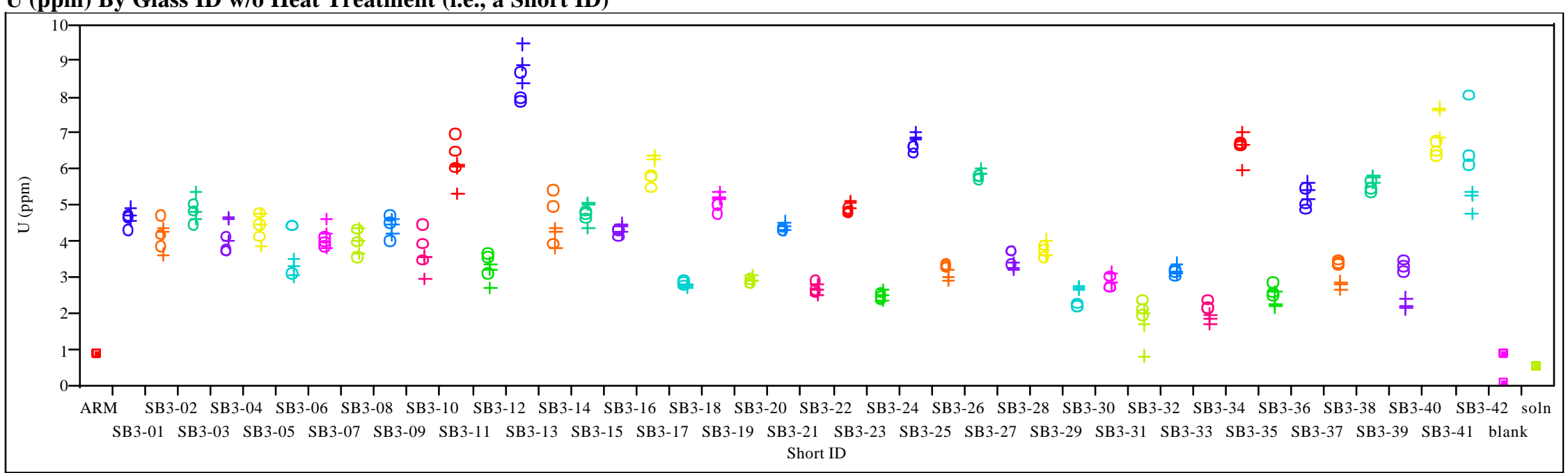




\section{Exhibit I.5. SRTC-ML Mesaurements of PCT Leachate Solutions by SB3 Phase 1 Glass ID without EA}

(Plus - Quenched; Circle - Centerline Canister Cooled (ccc))

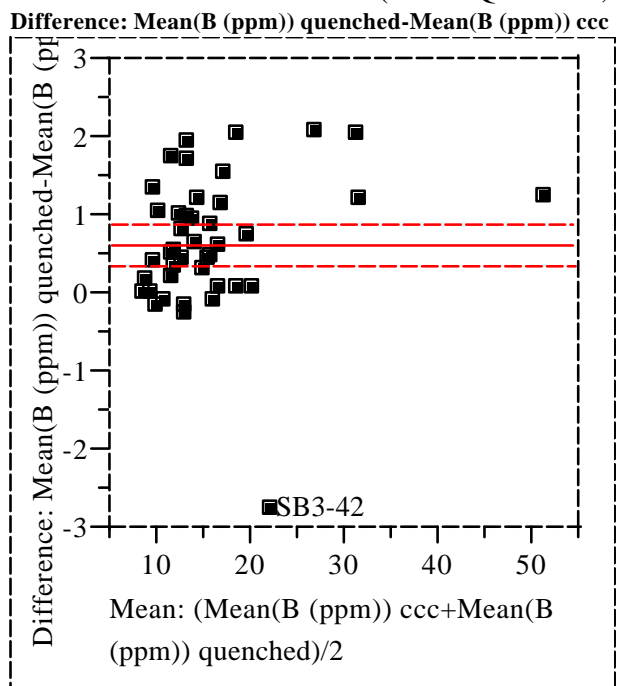

Mean $(\mathrm{B}(\mathrm{ppm}))$ quenched $\quad 16.1085$

Mean (B (ppm)) ccc $\quad 15.4787$

Mean Difference $\quad 0.62977$

Std Error

Upper95\%

Lower95\%

$\mathrm{N}$

Correlation

Difference: Mean $(\mathrm{Li}(\mathrm{ppm}))$ quenched-Mean $(\mathrm{Li}(\mathrm{ppm}))$ ccc

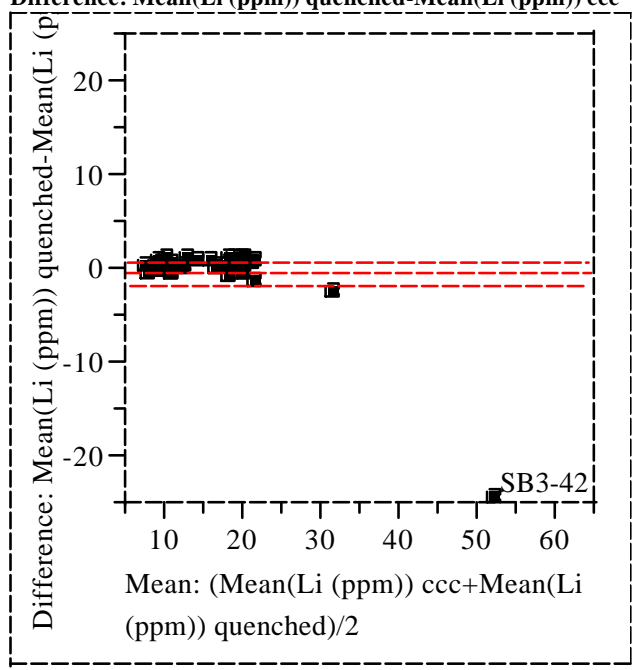

$\operatorname{Mean}(\mathrm{Li}(\mathrm{ppm}))$ quenched

Mean $(\operatorname{Li}(\mathrm{ppm})) \mathrm{ccc} \quad 15.5572$

Mean Difference $\quad-0.5173$

Std Error

Upper95\%

Lower95\%

$\mathrm{N}$

Correlation
t-Ratio $\quad-0.86492$

DF $\quad 41$

Prob $>|\mathrm{t}| \quad 0.3921$

Prob $>\mathrm{t} \quad 0.8039$

Prob $<\mathrm{t} \quad 0.1961$
Difference: Mean(Na (ppm)) quenched-Mean(Na (ppm)) ccc

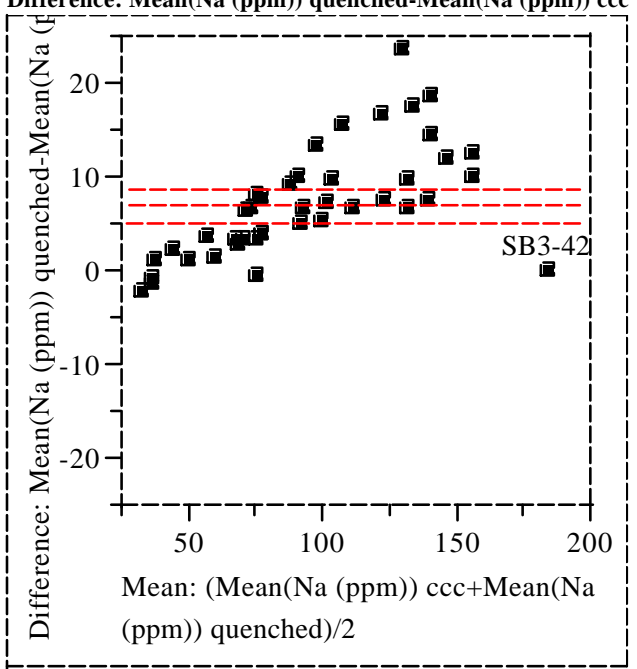

$\begin{array}{lrlr}\text { Mean }(\mathrm{Na}(\mathrm{ppm})) \text { quenched } & 97.9993 & \mathrm{t}-\text { Ratio } & 7.697725 \\ \text { Mean }(\mathrm{Na}(\mathrm{ppm})) \text { ccc } & 91.0137 & \mathrm{DF} & 41 \\ \text { Mean Difference } & 6.98559 & \text { Prob }>|\mathrm{t}| & <.0001 \\ \text { Std Error } & 0.90749 & \text { Prob }>\mathrm{t} & <.0001 \\ \text { Upper95\% } & 8.8183 & \text { Prob }<\mathrm{t} & 1.0000 \\ \text { Lower95\% } & 5.15288 & & \\ \mathrm{~N} & 42 & & \\ \text { Correlation } & 0.99283 & & \end{array}$

Difference: Mean $(\mathrm{Si}(\mathrm{ppm}))$ quenched-Mean(Si (ppm)) ccc

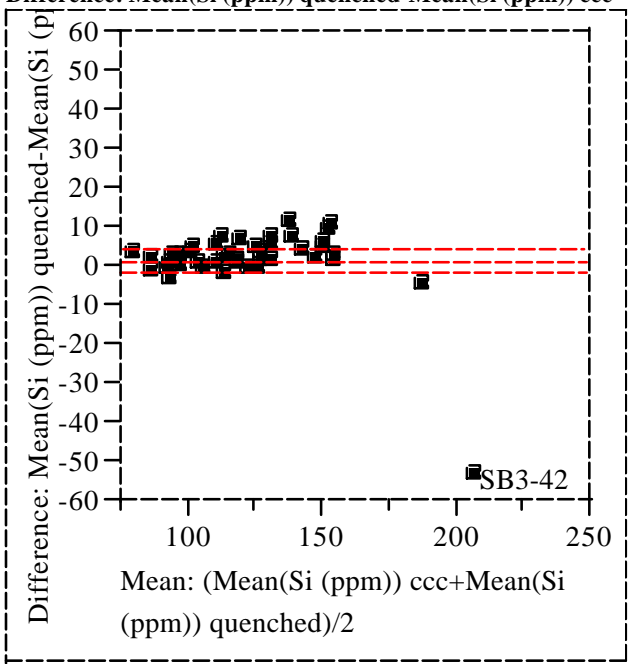

$\begin{array}{lrlr}\text { Mean }(\mathrm{Si}(\mathrm{ppm})) \text { quenched } & 123.251 & \mathrm{t}-\text { Ratio } & 0.799233 \\ \text { Mean }(\mathrm{Si}(\mathrm{ppm})) \mathrm{ccc} & 122.095 & \mathrm{DF} & 41 \\ \text { Mean Difference } & 1.15611 & \text { Prob }>|\mathrm{t}| & 0.4288 \\ \text { Std Error } & 1.44652 & \text { Prob }>\mathrm{t} & 0.2144 \\ \text { Upper95\% } & 4.07742 & \text { Prob }<\mathrm{t} & 0.7856 \\ \text { Lower95\% } & -1.7652 & & \\ \mathrm{~N} & 42 & & \\ \text { Correlation } & 0.94874 & & \end{array}$




\section{Exhibit I.5. SRTC-ML Mesaurements of PCT Leachate Solutions by SB3 Phase 1 Glass ID without EA}

(Plus - Quenched; Circle - Centerline Canister Cooled (ccc))

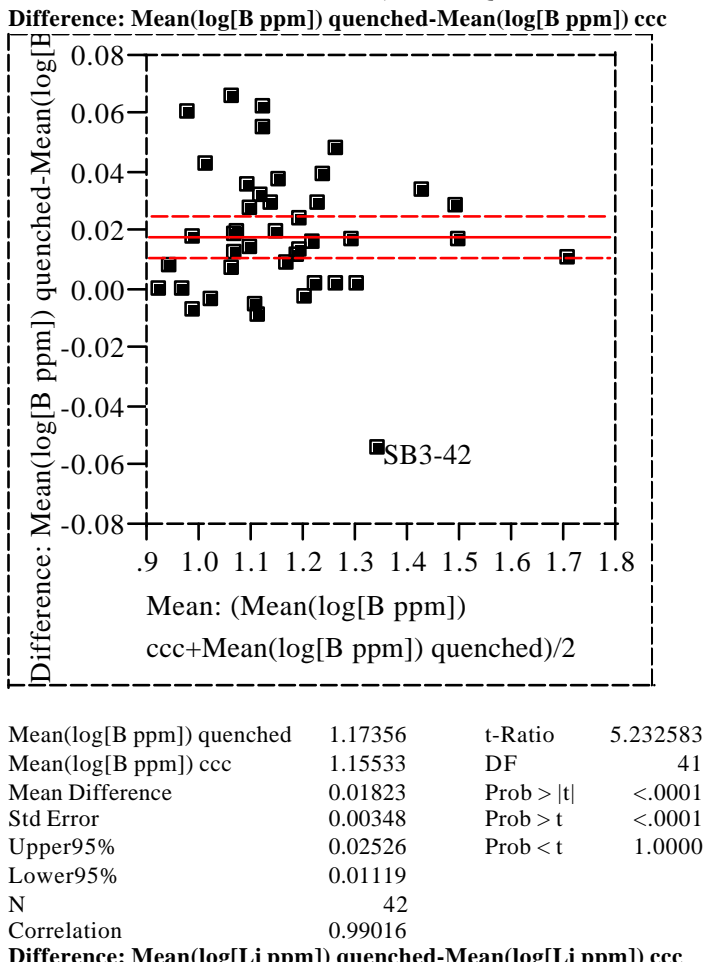

Difference: Mean $(\log [\mathrm{Li}$ ppm] $)$ quenched-Mean $(\log [\mathrm{Li} \mathrm{ppm}])$ cc

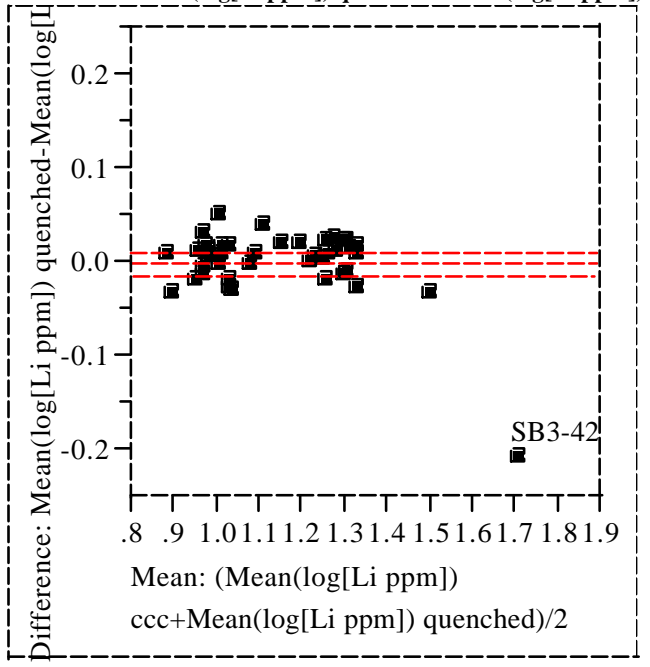

$\begin{array}{lrlr}\text { Mean }(\log [\mathrm{Li} \mathrm{ppm}]) \text { quenched } & 1.14254 & \mathrm{t}-\text { Ratio } & -0.39455 \\ \text { Mean }(\log [\mathrm{Li} \mathrm{ppm}]) \text { ccc } & 1.14486 & \text { DF } & 41 \\ \text { Mean Difference } & -0.0023 & \text { Prob }>|\mathrm{t}| & 0.6952 \\ \text { Std Error } & 0.00588 & \text { Prob }>\mathrm{t} & 0.6524 \\ \text { Upper95\% } & 0.00956 & \text { Prob }<\mathrm{t} & 0.3476 \\ \text { Lower95\% } & -0.0142 & & \\ \text { N } & 42 & & \\ \text { Correlation } & 0.98158 & & \end{array}$

Difference: $\operatorname{Mean}(\log [\underline{\mathrm{Na}} \underline{\mathrm{ppm}}])$ quenched-Mean $(\log [\underline{\mathrm{Na}} \underline{\mathrm{ppm}}])$ ccc

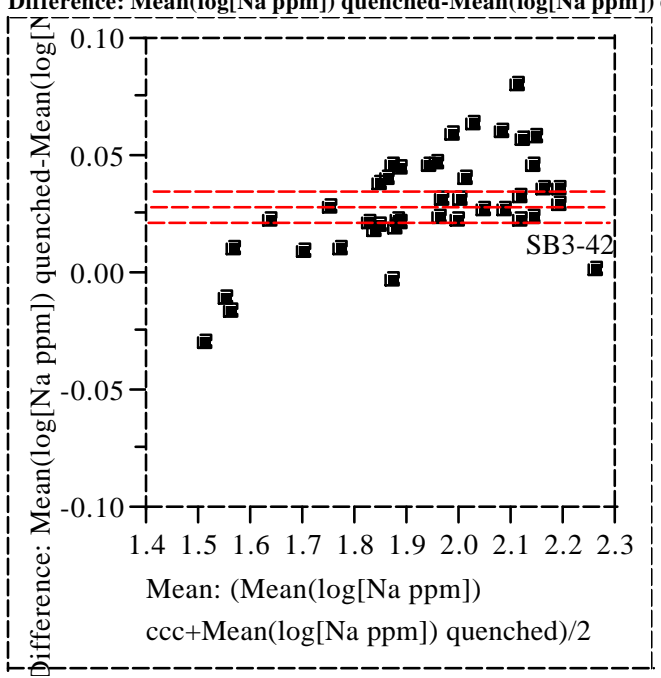

$\begin{array}{lrlr}\text { Mean }(\log [\mathrm{Na} \mathrm{ppm}]) \text { quenched } & 1.95091 & \mathrm{t}-\text { Ratio } & 8.264532 \\ \text { Mean }(\log [\mathrm{Na} \mathrm{ppm}]) \text { ccc } & 1.92286 & \mathrm{DF} & 41 \\ \text { Mean Difference } & 0.02805 & \text { Prob }>|\mathrm{t}| & <.0001 \\ \text { Std Error } & 0.00339 & \text { Prob }>\mathrm{t} & <.0001 \\ \text { Upper95\% } & 0.03491 & \text { Prob }<\mathrm{t} & 1.0000 \\ \text { Lower95\% } & 0.0212 & & \\ \text { N } & 42 & & \\ \text { Correlation } & 0.99572 & & \end{array}$

Difference: Mean $(\log [\mathrm{Si}$ ppm] $)$ quenched-Mean $(\log [\mathrm{Si}$ ppm] $)$ ccc

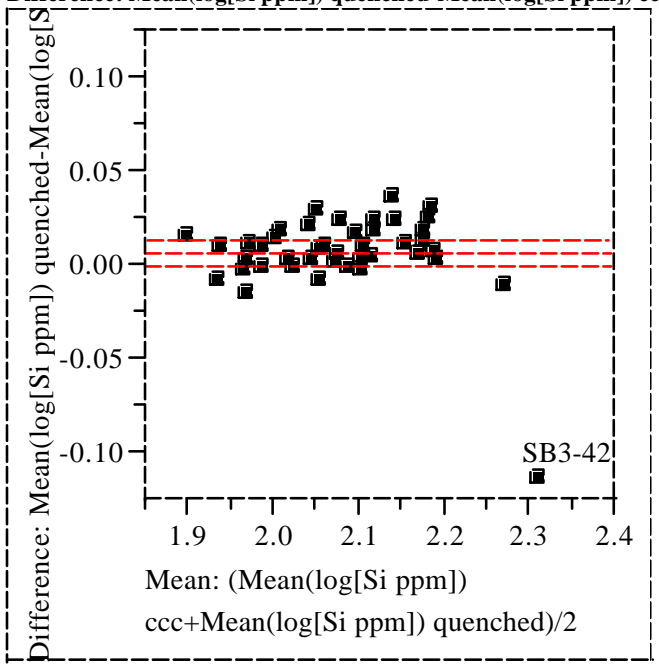

$\begin{array}{lrlr}\text { Mean }(\log [\mathrm{Si} \mathrm{ppm}]) \text { quenched } & 2.0816 & \mathrm{t}-\text { Ratio } & 1.635174 \\ \text { Mean }(\log [\mathrm{Si} \mathrm{ppm}]) \mathrm{ccc} & 2.07601 & \mathrm{DF} & 41 \\ \text { Mean Difference } & 0.0056 & \text { Prob }>|\mathrm{t}| & 0.1097 \\ \text { Std Error } & 0.00342 & \text { Prob }>\mathrm{t} & 0.0548 \\ \text { Upper95\% } & 0.01251 & \text { Prob }<\mathrm{t} & 0.9452 \\ \text { Lower95\% } & -0.0013 & & \\ \mathrm{~N} & 42 & & \\ \text { Correlation } & 0.97274 & & \end{array}$




\section{Exhibit I.5. SRTC-ML Mesaurements of PCT Leachate Solutions by SB3 Phase 1 Glass ID without EA}

(Plus - Quenched; Circle - Centerline Canister Cooled (ccc))

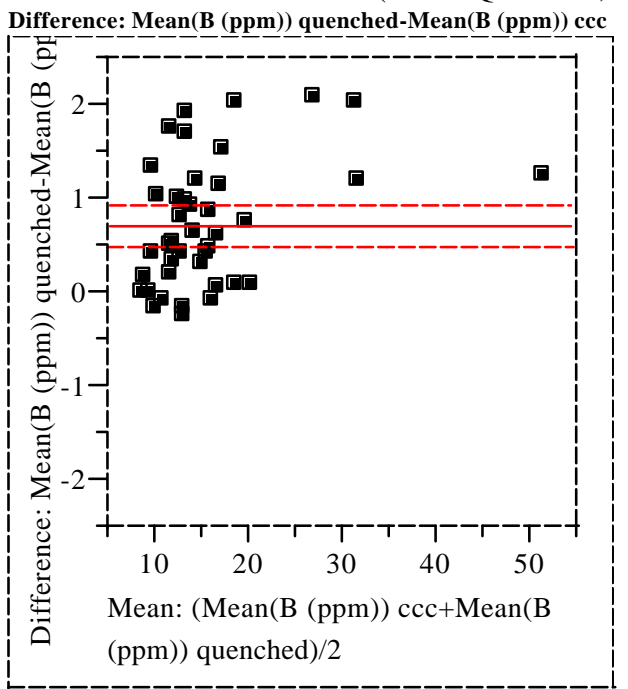

$\begin{array}{lrlr}\text { Mean }(\mathrm{B}(\mathrm{ppm})) \text { quenched } & 16 & \mathrm{t}-\text { Ratio } & 6.760471 \\ \text { Mean }(\mathrm{B}(\mathrm{ppm})) \text { ccc } & 15.2872 & \mathrm{DF} & 40 \\ \text { Mean Difference } & 0.71289 & \text { Prob }>|\mathrm{t}| & <.0001 \\ \text { Std Error } & 0.10545 & \text { Prob }>\mathrm{t} & <.0001 \\ \text { Upper95\% } & 0.92601 & \text { Prob }<\mathrm{t} & 1.0000 \\ \text { Lower95\% } & 0.49977 & & \\ \mathrm{~N} & 41 & & \\ \text { Correlation } & 0.99674 & & \\ \text { Difference: Mean }(\mathbf{L i}(\mathbf{p p m})) \text { quenched-Mean }(\mathbf{L i}(\mathbf{p p m})) \text { ccc }\end{array}$

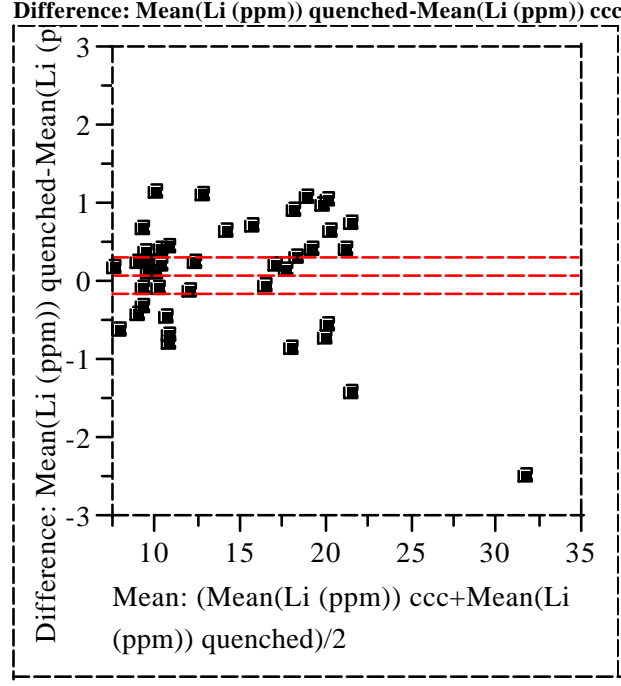

$\begin{array}{lrlr}\text { Mean }(\mathrm{Li}(\mathrm{ppm})) \text { quenched } & 14.4351 & \mathrm{t}-\mathrm{Ratio} & 0.616012 \\ \text { Mean }(\mathrm{Li}(\mathrm{ppm})) \mathrm{ccc} & 14.3648 & \mathrm{DF} & 40 \\ \text { Mean Difference } & 0.07033 & \text { Prob }>|\mathrm{t}| & 0.5414 \\ \text { Std Error } & 0.11416 & \text { Prob }>\mathrm{t} & 0.2707 \\ \text { Upper95\% } & 0.30106 & \text { Prob }<\mathrm{t} & 0.7293 \\ \text { Lower95\% } & -0.1604 & & \\ \text { N } & 41 & & \\ \text { Correlation } & 0.99124 & & \end{array}$

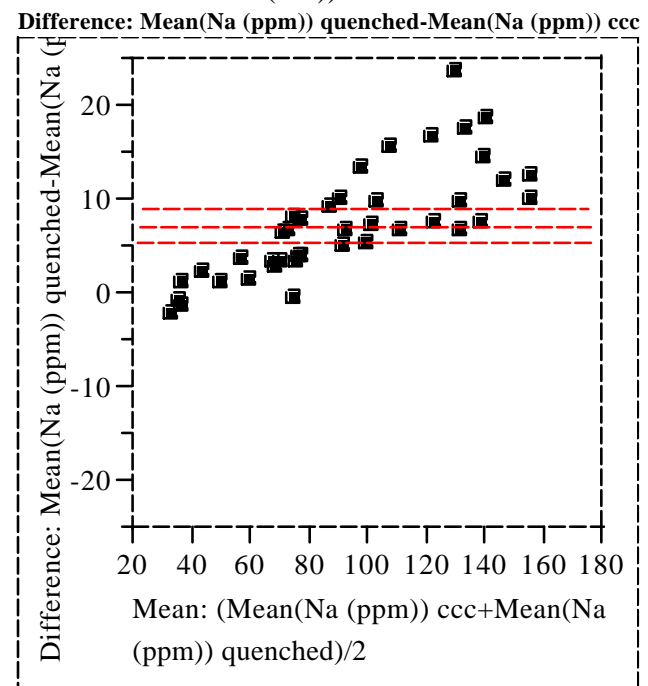

$\begin{array}{lrlr}\text { Mean }(\mathrm{Na}(\mathrm{ppm})) \text { quenched } & 95.9044 & \mathrm{t}-\text { Ratio } & 7.834761 \\ \text { Mean }(\mathrm{Na}(\mathrm{ppm})) \mathrm{ccc} & 88.7484 & \mathrm{DF} & 40 \\ \text { Mean Difference } & 7.15597 & \text { Prob }>|\mathrm{t}| & <.0001 \\ \text { Std Error } & 0.91336 & \text { Prob }>\mathrm{t} & <.0001 \\ \text { Upper95\% } & 9.00194 & \text { Prob }<\mathrm{t} & 1.0000 \\ \text { Lower95\% } & 5.31 & & \\ \mathrm{~N} & 41 & & \\ \text { Correlation } & 0.99465 & & \end{array}$

Difference: Mean(Si (ppm)) quenched-Mean $(\mathrm{Si}(\mathrm{ppm}))$ ccc

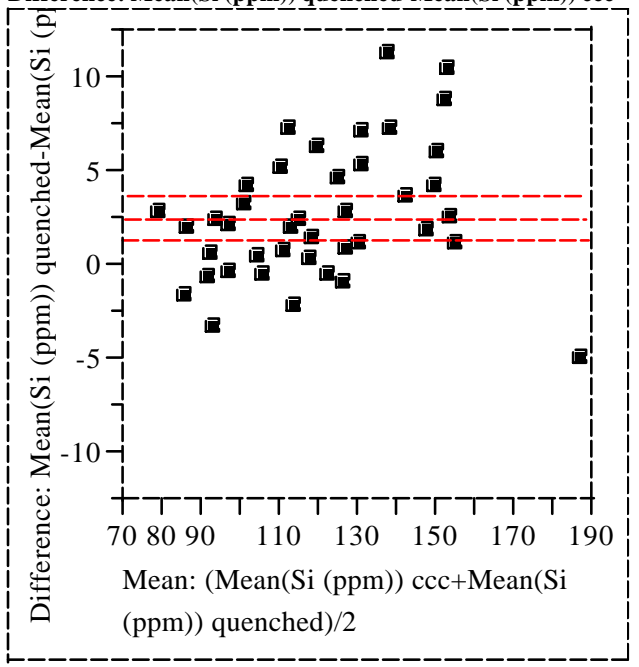

$\begin{array}{lrlr}\text { Mean }(\mathrm{Si}(\mathrm{ppm})) \text { quenched } & 121.88 & \mathrm{t}-\text { Ratio } & 4.529057 \\ \text { Mean }(\mathrm{Si}(\mathrm{ppm})) \mathrm{ccc} & 119.382 & \mathrm{DF} & 40 \\ \text { Mean Difference } & 2.49869 & \text { Prob }>|\mathrm{t}| & <.0001 \\ \text { Std Error } & 0.5517 & \text { Prob }>\mathrm{t} & <.0001 \\ \text { Upper95\% } & 3.61373 & \text { Prob }<\mathrm{t} & 1.0000 \\ \text { Lower95\% } & 1.38366 & & \\ \mathrm{~N} & 41 & & \\ \text { Correlation } & 0.98985 & & \end{array}$




\section{Exhibit I.5. SRTC-ML Mesaurements of PCT Leachate Solutions by SB3 Phase 1 Glass ID without EA}

(Plus - Quenched; Circle - Centerline Canister Cooled (ccc))

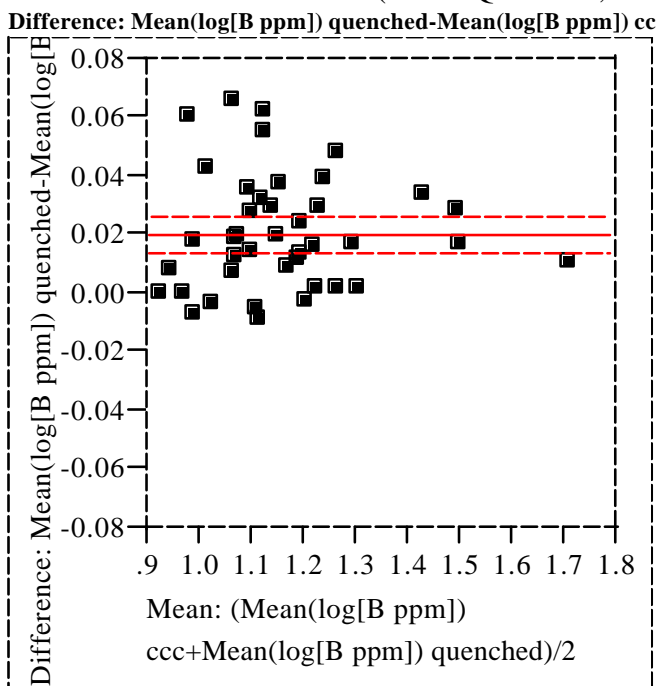

\begin{tabular}{|c|c|c|c|}
\hline Mean $(\log [\mathrm{B}$ ppm $])$ quenched & 1.17016 & t-Ratio & 6.527129 \\
\hline Mean $(\log [\mathrm{B}$ ppm $]) \mathrm{ccc}$ & 1.15015 & DF & 40 \\
\hline Mean Difference & 0.02001 & Prob $>|t|$ & $<.0001$ \\
\hline Std Error & 0.00307 & Prob $>t$ & $<.0001$ \\
\hline Upper95\% & 0.02621 & Prob $<\mathrm{t}$ & 1.0000 \\
\hline Lower95\% & 0.01382 & & \\
\hline $\mathrm{N}$ & 41 & & \\
\hline Correlation & 0.99246 & & \\
\hline
\end{tabular}

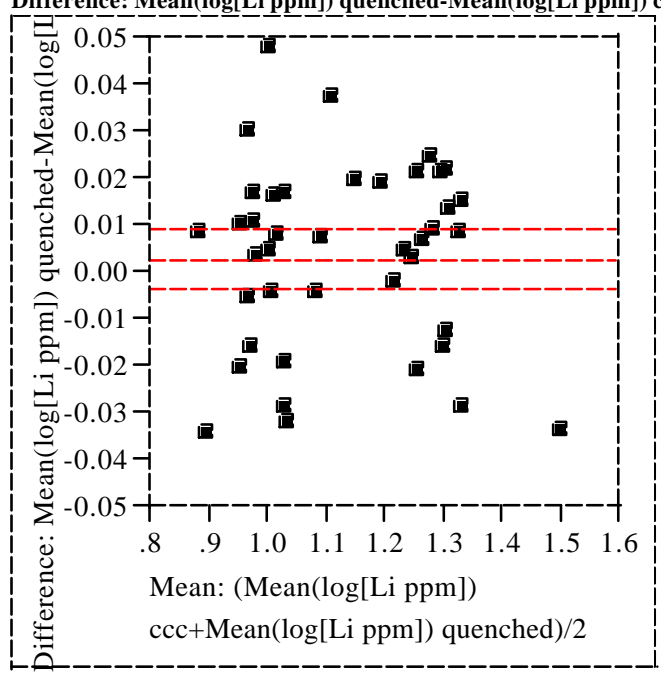

$\begin{array}{lrlr}\text { Mean }(\log [\mathrm{Li} \mathrm{ppm}]) \text { quenched } & 1.13138 & \mathrm{t}-\text { Ratio } & 0.87388 \\ \text { Mean(log[Li ppm] ccc } & 1.12866 & \text { DF } & 40 \\ \text { Mean Difference } & 0.00272 & \text { Prob }>|\mathrm{t}| & 0.3874 \\ \text { Std Error } & 0.00311 & \text { Prob }>\mathrm{t} & 0.1937 \\ \text { Upper95\% } & 0.009 & \text { Prob }<\mathrm{t} & 0.8063 \\ \text { Lower95\% } & -0.0036 & & \\ \text { N } & 41 & & \\ \text { Correlation } & 0.99196 & & \end{array}$

Difference: Mean $(\log [$ Na ppm] $)$ quenched-Mean $(\log [\underline{N a}$ ppm $])$ ccc

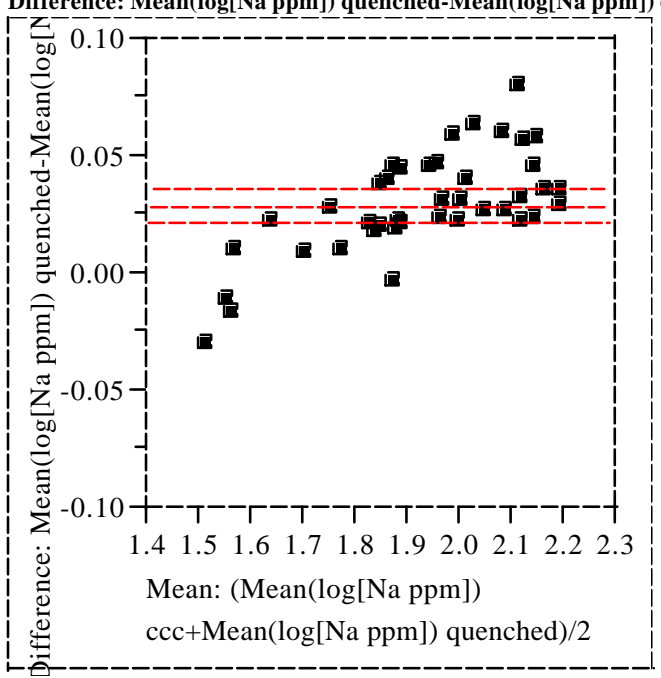

$\begin{array}{lrlr}\text { Mean }(\log [\mathrm{Na} \mathrm{ppm}]) \text { quenched } & 1.94326 & \mathrm{t}-\text { Ratio } & 8.431366 \\ \text { Mean }(\log [\mathrm{Na} \mathrm{ppm}]) \mathrm{ccc} & 1.91453 & \mathrm{DF} & 40 \\ \text { Mean Difference } & 0.02873 & \text { Prob }>|\mathrm{t}| & <.0001 \\ \text { Std Error } & 0.00341 & \text { Prob }>\mathrm{t} & <.0001 \\ \text { Upper95\% } & 0.03562 & \text { Prob }<\mathrm{t} & 1.0000 \\ \text { Lower95\% } & 0.02184 & & \\ \mathrm{~N} & 41 & & \\ \text { Correlation } & 0.99636 & & \end{array}$

Difference: $\mathrm{Mean}(\log [\mathrm{Si}$ ppm] $)$ quenched-Mean $(\log [\mathrm{Si} \mathrm{ppm}])$ ccc

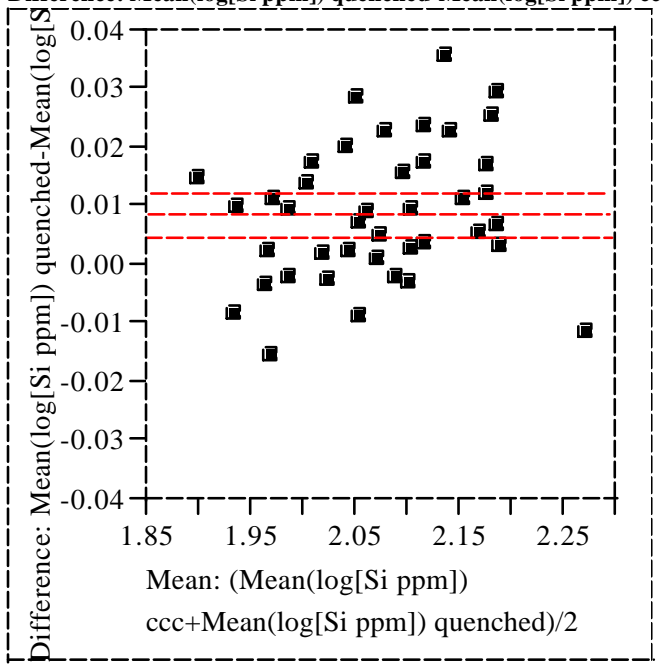

$\begin{array}{lrlr}\text { Mean }(\log [\mathrm{Si} \mathrm{ppm}]) \text { quenched } & 2.0774 & \mathrm{t}-\text { Ratio } & 4.660801 \\ \text { Mean }(\log [\mathrm{Si} p \mathrm{ppm}]) \mathrm{ccc} & 2.06888 & \mathrm{DF} & 40 \\ \text { Mean Difference } & 0.00852 & \text { Prob }>|\mathrm{t}| & <.0001 \\ \text { Std Error } & 0.00183 & \text { Prob }>\mathrm{t} & <.0001 \\ \text { Upper95\% } & 0.01221 & \text { Prob }<\mathrm{t} & 1.0000 \\ \text { Lower95\% } & 0.00482 & & \\ \mathrm{~N} & 41 & & \\ \text { Correlation } & 0.99118 & & \end{array}$


Immobilization Technology Section

WSRC-TR-2002-00549

Savannah River Technology Center

Rev. 0

Westinghouse Savannah River Company

\section{Exhibit I.8 Correlations and Scatterplots of Normalized PCTs Over All Compositional Views and Heat Treatments}

\section{Correlations}

$\begin{array}{ccccc} & \log N L(B)(g / L) & \log N L(L i)(g / L) & \log N L(N a)(g / L) & \log N L(S i)(g / L) \\ \log N L(B)(g / L) & 1.0000 & 0.9653 & 0.9281 & 0.8894 \\ \log N L(L i)(g / L) & 0.9653 & 1.0000 & 0.8880 & 0.9182 \\ \log N L(N a)(g / L) & 0.9281 & 0.8880 & 1.0000 & 0.9315 \\ \log N L(S i)(g / L) & 0.8894 & 0.9182 & 0.9315 & 1.0000\end{array}$

5 rows not used due to missing values.

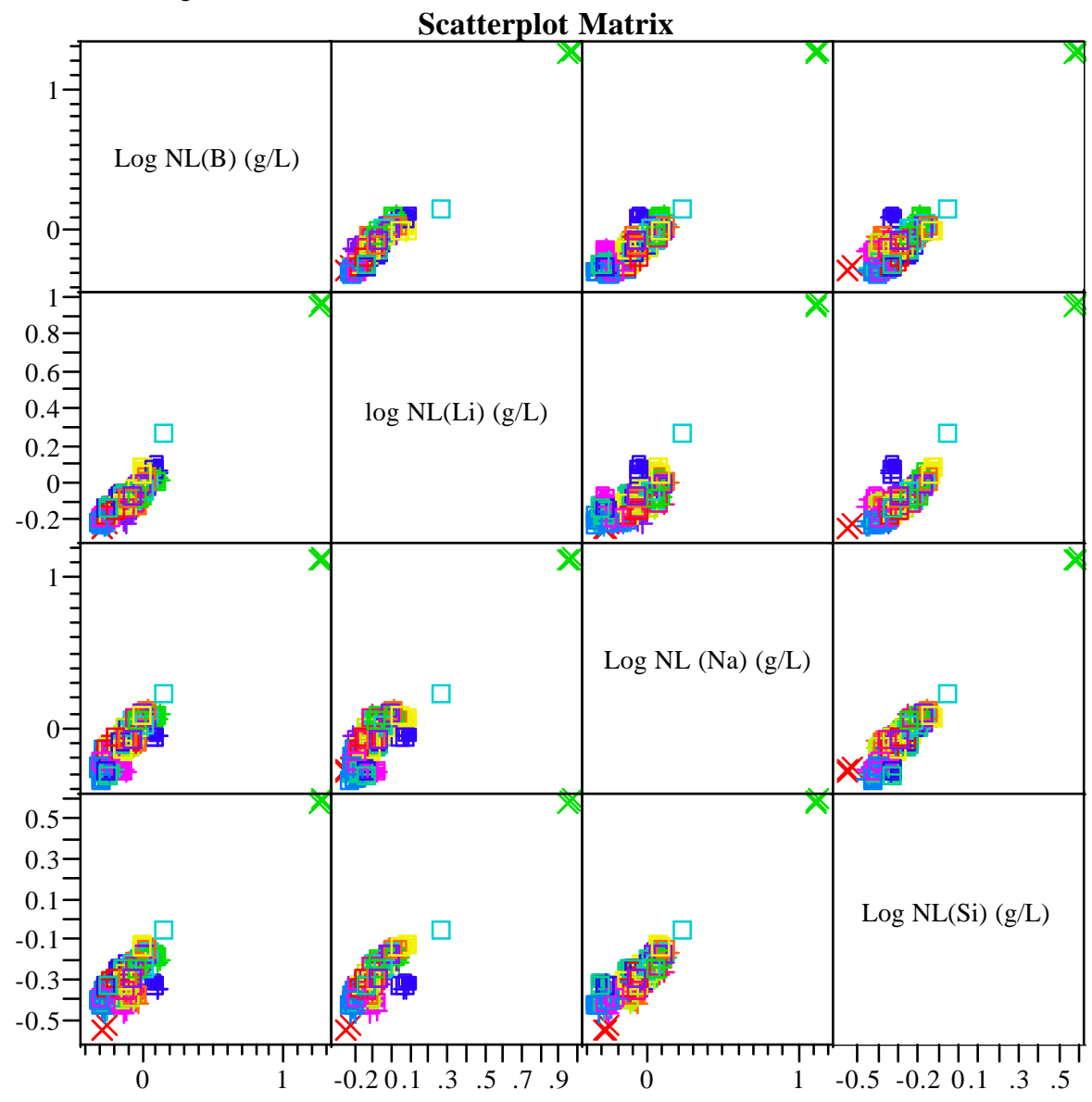


Immobilization Technology Section

WSRC-TR-2002-00549

Savannah River Technology Center

Rev. 0

Westinghouse Savannah River Company

\section{Appendix J}

$\Delta \mathrm{G}_{\mathrm{P}}$ Delta as a Function Fe Concentration and Redox 
Table J.1. Predicted $\Delta \mathrm{G}_{\mathrm{P}}$ Delta as a Function of Fe Concentration in Glass and Redox.

\begin{tabular}{|c|c|c|c|c|c|c|c|c|c|c|c|c|c|c|c|}
\hline & \multicolumn{15}{|c|}{ Fe Concentration (wt\%) in Glass } \\
\hline $\operatorname{Redox}(\mathrm{f})$ & 1 & 2 & 3 & 4 & 5 & 6 & 1 & 8 & 9 & 10 & 11 & 12 & 13 & 14 & 15 \\
\hline 0.00 & 0000 & .0000 & \begin{tabular}{|l|l}
0.0000 \\
\end{tabular} & 0.0000 & 0.0000 & \begin{tabular}{|l|l}
0.0000 \\
\end{tabular} & \begin{tabular}{|l|l}
0.0000 \\
\end{tabular} & 0.0000 & \begin{tabular}{|l|}
0.0000 \\
\end{tabular} & 000 & \begin{tabular}{|l|l}
0.0000 \\
\end{tabular} & 0000 & \begin{tabular}{|l|l}
0.00000 \\
\end{tabular} & \begin{tabular}{|l|l}
0.0000 \\
\end{tabular} & 0000 \\
\hline 0.01 & 051 & 0102 & -0.0154 & -0.0205 & 0256 & 307 & 59 & -0.0410 & 6 & 0512 & -0.0564 & .0615 & .0666 & .0717 & 0768 \\
\hline .02 & 102 & $\overline{05}$ & 7 & 0 & 2 & 0615 & 7 & 20 & 22 & 25 & 1127 & 30 & 32 & 1434 & -0.1537 \\
\hline 0.03 & & 307 & \begin{tabular}{|l|}
-0.0461 \\
\end{tabular} & 0615 & 0768 & 9922 & \begin{tabular}{|l|}
-0.1076 \\
\end{tabular} & & & & -0.169 & & 1998 & 0.2152 & \\
\hline 0.04 & 0205 & -0.0410 & \begin{tabular}{|l}
-0.0615 \\
\end{tabular} & -0.0820 & -0.1025 & \begin{tabular}{|l}
-0.1230 \\
\end{tabular} & \begin{tabular}{|l}
-0.1434 \\
\end{tabular} & -0.1639 & -0.1844 & \begin{tabular}{|l}
-0.2049 \\
\end{tabular} & \begin{tabular}{|l}
-0.2254 \\
\end{tabular} & \begin{tabular}{|l}
-0.2459 \\
\end{tabular} & \begin{tabular}{|l}
-0.2664 \\
\end{tabular} & -0.2869 & -0.3074 \\
\hline 0.05 & 0.0256 & -0.0512 & -0.0768 & -0.1025 & -0.1281 & -0.1537 & -0.1793 & \begin{tabular}{|l|}
-0.2049 \\
\end{tabular} & -0.2305 & \begin{tabular}{|l|}
-0.2561 \\
\end{tabular} & -0.2818 & -0.3074 & -0.3330 & -0.3586 & -0.3842 \\
\hline 0.06 & 307 & 0615 & .0922 & .1230 & .1537 & -0.1844 & -0.2152 & 2459 & .2766 & -0.3074 & -0.3381 & -0.3689 & -0.3996 & 0.4303 & -0.4611 \\
\hline 0.07 & \begin{tabular}{|l|}
-0.0359 \\
\end{tabular} & \begin{tabular}{|l|}
-0.0717 \\
\end{tabular} & \begin{tabular}{|l|}
-0.1076 \\
\end{tabular} & -0.1434 & -0.1793 & \begin{tabular}{|l|}
-0.2152 \\
\end{tabular} & \begin{tabular}{|l|}
-0.2510 \\
\end{tabular} & \begin{tabular}{|l|}
-0.2869 \\
\end{tabular} & -0.3227 & \begin{tabular}{|l|}
-0.3586 \\
\end{tabular} & \begin{tabular}{|l|}
-0.3945 \\
\end{tabular} & \begin{tabular}{|l|}
-0.4303 \\
\end{tabular} & \begin{tabular}{|l|}
-0.4662 \\
\end{tabular} & \begin{tabular}{|l|}
-0.5020 \\
\end{tabular} & -0.5379 \\
\hline 0.08 & 0410 & .0820 & .1230 & 1639 & 2049 & 0.2459 & 69 & 79 & 0.3689 & \begin{tabular}{|l|l}
-0.4098 \\
\end{tabular} & 4508 & \begin{tabular}{|l|}
-0.4918 \\
\end{tabular} & \begin{tabular}{|l|}
-0.5328 \\
\end{tabular} & 0.5738 & -0.6148 \\
\hline 0.09 & -0.0461 & -0.0922 & -0.1383 & -0.1844 & -0.2305 & -0.2766 & -0.3227 & \begin{tabular}{|l|}
-0.3689 \\
\end{tabular} & -0.4150 & -0.4611 & -0.5072 & -0.5533 & -0.5994 & -0.6455 & -0.6916 \\
\hline 10 & & 1025 & & 49 & 2561 & -0 & & & -0.4 & & .5635 & & -0. & .7172 & \\
\hline 0.11 & \begin{tabular}{|l|}
-0.0564 \\
\end{tabular} & \begin{tabular}{|l|}
-0.1127 \\
\end{tabular} & \begin{tabular}{|l|}
-0.1691 \\
\end{tabular} & -0.2254 & -0.2818 & \begin{tabular}{|l|}
-0.3381 \\
\end{tabular} & \begin{tabular}{|l}
-0.3945 \\
\end{tabular} & -0.4508 & -0.5072 & \begin{tabular}{|l}
-0.5635 \\
\end{tabular} & \begin{tabular}{|c|}
-0.6199 \\
\end{tabular} & \begin{tabular}{|l}
-0.6762 \\
\end{tabular} & \begin{tabular}{|l}
-0.7326 \\
\end{tabular} & \begin{tabular}{|l}
-0.7889 \\
\end{tabular} & -0.8453 \\
\hline 0.12 & -0.0615 & -0.1230 & -0.1844 & -0.2459 & -0.3074 & \begin{tabular}{|l|}
-0.3689 \\
\end{tabular} & -0.4303 & \begin{tabular}{|l|}
-0.4918 \\
\end{tabular} & \begin{tabular}{|l}
-0.5533 \\
\end{tabular} & 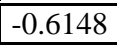 & -0.6762 & -0.7377 & -0.7992 & -0.8607 & -0.9221 \\
\hline-0 & 666 & -0.1332 & -0.19 & -0.2664 & \begin{tabular}{|l}
-0.3330 \\
\end{tabular} & -0.3 & -0.4 & 8 & -0.5 & \begin{tabular}{|l|}
-0.6660 \\
\end{tabular} & \begin{tabular}{|l|}
-0.7326 \\
\end{tabular} & \begin{tabular}{|l|}
-0.7992 \\
\end{tabular} & \begin{tabular}{|l|}
-0.8658 \\
\end{tabular} & -0.9324 & 0.9990 \\
\hline 0.14 & \begin{tabular}{|l|l}
-0.0717 \\
\end{tabular} & $\begin{array}{l}-0.1434 \\
\end{array}$ & \begin{tabular}{|l|l|}
-0.2152 \\
\end{tabular} & -0.2869 & -0.3586 & $\begin{array}{l}-0.4303 \\
\end{array}$ & $\begin{array}{l}-0.5020 \\
\end{array}$ & .5738 & \begin{tabular}{|l|}
-0.6455 \\
\end{tabular} & $\begin{array}{l}-0.7172 \\
\end{array}$ & \begin{tabular}{|l}
-0.7889 \\
\end{tabular} & \begin{tabular}{|l|}
-0.8607 \\
\end{tabular} & \begin{tabular}{|l|}
-0.9324 \\
\end{tabular} & -1.0041 & -1.0758 \\
\hline 0.15 & -0.0768 & -0.1537 & -0.2305 & -0.3074 & -0.3842 & -0.4611 & \begin{tabular}{|l}
-0.5379 \\
\end{tabular} & -0.6148 & -0.6916 & -0.7684 & -0.8453 & -0.9221 & -0.9990 & -1.0758 & -1.1527 \\
\hline 0.16 & \begin{tabular}{|l}
-0.0820 \\
\end{tabular} & 1639 & \begin{tabular}{|l|}
-0.2459 \\
\end{tabular} & -0.3279 & \begin{tabular}{|l|}
-0.4098 \\
\end{tabular} & -0.4 & & & \begin{tabular}{|l|}
-0.7377 \\
\end{tabular} & \begin{tabular}{|l}
-0.8197 \\
\end{tabular} & \begin{tabular}{|l}
-0.9016 \\
\end{tabular} & \begin{tabular}{|l|}
-0.9836 \\
\end{tabular} & -1.0656 & -1.1475 & -1.2295 \\
\hline 17 & 58 & -0.1742 & \begin{tabular}{|l}
-0.2613 \\
\end{tabular} & -0.3484 & \begin{tabular}{|l}
-0.4354 \\
\end{tabular} & \begin{tabular}{|l}
-0.5225 \\
\end{tabular} & -0.6 & -0.6967 & $\mid-0.7$ & \begin{tabular}{|l}
-0.8709 \\
\end{tabular} & -0.9580 & -1.0451 & -1.1322 & -1.2193 & -1.3063 \\
\hline .18 & \begin{tabular}{|l|}
-0.0922 \\
\end{tabular} & -0.1844 & \begin{tabular}{|l|l}
-0.2766 \\
\end{tabular} & -0.3689 & -0.4611 & \begin{tabular}{|l}
-0.5533 \\
\end{tabular} & -0.6 & \begin{tabular}{|l|l}
-0.7377 \\
\end{tabular} & \begin{tabular}{|l|}
-0.8299 \\
\end{tabular} & \begin{tabular}{|l}
-0.9221 \\
\end{tabular} & \begin{tabular}{|l}
-1.0143 \\
\end{tabular} & \begin{tabular}{|l}
-1.1066 \\
\end{tabular} & \begin{tabular}{|l}
-1.1988 \\
\end{tabular} & \begin{tabular}{|l|}
-1.2910 \\
\end{tabular} & -1.3832 \\
\hline 0.19 & -0.0973 & -0.1947 & -0.2920 & -0.3893 & -0.4867 & -0.5840 & -0.6814 & \begin{tabular}{|l|l|} 
& -0.7787 \\
\end{tabular} & -0.8760 & -0.9734 & -1.0707 & -1.1680 & -1.2654 & -1.3627 & -1.4600 \\
\hline & & & \begin{tabular}{|l|}
-0.3074 \\
\end{tabular} & -0.4098 & \begin{tabular}{|l|}
-0.5123 \\
\end{tabular} & & & & & & \begin{tabular}{|l}
-1.1270 \\
\end{tabular} & \begin{tabular}{|l}
-1.2295 \\
\end{tabular} & \begin{tabular}{|l}
-1.3320 \\
\end{tabular} & \begin{tabular}{|l}
-1.4344 \\
\end{tabular} & \begin{tabular}{|l|l|}
-1.5369 \\
\end{tabular} \\
\hline-2 & J & 2 & \begin{tabular}{|l|}
-0.3227 \\
\end{tabular} & -0.4303 & -0.5379 & -0.6 & \begin{tabular}{|l|}
-0.7 \\
\end{tabular} & 07 & \begin{tabular}{|l|}
-0.9682 \\
\end{tabular} & -1.0758 & \begin{tabular}{|l}
-1.1834 \\
\end{tabular} & \begin{tabular}{|l}
-1.2910 \\
\end{tabular} & \begin{tabular}{|l}
-1.3986 \\
\end{tabular} & \begin{tabular}{|l|}
-1.5061 \\
\end{tabular} & -1.6137 \\
\hline 0.22 & \begin{tabular}{|l|l|l|l|}
-0.127 \\
\end{tabular} & \begin{tabular}{|l|l}
-0.2254 \\
\end{tabular} & \begin{tabular}{|l|}
-0.3381 \\
\end{tabular} & -0.4508 & \begin{tabular}{|l|}
0.5635 \\
\end{tabular} & \begin{tabular}{|l|l|}
-0.6762 \\
\end{tabular} & \begin{tabular}{|l}
-0.7889 \\
\end{tabular} & \begin{tabular}{|l}
-0.9016 \\
\end{tabular} & \begin{tabular}{|l}
-1.0143 \\
\end{tabular} & \begin{tabular}{|l}
-1.1270 \\
\end{tabular} & \begin{tabular}{|l}
-1.2398 \\
\end{tabular} & \begin{tabular}{|l}
-1.3525 \\
\end{tabular} & $\begin{array}{l}-1.4652 \\
\end{array}$ & \begin{tabular}{|l}
-1.5779 \\
\end{tabular} & -1.6906 \\
\hline 0.23 & \begin{tabular}{|l|}
-0.1178 \\
\end{tabular} & -0.2357 & -0.3535 & -0.4713 & \begin{tabular}{|l|}
-0.5891 \\
\end{tabular} & \begin{tabular}{|l|}
-0.7070 \\
\end{tabular} & \begin{tabular}{|l|}
-0.8248 \\
\end{tabular} & \begin{tabular}{|l|}
-0.9426 \\
\end{tabular} & \begin{tabular}{|l|}
-1.0604 \\
\end{tabular} & \begin{tabular}{|l|}
-1.1783 \\
\end{tabular} & \begin{tabular}{|l|}
-1.2961 \\
\end{tabular} & \begin{tabular}{|l|}
-1.4139 \\
\end{tabular} & -1.5318 & -1.6496 & \begin{tabular}{|l|}
-1.7674 \\
\end{tabular} \\
\hline 0.24 & & & \begin{tabular}{|l|}
-0.3689 \\
\end{tabular} & -0.4918 & \begin{tabular}{|l|l|}
-0.6148 \\
\end{tabular} & & & & \begin{tabular}{|l|}
-1.1066 \\
\end{tabular} & & -1.3525 & \begin{tabular}{|l}
-1.4754 \\
\end{tabular} & \begin{tabular}{|l}
-1.5984 \\
\end{tabular} & \begin{tabular}{|l}
-1.7213 \\
\end{tabular} & -1.8443 \\
\hline 025 & 170 & \begin{tabular}{|l}
-0.2561 \\
\end{tabular} & \begin{tabular}{|l}
-0.3842 \\
\end{tabular} & -0.5123 & -0.6404 & \begin{tabular}{|l|}
-0.7684 \\
\end{tabular} & -0.8 & 46 & \begin{tabular}{|l}
-1.1527 \\
\end{tabular} & \begin{tabular}{|l|}
-1.2807 \\
\end{tabular} & -1.4088 & \begin{tabular}{|l}
-1.5369 \\
\end{tabular} & -1.6650 & \begin{tabular}{|l}
-1.7930 \\
\end{tabular} & -19211 \\
\hline 0.26 & \begin{tabular}{|l|l|}
-0.1332 \\
\end{tabular} & \begin{tabular}{|l|}
-0.2664 \\
\end{tabular} & \begin{tabular}{|l|}
-0.3996 \\
\end{tabular} & -0.5328 & \begin{tabular}{|l|}
-0.6660 \\
\end{tabular} & \begin{tabular}{|l}
-0.7992 \\
\end{tabular} & \begin{tabular}{|l}
-0.9324 \\
\end{tabular} & -1.0656 & -1.1988 & \begin{tabular}{|l}
-1.3320 \\
\end{tabular} & \begin{tabular}{|l|}
-1.4652 \\
\end{tabular} & \begin{tabular}{|l}
-1.5984 \\
\end{tabular} & \begin{tabular}{|l}
-1.7316 \\
\end{tabular} & \begin{tabular}{|l|}
-1.8647 \\
\end{tabular} & \begin{tabular}{|l|l}
-1.9979 \\
\end{tabular} \\
\hline 0.27 & \begin{tabular}{|l}
-0.1383 \\
\end{tabular} & \begin{tabular}{|l|}
-0.2766 \\
\end{tabular} & -0.4150 & -0.5533 & \begin{tabular}{|l|}
-0.6916 \\
\end{tabular} & \begin{tabular}{|l|}
-0.8299 \\
\end{tabular} & \begin{tabular}{|l|}
-0.9682 \\
\end{tabular} & \begin{tabular}{|l|l|}
-1.1066 \\
\end{tabular} & \begin{tabular}{|l|}
-1.2449 \\
\end{tabular} & \begin{tabular}{|l|}
-1.3832 \\
\end{tabular} & -1.5215 & -1.6598 & \begin{tabular}{|l|}
-1.7982 \\
\end{tabular} & -1.9365 & \begin{tabular}{|l|}
-2.0748 \\
\end{tabular} \\
\hline 0.28 & & -0.2869 & -0.43 & -0.5738 & -0.7172 & -0.8 & & & & & \begin{tabular}{|l}
-1.5779 \\
\end{tabular} & \begin{tabular}{|l}
-1.7213 \\
\end{tabular} & \begin{tabular}{|l|}
-1.8647 \\
\end{tabular} & \begin{tabular}{|l}
-2.0082 \\
\end{tabular} & 2.1516 \\
\hline 020 & & \begin{tabular}{|l|}
-0.2971 \\
\end{tabular} & \begin{tabular}{|l|}
-0.4457 \\
\end{tabular} & -0.5943 & \begin{tabular}{|l|}
-0.7428 \\
\end{tabular} & & & & & & \begin{tabular}{|l|}
-1.6342 \\
\end{tabular} & \begin{tabular}{|l|}
-1.7828 \\
\end{tabular} & \begin{tabular}{|l|}
-1.9313 \\
\end{tabular} & \begin{tabular}{|l|}
-2.0799 \\
\end{tabular} & -2.2285 \\
\hline 0.30 & \begin{tabular}{|l|}
-0.1537 \\
\end{tabular} & \begin{tabular}{|c|}
-0.3074 \\
\end{tabular} & \begin{tabular}{|c|}
-0.4611 \\
\end{tabular} & -0.6148 & -0.7684 & -0.9221 & -1.0758 & -1.2295 & \begin{tabular}{|l|}
-1.3832 \\
\end{tabular} & \begin{tabular}{|l|}
-1.5369 \\
\end{tabular} & -1.6906 & -1.8443 & \begin{tabular}{|l|}
-1.9979 \\
\end{tabular} & -2.1516 & -2.3053 \\
\hline 0.31 & \begin{tabular}{|c|c|}
-0.158 \\
\end{tabular} & \begin{tabular}{|l}
-0.3176 \\
\end{tabular} & \begin{tabular}{|l|l}
-0.4764 \\
\end{tabular} & -0.6352 & 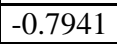 & \begin{tabular}{|l}
-0.9529 \\
\end{tabular} & -1.1117 & $\begin{array}{l}-1.2705 \\
\end{array}$ & \begin{tabular}{|l}
-1.4293 \\
\end{tabular} & \begin{tabular}{|l|}
-1.5881 \\
\end{tabular} & \begin{tabular}{|l}
-1.7469 \\
\end{tabular} & \begin{tabular}{|l}
-1.9057 \\
\end{tabular} & \begin{tabular}{|l|}
-2.0645 \\
\end{tabular} & -2.2234 & -2.3822 \\
\hline 0.32 & & \begin{tabular}{|l}
-0.3279 \\
\end{tabular} & \begin{tabular}{|l|}
-0.4918 \\
\end{tabular} & -0.65 & \begin{tabular}{|l|}
-0.8197 \\
\end{tabular} & \begin{tabular}{|l}
-0.9836 \\
\end{tabular} & \begin{tabular}{|l|}
-1.1475 \\
\end{tabular} & & \begin{tabular}{|l}
-1.4754 \\
\end{tabular} & -1.6 & -1.8033 & -1.9672 & \begin{tabular}{|l|}
-2.1311 \\
\end{tabular} & 2.2951 & \\
\hline 0.33 & \begin{tabular}{|l|l|}
-0.1691 \\
\end{tabular} & \begin{tabular}{|l}
-0.3381 \\
\end{tabular} & \begin{tabular}{|l|}
-0.5072 \\
\end{tabular} & -0.6762 & \begin{tabular}{|l}
-0.8453 \\
\end{tabular} & -1.0143 & -1.1834 & -1.3525 & 1.5215 & -1.6906 & 1.8596 & 2.0287 & .1991 & 2.3668 & 2.535 \\
\hline
\end{tabular}

